\title{
Twenty-two new species in the genus
}

\section{Hyphantrophaga Townsend (Diptera: Tachinidae)}

\section{from Area de Conservación Guanacaste, with a key to the species of Mesoamerica}

\author{
AJ Fleming ${ }^{\ddagger}$, D. Monty Wood ${ }^{\ddagger}$, M. Alex Smith`, Tanya Dapkeyl, Winnie Hallwachs', Daniel Janzen' \\ $\ddagger$ Agriculture Agri-Food Canada, Ottawa, Canada \\ $\S$ University of Guelph, Guelph, Canada \\ | Department of Biology, University of Pennsylvania, Philadelphia, United States of America
}

Corresponding author: AJ Fleming (ajfleming604@gmail.com)

Academic editor: Daniel Whitmore

Received: 05 Sep 2018 | Accepted: 18 Jun 2019 | Published: 28 Jun 2019

Citation: Fleming A, Wood D, Smith M, Dapkey T, Hallwachs W, Janzen D (2019) Twenty-two new species in the genus Hyphantrophaga Townsend (Diptera: Tachinidae) from Area de Conservación Guanacaste, with a key to the species of Mesoamerica. Biodiversity Data Journal 7: e29553. https://doi.org/10.3897/BDJ.7.e29553

ZooBank: urn:Isid:zoobank.org:pub:A13FD7BA-D8FA-4F9D-9B80-C918AFF62851

\section{Abstract}

\section{Background}

We describe 22 new species in the genus Hyphantrophaga Townsend, 1892 (Diptera: Tachinidae) from Area de Conservación Guanacaste (ACG) in north-western Costa Rica. All species were reared from an ongoing inventory of wild-caught caterpillars spanning a variety of families (Lepidoptera: Bombycidae, Crambidae, Depressariidae, Doidae, Erebidae, Euteliidae, Gelechiidae, Geometridae, Hedylidae, Hesperiidae, Immidae, Lasiocampidae, Limacodidae, Megalopygidae, Mimaloniidae, Noctuidae, Nolidae, Notodontidae, Nymphalidae, Papilionidae, Pieridae, Phiditiidae, Pterophoridae, Pyralidae, Riodinidae, Saturniidae, Sphingidae, Thyrididae, Tortricidae and Zygaenidae). We provide a morphological description of each species together with information on life history, molecular data and photographic documentation. In addition to the new species, we 
provide a redescription of the genus, as well as the redescription of three previously described species, which were also collected within ACG during this study: Hyphantrophaga angustata (van der Wulp), Hyphantrophaga myersi (Aldrich) and Hyphantrophaga virilis (Aldrich \& Webber).

\section{New information}

The following 22 new species of Hyphantrophaga are described: Hyphantrophaga adrianguadamuzi Fleming \& Wood sp. n., Hyphantrophaga albopilosa Fleming \& Wood sp. n., Hyphantrophaga anacordobae Fleming \& Wood sp. n., Hyphantrophaga calixtomoragai Fleming \& Wood sp. n., Hyphantrophaga calva Fleming \& Wood sp. n.., Hyphantrophaga ciriloumanai Fleming \& Wood sp. n., Hyphantrophaga danausophaga Fleming \& Wood sp. n., Hyphantrophaga diniamartinezae Fleming \& Wood sp. n., Hyphantrophaga duniagarciae Fleming \& Wood sp. n., Hyphantrophaga edwinapui Fleming \& Wood sp. n., Hyphantrophaga eldaarayae Fleming \& Wood sp. n., Hyphantrophaga eliethcantillanoe Fleming \& Wood sp. n., Hyphantrophaga gilberthampiei Fleming \& Wood sp. n., Hyphantrophaga guillermopereirai Fleming \& Wood sp. n., Hyphantrophaga hazelcambroneroae Fleming \& Wood sp. n., Hyphantrophaga luciariosae Fleming \& Wood sp. n., Hyphantrophaga manuelriosi Fleming \& Wood sp. n., Hyphantrophaga morphophaga Fleming \& Wood sp. n., Hyphantrophaga nigricauda Fleming \& Wood sp. n., Hyphantrophaga osvaldoespinozai Fleming \& Wood sp. n., Hyphantrophaga pabloumanai Fleming \& Wood sp. n. and Hyphantrophaga similis Fleming \& Wood sp. $\mathbf{n}$.

The following are proposed by Wood as new synonyms of Hyphantrophaga Townsend, 1892: Brachymasicera Townsend, 1911 syn. n., Ommasicera Townsend, 1911 syn. n., Ophirosturmia Townsend, 1911 syn. n., Patillalia Curran, 1934 syn. n. and Ypophaemyiops Townsend, 1935 syn. $\mathbf{n}$.

The following nine new combinations are proposed as a result of the new synonymies: Hyphantrophaga adamsoni (Thompson, 1963), comb. n., Hyphantrophaga fasciata (Curran, 1934), comb. n., Hyphantrophaga glauca (Giglio-Tos, 1893), comb. n., Hyphantrophaga gowdeyi (Curran, 1926), comb. n., Hyphantrophaga myersi (Aldrich, 1933), comb. n., Hyphantrophaga nigripes (Townsend, 1928), comb. n., Hyphantrophaga optica (Schiner, 1868), comb. n., Hyphantrophaga polita (Townsend, 1911), comb. n., Hyphantrophaga subpolita (Townsend, 1912), comb. n.

\section{Keywords}

caterpillar, tropical, Goniini, parasitoid, fly, rain forest, dry forest, cloud forest, ACG 


\section{Introduction}

The New World genus Hyphantrophaga (Exoristinae: Goniini) was erected by Townsend (1892). He initially described the type species, Meigenia hyphantriae Townsend, 1891, reluctantly placing it in the genus Meigenia Robineau-Desvoidy, 1830; one year later (Townsend 1892), after further analysis and clarification, he changed his mind and moved $M$. hyphantriae to the new genus Hyphantrophaga. The original description of $M$. hyphantriae was based on four specimens (three females, one male) reared from Hyphantria cunea (Drury, 1773) (Lepidoptera: Erebidae) collected during his time at Las Cruces, New Mexico. When Townsend (1892) described the new genus, he realised that what he had initially thought was a male was, in fact, a female. As a result, the new genus Hyphantrophaga was based on four female syntypes of which only two remain, deposited in SEMK and USNM, respectively. In the same work, he also added the description of two males collected later, in 1891.

In an afterword to the description, Townsend suggested that $M$. hyphantriae could be differentiated from all other species of Meigenia by the lack of discal setae present on all abdominal segments with the exception of T5 and the morphology of the face (two character states useful in differentiating species, but that we now see as variable within the genus). As in Houghia Coquillett, 1897, the accurate identification of Hyphantrophaga is based on a characteristic "gestalt" that comprises the combination of features within the genus and the fact that the females lay microtype eggs.

Since its original description, the genus has seen many changes. Sellers (1943) made Hyphantrophaga a junior synonym of Zenillia Robineau-Desvoidy, 1830. Twenty-two years later, Sabrosky and Arnaud (1965) treated the genus as valid (without officially resurrecting it), raising the number of valid species to three. It was not until much later that O'Hara and Wood (1998) officially resurrected the genus, acknowledging some of the changes to the generic limits suggested by Sellers (1943). They also synonimised Eusisyropa Townsend, 1908 and increased the number of valid species of Hyphantrophaga from three to 15, with 10 of these belonging to the Neotropical fauna.

Hyphantrophaga belongs to the tribe Goniini; females of all members of this tribe lay "microtype" eggs directly on the foliage, often in direct proximity to or around the host caterpillar. As the host feeds, the tachinid egg is consumed along with the leaf fragments. Upon hatching, the first instar larva traverses the gut wall and finds its way to a "safe" space within the caterpillar's body, where it remains until it eventually kills its host and continues feeding on the carcass until it pupates. Only very rarely does the larva not kill the caterpillar (e.g. DeVries 1984 recorded a reared adult butterfly from a caterpillar that had already produced viable tachinid larvae).

All of the new species of Hyphantrophaga reared from Area de Conservaci ón Guanacaste (ACG) described in this paper are based on differences in external morphology, male terminalia, CO1 (cox1 or cytochrome oxidase 1) gene sequences and on comparison by AJF and DMW with other named species of Hyphantrophaga from other regions. It is 
important to note, however, that these new species are not to be taken as an indication of the total number of species of Hyphantrophaga, even in such a small country as Costa Rica. Comparisons of tachinids collected during the ACG inventory with those present in the national collection in the Museo Nacional de Costa Rica (formerly INBIO) show minimal overlap in species, suggesting that the tachinid fauna in other parts of the country is quite different from that of ACG and requires much additional study. Our study provides the descriptions of 22 new species of Hyphantrophaga; we also synonymise five genera under Hyphantrophaga, leading to nine new combinations of species names under that genus and thereby increasing the total number of species in the genus from 15 to 46 . There may also be a small number of apparent species of Hyphantrophaga that have been reared by the ACG inventory and which at present can only be distinguished by their gene sequences (henceforth referred to as DNA barcodes) and host records or of which there is insufficient material to make an accurate diagnosis. We have elected to leave such species for potential later description once additional material is available.

The present study is part of a larger group of studies documenting the tachinid species living within ACG (http://www.acguanacaste.ac.cr) and providing names for new species as they are discovered (Fleming et al. 2014b, Fleming et al. 2014a, Fleming et al. 2015a, Fleming et al. 2015c, Fleming et al. 2015b, Fleming et al. 2015d, Fleming et al. 2016a, Fleming et al. 2016b, Fleming et al. 2017). This series of taxonomic papers will represent a baseline for further, detailed ecological and behavioural accounts and studies extending across ACG ecological groups, whole ecosystems and taxonomic assemblages much larger than a genus.

\section{Materials and methods}

\section{Project aims and rearing intensity}

All flies and rearing information described here were collected by the ongoing ACG inventory of the caterpillars, their food plants and their parasitoids, throughout the major ACG terrestrial ecosystems (Smith et al. 2006, Smith et al. 2007, Smith et al. 2008, Janzen et al. 2009, Janzen and Hallwachs 2011, Smith et al. 2009, Smith et al. 2012, Rodriguez et al. 2012, Fleming et al. 2014a, Janzen et al. 2016). The parasitoid rearing methods are described at http://janzen.bio.upenn.edu/caterpillars/methodology/how/parasitoid husban dry.htm. This inventory has reared more than 750,000+ wild-caught caterpillars since its inception (Janzen et al. 2009, Janzen and Hallwachs 2011, Fernandez-Triana et al. 2014). This effort is continuing to provide an unprecedented amount of data, providing an invaluable tri-trophic image on parasitoid biology including parasitoids, hosts and host plants. All frequencies of parasitisation reported here need to be considered against this background inventory.

The scope of our treatment of the genus Hyphantrophaga is limited to those species found in the Mesoamerican region, from the Isthmus of Tehuantepec in southern Mexico to the Darién gap along the southern border of Panama with Colombia. While we took into 
account all known species in our comparisons and diagnoses of the new species, only the species distributed within this region are included in the key.

\section{Imaging and dissections}

The species accounts and descriptions presented in this paper are complemented by a series of colour photos, used to illustrate the morphological differences and similarities amongst the species. The morphological terminology used follows Cumming and Wood (2009). The characters in our descriptions are presented in order of appearance on the body from the front to the rear and arranged under the headings Head, Thorax, Abdomen and Male terminalia. All dissections and photography were carried out following the methods detailed by Fleming et al. (2014a). Measurements and examples of anatomical landmarks discussed herein are illustrated in Fig. 1. Whenever possible, males were selected preferentially as the holotype, since they bear the most differences in external morphology and are thus better for distinguishing the species. Note that, in cases where only one male was available, this was designated as the holotype and was not subjected to dissection.

\section{Voucher specimen management}

The management of voucher specimens has been detailed in previous papers in this series (Fleming et al. 2014a). In brief, caterpillars reared from the ACG inventory receive a unique voucher code in the format yy-SRNP-xxxxx. Parasitoids emerging from a caterpillar receive the same voucher code; when/if they are later individually processed for DNA barcoding, each receives a second, unique voucher code in the format DHJPARxxxxxxx. The associated data for each voucher code are available at: http://janzen.bio.upenn.edu/ caterpillars/database.lasso. All associated data and successful barcodes are permanently and publicly deposited in the Barcode of Life Data System (BOLD) (Ratnasingham and Hebert 2007), with a select set of these data also subsequently migrated to GenBank. Each barcoded specimen also receives accession numbers from the Barcode of Life Data System (BOLD) and GenBank, respectively. The dynamic nature of the inventory means that it is continually adding new specimens, which can be found by searching for the genus Hyphantrophaga in BOLD.

All inventoried specimens, discussed herein, were collected under Costa Rican government research permits issued to $\mathrm{DHJ}$ and the Tachinidae samples were exported under permit by $\mathrm{DHJ}$ from Costa Rica to their final depository in the CNC. Tachinid identifications for the inventory are conducted by $\mathrm{DHJ}$ in coordination with a) visual inspection of morphology by AJF and DMW, b) DNA barcoding by MAS and BIO and c) databasing and association with host caterpillars by $\mathrm{DHJ}$ and $\mathrm{WH}$, through the inventory itself. 


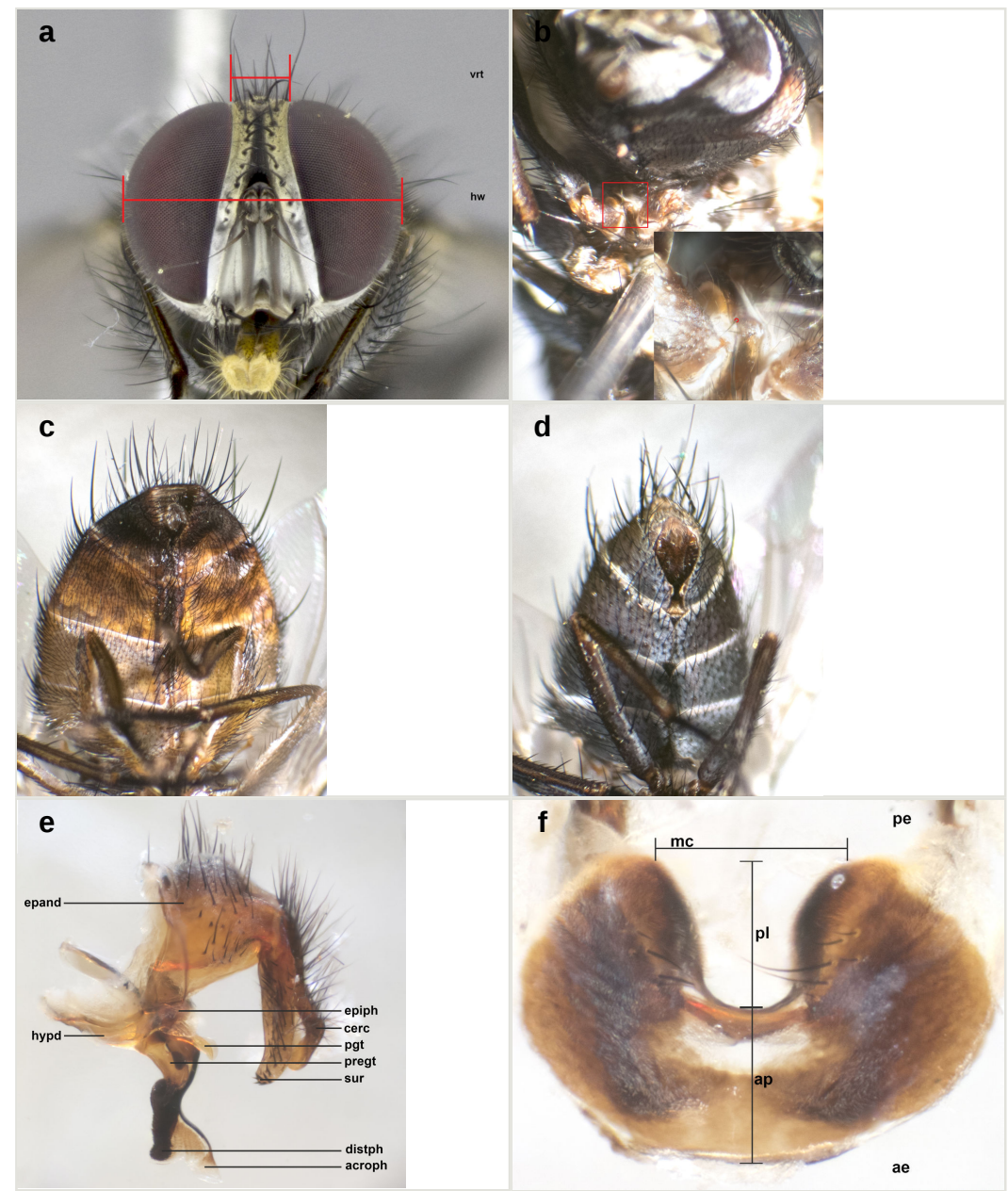

Figure 1.

Examples of measurements and landmark anatomical features.

a: Sample of measured areas from front of head as shown on Hyphantrophaga virilis (Aldrich \& Webber, 1924); vrt, vertex; hw, head width doi

b: Detailed view of hind coxa showing setose hind margin in Hyphantrophaga virilis doi

c: Ventral view of abdomen in Hyphantrophaga albopilosa sp. $\mathbf{n}$. showing presence of sex patches on T4 and T5 doi

d: Ventral view of abdomen in Hyphantrophaga guillermopereirai sp. $\mathbf{n}$. showing absence of sex patches doi

e: Detailed lateral view of male genitalia of Hyphantrophaga nigricauda sp. $\mathbf{n}$. Abbreviations: acroph $=$ acrophallus; cerc = cercus; distph $=$ distiphallus; epand $=$ epandrium; epiph = epiphallus; hypd = hypandrium; pgt = postgonite; pregt = pregonite; sur = surstylus doi

f: Detailed ventral view of sternite 5 of Hyphantrophaga myersi (Aldrich, 1933). Abbreviations: ae $=$ anterior edge; $a p=$ anterior plate $; \mathrm{mc}=$ median cleft; $\mathrm{pe}=$ posterior edge; $\mathrm{pl}=$ posterior lobes doi 
The date of capture cited for each specimen is the date of eclosion of the fly and not the date of capture of the caterpillar. Eclosion date is much more representative of the time when that fly species is on the wing than is the time of capture of the parasitised caterpillar. The "collector" is the parataxonomist who found the caterpillar, rather than the person who later retrieved the newly eclosed fly and processed it by freezing, pinning, labelling and oven-drying. The type-series of the newly-described species are housed in the Diptera collection of the Canadian National Collection (CNC).

\section{Acronyms for Depositories}

AMNH American Museum of Natural History, New York, New York, USA

CAS California Academy of Sciences, San Francisco, California, USA

CNC Canadian National Collection of Insects, Arachnids and Nematodes, Ottawa, Canada

MCZ Museum of Comparative Zoology, Harvard University, Cambridge, Massachusetts, USA

MACN Museo Argentino de Ciencias Naturales Bernardino Rivadavia, Buenos Aires, Argentina

MNHN Muséum National d'Histoire Naturelle, Paris, France

NHMUK Natural History Museum, London, United Kingdom (formerly British Museum (Natural History))

NHMW Naturhistorisches Museum Wien, Vienna, Austria

SEMK Snow Entomological Museum, University of Kansas, Lawrence, Kansas, USA

USNM National Museum of Natural History, Washington, D.C., U.S.A. (formerly United States National Museum)

\section{Interim names for undescribed host species}

As in the other papers in this series, our convention for naming undescribed host species follows a standardised, interim naming system used for taxonomic units considered as distinct species and identified by DNA barcodes. Interim names are given in the format " Eois Janzen52" or "Caviria reginaDHJ01", where the "species epithet" is either composed of the name of the taxonomist who identified the species and a number or the name of a species-group followed by a code. This prevents confusion with already described species while maintaining traceability of each undescribed species within the ACG project. 


\section{DNA barcoding}

We generated DNA extracts from single legs using a standard glass fibre protocol (Ivanova et al. 2006), using the standard DNA barcode region (5' cytochrome c oxidase I (COI) gene) for all specimens of ACG Hyphantrophaga. The DNA barcodes (658 bp near the 5' terminus of the $\mathrm{COI}$ gene) were amplified using general insect primers, using standard protocols for both production and quality control (Smith et al. 2006, Smith et al. 2007, Smith et al. 2008, Smith et al. 2009, Smith et al. 2012). All DNA sequences, trace files and accessions were deposited in the Barcode of Life Data System (BOLD) (Ratnasingham and Hebert 2007). Metadata (including GenBank accession codes) associated with each sequence can be consulted on BOLD, by using the persistent DOI dx.doi.org/10.5883/DSASACGHYP.

In some cases of ecological specialisation displaying only slight (or in one case, no) barcode divergences, we amplified the internal transcribed spacer regions (ITS1 \& ITS2) of the ribosomal RNA. Primers for ITS1 occur in conserved protein coding areas (18S and 5.8S). PCR reactions were carried out in $12.5 \mathrm{ml}$ reaction volumes containing: $2.5 \mathrm{mM}$ $\mathrm{MgCl}_{2}, 25 \mathrm{pmol}$ of each primer, $50 \mathrm{mM}$ dNTPs, $10 \mathrm{mM}$ Tris. $\mathrm{HCl}(\mathrm{pH} 8.3), 50 \mathrm{mM} \mathrm{KCl}$, 10-20 ng (1-2 ml) of genomic DNA and 1 unit of Platinum TaqDNA polymerase, using a thermocycling profile of 1 cycle of 2 min at $94^{\circ} \mathrm{C}, 40$ cycles of $40 \mathrm{sec}$ at $94^{\circ} \mathrm{C}, 40 \mathrm{sec}$ at $67^{\circ}$ $\mathrm{C}$ and $2 \mathrm{~min}$ at $72^{\circ} \mathrm{C}$, with a final step of $5 \mathrm{~min}$ at $72^{\circ} \mathrm{C}$. We directly sequenced the amplifications using the primers CAS18Fs1 and CAS5p8sB1d (Ji et al. 2003). Amplicons were evaluated using Sequencher version 5.0 (Gene Codes), examined by eye using Bioedit (Hall 1999) and aligned using Muscle (Edgar 2004).

\section{Hyphantrophaga morphophaga species group}

Our analysis of the genus Hyphantrophaga required us to create a special group to separate a pair of cryptic species, including two of the new species described herein, Hyphantrophaga morphophaga sp. n. and Hyphantrophaga danausophaga sp. $\mathbf{n}$. As of the writing of this paper, species in this group are distinguishable only by life history and behaviour. The implications and diagnosis of this special group, termed here the " Hyphantrophaga morphophaga species group", will be discussed in the analysis section below.

The species, belonging to this group, share morphological and molecular similarities but differ considerably in their host preference, life history and habitat. Both species share the following combination of character states: pedicel brown to black, concolorous with first flagellomere; thorax hirsute, covered in black setulae throughout; three katepisternal setae; median marginal setae present on all tergites including ST1+2; discal setae absent on all but T5; males with distinctive sex patch; and hind coxa either setose or bare. 


\section{Taxon treatments}

\section{Hyphantrophaga Townsend, 1892}

\section{Nomenclature}

Hyphantrophaga Townsend, 1892: 247. Type species: Meigenia hyphantriae Townsend, 1891, by original designation.

Eusisyropa Townsend, 1908: 97. Type species: Tachina blanda Osten Sacken, 1887, by monotypy. Synonymy by O'Hara and Wood 1998

Brachymasicera Townsend, 1911: 133. Type species: Brachymasicera polita Townsend, 1911, by original designation. Syn. $\mathbf{n}$.

Ommasicera Townsend, 1911: 145. Type species: Ommasicera chaetosa Townsend, 1911, by monotypy. Syn. $\mathbf{n}$. [Original description of genus based on female reproductive system, full description of the adult was not provided until Townsend 1912: 337.]

Oomasicera. Incorrect subsequent spelling of Ommasicera Townsend, 1911 (Guimaraes 1971: 204).

Ophirosturmia Townsend, 1911: 133. Type species: Ophirosturmia cincta Townsend, 1911, by original designation. Syn. $\mathbf{n}$.

Patillalia Curran, 1934: 459. Type species: Patillalia fasciata Curran, 1934, by original designation. Syn. $\mathbf{n}$.

Ypophaemyiops Townsend, 1935: 233. Type species: Prophryno myersi Aldrich, 1933, by original designation. Syn. $\mathbf{n}$.

\section{Other species included in Hyphantrophaga Townsend, 1892}

adamsoni Thompson, 1963: 293 (Zenillia). Holotype female (CNC). Type locality: Trinidad, St. Augustine. Comb. $\mathbf{n}$.

angustata van der Wulp, 1890: 70 (Exorista). Holotype male (NHMUK). Type locality: Mexico, Guerrero, Chilpancingo, $4600 \mathrm{ft}$.

coquilletti Aldrich \& Webber, 1924: 18 (Zenillia). Holotype male (USNM). Type locality: USA, Texas, Belfrage.

auratofrontalis Brèthes, 1908: 475 (Exorista). Syntypes male and female (MACN). Type locality: Argentina, Buenos Aires.

autographae Sellers, 1943: 23 (Zenillia). Holotype male (USNM). Type locality: Cuba, Baraguá. 
blanda Osten Sacken, 1887: 162 (Tachina (Exorista)). Holotype female (MCZ). Type locality: unknown (Massachusetts according to Townsend 1941:266.

boarmiae Coquillett, 1897: 95 (Exorista). Lectotype female (USNM), by designation of Aldrich and Webber 1924: 39. Type locality: USA, Massachusetts, Cotuit.

hypenae Coquillett in Howard 1897: 47 (Exorista). Nomen nudum

proserpina Williston, 1889: 1919 (Exorista, as subspecies of blanda). Holotype male (depository unknown). Type locality: unknown.

blandita Coquillett, 1897: 96 (Exorista). Holotype female (USNM). Type locality: USA, New Hampshire, Franconia.

blandoides Thompson, 1963: 297 (Eusisyropa). Holotype female (CNC). Type locality: Trinidad, Sta. Cruz Valley.

brasiliensis Moreira, 1915: 227 (Masicera). Type status unclear, depository unknown. Type locality: Brazil, Rio de Janeiro. Nomen dubium

chaetosa Townsend, 1911: 145 [description based on female reproductive system; full description of adult in Townsend 1912: 337] (Ommasicera). Holotype female (USNM). Type locality: Peru, Piura, Valle del Río Chira, Sullana. Comb. n.

collina Reinhard, 1944: 68 (Zenillia). Holotype male (SEMK). Type locality: USA, Arizona, Chiricahua Mountains.

euchaetiae Sellers, 1943: 13 (Zenillia). Holotype male (USNM). Type locality: USA, New York, Clayton.

fasciata Curran, 1934: 469 (Patillalia). Holotype female (AMNH). Type locality: Panama, Canal Zone, Patilla Point. Comb. $\mathbf{n}$.

glauca Giglio-Tos, 1893: 6 (Masicera). Holotype female (MZUT). Type locality: Mexico.

gowdeyi Curran, 1926: 112 (Zenillia). Holotype female (AMNH). Type locality: Jamaica, St. Andrew Parish, Cinchona Botanical Gardens (as Hill Gardens). Comb. n.

hyphantriae Townsend, 1891: 176 (Meigenia). Lectotype male (USNM), by present designation of Wood. Type locality: USA, New Mexico, Las Cruces.

ceratomiae Coquillett, 1897: 101 (Exorista). Holotype male (USNM). Type locality: USA, Texas, Fort Worth.

desmiae Sellers, 1943: 16 (Zenillia). Holotype male (USNM). Type locality: USA, California, Exeter. 
myersi Aldrich, 1933: 173 (Prophryno). Holotype male (NHMUK). Type locality: Guyana (as British Guiana), Pakeraima Mts., Upper Ireng River. Comb. n.

nigripes Townsend, 1928: 159 (Brachymasicera). Holotype female (USNM). Type locality: Peru, Chiclayo, Pomalca. Comb. n.

niveifacies Macquart, 1851a: 162 [also Macquart, 1851b: 189] (Exorista). 2 syntypes: 1 male, 1 female (MNHN). Type locality: Brazil, Bahia, Salvador (as "Bahia").

optica Schiner, 1868: 327 (Exorista). Holotype female (NHMW). Type locality: Brazil. Comb. $n$.

polita Townsend, 1911: 143 (Brachymasicera). Holotype female (USNM). Type locality: Peru, Piura. Comb. $\mathbf{n}$.

scolex Reinhard, 1953: 56 (Zenillia) Holotype female (CAS). Type locality: USA, California, Los Angeles County, Tanbark Flat.

sellersi Sabrosky, 1983: 254 (Eusisyropa). 12 syntypes: 6 males, 6 females (USNM). Type locality: USA, Mississippi, Oxford; [new name for boarmiae of authors, not Coquillett, 1897 (Sabrosky 1983); name made available by Sabrosky (1983) in a bibliographic reference to the diagnosis of boarmiae in the Sellers' key (Sellers 1943: 6-7) to the species of Zenillia].

subpolita Townsend, 1912: 341 (Brachymasicera). Holotype female (USNM). Type locality: Peru, Piura. Comb. n.

tucumanensis Sellers, 1943: 21 (Zenillia). Holotype male (USNM). Type locality: Argentina, Tucuman.

virilis Aldrich \& Webber, 1924: 40 (Zenillia). Holotype male (USNM). Type locality: USA, Illinois, Chicago [as New York, Rye, in error - see Arnaud 1963: 116.

\section{Type species}

Meigenia hyphantriae Townsend, 1891 - Townsend 1891 [176].

\section{Description}

Male. Head (Fig. 1a): vertex 1/4-1/3 of head width; 1-3 reclinate upper orbital setae; ocellar setae proclinate, well-developed, long and arising either beside or behind anterior ocellus; eye haired in all species; parafacial bare; fronto-orbital plate ranging from shiny silver or gold to brownish with a silver sheen and displaying varying degrees of hirsuteness, with setulae not extending below lowest frontal seta; lower margin of face level with vibrissa, thus not visible in profile; facial ridge bare in most species with two notable exceptions, $H$. hazelcambroneroae sp. n. and $H$. myersi, in which the facial ridge is setulose; arista ranging from bare to minutely pubescent, usually distinctly thickened on basal $1 / 4$ or $1 / 5$, ranging in colour from orange to dark brown/black. 
Thorax: ranging from bright gold tomentose to dull grey; 2-4 prominent dorsal vittae, which can be thick and unbroken or thin and only scarcely visible under certain angles of light; prosternum setose; proepisternum with 1-5 main setae surrounded by a brush of shorter, weaker, hairlike setulae; postpronotum with 3-6 setae arranged in a triangle; chaetotaxy: acrostichal setae 3-4:3; dorsocentral setae 3-4:3-4 (only three exceptions displaying three postsutural dorsocentral setae: $H$. calva sp. n., $H$. fasciata, $H$. hazelcambroneroae sp. n.); intra-alar setae 2-4:3; supra-alar setae $2: 3$; 2-3 katepisternal setae; scutellum with four pairs of marginal setae (basal, lateral, subapical and apical); basal scutellar setae often longer than or subequal to lateral scutellar setae; subapical setae typically the strongest of the scutellar marginal setae, ranging from slightly curved and medially convergent to parallel, straight or divergent; ranging in length from equal to or longer than basal scutellar setae; apical setae crossed and short, usually $1 / 4$ length of subapical setae, slightly upturned, at a slight upward angle compared to the plane of the rest of the marginal scutellar setae. Legs: ranging in ground colour from yellow to black; hind coxa bare or setose, with a single seta along dorsal margin (Fig. 1b) (this character is sexually dimorphic within $H$. calva sp. n. and variable in both sexes of $H$. proxima sp. n. and $H$. vicina sp. n.). Wings: pale translucent, not strongly infuscate; vein $R_{4+5}$ setose, with only $2-3$ setulae at base. Abdomen (Fig. 1c, d): ground colour ranging from black to different tonalities of brown to orange; mid-dorsal depression on ST1+2 ranging from reaching halfway across the syntergite to almost reaching the hind margin; median marginal setae present on T3 and T4 and often on ST1+2; median discal setae most often confined to $\mathrm{T} 5$, but this varies amongst species, with some species displaying median discal setae on T3, T4 and T5; the presence of a sex patch is variable amongst species, ranging from absent to present anywhere between T3-T5. Terminalia (Fig. 1e, f): sternite 5 with a deeply excavated median cleft along posterior edge, smoothly U-shaped, with margins covered in dense tomentum; posterior lobes rounded apically, either bare with multiple fine hairlike setulae or with 2-3 strong setae surrounded by many shorter, weaker setulae. Anterior plate of sternite 5 subequal to or longer than posterior lobes; unsclerotised "window" on anterior plate of sternite 5 ranging from absent to almost entirely transparent, directly basal to posterior lobes; the shape of the window, as well as its presence, varies between species. Cerci in posterior view variable between species, ranging from rectangular, digitiform, to triangular; either longer than or only slightly shorter than surstyli; blunt and rounded at apex to apically pointed, either completely separate medially to fused along most of their length; in lateral view often with a strong downward curve at apex, giving it a clubbed appearance; densely setulose along basal $2 / 3$, ventrally setose along entire length. Surstylus in lateral view almost parallel sided along its length, sometimes ending in a slightly downcurved apex, making the structure appear bladelike; when viewed dorsally, the surstyli range from being slightly divergent to slightly convergent or with inward-curved apices, but never strongly convergent. Pregonite usually broad, well-developed, apically squared off or rounded, usually blunt, typically devoid of setulae. Postgonite slightly narrowed, up to $1 / 3$ as wide as pregonite, sharply pointed and curved at apex, typically short and scythelike, with few exceptions in which the postgonite is subequal in length to the pregonite. Epiphallus well-developed and apically hooked. Distiphallus broadly cone- 
shaped (in some species this cone or flare is much more pronounced, in others it is square or barrel-shaped), with a slender median longitudinal sclerotised reinforcement on its posterior surface and a broad, anterolateral sclerotised acrophallus on anterior surface near apex.

Female. As male except in the following traits: head with two pairs of proclinate orbital setae. Abdomen slightly more globose than in male; T5 folded over into a narrow slit, a trait stereotypical of the tribe Goniini. In cases where sexual dimorphism was observed, the differing character states are mentioned in the species descriptions.

\section{Diagnosis}

Hyphantrophaga, as all other Goniini, is difficult to characterise to tribe based on morphological character states and can only be reliably ascribed to this tribe (sensu Herting 1984) based on its microtype ovipary. However, Hyphantrophaga does possess a combination of traits that can be considered stereotypical of the group: prosternum setose; males of all species with two pairs of well-developed reclinate upper orbital setae, proclinate orbital setae only present in females (a character state that distinguishes them from males of Houghia Coquillet and Carcelia Robineau-Desvoidy, in which orbital setae are absent or proclinate); the ocellar setae are always proclinate and in most species arise beside the anterior ocellus; the parafacial, katepimeron and the upper half or more of the facial ridge, are bare; 3-4 well-developed and evenlyspaced postsutural supra-alar setae, the anteriormost being stouter than the first postsutural dorsocentral seta; median discal setae present only on abdominal T5 (in most species); the three major setae of the postpronotum are arranged in a triangle; wings lacking costal spine; hind coxa can be setose or bare (in some cases this character state can be sexually variable within species). The height of the gena is about $1 / 5$ to $1 / 10$ the height of the head, which approaches the condition found in some Houghia and differentiates Hyphantrophaga from the members of Carcelia. The eyes of all species of Hyphantrophaga are haired; however, in a few species, the ommatrichia can be short and sparse. The 22 species of Hyphantrophaga described herein can be identified to genus using the keys in both Wood 1987 and Wood and Zumbado 2010.

\section{Distribution}

Ubiquitous throughout the New World, inhabiting a wide variety of ecosystems from south-eastern Canada and the north-eastern USA, west to California and south to Argentina and Brazil.

\section{Ecology}

Within the ACG inventory, Hyphantrophaga has been reared from a wide variety of Lepidoptera hosts throughout the diverse ecosystems of the research area, including: Bombycidae, Crambidae, Depressariidae, Doidae, Erebidae, Euteliidae, Gelechiidae, Geometridae, Hedylidae, Hesperiidae, Immidae, Lasiocampidae, Limacodidae, 
Megalopygidae, Mimaloniidae, Noctuidae, Nolidae, Notodontidae, Nymphalidae, Papilionidae, Pieridae, Pterophoridae, Pyralidae, Riodinidae, Saturniidae, Sphingidae, Thyrididae, Tortricidae and Zygaenidae.

\section{Taxon discussion}

Hyphantrophaga brasiliensis (Moreira, 1915) is treated as a nomen dubium within Hyphantrophaga, as it has proven impossible to ascertain the repository or even the existence of the type material.

\section{Hyphantrophaga adrianguadamuzi Fleming \& Wood, sp. n.}

- ZooBank urn:Isid:zoobank.org:act:AC7B0563-B5FD-40F4-924B-D187838172B4

\section{Materials}

Holotype:

a. $\quad$ scientificName: Hyphantrophaga adrianguadamuzi; phylum: Arthropoda; class: Insecta; order: Diptera; family: Tachinidae; genus: Hyphantrophaga; specificEpithet: adrianguadamuzi; scientificNameAuthorship: Fleming \& Wood, 2018; continent: Central America; country: Costa Rica; countryCode: CR; stateProvince: Guanacaste; county: Sector Santa Rosa; locality: Area de Conservacion Guanacaste; verbatimLocality: Area Administrativa; verbatimElevation: 295; verbatimLatitude: 10.8376; verbatimLongitude: -85.6187; verbatimCoordinateSystem: Decimal; decimalLatitude: 10.8376; decimalLongitude: -85.6187; samplingProtocol: Reared from the larva of the Pyralidae, Paridnea holophaealisDHJ02; verbatimEventDate: 07Sep-2004; individualID: DHJPAR0007297; individualCount: 1; sex: male; lifeStage: adult; preparations: pinned; catalogNumber: DHJPAR0007297; occurrenceDetails: htt p://janzen.sas.upenn.edu; recordedBy: D.H. Janzen, W. Hallwachs, \& Ruth Franco; otherCatalog Numbers: ASTAT069-06,04-SRNP-13432, BOLD:ABZ0529; identifiedBy: AJ Fleming; dateldentified: 2017; language: en; institutionCode: CNC; collectionCode: Insects; basisOfRecord: Pinned Specimen

\section{Paratypes:}

a. $\quad$ scientificName: Hyphantrophaga adrianguadamuzi; phylum: Arthropoda; class: Insecta; order: Diptera; family: Tachinidae; genus: Hyphantrophaga; specificEpithet: adrianguadamuzi; scientificNameAuthorship: Fleming \& Wood, 2018; continent: Central America; country: Costa Rica; countryCode: CR; stateProvince: Guanacaste; county: Sector Santa Rosa; locality: Area de Conservacion Guanacaste; verbatimLocality: Camino Borrachos; verbatimElevation: 295; verbatimLatitude: 10.8429; verbatimLongitude: -85.6161; verbatimCoordinateSystem: Decimal; decimalLatitude: 10.8429; decimalLongitude: -85.6161; samplingProtocol: Reared from the larva of the Pyralidae, Paridnea holophaealis; verbatimEventDate: 06-Aug-2009; individualID: DHJPAR0037527; individualCount: 1; sex: male; lifeStage: adult; preparations: pinned; catalogNumber: DHJPAR0037527; occurrenceDetails: http:// janzen.sas.upenn.edu; recordedBy: D.H. Janzen, W. Hallwachs, \& Guillermo Pereira; otherCatalogNumbers: ASHYC4272-10,09-SRNP-14043, BOLD:ABZ0529; identifiedBy: AJ Fleming; dateldentified: 2017; language: en; institutionCode: CNC; collectionCode: Insects; basisOfRecord: Pinned Specimen 
b. scientificName: Hyphantrophaga adrianguadamuzi; phylum: Arthropoda; class: Insecta; order: Diptera; family: Tachinidae; genus: Hyphantrophaga; specificEpithet: adrianguadamuzi; scientificNameAuthorship: Fleming \& Wood, 2018; continent: Central America; country: Costa Rica; countryCode: CR; stateProvince: Guanacaste; county: Sector Mundo Nuevo; locality: Area de Conservacion Guanacaste; verbatimLocality: Camino Pozo Tres; verbatimElevation: 733; verbatimLatitude: 10.7708; verbatimLongitude: -85.3742; verbatimCoordinateSystem: Decimal; decimalLatitude: 10.7708; decimalLongitude: -85.3742; samplingProtocol: Reared from the larva of the Pyralidae, Paridnea holophaealis; verbatimEventDate: 16-Aug-2009; individualID: DHJPAR0035662; individualCount: 1; sex: male; lifeStage: adult; preparations: pinned; catalogNumber: DHJPAR0035662; occurrenceDetails: http:// janzen.sas.upenn.edu; recordedBy: D.H. Janzen, W. Hallwachs, \& Jose Cortez; otherCatalogNumbers: ASHYD1043-09,09-SRNP-56575, BOLD:ABZ0529; identifiedBy: AJ Fleming; dateldentified: 2017; language: en; institutionCode: CNC; collectionCode: Insects; basisOfRecord: Pinned Specimen

c. ScientificName: Hyphantrophaga adrianguadamuzi; phylum: Arthropoda; class: Insecta; order: Diptera; family: Tachinidae; genus: Hyphantrophaga; specificEpithet: adrianguadamuzi; scientificNameAuthorship: Fleming \& Wood, 2018; continent: Central America; country: Costa Rica; countryCode: CR; stateProvince: Guanacaste; county: Sector Santa Rosa; locality: Area de Conservacion Guanacaste; verbatimLocality: Area Administrativa; verbatimElevation: 295; verbatimLatitude: 10.8376; verbatimLongitude: -85.6187; verbatimCoordinateSystem: Decimal; decimalLatitude: 10.8376; decimalLongitude: -85.6187; samplingProtocol: Reared from the larva of the Pyralidae, Paridnea holophaealis; verbatimEventDate: 29-Aug-2009; individualID: DHJPAR0035660; individualCount: 1; sex: female; lifeStage: adult; preparations: pinned; catalogNumber: DHJPAR0035660; occurrenceDetails: http:// janzen.sas.upenn.edu; recordedBy: D.H. Janzen, W. Hallwachs, \& Johan Vargas; otherCatalogNumbers: ASHYD1041-09,09-SRNP-14511, BOLD:ABZ0529; identifiedBy: AJ Fleming; dateldentified: 2017; language: en; institutionCode: CNC; collectionCode: Insects; basisOfRecord: Pinned Specimen

d. ScientificName: Hyphantrophaga adrianguadamuzi; phylum: Arthropoda; class: Insecta; order: Diptera; family: Tachinidae; genus: Hyphantrophaga; specificEpithet: adrianguadamuzi; scientificNameAuthorship: Fleming \& Wood, 2018; continent: Central America; country: Costa Rica; countryCode: CR; stateProvince: Guanacaste; county: Sector Santa Rosa; locality: Area de Conservacion Guanacaste; verbatimLocality: Area Administrativa; verbatimElevation: 295; verbatimLatitude: 10.8376; verbatimLongitude: -85.6187; verbatimCoordinateSystem: Decimal; decimalLatitude: 10.8376; decimalLongitude: -85.6187; samplingProtocol: Reared from the larva of the Pyralidae, Paridnea holophaealis; verbatimEventDate: 24-Nov-2011; individualID: DHJPAR0046407; individualCount: 1; sex: female; lifeStage: adult; preparations: pinned; catalogNumber: DHJPAR0046407; occurrenceDetails: http:// janzen.sas.upenn.edu; recordedBy: D.H. Janzen, W. Hallwachs, \& Guillermo Pereira; otherCatalog Numbers: ACGBA580-12,11-SRNP-14585, BOLD:ABZ0529; identifiedBy: AJ Fleming; dateldentified: 2017; language: en; institutionCode: CNC; collectionCode: Insects; basisOfRecord: Pinned Specimen

\section{Description}

Male (Fig. 2). Length: 7-8 mm. Head (Fig. 2b): vertex 1/4 of head width; two reclinate upper orbital setae; ocellar setae arising behind anterior ocellus; ocellar triangle with a 
very slight gold tinge compared to rest of fronto-orbital plate; fronto-orbital plate shiny silver and setulose, setulae not extending below lowest frontal seta; parafacial bare and shiny silver; facial ridge bare; eye densely haired; pedicel black, concolorous with postpedicel; arista distinctly thickened on basal 1/3-1/4; palpus light brown basally, apically orange, haired throughout. Thorax (Fig. 2a, c): brassy tomentose dorsally, contrasting with silver grey laterally; four thin yet prominent dorsal vittae, outermost two broken across suture, innermost pair unbroken, not reaching beyond 2nd postsutural dorsocentral seta; postpronotum with three setae arranged in a triangle; chaetotaxy: acrostichal setae 3:3; dorsocentral setae 3:4; intra-alar setae 2:3; supra-alar setae 2:3; two katepisternal setae; basal scutellar setae subequal in length to lateral scutellar setae; subapical scutellar setae strongest and longest of marginal scutellar setae, strongly divergent; apical scutellar setae $1 / 3$ of length of basal scutellar setae crossed apically; one pair of discal scutellar setae set as widely apart as subapical scutellar setae; scutellum concolorous with scutum. Legs (Fig. 2c): black in ground colour with dense covering of black hairs, making them appear darker; fore femur with dense silver tomentum on posterodorsal surface; hind coxa setose. Wing (Fig. 2a): hyaline, very slightly infuscate at base; vein $\mathrm{R}_{4+5}$ with two setulae at base. Abdomen (Fig. 2a, c): ground colour light brown; middorsal depression on ST1+2 not reaching hind margin; median marginal setae present on ST1+2-T3; a complete row of marginal setae present on T4 and T5; discal setae present on T3-T5; sex patch absent; distinct tomentose bands along anterior 4/5 of T3-T5. Terminalia (Fig. 2d, e, f): anterior edge of sternite 5 (Fig. 2f) with a deeply curved medial depression, posterior margin with a deep median cleft, smoothly U-shaped. Anterior plate of sternite 5 subequal to length of apical lobes; unsclerotised "window" ovoid to slightly rectangular, as wide as median cleft. Lateral lobe of sternite slightly pointed apically and with many short setae of equal length. Anterior plate of sternite 5 shorter than apical lobes. Cerci in posterior view (Fig. 2d) triangular with external edges inwardly wedged, slightly shorter than surstyli, blunt and rounded off towards apex, completely separate medially, diverging slightly at tips; in lateral view with a strong, almost 90 degree bend $1 / 5$ along their length. Surstylus in lateral view (Fig. 2e) almost parallel-sided along its length, ending in a slightly downcurved apex, making the structure appear fingerlike; basal half twice as wide as apical half; surstylus appearing fused with epandrium. Surstyli when viewed dorsally strongly divergent and wide open. Pregonite well-developed, $1 / 2$ as long as distiphallus, squared off, ending in a trumpet-like, flared apex. Postgonite elongate and slender, horn-shaped, subequal in length to pregonite, slightly narrowed towards apex, not strongly curved. Distiphallus sail-shaped, apically flared, with a slender median longitudinal sclerotised reinforcement on its posterior surface and a broad, anterolateral, sclerotised acrophallus on each side, joining the plate of opposite side on anterior surface near apex.

Female. Length: 6-9 mm. As male, differing only by the presence of two pairs of proclinate orbital setae. 


\section{Diagnosis}

Hyphantrophaga adrianguadamuzi sp. n. can be distinguished from all other Hyphantrophaga species by the following combination of traits: pedicel black (concolorous with postpedicel), palpus brown basally and orange apically, two katepisternal setae, hind coxa setose, colouration of T5 matching rest of tergites, median marginal setae absent on ST1+2 and discal setae present on T3-T5.

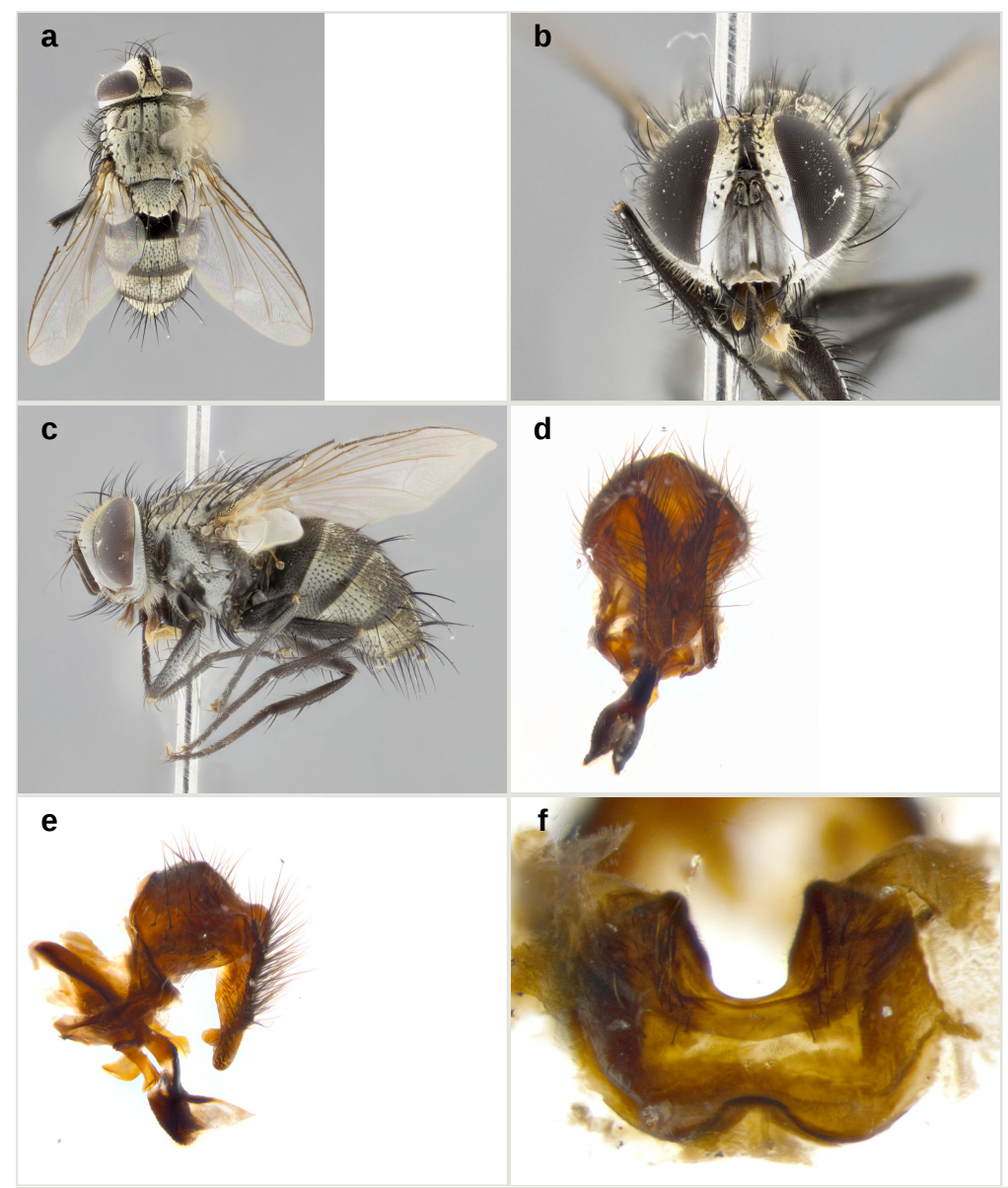

Figure 2.

Hyphantrophaga adrianguadamuzi sp. n.; a-c: habitus; holotype male, voucher $\mathrm{n}$. DHJPAR0007297; d-f: terminalia; male paratype, voucher n. DHJPAR0037527.
a: dorsal view doi
b: frontal view doi
c: lateral view doi
d: dorsal view doi
e: lateral view doi
f: sternite 5 , ventral view doi 


\section{Etymology}

Hyphantrophaga adrianguadamuzi sp. $\mathbf{n}$. is named in recognition of Adrian Guadamuz Chavarría's dedication and work in finding and rearing the ACG caterpillars that contained tachinid larvae.

\section{Distribution}

Costa Rica, ACG, Guanacaste Province, 295-733 m elevation.

\section{Ecology}

Hyphantrophaga adrianguadamuzi sp. $\mathbf{n}$. has been reared five times from one species of Lepidoptera in the family Pyralidae, Paridnea holophealis Ragonot, 1892, in dry forest and dry-rain lowland intergrade.

\section{Hyphantrophaga albopilosa Fleming \& Wood, sp. n.}

- ZooBank urn:Isid:zoobank.org:act:1BCF3C29-CCCF-4AF3-9372-6BB7FA910C87

\section{Materials}

Holotype:

a. $\quad$ scientificName: Hyphantrophaga albopilosa; phylum: Arthropoda; class: Insecta; order: Diptera; family: Tachinidae; genus: Hyphantrophaga; specificEpithet: albopilosa; scientificNameAuthorship: Fleming \& Wood, 2018; continent: Central America; country: Costa Rica; countryCode: CR; stateProvince: Guanacaste; county: Sector Pitilla; locality: Area de Conservacion Guanacaste; verbatimLocality: Sendero Laguna; verbatimElevation: 680; verbatimLatitude: 10.9888; verbatimLongitude: -85.4234; verbatimCoordinateSystem: Decimal; decimalLatitude: 10.9888; decimalLongitude: -85.4234; samplingProtocol: Reared from the larva of the Sphingidae, Adhemarius ypsilon; verbatimEventDate: 18-Aug-2004; individualID: DHJPAR0007442; individualCount: 1; sex: male; lifeStage: adult; preparations: pinned; catalogNumber: DHJPAR0007442; occurrenceDetails: http://janzen.sas.upenn.edu; recordedBy: D.H. Janzen, W. Hallwachs \& Manuel Rios; otherCatalogNumbers: ASTAT214-06, 04SRNP-34031, BOLD:AAF3886; identifiedBy: AJ Fleming; dateldentified: 2017; language: en; institutionCode: CNC; collectionCode: Insects; basisOfRecord: Pinned Specimen

\section{Paratypes:}

a. scientificName: Hyphantrophaga albopilosa; phylum: Arthropoda; class: Insecta; order: Diptera; family: Tachinidae; genus: Hyphantrophaga; specificEpithet: albopilosa; scientificNameAuthorship: Fleming \& Wood, 2018; continent: Central America; country: Costa Rica; countryCode: CR; stateProvince: Alajuela; county: Sector San Cristobal; locality: Area de Conservacion Guanacaste; verbatimLocality: Finca San Gabriel; verbatimElevation: 645; verbatimLatitude: 10.8777 ; verbatimLongitude: -85.3934; verbatimCoordinateSystem: Decimal; decimalLatitude: 10.8777 ; decimalLongitude: -85.3934; samplingProtocol: Reared from the larva of the Sphingidae, Adhemarius ypsilon; verbatimEventDate: 12-Jan-2014; individualID: DHJPAR0054045; individualCount: 1; sex: female; lifeStage: adult; preparations: pinned; catalogNumber: 
DHJPAR0054045; occurrenceDetails: http://janzen.sas.upenn.edu; recordedBy: D.H. Janzen, W. Hallwachs \& Elda Araya; otherCatalogNumbers: ASHYD3213-14, 13SRNP-7080, BOLD:AAF3886; identifiedBy: AJ Fleming; dateldentified: 2017; language: en; institutionCode: CNC; collectionCode: Insects; basisOfRecord: Pinned Specimen

b. ScientificName: Hyphantrophaga albopilosa; phylum: Arthropoda; class: Insecta; order: Diptera; family: Tachinidae; genus: Hyphantrophaga; specificEpithet: albopilosa; scientificNameAuthorship: Fleming \& Wood, 2018; continent: Central America; country: Costa Rica; countryCode: CR; stateProvince: Alajuela; county: Sector San Cristobal; locality: Area de Conservacion Guanacaste; verbatimLocality: Sendero Corredor; verbatimElevation: 620; verbatimLatitude: 10.8787; verbatimLongitude: -85.3896; verbatimCoordinateSystem: Decimal; decimalLatitude: 10.8787 ; decimalLongitude: -85.3896; samplingProtocol: Reared from the larva of the Sphingidae, Adhemarius ypsilon; verbatimEventDate: 29-Nov-2006; individualID: DHJPAR0016631; individualCount: 1; sex: female; lifeStage: adult; preparations: pinned; catalogNumber: DHJPAR0016631; occurrenceDetails: http://janzen.sas.upenn.edu; recordedBy: D.H. Janzen, W. Hallwachs \& Elda Araya; otherCatalog Numbers: ASTAP936-07, 06SRNP-8879, BOLD:AAF3886; identifiedBy: AJ Fleming; dateldentified: 2017; language: en; institutionCode: CNC; collectionCode: Insects; basisOfRecord: Pinned Specimen

c. ScientificName: Hyphantrophaga albopilosa; phylum: Arthropoda; class: Insecta; order: Diptera; family: Tachinidae; genus: Hyphantrophaga; specificEpithet: albopilosa; scientificNameAuthorship: Fleming \& Wood, 2018; continent: Central America; country: Costa Rica; countryCode: CR; stateProvince: Alajuela; county: Sector San Cristobal; locality: Dos Rios; verbatimLocality: Finca San Gabriel; verbatimElevation: 645; verbatimLatitude: 10.8777; verbatimLongitude: -85.3934 ; verbatimCoordinateSystem: Decimal; decimalLatitude: 10.8777; decimalLongitude: -85.3934; samplingProtocol: Reared from the larva of the Sphingidae, Adhemarius ypsilon; verbatimEventDate: 22Aug-2004; individualID: DHJPAR0007441; individualCount: 1; sex: female; lifeStage: adult; preparations: pinned; catalogNumber: DHJPAR0007441; occurrenceDetails: htt p://janzen.sas.upenn.edu; recordedBy: D.H. Janzen, W. Hallwachs \& Osvaldo Espinoza; otherCatalogNumbers: ASTAT213-06, 04-SRNP-3482, BOLD:AAF3886; identifiedBy: AJ Fleming; dateldentified: 2017; language: en; institutionCode: CNC; collectionCode: Insects; basisOfRecord: Pinned Specimen

d. ScientificName: Hyphantrophaga albopilosa; phylum: Arthropoda; class: Insecta; order: Diptera; family: Tachinidae; genus: Hyphantrophaga; specificEpithet: albopilosa; scientificNameAuthorship: Fleming \& Wood, 2018; continent: Central America; country: Costa Rica; countryCode: CR; stateProvince: Guanacaste; county: Sector Pitilla; locality: Area de Conservacion Guanacaste; verbatimLocality: Amonias; verbatimElevation: 390; verbatimLatitude: 11.0425; verbatimLongitude: -85.4034; verbatimCoordinateSystem: Decimal; decimalLatitude: 11.0425 ; decimalLongitude: -85.4034; samplingProtocol: Reared from the larva of the Sphingidae, Adhemarius ypsilon; verbatimEventDate: 08-Sep-2012; individualID: DHJPAR0050333; individualCount: 1; sex: male; lifeStage: adult; preparations: pinned; catalogNumber: DHJPAR0050333; occurrenceDetails: http://janzen.sas.upenn.edu; recordedBy: D.H. Janzen, W. Hallwachs \& Freddy Quesada; otherCatalogNumbers: ACGAZ1647-12, 12SRNP-31215, BOLD:AAF3886; identifiedBy: AJ Fleming; dateldentified: 2017; language: en; institutionCode: CNC; collectionCode: Insects; basisOfRecord: Pinned Specimen 


\section{Description}

Male (Fig. 3). Length: 7-9 mm. Head (Fig. 3b): vertex 1/5 of head width; one pair of reclinate upper orbital setae; ocellar setae arising behind anterior ocellus; ocellar triangle silver (slight gold tinge present but overall concolorous with fronto-orbital plate); fronto-orbital plate shiny silver and setulose, setulae not extending below lowest frontal seta; parafacial brilliant silver and bare; facial ridge bare; eye with short sparse ommatrichia up to $2 \mathrm{X}$ as long as one ommatidium; pedicel brownish-black with some orange, concolorous with postpedicel; arista brown, very minutely pubescent, distinctly thickened on basal 1/3-1/4; palpus yellow and densely haired, apically clubbed. Thorax (Fig. 3a, c): bright brassy-gold tomentose dorsally, contrasting with silver grey laterally; densely covered in blond setulae along anterior and lateral surfaces, dorsally with dense dark setulae interspersed amongst setae; four thin yet prominent dorsal vittae, outermost two broken across suture, innermost pair unbroken, reaching just beyond 2nd postsutural dorsocentral seta; postpronotum with 4-5 setae arranged in a triangle; chaetotaxy: acrostichal setae 3:3; dorsocentral setae 3:4; intra-alar setae 2:3; supra-alar setae 2:3; two katepisternal setae; basal scutellar setae subequal in length to subapical scutellar setae; lateral scutellar setae less than $1 / 2$ as long as subapical scutellar setae, curving inwards medially; apical scutellar setae subequal in length to lateral scutellar setae, crossed apically; one pair of discal scutellar setae more widely set than apical setae but more narrowly set than subapical setae; scutellum concolorous with scutum. Legs (Fig. 3c): brilliant yellow in ground colour; fore femur with dense silver tomentum on posterodorsal surface; hind coxa bare. Wing (Fig. 3a): pale translucent, hyaline, not distinctly infuscate; vein $\mathrm{R}_{4+5}$ with 2-3 setulae at base. Abdomen (Fig. 3a, c): ground colour yellow; middorsal depression on ST1+2 almost reaching hind margin; median marginal setae absent on ST1+2, present on T3; a complete row of marginal setae present on T4; discal setae only on T5; sex patch covering ventral surfaces of T4-T5; distinct brassy tomentose bands along anterior edge of T3 and T4, broken medially by a dorsocentral stripe and covering almost $80 \%$ of tergites; T5 with brassy tomentum throughout. Terminalia (Fig. 3d, e, f): anterior margin of sternite 5 (Fig. 3f) with a deeply curved medial depression, posterior margin with a deep median cleft, smoothly U-shaped. Lateral lobe of sternite slightly pointed apically and with many short setulae of equal length. Anterior plate of sternite 5 shorter than apical lobes. Unsclerotised "window" anterior to median cleft broadly rounded, stalked and convex reminiscent of a mushroom cap, extending as widely as median cleft. Cerci in posterior view (Fig. 3d) triangular with external edges inwardly wedged, slightly shorter than surstyli, blunt and rounded off towards apex, completely separate medially, diverging slightly at tips; in lateral view with a strong, almost 90 degree bend $1 / 5$ along their length. Surstylus in lateral view (Fig. 3e) almost parallel-sided along its length, ending in a slightly downcurved apex, making the structure appear fingerlike; basal half, twice as wide as apical half; surstylus appearing fused with epandrium. Surstyli when viewed dorsally strongly divergent and wide open. Pregonite welldeveloped, $1 / 2$ as long as distiphallus, squared off, ending in a trumpet-like, flared apex. Postgonite elongate and slender, horn-shaped, subequal in length to pregonite, slightly narrowed towards apex, not strongly curved. Distiphallus sail-shaped, apically 
flared, with a slender median longitudinal sclerotised reinforcement on its posterior surface and a broad, anterolateral, sclerotised acrophallus on each side, joining the plate of opposite side on anterior surface near apex.
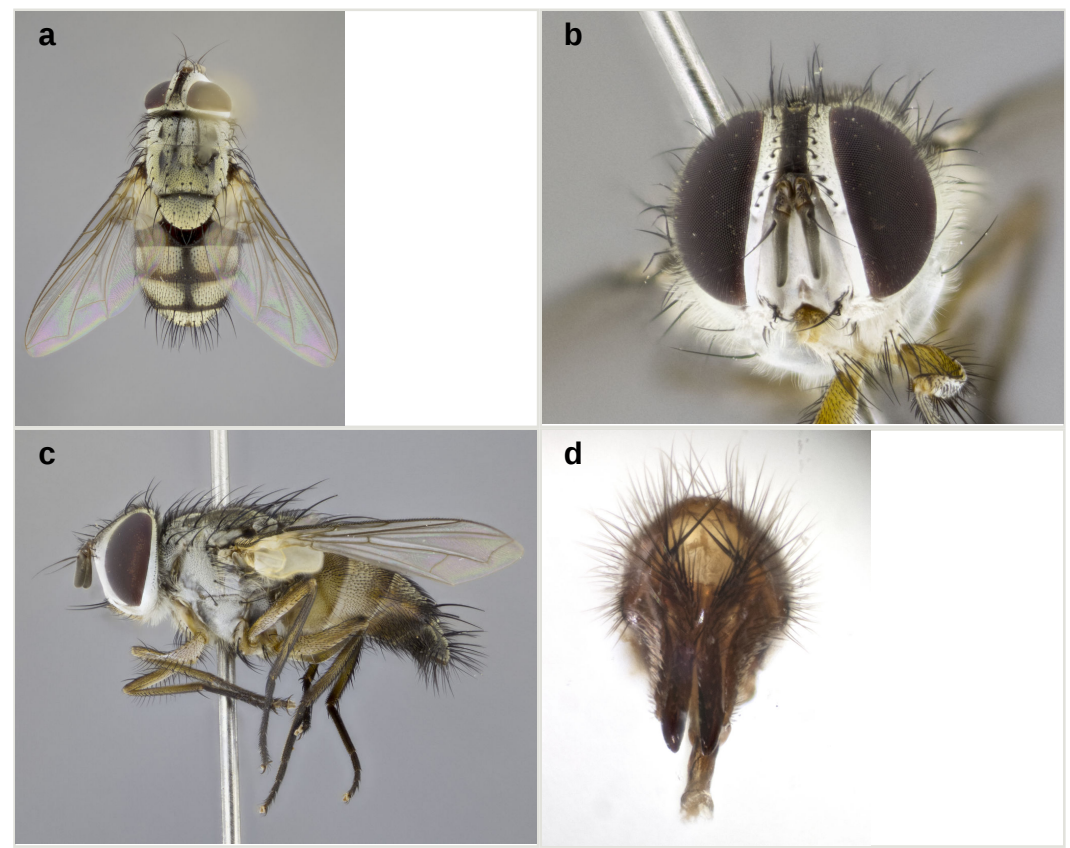

d

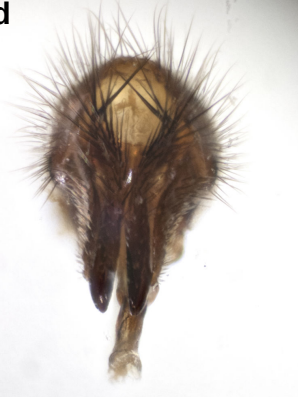

e

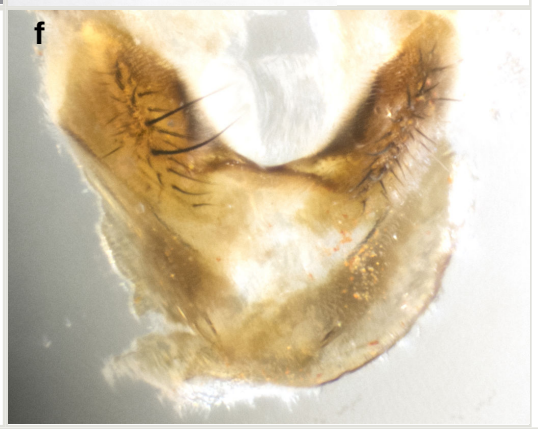

Figure 3.

Hyphantrophaga albopilosa sp. $\mathbf{n}$.; a-c: habitus; holotype male, voucher $\mathrm{n}$. DHJPAR0007442; d-f: terminalia; male paratype, voucher n. 04-SRNP-34031.
a: dorsal view doi
b: frontal view doi
c: lateral view doi
d: dorsal view doi
e: lateral view doi
f: sternite 5 , ventral view doi 
Female. Length: 7-9 mm. As male, differing only by the presence of two pairs of proclinate orbital setae.

\section{Diagnosis}

Hyphantrophaga albopilosa sp. n. can be distinguished from all other Hyphantrophaga species by the following combination of traits: brilliant silver parafacial, two katepisternal setae, dense covering of blond setulae along lateral surface of thorax, brilliant yellow legs, hind coxa bare and abdomen yellow in ground colour.

\section{Etymology}

From the Latin adjective "albopilosa", meaning "white-haired", in reference to its blond thoracic setulae.

\section{Distribution}

Costa Rica, ACG, Alajuela and Guanacaste Provinces, 390-645 m elevation.

\section{Ecology}

Hyphantrophaga albopilosa sp. $\mathbf{n}$. has been reared six times from a single species of Lepidoptera in the family Sphingidae, Adhemarius ypsilon (Rothschild \& Jordan, 1903), in rain forest.

\section{Hyphantrophaga anacordobae Fleming \& Wood, sp. n.}

- ZooBank urn:Isid:zoobank.org:act:5F5907DE-B5AD-4947-823F-527624FDE70B

\section{Materials}

Holotype:

a. ScientificName: Hyphantrophaga anacordobae; phylum: Arthropoda; class: Insecta; order: Diptera; family: Tachinidae; genus: Hyphantrophaga; specificEpithet: anacordobae; scientific NameAuthorship: Fleming \& Wood, 2018; continent: Central America; country: Costa Rica; countryCode: CR; stateProvince: Guanacaste; county: Sector Cacao; locality: Area de Conservacion Guanacaste; verbatimLocality: Sendero Ponderosa; verbatimElevation: 1060; verbatimLatitude: 10.9146; verbatimLongitude: -85.4626; verbatimCoordinateSystem: Decimal; decimalLatitude: 10.9146; decimalLongitude: -85.4626; samplingProtocol: Reared from the larva of the Nymphalidae, Morpho justitiae; verbatimEventDate: 04-Nov-2002; individualID: DHJPAR0007484; individualCount: 1; sex: male; lifeStage: adult; preparations: pinned; catalogNumber: DHJPAR0007484; occurrenceDetails: http://janzen.sas.upenn.edu; recordedBy: D.H. Janzen, W. Hallwachs \& Freddy Quesada; otherCatalogNumbers: ASTAT256-06, 02-SRNP-23842, BOLD:AAC5448; identifiedBy: AJ Fleming; dateldentified: 2017; language: en; institutionCode: CNC; collectionCode: Insects; basisOfRecord: Pinned Specimen 


\section{Paratypes:}

a. $\quad$ scientificName: Hyphantrophaga anacordobae; phylum: Arthropoda; class: Insecta; order: Diptera; family: Tachinidae; genus: Hyphantrophaga; specificEpithet: anacordobae; scientificNameAuthorship: Fleming \& Wood, 2018; continent: Central America; country: Costa Rica; countryCode: CR; stateProvince: Guanacaste; county: Sector Santa Maria; locality: Area de Conservacion Guanacaste; verbatimLocality: Estacion Santa Maria; verbatimElevation: 840; verbatimLatitude: 10.7645; verbatimLongitude: -85.3116; verbatimCoordinateSystem: Decimal; decimalLatitude: 10.7645; decimalLongitude: -85.3116; samplingProtocol: Reared from the larva of the Nymphalidae, Morpho justitiae; verbatimEventDate: 16-Nov-1995; individualID: DHJPAR0007481; individualCount: 1; sex: female; lifeStage: adult; preparations: pinned; catalogNumber: DHJPAR0007481; occurrenceDetails: http:// janzen.sas.upenn.edu; recordedBy: D.H. Janzen, W. Hallwachs \& gusaneros; otherCatalog Numbers: ASTAT253-06, 95-SRNP-10486, BOLD:AAC5448; identifiedBy: AJ Fleming; dateldentified: 2017; language: en; institutionCode: CNC; collectionCode: Insects; basisOfRecord: Pinned Specimen

b. ScientificName: Hyphantrophaga anacordobae; phylum: Arthropoda; class: Insecta; order: Diptera; family: Tachinidae; genus: Hyphantrophaga; specificEpithet: anacordobae; scientificNameAuthorship: Fleming \& Wood, 2018; continent: Central America; country: Costa Rica; countryCode: CR; stateProvince: Guanacaste; county: Sector Cacao; locality: Area de Conservacion Guanacaste; verbatimLocality: Sendero Ponderosa; verbatimElevation: 1060; verbatimLatitude: 10.9146; verbatimLongitude: -85.4626; verbatimCoordinateSystem: Decimal; decimalLatitude: 10.9146; decimalLongitude: -85.4626; samplingProtocol: Reared from the larva of the Nymphalidae, Morpho justitiae; verbatimEventDate: 14-Nov-2002; individualID: DHJPAR0007482; individualCount: 1; sex: male; lifeStage: adult; preparations: pinned; catalog Number: DHJPAR0007482; occurrenceDetails: http://janzen.sas.upenn.edu; recordedBy: D.H. Janzen, W. Hallwachs \& Mariano Pereira; otherCatalogNumbers: ASTAT254-06, 02-SRNP-23679, BOLD:AAC5448; identifiedBy: AJ Fleming; dateldentified: 2017; language: en; institutionCode: CNC; collectionCode: Insects; basisOfRecord: Pinned Specimen

c. ScientificName: Hyphantrophaga anacordobae; phylum: Arthropoda; class: Insecta; order: Diptera; family: Tachinidae; genus: Hyphantrophaga; specificEpithet: anacordobae; scientificNameAuthorship: Fleming \& Wood, 2018; continent: Central America; country: Costa Rica; countryCode: CR; stateProvince: Guanacaste; county: Sector Cacao; locality: Area de Conservacion Guanacaste; verbatimLocality: Sendero Ponderosa; verbatimElevation: 1060; verbatimLatitude: 10.9146; verbatimLongitude: -85.4626; verbatimCoordinateSystem: Decimal; decimalLatitude: 10.9146; decimalLongitude: -85.4626; samplingProtocol: Reared from the larva of the Nymphalidae, Morpho justitiae; verbatimEventDate: 11-Oct-2002; individualID: DHJPAR0007483; individualCount: 1; sex: female; lifeStage: adult; preparations: pinned; catalogNumber: DHJPAR0007483; occurrenceDetails: http:// janzen.sas.upenn.edu; recordedBy: D.H. Janzen, W. Hallwachs \& Mariano Pereira; otherCatalog Numbers: ASTAT255-06, 02-SRNP-23680, BOLD:AAC5448; identifiedBy: AJ Fleming; dateldentified: 2017; language: en; institutionCode: CNC; collectionCode: Insects; basisOfRecord: Pinned Specimen

d. $\quad$ scientificName: Hyphantrophaga anacordobae; phylum: Arthropoda; class: Insecta; order: Diptera; family: Tachinidae; genus: Hyphantrophaga; specificEpithet: anacordobae; scientificNameAuthorship: Fleming \& Wood, 2018; continent: Central America; country: Costa Rica; countryCode: CR; stateProvince: Guanacaste; county: 
Sector Santa Maria; locality: Area de Conservacion Guanacaste; verbatimLocality: Estacion Santa Maria; verbatimElevation: 840; verbatimLatitude: 10.7645; verbatimLongitude: -85.3116; verbatimCoordinateSystem: Decimal; decimalLatitude: 10.7645; decimalLongitude: -85.3116; samplingProtocol: Reared from the larva of the Nymphalidae, Morpho justitiae; verbatimEventDate: 19-Nov-1995; individualID: DHJPAR0007480; individualCount: 1; sex: female; lifeStage: adult; preparations: pinned; catalogNumber: DHJPAR0007480; occurrenceDetails: http:// janzen.sas.upenn.edu; recordedBy: D.H. Janzen, W. Hallwachs \& gusaneros; otherCatalog Numbers: ASTAT252-06, 95-SRNP-10486.1, BOLD:AAC5448; identifiedBy: AJ Fleming; dateldentified: 2017; language: en; institutionCode: CNC; collectionCode: Insects; basisOfRecord: Pinned Specimen

e. $\quad$ scientificName: Hyphantrophaga anacordobae; phylum: Arthropoda; class: Insecta; order: Diptera; family: Tachinidae; genus: Hyphantrophaga; specificEpithet: anacordobae; scientificNameAuthorship: Fleming \& Wood, 2018; continent: Central America; country: Costa Rica; countryCode: CR; stateProvince: Guanacaste; county: Sector Cacao; locality: Area de Conservacion Guanacaste; verbatimLocality: Sendero Ponderosa; verbatimElevation: 1060; verbatimLatitude: 10.9146; verbatimLongitude: -85.4626; verbatimCoordinateSystem: Decimal; decimalLatitude: 10.9146; decimalLongitude: -85.4626; samplingProtocol: Reared from the larva of the Nymphalidae, Morpho justitiae; verbatimEventDate: 16-Nov-2002; individualID: DHJPAR0007485; individualCount: 1; sex: male; lifeStage: adult; preparations: pinned; catalogNumber: DHJPAR0007485; occurrenceDetails: http://janzen.sas.upenn.edu; recordedBy: D.H. Janzen, W. Hallwachs \& Guillermo Pereira; otherCatalogNumbers: ASTAT257-06, 02-SRNP-23678, BOLD:AAC5448; identifiedBy: AJ Fleming; dateldentified: 2017; language: en; institutionCode: CNC; collectionCode: Insects; basisOfRecord: Pinned Specimen

f. $\quad$ scientificName: Hyphantrophaga anacordobae; phylum: Arthropoda; class: Insecta; order: Diptera; family: Tachinidae; genus: Hyphantrophaga; specificEpithet: anacordobae; scientificNameAuthorship: Fleming \& Wood, 2018; continent: Central America; country: Costa Rica; countryCode: CR; stateProvince: Guanacaste; county: Sector Cacao; locality: Area de Conservacion Guanacaste; verbatimLocality: Sendero Ponderosa; verbatimElevation: 1060; verbatimLatitude: 10.9146; verbatimLongitude: -85.4626; verbatimCoordinateSystem: Decimal; decimalLatitude: 10.9146; decimalLongitude: -85.4626; samplingProtocol: Reared from the larva of the Nymphalidae, Morpho justitiae; verbatimEventDate: 28-Jan-2003; individualID: DHJPAR0007486; individualCount: 1; sex: female; lifeStage: adult; preparations: pinned; catalogNumber: DHJPAR0007486; occurrenceDetails: http:// janzen.sas.upenn.edu; recordedBy: D.H. Janzen, W. Hallwachs \& Freddy Quesada; otherCatalog Numbers: ASTAT258-06, 02-SRNP-24540.7, BOLD:AAC5448; identifiedBy: AJ Fleming; dateldentified: 2017; language: en; institutionCode: CNC; collectionCode: Insects; basisOfRecord: Pinned Specimen

g. ScientificName: Hyphantrophaga anacordobae; phylum: Arthropoda; class: Insecta; order: Diptera; family: Tachinidae; genus: Hyphantrophaga; specificEpithet: anacordobae; scientificNameAuthorship: Fleming \& Wood, 2018; continent: Central America; country: Costa Rica; countryCode: CR; stateProvince: Guanacaste; county: Sector Cacao; locality: Area de Conservacion Guanacaste; verbatimLocality: Sendero Ponderosa; verbatimElevation: 1060; verbatimLatitude: 10.9146; verbatimLongitude: -85.4626; verbatimCoordinateSystem: Decimal; decimalLatitude: 10.9146; decimalLongitude: -85.4626; samplingProtocol: Reared from the larva of the Nymphalidae, Morpho justitiae; verbatimEventDate: 31-Jan-2003; individualID: 
DHJPAR0007487; individualCount: 1; sex: male; lifeStage: adult; preparations: pinned; catalogNumber: DHJPAR0007487; occurrenceDetails: http://janzen.sas.upenn.edu; recordedBy: D.H. Janzen, W. Hallwachs \& Harry Ramirez; otherCatalogNumbers: ASTAT259-06, 02-SRNP-24536.7, BOLD:AAC5448; identifiedBy: AJ Fleming; dateldentified: 2017; language: en; institutionCode: CNC; collectionCode: Insects; basisOfRecord: Pinned Specimen

h. $\quad$ scientificName: Hyphantrophaga anacordobae; phylum: Arthropoda; class: Insecta; order: Diptera; family: Tachinidae; genus: Hyphantrophaga; specificEpithet: anacordobae; scientificNameAuthorship: Fleming \& Wood, 2018; continent: Central America; country: Costa Rica; countryCode: CR; stateProvince: Guanacaste; county: Sector Cacao; locality: Area de Conservacion Guanacaste; verbatimLocality: Sendero Ponderosa; verbatimElevation: 1060; verbatimLatitude: 10.9146; verbatimLongitude: -85.4626; verbatimCoordinateSystem: Decimal; decimalLatitude: 10.9146; decimalLongitude: -85.4626; samplingProtocol: Reared from the larva of the Nymphalidae, Morpho justitiae; verbatimEventDate: 11-Dec-2002; individualID: DHJPAR0007488; individualCount: 1; sex: male; lifeStage: adult; preparations: pinned; catalogNumber: DHJPAR0007488; occurrenceDetails: http://janzen.sas.upenn.edu; recordedBy: D.H. Janzen, W. Hallwachs \& Mariano Pereira; otherCatalogNumbers: ASTAT260-06, 02-SRNP-24200, BOLD:AAC5448; identifiedBy: AJ Fleming; dateldentified: 2017; language: en; institutionCode: CNC; collectionCode: Insects; basisOfRecord: Pinned Specimen

i. ScientificName: Hyphantrophaga anacordobae; phylum: Arthropoda; class: Insecta; order: Diptera; family: Tachinidae; genus: Hyphantrophaga; specificEpithet: anacordobae; scientificNameAuthorship: Fleming \& Wood, 2018; continent: Central America; country: Costa Rica; countryCode: CR; stateProvince: Guanacaste; county: Sector Cacao; locality: Area de Conservacion Guanacaste; verbatimLocality: Sendero Ponderosa; verbatimElevation: 1060; verbatimLatitude: 10.9146; verbatimLongitude: -85.4626; verbatim CoordinateSystem: Decimal; decimalLatitude: 10.9146; decimalLongitude: -85.4626; samplingProtocol: Reared from the larva of the Nymphalidae, Morpho justitiae; verbatimEventDate: 21-Feb-2003; individualID: DHJPAR0007489; individualCount: 1; sex: female; lifeStage: adult; preparations: pinned; catalogNumber: DHJPAR0007489; occurrenceDetails: http:// janzen.sas.upenn.edu; recordedBy: D.H. Janzen, W. Hallwachs \& Mariano Pereira; otherCatalog Numbers: ASTAT261-06, 02-SRNP-24542, BOLD:AAC5448; identifiedBy: AJ Fleming; dateldentified: 2017; language: en; institutionCode: CNC; collectionCode: Insects; basisOfRecord: Pinned Specimen

j. $\quad$ scientificName: Hyphantrophaga anacordobae; phylum: Arthropoda; class: Insecta; order: Diptera; family: Tachinidae; genus: Hyphantrophaga; specificEpithet: anacordobae; scientificNameAuthorship: Fleming \& Wood, 2018; continent: Central America; country: Costa Rica; countryCode: CR; stateProvince: Guanacaste; county: Sector Del Oro; locality: Area de Conservacion Guanacaste; verbatimLocality: Monte Cristo; verbatimElevation: 525; verbatimLatitude: 11.0137; verbatimLongitude: -85.4253; verbatimCoordinateSystem: Decimal; decimalLatitude: 11.0137; decimalLongitude: -85.4253; samplingProtocol: Reared from the larva of the Nymphalidae, Morpho justitiae; verbatimEventDate: 26-May-2007; individualID: DHJPAR0019597; individualCount: 1; sex: female; lifeStage: adult; preparations: pinned; catalogNumber: DHJPAR0019597; occurrenceDetails: http:// janzen.sas.upenn.edu; recordedBy: D.H. Janzen, W. Hallwachs \& Roster Moraga; otherCatalogNumbers: ASTAB145-07, 07-SRNP-21398, BOLD:AAC5448; identifiedBy: 
AJ Fleming; dateldentified: 2017; language: en; institutionCode: CNC; collectionCode: Insects; basisOfRecord: Pinned Specimen

k. $\quad$ scientificName: Hyphantrophaga anacordobae; phylum: Arthropoda; class: Insecta; order: Diptera; family: Tachinidae; genus: Hyphantrophaga; specificEpithet: anacordobae; scientificNameAuthorship: Fleming \& Wood, 2018; continent: Central America; country: Costa Rica; countryCode: CR; stateProvince: Alajuela; county: Sector San Cristobal; locality: Area de Conservacion Guanacaste; verbatimLocality: Tajo Angeles; verbatimElevation: 540; verbatimLatitude: 10.8647; verbatimLongitude: -85.4153; verbatimCoordinateSystem: Decimal; decimalLatitude: 10.8647; decimalLongitude: -85.4153; samplingProtocol: Reared from the larva of the Nymphalidae, Morpho amathonte; verbatimEventDate: 24-Nov-2010; individualID: DHJPAR0040955; individualCount: 1; sex: male; lifeStage: adult; preparations: pinned; catalogNumber: DHJPAR0040955; occurrenceDetails: http://janzen.sas.upenn.edu; recordedBy: D.H. Janzen, W. Hallwachs \& Carolina Cano; otherCatalogNumbers: ASHYF870-11, 10-SRNP-4655, BOLD:AAC5448; identifiedBy: AJ Fleming; dateldentified: 2017; language: en; institutionCode: CNC; collectionCode: Insects; basisOfRecord: Pinned Specimen

I. scientificName: Hyphantrophaga anacordobae; phylum: Arthropoda; class: Insecta; order: Diptera; family: Tachinidae; genus: Hyphantrophaga; specificEpithet: anacordobae; scientificNameAuthorship: Fleming \& Wood, 2018; continent: Central America; country: Costa Rica; countryCode: CR; stateProvince: Guanacaste; county: Sector Pitilla; locality: Area de Conservacion Guanacaste; verbatimLocality: Sendero Cuestona; verbatimEventDate: 09-Oct-2016; individualID: DHJPAR0059877; individualCount: 1; sex: male; lifeStage: adult; preparations: pinned; catalogNumber: DHJPAR0059877; occurrenceDetails: http://janzen.sas.upenn.edu; recordedBy: D.H. Janzen, W. Hallwachs \& Manuel Rios; otherCatalogNumbers: ACGBA6298-16, 16SRNP-31601, BOLD:AAC5448; identifiedBy: AJ Fleming; dateldentified: 2107; language: en; institutionCode: CNC; collectionCode: Insects; basisOfRecord: Pinned Specimen

\section{Description}

Male (Fig. 4). Length: 9-12 mm. Head (Fig. 4b): vertex 1/4 of head width; two pairs of reclinate upper orbital setae; ocellar setae arising behind anterior ocellus; ocellar triangle black medially, brassy/silver laterally; fronto-orbital plate shiny silver and densely setulose, setulae not extending below lowest frontal seta; parafacial silver and bare; eye densely haired; facial ridge bare; pedicel black, concolorous with postpedicel; arista dark brown, very minutely pubescent, distinctly thickened on basal 1/3-1/4; palpus yellow and densely haired so as to appear darkened, apically with a slight clubbed appearance. Thorax (Fig. 4a, c): slightly brassy tomentose dorsally, contrasting with dark silver grey laterally; densely covered in black setulae along all surfaces, interspersed amongst setae; four thick and prominent dorsal vittae, outermost two broken across suture, innermost pair unbroken, reaching just beyond 2nd postsutural dorsocentral seta; postpronotum with 5-6 setae arranged in a triangle; chaetotaxy: acrostichal setae 3:3; dorsocentral setae 3:4; intra-alar setae 2-3:3; supraalar setae 2:3; three katepisternal setae; basal scutellar setae subequal to slightly shorter than subapical scutellar setae; lateral scutellar setae subequal in length to apical setae, with a strong inward curve medially; apical scutellar setae $1 / 2$ as long as 
subapical scutellar setae, crossed apically; one pair of discal scutellar setae set as widely apart as subapical setae; scutellum darkened across basal $20 \%$, remainder concolorous with scutum. Legs (Fig. 4c): ground colour black; fore femur with dense silver tomentum on posterodorsal surface; hind coxa bare. Wing (Fig. 4a): pale translucent, hyaline, not distinctly infuscate; vein $\mathrm{R}_{4+5}$ with 3-4 setulae at base. Abdomen (Fig. 4a, c): ground colour dark reddish-brown, appearing black; middorsal depression on ST1+2 almost reaching hind margin; median marginal setae absent on ST1+2, present on T3; complete row of marginal setae present on T4; discal setae only on T5; sex patch covering $50 \%$ of the laterodorsal surface of T4 and the ventral surfaces of T4-T5; distinct brassy tomentose bands along anterior edge of T3 and T4, broken medially by a dorsocentral stripe and covering almost $40 \%$ of tergites; T5 with brassy tomentum throughout. Terminalia (Fig. 4d, e, f): sternite 5 (Fig. 4f) with a deeply excavated median cleft, squared off U-shaped, margins covered in dense tomentum. Lateral lobes of sternite rounded triangular apically, with 2-3 strong setae surrounded by many shorter, weaker setulae. Anterior plate of sternite 5 subequal to length of apical lobes; unsclerotised "window" slightly rectangular, two palmlike arms extending as wide as median cleft. Lateral lobe of sternite slightly pointed apically and with many short setae of equal length. Cerci in posterior view (Fig. 4d) slightly triangular and subequal in length to surstyli, blunt and rounded at apex, completely separate medially, appearing but not divergent; in lateral view with a strong downward angle commencing at midpoint; densely setulose along basal 2/3 dorsally, setulose ventrally along entire length (visible in lateral view). Surstylus in lateral view (Fig. 4e) almost parallel-sided along its length, ending in a slightly downcurved apex, making the structure appear blade-like; surstylus appearing to be fused with epandrium; when viewed dorsally surstyli appearing concave with a bulbous base where surstyli meet epandrium, inner margin straight slightly divergent at apices. Pregonite short, 0.5 times as long as distiphallus, bare and rounded off apically. Postgonite slightly narrow, 1/3 as wide as pregonite, sharply pointed and curved, vaguely C-shaped. Distiphallus $\sim 1.5$ times as long as basiphallus. Distiphallus sail-shaped, with a slender median longitudinal sclerotised reinforcement on its posterior surface and a broad, anterolateral, sclerotised acrophallus on each side, joining the plate of opposite side on anterior surface near apex.

Female. Length: 8-11 mm. As male, differing only by the presence of two pairs of proclinate orbital setae.

\section{Diagnosis}

Hyphantrophaga anacordobae sp. n. can be distinguished from all other Hyphantrophaga species by the following combination of traits: three katepisternal setae, legs entirely black, hind coxa bare, ground colour of abdomen dark reddishbrown (appearing black under certain angles of light) and median marginal setae absent from ST1+2. 


\section{Etymology}

Hyphantrophaga anacordobae sp. $\mathbf{n}$. is named in recognition of Anabelle Córdoba Alemán's dedication and work in finding and rearing the ACG caterpillars that contained tachinid larvae.

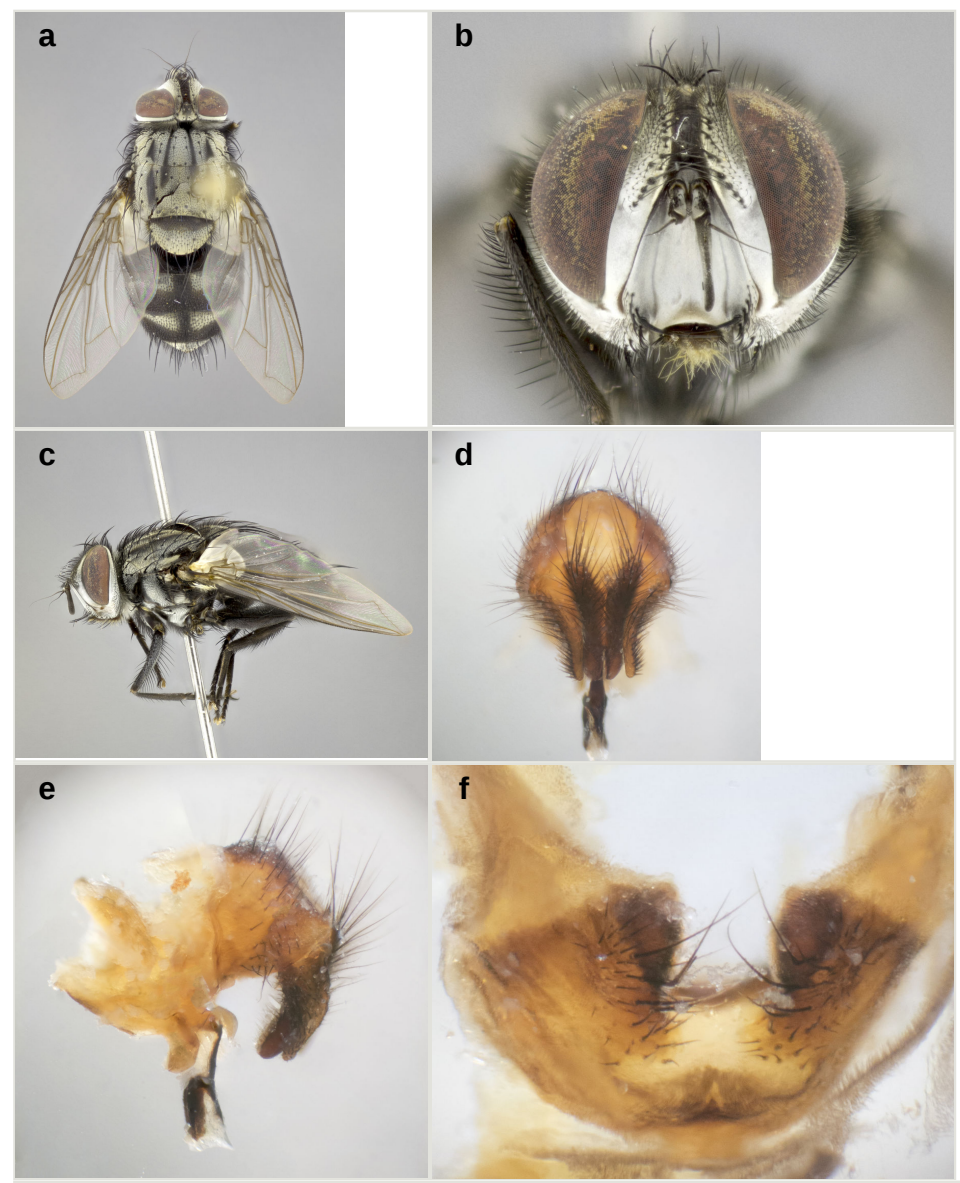

Figure 4.

Hyphantrophaga anacordobae sp. n.; a-c: habitus; holotype male, voucher $\mathrm{n}$. DHJPAR0007484; d-f: terminalia; male paratype, voucher n. DHJPAR0007487.
a: dorsal view doi
b: frontal view doi
c: lateral view doi
d: dorsal view doi
e: lateral view doi
f: sternite 5 , ventral view doi 


\section{Distribution}

Costa Rica, ACG, Guanacaste Province, 525-1060 m elevation.

\section{Ecology}

Hyphantrophaga anacordobae sp. $\mathbf{n}$. has been reared 13 times from two species of Lepidoptera in the family Nymphalidae, Morpho helenor (Cramer, 1776) and Morpho justitiae (Salvin \& Godman, 1868), in rain forest, cloud forest and dry-rain lowland intergrade.

\section{Hyphantrophaga angustata (van der Wulp, 1890)}

\section{Materials}

Holotype:

a. scientificName: Hyphantrophaga angustata; phylum: Arthropoda; class: Insecta; order: Diptera; family: Tachinidae; genus: Exorista; specificEpithet: angustata; scientificNameAuthorship: (van der Wulp, 1890); continent: Central America; country: Mexico; countryCode: MX; stateProvince: Guerrero; county: Chilpancingo; verbatimElevation: 4600; individualCount: 1 ; sex: male; lifeStage: adult; preparations: pinned; recordedBy: H.H. Smith; identifiedBy: Wood; dateldentified: 2015; language: en; institutionCode: NHMUK; collectionCode: Insects; basisOfRecord: Pinned Specimen

\section{Other materials:}

a. scientificName: Hyphantrophaga angustata; phylum: Arthropoda; class: Insecta; order: Diptera; family: Tachinidae; genus: Zenillia; specificEpithet: coquilletti; scientificNameAuthorship: (Aldrich \& Webber, 1924); continent: North America; country: United States of America; countryCode: US; stateProvince: Texas; county: Belfrage; individualID: 25702; individualCount: 1; sex: male; lifeStage: adult; preparations: pinned; recordedBy: C.V. Riley; otherCatalogNumbers: Type. No. 25702 USNM; identifiedBy: AJ Fleming; dateldentified: 2017; language: en; institutionCode: USNM; collectionCode: Insects; basisOfRecord: Pinned Specimen

b. ScientificName: Hyphantrophaga angustata; phylum: Arthropoda; class: Insecta; order: Diptera; family: Tachinidae; genus: Hyphantrophaga; specificEpithet: angustata; scientificNameAuthorship: (van der Wulp, 1890); continent: Central America; country: Costa Rica; countryCode: CR; stateProvince: Guanacaste; county: Sector Mundo Nuevo; locality: Area de Conservacion Guanacaste; verbatimLocality: Estacion La Perla; verbatimElevation: 325 ; verbatimLatitude: 10.7674; verbatimLongitude: -85.4331; verbatimCoordinateSystem: Decimal; decimalLatitude: 10.7674; decimalLongitude: -85.4331; samplingProtocol: Reared from the larva of the Thyrididae, Dysodia immargo; verbatimEventDate: 17-Aug-2013; individualID: DHJPAR0052588; individualCount: 1; sex: male; lifeStage: adult; preparations: pinned; catalogNumber: DHJPAR0052588; occurrenceDetails: http://janzen.sas.upenn.edu; recordedBy: D.H. Janzen, W. Hallwachs \& Jose Cortez; otherCatalogNumbers: ASHYM1942-13, 13-SRNP-55696, BOLD:AAD0627; identifiedBy: AJ Fleming; dateldentified: 2017; language: en; institutionCode: CNC; collectionCode: Insects; basisOfRecord: Pinned Specimen 
c. scientificName: Hyphantrophaga angustata; phylum: Arthropoda; class: Insecta; order: Diptera; family: Tachinidae; genus: Hyphantrophaga; specificEpithet: angustata; scientificNameAuthorship: (van der Wulp, 1890); continent: Central America; country: Costa Rica; countryCode: CR; stateProvince: Guanacaste; county: Sector Santa Rosa; locality: Area de Conservacion Guanacaste; verbatimLocality: Bosque San Emilio; verbatimElevation: 300; verbatimLatitude: 10.8439; verbatimLongitude: -85.6138; verbatimCoordinateSystem: Decimal; decimalLatitude: 10.8439; decimalLongitude: -85.6138; samplingProtocol: Reared from the larva of the Thyrididae, Dysodia speculifera; verbatimEventDate: 14-Oct-2001; individualID: DHJPAR0007320; individualCount: 1; sex: female; lifeStage: adult; preparations: pinned; catalogNumber: DHJPAR0007320; occurrenceDetails: http://janzen.sas.upenn.edu; recordedBy: D.H. Janzen, W. Hallwachs \& gusaneros; otherCatalogNumbers: ASTAT092-06, 01SRNP-14940, BOLD:AAD0627; identifiedBy: AJ Fleming; dateldentified: 2017; language: en; institutionCode: CNC; collectionCode: Insects; basisOfRecord: Pinned Specimen

d. scientificName: Hyphantrophaga angustata; phylum: Arthropoda; class: Insecta; order: Diptera; family: Tachinidae; genus: Hyphantrophaga; specificEpithet: angustata; scientificNameAuthorship: (van der Wulp, 1890); continent: Central America; country: Costa Rica; countryCode: CR; stateProvince: Guanacaste; county: Sector Santa Rosa; locality: Area de Conservacion Guanacaste; verbatimLocality: Area Administrativa; verbatimElevation: 295; verbatimLatitude: 10.8376; verbatimLongitude: -85.6187 ; verbatimCoordinateSystem: Decimal; decimalLatitude: 10.8376 ; decimalLongitude: -85.6187; samplingProtocol: Reared from the larva of the Thyrididae, Dysodia speculifera; verbatimEventDate: 15-Aug-2002; individualID: DHJPAR0007317; individualCount: 1; sex: male; lifeStage: adult; preparations: pinned; catalogNumber: DHJPAR0007317; occurrenceDetails: http://janzen.sas.upenn.edu; recordedBy: D.H. Janzen \& W. Hallwachs; otherCatalogNumbers: ASTAT089-06, 02-SRNP-12711, BOLD:AAD0627; identifiedBy: AJ Fleming; dateldentified: 2017; language: en; institutionCode: CNC; collectionCode: Insects; basisOfRecord: Pinned Specimen

e. ScientificName: Hyphantrophaga angustata; phylum: Arthropoda; class: Insecta; order: Diptera; family: Tachinidae; genus: Hyphantrophaga; specificEpithet: angustata; scientificNameAuthorship: (van der Wulp, 1890); continent: Central America; country: Costa Rica; countryCode: CR; stateProvince: Guanacaste; county: Sector Santa Rosa; locality: Area de Conservacion Guanacaste; verbatimLocality: Area Administrativa; verbatimElevation: 295; verbatimLatitude: 10.8376; verbatimLongitude: -85.6187; verbatimCoordinateSystem: Decimal; decimalLatitude: 10.8376; decimalLongitude: -85.6187; samplingProtocol: Reared from the larva of the Thyrididae, Dysodia speculifera; verbatimEventDate: 04-Aug-2002; individualID: DHJPAR0007323; individualCount: 1; sex: male; lifeStage: adult; preparations: pinned; catalogNumber: DHJPAR0007323; occurrenceDetails: http://janzen.sas.upenn.edu; recordedBy: D.H. Janzen \& W. Hallwachs; otherCatalogNumbers: ASTAT095-06, 02-SRNP-12709, BOLD:AAD0627; identifiedBy: AJ Fleming; dateldentified: 2017; language: en; institutionCode: CNC; collectionCode: Insects; basisOfRecord: Pinned Specimen

f. ScientificName: Hyphantrophaga angustata; phylum: Arthropoda; class: Insecta; order: Diptera; family: Tachinidae; genus: Hyphantrophaga; specificEpithet: angustata; scientificNameAuthorship: (van der Wulp, 1890); continent: Central America; country: Costa Rica; countryCode: CR; stateProvince: Guanacaste; county: Sector Santa Rosa; locality: Area de Conservacion Guanacaste; verbatimLocality: Bosque San Emilio; verbatimElevation: 300; verbatimLatitude: 10.8439; verbatimLongitude: -85.6138; verbatimCoordinateSystem: Decimal; decimalLatitude: 10.8439; decimalLongitude: 
-85.6138; samplingProtocol: Reared from the larva of the Thyrididae, Dysodia speculifera; verbatimEventDate: 25-Aug-2001; individualID: DHJPAR0007319; individualCount: 1; sex: male; lifeStage: adult; preparations: pinned; catalogNumber: DHJPAR0007319; occurrenceDetails: http://janzen.sas.upenn.edu; recordedBy: D.H. Janzen, W. Hallwachs \& gusaneros; otherCatalogNumbers: ASTAT091-06, 01SRNP-14941, BOLD:AAD0627; identifiedBy: AJ Fleming; dateldentified: 2017; language: en; institutionCode: CNC; collectionCode: Insects; basisOfRecord: Pinned Specimen

g. scientificName: Hyphantrophaga angustata; phylum: Arthropoda; class: Insecta; order: Diptera; family: Tachinidae; genus: Hyphantrophaga; specificEpithet: angustata; scientificNameAuthorship: (van der Wulp, 1890); continent: Central America; country: Costa Rica; countryCode: CR; stateProvince: Guanacaste; county: Sector Santa Rosa; locality: Area de Conservacion Guanacaste; verbatimLocality: Bosque San Emilio; verbatimElevation: 300; verbatimLatitude: 10.8439; verbatimLongitude: -85.6138; verbatimCoordinateSystem: Decimal; decimalLatitude: 10.8439; decimalLongitude: -85.6138; samplingProtocol: Reared from the larva of the Thyrididae, Dysodia speculifera; verbatimEventDate: 13-Oct-2001; individualID: DHJPAR0007321; individualCount: 1; sex: female; lifeStage: adult; preparations: pinned; catalogNumber: DHJPAR0007321; occurrenceDetails: http://janzen.sas.upenn.edu; recordedBy: D.H. Janzen, W. Hallwachs \& gusaneros; otherCatalogNumbers: ASTAT093-06, 01SRNP-14942, BOLD:AAD0627; identifiedBy: AJ Fleming; dateldentified: 2017; language: en; institutionCode: CNC; collectionCode: Insects; basisOfRecord: Pinned Specimen

h. ScientificName: Hyphantrophaga angustata; phylum: Arthropoda; class: Insecta; order: Diptera; family: Tachinidae; genus: Hyphantrophaga; specificEpithet: angustata; scientificNameAuthorship: (van der Wulp, 1890); continent: Central America; country: Costa Rica; countryCode: CR; stateProvince: Guanacaste; county: Sector Santa Rosa; locality: Area de Conservacion Guanacaste; verbatimLocality: Area Administrativa; verbatimElevation: 295; verbatimLatitude: 10.8376; verbatimLongitude: -85.6187; verbatimCoordinateSystem: Decimal; decimalLatitude: 10.8376 ; decimalLongitude: -85.6187; samplingProtocol: Reared from the larva of the Thyrididae, Dysodia speculifera; verbatimEventDate: 30-Sep-2002; individualID: DHJPAR0007322; individualCount: 1; lifeStage: adult; preparations: pinned; catalogNumber: DHJPAR0007322; occurrenceDetails: http://janzen.sas.upenn.edu; recordedBy: D.H. Janzen \& W. Hallwachs; otherCatalogNumbers: ASTAT094-06, 02-SRNP-12710,; identifiedBy: AJ Fleming; dateldentified: 2017; language: en; institutionCode: CNC; collectionCode: Insects; basisOfRecord: Pinned Specimen

i. ScientificName: Hyphantrophaga angustata; phylum: Arthropoda; class: Insecta; order: Diptera; family: Tachinidae; genus: Hyphantrophaga; specificEpithet: angustata; scientificNameAuthorship: (van der Wulp, 1890); continent: Central America; country: Costa Rica; countryCode: CR; stateProvince: Guanacaste; county: Sector Santa Rosa; locality: Area de Conservacion Guanacaste; verbatimLocality: Luces; verbatimElevation: 300; verbatimLatitude: 10.8536; verbatimLongitude: -85.6094; verbatimCoordinateSystem: Decimal; decimalLatitude: 10.8536 ; decimalLongitude: -85.6094; samplingProtocol: Reared from the larva of the Thyrididae, Dysodia speculifera; verbatimEventDate: 24-Jul-1990; individualID: DHJPAR0007324; individualCount: 1; lifeStage: adult; preparations: pinned; catalog Number: DHJPAR0007324; occurrenceDetails: http://janzen.sas.upenn.edu; recordedBy: D.H. Janzen, W. Hallwachs \& gusaneros; otherCatalogNumbers: ASTAT096-06, 90- 
SRNP-975, BOLD:AAD0627; identifiedBy: AJ Fleming; dateldentified: 2017; language: en; institutionCode: CNC; collectionCode: Insects; basisOfRecord: Pinned Specimen

j. $\quad$ scientificName: Hyphantrophaga angustata; phylum: Arthropoda; class: Insecta; order: Diptera; family: Tachinidae; genus: Hyphantrophaga; specificEpithet: angustata; scientificNameAuthorship: (van der Wulp, 1890); continent: Central America; country: Costa Rica; countryCode: CR; stateProvince: Guanacaste; county: Sector Santa Rosa; locality: Area de Conservacion Guanacaste; verbatimLocality: Area Administrativa; verbatimElevation: 295; verbatimLatitude: 10.8376; verbatimLongitude: -85.6187; verbatimCoordinateSystem: Decimal; decimalLatitude: 10.8376; decimalLongitude: -85.6187; samplingProtocol: Reared from the larva of the Thyrididae, Dysodia speculifera; verbatimEventDate: 18-Jun-2003; individualID: DHJPAR0007318; individualCount: 1; lifeStage: adult; preparations: pinned; catalogNumber: DHJPAR0007318; occurrenceDetails: http://janzen.sas.upenn.edu; recordedBy: D.H. Janzen \& W. Hallwachs; otherCatalogNumbers: ASTAT090-06, 02-SRNP-12713, BOLD:AAD0627; identifiedBy: AJ Fleming; dateldentified: 2017; language: en; institutionCode: CNC; collectionCode: Insects; basisOfRecord: Pinned Specimen

\section{Description}

Male (Fig. 5). Length 10-12 mm. Head (Fig. 5b): vertex 1/3 of head width; three pairs of reclinate upper orbital setae; ocellar setae arising behind anterior ocellus; ocellar triangle slightly gold-tinged compared to rest of fronto-orbital plate; fronto-orbital plate shiny silver and setulose, setulae not extending below lowest frontal seta; parafacial silver and bare; eye densely haired; facial ridge bare; pedicel black, with orange at base where it joins the postpedicel, remainder concolorous with postpedicel; arista orange basally, very minutely pubescent, distinctly thickened on basal $1 / 3-1 / 4$; palpus dark brown and haired basally, apically orange and bare. Thorax (Fig. 5a, c): silver grey tomentose dorsally and laterally; four thin dorsal vittae, outermost two broken across suture, innermost pair unbroken, reaching just beyond 1st postsutural dorsocentral seta; postpronotum with 4-5 setae arranged in a triangle; chaetotaxy: acrostichal setae 3-4:3; dorsocentral setae 3-4:3; intra-alar setae 3:3; supra-alar setae 2:3; three katepisternal setae; lateral scutellar setae subequal in length to basal scutellar setae; subapical scutellar setae strongest and longest of marginal scutellar setae, strongly divergent; apical scutellar setae subequal to lateral scutellar setae 2/3 length of subapical scutellar setae, crossed apically; one pair of discal scutellar setae set more widely set than apical scutellar setae but more narrowly than subapical scutellar setae; scutellum very slightly darkened across basal $20 \%$, remainder concolorous with scutum. Legs (Fig. 5c): yellow in ground colour with dense covering of black hairs, making them appear darker; fore femur with dense silver tomentum on posterodorsal surface; hind coxa bare. Wing (Fig. 5a): pale translucent, slightly infuscate; vein $\mathrm{R}_{4+5}$ with two setulae at base. Abdomen (Fig. $5 \mathrm{a}, \mathrm{c}$ ): ground colour black; mid-dorsal depression on ST1+2 not reaching hind margin; median marginal setae present on ST1+2 and T3; a complete row of marginal setae present on T4; discal setae only on T5; sex patch covering ventral surfaces of T4-T5; distinct tomentose bands along anterior $2 / 3$ of T3 and T4, broken medially by a dorsocentral stripe; T5 with silver tomentum covering anterior $1 / 3$, apparent only laterally but appearing as if it covers entire tergite under certain angles of light. Terminalia (Fig. $5 d$, 
e, f): anterior plate of sternite 5 (Fig. $5 f$ ) with broadly rounded edge, with a very slight, almost absent medial depression; posterior edge with a deeply excavated median cleft, smoothly U-shaped, margins covered in dense tomentum; unsclerotised "window" anterior to median cleft umbonate, $2 \mathrm{X}$ as wide as median cleft. Posterior lobes of sternite rounded apically, with 4-6 strong setae surrounded by many shorter, weaker setulae; anterior plate of sternite $2 \mathrm{X}$ as long as length of posterior lobes. Cerci in posterior view (Fig. 5d) long, slender and digitiform, longer than surstyli; blunt and rounded at apex, completely separate medially; densely setulose along basal $2 / 3$ dorsally, setulose ventrally along entire length (visible in lateral view) ; in lateral view (Fig. 5e) with a slight downward curve apically. Surstylus in lateral view almost parallelsided along its length, with a rounded apex, giving the whole structure a spatulate appearance; when viewed dorsally the surstyli appearing to converge slightly. Pregonite broad and well-developed, tapering to a rounded, slightly blunted apex; devoid of setulae. Postgonite narrow up to $1 / 3$, as wide as pregonite, sharply pointed and curved at apex, scythe-like and subequal in length to pregonite. Epiphallus welldeveloped and apically hooked. Distiphallus rectangular in shape, with a slender median longitudinal sclerotised reinforcement on its posterior surface and a broad, anterolateral, sclerotised acrophallus, joining on both sides of anterior surface near apex.

Female. Length: 8-11 mm. As male, differing only by the presence of two pairs of proclinate orbital setae.

\section{Diagnosis}

Hyphantrophaga angustata (van der Wulp) can be distinguished from all other Hyphantrophaga species by the following combination of traits: pedicel orange at base, palpus reddish-orange apically, black basally, three katepisternal setae, legs yellow with dense tomentum making them appear black, hind coxa bare, abdominal ground colour black, abdomen with silver tomentum, T5 with silver tomentum ventrolaterally, median marginal setae present on ST1+2.

\section{Distribution}

From south-western USA south to Costa Rica; Costa Rica, ACG, Guanacaste Province, 295-325 m elevation.

\section{Ecology}

Within ACG inventory, Hyphantrophaga angustata (van der Wulp) has been reared 10 times from two species of Lepidoptera in the family Thyrididae, Dysodia speculifera (Sepp, 1852) and Dysodia immargo Dyar, 1913, in dry forest and dry-rain lowland intergrade. 


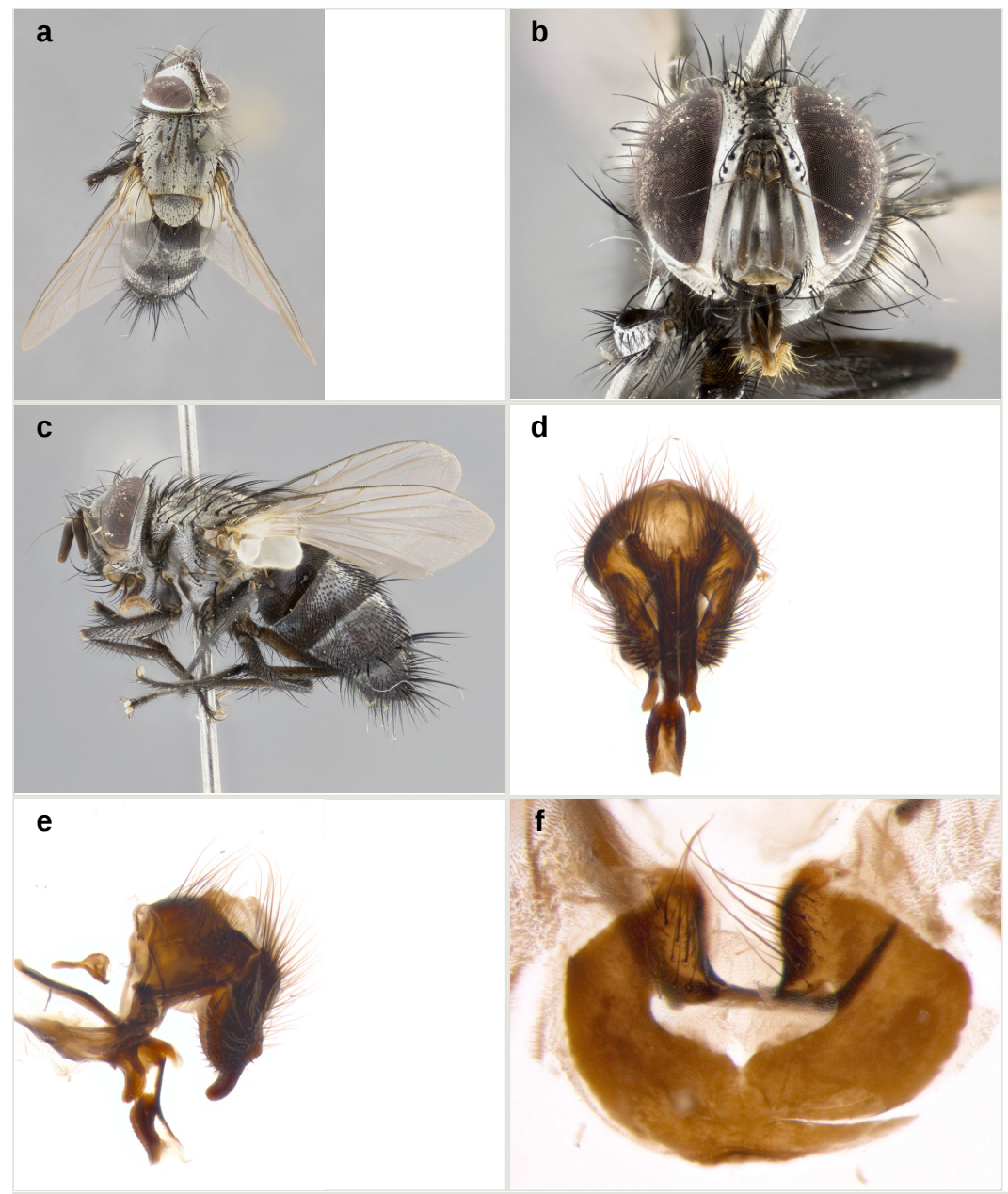

Figure 5.

Hyphantrophaga angustata (van der Wulp, 1890); a-c: habitus; male (ACG), voucher n. DHJPAR0007323; d-f: terminalia; male (ACG), voucher n. DHJPAR0007324.

a: dorsal view doi

b: frontal view doi

c: lateral view doi

d: dorsal view doi

e: lateral view doi

f: sternite 5 , ventral view doi 


\section{Hyphantrophaga calixtomoragai Fleming \& Wood, sp. n.}

- ZooBank urn:Isid:Zoobank.org:act:14DA9F7F-80ED-4D69-93CD-C185F76FC148

\section{Materials}

Holotype:

a. $\quad$ scientificName: Hyphantrophaga calixtomoragai; phylum: Arthropoda; class: Insecta; order: Diptera; family: Tachinidae; genus: Hyphantrophaga; specificEpithet: calixtomoragai; scientificNameAuthorship: Fleming \& Wood, 2018; continent: Central America; country: Costa Rica; countryCode: CR; stateProvince: Guanacaste; county: Sector Santa Rosa; locality: Area de Conservacion Guanacaste; verbatimLocality: Chiringon; verbatimElevation: 250; verbatimLatitude: 10.8388; verbatimLongitude: -85.601; verbatimCoordinateSystem: Decimal; decimalLatitude: 10.8388; decimalLongitude: -85.601; samplingProtocol: Reared from the larva of the Erebidae, Melipotis perpendicularisDHJ02; verbatimEventDate: 24-Jun-1992; individualID: DHJPAR0007342; individualCount: 1; sex: male; lifeStage: adult; preparations: pinned; catalogNumber: DHJPAR0007342; occurrenceDetails: http://janzen.sas.upenn.edu; recordedBy: D.H. Janzen, W. Hallwachs \& Freddy Quesada; otherCatalogNumbers: ASTAT114-06, 92-SRNP-1048.11, BOLD:ACE3368; identifiedBy: AJ Fleming; dateldentified: 2017; language: en; institutionCode: CNC; collectionCode: Insects; basisOfRecord: Pinned Specimen

\section{Paratypes:}

a. ScientificName: Hyphantrophaga calixtomoragai; phylum: Arthropoda; class: Insecta; order: Diptera; family: Tachinidae; genus: Hyphantrophaga; specificEpithet: calixtomoragai; scientificNameAuthorship: Fleming \& Wood, 2018; continent: Central America; country: Costa Rica; countryCode: CR; stateProvince: Guanacaste; county: Sector Santa Rosa; locality: Area de Conservacion Guanacaste; verbatimLocality: Quebrada Costa Rica; verbatimElevation: 275; verbatimLatitude: 10.8274; verbatimLongitude: -85.6365 ; verbatimCoordinateSystem: Decimal; decimalLatitude: 10.8274; decimalLongitude: -85.6365; samplingProtocol: Reared from the larva of the Erebidae, Metria leucoplaga; verbatimEventDate: 28-Apr-1992; individualID:

DHJPAR0007343; individualCount: 1; sex: female; lifeStage: adult; preparations: pinned; catalogNumber: DHJPAR0007343; occurrenceDetails: http:// janzen.sas.upenn.edu; recordedBy: D.H. Janzen, W. Hallwachs \& gusaneros; otherCatalogNumbers: ASTAT115-06, 91-SRNP-522, BOLD:ACE3368; identifiedBy: AJ Fleming; dateldentified: 2017; language: en; institutionCode: CNC; collectionCode: Insects; basisOfRecord: Pinned Specimen

b. $\quad$ scientificName: Hyphantrophaga calixtomoragai; phylum: Arthropoda; class: Insecta; order: Diptera; family: Tachinidae; genus: Hyphantrophaga; specificEpithet: calixtomoragai; scientificNameAuthorship: Fleming \& Wood, 2018; continent: Central America; country: Costa Rica; countryCode: CR; stateProvince: Guanacaste; county: Sector Santa Rosa; locality: Area de Conservacion Guanacaste; verbatimLocality: Vado Poza Salada; verbatimElevation: 8; verbatimLatitude: 10.798; verbatimLongitude: -85.6498; verbatimCoordinateSystem: Decimal; decimalLatitude: 10.798; decimalLongitude: -85.6498; samplingProtocol: Reared from the larva of the Erebidae, Toxonprucha Poole01; verbatimEventDate: 08-Apr-2006; individualID:

DHJPAR0019834; individualCount: 1; sex: male; lifeStage: adult; preparations: pinned; catalogNumber: DHJPAR0019834; occurrenceDetails: http://janzen.sas.upenn.edu; recordedBy: D.H. Janzen, W. Hallwachs \& Mariano Pereira; otherCatalogNumbers: 
ASTAB382-07, 06-SRNP-15096, BOLD:ACE3368; identifiedBy: AJ Fleming; dateldentified: 2017; language: en; institutionCode: CNC; collectionCode: Insects; basisOfRecord: Pinned Specimen

c. scientificName: Hyphantrophaga calixtomoragai; phylum: Arthropoda; class: Insecta; order: Diptera; family: Tachinidae; genus: Hyphantrophaga; specificEpithet: calixtomoragai; scientificNameAuthorship: Fleming \& Wood, 2018; continent: Central America; country: Costa Rica; countryCode: CR; stateProvince: Guanacaste; county: Sector Mundo Nuevo; locality: Area de Conservacion Guanacaste; verbatimLocality: Estacion La Perla; verbatimElevation: 325; verbatimLatitude: 10.7674; verbatimLongitude: -85.4331; verbatimCoordinateSystem: Decimal; decimalLatitude: 10.7674; decimalLongitude: -85.4331; samplingProtocol: Reared from the larva of the Erebidae, Ramphia albizona; verbatimEventDate: 08-Aug-2013; individualID:

DHJPAR0052586; individualCount: 1; sex: male; lifeStage: adult; preparations: pinned; catalogNumber: DHJPAR0052586; occurrenceDetails: http://janzen.sas.upenn.edu; recordedBy: D.H. Janzen, W. Hallwachs \& Mariano Pereira; otherCatalogNumbers: ASHYM1940-13, 13-SRNP-55372, BOLD:ACE3368; identifiedBy: AJ Fleming; dateldentified: 2017; language: en; institutionCode: CNC; collectionCode: Insects; basisOfRecord: Pinned Specimen

d. $\quad$ scientificName: Hyphantrophaga calixtomoragai; phylum: Arthropoda; class: Insecta; order: Diptera; family: Tachinidae; genus: Hyphantrophaga; specificEpithet: calixtomoragai; scientificNameAuthorship: Fleming \& Wood, 2018; continent: Central America; country: Costa Rica; countryCode: CR; stateProvince: Guanacaste; county: Sector Mundo Nuevo; locality: Area de Conservacion Guanacaste; verbatimLocality: Estacion La Perla; verbatimElevation: 325; verbatimLatitude: 10.7674; verbatimLongitude: -85.4331; verbatimCoordinateSystem: Decimal; decimalLatitude: 10.7674; decimalLongitude: -85.4331; samplingProtocol: Reared from the larva of the Erebidae, Ramphia albizona; verbatimEventDate: 05-Mar-2014; individualID: DHJPAR0055058; individualCount: 1; sex: male; lifeStage: adult; preparations: pinned; catalogNumber: DHJPAR0055058; occurrenceDetails: http://janzen.sas.upenn.edu; recordedBy: D.H. Janzen, W. Hallwachs \& gusaneros; otherCatalogNumbers: ASHYH1605-14, 13-SRNP-55366, BOLD:ACE3368; identifiedBy: AJ Fleming; dateldentified: 2017; language: en; institutionCode: CNC; collectionCode: Insects; basisOfRecord: Pinned Specimen

e. $\quad$ scientificName: Hyphantrophaga calixtomoragai; phylum: Arthropoda; class: Insecta; order: Diptera; family: Tachinidae; genus: Hyphantrophaga; specificEpithet: calixtomoragai; scientificNameAuthorship: Fleming \& Wood, 2018; continent: Central America; country: Costa Rica; countryCode: CR; stateProvince: Guanacaste; county: Sector Mundo Nuevo; locality: Area de Conservacion Guanacaste; verbatimLocality: Estacion La Perla; verbatimElevation: 325; verbatimLatitude: 10.7674; verbatimLongitude: -85.4331; verbatimCoordinateSystem: Decimal; decimalLatitude: 10.7674; decimalLongitude: -85.4331; samplingProtocol: Reared from the larva of the Erebidae, Ramphia albizona; verbatimEventDate: 22-Feb-2014; individualID: DHJPAR0055059; individualCount: 1; sex: female; lifeStage: adult; preparations: pinned; catalogNumber: DHJPAR0055059; occurrenceDetails: http:// janzen.sas.upenn.edu; recordedBy: D.H. Janzen, W. Hallwachs \& Guillermo Pereira; otherCatalogNumbers: ASHYH1606-14, 13-SRNP-55364, BOLD:ACE3368; identifiedBy: AJ Fleming; dateldentified: 2017; language: en; institutionCode: CNC; collectionCode: Insects; basisOfRecord: Pinned Specimen

f. ScientificName: Hyphantrophaga calixtomoragai; phylum: Arthropoda; class: Insecta; order: Diptera; family: Tachinidae; genus: Hyphantrophaga; specificEpithet: 
calixtomoragai; scientificNameAuthorship: Fleming \& Wood, 2018; continent: Central America; country: Costa Rica; countryCode: CR; stateProvince: Guanacaste; county: Sector Mundo Nuevo; locality: Area de Conservacion Guanacaste; verbatimLocality: Sendero Aguacate; verbatimElevation: 335; verbatimLatitude: 10.769; verbatimLongitude: -85.4346 ; verbatimCoordinateSystem: Decimal; decimalLatitude: 10.769; decimalLongitude: -85.4346; samplingProtocol: Reared from the larva of the Erebidae, Smyra stipatura; verbatimEventDate: 31-Jul-2014; individualID: DHJPAR0055854; individualCount: 1; sex: female; lifeStage: adult; preparations: pinned; catalogNumber: DHJPAR0055854; occurrenceDetails: http:// janzen.sas.upenn.edu; recordedBy: D.H. Janzen, W. Hallwachs \& Freddy Quesada; otherCatalogNumbers: ASHYH2586-14, 14-SRNP-55795, BOLD:ACE3368; identifiedBy: AJ Fleming; dateldentified: 2017; language: en; institutionCode: CNC; collectionCode: Insects; basisOfRecord: Pinned Specimen

g. $\quad$ scientificName: Hyphantrophaga calixtomoragai; phylum: Arthropoda; class: Insecta; order: Diptera; family: Tachinidae; genus: Hyphantrophaga; specificEpithet: calixtomoragai; scientificNameAuthorship: Fleming \& Wood, 2018; continent: Central America; country: Costa Rica; countryCode: CR; stateProvince: Guanacaste; county: Sector Mundo Nuevo; locality: Area de Conservacion Guanacaste; verbatimLocality: Sendero Aguacate; verbatimElevation: 335; verbatimLatitude: 10.769; verbatimLongitude: -85.4346; verbatimCoordinateSystem: Decimal; decimalLatitude: 10.769; decimalLongitude: -85.4346; samplingProtocol: Reared from the larva of the Erebidae, Smyra stipatura; verbatimEventDate: 03-Jul-2014; individualID: DHJPAR0055856; individualCount: 1; sex: male; lifeStage: adult; preparations: pinned; catalogNumber: DHJPAR0055856; occurrenceDetails: http://janzen.sas.upenn.edu; recordedBy: D.H. Janzen, W. Hallwachs \& Harry Ramirez; otherCatalogNumbers: ASHYH2588-14, 14-SRNP-55796, BOLD:ACE3368; identifiedBy: AJ Fleming; dateldentified: 2017; language: en; institutionCode: CNC; collectionCode: Insects; basisOfRecord: Pinned Specimen

\section{Description}

Male (Fig. 6). Length: 8-12 mm. Head (Fig. 6b): vertex 1/4 of head width; two pairs of reclinate upper orbital setae; ocellar setae arising behind anterior ocellus; ocellar triangle gold; fronto-orbital plate brilliant silver and setulose, setulae not extending below lowest frontal seta; parafacial shiny silver and bare; eye densely haired; facial ridge bare; pedicel orange, postpedicel dark brown-black; arista brown, very minutely pubescent, distinctly thickened on basal 1/3-1/4; palpus orange, clubbed and densely haired apically. Thorax (Fig. 6a, c): silver tomentose dorsally and laterally; densely covered with many short black setulae along anterior and lateral surfaces, interspersed amongst the setae; four thin dorsal vittae, outermost two broken across suture, innermost pair unbroken, almost reaching 2nd postsutural dorsocentral seta; postpronotum with 4-5 setae arranged in a triangle; chaetotaxy: acrostichal setae 3:3; dorsocentral setae 3-4:4; intra-alar setae 3-4:3; supra-alar setae 2:3; three katepisternal setae; basal scutellar setae curving inwards medially, as long as subapical setae; lateral scutellar setae less than $1 / 2$ as long as subapical scutellar setae; apical scutellar setae $1 / 3$ as long as subapical setae, crossed apically; one pair of discal scutellar setae set as widely apart as subapical scutellar setae; scutellum concolorous with scutum. Legs (Fig. 6c): brilliant yellow in ground colour except femora 
which appear reddish-brown; fore femur with dense silver tomentum on posterodorsal surface; hind coxa setose. Wing (Fig. 6a): pale translucent, hyaline, not distinctly infuscate; vein $\mathrm{R}_{4+5}$ with only 2-3 setulae at base. Abdomen (Fig. 6a, c): ground colour dark brown-black; middorsal depression on ST1+2 almost reaching hind margin; median marginal setae reduced but present on ST1+2, strong on T3; a complete row of marginal setae present on T4; discal setae only on T5; sex patch indistinct/absent; silver tomentose bands present along anterior edge of T3 and T4, unbroken medially and covering almost $90 \%$ of tergal surface; T5 with silver tomentum throughout. Terminalia (Fig. 6d, e, f): sternite 5 (Fig. 6f) with a deeply excavated median cleft, smoothly U-shaped, margins covered in dense tomentum. Lateral lobes of sternite rounded apically, devoid of any strong setae. Anterior plate of sternite 5 from subequal to slightly shorter than apical lobes; unsclerotised "window" anterior to median cleft rectangular, narrow and as wide as median cleft. Cerci in posterior view (Fig. 6d) subrectangular and slightly shorter than surstyli, blunt and slightly clubbed at apex, completely separate medially but not significantly divergent; in lateral view straight with a blunt apical hook slightly downwardly curved; densely setulose along entire length dorsally, setulose ventrally along entire length (visible in lateral view). Surstylus in lateral view (Fig. 6e) almost parallel-sided along its length, ending in a slightly pointed apex, making the structure appear blade-like; when viewed dorsally, surstyli appearing to point outwards, not strongly convergent. Pregonite broad and well-developed, tapering apically to a rounded, slightly blunted apex; devoid of setulae. Postgonite narrow, up to $1 / 2$ as wide as pregonite, sharply pointed and curved at apex, scythe-like and subequal in length to pregonite. Epiphallus well-developed and apically hooked. Distiphallus rectangular in shape, with a slender median longitudinal sclerotised reinforcement on its posterior surface and a broad, anterolateral, sclerotised acrophallus, joining on both sides of anterior surface near apex.

Female. Length: 9-12 mm. As male, differing only by the presence of two pairs of proclinate orbital setae.

\section{Diagnosis}

Hyphantrophaga calixtomoragai sp. n. can be distinguished from all other Hyphantrophaga species by the following combination of traits: ocellar triangle gold, fronto-orbital plate silver and setulose, pedicel orange, three katepisternal setae, hind coxa setose, abdominal tomentum silver throughout, median marginal setae present on ST1+2, discal setae absent from T3 and T4 and sex patch absent.

\section{Etymology}

Hyphantrophaga calixtomoragai sp. $\mathbf{n}$. is named in recognition of Calixto Moraga Medina's dedication and work at finding and rearing the ACG caterpillars that contained tachinid larvae. 

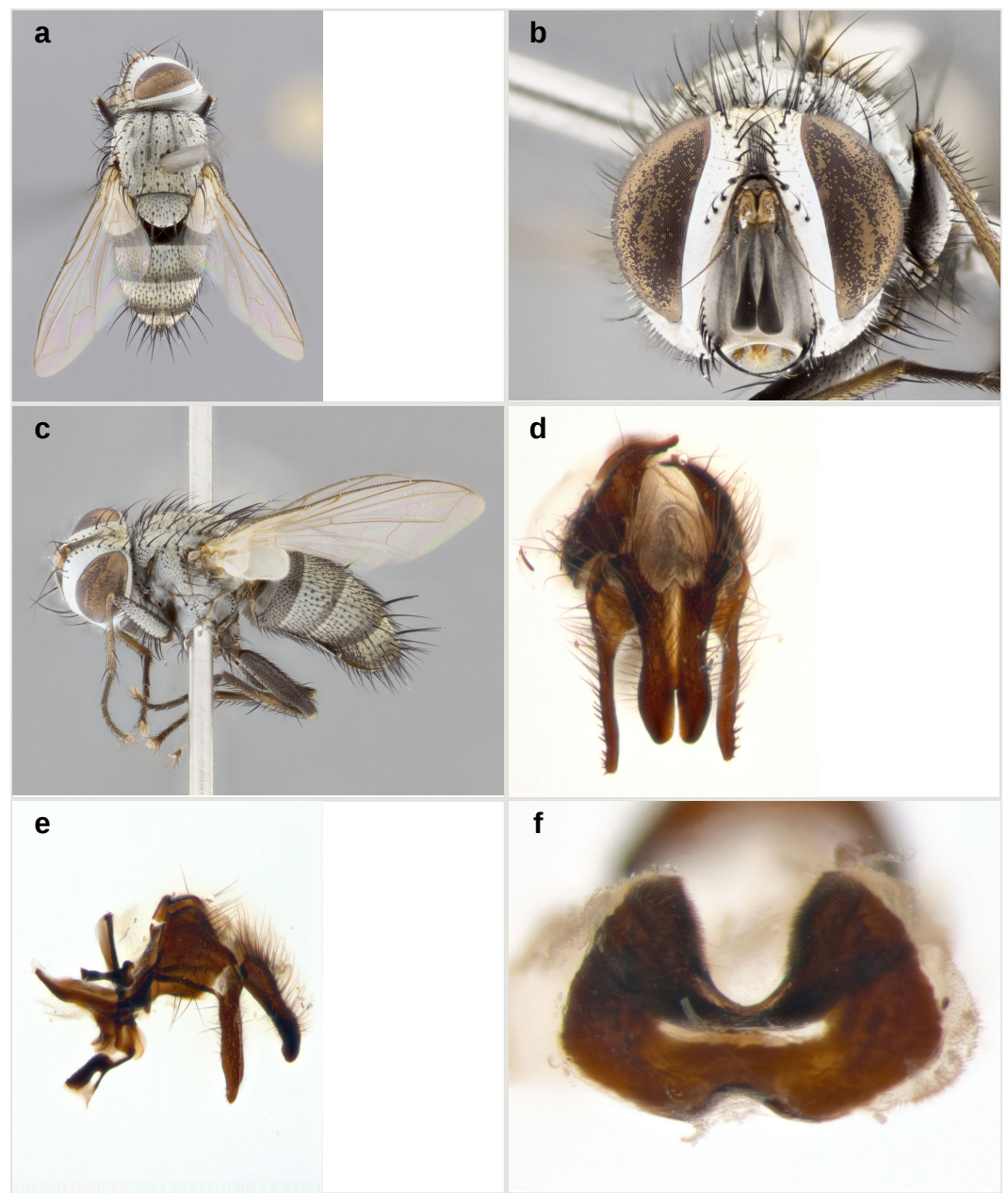

\section{Figure 6.}

Hyphantrophaga calixtomoragai sp. n.; a-c: habitus; holotype male, voucher $\mathrm{n}$. DHJPAR0007342; d-f: terminalia; male paratype, voucher n. DHJPAR0019834.
a: dorsal view doi
b: frontal view doi
c: lateral view doi
d: dorsal view doi
e: lateral view doi
f: sternite 5 , ventral view doi

\section{Distribution}

Costa Rica, ACG, Guanacaste Province, 8-215 m elevation. 


\section{Ecology}

Hyphantrophaga calixtomoragai sp. $\mathbf{n}$. has been reared six times from six different species of Lepidoptera in the family Erebidae, Metria leucoplaga (Hampson, 1910), Melipotis perpendicularisDHJ02, Toxonprucha Poole01, Abacena accincta Felder \& Rogenhofer, 1874, Ramphia albizona (Latreille, 1817) and Smyra stipatura (Walker, 1858); in dry forest and dry-rain lowland intergrades.

\section{Hyphantrophaga calva Fleming \& Wood, sp. n.}

\section{- ZooBank urn:Isid:zoobank.org:act:27A02B9A-6716-4EC2-B2B7-7129B6466B27}

\section{Materials}

\section{Holotype:}

a. $\quad$ scientificName: Hyphantrophaga calva; phylum: Arthropoda; class: Insecta; order: Diptera; family: Tachinidae; genus: Hyphantrophaga; specificEpithet: calva; scientificNameAuthorship: Fleming \& Wood, 2018; continent: Central America; country: Costa Rica; countryCode: CR; stateProvince: Alajuela; county: Sector San Cristobal; locality: Area de Conservacion Guanacaste; verbatimLocality: Puente Palma; verbatimElevation: 460; verbatimLatitude: 10.9163; verbatimLongitude: -85.3787; verbatimCoordinateSystem: Decimal; decimalLatitude: 10.9163; decimalLongitude: -85.3787; samplingProtocol: Reared from the larvae of the Depressariidae, Anadasmus Janzen11; verbatimEventDate: 14-Jun-2007; individualID: DHJPAR0019630; individualCount: 1; sex: male; lifeStage: adult; preparations: pinned; catalogNumber: DHJPAR0019630; occurrenceDetails: http://janzen.sas.upenn.edu; recordedBy: D.H. Janzen, W. Hallwachs \& Osvaldo Espinoza; otherCatalogNumbers: ASTAB178-07, 07SRNP-2267, BOLD:AAC5447; identifiedBy: AJ Fleming; dateldentified: 2017; language: en; institutionCode: CNC; collectionCode: Insects; basisOfRecord: Pinned Specimen

\section{Paratypes:}

a. ScientificName: Hyphantrophaga calva; phylum: Arthropoda; class: Insecta; order: Diptera; family: Tachinidae; genus: Hyphantrophaga; specificEpithet: calva; scientificNameAuthorship: Fleming \& Wood, 2018; continent: Central America; country: Costa Rica; countryCode: CR; stateProvince: Guanacaste; county: Sector Del Oro; locality: Area de Conservacion Guanacaste; verbatimLocality: Quebrada Oro; verbatimElevation: 290; verbatimLatitude: 11.0338; verbatimLongitude: -85.4771; verbatimCoordinateSystem: Decimal; decimalLatitude: 11.0338; decimalLongitude: -85.4771; samplingProtocol: Reared from the larvae of the Depressariidae, Anadasmus Janzen11; verbatimEventDate: 22-Dec-2005; individualID: DHJPAR0006678; individualCount: 1; sex: male; lifeStage: adult; preparations: pinned; catalogNumber: DHJPAR0006678; occurrenceDetails: http://janzen.sas.upenn.edu; recordedBy: D.H. Janzen, W. Hallwachs \& Lucia Rios; otherCatalogNumbers: ASTA856-06, 05SRNP-25287, BOLD:AAC5447; identifiedBy: AJ Fleming; dateldentified: 2017; language: en; institutionCode: CNC; collectionCode: Insects; basisOfRecord: Pinned Specimen

b. $\quad$ scientificName: Hyphantrophaga calva; phylum: Arthropoda; class: Insecta; order: Diptera; family: Tachinidae; genus: Hyphantrophaga; specificEpithet: calva; scientificNameAuthorship: Fleming \& Wood, 2018; continent: Central America; country: 
Costa Rica; countryCode: CR; stateProvince: Alajuela; county: Sector Rincon Rain Forest; locality: Area de Conservacion Guanacaste; verbatimLocality: Puente Rio Negro; verbatimElevation: 340; verbatimLatitude: 10.9038; verbatimLongitude: -85.3027; verbatimCoordinateSystem: Decimal; decimalLatitude: 10.9038; decimalLongitude: -85.3027; samplingProtocol: Reared from the larvae of the Depressariidae, Cerconota Janzen102; verbatimEventDate: 13-Feb-2004; individualID: DHJPAR0007491; individualCount: 1; sex: male; lifeStage: adult; preparations: pinned; catalogNumber: DHJPAR0007491; occurrenceDetails: http://janzen.sas.upenn.edu; recordedBy: D.H. Janzen, W. Hallwachs \& Minor Carmona; otherCatalogNumbers: ASTAT263-06, 04-SRNP-40127, BOLD:AAC5447; identifiedBy: AJ Fleming; dateldentified: 2017; language: en; institutionCode: CNC; collectionCode: Insects; basisOfRecord: Pinned Specimen

c. ScientificName: Hyphantrophaga calva; phylum: Arthropoda; class: Insecta; order: Diptera; family: Tachinidae; genus: Hyphantrophaga; specificEpithet: calva; scientificNameAuthorship: Fleming \& Wood, 2018; continent: Central America; country: Costa Rica; countryCode: CR; stateProvince: Alajuela; county: Sector Rincon Rain Forest; locality: Area de Conservacion Guanacaste; verbatimLocality: Puente Rio Negro; verbatimElevation: 340 ; verbatimLatitude: 10.9038; verbatimLongitude: -85.3027; verbatimCoordinateSystem: Decimal; decimalLatitude: 10.9038; decimalLongitude: -85.3027; samplingProtocol: Reared from the larvae of the Depressariidae, Cerconota Janzen102; verbatimEventDate: 14-Feb-2004; individualID: DHJPAR0007492; individualCount: 1; sex: male; lifeStage: adult; preparations: pinned; catalogNumber: DHJPAR0007492; occurrenceDetails: http://janzen.sas.upenn.edu; recordedBy: D.H. Janzen, W. Hallwachs \& Minor Carmona; otherCatalogNumbers: ASTAT264-06, 04-SRNP-40125, BOLD:AAC5447; identifiedBy: AJ Fleming; dateldentified: 2017; language: en; institutionCode: CNC; collectionCode: Insects; basisOfRecord: Pinned Specimen

d. ScientificName: Hyphantrophaga calva; phylum: Arthropoda; class: Insecta; order: Diptera; family: Tachinidae; genus: Hyphantrophaga; specificEpithet: calva; scientificNameAuthorship: Fleming \& Wood, 2018; continent: Central America; country: Costa Rica; countryCode: CR; stateProvince: Alajuela; county: Sector Rincon Rain Forest; locality: Area de Conservacion Guanacaste; verbatimLocality: Puente Rio Negro; verbatimElevation: 340 ; verbatimLatitude: 10.9038; verbatimLongitude: -85.3027; verbatimCoordinateSystem: Decimal; decimalLatitude: 10.9038; decimalLongitude: -85.3027; samplingProtocol: Reared from the larvae of the Depressariidae, Cerconota Janzen102; verbatimEventDate: 24-Feb-2004; individualID: DHJPAR0007493; individualCount: 1; sex: female; lifeStage: adult; preparations: pinned; catalogNumber: DHJPAR0007493; occurrenceDetails: http:// janzen.sas.upenn.edu; recordedBy: D.H. Janzen, W. Hallwachs \& Minor Carmona; otherCatalog Numbers: ASTAT265-06, 04-SRNP-40128, BOLD:AAC5447; identifiedBy: AJ Fleming; dateldentified: 2017; language: en; institutionCode: CNC; collectionCode: Insects; basisOfRecord: Pinned Specimen

e. $\quad$ scientificName: Hyphantrophaga calva; phylum: Arthropoda; class: Insecta; order: Diptera; family: Tachinidae; genus: Hyphantrophaga; specificEpithet: calva; scientificNameAuthorship: Fleming \& Wood, 2018; continent: Central America; country: Costa Rica; countryCode: CR; stateProvince: Alajuela; county: Sector Rincon Rain Forest; locality: Area de Conservacion Guanacaste; verbatimLocality: San Lucas; verbatimElevation: 320; verbatimLatitude: 10.9185; verbatimLongitude: -85.3034; verbatimCoordinateSystem: Decimal; decimalLatitude: 10.9185 ; decimalLongitude: -85.3034; samplingProtocol: Reared from the larvae of the Depressariidae, Cerconota 
Janzen102; verbatimEventDate: 31-Mar-2004; individualID: DHJPAR0007494; individualCount: 1; sex: male; lifeStage: adult; preparations: pinned; catalogNumber: DHJPAR0007494; occurrenceDetails: http://janzen.sas.upenn.edu; recordedBy: D.H. Janzen, W. Hallwachs \& Jose Perez; otherCatalogNumbers: ASTAT266-06, 04SRNP-40543, BOLD:AAC5447; identifiedBy: AJ Fleming; dateldentified: 2017; language: en; institutionCode: CNC; collectionCode: Insects; basisOfRecord: Pinned Specimen

f. ScientificName: Hyphantrophaga calva; phylum: Arthropoda; class: Insecta; order: Diptera; family: Tachinidae; genus: Hyphantrophaga; specificEpithet: calva; scientificNameAuthorship: Fleming \& Wood, 2018; continent: Central America; country: Costa Rica; countryCode: CR; stateProvince: Alajuela; county: Sector San Cristobal; locality: Area de Conservacion Guanacaste; verbatimLocality: Puente Palma; verbatimElevation: 460; verbatimLatitude: 10.9163; verbatimLongitude: -85.3787; verbatimCoordinateSystem: Decimal; decimalLatitude: 10.9163; decimalLongitude: -85.3787; sampling Protocol: Reared from the larvae of the Depressariidae, Anadasmus Janzen11; verbatimEventDate: 10-Nov-2004; individualID: DHJPAR0007495; individualCount: 1; sex: female; lifeStage: adult; preparations: pinned; catalog Number: DHJPAR0007495; occurrenceDetails: http://janzen.sas.upenn.edu; recordedBy: D.H. Janzen, W. Hallwachs \& Gloria Sihezar; otherCatalog Numbers: ASTAT267-06, 04SRNP-60321, BOLD:AAC5447; identifiedBy: AJ Fleming; dateldentified: 2017; language: en; institutionCode: CNC; collectionCode: Insects; basisOfRecord: Pinned Specimen

g. scientificName: Hyphantrophaga calva; phylum: Arthropoda; class: Insecta; order: Diptera; family: Tachinidae; genus: Hyphantrophaga; specificEpithet: calva; scientificNameAuthorship: Fleming \& Wood, 2018; continent: Central America; country: Costa Rica; countryCode: CR; stateProvince: Alajuela; county: Sector San Cristobal; locality: Area de Conservacion Guanacaste; verbatimLocality: Sendero Corredor; verbatimElevation: 620; verbatimLatitude: 10.8787; verbatimLongitude: -85.3896; verbatimCoordinateSystem: Decimal; decimalLatitude: 10.8787; decimalLongitude: -85.3896; samplingProtocol: Reared from the larvae of the Depressariidae, Anadasmus Janzen25; verbatimEventDate: 23-Oct-2004; individualID: DHJPAR0007496; individualCount: 1; sex: female; lifeStage: adult; preparations: pinned; catalogNumber: DHJPAR0007496; occurrenceDetails: http://janzen.sas.upenn.edu; recordedBy: D.H. Janzen, W. Hallwachs \& Elda Araya; otherCatalog Numbers: ASTAT268-06, 04SRNP-4735, BOLD:AAC5447; identifiedBy: AJ Fleming; dateldentified: 2017; language: en; institutionCode: CNC; collectionCode: Insects; basisOfRecord: Pinned Specimen

h. ScientificName: Hyphantrophaga calva; phylum: Arthropoda; class: Insecta; order: Diptera; family: Tachinidae; genus: Hyphantrophaga; specificEpithet: calva; scientificNameAuthorship: Fleming \& Wood, 2018; continent: Central America; country: Costa Rica; countryCode: CR; stateProvince: Alajuela; county: Sector Rincon Rain Forest; locality: Area de Conservacion Guanacaste; verbatimLocality: Estacion Caribe; verbatimElevation: 415; verbatimLatitude: 10.9019; verbatimLongitude: -85.2749; verbatimCoordinateSystem: Decimal; decimalLatitude: 10.9019; decimalLongitude: -85.2749; sampling Protocol: Reared from the larvae of the Depressariidae, Anadasmus Janzen11; verbatimEventDate: 20-May-2005; individualID: DHJPAR0008084; individualCount: 1; sex: female; lifeStage: adult; preparations: pinned; catalog Number: DHJPAR0008084; occurrenceDetails: http://janzen.sas.upenn.edu; recordedBy: D.H. Janzen, W. Hallwachs \& Minor Carmona; otherCatalogNumbers: ASTAT856-06, 05SRNP-41074, BOLD:AAC5447; identifiedBy: AJ Fleming; dateldentified: 2017; 
language: en; institutionCode: CNC; collectionCode: Insects; basisOfRecord: Pinned Specimen

i. ScientificName: Hyphantrophaga calva; phylum: Arthropoda; class: Insecta; order: Diptera; family: Tachinidae; genus: Hyphantrophaga; specificEpithet: calva; scientificNameAuthorship: Fleming \& Wood, 2018; continent: Central America; country: Costa Rica; countryCode: CR; stateProvince: Alajuela; county: Sector Rincon Rain Forest; locality: Area de Conservacion Guanacaste; verbatimLocality: San Lucas; verbatimElevation: 320; verbatimLatitude: 10.9185; verbatimLongitude: -85.3034; verbatimCoordinateSystem: Decimal; decimalLatitude: 10.9185; decimalLongitude: -85.3034; samplingProtocol: Reared from the larvae of the Depressariidae, Anadasmus Janzen11; verbatimEventDate: 12-Nov-2003; individualID: DHJPAR0003327; individualCount: 1; sex: female; lifeStage: adult; preparations: pinned; catalog Number: DHJPAR0003327; occurrenceDetails: http://janzen.sas.upenn.edu; recordedBy: D.H. Janzen, W. Hallwachs \& Carolina Cano; otherCatalogNumbers: ASTA234-05, 03SRNP-13029.1, BOLD:AAC5447; identifiedBy: AJ Fleming; dateldentified: 2017; language: en; institutionCode: CNC; collectionCode: Insects; basisOfRecord: Pinned Specimen

j. $\quad$ scientific Name: Hyphantrophaga calva; phylum: Arthropoda; class: Insecta; order: Diptera; family: Tachinidae; genus: Hyphantrophaga; specificEpithet: calva; scientificNameAuthorship: Fleming \& Wood, 2018; continent: Central America; country: Costa Rica; countryCode: CR; stateProvince: Alajuela; county: Brasilia; locality: Area de Conservacion Guanacaste; verbatimLocality: Brisanta; verbatimElevation: 290; verbatimLatitude: 11.0274; verbatimLongitude: -85.3368 ; verbatimCoordinateSystem: Decimal; decimalLatitude: 11.0274; decimalLongitude: -85.3368; samplingProtocol: Reared from the larvae of the Depressariidae, Cerconota Janzen707; verbatimEventDate: 30-Jul-2007; individualID: DHJPAR0021046; individualCount: 1; sex: female; lifeStage: adult; preparations: pinned; catalogNumber: DHJPAR0021046; occurrenceDetails: http://janzen.sas.upenn.edu; recordedBy: D.H. Janzen, W. Hallwachs \& Duvalier Briceno; otherCatalogNumbers: ASTA1389-07, 07-SRNP-65192, BOLD:AAC5447; identifiedBy: AJ Fleming; dateldentified: 2017; language: en; institutionCode: CNC; collectionCode: Insects; basisOfRecord: Pinned Specimen

k. ScientificName: Hyphantrophaga calva; phylum: Arthropoda; class: Insecta; order: Diptera; family: Tachinidae; genus: Hyphantrophaga; specificEpithet: calva; scientificNameAuthorship: Fleming \& Wood, 2018; continent: Central America; country: Costa Rica; countryCode: CR; stateProvince: Alajuela; county: Sector Rincon Rain Forest; locality: Area de Conservacion Guanacaste; verbatimLocality: Estacion Caribe; verbatimElevation: 415; verbatimLatitude: 10.9019; verbatimLongitude: -85.2749; verbatimCoordinateSystem: Decimal; decimalLatitude: 10.9019; decimalLongitude: -85.2749; samplingProtocol: Reared from the larvae of the Depressariidae, Anadasmus Janzen11; verbatimEventDate: 18-Jul-2009; individualID: DHJPAR0035699; individualCount: 1; sex: female; lifeStage: adult; preparations: pinned; catalogNumber: DHJPAR0035699; occurrenceDetails: http://janzen.sas.upenn.edu; recordedBy: D.H. Janzen, W. Hallwachs \& Pablo Umaña Calderon; otherCatalogNumbers: ASHYD1080-09, 09-SRNP-41475, BOLD:AAC5447; identifiedBy: AJ Fleming; dateldentified: 2017; language: en; institutionCode: CNC; collectionCode: Insects; basisOfRecord: Pinned Specimen

I. scientificName: Hyphantrophaga calva; phylum: Arthropoda; class: Insecta; order: Diptera; family: Tachinidae; genus: Hyphantrophaga; specificEpithet: calva; scientificNameAuthorship: Fleming \& Wood, 2018; continent: Central America; country: Costa Rica; countryCode: CR; stateProvince: Alajuela; county: Sector Rincon Rain 
Forest; locality: Area de Conservacion Guanacaste; verbatimLocality: Sendero Parcelas; verbatimElevation: 375 ; verbatimLatitude: 10.9078; verbatimLongitude: -85.2914; verbatimCoordinateSystem: Decimal; decimalLatitude: 10.9078; decimalLongitude: -85.2914; samplingProtocol: Reared from the larvae of the Depressariidae, Anadasmus Janzen11; verbatimEventDate: 18-Jul-2010; individualID: DHJPAR0040132; individualCount: 1; sex: female; lifeStage: adult; preparations: pinned; catalogNumber: DHJPAR0040132; occurrenceDetails: http:// janzen.sas.upenn.edu; recordedBy: D.H. Janzen, W. Hallwachs \& Jose Perez; otherCatalogNumbers: ASHYE2299-11, 10-SRNP-42130, BOLD:AAC5447; identifiedBy: AJ Fleming; dateldentified: 2017; language: en; institutionCode: CNC; collectionCode: Insects; basisOfRecord: Pinned Specimen

m. scientificName: Hyphantrophaga calva; phylum: Arthropoda; class: Insecta; order: Diptera; family: Tachinidae; genus: Hyphantrophaga; specificEpithet: calva; scientificNameAuthorship: Fleming \& Wood, 2018; continent: Central America; country: Costa Rica; countryCode: CR; stateProvince: Guanacaste; county: Sector Pitilla; locality: Area de Conservacion Guanacaste; verbatimLocality: Medrano; verbatimElevation: 380; verbatimLatitude: 11.016; verbatimLongitude: -85.3805; verbatimCoordinateSystem: Decimal; decimalLatitude: 11.016; decimalLongitude: -85.3805; samplingProtocol: Reared from the larvae of the Depressariidae, Anadasmus Janzen11; verbatimEventDate: 18-Mar-2011; individualID: DHJPAR0042269; individualCount: 1; sex: female; lifeStage: adult; preparations: pinned; catalogNumber: DHJPAR0042269; occurrenceDetails: http://janzen.sas.upenn.edu; recordedBy: D.H. Janzen, W. Hallwachs \& Ricardo Calero; otherCatalogNumbers: ASHYH033-11, 11SRNP-70323, BOLD:AAC5447; identifiedBy: AJ Fleming; dateldentified: 2017; language: en; institutionCode: CNC; collectionCode: Insects; basisOfRecord: Pinned Specimen

n. scientificName: Hyphantrophaga calva; phylum: Arthropoda; class: Insecta; order: Diptera; family: Tachinidae; genus: Hyphantrophaga; specificEpithet: calva; scientificNameAuthorship: Fleming \& Wood, 2018; continent: Central America; country: Costa Rica; countryCode: CR; stateProvince: Guanacaste; county: Sector Pitilla; locality: Area de Conservacion Guanacaste; verbatimLocality: Bullas; verbatimElevation: 440; verbatimLatitude: 10.9867; verbatimLongitude: -85.385; verbatimCoordinateSystem: Decimal; decimalLatitude: 10.9867; decimalLongitude: -85.385; sampling Protocol: Reared from the larvae of the Depressariidae, Anadasmus Janzen11; verbatimEventDate: 09-Mar-2011; individualID: DHJPAR0042298; individualCount: 1; sex: male; lifeStage: adult; preparations: pinned; catalogNumber: DHJPAR0042298; occurrenceDetails: http://janzen.sas.upenn.edu; recordedBy: D.H. Janzen, W. Hallwachs \& Duvalier Briceno; otherCatalogNumbers: ASHYH062-11, 11SRNP-65093, BOLD:AAC5447; identifiedBy: AJ Fleming; dateldentified: 2017; language: en; institutionCode: CNC; collectionCode: Insects; basisOfRecord: Pinned Specimen

o. $\quad$ scientificName: Hyphantrophaga calva; phylum: Arthropoda; class: Insecta; order: Diptera; family: Tachinidae; genus: Hyphantrophaga; specificEpithet: calva; scientificNameAuthorship: Fleming \& Wood, 2018; continent: Central America; country: Costa Rica; countryCode: CR; stateProvince: Alajuela; county: Sector Rincon Rain Forest; locality: Area de Conservacion Guanacaste; verbatimLocality: San Lucas; verbatimElevation: 320; verbatimLatitude: 10.9185; verbatimLongitude: -85.3034; verbatimCoordinateSystem: Decimal; decimalLatitude: 10.9185; decimalLongitude: -85.3034; samplingProtocol: Reared from the larvae of the Depressariidae, Anadasmus Janzen11; verbatimEventDate: 12-Oct-2011; individualID: DHJPAR0045566; 
individualCount: 1; sex: male; lifeStage: adult; preparations: pinned; catalogNumber: DHJPAR0045566; occurrenceDetails: http://janzen.sas.upenn.edu; recordedBy: D.H. Janzen, W. Hallwachs \& Anabelle Cordoba; otherCatalogNumbers: ACGAZ755-11, 11SRNP-43978, BOLD:AAC5447; identifiedBy: AJ Fleming; dateldentified: 2017; language: en; institutionCode: CNC; collectionCode: Insects; basisOfRecord: Pinned Specimen

p. $\quad$ scientificName: Hyphantrophaga calva; phylum: Arthropoda; class: Insecta; order: Diptera; family: Tachinidae; genus: Hyphantrophaga; specificEpithet: calva; scientificNameAuthorship: Fleming \& Wood, 2018; continent: Central America; country: Costa Rica; countryCode: CR; stateProvince: Alajuela; county: Sector Rincon Rain Forest; locality: Area de Conservacion Guanacaste; verbatimLocality: San Lucas; verbatimElevation: 320; verbatimLatitude: 10.9185; verbatimLongitude: -85.3034; verbatimCoordinateSystem: Decimal; decimalLatitude: 10.9185; decimalLongitude: -85.3034; samplingProtocol: Reared from the larvae of the Depressariidae, Anadasmus Janzen11; verbatimEventDate: 29-Sep-2011; individualID: DHJPAR0045573; individualCount: 1; sex: male; lifeStage: adult; preparations: pinned; catalogNumber: DHJPAR0045573; occurrenceDetails: http://janzen.sas.upenn.edu; recordedBy: D.H. Janzen, W. Hallwachs \& Anabelle Cordoba; otherCatalogNumbers: ACGAZ762-11, 11SRNP-43974, BOLD:AAC5447; identifiedBy: AJ Fleming; dateldentified: 2017; language: en; institutionCode: CNC; collectionCode: Insects; basisOfRecord: Pinned Specimen

q. $\quad$ scientificName: Hyphantrophaga calva; phylum: Arthropoda; class: Insecta; order: Diptera; family: Tachinidae; genus: Hyphantrophaga; specificEpithet: calva; scientificNameAuthorship: Fleming \& Wood, 2018; continent: Central America; country: Costa Rica; countryCode: CR; stateProvince: Alajuela; county: Sector Rincon Rain Forest; locality: Area de Conservacion Guanacaste; verbatimLocality: Camino Albergue Oscar; verbatimElevation: 560; verbatimLatitude: 10.8774; verbatimLongitude: -85.3236; verbatimCoordinateSystem: Decimal; decimalLatitude: 10.8774; decimalLongitude: -85.3236; samplingProtocol: Reared from the larvae of the Depressariidae, Anadasmus Janzen11; verbatimEventDate: 22-Nov-2011; individualID: DHJPAR0046412; individualCount: 1; sex: male; lifeStage: adult; preparations: pinned; catalogNumber: DHJPAR0046412; occurrenceDetails: http://janzen.sas.upenn.edu; recordedBy: D.H. Janzen, W. Hallwachs \& Osvaldo Espinoza; otherCatalog Numbers: ACGBA585-12, 11-SRNP-4193, BOLD:AAC5447; identifiedBy: AJ Fleming; dateldentified: 2017; language: en; institutionCode: CNC; collectionCode: Insects; basisOfRecord: Pinned Specimen

r. ScientificName: Hyphantrophaga calva; phylum: Arthropoda; class: Insecta; order: Diptera; family: Tachinidae; genus: Hyphantrophaga; specificEpithet: calva; scientificNameAuthorship: Fleming \& Wood, 2018; continent: Central America; country: Costa Rica; countryCode: CR; stateProvince: Alajuela; county: Sector Rincon Rain Forest; locality: Area de Conservacion Guanacaste; verbatimLocality: San Lucas; verbatimElevation: 320; verbatimLatitude: 10.9185; verbatimLongitude: -85.3034; verbatimCoordinateSystem: Decimal; decimalLatitude: 10.9185; decimalLongitude: -85.3034; samplingProtocol: Reared from the larvae of the Depressariidae, Anadasmus Janzen11; verbatimEventDate: 25-Aug-2012; individualID: DHJPAR0049599; individualCount: 1; sex: male; lifeStage: adult; preparations: pinned; catalogNumber: DHJPAR0049599; occurrenceDetails: http://janzen.sas.upenn.edu; recordedBy: D.H. Janzen, W. Hallwachs \& Anabelle Cordoba; otherCatalogNumbers: ASHYB2393-12, 12-SRNP-44077, BOLD:AAC5447; identifiedBy: AJ Fleming; dateldentified: 2017; 
language: en; institutionCode: CNC; collectionCode: Insects; basisOfRecord: Pinned Specimen

s. ScientificName: Hyphantrophaga calva; phylum: Arthropoda; class: Insecta; order: Diptera; family: Tachinidae; genus: Hyphantrophaga; specificEpithet: calva; scientificNameAuthorship: Fleming \& Wood, 2018; continent: Central America; country: Costa Rica; countryCode: CR; stateProvince: Alajuela; county: Sector Rincon Rain Forest; locality: Area de Conservacion Guanacaste; verbatimLocality: San Lucas; verbatimElevation: 320; verbatimLatitude: 10.9185; verbatimLongitude: -85.3034; verbatimCoordinateSystem: Decimal; decimalLatitude: 10.9185; decimalLongitude: -85.3034; samplingProtocol: Reared from the larvae of the Depressariidae, Anadasmus Janzen11; verbatimEventDate: 26-Aug-2012; individualID: DHJPAR0050265; individualCount: 1; sex: female; lifeStage: adult; preparations: pinned; catalogNumber: DHJPAR0050265; occurrenceDetails: http://janzen.sas.upenn.edu; recordedBy: D.H. Janzen, W. Hallwachs \& Anabelle Cordoba; otherCatalogNumbers: ACGAZ1579-12, 12-SRNP-44076, BOLD:AAC5447; identifiedBy: AJ Fleming; dateldentified: 2017; language: en; institutionCode: CNC; collectionCode: Insects; basisOfRecord: Pinned Specimen

t. ScientificName: Hyphantrophaga calva; phylum: Arthropoda; class: Insecta; order: Diptera; family: Tachinidae; genus: Hyphantrophaga; specificEpithet: calva; scientificNameAuthorship: Fleming \& Wood, 2018; continent: Central America; country: Costa Rica; countryCode: CR; stateProvince: Alajuela; county: Sector Rincon Rain Forest; Iocality: Area de Conservacion Guanacaste; verbatimLocality: San Lucas; verbatimElevation: 320; verbatimLatitude: 10.9185; verbatimLongitude: -85.3034; verbatimCoordinateSystem: Decimal; decimalLatitude: 10.9185; decimalLongitude: -85.3034; samplingProtocol: Reared from the larvae of the Depressariidae, Anadasmus Janzen11; verbatimEventDate: 26-Aug-2012; individualID: DHJPAR0050273; individualCount: 1; sex: female; lifeStage: adult; preparations: pinned; catalogNumber: DHJPAR0050273; occurrenceDetails: http://janzen.sas.upenn.edu; recordedBy: D.H. Janzen, W. Hallwachs \& Anabelle Cordoba; otherCatalogNumbers: ACGAZ1587-12, 12-SRNP-44078, BOLD:AAC5447; identifiedBy: AJ Fleming; dateldentified: 2017; language: en; institutionCode: CNC; collectionCode: Insects; basisOfRecord: Pinned Specimen

u. ScientificName: Hyphantrophaga calva; phylum: Arthropoda; class: Insecta; order: Diptera; family: Tachinidae; genus: Hyphantrophaga; specificEpithet: calva; scientificNameAuthorship: Fleming \& Wood, 2018; continent: Central America; country: Costa Rica; countryCode: CR; stateProvince: Guanacaste; county: Sector Pitilla; Iocality: Area de Conservacion Guanacaste; verbatimLocality: Casa Roberto;

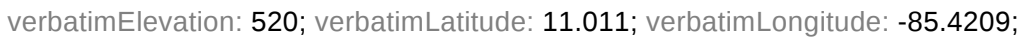
verbatimCoordinateSystem: Decimal; decimalLatitude: 11.011; decimalLongitude: -85.4209; samplingProtocol: Reared from the larvae of the Depressariidae, Chlamydastis phytopteraEPR03; verbatimEventDate: 18-Jan-2013; individualID: DHJPAR0050501; individualCount: 1; sex: female; lifeStage: adult; preparations: pinned; catalogNumber: DHJPAR0050501; occurrenceDetails: http:// janzen.sas.upenn.edu; recordedBy: D.H. Janzen, W. Hallwachs \& Manuel Rios; otherCatalogNumbers: ACGBA3093-13, 12-SRNP-31968, BOLD:AAC5447; identifiedBy: AJ Fleming; dateldentified: 2017; language: en; institutionCode: CNC; collectionCode: Insects; basisOfRecord: Pinned Specimen

v. ScientificName: Hyphantrophaga calva; phylum: Arthropoda; class: Insecta; order: Diptera; family: Tachinidae; genus: Hyphantrophaga; specificEpithet: calva; scientificNameAuthorship: Fleming \& Wood, 2018; continent: Central America; country: 
Costa Rica; countryCode: CR; stateProvince: Alajuela; county: Sector Rincon Rain Forest; locality: Area de Conservacion Guanacaste; verbatimLocality: Finca Esmeralda; verbatimElevation: 123; verbatimLatitude: 10.9355; verbatimLongitude: -85.2531; verbatimCoordinateSystem: Decimal; decimalLatitude: 10.9355; decimalLongitude: -85.2531; samplingProtocol: Reared from the larvae of the Depressariidae, Anadasmus Janzen11; verbatimEventDate: 16-Nov-2012; individualID: DHJPAR0050544; individualCount: 1; sex: female; lifeStage: adult; preparations: pinned; catalogNumber: DHJPAR0050544; occurrenceDetails: http:// janzen.sas.upenn.edu; recordedBy: D.H. Janzen, W. Hallwachs \& Mercedes Moraga; otherCatalogNumbers: ACGBA3136-13, 12-SRNP-77287, BOLD:AAC5447; identifiedBy: AJ Fleming; dateldentified: 2017; language: en; institutionCode: CNC; collectionCode: Insects; basisOfRecord: Pinned Specimen

w. ScientificName: Hyphantrophaga calva; phylum: Arthropoda; class: Insecta; order: Diptera; family: Tachinidae; genus: Hyphantrophaga; specificEpithet: calva; scientificNameAuthorship: Fleming \& Wood, 2018; continent: Central America; country: Costa Rica; countryCode: CR; stateProvince: Alajuela; county: Sector Rincon Rain Forest; locality: Area de Conservacion Guanacaste; verbatimLocality: Quebrada Escondida; verbatimElevation: 420; verbatimLatitude: 10.8993; verbatimLongitude: -85.2749; verbatimCoordinateSystem: Decimal; decimalLatitude: 10.8993; decimalLongitude: -85.2749; samplingProtocol: Reared from the larvae of the Depressariidae, Anadasmus Janzen11; verbatimEventDate: 06-Feb-2013; individualID: DHJPAR0050624; individualCount: 1; sex: female; lifeStage: adult; preparations: pinned; catalogNumber: DHJPAR0050624; occurrenceDetails: http:// janzen.sas.upenn.edu; recordedBy: D.H. Janzen, W. Hallwachs \& Jose Perez; otherCatalogNumbers: ACGBA3216-13, 13-SRNP-40038, BOLD:AAC5447; identifiedBy: AJ Fleming; dateldentified: 2017; language: en; institutionCode: CNC; collectionCode: Insects; basisOfRecord: Pinned Specimen

x. ScientificName: Hyphantrophaga calva; phylum: Arthropoda; class: Insecta; order: Diptera; family: Tachinidae; genus: Hyphantrophaga; specificEpithet: calva; scientificNameAuthorship: Fleming \& Wood, 2018; continent: Central America; country: Costa Rica; countryCode: CR; stateProvince: Alajuela; county: Sector Rincon Rain Forest; locality: Area de Conservacion Guanacaste; verbatimLocality: Quebrada Bambu; verbatimElevation: 109; verbatimLatitude: 10.9301; verbatimLongitude: -85.2521; verbatimCoordinateSystem: Decimal; decimalLatitude: 10.9301; decimalLongitude: -85.2521; samplingProtocol: Reared from the larvae of the Depressariidae, Anadasmus Janzen30; verbatimEventDate: 24-Dec-2012; individualID: DHJPAR0050651; individualCount: 1; sex: female; lifeStage: adult; preparations: pinned; catalogNumber: DHJPAR0050651; occurrenceDetails: http:// janzen.sas.upenn.edu; recordedBy: D.H. Janzen, W. Hallwachs \& Cirilo Umaña; otherCatalogNumbers: ACGBA3243-13, 12-SRNP-77921, BOLD:AAC5447; identifiedBy: AJ Fleming; dateldentified: 2017; language: en; institutionCode: CNC; collectionCode: Insects; basisOfRecord: Pinned Specimen

y. $\quad$ scientificName: Hyphantrophaga calva; phylum: Arthropoda; class: Insecta; order: Diptera; family: Tachinidae; genus: Hyphantrophaga; specificEpithet: calva; scientificNameAuthorship: Fleming \& Wood, 2018; continent: Central America; country: Costa Rica; countryCode: CR; stateProvince: Alajuela; county: Sector Rincon Rain Forest; locality: Area de Conservacion Guanacaste; verbatimLocality: Palomo; verbatimElevation: 96; verbatimLatitude: 10.9619; verbatimLongitude: -85.2804; verbatimCoordinateSystem: Decimal; decimalLatitude: 10.9619; decimalLongitude: -85.2804; samplingProtocol: Reared from the larvae of the Depressariidae, Stenoma 
aterpes; individualID: DHJPAR0055031; individualCount: 1; sex: male; lifeStage: adult; preparations: pinned; catalogNumber: DHJPAR0055031; occurrenceDetails: http:// janzen.sas.upenn.edu; recordedBy: D.H. Janzen, W. Hallwachs \& Keiner Aragon; otherCatalogNumbers: ASHYH1578-14, 14-SRNP-45343, BOLD:AAC5447; identifiedBy: AJ Fleming; dateldentified: 2017; language: en; institutionCode: CNC; collectionCode: Insects; basisOfRecord: Pinned Specimen

z. scientificName: Hyphantrophaga calva; phylum: Arthropoda; class: Insecta; order: Diptera; family: Tachinidae; genus: Hyphantrophaga; specificEpithet: calva; scientificNameAuthorship: Fleming \& Wood, 2018; continent: Central America; country: Costa Rica; countryCode: CR; stateProvince: Guanacaste; county: Sector Rincon Rain Forest; locality: Area de Conservacion Guanacaste; verbatimLocality: Quebrada Bambu; verbatimElevation: 109; verbatimLatitude: 10.9301; verbatimLongitude: -85.2521; verbatimCoordinateSystem: Decimal; decimalLatitude: 10.9301; decimalLongitude: -85.2521; samplingProtocol: Reared from the larvae of the Depressariidae, same as 05-SRNP-24825; verbatimEventDate: 28-Jul-2017; individualID: DHJPAR0061376; individualCount: 1; sex: female; lifeStage: adult; preparations: pinned; catalogNumber: DHJPAR0061376; occurrenceDetails: http:// janzen.sas.upenn.edu; recordedBy: D.H. Janzen, W. Hallwachs \& Cirilo Umaña; otherCatalogNumbers: ACGBA7759-17, 17-SRNP-75888, BOLD:AAC5447; identifiedBy: AJ Fleming; dateldentified: 2017; language: en; institutionCode: CNC; collectionCode: Insects; basisOfRecord: Pinned Specimen

\section{Description}

Male (Fig. 7). Length: 7-11 mm. Head (Fig. 7b): vertex 2/9 of head width; two pairs of reclinate upper orbital setae; ocellar setae arising behind anterior ocellus; ocellar triangle gold; fronto-orbital plate shiny silver with a slight brassy-gold tinge on upper $3 / 4$, setulose, setulae not extending below lowest frontal seta; parafacial silver and bare; eye with short sparse ommatrichia up to $2 \mathrm{X}$ as long as one ommatidium; facial ridge bare; pedicel brownish-black, concolorous with postpedicel; arista brown, very minutely pubescent, distinctly thickened on basal 1/8; palpus dark orange and haired apically, oar-shaped. Thorax (Fig. 7a, c): brassy tomentose dorsally, contrasting with dark silver grey laterally; densely covered in black setulae on all surfaces; four thin dorsal vittae, outermost two broken across suture, innermost pair unbroken, reaching 2nd postsutural dorsocentral seta; postpronotum with 3-4 setae arranged in a triangle; chaetotaxy: acrostichal setae 3:3; dorsocentral setae 3:3; intra-alar setae 2:3; supraalar setae 2:3; two katepisternal setae; basal scutellar setae subequal in length to subapical scutellar setae; lateral scutellar setae less than $2 / 3$ as long as subapical setae, curving inwards medially; apical scutellar setae short, crossed apically; one pair of discal scutellar setae more widely set than subapical setae; scutellum very slightly darkened across basal 20\%, remainder concolorous with scutum. Legs (Fig. 7c): femora black in ground colour, remaining segments yellow in ground colour, covered in dark hairs; fore femur with dense silver tomentum on posterodorsal surface; hind coxa bare. Wing (Fig. 7a): pale translucent, hyaline, not distinctly infuscate; vein $R_{4+5}$ with only 2-3 setulae at base. Abdomen (Fig. $7 \mathrm{a}, \mathrm{c}$ ): ground colour dorsally black, yelloworange ventrally; mid-dorsal depression on ST1+2 almost reaching hind margin; median marginal setae present on ST1+2-T3; a complete row of marginal setae 
present on T4 and T5; discal setae only on present T5; sex patch covering ventral surfaces of T4-T5; distinct brassy-silver tomentose bands along anterior edge of tergites, covering $50 \%$ of T3 and almost $80 \%$ of T4; T5 brassy tomentose throughout. Terminalia (Fig. 7d, e, f): sternite 5 (Fig. 7f) with a deeply excavated median cleft, smoothly U-shaped, margins covered in dense tomentum. Lateral lobes of sternite rounded apically, 3-5 strong setae surrounded by many shorter, weaker setulae. Anterior plate of sternite 5 from subequal to slightly longer than apical lobes; unsclerotised "window" anterior to median cleft absent. Cerci in posterior view (Fig. 7d) rectangular and slightly shorter than surstyli, blunt and rounded at apex, completely separate medially but not divergent; in lateral view with a strong downward curve in apical 1/3; densely setulose along basal 2/3 dorsally, setulose ventrally along entire length (visible in lateral view). Surstylus in lateral view (Fig. 7e) almost triangular, ending in a slightly downcurved apex, making the structure appear blade-like; when viewed dorsally, surstyli appearing to point outward, not strongly convergent. Pregonite short, not well-developed, $1 / 3$ as long as distiphallus, trumpet-like, at apex. Postgonite slightly narrow, $1 / 3$ as wide as pregonite, sharply pointed and curved at apex. Distiphallus tubular with a slender median longitudinal sclerotised reinforcement on its posterior surface and a broad, anterolateral, sclerotised acrophallus on each side, joining the plate of opposite side on anterior surface near apex.

Female. Length: 8-11 mm. As males, differing by the presence of two pairs of proclinate orbital setae and the presence of setae on the hind coxa.

\section{Diagnosis}

Hyphantrophaga calva sp. n. can be distinguished from all other Hyphantrophaga species by the following combination of traits: thorax with three postsutural dorsocentral setae, two katepisternal setae, hind coxa bare in males, setose in females, median marginal setae present on ST1+2, discal setae only present on T5.

\section{Etymology}

From the Latin adjective "calvus", meaning "bald", in reference to the lack of hairs on the hind coxa in the males of the species.

\section{Distribution}

Costa Rica, ACG, Alajuela and Guanacaste Provinces, 96-620 m elevation.

\section{Ecology}

Hyphantrophaga calva sp. $\mathbf{n}$. has been reared 13 times from seven species of Lepidoptera in the family Depressariidae, Andasmus Janzen11, Andasmus Janzen25, Andasmus Janzen30, Cerconota Janzen102, Cerconota Janzen707, Chlamydastis christhompsoni (m.s. name), Stenoma aterpes Walsingham, 1913; in rain forest and dry-rain lowland intergrade. 

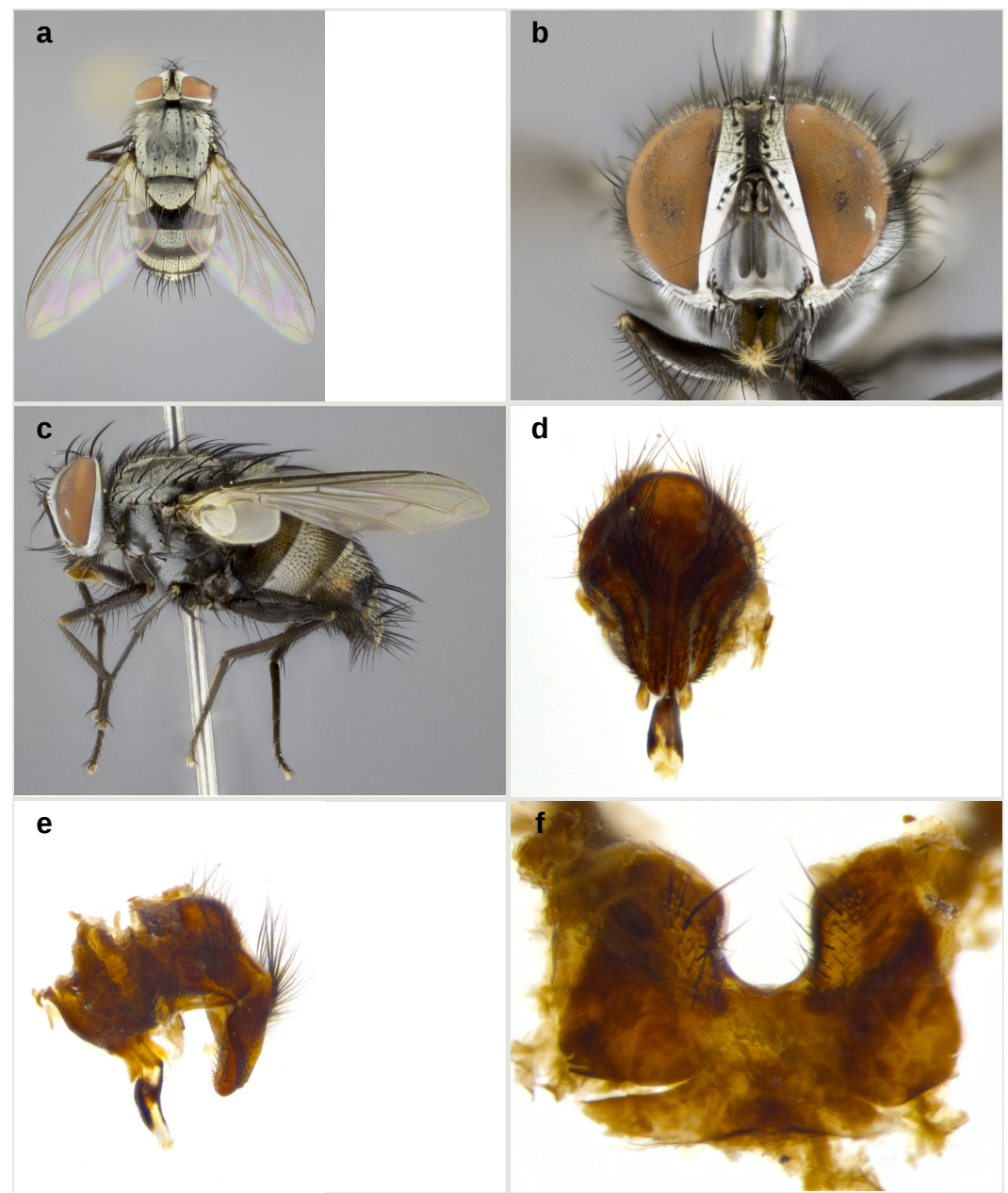

Figure 7.

Hyphantrophaga calva sp. n.; a-c: habitus; holotype male, voucher n. DHJPAR0019630; d-f: terminalia; male paratype, voucher n. DHJPAR0046412.
a: dorsal view doi
b: frontal view doi
c: lateral view doi
d: dorsal view doi
e: lateral view doi
f: sternite 5 , ventral view doi 


\section{Hyphantrophaga ciriloumanai Fleming \& Wood, sp. n.}

- ZooBank urn:Isid:zoobank.org:act:10820514-749C-4741-82BF-3570BAD441DB

\section{Materials}

Holotype:

a. $\quad$ scientificName: Hyphantrophaga ciriloumanai; phylum: Arthropoda; class: Insecta; order: Diptera; family: Tachinidae; genus: Hyphantrophaga; specificEpithet: ciriloumanai; scientificNameAuthorship: Fleming \& Wood, 2018; continent: Central America; country: Costa Rica; countryCode: CR; stateProvince: Alajuela; county: Sector Rincon Rain Forest; locality: Area de Conservacion Guanacaste; verbatimLocality: Estacion Caribe; verbatimElevation: 415; verbatimLatitude: 10.9019; verbatimLongitude: -85.2749; verbatimCoordinateSystem: Decimal; decimalLatitude: 10.9019; decimalLongitude: -85.2749; samplingProtocol: Reared from the larva of the Depressariidae, Anadasmus Janzen25; verbatimEventDate: 24-Aug-2007; individualID: DHJPAR0021010; individualCount: 1; sex: male; lifeStage: adult; preparations: pinned; catalogNumber: DHJPAR0021010; occurrenceDetails: http://janzen.sas.upenn.edu; recordedBy: D.H. Janzen, W. Hallwachs \& Jose Perez; otherCatalogNumbers: ASTA1353-07, 07-SRNP-42179, BOLD:AAA1908; identifiedBy: AJ Fleming; dateldentified: 2017; language: en; institutionCode: CNC; collectionCode: Insects; basisOfRecord: Pinned Specimen

\section{Paratypes:}

a. ScientificName: Hyphantrophaga ciriloumanai; phylum: Arthropoda; class: Insecta; order: Diptera; family: Tachinidae; genus: Hyphantrophaga; specificEpithet: ciriloumanai; scientificNameAuthorship: Fleming \& Wood, 2018; continent: Central America; country: Costa Rica; countryCode: CR; stateProvince: Alajuela; county: Sector San Cristobal; locality: Area de Conservacion Guanacaste; verbatimLocality: Quebrada San Francisco; verbatimElevation: 690; verbatimLatitude: 10.8725; verbatimLongitude: -85.3793; verbatimCoordinateSystem: Decimal; decimalLatitude: 10.8725; decimalLongitude: -85.3793; samplingProtocol: Reared from the larva of the Depressariidae, Anadasmus Janzen25; verbatimEventDate: 20-Nov-2004; individualID: DHJPAR0007288; individualCount: 1; sex: male; lifeStage: adult; preparations: pinned; catalogNumber: DHJPAR0007288; occurrenceDetails: http://janzen.sas.upenn.edu; recordedBy: D.H. Janzen, W. Hallwachs \& Osvaldo Espinoza; otherCatalogNumbers: ASTAT060-06, 04-SRNP-60408, BOLD:AAA1908; identifiedBy: AJ Fleming; dateldentified: 2017; language: en; institutionCode: CNC; collectionCode: Insects; basisOfRecord: Pinned Specimen

b. ScientificName: Hyphantrophaga ciriloumanai; phylum: Arthropoda; class: Insecta; order: Diptera; family: Tachinidae; genus: Hyphantrophaga; specificEpithet: ciriloumanai; scientificNameAuthorship: Fleming \& Wood, 2018; continent: Central America; country: Costa Rica; countryCode: CR; stateProvince: Alajuela; county: Sector San Cristobal; locality: Area de Conservacion Guanacaste; verbatimLocality: Quebrada Cementerio; verbatimElevation: 700; verbatimLatitude: 10.8712; verbatimLongitude: -85.3875 ; verbatimCoordinateSystem: Decimal; decimalLatitude: 10.8712; decimalLongitude: -85.3875 ; samplingProtocol: Reared from the larva of the Depressariidae, Stenoma convexicostata; verbatimEventDate: 28-Aug-2001; individualID: DHJPAR0011465; individualCount: 1; sex: male; lifeStage: adult; preparations: pinned; catalogNumber: DHJPAR0011465; occurrenceDetails: http:// janzen.sas.upenn.edu; recordedBy: D.H. Janzen, W. Hallwachs \& Carolina Cano; 
otherCatalog Numbers: ASTAQ852-06, 01-SRNP-2659, BOLD:AAA1908; identifiedBy: AJ Fleming; dateldentified: 2017; language: en; institutionCode: CNC; collectionCode: Insects; basisOfRecord: Pinned Specimen

c. $\quad$ scientificName: Hyphantrophaga ciriloumanai; phylum: Arthropoda; class: Insecta; order: Diptera; family: Tachinidae; genus: Hyphantrophaga; specificEpithet: ciriloumanai; scientificNameAuthorship: Fleming \& Wood, 2018; continent: Central America; country: Costa Rica; countryCode: CR; stateProvince: Alajuela; county: Sector San Cristobal; locality: Area de Conservacion Guanacaste; verbatimLocality: Quebrada Cementerio; verbatimElevation: 700; verbatimLatitude: 10.8712; verbatimLongitude: -85.3875; verbatimCoordinateSystem: Decimal; decimalLatitude: 10.8712; decimalLongitude: -85.3875 ; samplingProtocol: Reared from the larva of the Depressariidae, Stenoma convexicostata; verbatimEventDate: 19-Aug-2001; individualID: DHJPAR0011466; individualCount: 1; sex: female; lifeStage: adult; preparations: pinned; catalogNumber: DHJPAR0011466; occurrenceDetails: http:// janzen.sas.upenn.edu; recordedBy: D.H. Janzen, W. Hallwachs \& Carolina Cano; otherCatalogNumbers: ASTAQ853-06, 01-SRNP-2561, BOLD:AAA1908; identifiedBy: AJ Fleming; dateldentified: 2017; language: en; institutionCode: CNC; collectionCode: Insects; basisOfRecord: Pinned Specimen

d. $\quad$ scientificName: Hyphantrophaga ciriloumanai; phylum: Arthropoda; class: Insecta; order: Diptera; family: Tachinidae; genus: Hyphantrophaga; specificEpithet: ciriloumanai; scientificNameAuthorship: Fleming \& Wood, 2018; continent: Central America; country: Costa Rica; countryCode: CR; stateProvince: Alajuela; county: Sector San Cristobal; locality: Area de Conservacion Guanacaste; verbatimLocality: Quebrada Cementerio; verbatimElevation: 700; verbatimLatitude: 10.8712; verbatimLongitude: -85.3875 ; verbatimCoordinateSystem: Decimal; decimalLatitude: 10.8712; decimalLongitude: -85.3875; samplingProtocol: Reared from the larva of the Depressariidae, Anadasmus Janzen11; verbatimEventDate: 03-Sep-2006; individualID: DHJPAR0016239; individualCount: 1; sex: male; lifeStage: adult; preparations: pinned; catalogNumber: DHJPAR0016239; occurrenceDetails: http://janzen.sas.upenn.edu; recordedBy: D.H. Janzen, W. Hallwachs \& Gloria Sihezar; otherCatalogNumbers: ASTAP268-06, 06-SRNP-6396, BOLD:AAA1908; identifiedBy: AJ Fleming; dateldentified: 2017; language: en; institutionCode: CNC; collectionCode: Insects; basisOfRecord: Pinned Specimen

e. $\quad$ scientificName: Hyphantrophaga ciriloumanai; phylum: Arthropoda; class: Insecta; order: Diptera; family: Tachinidae; genus: Hyphantrophaga; specificEpithet: ciriloumanai; scientificNameAuthorship: Fleming \& Wood, 2018; continent: Central America; country: Costa Rica; countryCode: CR; stateProvince: Alajuela; county: Sector San Cristobal; locality: Area de Conservacion Guanacaste; verbatimLocality: Finca San Gabriel; verbatimElevation: 645; verbatimLatitude: 10.8777 ; verbatimLongitude: -85.3934; verbatimCoordinateSystem: Decimal; decimalLatitude: 10.8777; decimalLongitude: -85.3934; samplingProtocol: Reared from the larva of the Depressariidae, Anadasmus Janzen25; verbatimEventDate: 07-Mar-2007; individualID: DHJPAR0017158; individualCount: 1; sex: female; lifeStage: adult; preparations: pinned; catalogNumber: DHJPAR0017158; occurrenceDetails: http:// janzen.sas.upenn.edu; recordedBy: D.H. Janzen, W. Hallwachs \& Anabelle Cordoba; otherCatalogNumbers: ASTAP596-07, 07-SRNP-464, BOLD:AAA1908; identifiedBy: AJ Fleming; dateldentified: 2017; language: en; institutionCode: CNC; collectionCode: Insects; basisOfRecord: Pinned Specimen

f. ScientificName: Hyphantrophaga ciriloumanai; phylum: Arthropoda; class: Insecta; order: Diptera; family: Tachinidae; genus: Hyphantrophaga; specificEpithet: 
ciriloumanai; scientificNameAuthorship: Fleming \& Wood, 2018; continent: Central America; country: Costa Rica; countryCode: CR; stateProvince: Alajuela; county: Sector San Cristobal; locality: Area de Conservacion Guanacaste; verbatimLocality: Estacion San Ramon; verbatimElevation: 660; verbatimLatitude: 10.8835; verbatimLongitude: -85.4097; verbatimCoordinateSystem: Decimal; decimalLatitude: 10.8835; decimalLongitude: -85.4097; samplingProtocol: Reared from the larva of the Depressariidae, Anadasmus Janzen25; verbatimEventDate: 28-May-2007; individualID: DHJPAR0019707; individualCount: 1; sex: female; lifeStage: adult; preparations: pinned; catalogNumber: DHJPAR0019707; occurrenceDetails: http:// janzen.sas.upenn.edu; recordedBy: D.H. Janzen, W. Hallwachs \& Gloria Sihezar; otherCatalog Numbers: ASTAB255-07, 07-SRNP-2003, BOLD:AAA1908; identifiedBy: AJ Fleming; dateldentified: 2017; language: en; institutionCode: CNC; collectionCode: Insects; basisOfRecord: Pinned Specimen

g. ScientificName: Hyphantrophaga ciriloumanai; phylum: Arthropoda; class: Insecta; order: Diptera; family: Tachinidae; genus: Hyphantrophaga; specificEpithet: ciriloumanai; scientificNameAuthorship: Fleming \& Wood, 2018; continent: Central America; country: Costa Rica; countryCode: CR; stateProvince: Alajuela; county: Sector Rincon Rain Forest; locality: Area de Conservacion Guanacaste; verbatimLocality: Estacion Caribe; verbatimElevation: 415; verbatimLatitude: 10.9019; verbatimLongitude: -85.2749; verbatimCoordinateSystem: Decimal; decimalLatitude: 10.9019; decimalLongitude: -85.2749; samplingProtocol: Reared from the larva of the Depressariidae, Anadasmus Janzen25; verbatimEventDate: 27-Aug-2007; individualID: DHJPAR0020971; individualCount: 1; sex: female; lifeStage: adult; preparations: pinned; catalogNumber: DHJPAR0020971; occurrenceDetails: http:// janzen.sas.upenn.edu; recordedBy: D.H. Janzen, W. Hallwachs \& Jose Perez; otherCatalog Numbers: ASTA1314-07, 07-SRNP-42176, BOLD:AAA1908; identifiedBy: AJ Fleming; dateldentified: 2017; language: en; institutionCode: CNC; collectionCode: Insects; basisOfRecord: Pinned Specimen

h. $\quad$ scientificName: Hyphantrophaga ciriloumanai; phylum: Arthropoda; class: Insecta; order: Diptera; family: Tachinidae; genus: Hyphantrophaga; specificEpithet: ciriloumanai; scientificNameAuthorship: Fleming \& Wood, 2018; continent: Central America; country: Costa Rica; countryCode: CR; stateProvince: Guanacaste; county: Sector Pitilla; locality: Area de Conservacion Guanacaste; verbatimLocality: Estacion Pitilla; verbatimElevation: 675; verbatimLatitude: 10.9893; verbatimLongitude: -85.4258; verbatimCoordinateSystem: Decimal; decimalLatitude: 10.9893; decimalLongitude: -85.4258; samplingProtocol: Reared from the larva of the Depressariidae, Anadasmus Janzen42; verbatimEventDate: 05-Apr-2004; individualID: DHJPAR0007270; individualCount: 1; sex: male; lifeStage: adult; preparations: pinned; catalog Number: DHJPAR0007270; occurrenceDetails: http://janzen.sas.upenn.edu; recordedBy: D.H. Janzen, W. Hallwachs \& Petrona Rios; otherCatalogNumbers: ASTAT042-06, 04-SRNP-31074, BOLD:AAA1908; identifiedBy: AJ Fleming; dateldentified: 2017; language: en; institutionCode: CNC; collectionCode: Insects; basisOfRecord: Pinned Specimen

i. $\quad$ scientificName: Hyphantrophaga ciriloumanai; phylum: Arthropoda; class: Insecta; order: Diptera; family: Tachinidae; genus: Hyphantrophaga; specificEpithet: ciriloumanai; scientificNameAuthorship: Fleming \& Wood, 2018; continent: Central America; country: Costa Rica; countryCode: CR; stateProvince: Guanacaste; county: Sector El Hacha; locality: Area de Conservacion Guanacaste; verbatimLocality: Estacion Los Almendros; verbatimElevation: 290; verbatimLatitude: 11.0323; verbatimLongitude: -85.5278; verbatimCoordinateSystem: Decimal; decimalLatitude: 
11.0323; decimalLongitude: -85.5278; samplingProtocol: Reared from the larva of the Pyralidae, Accinctapubes albifasciataDHJ01; verbatimEventDate: 22-Dec-2001; individualID: DHJPAR0022956; individualCount: 1; sex: male; lifeStage: adult; preparations: pinned; catalogNumber: DHJPAR0022956; occurrenceDetails: http:// janzen.sas.upenn.edu; recordedBy: D.H. Janzen, W. Hallwachs \& Lucia Rios; otherCatalogNumbers: ASTAW120-08, 01-SRNP-24061, BOLD:AAA1908; identifiedBy: AJ Fleming; dateldentified: 2017; language: en; institutionCode: CNC; collectionCode: Insects; basisOfRecord: Pinned Specimen

j. $\quad$ scientificName: Hyphantrophaga ciriloumanai; phylum: Arthropoda; class: Insecta; order: Diptera; family: Tachinidae; genus: Hyphantrophaga; specificEpithet: ciriloumanai; scientificNameAuthorship: Fleming \& Wood, 2018; continent: Central America; country: Costa Rica; countryCode: CR; stateProvince: Alajuela; county: Sector San Cristobal; locality: Area de Conservacion Guanacaste; verbatimLocality: Sendero Perdido; verbatimElevation: 620; verbatimLatitude: 10.8794; verbatimLongitude: -85.3861; verbatimCoordinateSystem: Decimal; decimalLatitude: 10.8794; decimalLongitude: -85.3861; samplingProtocol: Reared from the larva of the Pyralidae, Deuterollyta oediperalisDHJ02; verbatimEventDate: 24-Aug-2008; individualID: DHJPAR0029604; individualCount: 1; sex: female; lifeStage: adult; preparations: pinned; catalogNumber: DHJPAR0029604; occurrenceDetails: http:// janzen.sas.upenn.edu; recordedBy: D.H. Janzen, W. Hallwachs \& Elda Araya; otherCatalog Numbers: ASHYM1025-09, 08-SRNP-4436, BOLD:AAA1908; identifiedBy: AJ Fleming; dateldentified: 2017; language: en; institutionCode: CNC; collectionCode: Insects; basisOfRecord: Pinned Specimen

k. ScientificName: Hyphantrophaga ciriloumanai; phylum: Arthropoda; class: Insecta; order: Diptera; family: Tachinidae; genus: Hyphantrophaga; specificEpithet: ciriloumanai; scientificNameAuthorship: Fleming \& Wood, 2018; continent: Central America; country: Costa Rica; countryCode: CR; stateProvince: Alajuela; county: Sector San Cristobal; locality: Area de Conservacion Guanacaste; verbatimLocality: Sendero Perdido; verbatimElevation: 620; verbatimLatitude: 10.8794; verbatimLongitude: -85.3861; verbatimCoordinateSystem: Decimal; decimalLatitude: 10.8794; decimalLongitude: -85.3861; samplingProtocol: Reared from the larva of the Pyralidae, Deuterollyta oediperalisDHJ02; verbatimEventDate: 22-Aug-2008; individualID: DHJPAR0029607; individualCount: 1; sex: male; lifeStage: adult; preparations: pinned; catalogNumber: DHJPAR0029607; occurrenceDetails: http:// janzen.sas.upenn.edu; recordedBy: D.H. Janzen, W. Hallwachs \& Elda Araya; otherCatalog Numbers: ASHYM1028-09, 08-SRNP-4437, BOLD:AAA1908; identifiedBy: AJ Fleming; dateldentified: 2017; language: en; institutionCode: CNC; collectionCode: Insects; basisOfRecord: Pinned Specimen

I. ScientificName: Hyphantrophaga ciriloumanai; phylum: Arthropoda; class: Insecta; order: Diptera; family: Tachinidae; genus: Hyphantrophaga; specificEpithet: ciriloumanai; scientificNameAuthorship: Fleming \& Wood, 2018; continent: Central America; country: Costa Rica; countryCode: CR; stateProvince: Alajuela; county: Sector San Cristobal; locality: Area de Conservacion Guanacaste; verbatimLocality: Sendero Perdido; verbatimElevation: 620; verbatimLatitude: 10.8794; verbatimLongitude: -85.3861; verbatimCoordinateSystem: Decimal; decimalLatitude: 10.8794; decimalLongitude: -85.3861; samplingProtocol: Reared from the larva of the Pyralidae, Deuterollyta oediperalisDHJ02; verbatimEventDate: 30-Aug-2008; individualID: DHJPAR0029619; individualCount: 1; sex: female; lifeStage: adult; preparations: pinned; catalogNumber: DHJPAR0029619; occurrenceDetails: http:// janzen.sas.upenn.edu; recordedBy: D.H. Janzen, W. Hallwachs \& Elda Araya; 
otherCatalog Numbers: ASHYM1040-09, 08-SRNP-4439, BOLD:AAA1908; identifiedBy: AJ Fleming; dateldentified: 2017; language: en; institutionCode: CNC; collectionCode: Insects; basisOfRecord: Pinned Specimen

m. $\quad$ scientificName: Hyphantrophaga ciriloumanai; phylum: Arthropoda; class: Insecta; order: Diptera; family: Tachinidae; genus: Hyphantrophaga; specificEpithet: ciriloumanai; scientificNameAuthorship: Fleming \& Wood, 2018; continent: Central America; country: Costa Rica; countryCode: CR; stateProvince: Alajuela; county: Sector Rincon Rain Forest; locality: Area de Conservacion Guanacaste; verbatimLocality: Rio Francia Arriba; verbatimElevation: 400; verbatimLatitude: 10.8967; verbatimLongitude: -85.29; verbatimCoordinateSystem: Decimal; decimalLatitude: 10.8967; decimalLongitude: -85.29; samplingProtocol: Reared from the larva of the Depressariidae, Anadasmus Janzen25; verbatimEventDate: 10Nov-2008; individualID: DHJPAR0029992; individualCount: 1; sex: male; lifeStage: adult; preparations: pinned; catalogNumber: DHJPAR0029992; occurrenceDetails: htt p://janzen.sas.upenn.edu; recordedBy: D.H. Janzen, W. Hallwachs \& Jose Perez; otherCatalogNumbers: ASHYB736-09, 08-SRNP-41992, BOLD:AAA1908; identifiedBy: AJ Fleming; dateldentified: 2017; language: en; institutionCode: CNC; collectionCode: Insects; basisOfRecord: Pinned Specimen

n. $\quad$ scientificName: Hyphantrophaga ciriloumanai; phylum: Arthropoda; class: Insecta; order: Diptera; family: Tachinidae; genus: Hyphantrophaga; specificEpithet: ciriloumanai; scientificNameAuthorship: Fleming \& Wood, 2018; continent: Central America; country: Costa Rica; countryCode: CR; stateProvince: Alajuela; county: Brasilia; locality: Area de Conservacion Guanacaste; verbatimLocality: Moga; verbatimElevation: 320; verbatimLatitude: 11.0123; verbatimLongitude: -85.3493; verbatimCoordinateSystem: Decimal; decimalLatitude: 11.0123; decimalLongitude: -85.3493; samplingProtocol: Reared from the larva of the Depressariidae, Anadasmus Janzen51; verbatimEventDate: 07-Feb-2009; individualID: DHJPAR0030228; individualCount: 1; sex: male; lifeStage: adult; preparations: pinned; catalogNumber: DHJPAR0030228; occurrenceDetails: http://janzen.sas.upenn.edu; recordedBy: D.H. Janzen, W. Hallwachs \& Duvalier Briceno; otherCatalogNumbers: ASHYB972-09, 09SRNP-65048, BOLD:AAA1908; identifiedBy: AJ Fleming; dateldentified: 2017; language: en; institutionCode: CNC; collectionCode: Insects; basisOfRecord: Pinned Specimen

o. $\quad$ scientificName: Hyphantrophaga ciriloumanai; phylum: Arthropoda; class: Insecta; order: Diptera; family: Tachinidae; genus: Hyphantrophaga; specificEpithet: ciriloumanai; scientificNameAuthorship: Fleming \& Wood, 2018; continent: Central America; country: Costa Rica; countryCode: CR; stateProvince: Alajuela; county: Sector Rincon Rain Forest; locality: Area de Conservacion Guanacaste; verbatimLocality: San Lucas; verbatimElevation: 320; verbatimLatitude: 10.9185; verbatimLongitude: -85.3034; verbatimCoordinateSystem: Decimal; decimalLatitude: 10.9185; decimalLongitude: -85.3034; samplingProtocol: Reared from the larva of the Depressariidae, Anadasmus Janzen28; verbatimEventDate: 14-Jul-2009; individualID: DHJPAR0035708; individualCount: 1; sex: female; lifeStage: adult; preparations: pinned; catalogNumber: DHJPAR0035708; occurrenceDetails: http:// janzen.sas.upenn.edu; recordedBy: D.H. Janzen, W. Hallwachs \& Anabelle Cordoba; otherCatalogNumbers: ASHYD1089-09, 09-SRNP-41293, BOLD:AAA1908; identifiedBy: AJ Fleming; dateldentified: 2017; language: en; institutionCode: CNC; collectionCode: Insects; basisOfRecord: Pinned Specimen

p. ScientificName: Hyphantrophaga ciriloumanai; phylum: Arthropoda; class: Insecta; order: Diptera; family: Tachinidae; genus: Hyphantrophaga; specificEpithet: 
ciriloumanai; scientificNameAuthorship: Fleming \& Wood, 2018; continent: Central America; country: Costa Rica; countryCode: CR; stateProvince: Alajuela; county: Sector San Cristobal; locality: Area de Conservacion Guanacaste; verbatimLocality: Quebrada Cementerio; verbatimElevation: 700; verbatimLatitude: 10.8712; verbatimLongitude: -85.3875 ; verbatimCoordinateSystem: Decimal; decimalLatitude: 10.8712; decimalLongitude: -85.3875; samplingProtocol: Reared from the larva of the Depressariidae, Anadasmus Janzen11; verbatimEventDate: 03-Aug-2009; individualID: DHJPAR0035717; individualCount: 1; sex: female; lifeStage: adult; preparations: pinned; catalogNumber: DHJPAR0035717; occurrenceDetails: http:// janzen.sas.upenn.edu; recordedBy: D.H. Janzen, W. Hallwachs \& Osvaldo Espinoza; otherCatalog Numbers: ASHYD1098-09, 09-SRNP-3444, BOLD:AAA1908; identifiedBy: AJ Fleming; dateldentified: 2017; language: en; institutionCode: CNC; collectionCode: Insects; basisOfRecord: Pinned Specimen

q. $\quad$ scientificName: Hyphantrophaga ciriloumanai; phylum: Arthropoda; class: Insecta; order: Diptera; family: Tachinidae; genus: Hyphantrophaga; specificEpithet: ciriloumanai; scientificNameAuthorship: Fleming \& Wood, 2018; continent: Central America; country: Costa Rica; countryCode: CR; stateProvince: Alajuela; county: Sector San Cristobal; locality: Area de Conservacion Guanacaste; verbatimLocality: Quebrada Cementerio; verbatimElevation: 700; verbatimLatitude: 10.8712; verbatimLongitude: -85.3875; verbatimCoordinateSystem: Decimal; decimalLatitude: 10.8712; decimalLongitude: -85.3875; samplingProtocol: Reared from the larva of the Depressariidae, Anadasmus Janzen11; verbatimEventDate: 07-Jul-2009; individualID: DHJPAR0035721; individualCount: 1; sex: male; lifeStage: adult; preparations: pinned; catalogNumber: DHJPAR0035721; occurrenceDetails: http://janzen.sas.upenn.edu; recordedBy: D.H. Janzen, W. Hallwachs \& Osvaldo Espinoza; otherCatalogNumbers: ASHYD1102-09, 09-SRNP-3436,; identifiedBy: AJ Fleming; dateldentified: 2017; language: en; institutionCode: CNC; collectionCode: Insects; basisOfRecord: Pinned Specimen

r. ScientificName: Hyphantrophaga ciriloumanai; phylum: Arthropoda; class: Insecta; order: Diptera; family: Tachinidae; genus: Hyphantrophaga; specificEpithet: ciriloumanai; scientificNameAuthorship: Fleming \& Wood, 2018; continent: Central America; country: Costa Rica; countryCode: CR; stateProvince: Alajuela; county: Sector San Cristobal; locality: Area de Conservacion Guanacaste; verbatimLocality: Quebrada Cementerio; verbatimElevation: 700; verbatimLatitude: 10.8712; verbatimLongitude: -85.3875 ; verbatimCoordinateSystem: Decimal; decimalLatitude: 10.8712; decimalLongitude: -85.3875; samplingProtocol: Reared from the larva of the Depressariidae, Anadasmus Janzen11; verbatimEventDate: 08-Aug-2009; individualID: DHJPAR0035733; individualCount: 1; sex: male; lifeStage: adult; preparations: pinned; catalog Number: DHJPAR0035733; occurrenceDetails: http://janzen.sas.upenn.edu; recordedBy: D.H. Janzen, W. Hallwachs \& Osvaldo Espinoza; otherCatalog Numbers: ASHYD1114-09, 09-SRNP-3450, BOLD:AAA1908; identifiedBy: AJ Fleming; dateldentified: 2017; language: en; institutionCode: CNC; collectionCode: Insects; basisOfRecord: Pinned Specimen

s. $\quad$ scientificName: Hyphantrophaga ciriloumanai; phylum: Arthropoda; class: Insecta; order: Diptera; family: Tachinidae; genus: Hyphantrophaga; specificEpithet: ciriloumanai; scientificNameAuthorship: Fleming \& Wood, 2018; continent: Central America; country: Costa Rica; countryCode: CR; stateProvince: Alajuela; county: Sector San Cristobal; locality: Area de Conservacion Guanacaste; verbatimLocality: Finca San Gabriel; verbatimElevation: 645; verbatimLatitude: 10.8777 ; verbatimLongitude: -85.3934; verbatimCoordinateSystem: Decimal; decimalLatitude: 
10.8777; decimalLongitude: -85.3934; samplingProtocol: Reared from the larva of the Depressariidae, Anadasmus Janzen25; verbatimEventDate: 21-Aug-2009; individualID: DHJPAR0035734; individualCount: 1; sex: female; lifeStage: adult; preparations: pinned; catalogNumber: DHJPAR0035734; occurrenceDetails: http:// janzen.sas.upenn.edu; recordedBy: D.H. Janzen, W. Hallwachs \& Osvaldo Espinoza; otherCatalog Numbers: ASHYD1115-09, 09-SRNP-3845, BOLD:AAA1908; identifiedBy: AJ Fleming; dateldentified: 2017; language: en; institutionCode: CNC; collectionCode: Insects; basisOfRecord: Pinned Specimen

t. $\quad$ scientificName: Hyphantrophaga ciriloumanai; phylum: Arthropoda; class: Insecta; order: Diptera; family: Tachinidae; genus: Hyphantrophaga; specificEpithet: ciriloumanai; scientificNameAuthorship: Fleming \& Wood, 2018; continent: Central America; country: Costa Rica; countryCode: CR; stateProvince: Alajuela; county: Sector Rincon Rain Forest; locality: Area de Conservacion Guanacaste; verbatimLocality: Sendero Rincon; verbatimElevation: 430; verbatimLatitude: 10.8962; verbatimLongitude: -85.2777; verbatimCoordinateSystem: Decimal; decimalLatitude: 10.8962; decimalLongitude: -85.2777; samplingProtocol: Reared from the larva of the Depressariidae, Stenoma Janzen06; verbatimEventDate: 02-Aug-2009; individualID: DHJPAR0035846; individualCount: 1; sex: female; lifeStage: adult; preparations: pinned; catalogNumber: DHJPAR0035846; occurrenceDetails: http:// janzen.sas.upenn.edu; recordedBy: D.H. Janzen, W. Hallwachs \& Pablo Umaña Calderon; otherCatalog Numbers: ASHYD1227-09, 09-SRNP-41403, BOLD:AAA1908; identifiedBy: AJ Fleming; dateldentified: 2017; language: en; institutionCode: CNC; collectionCode: Insects; basisOfRecord: Pinned Specimen

u. $\quad$ scientificName: Hyphantrophaga ciriloumanai; phylum: Arthropoda; class: Insecta; order: Diptera; family: Tachinidae; genus: Hyphantrophaga; specificEpithet: ciriloumanai; scientificNameAuthorship: Fleming \& Wood, 2018; continent: Central America; country: Costa Rica; countryCode: CR; stateProvince: Alajuela; county: Sector Rincon Rain Forest; locality: Area de Conservacion Guanacaste; verbatimLocality: Cafecito; verbatimElevation: 455; verbatimLatitude: 10.944; verbatimLongitude: -85.3174; verbatimCoordinateSystem: Decimal; decimalLatitude: 10.944; decimalLongitude: -85.3174; samplingProtocol: Reared from the larva of the Depressariidae, Anadasmus Janzen16; verbatimEventDate: 29-Aug-2009; individualID: DHJPAR0035851; individualCount: 1; sex: female; lifeStage: adult; preparations: pinned; catalogNumber: DHJPAR0035851; occurrenceDetails: http:// janzen.sas.upenn.edu; recordedBy: D.H. Janzen, W. Hallwachs \& Noe Castillo; otherCatalogNumbers: ASHYD1232-09, 09-SRNP-69674, BOLD:AAA1908; identifiedBy: AJ Fleming; dateldentified: 2017; language: en; institutionCode: CNC; collectionCode: Insects; basisOfRecord: Pinned Specimen

v. ScientificName: Hyphantrophaga ciriloumanai; phylum: Arthropoda; class: Insecta; order: Diptera; family: Tachinidae; genus: Hyphantrophaga; specificEpithet: ciriloumanai; scientificNameAuthorship: Fleming \& Wood, 2018; continent: Central America; country: Costa Rica; countryCode: CR; stateProvince: Alajuela; county: Sector San Cristobal; locality: Area de Conservacion Guanacaste; verbatimLocality: Quebrada Cementerio; verbatimElevation: 700; verbatimLatitude: 10.8712; verbatimLongitude: -85.3875 ; verbatimCoordinateSystem: Decimal; decimalLatitude: 10.8712; decimalLongitude: -85.3875; samplingProtocol: Reared from the larva of the Depressariidae, Anadasmus Janzen11; verbatimEventDate: 21-Oct-2009; individualID: DHJPAR0036550; individualCount: 1; sex: male; lifeStage: adult; preparations: pinned; catalog Number: DHJPAR0036550; occurrenceDetails: http://janzen.sas.upenn.edu; recordedBy: D.H. Janzen, W. Hallwachs \& Osvaldo Espinoza; otherCatalogNumbers: 
ASHYE1461-09, 09-SRNP-4831, BOLD:AAA1908; identifiedBy: AJ Fleming; dateldentified: 2017; language: en; institutionCode: CNC; collectionCode: Insects; basisOfRecord: Pinned Specimen

w. ScientificName: Hyphantrophaga ciriloumanai; phylum: Arthropoda; class: Insecta; order: Diptera; family: Tachinidae; genus: Hyphantrophaga; specificEpithet: ciriloumanai; scientificNameAuthorship: Fleming \& Wood, 2018; continent: Central America; country: Costa Rica; countryCode: CR; stateProvince: Alajuela; county: Sector Rincon Rain Forest; locality: Area de Conservacion Guanacaste; verbatimLocality: San Lucas; verbatimElevation: 320; verbatimLatitude: 10.9185; verbatimLongitude: -85.3034; verbatimCoordinateSystem: Decimal; decimalLatitude: 10.9185; decimalLongitude: -85.3034; samplingProtocol: Reared from the larva of the Depressariidae, Anadasmus Janzen25; verbatimEventDate: 12-Sep-2009; individualID: DHJPAR0036562; individualCount: 1; sex: male; lifeStage: adult; preparations: pinned; catalogNumber: DHJPAR0036562; occurrenceDetails: http://janzen.sas.upenn.edu; recordedBy: D.H. Janzen, W. Hallwachs \& Pablo Umaña Calderon; otherCatalog Numbers: ASHYE1473-09, 09-SRNP-42159, BOLD:AAA1908; identifiedBy: AJ Fleming; dateldentified: 2017; language: en; institutionCode: CNC; collectionCode: Insects; basisOfRecord: Pinned Specimen

x. $\quad$ scientificName: Hyphantrophaga ciriloumanai; phylum: Arthropoda; class: Insecta; order: Diptera; family: Tachinidae; genus: Hyphantrophaga; specificEpithet: ciriloumanai; scientificNameAuthorship: Fleming \& Wood, 2018; continent: Central America; country: Costa Rica; countryCode: CR; stateProvince: Alajuela; county: Sector San Cristobal; locality: Area de Conservacion Guanacaste; verbatimLocality: Finca San Gabriel; verbatimElevation: 645; verbatimLatitude: 10.8777 ; verbatimLongitude: -85.3934; verbatimCoordinateSystem: Decimal; decimalLatitude: 10.8777; decimalLongitude: -85.3934; samplingProtocol: Reared from the larva of the Depressariidae, Anadasmus Janzen25; verbatimEventDate: 27-Aug-2009; individualID: DHJPAR0036577; individualCount: 1; sex: female; lifeStage: adult; preparations: pinned; catalogNumber: DHJPAR0036577; occurrenceDetails: http:// janzen.sas.upenn.edu; recordedBy: D.H. Janzen, W. Hallwachs \& Osvaldo Espinoza; otherCatalogNumbers: ASHYE1488-09, 09-SRNP-3858, BOLD:AAA1908; identifiedBy: AJ Fleming; dateldentified: 2017; language: en; institutionCode: CNC; collectionCode: Insects; basisOfRecord: Pinned Specimen

y. $\quad$ scientificName: Hyphantrophaga ciriloumanai; phylum: Arthropoda; class: Insecta; order: Diptera; family: Tachinidae; genus: Hyphantrophaga; specificEpithet: ciriloumanai; scientificNameAuthorship: Fleming \& Wood, 2018; continent: Central America; country: Costa Rica; countryCode: CR; stateProvince: Alajuela; county: Sector San Cristobal; locality: Area de Conservacion Guanacaste; verbatimLocality: Finca San Gabriel; verbatimElevation: 645; verbatimLatitude: 10.8777 ; verbatimLongitude: -85.3934; verbatimCoordinateSystem: Decimal; decimalLatitude: 10.8777; decimalLongitude: -85.3934; samplingProtocol: Reared from the larva of the Depressariidae, Anadasmus Janzen25; verbatimEventDate: 01-Dec-2009; individualID: DHJPAR0037338; individualCount: 1; sex: female; lifeStage: adult; preparations: pinned; catalogNumber: DHJPAR0037338; occurrenceDetails: http:// janzen.sas.upenn.edu; recordedBy: D.H. Janzen, W. Hallwachs \& Elda Araya; otherCatalogNumbers: ASHYC4083-10, 09-SRNP-5595, BOLD:AAA1908; identifiedBy: AJ Fleming; dateldentified: 2017; language: en; institutionCode: CNC; collectionCode: Insects; basisOfRecord: Pinned Specimen

z. ScientificName: Hyphantrophaga ciriloumanai; phylum: Arthropoda; class: Insecta; order: Diptera; family: Tachinidae; genus: Hyphantrophaga; specificEpithet: 
ciriloumanai; scientificNameAuthorship: Fleming \& Wood, 2018; continent: Central America; country: Costa Rica; countryCode: CR; stateProvince: Alajuela; county: Sector San Cristobal; locality: Area de Conservacion Guanacaste; verbatimLocality: Puente Palma; verbatimElevation: 460; verbatimLatitude: 10.9163; verbatimLongitude: -85.3787; verbatimCoordinateSystem: Decimal; decimalLatitude: 10.9163; decimalLongitude: -85.3787; samplingProtocol: Reared from the larva of the Depressariidae, Anadasmus Janzen51; verbatimEventDate: 31-Jan-2010; individualID: DHJPAR0037481; individualCount: 1; sex: male; lifeStage: adult; preparations: pinned; catalogNumber: DHJPAR0037481; occurrenceDetails: http://janzen.sas.upenn.edu; recordedBy: D.H. Janzen, W. Hallwachs \& Elda Araya; otherCatalogNumbers: ASHYC4226-10, 09-SRNP-7106, BOLD:AAA1908; identifiedBy: AJ Fleming; dateldentified: 2017; language: en; institutionCode: CNC; collectionCode: Insects; basisOfRecord: Pinned Specimen

aa. scientificName: Hyphantrophaga ciriloumanai; phylum: Arthropoda; class: Insecta; order: Diptera; family: Tachinidae; genus: Hyphantrophaga; specificEpithet: ciriloumanai; scientificNameAuthorship: Fleming \& Wood, 2018; continent: Central America; country: Costa Rica; countryCode: CR; stateProvince: Alajuela; county: Sector Rincon Rain Forest; locality: Area de Conservacion Guanacaste; verbatimLocality: Jacobo; verbatimElevation: 461; verbatimLatitude: 10.9408; verbatimLongitude: -85.3177; verbatimCoordinateSystem: Decimal; decimalLatitude; 10.9408; decimalLongitude: -85.3177; samplingProtocol: Reared from the larva of the Depressariidae, Anadasmus Janzen16; verbatimEventDate: 31-Oct-2009; individualID: DHJPAR0037563; individualCount: 1; sex: female; lifeStage: adult; preparations: pinned; catalogNumber: DHJPAR0037563; occurrenceDetails: http:// janzen.sas.upenn.edu; recordedBy: D.H. Janzen, W. Hallwachs \& Maricruz Castillo; otherCatalogNumbers: ASHYC4308-10, 09-SRNP-80100, BOLD:AAA1908; identifiedBy: AJ Fleming; dateldentified: 2017; language: en; institutionCode: CNC; collectionCode: Insects; basisOfRecord: Pinned Specimen

ab. ScientificName: Hyphantrophaga ciriloumanai; phylum: Arthropoda; class: Insecta; order: Diptera; family: Tachinidae; genus: Hyphantrophaga; specificEpithet: ciriloumanai; scientificNameAuthorship: Fleming \& Wood, 2018; continent: Central America; country: Costa Rica; countryCode: CR; stateProvince: Guanacaste; county: Sector Pitilla; locality: Area de Conservacion Guanacaste; verbatimLocality: Sendero Laguna; verbatimElevation: 680; verbatimLatitude: 10.9888; verbatimLongitude: -85.4234; verbatimCoordinateSystem: Decimal; decimalLatitude: 10.9888; decimalLongitude: -85.4234; samplingProtocol: Reared from the larva of the Depressariidae, Anadasmus Janzen41; verbatimEventDate: 21-Aug-2009; individualID: DHJPAR0040175; individualCount: 1; sex: male; lifeStage: adult; preparations: pinned; catalogNumber: DHJPAR0040175; occurrenceDetails: http://janzen.sas.upenn.edu; recordedBy: D.H. Janzen, W. Hallwachs \& Calixto Moraga; otherCatalog Numbers: ASHYE2342-11, 09-SRNP-32287, BOLD:AAA1908; identifiedBy: AJ Fleming; dateldentified: 2017; language: en; institutionCode: CNC; collectionCode: Insects; basisOfRecord: Pinned Specimen

\section{Other materials:}

a. scientificName: Hyphantrophaga ciriloumanai; phylum: Arthropoda; class: Insecta; order: Diptera; family: Tachinidae; genus: Hyphantrophaga; specificEpithet: ciriloumanai; scientificNameAuthorship: Fleming \& Wood, 2018; continent: Central America; country: Costa Rica; countryCode: CR; stateProvince: Alajuela; county: Sector San Cristobal; locality: Area de Conservacion Guanacaste; verbatimLocality: 
Tajo Angeles; verbatimElevation: 540; verbatimLatitude: 10.8647; verbatimLongitude: -85.4153; verbatimCoordinateSystem: Decimal; decimalLatitude: 10.8647; decimalLongitude: -85.4153; samplingProtocol: Reared from the larva of the Depressariidae, Anadasmus Janzen42; verbatimEventDate: 22-Nov-2010; individualID: DHJPAR0040927; individualCount: 1; lifeStage: adult; preparations: pinned; catalogNumber: DHJPAR0040927; occurrenceDetails: http://janzen.sas.upenn.edu; recordedBy: D.H. Janzen, W. Hallwachs \& Gloria Sihezar; otherCatalogNumbers: ASHYF842-11, 10-SRNP-6211, BOLD:AAA1908; identifiedBy: AJ Fleming; dateldentified: 2017; language: en; institutionCode: CNC; collectionCode: Insects; basisOfRecord: Pinned Specimen

b. $\quad$ scientificName: Hyphantrophaga ciriloumanai; phylum: Arthropoda; class: Insecta; order: Diptera; family: Tachinidae; genus: Hyphantrophaga; specificEpithet: ciriloumanai; scientificNameAuthorship: Fleming \& Wood, 2018; continent: Central America; country: Costa Rica; countryCode: CR; stateProvince: Alajuela; county: Sector San Cristobal; locality: Area de Conservacion Guanacaste; verbatimLocality: Tajo Angeles; verbatimElevation: 540; verbatimLatitude: 10.8647; verbatimLongitude: -85.4153; verbatimCoordinateSystem: Decimal; decimalLatitude: 10.8647; decimalLongitude: -85.4153; samplingProtocol: Reared from the larva of the Depressariidae, Anadasmus Janzen42; verbatimEventDate: 20-Nov-2010; individualID: DHJPAR0040928; individualCount: 1; lifeStage: adult; preparations: pinned; catalogNumber: DHJPAR0040928; occurrenceDetails: http://janzen.sas.upenn.edu; recordedBy: D.H. Janzen, W. Hallwachs \& Gloria Sihezar; otherCatalogNumbers: ASHYF843-11, 10-SRNP-6212, BOLD:AAA1908; identifiedBy: AJ Fleming; dateldentified: 2017; language: en; institutionCode: CNC; collectionCode: Insects; basisOfRecord: Pinned Specimen

c. $\quad$ scientificName: Hyphantrophaga ciriloumanai; phylum: Arthropoda; class: Insecta; order: Diptera; family: Tachinidae; genus: Hyphantrophaga; specificEpithet: ciriloumanai; scientificNameAuthorship: Fleming \& Wood, 2018; continent: Central America; country: Costa Rica; countryCode: CR; stateProvince: Alajuela; county: Sector San Cristobal; locality: Area de Conservacion Guanacaste; verbatimLocality: Tajo Angeles; verbatimElevation: 540; verbatimLatitude: 10.8647; verbatimLongitude: -85.4153; verbatimCoordinateSystem: Decimal; decimalLatitude: 10.8647; decimalLongitude: -85.4153; samplingProtocol: Reared from the larva of the Depressariidae, Anadasmus Janzen25; verbatimEventDate: 02-Dec-2010; individualID: DHJPAR0040935; individualCount: 1; lifeStage: adult; preparations: pinned; catalogNumber: DHJPAR0040935; occurrenceDetails: http://janzen.sas.upenn.edu; recordedBy: D.H. Janzen, W. Hallwachs \& Gloria Sihezar; otherCatalogNumbers: ASHYF850-11, 10-SRNP-6221, BOLD:AAA1908; identifiedBy: AJ Fleming; dateldentified: 2017; language: en; institutionCode: CNC; collectionCode: Insects; basisOfRecord: Pinned Specimen

d. scientificName: Hyphantrophaga ciriloumanai; phylum: Arthropoda; class: Insecta; order: Diptera; family: Tachinidae; genus: Hyphantrophaga; specificEpithet: ciriloumanai; scientificNameAuthorship: Fleming \& Wood, 2018; continent: Central America; country: Costa Rica; countryCode: CR; stateProvince: Alajuela; county: Sector San Cristobal; locality: Area de Conservacion Guanacaste; verbatimLocality: Tajo Angeles; verbatimElevation: 540; verbatimLatitude: 10.8647; verbatimLongitude: -85.4153; verbatimCoordinateSystem: Decimal; decimalLatitude: 10.8647; decimalLongitude: -85.4153; samplingProtocol: Reared from the larva of the Depressariidae, Anadasmus Janzen17; verbatimEventDate: 20-Feb-2011; individualID: DHJPAR0042651; individualCount: 1; lifeStage: adult; preparations: pinned; 
catalogNumber: DHJPAR0042651; occurrenceDetails: http://janzen.sas.upenn.edu; recordedBy: D.H. Janzen, W. Hallwachs \& Osvaldo Espinoza; otherCatalogNumbers: ASHYH409-11, 11-SRNP-70, BOLD:AAA1908; identifiedBy: AJ Fleming; dateldentified: 2017; language: en; institutionCode: CNC; collectionCode: Insects; basisOfRecord: Pinned Specimen

e. $\quad$ scientificName: Hyphantrophaga ciriloumanai; phylum: Arthropoda; class: Insecta; order: Diptera; family: Tachinidae; genus: Hyphantrophaga; specificEpithet: ciriloumanai; scientificNameAuthorship: Fleming \& Wood, 2018; continent: Central America; country: Costa Rica; countryCode: CR; stateProvince: Guanacaste; county: Sector Mundo Nuevo; locality: Area de Conservacion Guanacaste; verbatimLocality: Cerro Gongora Pelado; verbatimElevation: 740; verbatimLatitude: 10.7631; verbatimLongitude: -85.4133; verbatimCoordinateSystem: Decimal; decimalLatitude: 10.7631; decimalLongitude: -85.4133; samplingProtocol: Reared from the larva of the Depressariidae, Anadasmus Janzen26; verbatimEventDate: 28-Jan-2012; individualID: DHJPAR0046574; individualCount: 1; sex: female; lifeStage: adult; preparations: pinned; catalogNumber: DHJPAR0046574; occurrenceDetails: http:// janzen.sas.upenn.edu; recordedBy: D.H. Janzen, W. Hallwachs \& Jose Cortez; otherCatalog Numbers: ACGBA747-12, 11-SRNP-57582, BOLD:AAA1908; identifiedBy: AJ Fleming; dateldentified: 2017; language: en; institutionCode: CNC; collectionCode: Insects; basisOfRecord: Pinned Specimen

f. $\quad$ scientificName: Hyphantrophaga ciriloumanai; phylum: Arthropoda; class: Insecta; order: Diptera; family: Tachinidae; genus: Hyphantrophaga; specificEpithet: ciriloumanai; scientificNameAuthorship: Fleming \& Wood, 2018; continent: Central America; country: Costa Rica; countryCode: CR; stateProvince: Alajuela; county: Sector San Cristobal; locality: Area de Conservacion Guanacaste; verbatimLocality: Sendero Vivero; verbatimElevation: 730; verbatimLatitude: 10.8674; verbatimLongitude: -85.3874; verbatimCoordinateSystem: Decimal; decimalLatitude: 10.8674; decimalLongitude: -85.3874; samplingProtocol: Reared from the larva of the Depressariidae, Anadasmus Janzen28; verbatimEventDate: 17-Nov-2012; individuallD: DHJPAR0050694; individualCount: 1; sex: female; lifeStage: adult; preparations: pinned; catalogNumber: DHJPAR0050694; occurrenceDetails: http:// janzen.sas.upenn.edu; recordedBy: D.H. Janzen, W. Hallwachs \& Elda Araya; otherCatalog Numbers: ACGBA3286-13, 12-SRNP-4461, BOLD:AAA1908; identifiedBy: AJ Fleming; dateldentified: 2017; language: en; institutionCode: CNC; collectionCode: Insects; basisOfRecord: Pinned Specimen

g. ScientificName: Hyphantrophaga ciriloumanai; phylum: Arthropoda; class: Insecta; order: Diptera; family: Tachinidae; genus: Hyphantrophaga; specificEpithet: ciriloumanai; scientificNameAuthorship: Fleming \& Wood, 2018; continent: Central America; country: Costa Rica; countryCode: CR; stateProvince: Alajuela; county: Sector San Cristobal; locality: Area de Conservacion Guanacaste; verbatimLocality: Sendero Vivero; verbatimElevation: 730; verbatimLatitude: 10.8674; verbatimLongitude: -85.3874; verbatimCoordinateSystem: Decimal; decimalLatitude: 10.8674; decimalLongitude: -85.3874; samplingProtocol: Reared from the larva of the Depressariidae, Anadasmus Janzen28; verbatimEventDate: 21-Nov-2012; individualID: DHJPAR0050697; individualCount: 1; sex: female; lifeStage: adult; preparations: pinned; catalogNumber: DHJPAR0050697; occurrenceDetails: http:// janzen.sas.upenn.edu; recordedBy: D.H. Janzen, W. Hallwachs \& Elda Araya; otherCatalogNumbers: ACGBA3289-13, 12-SRNP-4462, BOLD:AAA1908; identifiedBy: AJ Fleming; dateldentified: 2017; language: en; institutionCode: CNC; collectionCode: Insects; basisOfRecord: Pinned Specimen 
h. scientificName: Hyphantrophaga ciriloumanai; phylum: Arthropoda; class: Insecta; order: Diptera; family: Tachinidae; genus: Hyphantrophaga; specificEpithet: ciriloumanai; scientificNameAuthorship: Fleming \& Wood, 2018; continent: Central America; country: Costa Rica; countryCode: CR; stateProvince: Guanacaste; county: Sector Rincon Rain Forest; locality: Area de Conservacion Guanacaste; verbatimLocality: Camino Rio Nino; verbatimElevation: 326; verbatimLatitude: 10.9707; verbatimLongitude: -85.3143 ; verbatimCoordinateSystem: Decimal; decimalLatitude: 10.9707; decimalLongitude: -85.3143; samplingProtocol: Reared from the larva of the Depressariidae, Anadasmus Janzen17; verbatimEventDate: 25-Jul-2017; individualID: DHJPAR0061299; individualCount: 1; lifeStage: adult; preparations: pinned; catalogNumber: DHJPAR0061299; occurrenceDetails: http://janzen.sas.upenn.edu; recordedBy: D.H. Janzen, W. Hallwachs \& Anabelle Cordoba; otherCatalogNumbers: ACGBA7682-17, 17-SRNP-27005, BOLD:AAA1908; identifiedBy: AJ Fleming; dateldentified: 2017; language: en; institutionCode: CNC; collectionCode: Insects; basisOfRecord: Pinned Specimen

i. $\quad$ scientificName: Hyphantrophaga ciriloumanai; phylum: Arthropoda; class: Insecta; order: Diptera; family: Tachinidae; genus: Hyphantrophaga; specificEpithet: ciriloumanai; scientificNameAuthorship: Fleming \& Wood, 2018; continent: Central America; country: Costa Rica; countryCode: CR; stateProvince: Guanacaste; county: Sector Rincon Rain Forest; locality: Area de Conservacion Guanacaste; verbatimLocality: Camino Rio Nino; verbatimElevation: 326; verbatimLatitude: 10.9707; verbatimLongitude: -85.3143; verbatimCoordinateSystem: Decimal; decimalLatitude: 10.9707; decimalLongitude: -85.3143; samplingProtocol: Reared from the larva of the Depressariidae, Anadasmus Janzen17; verbatimEventDate: 30-Jul-2017; individualID: DHJPAR0061302; individualCount: 1; lifeStage: adult; preparations: pinned; catalogNumber: DHJPAR0061302; occurrenceDetails: http://janzen.sas.upenn.edu; recordedBy: D.H. Janzen, W. Hallwachs \& Anabelle Cordoba; otherCatalog Numbers: ACGBA7685-17, 17-SRNP-27002, BOLD:AAA1908; identifiedBy: AJ Fleming; dateldentified: 2017; language: en; institutionCode: CNC; collectionCode: Insects; basisOfRecord: Pinned Specimen

j. $\quad$ scientificName: Hyphantrophaga ciriloumanai; phylum: Arthropoda; class: Insecta; order: Diptera; family: Tachinidae; genus: Hyphantrophaga; specificEpithet: ciriloumanai; scientificNameAuthorship: Fleming \& Wood, 2018; continent: Central America; country: Costa Rica; countryCode: CR; stateProvince: Guanacaste; county: Sector Rincon Rain Forest; locality: Area de Conservacion Guanacaste; verbatimLocality: Quebrada Bambu; verbatimElevation: 109; verbatimLatitude: 10.9301; verbatimLongitude: -85.2521; verbatimCoordinateSystem: Decimal; decimalLatitude: 10.9301; decimalLongitude: -85.2521; samplingProtocol: Reared from the larva of the Depressariidae, Anadasmus Janzen25; verbatimEventDate: 22Jun-2017; individualID: DHJPAR0061395; individualCount: 1; lifeStage: adult; preparations: pinned; catalogNumber: DHJPAR0061395; occurrenceDetails: http:// janzen.sas.upenn.edu; recordedBy: D.H. Janzen, W. Hallwachs \& Cirilo Umaña; otherCatalogNumbers: ACGBA7778-17, 17-SRNP-75609, BOLD:AAA1908; identifiedBy: AJ Fleming; dateldentified: 2017; language: en; institutionCode: CNC; collectionCode: Insects; basisOfRecord: Pinned Specimen

\section{Description}

Male (Fig. 8). Length: 7-8 mm. Head (Fig. 8b): vertex 1/4 of head width; two pairs of reclinate upper orbital setae; ocellar setae arising behind anterior ocellus; ocellar 
triangle gold; fronto-orbital plate shiny silver with brilliant gold at vertex and in a narrow strip along frontal setae, sparsely setulose, setulae not extending below lowest frontal seta; parafacial silver and bare; eye densely haired; facial ridge bare; pedicel black, concolorous with postpedicel; arista black, very minutely pubescent, distinctly thickened on basal 1/3-1/4; palpus yellow and haired apically, oar-shaped. Thorax (Fig. 8a, c): brilliant gold tomentose dorsally, contrasting with silver grey laterally; dorsally with dense dark setulae interspersed amongst setae; four thin dorsal vittae, outermost two broken across suture, innermost pair narrowly broken across suture, reaching 2 nd postsutural dorsocentral seta; postpronotum with 3-5 setae arranged in a triangle; chaetotaxy: acrostichal setae 3:3; dorsocentral setae 3:4; intra-alar setae 2:3; supra-alar setae 2:3; two katepisternal setae; basal scutellar setae subequal to subapical setae with a strong inward curve; lateral scutellar setae $2 / 3$ as long as subapical setae, slightly curving inwards medially; apical scutellar setae $1 / 3$ as long as subapical setae, crossed apically; one pair of discal scutellar setae set as widely apart as subapical setae; scutellum concolorous with scutum. Legs (Fig. 8c): black ground colour; fore femur with dense silver tomentum on posterodorsal surface; hind coxa setose. Wing (Fig. 8a): pale translucent, hyaline, only slightly infuscate near base; vein $\mathrm{R}_{4+5}$ with 2-3 setulae at base. Abdomen (Fig. 8a, c): ground colour black; middorsal depression on ST1+2 reaching hind margin; median marginal setae present on ST1+2T3; a complete row of marginal setae present on T4; discal setae present on T3-T5; sex patch absent; distinct gold tomentose bands along anterior edge of T3 and T4, unbroken medially and covering almost $80 \%$ of tergites; T5 with gold tomentum throughout. Terminalia (Fig. 8d, e, f): sternite 5 (Fig. 8f) with a deeply excavated median cleft, narrow U-shaped, margins covered in dense tomentum. Lateral lobes of sternite rounded apically, 3-5 strong setae surrounded by many shorter, weaker setulae. Anterior plate of sternite 5 ranging from subequal to $1 / 2$ as long as apical lobes; unsclerotised "window" anterior to median cleft absent. Cerci in posterior view (Fig. 8d) rectangular and slightly shorter than surstyli, very slightly clubbed and rounded at apex, completely separate medially but not divergent; in lateral view with a strong downward curve in apical 1/3; densely setulose along basal 2/3 dorsally, setulose ventrally along entire length (visible in lateral view). Surstylus in lateral view (Fig. 8e) slender, almost evenly parallel sided, ending in a slightly downcurved apex, making the structure appear blade-like; when viewed dorsally, surstyli appearing to point outward, not strongly convergent. Pregonite short, not well-developed, 1/3 as long as distiphallus, trumpet-like, at apex. Postgonite slightly narrow, 1/3 as wide as pregonite, sharply pointed and curved at apex. Distiphallus sail-shaped, with a slender median longitudinal sclerotised reinforcement on its posterior surface and a broad, anterolateral, sclerotised acrophallus on each side, joining the plate of opposite side on anterior surface near apex.

Female. Length: $7 \mathrm{~mm}$. As male, differing only by the presence of two pairs of proclinate orbital setae. 


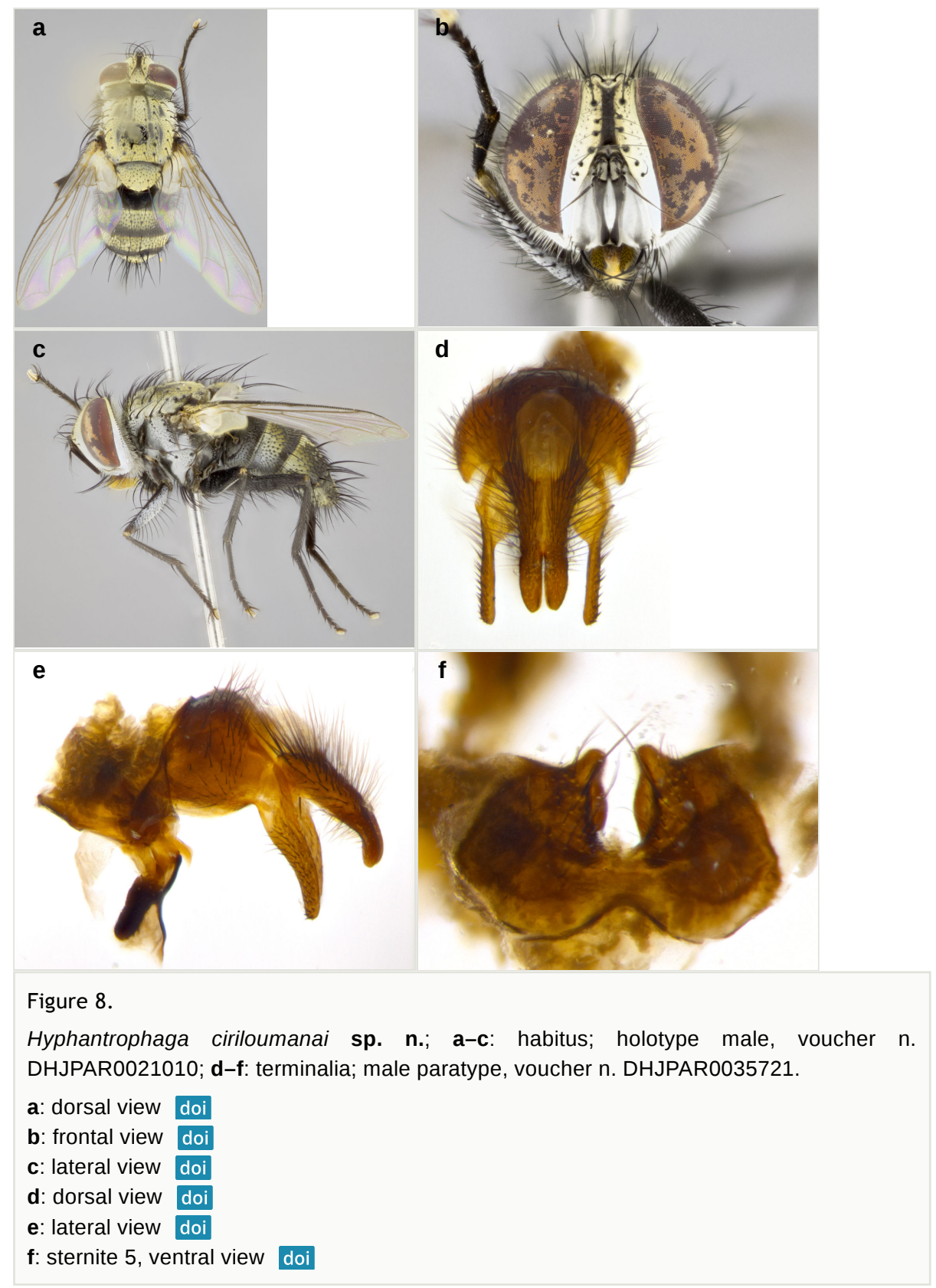

\section{Diagnosis}

Hyphantrophaga ciriloumanai sp. n. can be distinguished from all other Hyphantrophaga species by the following combination of traits: fronto-orbital plate gold tomentose, pedicel and arista black, thorax gold dorsally, silver/grey tomentose laterally, four thoracic vittae, inner pair broken at suture, four postsutural dorsocentral 
setae, legs black, hind coxa setose, abdominal tomentum gold, covering more than $50 \%$ of tergal surface, median marginal setae present on ST1+2, discal setae present T3-T5.

\section{Etymology}

Hyphantrophaga ciriloumanai sp. $\mathbf{n}$. is named in recognition of Cirilo Umaña Dominguez's dedication and work in finding and rearing the ACG caterpillars that contained tachinid larvae.

\section{Distribution}

Costa Rica, ACG, Alajuela and Guanacaste Provinces, 320-740 m elevation.

\section{Ecology}

Hyphantrophaga ciriloumanai sp. $\mathbf{n}$. has been reared 37 times from 12 species of Lepidoptera in the following families: Depressariidae, (Andasmus Janzen11, Andasmus Janzen16, Andasmus Janzen17, Andasmus Janzen25, Andasmus Janzen26, Andasmus Janzen28, Andasmus Janzen41, Andasmus Janzen42, Andasmus Janzen51, Stenoma convexicosta, Stenoma Janzen06) and Pyralidae, (Accinctapubes albifasciataDHJ01, Deuterollyta oediperalisDHJ02); in rain forest, dry forest and dryrain lowland intergrade.

\section{Hyphantrophaga danausophaga Fleming \& Wood, sp. n.}

- ZooBank urn:Isid:Zoobank.org:act:0E6B3A67-A03B-4113-9031-78E40CF00FC3

\section{Materials}

Holotype:

a. $\quad$ scientificName: Hyphantrophaga danausophaga; phylum: Arthropoda; class: Insecta; order: Diptera; family: Tachinidae; genus: Hyphantrophaga; specificEpithet: danausophaga; scientificNameAuthorship: Fleming \& Wood, 2018; continent: Central America; country: Costa Rica; countryCode: CR; stateProvince: Alajuela; county: Sector San Cristobal; locality: Area de Conservacion Guanacaste; verbatimLocality: Sendero Pinyal; verbatimElevation: 630; verbatimLatitude: 10.8716; verbatimLongitude: -85.3933; verbatimCoordinateSystem: Decimal; decimalLatitude: 10.8716; decimalLongitude: -85.3933; samplingProtocol: Reared from the larva of the Nymphalidae, Danaus plexippus; verbatimEventDate: 04-May-2006; individualID:

DHJPAR0010000; individualCount: 1; sex: male; lifeStage: adult; preparations: pinned; catalogNumber: DHJPAR0010000; occurrenceDetails: http://janzen.sas.upenn.edu; recordedBy: D.H. Janzen, W. Hallwachs \& Anabelle Cordoba; otherCatalog Numbers: ASTAV601-06, 06-SRNP-2806, BOLD:AAA5134; identifiedBy: AJ Fleming; dateldentified: 2017; language: en; institutionCode: CNC; collectionCode: Insects; basisOfRecord: Pinned Specimen 


\section{Paratypes:}

a. $\quad$ scientificName: Hyphantrophaga danausophaga; phylum: Arthropoda; class: Insecta; order: Diptera; family: Tachinidae; genus: Hyphantrophaga; specificEpithet: danausophaga; scientificNameAuthorship: Fleming \& Wood, 2018; continent: Central America; country: Costa Rica; countryCode: CR; stateProvince: Alajuela; county: Sector San Cristobal; locality: Area de Conservacion Guanacaste; verbatimLocality: Sendero Vivero; verbatimElevation: 730; verbatimLatitude: 10.8674; verbatimLongitude: -85.3874; verbatimCoordinateSystem: Decimal; decimalLatitude: 10.8674; decimalLongitude: -85.3874; samplingProtocol: Reared from the larva of the Nymphalidae, Danaus plexippus; verbatimEventDate: 30-Nov-2005; individualID: DHJPAR0005447; individualCount: 1; sex: male; lifeStage: adult; preparations: pinned; catalogNumber: DHJPAR0005447; occurrenceDetails: http://janzen.sas.upenn.edu; recordedBy: D.H. Janzen, W. Hallwachs \& Gloria Sihezar; otherCatalogNumbers: ASTA567-06, 05-SRNP-6883, BOLD:AAA5134; identifiedBy: AJ Fleming; dateldentified: 2017; language: en; institutionCode: USNM; collectionCode: Insects; basisOfRecord: Pinned Specimen

b. $\quad$ scientificName: Hyphantrophaga danausophaga; phylum: Arthropoda; class: Insecta; order: Diptera; family: Tachinidae; genus: Hyphantrophaga; specificEpithet: danausophaga; scientificNameAuthorship: Fleming \& Wood, 2018; continent: Central America; country: Costa Rica; countryCode: CR; stateProvince: Guanacaste; county: Sector Mundo Nuevo; locality: Area de Conservacion Guanacaste; verbatimLocality: Porton Rivas; verbatimElevation: 570 ; verbatimLatitude: 10.7586 ; verbatimLongitude: -85.3727; verbatimCoordinateSystem: Decimal; decimalLatitude: 10.7586; decimalLongitude: -85.3727; samplingProtocol: Reared from the larva of the Nymphalidae, Lycorea atergatis; verbatimEventDate: 06-Jan-2006; individualID: DHJPAR0005539; individualCount: 1; sex: male; lifeStage: adult; preparations: pinned; catalog Number: DHJPAR0005539; occurrenceDetails: http://janzen.sas.upenn.edu; recordedBy: D.H. Janzen, W. Hallwachs \& Jose Alberto Sanchez; otherCatalogNumbers: ASTA658-06, 05-SRNP-66253, BOLD:AAA5134; identifiedBy: AJ Fleming; dateldentified: 2017; language: en; institutionCode: BMNH; collectionCode: Insects; basisOfRecord: Pinned Specimen

c. ScientificName: Hyphantrophaga danausophaga; phylum: Arthropoda; class: Insecta; order: Diptera; family: Tachinidae; genus: Hyphantrophaga; specificEpithet: danausophaga; scientificNameAuthorship: Fleming \& Wood, 2018; continent: Central America; country: Costa Rica; countryCode: CR; stateProvince: Guanacaste; county: Sector Mundo Nuevo; locality: Area de Conservacion Guanacaste; verbatimLocality: Porton Rivas; verbatimElevation: 570; verbatimLatitude: 10.7586; verbatimLongitude: -85.3727; verbatimCoordinateSystem: Decimal; decimalLatitude: 10.7586; decimalLongitude: -85.3727; samplingProtocol: Reared from the larva of the Nymphalidae, Lycorea atergatis; verbatimEventDate: 31-Dec-2005; individualID: DHJPAR0006686; individualCount: 1; sex: male; lifeStage: adult; preparations: pinned; catalogNumber: DHJPAR0006686; occurrenceDetails: http://janzen.sas.upenn.edu; recordedBy: D.H. Janzen, W. Hallwachs \& Jose Alberto Sanchez; otherCatalogNumbers: ASTA864-06, 05-SRNP-66243,; identifiedBy: AJ Fleming; dateldentified: 2017; language: en; institutionCode: CNC; collectionCode: Insects; basisOfRecord: Pinned Specimen

d. ScientificName: Hyphantrophaga danausophaga; phylum: Arthropoda; class: Insecta; order: Diptera; family: Tachinidae; genus: Hyphantrophaga; specificEpithet: danausophaga; scientificNameAuthorship: Fleming \& Wood, 2018; continent: Central America; country: Costa Rica; countryCode: CR; stateProvince: Alajuela; county: 
Sector San Cristobal; locality: Area de Conservacion Guanacaste; verbatimLocality: Puente Palma; verbatimElevation: 460; verbatimLatitude: 10.9163; verbatimLongitude: -85.3787; verbatimCoordinateSystem: Decimal; decimalLatitude: 10.9163; decimalLongitude: -85.3787; samplingProtocol: Reared from the larva of the Nymphalidae, Danaus plexippus; verbatimEventDate: 11-Aug-2004; individualID: DHJPAR0007382; individualCount: 1; sex: female; lifeStage: adult; preparations: pinned; catalogNumber: DHJPAR0007382; occurrenceDetails: http:// janzen.sas.upenn.edu; recordedBy: D.H. Janzen, W. Hallwachs \& Osvaldo Espinoza; otherCatalog Numbers: ASTAT154-06, 04-SRNP-3571, BOLD:AAA5134; identifiedBy: AJ Fleming; dateldentified: 2017; language: en; institutionCode: CNC; collectionCode: Insects; basisOfRecord: Pinned Specimen

e. $\quad$ scientificName: Hyphantrophaga danausophaga; phylum: Arthropoda; class: Insecta; order: Diptera; family: Tachinidae; genus: Hyphantrophaga; specificEpithet: danausophaga; scientificNameAuthorship: Fleming \& Wood, 2018; continent: Central America; country: Costa Rica; countryCode: CR; stateProvince: Alajuela; county: Sector San Cristobal; locality: Area de Conservacion Guanacaste; verbatimLocality: Vado Rio Cucaracho; verbatimElevation: 640; verbatimLatitude: 10.8702; verbatimLongitude: -85.3915; verbatimCoordinateSystem: Decimal; decimalLatitude: 10.8702; decimalLongitude: -85.3915; samplingProtocol: Reared from the larva of the Nymphalidae, Danaus plexippus; verbatimEventDate: 16-Mar-2000; individualID: DHJPAR0007412; individualCount: 1; sex: male; lifeStage: adult; preparations: pinned; catalogNumber: DHJPAR0007412; occurrenceDetails: http://janzen.sas.upenn.edu; recordedBy: D.H. Janzen, W. Hallwachs \& Gloria Sihezar; otherCatalog Numbers: ASTAT184-06, 00-SRNP-474, BOLD:AAA5134; identifiedBy: AJ Fleming; dateldentified: 2017; language: en; institutionCode: CNC; collectionCode: Insects; basisOfRecord: Pinned Specimen

f. ScientificName: Hyphantrophaga danausophaga; phylum: Arthropoda; class: Insecta; order: Diptera; family: Tachinidae; genus: Hyphantrophaga; specificEpithet: danausophaga; scientificNameAuthorship: Fleming \& Wood, 2018; continent: Central America; country: Costa Rica; countryCode: CR; stateProvince: Alajuela; county: Sector San Cristobal; locality: Area de Conservacion Guanacaste; verbatimLocality: Vado Rio Cucaracho; verbatimElevation: 640; verbatimLatitude: 10.8702; verbatimLongitude: -85.3915; verbatimCoordinateSystem: Decimal; decimalLatitude: 10.8702; decimalLongitude: -85.3915; samplingProtocol: Reared from the larva of the Nymphalidae, Danaus plexippus; verbatimEventDate: 14-Mar-2000; individualID: DHJPAR0007413; individualCount: 1; sex: female; lifeStage: adult; preparations: pinned; catalogNumber: DHJPAR0007413; occurrenceDetails: http:// janzen.sas.upenn.edu; recordedBy: D.H. Janzen, W. Hallwachs \& Osvaldo Espinoza; otherCatalogNumbers: ASTAT185-06, 00-SRNP-449, BOLD:AAA5134; identifiedBy: AJ Fleming; dateldentified: 2017; language: en; institutionCode: CNC; collectionCode: Insects; basisOfRecord: Pinned Specimen

g. ScientificName: Hyphantrophaga danausophaga; phylum: Arthropoda; class: Insecta; order: Diptera; family: Tachinidae; genus: Hyphantrophaga; specificEpithet: danausophaga; scientificNameAuthorship: Fleming \& Wood, 2018; continent: Central America; country: Costa Rica; countryCode: CR; stateProvince: Alajuela; county: Sector San Cristobal; locality: Area de Conservacion Guanacaste; verbatimLocality: Puente Palma; verbatimElevation: 460; verbatimLatitude: 10.9163; verbatimLongitude: -85.3787; verbatimCoordinateSystem: Decimal; decimalLatitude: 10.9163; decimalLongitude: -85.3787; samplingProtocol: Reared from the larva of the Nymphalidae, Danaus plexippus; verbatimEventDate: 12-Aug-2005; individualID: 
DHJPAR0007414; individualCount: 1; sex: male; lifeStage: adult; preparations: pinned; catalogNumber: DHJPAR0007414; occurrenceDetails: http://janzen.sas.upenn.edu; recordedBy: D.H. Janzen, W. Hallwachs \& Anabelle Cordoba; otherCatalog Numbers: ASTAT186-06, 05-SRNP-4056, BOLD:AAA5134; identifiedBy: AJ Fleming; dateldentified: 2017; language: en; institutionCode: CNC; collectionCode: Insects; basisOfRecord: Pinned Specimen

h. $\quad$ scientificName: Hyphantrophaga danausophaga; phylum: Arthropoda; class: Insecta; order: Diptera; family: Tachinidae; genus: Hyphantrophaga; specificEpithet: danausophaga; scientificNameAuthorship: Fleming \& Wood, 2018; continent: Central America; country: Costa Rica; countryCode: CR; stateProvince: Alajuela; county: Sector San Cristobal; locality: Area de Conservacion Guanacaste; verbatimLocality: Vado Rio Cucaracho; verbatimElevation: 640; verbatimLatitude: 10.8702; verbatimLongitude: -85.3915; verbatimCoordinateSystem: Decimal; decimalLatitude: 10.8702; decimalLongitude: -85.3915; samplingProtocol: Reared from the larva of the Nymphalidae, Danaus plexippus; verbatimEventDate: 24-Mar-2000; individualID: DHJPAR0007415; individualCount: 1; sex: female; lifeStage: adult; preparations: pinned; catalogNumber: DHJPAR0007415; occurrenceDetails: http:// janzen.sas.upenn.edu; recordedBy: D.H. Janzen, W. Hallwachs \& Osvaldo Espinoza; otherCatalog Numbers: ASTAT187-06, 00-SRNP-618, BOLD:AAA5134; identifiedBy: AJ Fleming; dateldentified: 2017; language: en; institutionCode: CNC; collectionCode: Insects; basisOfRecord: Pinned Specimen

i. ScientificName: Hyphantrophaga danausophaga; phylum: Arthropoda; class: Insecta; order: Diptera; family: Tachinidae; genus: Hyphantrophaga; specificEpithet: danausophaga; scientificNameAuthorship: Fleming \& Wood, 2018; continent: Central America; country: Costa Rica; countryCode: CR; stateProvince: Alajuela; county: Sector San Cristobal; locality: Area de Conservacion Guanacaste; verbatimLocality: Vado Rio Cucaracho; verbatimElevation: 640; verbatimLatitude: 10.8702; verbatimLongitude: -85.3915; verbatimCoordinateSystem: Decimal; decimalLatitude: 10.8702; decimalLongitude: -85.3915; samplingProtocol: Reared from the larva of the Nymphalidae, Danaus plexippus; verbatimEventDate: 21-Mar-2000; individuallD: DHJPAR0007416; individualCount: 1; sex: female; lifeStage: adult; preparations: pinned; catalogNumber: DHJPAR0007416; occurrenceDetails: http:// janzen.sas.upenn.edu; recordedBy: D.H. Janzen, W. Hallwachs \& Osvaldo Espinoza; otherCatalogNumbers: ASTAT188-06, 00-SRNP-614, BOLD:AAA5134; identifiedBy: AJ Fleming; dateldentified: 2017; language: en; institutionCode: CNC; collectionCode: Insects; basisOfRecord: Pinned Specimen

j. $\quad$ scientificName: Hyphantrophaga danausophaga; phylum: Arthropoda; class: Insecta; order: Diptera; family: Tachinidae; genus: Hyphantrophaga; specificEpithet: danausophaga; scientificNameAuthorship: Fleming \& Wood, 2018; continent: Central America; country: Costa Rica; countryCode: CR; stateProvince: Alajuela; county: Sector San Cristobal; locality: Area de Conservacion Guanacaste; verbatimLocality: Sendero Pinyal; verbatimElevation: 630; verbatimLatitude: 10.8716; verbatimLongitude: -85.3933; verbatimCoordinateSystem: Decimal; decimalLatitude: 10.8716; decimalLongitude: -85.3933; samplingProtocol: Reared from the larva of the Nymphalidae, Danaus plexippus; verbatimEventDate: 09-Jul-1998; individualID: DHJPAR0007417; individualCount: 1; sex: male; lifeStage: adult; preparations: pinned; catalogNumber: DHJPAR0007417; occurrenceDetails: http://janzen.sas.upenn.edu; recordedBy: D.H. Janzen, W. Hallwachs \& Gloria Sihezar; otherCatalogNumbers: ASTAT189-06, 98-SRNP-7256, BOLD:AAA5134; identifiedBy: AJ Fleming; 
dateldentified: 2017; Ianguage: en; institutionCode: CNC; collectionCode: Insects; basisOfRecord: Pinned Specimen

k. ScientificName: Hyphantrophaga danausophaga; phylum: Arthropoda; class: Insecta; order: Diptera; family: Tachinidae; genus: Hyphantrophaga; specificEpithet: danausophaga; scientificNameAuthorship: Fleming \& Wood, 2018; continent: Central America; country: Costa Rica; countryCode: CR; stateProvince: Alajuela; county: Sector San Cristobal; locality: Area de Conservacion Guanacaste; verbatimLocality: Sendero Pinyal; verbatimElevation: 630; verbatimLatitude: 10.8716; verbatimLongitude: -85.3933; verbatimCoordinateSystem: Decimal; decimalLatitude: 10.8716; decimalLongitude: -85.3933; samplingProtocol: Reared from the larva of the Nymphalidae, Danaus plexippus; verbatimEventDate: 09-Aug-1998; individualID: DHJPAR0007418; individualCount: 1; sex: female; lifeStage: adult; preparations: pinned; catalogNumber: DHJPAR0007418; occurrenceDetails: http:// janzen.sas.upenn.edu; recordedBy: D.H. Janzen, W. Hallwachs \& Gloria Sihezar; otherCatalogNumbers: ASTAT190-06, 98-SRNP-7255,; identifiedBy: AJ Fleming; dateldentified: 2017; language: en; institutionCode: CNC; collectionCode: Insects; basisOfRecord: Pinned Specimen

I. scientificName: Hyphantrophaga danausophaga; phylum: Arthropoda; class: Insecta; order: Diptera; family: Tachinidae; genus: Hyphantrophaga; specificEpithet: danausophaga; scientificNameAuthorship: Fleming \& Wood, 2018; continent: Central America; country: Costa Rica; countryCode: CR; stateProvince: Alajuela; county: Sector San Cristobal; locality: Area de Conservacion Guanacaste; verbatimLocality: Melina Bufalo; verbatimElevation: 560; verbatimLatitude: 10.884; verbatimLongitude: -85.386; verbatimCoordinateSystem: Decimal; decimalLatitude: 10.884; decimalLongitude: -85.386; samplingProtocol: Reared from the larva of the Nymphalidae, Danaus plexippus; verbatimEventDate: 16-Mar-2000; individualID: DHJPAR0007419; individualCount: 1; sex: male; lifeStage: adult; preparations: pinned; catalogNumber: DHJPAR0007419; occurrenceDetails: http://janzen.sas.upenn.edu; recordedBy: D.H. Janzen, W. Hallwachs \& Carolina Cano; otherCatalogNumbers: ASTAT191-06, 00-SRNP-412, BOLD:AAA5134; identifiedBy: AJ Fleming; dateldentified: 2017; language: en; institutionCode: CNC; collectionCode: Insects; basisOfRecord: Pinned Specimen

m. scientificName: Hyphantrophaga danausophaga; phylum: Arthropoda; class: Insecta; order: Diptera; family: Tachinidae; genus: Hyphantrophaga; specificEpithet: danausophaga; scientificNameAuthorship: Fleming \& Wood, 2018; continent: Central America; country: Costa Rica; countryCode: CR; stateProvince: Alajuela; county: Sector San Cristobal; locality: Area de Conservacion Guanacaste; verbatimLocality: Quebrada Cementerio; verbatimElevation: 700; verbatimLatitude: 10.8712; verbatimLongitude: -85.3875 ; verbatimCoordinateSystem: Decimal; decimalLatitude: 10.8712; decimalLongitude: -85.3875; samplingProtocol: Reared from the larva of the Nymphalidae, Danaus plexippus; verbatimEventDate: 05-Apr-2000; individualID: DHJPAR0007420; individualCount: 1; sex: female; lifeStage: adult; preparations: pinned; catalogNumber: DHJPAR0007420; occurrenceDetails: http:// janzen.sas.upenn.edu; recordedBy: D.H. Janzen, W. Hallwachs \& Freddy Quesada; otherCatalog Numbers: ASTAT192-06, 00-SRNP-774, BOLD:AAA5134; identifiedBy: AJ Fleming; dateldentified: 2017; language: en; institutionCode: CNC; collectionCode: Insects; basisOfRecord: Pinned Specimen

n. ScientificName: Hyphantrophaga danausophaga; phylum: Arthropoda; class: Insecta; order: Diptera; family: Tachinidae; genus: Hyphantrophaga; specificEpithet: danausophaga; scientificNameAuthorship: Fleming \& Wood, 2018; continent: Central 
America; country: Costa Rica; countryCode: CR; stateProvince: Alajuela; county: Sector San Cristobal; locality: Area de Conservacion Guanacaste; verbatimLocality: Vado Rio Cucaracho; verbatimElevation: 640; verbatimLatitude: 10.8702; verbatimLongitude: -85.3915; verbatimCoordinateSystem: Decimal; decimalLatitude: 10.8702; decimalLongitude: -85.3915; samplingProtocol: Reared from the larva of the Nymphalidae, Danaus plexippus; verbatimEventDate: 14-Mar-2000; individualID: DHJPAR0007421; individualCount: 1; sex: male; lifeStage: adult; preparations: pinned; catalogNumber: DHJPAR0007421; occurrenceDetails: http://janzen.sas.upenn.edu; recordedBy: D.H. Janzen, W. Hallwachs \& Gloria Sihezar; otherCatalogNumbers: ASTAT193-06, 00-SRNP-473, BOLD:AAA5134; identifiedBy: AJ Fleming; dateldentified: 2017; language: en; institutionCode: CNC; collectionCode: Insects; basisOfRecord: Pinned Specimen

o. $\quad$ scientificName: Hyphantrophaga danausophaga; phylum: Arthropoda; class: Insecta; order: Diptera; family: Tachinidae; genus: Hyphantrophaga; specificEpithet: danausophaga; scientificNameAuthorship: Fleming \& Wood, 2018; continent: Central America; country: Costa Rica; countryCode: CR; stateProvince: Alajuela; county: Sector San Cristobal; locality: Area de Conservacion Guanacaste; verbatimLocality: Vado Rio Cucaracho; verbatimElevation: 640; verbatimLatitude: 10.8702; verbatimLongitude: -85.3915; verbatimCoordinateSystem: Decimal; decimalLatitude: 10.8702; decimalLongitude: -85.3915; samplingProtocol: Reared from the larva of the Nymphalidae, Danaus plexippus; verbatimEventDate: 19-Mar-2000; individualID: DHJPAR0007422; individualCount: 1; sex: female; lifeStage: adult; preparations: pinned; catalogNumber: DHJPAR0007422; occurrenceDetails: http:// janzen.sas.upenn.edu; recordedBy: D.H. Janzen, W. Hallwachs \& Carolina Cano; otherCatalogNumbers: ASTAT194-06, 00-SRNP-551, BOLD:AAA5134; identifiedBy: AJ Fleming; dateldentified: 2017; language: en; institutionCode: CNC; collectionCode: Insects; basisOfRecord: Pinned Specimen

p. $\quad$ scientificName: Hyphantrophaga danausophaga; phylum: Arthropoda; class: Insecta; order: Diptera; family: Tachinidae; genus: Hyphantrophaga; specificEpithet: danausophaga; scientificNameAuthorship: Fleming \& Wood, 2018; continent: Central America; country: Costa Rica; countryCode: CR; stateProvince: Alajuela; county: Sector San Cristobal; locality: Area de Conservacion Guanacaste; verbatimLocality: Melina Bufalo; verbatimElevation: 560 ; verbatimLatitude: 10.884 ; verbatimLongitude: -85.386; verbatimCoordinateSystem: Decimal; decimalLatitude: 10.884; decimalLongitude: -85.386; samplingProtocol: Reared from the larva of the Nymphalidae, Danaus plexippus; verbatimEventDate: 19-Mar-2000; individualID: DHJPAR0007423; individualCount: 1; sex: male; lifeStage: adult; preparations: pinned; catalogNumber: DHJPAR0007423; occurrenceDetails: http://janzen.sas.upenn.edu; recordedBy: D.H. Janzen, W. Hallwachs \& Carolina Cano; otherCatalogNumbers: ASTAT195-06, 00-SRNP-413,; identifiedBy: AJ Fleming; dateldentified: 2017; language: en; institutionCode: CNC; collectionCode: Insects; basisOfRecord: Pinned Specimen

q. $\quad$ scientificName: Hyphantrophaga danausophaga; phylum: Arthropoda; class: Insecta; order: Diptera; family: Tachinidae; genus: Hyphantrophaga; specificEpithet: danausophaga; scientificNameAuthorship: Fleming \& Wood, 2018; continent: Central America; country: Costa Rica; countryCode: CR; stateProvince: Alajuela; county: Sector San Cristobal; locality: Area de Conservacion Guanacaste; verbatimLocality: Vado Rio Cucaracho; verbatimElevation: 640; verbatimLatitude: 10.8702; verbatimLongitude: -85.3915; verbatimCoordinateSystem: Decimal; decimalLatitude: 10.8702; decimalLongitude: -85.3915; samplingProtocol: Reared from the larva of the 
Nymphalidae, Danaus plexippus; verbatimEventDate: 05-Mar-2000; individualID: DHJPAR0007424; individualCount: 1; sex: male; lifeStage: adult; preparations: pinned; catalogNumber: DHJPAR0007424; occurrenceDetails: http://janzen.sas.upenn.edu; recordedBy: D.H. Janzen, W. Hallwachs \& Osvaldo Espinoza; otherCatalogNumbers: ASTAT196-06, 00-SRNP-446, BOLD:AAA5134; identifiedBy: AJ Fleming; dateldentified: 2017; language: en; institutionCode: CNC; collectionCode: Insects; basisOfRecord: Pinned Specimen

r. scientificName: Hyphantrophaga danausophaga; phylum: Arthropoda; class: Insecta; order: Diptera; family: Tachinidae; genus: Hyphantrophaga; specificEpithet: danausophaga; scientificNameAuthorship: Fleming \& Wood, 2018; continent: Central America; country: Costa Rica; countryCode: CR; stateProvince: Guanacaste; county: Sector Cacao; locality: Area de Conservacion Guanacaste; verbatimLocality: Quebrada Otilio; verbatimElevation: 550; verbatimLatitude: 10.89; verbatimLongitude: -85.4797; verbatimCoordinateSystem: Decimal; decimalLatitude: 10.89; decimalLongitude: -85.4797; samplingProtocol: Reared from the larva of the Nymphalidae, Lycorea atergatis; verbatimEventDate: 18-Aug-2004; individualID: DHJPAR0007425; individualCount: 1; sex: female; lifeStage: adult; preparations: pinned; catalogNumber: DHJPAR0007425; occurrenceDetails: http:// janzen.sas.upenn.edu; recordedBy: D.H. Janzen, W. Hallwachs \& Harry Ramirez; otherCatalog Numbers: ASTAT197-06, 04-SRNP-47445,; identifiedBy: AJ Fleming; dateldentified: 2017; language: en; institutionCode: CNC; collectionCode: Insects; basisOfRecord: Pinned Specimen

s. $\quad$ scientificName: Hyphantrophaga danausophaga; phylum: Arthropoda; class: Insecta; order: Diptera; family: Tachinidae; genus: Hyphantrophaga; specificEpithet: danausophaga; scientificNameAuthorship: Fleming \& Wood, 2018; continent: Central America; country: Costa Rica; countryCode: CR; stateProvince: Alajuela; county: Sector San Cristobal; locality: Area de Conservacion Guanacaste; verbatimLocality: Melina Bufalo; verbatimElevation: 560; verbatimLatitude: 10.884; verbatimLongitude: -85.386; verbatimCoordinateSystem: Decimal; decimalLatitude: 10.884; decimalLongitude: -85.386; samplingProtocol: Reared from the larva of the Nymphalidae, Danaus plexippus; verbatimEventDate: 17-Mar-2000; individualID: DHJPAR0007426; individualCount: 1; sex: male; lifeStage: adult; preparations: pinned; catalogNumber: DHJPAR0007426; occurrenceDetails: http://janzen.sas.upenn.edu; recordedBy: D.H. Janzen, W. Hallwachs \& Carolina Cano; otherCatalogNumbers: ASTAT198-06, 00-SRNP-410,; identifiedBy: AJ Fleming; dateldentified: 2017; language: en; institutionCode: CNC; collectionCode: Insects; basisOfRecord: Pinned Specimen

t. ScientificName: Hyphantrophaga danausophaga; phylum: Arthropoda; class: Insecta; order: Diptera; family: Tachinidae; genus: Hyphantrophaga; specificEpithet: danausophaga; scientificNameAuthorship: Fleming \& Wood, 2018; continent: Central America; country: Costa Rica; countryCode: CR; stateProvince: Alajuela; county: Sector San Cristobal; locality: Area de Conservacion Guanacaste; verbatimLocality: Quebrada Cementerio; verbatimElevation: 700; verbatimLatitude: 10.8712; verbatimLongitude: -85.3875 ; verbatimCoordinateSystem: Decimal; decimalLatitude: 10.8712; decimalLongitude: -85.3875; samplingProtocol: Reared from the larva of the Nymphalidae, Danaus plexippus; verbatimEventDate: 05-Apr-2000; individualID: DHJPAR0007427; individualCount: 1; sex: male; lifeStage: adult; preparations: pinned; catalog Number: DHJPAR0007427; occurrenceDetails: http://janzen.sas.upenn.edu; recordedBy: D.H. Janzen, W. Hallwachs \& Osvaldo Espinoza; otherCatalogNumbers: ASTAT199-06, 00-SRNP-780, BOLD:AAA5134; identifiedBy: AJ Fleming; 
dateldentified: 2017; language: en; institutionCode: CNC; collectionCode: Insects; basisOfRecord: Pinned Specimen

u. $\quad$ scientificName: Hyphantrophaga danausophaga; phylum: Arthropoda; class: Insecta; order: Diptera; family: Tachinidae; genus: Hyphantrophaga; specificEpithet: danausophaga; scientificNameAuthorship: Fleming \& Wood, 2018; continent: Central America; country: Costa Rica; countryCode: CR; stateProvince: Alajuela; county: Sector San Cristobal; locality: Area de Conservacion Guanacaste; verbatimLocality: Sendero Vivero; verbatimElevation: 730; verbatimLatitude: 10.8674;

verbatimLongitude: -85.3874; verbatimCoordinateSystem: Decimal; decimalLatitude: 10.8674; decimalLongitude: -85.3874; samplingProtocol: Reared from the larva of the Nymphalidae, Danaus plexippus; verbatimEventDate: 13-Mar-2000; individualID: DHJPAR0007428; individualCount: 1; sex: female; lifeStage: adult; preparations: pinned; catalogNumber: DHJPAR0007428; occurrenceDetails: http:// janzen.sas.upenn.edu; recordedBy: D.H. Janzen, W. Hallwachs \& Carolina Cano; otherCatalogNumbers: ASTAT200-06, 00-SRNP-400, BOLD:AAA5134; identifiedBy: AJ Fleming; dateldentified: 2017; language: en; institutionCode: CNC; collectionCode: Insects; basisOfRecord: Pinned Specimen

v. ScientificName: Hyphantrophaga danausophaga; phylum: Arthropoda; class: Insecta; order: Diptera; family: Tachinidae; genus: Hyphantrophaga; specificEpithet: danausophaga; scientificNameAuthorship: Fleming \& Wood, 2018; continent: Central America; country: Costa Rica; countryCode: CR; stateProvince: Alajuela; county: Sector San Cristobal; locality: Area de Conservacion Guanacaste; verbatimLocality: Vado Rio Cucaracho; verbatimElevation: 640; verbatimLatitude: 10.8702; verbatimLongitude: -85.3915; verbatimCoordinateSystem: Decimal; decimalLatitude: 10.8702; decimalLongitude: -85.3915; samplingProtocol: Reared from the larva of the Nymphalidae, Danaus plexippus; verbatimEventDate: 21-Mar-2000; individualID: DHJPAR0007429; individualCount: 1; sex: male; lifeStage: adult; preparations: pinned; catalogNumber: DHJPAR0007429; occurrenceDetails: http://janzen.sas.upenn.edu; recordedBy: D.H. Janzen, W. Hallwachs \& Osvaldo Espinoza; otherCatalogNumbers: ASTAT201-06, 00-SRNP-477,; identifiedBy: AJ Fleming; dateldentified: 2017; language: en; institutionCode: CNC; collectionCode: Insects; basisOfRecord: Pinned Specimen

w. scientificName: Hyphantrophaga danausophaga; phylum: Arthropoda; class: Insecta; order: Diptera; family: Tachinidae; genus: Hyphantrophaga; specificEpithet: danausophaga; scientificNameAuthorship: Fleming \& Wood, 2018; continent: Central America; country: Costa Rica; countryCode: CR; stateProvince: Guanacaste; county: Sector Cacao; locality: Area de Conservacion Guanacaste; verbatimLocality: Sendero Maritza; verbatimElevation: 760; verbatimLatitude: 10.9364; verbatimLongitude: -85.4776; verbatimCoordinateSystem: Decimal; decimalLatitude: 10.9364; decimalLongitude: -85.4776; samplingProtocol: Reared from the larva of the Nymphalidae, Danaus plexippus; verbatimEventDate: 18-Apr-2002; individualID: DHJPAR0007430; individualCount: 1; sex: male; lifeStage: adult; preparations: pinned; catalogNumber: DHJPAR0007430; occurrenceDetails: http://janzen.sas.upenn.edu; recordedBy: D.H. Janzen, W. Hallwachs \& Harry Ramirez; otherCatalog Numbers: ASTAT202-06, 02-SRNP-8502,; identifiedBy: AJ Fleming; dateldentified: 2017; language: en; institutionCode: CNC; collectionCode: Insects; basisOfRecord: Pinned Specimen

x. ScientificName: Hyphantrophaga danausophaga; phylum: Arthropoda; class: Insecta; order: Diptera; family: Tachinidae; genus: Hyphantrophaga; specificEpithet: danausophaga; scientificNameAuthorship: Fleming \& Wood, 2018; continent: Central 
America; country: Costa Rica; countryCode: CR; stateProvince: Guanacaste; county: Sector Del Oro; locality: Area de Conservacion Guanacaste; verbatimLocality: Quebrada Romero; verbatimElevation: 490; verbatimLatitude: 11.0052; verbatimLongitude: -85.474; verbatimCoordinateSystem: Decimal; decimalLatitude: 11.0052; decimalLongitude: -85.474; samplingProtocol: Reared from the larva of the Nymphalidae, Danaus plexippus; verbatimEventDate: 14-Mar-2002; individualID: DHJPAR0007431; individualCount: 1; sex: female; lifeStage: adult; preparations: pinned; catalogNumber: DHJPAR0007431; occurrenceDetails: http:// janzen.sas.upenn.edu; recordedBy: D.H. Janzen, W. Hallwachs \& Guillermo Pereira; otherCatalogNumbers: ASTAT203-06, 02-SRNP-5392, BOLD:AAA5134; identifiedBy: AJ Fleming; dateldentified: 2017; language: en; institutionCode: CNC; collectionCode: Insects; basisOfRecord: Pinned Specimen

y. $\quad$ scientificName: Hyphantrophaga danausophaga; phylum: Arthropoda; class: Insecta; order: Diptera; family: Tachinidae; genus: Hyphantrophaga; specificEpithet: danausophaga; scientificNameAuthorship: Fleming \& Wood, 2018; continent: Central America; country: Costa Rica; countryCode: CR; stateProvince: Alajuela; county: Sector Rincon Rain Forest; locality: Area de Conservacion Guanacaste; verbatimLocality: Estacion Caribe; verbatimElevation: 415; verbatimLatitude: 10.9019; verbatimLongitude: -85.2749; verbatimCoordinateSystem: Decimal; decimalLatitude: 10.9019; decimalLongitude: -85.2749; samplingProtocol: Reared from the larva of the Nymphalidae, Danaus plexippus; verbatimEventDate: 09-May-2002; individuallD:

DHJPAR0007432; individualCount: 1; sex: male; lifeStage: adult; preparations: pinned; catalogNumber: DHJPAR0007432; occurrenceDetails: http://janzen.sas.upenn.edu; recordedBy: D.H. Janzen, W. Hallwachs \& Fraysi Vargas; otherCatalogNumbers: ASTAT204-06, 02-SRNP-6987,; identifiedBy: AJ Fleming; dateldentified: 2017; language: en; institutionCode: CNC; collectionCode: Insects; basisOfRecord: Pinned Specimen

z. $\quad$ scientificName: Hyphantrophaga danausophaga; phylum: Arthropoda; class: Insecta; order: Diptera; family: Tachinidae; genus: Hyphantrophaga; specificEpithet: danausophaga; scientificNameAuthorship: Fleming \& Wood, 2018; continent: Central America; country: Costa Rica; countryCode: CR; stateProvince: Guanacaste; county: Sector Cacao; locality: Area de Conservacion Guanacaste; verbatimLocality: Estacion Cacao; verbatimElevation: 1150; verbatimLatitude: 10.9269; verbatimLongitude: -85.4682; verbatimCoordinateSystem: Decimal; decimalLatitude: 10.9269; decimalLongitude: -85.4682; samplingProtocol: Reared from the larva of the Nymphalidae, Danaus plexippus; verbatimEventDate: 17-Oct-2003; individualID: DHJPAR0007433; individualCount: 1; lifeStage: adult; preparations: pinned; catalogNumber: DHJPAR0007433; occurrenceDetails: http://janzen.sas.upenn.edu; recordedBy: D.H. Janzen, W. Hallwachs \& Freddy Quesada; otherCatalog Numbers: ASTAT205-06, 03-SRNP-22759, BOLD:AAA5134; identifiedBy: AJ Fleming; dateldentified: 2017; language: en; institutionCode: CNC; collectionCode: Insects; basisOfRecord: Pinned Specimen

aa. $\quad$ scientificName: Hyphantrophaga danausophaga; phylum: Arthropoda; class: Insecta; order: Diptera; family: Tachinidae; genus: Hyphantrophaga; specificEpithet: danausophaga; scientificNameAuthorship: Fleming \& Wood, 2018; continent: Central America; country: Costa Rica; countryCode: CR; stateProvince: Guanacaste; county: Sector Del Oro; locality: Area de Conservacion Guanacaste; verbatimLocality: Quebrada Raiz; verbatimElevation: 280; verbatimLatitude: 11.0287; verbatimLongitude: -85.4867 ; verbatimCoordinateSystem: Decimal; decimalLatitude: 11.0287; decimalLongitude: -85.4867; samplingProtocol: Reared from the larva of the 
Nymphalidae, Danaus plexippus; verbatimEventDate: 28-Jun-2004; individualID: DHJPAR0007434; individualCount: 1; sex: female; lifeStage: adult; preparations: pinned; catalogNumber: DHJPAR0007434; occurrenceDetails: http:// janzen.sas.upenn.edu; recordedBy: D.H. Janzen, W. Hallwachs \& Lucia Rios; otherCatalog Numbers: ASTAT206-06, 04-SRNP-22623, BOLD:AAA5134; identifiedBy: AJ Fleming; dateldentified: 2017; language: en; institutionCode: CNC; collectionCode: Insects; basisOfRecord: Pinned Specimen

ab. $\quad$ scientificName: Hyphantrophaga danausophaga; phylum: Arthropoda; class: Insecta; order: Diptera; family: Tachinidae; genus: Hyphantrophaga; specificEpithet: danausophaga; scientificNameAuthorship: Fleming \& Wood, 2018; continent: Central America; country: Costa Rica; countryCode: CR; stateProvince: Alajuela; county: Sector Rincon Rain Forest; locality: Area de Conservacion Guanacaste; verbatimLocality: San Lucas; verbatimElevation: 320; verbatimLatitude: 10.9185; verbatimLongitude: -85.3034; verbatimCoordinateSystem: Decimal; decimalLatitude: 10.9185; decimalLongitude: -85.3034; samplingProtocol: Reared from the larva of the Nymphalidae, Danaus plexippus; verbatimEventDate: 16-Jan-2004; individualID: DHJPAR0007435; individualCount: 1; sex: female; lifeStage: adult; preparations: pinned; catalogNumber: DHJPAR0007435; occurrenceDetails: http:// janzen.sas.upenn.edu; recordedBy: D.H. Janzen, W. Hallwachs \& Minor Carmona; otherCatalogNumbers: ASTAT207-06, 03-SRNP-31832,; identifiedBy: AJ Fleming; dateldentified: 2017; language: en; institutionCode: CNC; collectionCode: Insects; basisOfRecord: Pinned Specimen

ac. ScientificName: Hyphantrophaga danausophaga; phylum: Arthropoda; class: Insecta; order: Diptera; family: Tachinidae; genus: Hyphantrophaga; specificEpithet: danausophaga; scientificNameAuthorship: Fleming \& Wood, 2018; continent: Central America; country: Costa Rica; countryCode: CR; stateProvince: Guanacaste; county: Sector Cacao; locality: Area de Conservacion Guanacaste; verbatimLocality: Cuesta Caimito; verbatimElevation: 640 ; verbatimLatitude: 10.8908; verbatimLongitude: -85.4719; verbatimCoordinateSystem: Decimal; decimalLatitude: 10.8908; decimalLongitude: -85.4719; samplingProtocol: Reared from the larva of the Nymphalidae, Danaus plexippus; verbatimEventDate: 26-Jun-2005; individualID: DHJPAR0007436; individualCount: 1; sex: female; lifeStage: adult; preparations: pinned; catalogNumber: DHJPAR0007436; occurrenceDetails: http:// janzen.sas.upenn.edu; recordedBy: D.H. Janzen, W. Hallwachs \& Manuel Pereira; otherCatalog Numbers: ASTAT208-06, 05-SRNP-45425, BOLD:AAA5134; identifiedBy: AJ Fleming; dateldentified: 2017; language: en; institutionCode: CNC; collectionCode: Insects; basisOfRecord: Pinned Specimen

ad. ScientificName: Hyphantrophaga danausophaga; phylum: Arthropoda; class: Insecta; order: Diptera; family: Tachinidae; genus: Hyphantrophaga; specificEpithet: danausophaga; scientificNameAuthorship: Fleming \& Wood, 2018; continent: Central America; country: Costa Rica; countryCode: CR; stateProvince: Alajuela; county: Sector Rincon Rain Forest; locality: Area de Conservacion Guanacaste; verbatimLocality: Sendero Venado; verbatimElevation: 420; verbatimLatitude: 10.8968; verbatimLongitude: -85.27; verbatimCoordinateSystem: Decimal; decimalLatitude: 10.8968; decimalLongitude: -85.27; samplingProtocol: Reared from the larva of the Nymphalidae, Danaus plexippus; verbatimEventDate: 17-May-2005; individualID: DHJPAR0007437; individualCount: 1; sex: male; lifeStage: adult; preparations: pinned; catalog Number: DHJPAR0007437; occurrenceDetails: http://janzen.sas.upenn.edu; recordedBy: D.H. Janzen, W. Hallwachs \& Minor Carmona; otherCatalogNumbers: ASTAT209-06, 05-SRNP-41041, BOLD:AAA5134; identifiedBy: AJ Fleming; 
dateldentified: 2017; Ianguage: en; institutionCode: CNC; collectionCode: Insects; basisOfRecord: Pinned Specimen

ae. $\quad$ scientificName: Hyphantrophaga danausophaga; phylum: Arthropoda; class: Insecta; order: Diptera; family: Tachinidae; genus: Hyphantrophaga; specificEpithet: danausophaga; scientificNameAuthorship: Fleming \& Wood, 2018; continent: Central America; country: Costa Rica; countryCode: CR; stateProvince: Alajuela; county: Sector San Cristobal; locality: Area de Conservacion Guanacaste; verbatimLocality: Sitio San Geronimo; verbatimElevation: 680; verbatimLatitude: 10.873; verbatimLongitude: -85.3814; verbatimCoordinateSystem: Decimal; decimalLatitude: 10.873; decimalLongitude: -85.3814; samplingProtocol: Reared from the larva of the Nymphalidae, Danaus plexippus; verbatimEventDate: 12-Mar-2005; individualID: DHJPAR0007438; individualCount: 1; sex: male; lifeStage: adult; preparations: pinned; catalogNumber: DHJPAR0007438; occurrenceDetails: http://janzen.sas.upenn.edu; recordedBy: D.H. Janzen, W. Hallwachs \& Elda Araya; otherCatalogNumbers: ASTAT210-06, 05-SRNP-744, BOLD:AAA5134; identifiedBy: AJ Fleming; dateldentified: 2017; language: en; institutionCode: CNC; collectionCode: Insects; basisOfRecord: Pinned Specimen

af. $\quad$ scientificName: Hyphantrophaga danausophaga; phylum: Arthropoda; class: Insecta; order: Diptera; family: Tachinidae; genus: Hyphantrophaga; specificEpithet: danausophaga; scientificNameAuthorship: Fleming \& Wood, 2018; continent: Central America; country: Costa Rica; countryCode: CR; stateProvince: Alajuela; county: Sector San Cristobal; locality: Area de Conservacion Guanacaste; verbatimLocality: Rio Blanco Abajo; verbatimElevation: 500; verbatimLatitude: 10.9004; verbatimLongitude: -85.3725 ; verbatimCoordinateSystem: Decimal; decimalLatitude: 10.9004; decimalLongitude: -85.3725; samplingProtocol: Reared from the larva of the Nymphalidae, Danaus plexippus; verbatimEventDate: 09-Apr-2003; individualID: DHJPAR0007439; individualCount: 1; sex: male; lifeStage: adult; preparations: pinned; catalogNumber: DHJPAR0007439; occurrenceDetails: http://janzen.sas.upenn.edu; recordedBy: D.H. Janzen, W. Hallwachs \& Minor Carmona; otherCatalogNumbers: ASTAT211-06, 03-SRNP-5767, BOLD:AAA5134; identifiedBy: AJ Fleming; dateldentified: 2017; language: en; institutionCode: CNC; collectionCode: Insects; basisOfRecord: Pinned Specimen

ag. scientificName: Hyphantrophaga danausophaga; phylum: Arthropoda; class: Insecta; order: Diptera; family: Tachinidae; genus: Hyphantrophaga; specificEpithet: danausophaga; scientificNameAuthorship: Fleming \& Wood, 2018; continent: Central America; country: Costa Rica; countryCode: CR; stateProvince: Alajuela; county: Sector San Cristobal; locality: Area de Conservacion Guanacaste; verbatimLocality: Vado Rio Cucaracho; verbatimElevation: 640; verbatimLatitude: 10.8702; verbatimLongitude: -85.3915; verbatimCoordinateSystem: Decimal; decimalLatitude: 10.8702; decimalLongitude: -85.3915; samplingProtocol: Reared from the larva of the Nymphalidae, Danaus plexippus; verbatimEventDate: 25-Mar-2006; individualID: DHJPAR0007122; individualCount: 1; lifeStage: adult; preparations: pinned; catalogNumber: DHJPAR0007122; occurrenceDetails: http://janzen.sas.upenn.edu; recordedBy: D.H. Janzen, W. Hallwachs \& Elda Araya; otherCatalogNumbers: ASTAV364-06, 06-SRNP-1738, BOLD:AAA5134; identifiedBy: AJ Fleming; dateldentified: 2017; language: en; institutionCode: CNC; collectionCode: Insects; basisOfRecord: Pinned Specimen

ah. ScientificName: Hyphantrophaga danausophaga; phylum: Arthropoda; class: Insecta; order: Diptera; family: Tachinidae; genus: Hyphantrophaga; specificEpithet: danausophaga; scientificNameAuthorship: Fleming \& Wood, 2018; continent: Central 
America; country: Costa Rica; countryCode: CR; stateProvince: Alajuela; county: Sector San Cristobal; locality: Area de Conservacion Guanacaste; verbatimLocality: Tajo Angeles; verbatimElevation: 540; verbatimLatitude: 10.8647; verbatimLongitude: -85.4153; verbatimCoordinateSystem: Decimal; decimalLatitude: 10.8647; decimalLongitude: -85.4153; samplingProtocol: Reared from the larva of the Nymphalidae, Danaus plexippus; verbatimEventDate: 25-Apr-2006; individualID: DHJPAR0009992; individualCount: 1; sex: male; lifeStage: adult; preparations: pinned; catalogNumber: DHJPAR0009992; occurrenceDetails: http://janzen.sas.upenn.edu; recordedBy: D.H. Janzen, W. Hallwachs \& Anabelle Cordoba; otherCatalog Numbers: ASTAV593-06, 06-SRNP-2664, BOLD:AAA5134; identifiedBy: AJ Fleming; dateldentified: 2017; language: en; institutionCode: CNC; collectionCode: Insects; basisOfRecord: Pinned Specimen

ai. ScientificName: Hyphantrophaga danausophaga; phylum: Arthropoda; class: Insecta; order: Diptera; family: Tachinidae; genus: Hyphantrophaga; specificEpithet: danausophaga; scientificNameAuthorship: Fleming \& Wood, 2018; continent: Central America; country: Costa Rica; countryCode: CR; stateProvince: Guanacaste; county: Sector Cacao; locality: Area de Conservacion Guanacaste; verbatimLocality: Cuesta Caimito; verbatimElevation: 640; verbatimLatitude: 10.8908; verbatimLongitude: -85.4719; verbatimCoordinateSystem: Decimal; decimalLatitude: 10.8908; decimalLongitude: -85.4719; samplingProtocol: Reared from the larva of the Nymphalidae, Danaus plexippus; verbatimEventDate: 13-Apr-2006; individualID: DHJPAR0010026; individualCount: 1; sex: male; lifeStage: adult; preparations: pinned; catalogNumber: DHJPAR0010026; occurrenceDetails: http://janzen.sas.upenn.edu; recordedBy: D.H. Janzen, W. Hallwachs \& Mariano Pereira; otherCatalogNumbers: ASTAV627-06, 06-SRNP-45198, BOLD:AAA5134; identifiedBy: AJ Fleming; dateldentified: 2017; language: en; institutionCode: CNC; collectionCode: Insects; basisOfRecord: Pinned Specimen

aj. $\quad$ scientificName: Hyphantrophaga danausophaga; phylum: Arthropoda; class: Insecta; order: Diptera; family: Tachinidae; genus: Hyphantrophaga; specificEpithet: danausophaga; scientificNameAuthorship: Fleming \& Wood, 2018; continent: Central America; country: Costa Rica; countryCode: CR; stateProvince: Guanacaste; county: Sector Cacao; locality: Area de Conservacion Guanacaste; verbatimLocality: Estacion Cacao; verbatimElevation: 1150; verbatimLatitude: 10.9269; verbatimLongitude: -85.4682; verbatimCoordinateSystem: Decimal; decimalLatitude: 10.9269; decimalLongitude: -85.4682; samplingProtocol: Reared from the larva of the Nymphalidae, Danaus gilippus; verbatimEventDate: 29-Mar-2011; individualID: DHJPAR0042587; individualCount: 1; lifeStage: adult; preparations: pinned; catalogNumber: DHJPAR0042587; occurrenceDetails: http://janzen.sas.upenn.edu; recordedBy: D.H. Janzen, W. Hallwachs \& Harry Ramirez; otherCatalogNumbers: ASHYH345-11, 11-SRNP-35111, BOLD:AAA5134; identifiedBy: AJ Fleming; dateldentified: 2017; language: en; institutionCode: CNC; collectionCode: Insects; basisOfRecord: Pinned Specimen

\section{Other materials:}

a. $\quad$ scientificName: Hyphantrophaga danausophaga; phylum: Arthropoda; class: Insecta; order: Diptera; family: Tachinidae; genus: Hyphantrophaga; specificEpithet: danausophaga; scientificNameAuthorship: Fleming \& Wood, 2018; continent: Central America; country: Costa Rica; countryCode: CR; stateProvince: Alajuela; county: Sector San Cristobal; locality: Area de Conservacion Guanacaste; verbatimLocality: Rio Areno; verbatimElevation: 460; verbatimLatitude: 10.9141; verbatimLongitude: 
-85.3817; verbatimCoordinateSystem: Decimal; decimalLatitude: 10.9141; decimalLongitude: -85.3817; samplingProtocol: Reared from the larva of the Nymphalidae, Danaus plexippus; verbatimEventDate: 16-Mar-2007; individualID: DHJPAR0017161; individualCount: 1; sex: female; lifeStage: adult; preparations: pinned; catalogNumber: DHJPAR0017161; occurrenceDetails: http:// janzen.sas.upenn.edu; recordedBy: D.H. Janzen, W. Hallwachs \& Anabelle Cordoba; otherCatalogNumbers: ASTAP599-07, 07-SRNP-760, BOLD:AAA5134; identifiedBy: AJ Fleming; dateldentified: 2017; language: en; institutionCode: CNC; collectionCode: Insects; basisOfRecord: Pinned Specimen

b. $\quad$ scientificName: Hyphantrophaga danausophaga; phylum: Arthropoda; class: Insecta; order: Diptera; family: Tachinidae; genus: Hyphantrophaga; specificEpithet: danausophaga; scientificNameAuthorship: Fleming \& Wood, 2018; continent: Central America; country: Costa Rica; countryCode: CR; stateProvince: Alajuela; county: Sector San Cristobal; locality: Area de Conservacion Guanacaste; verbatimLocality: Sendero Vivero; verbatimElevation: 730; verbatimLatitude: 10.8674; verbatimLongitude: -85.3874; verbatimCoordinateSystem: Decimal; decimalLatitude: 10.8674; decimalLongitude: -85.3874; samplingProtocol: Reared from the larva of the Nymphalidae, Danaus plexippus; verbatimEventDate: 18-Apr-2007; individualID: DHJPAR0019692; individualCount: 1; sex: female; lifeStage: adult; preparations: pinned; catalogNumber: DHJPAR0019692; occurrenceDetails: http:// janzen.sas.upenn.edu; recordedBy: D.H. Janzen, W. Hallwachs \& Anabelle Cordoba; otherCatalogNumbers: ASTAB240-07, 07-SRNP-1366, BOLD:AAA5134; identifiedBy: AJ Fleming; dateldentified: 2017; language: en; institutionCode: CNC; collectionCode: Insects; basisOfRecord: Pinned Specimen

c. ScientificName: Hyphantrophaga danausophaga; phylum: Arthropoda; class: Insecta; order: Diptera; family: Tachinidae; genus: Hyphantrophaga; specificEpithet: danausophaga; scientificNameAuthorship: Fleming \& Wood, 2018; continent: Central America; country: Costa Rica; countryCode: CR; stateProvince: Guanacaste; county: Sector Pitilla; locality: Area de Conservacion Guanacaste; verbatimLocality: Sendero Carica; verbatimElevation: 660; verbatimLatitude: 10.9928; verbatimLongitude: -85.4294; verbatimCoordinateSystem: Decimal; decimalLatitude: 10.9928; decimalLongitude: -85.4294; samplingProtocol: Reared from the larva of the Nymphalidae, Danaus plexippus; verbatimEventDate: 01-Apr-2007; individualID: DHJPAR0019735; individualCount: 1; sex: female; lifeStage: adult; preparations: pinned; catalogNumber: DHJPAR0019735; occurrenceDetails: http:// janzen.sas.upenn.edu; recordedBy: D.H. Janzen, W. Hallwachs \& Calixto Moraga; otherCatalogNumbers: ASTAB283-07, 07-SRNP-31425,; identifiedBy: AJ Fleming; dateldentified: 2017; language: en; institutionCode: CNC; collectionCode: Insects; basisOfRecord: Pinned Specimen

d. ScientificName: Hyphantrophaga danausophaga; phylum: Arthropoda; class: Insecta; order: Diptera; family: Tachinidae; genus: Hyphantrophaga; specificEpithet: danausophaga; scientificNameAuthorship: Fleming \& Wood, 2018; continent: Central America; country: Costa Rica; countryCode: CR; stateProvince: Guanacaste; county: Sector Pitilla; Iocality: Area de Conservacion Guanacaste; verbatimLocality: Sendero Carica; verbatimElevation: 660; verbatimLatitude: 10.9928; verbatimLongitude: -85.4294; verbatimCoordinateSystem: Decimal; decimalLatitude: 10.9928; decimalLongitude: -85.4294; samplingProtocol: Reared from the larva of the Nymphalidae, Danaus plexippus; verbatimEventDate: 01-Apr-2007; individualID: DHJPAR0019736; individualCount: 1; sex: female; lifeStage: adult; preparations: pinned; catalogNumber: DHJPAR0019736; occurrenceDetails: http:// 
janzen.sas.upenn.edu; recordedBy: D.H. Janzen, W. Hallwachs \& Calixto Moraga; otherCatalog Numbers: ASTAB284-07, 07-SRNP-31560, BOLD:AAA5134; identifiedBy: AJ Fleming; dateldentified: 2017; language: en; institutionCode: CNC; collectionCode: Insects; basisOfRecord: Pinned Specimen

e. $\quad$ scientificName: Hyphantrophaga danausophaga; phylum: Arthropoda; class: Insecta; order: Diptera; family: Tachinidae; genus: Hyphantrophaga; specificEpithet: danausophaga; scientificNameAuthorship: Fleming \& Wood, 2018; continent: Central America; country: Costa Rica; countryCode: CR; stateProvince: Guanacaste; county: Sector Pitilla; locality: Area de Conservacion Guanacaste; verbatimLocality: Sendero Carica; verbatimElevation: 660; verbatimLatitude: 10.9928; verbatimLongitude: -85.4294; verbatimCoordinateSystem: Decimal; decimalLatitude: 10.9928; decimalLongitude: -85.4294; samplingProtocol: Reared from the larva of the Nymphalidae, Danaus plexippus; verbatimEventDate: 31-Mar-2007; individualID: DHJPAR0019740; individualCount: 1; sex: male; lifeStage: adult; preparations: pinned; catalog Number: DHJPAR0019740; occurrenceDetails: http://janzen.sas.upenn.edu; recordedBy: D.H. Janzen, W. Hallwachs \& Calixto Moraga; otherCatalog Numbers: ASTAB288-07, 07-SRNP-31561, BOLD:AAA5134; identifiedBy: AJ Fleming; dateldentified: 2017; language: en; institutionCode: CNC; collectionCode: Insects; basisOfRecord: Pinned Specimen

f. $\quad$ scientificName: Hyphantrophaga danausophaga; phylum: Arthropoda; class: Insecta; order: Diptera; family: Tachinidae; genus: Hyphantrophaga; specificEpithet: danausophaga; scientificNameAuthorship: Fleming \& Wood, 2018; continent: Central America; country: Costa Rica; countryCode: CR; stateProvince: Guanacaste; county: Sector Pitilla; locality: Area de Conservacion Guanacaste; verbatimLocality: Estacion Pitilla; verbatimElevation: 675; verbatimLatitude: 10.9893; verbatimLongitude: -85.4258; verbatimCoordinateSystem: Decimal; decimalLatitude: 10.9893; decimalLongitude: -85.4258; samplingProtocol: Reared from the larva of the Nymphalidae, Danaus plexippus; verbatimEventDate: 29-Apr-2007; individualID: DHJPAR0019784; individualCount: 1; sex: male; lifeStage: adult; preparations: pinned; catalogNumber: DHJPAR0019784; occurrenceDetails: http://janzen.sas.upenn.edu; recordedBy: D.H. Janzen, W. Hallwachs \& Manuel Rios; otherCatalog Numbers: ASTAB332-07, 07-SRNP-31929, BOLD:AAA5134; identifiedBy: AJ Fleming; dateldentified: 2017; language: en; institutionCode: CNC; collectionCode: Insects; basisOfRecord: Pinned Specimen

g. $\quad$ scientificName: Hyphantrophaga danausophaga; phylum: Arthropoda; class: Insecta; order: Diptera; family: Tachinidae; genus: Hyphantrophaga; specificEpithet: danausophaga; scientificNameAuthorship: Fleming \& Wood, 2018; continent: Central America; country: Costa Rica; countryCode: CR; stateProvince: Guanacaste; county: Sector Horizontes; locality: Area de Conservacion Guanacaste; verbatimLocality: Quebrada San Pancho; verbatimElevation: 90; verbatimLatitude: 10.7477; verbatimLongitude: -85.5858; verbatimCoordinateSystem: Decimal; decimalLatitude: 10.7477; decimalLongitude: -85.5858; samplingProtocol: Reared from the larva of the Nymphalidae, Danaus plexippus; verbatimEventDate: 17-Jul-2007; individualID: DHJPAR0021826; individualCount: 1; lifeStage: adult; preparations: pinned; catalogNumber: DHJPAR0021826; occurrenceDetails: http://janzen.sas.upenn.edu; recordedBy: D.H. Janzen, W. Hallwachs \& Guillermo Pereira; otherCatalogNumbers: ASTAT964-07, 07-SRNP-14384, BOLD:AAA5134; identifiedBy: AJ Fleming; dateldentified: 2017; language: en; institutionCode: CNC; collectionCode: Insects; basisOfRecord: Pinned Specimen 
h. $\quad$ scientificName: Hyphantrophaga danausophaga; phylum: Arthropoda; class: Insecta; order: Diptera; family: Tachinidae; genus: Hyphantrophaga; specificEpithet: danausophaga; scientificNameAuthorship: Fleming \& Wood, 2018; continent: Central America; country: Costa Rica; countryCode: CR; stateProvince: Guanacaste; county: Sector Horizontes; locality: Area de Conservacion Guanacaste; verbatimLocality: Quebrada San Pancho; verbatimElevation: 90; verbatimLatitude: 10.7477; verbatimLongitude: -85.5858; verbatimCoordinateSystem: Decimal; decimalLatitude: 10.7477; decimalLongitude: -85.5858; samplingProtocol: Reared from the larva of the Nymphalidae, Danaus plexippus; verbatimEventDate: 17-Jul-2007; individualID: DHJPAR0021829; individualCount: 1; sex: female; lifeStage: adult; preparations: pinned; catalogNumber: DHJPAR0021829; occurrenceDetails: http:// janzen.sas.upenn.edu; recordedBy: D.H. Janzen, W. Hallwachs \& Guillermo Pereira; otherCatalogNumbers: ASTAT967-07, 07-SRNP-14379, BOLD:AAA5134; identifiedBy: AJ Fleming; dateldentified: 2017; language: en; institutionCode: CNC; collectionCode: Insects; basisOfRecord: Pinned Specimen

i. $\quad$ scientificName: Hyphantrophaga danausophaga; phylum: Arthropoda; class: Insecta; order: Diptera; family: Tachinidae; genus: Hyphantrophaga; specificEpithet:

danausophaga; scientificNameAuthorship: Fleming \& Wood, 2018; continent: Central America; country: Costa Rica; countryCode: CR; stateProvince: Guanacaste; county: Sector Del Oro; locality: Area de Conservacion Guanacaste; verbatimLocality: Jose Leon; verbatimElevation: 400; verbatimLatitude: 11.033; verbatimLongitude: -85.429; verbatimCoordinateSystem: Decimal; decimalLatitude: 11.033; decimalLongitude: -85.429; samplingProtocol: Reared from the larva of the Nymphalidae, Danaus plexippus; verbatimEventDate: 05-Feb-2009; individualID: DHJPAR0030196; individualCount: 1; sex: female; lifeStage: adult; preparations: pinned; catalogNumber: DHJPAR0030196; occurrenceDetails: http://janzen.sas.upenn.edu; recordedBy: D.H. Janzen, W. Hallwachs \& Lucia Rios; otherCatalogNumbers: ASHYB940-09, 09SRNP-20077, BOLD:AAA5134; identifiedBy: AJ Fleming; dateldentified: 2017; language: en; institutionCode: CNC; collectionCode: Insects; basisOfRecord: Pinned Specimen

j. $\quad$ scientificName: Hyphantrophaga danausophaga; phylum: Arthropoda; class: Insecta; order: Diptera; family: Tachinidae; genus: Hyphantrophaga; specificEpithet: danausophaga; scientificNameAuthorship: Fleming \& Wood, 2018; continent: Central America; country: Costa Rica; countryCode: CR; stateProvince: Guanacaste; county: Sector Del Oro; locality: Area de Conservacion Guanacaste; verbatimLocality: Jose Leon; verbatimElevation: 400; verbatimLatitude: 11.033; verbatimLongitude: -85.429; verbatimCoordinateSystem: Decimal; decimalLatitude: 11.033; decimalLongitude: -85.429; samplingProtocol: Reared from the larva of the Nymphalidae, Danaus plexippus; verbatimEventDate: 02-Feb-2009; individualID: DHJPAR0030197; individualCount: 1; sex: female; lifeStage: adult; preparations: pinned; catalogNumber: DHJPAR0030197; occurrenceDetails: http://janzen.sas.upenn.edu; recordedBy: D.H. Janzen, W. Hallwachs \& Lucia Rios; otherCatalogNumbers: ASHYB941-09, 09SRNP-20033, BOLD:AAA5134; identifiedBy: AJ Fleming; dateldentified: 2017; language: en; institutionCode: CNC; collectionCode: Insects; basisOfRecord: Pinned Specimen

k. $\quad$ scientificName: Hyphantrophaga danausophaga; phylum: Arthropoda; class: Insecta; order: Diptera; family: Tachinidae; genus: Hyphantrophaga; specificEpithet:

danausophaga; scientificNameAuthorship: Fleming \& Wood, 2018; continent: Central America; country: Costa Rica; countryCode: CR; stateProvince: Guanacaste; county: Sector Cacao; locality: Area de Conservacion Guanacaste; verbatimLocality: Estacion 
Cacao; verbatimElevation: 1150; verbatimLatitude: 10.9269; verbatimLongitude: -85.4682; verbatimCoordinateSystem: Decimal; decimalLatitude: 10.9269; decimalLongitude: -85.4682; samplingProtocol: Reared from the larva of the Nymphalidae, Danaus plexippus; verbatimEventDate: 11-May-2009; individualID: DHJPAR0034366; individualCount: 1; sex: female; lifeStage: adult; preparations: pinned; catalogNumber: DHJPAR0034366; occurrenceDetails: http:// janzen.sas.upenn.edu; recordedBy: D.H. Janzen, W. Hallwachs \& Dunia Garcia; otherCatalogNumbers: ASHYC1018-09, 09-SRNP-35278, BOLD:AAA5134; identifiedBy: AJ Fleming; dateldentified: 2017; language: en; institutionCode: CNC; collectionCode: Insects; basisOfRecord: Pinned Specimen

I. scientificName: Hyphantrophaga danausophaga; phylum: Arthropoda; class: Insecta; order: Diptera; family: Tachinidae; genus: Hyphantrophaga; specificEpithet: danausophaga; scientificNameAuthorship: Fleming \& Wood, 2018; continent: Central America; country: Costa Rica; countryCode: CR; stateProvince: Guanacaste; county: Sector Cacao; locality: Area de Conservacion Guanacaste; verbatimLocality: Estacion Cacao; verbatimElevation: 1150; verbatimLatitude: 10.9269; verbatimLongitude: -85.4682; verbatimCoordinateSystem: Decimal; decimalLatitude: 10.9269; decimalLongitude: -85.4682; samplingProtocol: Reared from the larva of the Nymphalidae, Danaus plexippus; verbatimEventDate: 22-May-2009; individualID: DHJPAR0034367; individualCount: 1; sex: female; lifeStage: adult; preparations: pinned; catalogNumber: DHJPAR0034367; occurrenceDetails: http:// janzen.sas.upenn.edu; recordedBy: D.H. Janzen, W. Hallwachs \& Manuel Pereira; otherCatalogNumbers: ASHYC1019-09, 09-SRNP-35356, BOLD:AAA5134; identifiedBy: AJ Fleming; dateldentified: 2017; language: en; institutionCode: CNC; collectionCode: Insects; basisOfRecord: Pinned Specimen

m. scientificName: Hyphantrophaga danausophaga; phylum: Arthropoda; class: Insecta; order: Diptera; family: Tachinidae; genus: Hyphantrophaga; specificEpithet: danausophaga; scientificNameAuthorship: Fleming \& Wood, 2018; continent: Central America; country: Costa Rica; countryCode: CR; stateProvince: Guanacaste; county: Sector Cacao; locality: Area de Conservacion Guanacaste; verbatimLocality: Estacion Cacao; verbatimElevation: 1150; verbatimLatitude: 10.9269; verbatimLongitude: -85.4682; verbatimCoordinateSystem: Decimal; decimalLatitude: 10.9269; decimalLongitude: -85.4682; samplingProtocol: Reared from the larva of the Nymphalidae, Danaus plexippus; verbatimEventDate: 10-May-2009; individualID: DHJPAR0034369; individualCount: 1; sex: male; lifeStage: adult; preparations: pinned; catalogNumber: DHJPAR0034369; occurrenceDetails: http://janzen.sas.upenn.edu; recordedBy: D.H. Janzen, W. Hallwachs \& Harry Ramirez; otherCatalog Numbers: ASHYC1021-09, 09-SRNP-35265, BOLD:AAA5134; identifiedBy: AJ Fleming; dateldentified: 2017; language: en; institutionCode: CNC; collectionCode: Insects; basisOfRecord: Pinned Specimen

n. ScientificName: Hyphantrophaga danausophaga; phylum: Arthropoda; class: Insecta; order: Diptera; family: Tachinidae; genus: Hyphantrophaga; specificEpithet: danausophaga; scientificNameAuthorship: Fleming \& Wood, 2018; continent: Central America; country: Costa Rica; countryCode: CR; stateProvince: Guanacaste; county: Sector Cacao; locality: Area de Conservacion Guanacaste; verbatimLocality: Estacion Cacao; verbatimElevation: 1150; verbatimLatitude: 10.9269; verbatimLongitude: -85.4682; verbatimCoordinateSystem: Decimal; decimalLatitude: 10.9269; decimalLongitude: -85.4682; samplingProtocol: Reared from the larva of the Nymphalidae, Danaus plexippus; verbatimEventDate: 18-May-2009; individualID: DHJPAR0034370; individualCount: 1; sex: male; lifeStage: adult; preparations: pinned; 
catalogNumber: DHJPAR0034370; occurrenceDetails: http://janzen.sas.upenn.edu; recordedBy: D.H. Janzen, W. Hallwachs \& Manuel Pereira; otherCatalogNumbers: ASHYC1022-09, 09-SRNP-35358, BOLD:AAA5134; identifiedBy: AJ Fleming; dateldentified: 2017; language: en; institutionCode: CNC; collectionCode: Insects; basisOfRecord: Pinned Specimen

o. ScientificName: Hyphantrophaga danausophaga; phylum: Arthropoda; class: Insecta; order: Diptera; family: Tachinidae; genus: Hyphantrophaga; specificEpithet: danausophaga; scientificNameAuthorship: Fleming \& Wood, 2018; continent: Central America; country: Costa Rica; countryCode: CR; stateProvince: Guanacaste; county: Sector Cacao; locality: Area de Conservacion Guanacaste; verbatimLocality: Estacion Cacao; verbatimElevation: 1150; verbatimLatitude: 10.9269; verbatimLongitude: -85.4682; verbatimCoordinateSystem: Decimal; decimalLatitude: 10.9269; decimalLongitude: -85.4682; samplingProtocol: Reared from the larva of the Nymphalidae, Danaus plexippus; verbatimEventDate: 20-May-2009; individualID: DHJPAR0034371; individualCount: 1; sex: female; lifeStage: adult; preparations: pinned; catalogNumber: DHJPAR0034371; occurrenceDetails: http:// janzen.sas.upenn.edu; recordedBy: D.H. Janzen, W. Hallwachs \& Manuel Pereira; otherCatalog Numbers: ASHYC1023-09, 09-SRNP-35342, BOLD:AAA5134; identifiedBy: AJ Fleming; dateldentified: 2017; language: en; institutionCode: CNC; collectionCode: Insects; basisOfRecord: Pinned Specimen

p. $\quad$ scientificName: Hyphantrophaga danausophaga; phylum: Arthropoda; class: Insecta; order: Diptera; family: Tachinidae; genus: Hyphantrophaga; specificEpithet: danausophaga; scientificNameAuthorship: Fleming \& Wood, 2018; continent: Central America; country: Costa Rica; countryCode: CR; stateProvince: Guanacaste; county: Sector Cacao; locality: Area de Conservacion Guanacaste; verbatimLocality: Estacion Cacao; verbatimElevation: 1150; verbatimLatitude: 10.9269; verbatimLongitude: -85.4682; verbatimCoordinateSystem: Decimal; decimalLatitude: 10.9269; decimalLongitude: -85.4682; samplingProtocol: Reared from the larva of the Nymphalidae, Danaus plexippus; verbatimEventDate: 05-May-2009; individualID: DHJPAR0034375; individualCount: 1; sex: female; lifeStage: adult; preparations: pinned; catalogNumber: DHJPAR0034375; occurrenceDetails: http:// janzen.sas.upenn.edu; recordedBy: D.H. Janzen, W. Hallwachs \& Manuel Pereira; otherCatalog Numbers: ASHYC1027-09, 09-SRNP-35341, BOLD:AAA5134; identifiedBy: AJ Fleming; dateldentified: 2017; language: en; institutionCode: CNC; collectionCode: Insects; basisOfRecord: Pinned Specimen

q. $\quad$ scientificName: Hyphantrophaga danausophaga; phylum: Arthropoda; class: Insecta; order: Diptera; family: Tachinidae; genus: Hyphantrophaga; specificEpithet: danausophaga; scientificNameAuthorship: Fleming \& Wood, 2018; continent: Central America; country: Costa Rica; countryCode: CR; stateProvince: Guanacaste; county: Sector Cacao; locality: Area de Conservacion Guanacaste; verbatimLocality: Estacion Cacao; verbatimElevation: 1150; verbatimLatitude: 10.9269; verbatimLongitude: -85.4682; verbatimCoordinateSystem: Decimal; decimalLatitude: 10.9269; decimalLongitude: -85.4682; samplingProtocol: Reared from the larva of the Nymphalidae, Danaus plexippus; verbatimEventDate: 22-May-2009; individualID: DHJPAR0034377; individualCount: 1; sex: female; lifeStage: adult; preparations: pinned; catalogNumber: DHJPAR0034377; occurrenceDetails: http:// janzen.sas.upenn.edu; recordedBy: D.H. Janzen, W. Hallwachs \& Manuel Pereira; otherCatalogNumbers: ASHYC1029-09, 09-SRNP-35356, BOLD:AAA5134; identifiedBy: AJ Fleming; dateldentified: 2017; language: en; institutionCode: CNC; collectionCode: Insects; basisOfRecord: Pinned Specimen 
r. scientificName: Hyphantrophaga danausophaga; phylum: Arthropoda; class: Insecta; order: Diptera; family: Tachinidae; genus: Hyphantrophaga; specificEpithet: danausophaga; scientificNameAuthorship: Fleming \& Wood, 2018; continent: Central America; country: Costa Rica; countryCode: CR; stateProvince: Guanacaste; county: Sector Cacao; locality: Area de Conservacion Guanacaste; verbatimLocality: Estacion Cacao; verbatimElevation: 1150; verbatimLatitude: 10.9269; verbatimLongitude: -85.4682; verbatimCoordinateSystem: Decimal; decimalLatitude: 10.9269; decimalLongitude: -85.4682; samplingProtocol: Reared from the larva of the Nymphalidae, Danaus plexippus; verbatimEventDate: 22-May-2009; individualID: DHJPAR0034379; individualCount: 1; sex: female; lifeStage: adult; preparations: pinned; catalogNumber: DHJPAR0034379; occurrenceDetails: http:// janzen.sas.upenn.edu; recordedBy: D.H. Janzen, W. Hallwachs \& Manuel Pereira; otherCatalog Numbers: ASHYC1031-09, 09-SRNP-35357, BOLD:AAA5134; identifiedBy: AJ Fleming; dateldentified: 2017; language: en; institutionCode: CNC; collectionCode: Insects; basisOfRecord: Pinned Specimen

s. $\quad$ scientificName: Hyphantrophaga danausophaga; phylum: Arthropoda; class: Insecta; order: Diptera; family: Tachinidae; genus: Hyphantrophaga; specificEpithet: danausophaga; scientificNameAuthorship: Fleming \& Wood, 2018; continent: Central America; country: Costa Rica; countryCode: CR; stateProvince: Alajuela; county: Sector San Cristobal; locality: Area de Conservacion Guanacaste; verbatimLocality: Finca San Gabriel; verbatimElevation: 645; verbatimLatitude: 10.8777 ; verbatimLongitude: -85.3934; verbatimCoordinateSystem: Decimal; decimalLatitude: 10.8777; decimalLongitude: -85.3934; samplingProtocol: Reared from the larva of the Nymphalidae, Danaus plexippus; verbatimEventDate: 06-Sep-2011; individualID: DHJPAR0045680; individualCount: 1; sex: female; lifeStage: adult; preparations: pinned; catalogNumber: DHJPAR0045680; occurrenceDetails: http:// janzen.sas.upenn.edu; recordedBy: D.H. Janzen, W. Hallwachs \& Christian Herrera; otherCatalog Numbers: ACGAZ869-11, 11-SRNP-3220, BOLD:AAA5134; identifiedBy: AJ Fleming; dateldentified: 2017; language: en; institutionCode: CNC; collectionCode: Insects; basisOfRecord: Pinned Specimen

t. ScientificName: Hyphantrophaga danausophaga; phylum: Arthropoda; class: Insecta; order: Diptera; family: Tachinidae; genus: Hyphantrophaga; specificEpithet: danausophaga; scientificNameAuthorship: Fleming \& Wood, 2018; continent: Central America; country: Costa Rica; countryCode: CR; stateProvince: Guanacaste; county: Sector Cacao; locality: Area de Conservacion Guanacaste; verbatimLocality: Estacion Cacao; verbatimElevation: 1150; verbatimLatitude: 10.9269; verbatimLongitude: -85.4682; verbatimCoordinateSystem: Decimal; decimalLatitude: 10.9269; decimalLongitude: -85.4682; samplingProtocol: Reared from the larva of the Nymphalidae, Danaus gilippus; verbatimEventDate: 14-Sep-2011; individualID: DHJPAR0046595; individualCount: 1; sex: female; lifeStage: adult; preparations: pinned; catalogNumber: DHJPAR0046595; occurrenceDetails: http:// janzen.sas.upenn.edu; recordedBy: D.H. Janzen, W. Hallwachs \& Manuel Pereira; otherCatalogNumbers: ACGBA768-12, 11-SRNP-35602, BOLD:AAA5134; identifiedBy: AJ Fleming; dateldentified: 2017; language: en; institutionCode: CNC; collectionCode: Insects; basisOfRecord: Pinned Specimen

u. $\quad$ scientificName: Hyphantrophaga danausophaga; phylum: Arthropoda; class: Insecta; order: Diptera; family: Tachinidae; genus: Hyphantrophaga; specificEpithet: danausophaga; scientificNameAuthorship: Fleming \& Wood, 2018; continent: Central America; country: Costa Rica; countryCode: CR; stateProvince: Guanacaste; county: Sector Cacao; locality: Area de Conservacion Guanacaste; verbatimLocality: Estacion 
Cacao; verbatimElevation: 1150; verbatimLatitude: 10.9269; verbatimLongitude: -85.4682; verbatimCoordinateSystem: Decimal; decimalLatitude: 10.9269; decimalLongitude: -85.4682; samplingProtocol: Reared from the larva of the Nymphalidae, Danaus gilippus; verbatimEventDate: 16-Sep-2011; individualID: DHJPAR0046596; individualCount: 1; sex: female; lifeStage: adult; preparations: pinned; catalogNumber: DHJPAR0046596; occurrenceDetails: http:// janzen.sas.upenn.edu; recordedBy: D.H. Janzen, W. Hallwachs \& Manuel Pereira; otherCatalogNumbers: ACGBA769-12, 11-SRNP-35603, BOLD:AAA5134; identifiedBy: AJ Fleming; dateldentified: 2017; language: en; institutionCode: CNC; collectionCode: Insects; basisOfRecord: Pinned Specimen

v. scientificName: Hyphantrophaga danausophaga; phylum: Arthropoda; class: Insecta; order: Diptera; family: Tachinidae; genus: Hyphantrophaga; specificEpithet: danausophaga; scientificNameAuthorship: Fleming \& Wood, 2018; continent: Central America; country: Costa Rica; countryCode: CR; stateProvince: Guanacaste; county: Sector Cacao; locality: Area de Conservacion Guanacaste; verbatimLocality: Estacion Cacao; verbatimElevation: 1150; verbatimLatitude: 10.9269; verbatimLongitude: -85.4682; verbatimCoordinateSystem: Decimal; decimalLatitude: 10.9269; decimalLongitude: -85.4682; samplingProtocol: Reared from the larva of the Nymphalidae, Danaus gilippus; verbatimEventDate: 14-Sep-2011; individualID: DHJPAR0046597; individualCount: 1; sex: female; lifeStage: adult; preparations: pinned; catalogNumber: DHJPAR0046597; occurrenceDetails: http:// janzen.sas.upenn.edu; recordedBy: D.H. Janzen, W. Hallwachs \& Manuel Pereira; otherCatalog Numbers: ACGBA770-12, 11-SRNP-35604, BOLD:AAA5134; identifiedBy: AJ Fleming; dateldentified: 2017; language: en; institutionCode: CNC; collectionCode: Insects; basisOfRecord: Pinned Specimen

w. ScientificName: Hyphantrophaga danausophaga; phylum: Arthropoda; class: Insecta; order: Diptera; family: Tachinidae; genus: Hyphantrophaga; specificEpithet: danausophaga; scientificNameAuthorship: Fleming \& Wood, 2018; continent: Central America; country: Costa Rica; countryCode: CR; stateProvince: Guanacaste; county: Sector Cacao; locality: Area de Conservacion Guanacaste; verbatimLocality: Estacion Cacao; verbatimElevation: 1150; verbatimLatitude: 10.9269; verbatimLongitude: -85.4682; verbatimCoordinateSystem: Decimal; decimalLatitude: 10.9269; decimalLongitude: -85.4682; samplingProtocol: Reared from the larva of the Nymphalidae, Danaus gilippus; verbatimEventDate: 16-Sep-2011; individualID: DHJPAR0046598; individualCount: 1; sex: female; lifeStage: adult; preparations: pinned; catalog Number: DHJPAR0046598; occurrenceDetails: http:// janzen.sas.upenn.edu; recordedBy: D.H. Janzen, W. Hallwachs \& Manuel Pereira; otherCatalogNumbers: ACGBA771-12, 11-SRNP-35605, BOLD:AAA5134; identifiedBy: AJ Fleming; dateldentified: 2017; language: en; institutionCode: CNC; collectionCode: Insects; basisOfRecord: Pinned Specimen

x. $\quad$ scientificName: Hyphantrophaga danausophaga; phylum: Arthropoda; class: Insecta; order: Diptera; family: Tachinidae; genus: Hyphantrophaga; specificEpithet: danausophaga; scientificNameAuthorship: Fleming \& Wood, 2018; continent: Central America; country: Costa Rica; countryCode: CR; stateProvince: Alajuela; county: Sector Rincon Rain Forest; locality: Area de Conservacion Guanacaste; verbatimLocality: Finca Esmeralda; verbatimElevation: 123; verbatimLatitude: 10.9355; verbatimLongitude: -85.2531; verbatimCoordinateSystem: Decimal; decimalLatitude: 10.9355; decimalLongitude: -85.2531; samplingProtocol: Reared from the larva of the Nymphalidae, Danaus plexippus; verbatimEventDate: 02-Apr-2012; individuallD: DHJPAR0050240; individualCount: 1; sex: male; lifeStage: adult; preparations: pinned; 
catalogNumber: DHJPAR0050240; occurrenceDetails: http://janzen.sas.upenn.edu; recordedBy: D.H. Janzen, W. Hallwachs \& Cirilo Umaña; otherCatalogNumbers: ACGAZ1554-12, 12-SRNP-75567, BOLD:AAA5134; identifiedBy: AJ Fleming; dateldentified: 2017; language: en; institutionCode: CNC; collectionCode: Insects; basisOfRecord: Pinned Specimen

y. $\quad$ scientificName: Hyphantrophaga danausophaga; phylum: Arthropoda; class: Insecta; order: Diptera; family: Tachinidae; genus: Hyphantrophaga; specificEpithet: danausophaga; scientificNameAuthorship: Fleming \& Wood, 2018; continent: Central America; country: Costa Rica; countryCode: CR; stateProvince: Alajuela; county: Sector Rincon Rain Forest; locality: Area de Conservacion Guanacaste; verbatimLocality: Finca Esmeralda; verbatimElevation: 123; verbatimLatitude: 10.9355; verbatimLongitude: -85.2531; verbatimCoordinateSystem: Decimal; decimalLatitude: 10.9355; decimalLongitude: -85.2531; samplingProtocol: Reared from the larva of the Nymphalidae, Danaus plexippus; verbatimEventDate: 26-Jan-2013; individualID: DHJPAR0050466; individualCount: 1; sex: female; lifeStage: adult; preparations: pinned; catalogNumber: DHJPAR0050466; occurrenceDetails: http:// janzen.sas.upenn.edu; recordedBy: D.H. Janzen, W. Hallwachs \& Cirilo Umaña; otherCatalogNumbers: ACGBA3058-13, 13-SRNP-75000, BOLD:AAA5134; identifiedBy: AJ Fleming; dateldentified: 2017; language: en; institutionCode: CNC; collectionCode: Insects; basisOfRecord: Pinned Specimen

z. $\quad$ scientificName: Hyphantrophaga danausophaga; phylum: Arthropoda; class: Insecta; order: Diptera; family: Tachinidae; genus: Hyphantrophaga; specificEpithet: danausophaga; scientificNameAuthorship: Fleming \& Wood, 2018; continent: Central America; country: Costa Rica; countryCode: CR; stateProvince: Alajuela; county: Sector Rincon Rain Forest; locality: Area de Conservacion Guanacaste; verbatimLocality: Finca Esmeralda; verbatimElevation: 123; verbatimLatitude: 10.9355; verbatimLongitude: -85.2531; verbatimCoordinateSystem: Decimal; decimalLatitude: 10.9355; decimalLongitude: -85.2531; samplingProtocol: Reared from the larva of the Nymphalidae, Danaus gilippus; verbatimEventDate: 17-Jan-2013; individualID: DHJPAR0050476; individualCount: 1; sex: female; lifeStage: adult; preparations: pinned; catalogNumber: DHJPAR0050476; occurrenceDetails: http:// janzen.sas.upenn.edu; recordedBy: D.H. Janzen, W. Hallwachs \& Cirilo Umaña; otherCatalogNumbers: ACGBA3068-13, 12-SRNP-78158, BOLD:AAA5134; identifiedBy: AJ Fleming; dateldentified: 2017; language: en; institutionCode: CNC; collectionCode: Insects; basisOfRecord: Pinned Specimen

aa. $\quad$ scientificName: Hyphantrophaga danausophaga; phylum: Arthropoda; class: Insecta; order: Diptera; family: Tachinidae; genus: Hyphantrophaga; specificEpithet: danausophaga; scientificNameAuthorship: Fleming \& Wood, 2018; continent: Central America; country: Costa Rica; countryCode: CR; stateProvince: Alajuela; county: Sector Rincon Rain Forest; locality: Area de Conservacion Guanacaste; verbatimLocality: Finca Esmeralda; verbatimElevation: 123; verbatimLatitude: 10.9355; verbatimLongitude: -85.2531; verbatimCoordinateSystem: Decimal; decimalLatitude: 10.9355; decimalLongitude: -85.2531; samplingProtocol: Reared from the larva of the Nymphalidae, Danaus gilippus; verbatimEventDate: 18-Jan-2013; individualID: DHJPAR0050479; individualCount: 1; sex: female; lifeStage: adult; preparations: pinned; catalogNumber: DHJPAR0050479; occurrenceDetails: http:// janzen.sas.upenn.edu; recordedBy: D.H. Janzen, W. Hallwachs \& Cirilo Umaña; otherCatalogNumbers: ACGBA3071-13, 12-SRNP-78157, BOLD:AAA5134; identifiedBy: AJ Fleming; dateldentified: 2017; language: en; institutionCode: CNC; collectionCode: Insects; basisOfRecord: Pinned Specimen 
ab. ScientificName: Hyphantrophaga danausophaga; phylum: Arthropoda; class: Insecta; order: Diptera; family: Tachinidae; genus: Hyphantrophaga; specificEpithet: danausophaga; scientificNameAuthorship: Fleming \& Wood, 2018; continent: Central America; country: Costa Rica; countryCode: CR; stateProvince: Alajuela; county: Sector Rincon Rain Forest; locality: Area de Conservacion Guanacaste; verbatimLocality: Finca Esmeralda; verbatimElevation: 123; verbatimLatitude: 10.9355; verbatimLongitude: -85.2531; verbatimCoordinateSystem: Decimal; decimalLatitude: 10.9355; decimalLongitude: -85.2531; samplingProtocol: Reared from the larva of the Nymphalidae, Danaus gilippus; verbatimEventDate: 10-Dec-2012; individualID: DHJPAR0050634; individualCount: 1; sex: male; lifeStage: adult; preparations: pinned; catalogNumber: DHJPAR0050634; occurrenceDetails: http://janzen.sas.upenn.edu; recordedBy: D.H. Janzen, W. Hallwachs \& Cirilo Umaña; otherCatalogNumbers: ACGBA3226-13, 12-SRNP-77661, BOLD:AAA5134; identifiedBy: AJ Fleming; dateldentified: 2017; language: en; institutionCode: CNC; collectionCode: Insects; basisOfRecord: Pinned Specimen

ac. ScientificName: Hyphantrophaga danausophaga; phylum: Arthropoda; class: Insecta; order: Diptera; family: Tachinidae; genus: Hyphantrophaga; specificEpithet: danausophaga; scientificNameAuthorship: Fleming \& Wood, 2018; continent: Central America; country: Costa Rica; countryCode: CR; stateProvince: Guanacaste; county: Sector El Hacha; locality: Area de Conservacion Guanacaste; verbatimLocality: Estacion Los Almendros; verbatimElevation: 290; verbatimLatitude: 11.0323; verbatimLongitude: -85.5278; verbatimCoordinateSystem: Decimal; decimalLatitude: 11.0323; decimalLongitude: -85.5278; samplingProtocol: Reared from the larva of the Nymphalidae, Danaus plexippus; verbatimEventDate: 19-Aug-2013; individualID: DHJPAR0052440; individualCount: 1; sex: male; lifeStage: adult; preparations: pinned; catalogNumber: DHJPAR0052440; occurrenceDetails: http://janzen.sas.upenn.edu; recordedBy: D.H. Janzen, W. Hallwachs \& Lucia Rios; otherCatalogNumbers: ASHYM1794-13, 13-SRNP-21416, BOLD:AAA5134; identifiedBy: AJ Fleming; dateldentified: 2017; language: en; institutionCode: CNC; collectionCode: Insects; basisOfRecord: Pinned Specimen

ad. ScientificName: Hyphantrophaga danausophaga; phylum: Arthropoda; class: Insecta; order: Diptera; family: Tachinidae; genus: Hyphantrophaga; specificEpithet: danausophaga; scientificNameAuthorship: Fleming \& Wood, 2018; continent: Central America; country: Costa Rica; countryCode: CR; stateProvince: Guanacaste; county: Sector El Hacha; locality: Area de Conservacion Guanacaste; verbatimLocality: Los Inocentes; verbatimElevation: 275 ; verbatimLatitude: 11.0324; verbatimLongitude: -85.4993; verbatimCoordinateSystem: Decimal; decimalLatitude: 11.0324; decimalLongitude: -85.4993; samplingProtocol: Reared from the larva of the Nymphalidae, Danaus plexippus; verbatimEventDate: 16-Aug-2013; individualID: DHJPAR0052445; individualCount: 1; sex: female; lifeStage: adult; preparations: pinned; catalogNumber: DHJPAR0052445; occurrenceDetails: http:// janzen.sas.upenn.edu; recordedBy: D.H. Janzen, W. Hallwachs \& Lucia Rios; otherCatalogNumbers: ASHYM1799-13, 13-SRNP-21291, BOLD:AAA5134; identifiedBy: AJ Fleming; dateldentified: 2017; language: en; institutionCode: CNC; collectionCode: Insects; basisOfRecord: Pinned Specimen

ae. $\quad$ scientificName: Hyphantrophaga danausophaga; phylum: Arthropoda; class: Insecta; order: Diptera; family: Tachinidae; genus: Hyphantrophaga; specificEpithet: danausophaga; scientificNameAuthorship: Fleming \& Wood, 2018; continent: Central America; country: Costa Rica; countryCode: CR; stateProvince: Guanacaste; county: Sector El Hacha; locality: Area de Conservacion Guanacaste; verbatimLocality: Los 
Inocentes; verbatimElevation: 275 ; verbatimLatitude: 11.0324; verbatimLongitude: -85.4993; verbatimCoordinateSystem: Decimal; decimalLatitude: 11.0324; decimalLongitude: -85.4993; samplingProtocol: Reared from the larva of the Nymphalidae, Danaus plexippus; verbatimEventDate: 10-Aug-2013; individualID: DHJPAR0052447; individualCount: 1; sex: male; lifeStage: adult; preparations: pinned; catalogNumber: DHJPAR0052447; occurrenceDetails: http://janzen.sas.upenn.edu; recordedBy: D.H. Janzen, W. Hallwachs \& Lucia Rios; otherCatalogNumbers: ASHYM1801-13, 13-SRNP-21325, BOLD:AAA5134; identifiedBy: AJ Fleming; dateldentified: 2017; language: en; institutionCode: CNC; collectionCode: Insects; basisOfRecord: Pinned Specimen

af. ScientificName: Hyphantrophaga danausophaga; phylum: Arthropoda; class: Insecta; order: Diptera; family: Tachinidae; genus: Hyphantrophaga; specificEpithet: danausophaga; scientificNameAuthorship: Fleming \& Wood, 2018; continent: Central America; country: Costa Rica; countryCode: CR; stateProvince: Guanacaste; county: Sector El Hacha; locality: Area de Conservacion Guanacaste; verbatimLocality: Los Inocentes; verbatimElevation: 275 ; verbatimLatitude: 11.0324; verbatimLongitude: -85.4993; verbatimCoordinateSystem: Decimal; decimalLatitude: 11.0324; decimalLongitude: -85.4993; samplingProtocol: Reared from the larva of the Nymphalidae, Danaus plexippus; verbatimEventDate: 08-Aug-2013; individualID: DHJPAR0052448; individualCount: 1; sex: female; lifeStage: adult; preparations: pinned; catalogNumber: DHJPAR0052448; occurrenceDetails: http:// janzen.sas.upenn.edu; recordedBy: D.H. Janzen, W. Hallwachs \& Lucia Rios; otherCatalogNumbers: ASHYM1802-13, 13-SRNP-21279, BOLD:AAA5134; identifiedBy: AJ Fleming; dateldentified: 2017; language: en; institutionCode: CNC; collectionCode: Insects; basisOfRecord: Pinned Specimen

ag. $\quad$ scientificName: Hyphantrophaga danausophaga; phylum: Arthropoda; class: Insecta; order: Diptera; family: Tachinidae; genus: Hyphantrophaga; specificEpithet: danausophaga; scientificNameAuthorship: Fleming \& Wood, 2018; continent: Central America; country: Costa Rica; countryCode: CR; stateProvince: Guanacaste; county: Sector EI Hacha; locality: Area de Conservacion Guanacaste; verbatimLocality: Los Inocentes; verbatimElevation: 275; verbatimLatitude: 11.0324; verbatimLongitude: -85.4993; verbatimCoordinateSystem: Decimal; decimalLatitude: 11.0324; decimalLongitude: -85.4993; samplingProtocol: Reared from the larva of the Nymphalidae, Danaus plexippus; verbatimEventDate: 06-Aug-2013; individualID: DHJPAR0052451; individualCount: 1; sex: female; lifeStage: adult; preparations: pinned; catalog Number: DHJPAR0052451; occurrenceDetails: http:// janzen.sas.upenn.edu; recordedBy: D.H. Janzen, W. Hallwachs \& Lucia Rios; otherCatalogNumbers: ASHYM1805-13, 13-SRNP-21288, BOLD:AAA5134; identifiedBy: AJ Fleming; dateldentified: 2017; language: en; institutionCode: CNC; collectionCode: Insects; basisOfRecord: Pinned Specimen

ah. ScientificName: Hyphantrophaga danausophaga; phylum: Arthropoda; class: Insecta; order: Diptera; family: Tachinidae; genus: Hyphantrophaga; specificEpithet: danausophaga; scientificNameAuthorship: Fleming \& Wood, 2018; continent: Central America; country: Costa Rica; countryCode: CR; stateProvince: Guanacaste; county: Sector El Hacha; locality: Area de Conservacion Guanacaste; verbatimLocality: Los Inocentes; verbatimElevation: 275 ; verbatimLatitude: 11.0324; verbatimLongitude: -85.4993; verbatimCoordinateSystem: Decimal; decimalLatitude: 11.0324; decimalLongitude: -85.4993; samplingProtocol: Reared from the larva of the Nymphalidae, Danaus plexippus; verbatimEventDate: 12-Aug-2013; individualID: DHJPAR0052453; individualCount: 1; sex: female; lifeStage: adult; preparations: 
pinned; catalogNumber: DHJPAR0052453; occurrenceDetails: http:// janzen.sas.upenn.edu; recordedBy: D.H. Janzen, W. Hallwachs \& Lucia Rios; otherCatalogNumbers: ASHYM1807-13, 13-SRNP-21283, BOLD:AAA5134; identifiedBy: AJ Fleming; dateldentified: 2017; language: en; institutionCode: CNC; collectionCode: Insects; basisOfRecord: Pinned Specimen

ai. ScientificName: Hyphantrophaga danausophaga; phylum: Arthropoda; class: Insecta; order: Diptera; family: Tachinidae; genus: Hyphantrophaga; specificEpithet: danausophaga; scientificNameAuthorship: Fleming \& Wood, 2018; continent: Central America; country: Costa Rica; countryCode: CR; stateProvince: Guanacaste; county: Sector El Hacha; locality: Area de Conservacion Guanacaste; verbatimLocality: Los Inocentes; verbatimElevation: 275 ; verbatimLatitude: 11.0324; verbatimLongitude: -85.4993; verbatimCoordinateSystem: Decimal; decimalLatitude: 11.0324; decimalLongitude: -85.4993; samplingProtocol: Reared from the larva of the Nymphalidae, Danaus plexippus; verbatimEventDate: 02-Aug-2013; individualID: DHJPAR0052483; individualCount: 1; sex: female; lifeStage: adult; preparations: pinned; catalogNumber: DHJPAR0052483; occurrenceDetails: http:// janzen.sas.upenn.edu; recordedBy: D.H. Janzen, W. Hallwachs \& Elieth Cantillano; otherCatalogNumbers: ASHYM1837-13, 13-SRNP-21247, BOLD:AAA5134; identifiedBy: AJ Fleming; dateldentified: 2017; language: en; institutionCode: CNC; collectionCode: Insects; basisOfRecord: Pinned Specimen

aj. ScientificName: Hyphantrophaga danausophaga; phylum: Arthropoda; class: Insecta; order: Diptera; family: Tachinidae; genus: Hyphantrophaga; specificEpithet: danausophaga; scientificNameAuthorship: Fleming \& Wood, 2018; continent: Central America; country: Costa Rica; countryCode: CR; stateProvince: Guanacaste; county: Sector El Hacha; locality: Area de Conservacion Guanacaste; verbatimLocality: Estacion Los Almendros; verbatimElevation: 290; verbatimLatitude: 11.0323; verbatimLongitude: -85.5278; verbatimCoordinateSystem: Decimal; decimalLatitude: 11.0323; decimalLongitude: -85.5278; samplingProtocol: Reared from the larva of the Nymphalidae, Danaus plexippus; verbatimEventDate: 16-Sep-2013; individualID: DHJPAR0053346; individualCount: 1; sex: female; lifeStage: adult; preparations: pinned; catalogNumber: DHJPAR0053346; occurrenceDetails: http:// janzen.sas.upenn.edu; recordedBy: D.H. Janzen, W. Hallwachs \& Lucia Rios; otherCatalogNumbers: ASHYM2700-13, 13-SRNP-21925, BOLD:AAA5134; identifiedBy: AJ Fleming; dateldentified: 2017; language: en; institutionCode: CNC; collectionCode: Insects; basisOfRecord: Pinned Specimen

ak. ScientificName: Hyphantrophaga danausophaga; phylum: Arthropoda; class: Insecta; order: Diptera; family: Tachinidae; genus: Hyphantrophaga; specificEpithet: danausophaga; scientificNameAuthorship: Fleming \& Wood, 2018; continent: Central America; country: Costa Rica; countryCode: CR; stateProvince: Guanacaste; county: Sector El Hacha; locality: Area de Conservacion Guanacaste; verbatimLocality: Los Inocentes; verbatimElevation: 275 ; verbatimLatitude: 11.0324; verbatimLongitude: -85.4993; verbatimCoordinateSystem: Decimal; decimalLatitude: 11.0324; decimalLongitude: -85.4993; samplingProtocol: Reared from the larva of the Nymphalidae, Danaus plexippus; verbatimEventDate: 11-Sep-2013; individualID: DHJPAR0053347; individualCount: 1; sex: male; lifeStage: adult; preparations: pinned; catalogNumber: DHJPAR0053347; occurrenceDetails: http://janzen.sas.upenn.edu; recordedBy: D.H. Janzen, W. Hallwachs \& Lucia Rios; otherCatalogNumbers: ASHYM2701-13, 13-SRNP-21855, BOLD:AAA5134; identifiedBy: AJ Fleming; dateldentified: 2017; language: en; institutionCode: CNC; collectionCode: Insects; basisOfRecord: Pinned Specimen 
al. scientificName: Hyphantrophaga danausophaga; phylum: Arthropoda; class: Insecta; order: Diptera; family: Tachinidae; genus: Hyphantrophaga; specificEpithet: danausophaga; scientificNameAuthorship: Fleming \& Wood, 2018; continent: Central America; country: Costa Rica; countryCode: CR; stateProvince: Guanacaste; county: Sector El Hacha; locality: Area de Conservacion Guanacaste; verbatimLocality: Los Inocentes; verbatimElevation: 275 ; verbatimLatitude: 11.0324; verbatimLongitude: -85.4993; verbatimCoordinateSystem: Decimal; decimalLatitude: 11.0324; decimalLongitude: -85.4993; samplingProtocol: Reared from the larva of the Nymphalidae, Danaus plexippus; verbatimEventDate: 14-Sep-2013; individuallD: DHJPAR0053348; individualCount: 1; sex: male; lifeStage: adult; preparations: pinned; catalogNumber: DHJPAR0053348; occurrenceDetails: http://janzen.sas.upenn.edu; recordedBy: D.H. Janzen, W. Hallwachs \& Lucia Rios; otherCatalogNumbers: ASHYM2702-13, 13-SRNP-21867, BOLD:AAA5134; identifiedBy: AJ Fleming; dateldentified: 2017; language: en; institutionCode: CNC; collectionCode: Insects; basisOfRecord: Pinned Specimen

am. $\quad$ scientificName: Hyphantrophaga danausophaga; phylum: Arthropoda; class: Insecta; order: Diptera; family: Tachinidae; genus: Hyphantrophaga; specificEpithet: danausophaga; scientificNameAuthorship: Fleming \& Wood, 2018; continent: Central America; country: Costa Rica; countryCode: CR; stateProvince: Guanacaste; county: Sector El Hacha; locality: Area de Conservacion Guanacaste; verbatimLocality: Los Inocentes; verbatimElevation: 275 ; verbatimLatitude: 11.0324; verbatimLongitude: -85.4993; verbatimCoordinateSystem: Decimal; decimalLatitude: 11.0324; decimalLongitude: -85.4993; samplingProtocol: Reared from the larva of the Nymphalidae, Danaus plexippus; verbatimEventDate: 17-Sep-2013; individualID: DHJPAR0053349; individualCount: 1; sex: male; lifeStage: adult; preparations: pinned; catalogNumber: DHJPAR0053349; occurrenceDetails: http://janzen.sas.upenn.edu; recordedBy: D.H. Janzen, W. Hallwachs \& Lucia Rios; otherCatalogNumbers: ASHYM2703-13, 13-SRNP-22005, BOLD:AAA5134; identifiedBy: AJ Fleming; dateldentified: 2017; language: en; institutionCode: CNC; collectionCode: Insects; basisOfRecord: Pinned Specimen

an. ScientificName: Hyphantrophaga danausophaga; phylum: Arthropoda; class: Insecta; order: Diptera; family: Tachinidae; genus: Hyphantrophaga; specificEpithet: danausophaga; scientificNameAuthorship: Fleming \& Wood, 2018; continent: Central America; country: Costa Rica; countryCode: CR; stateProvince: Guanacaste; county: Sector El Hacha; locality: Area de Conservacion Guanacaste; verbatimLocality: Los Inocentes; verbatimElevation: 275 ; verbatimLatitude: 11.0324 ; verbatimLongitude: -85.4993; verbatimCoordinateSystem: Decimal; decimalLatitude: 11.0324; decimalLongitude: -85.4993; samplingProtocol: Reared from the larva of the Nymphalidae, Danaus plexippus; verbatimEventDate: 20-Sep-2013; individualID: DHJPAR0053350; individualCount: 1; sex: male; lifeStage: adult; preparations: pinned; catalogNumber: DHJPAR0053350; occurrenceDetails: http://janzen.sas.upenn.edu; recordedBy: D.H. Janzen, W. Hallwachs \& Lucia Rios; otherCatalogNumbers: ASHYM2704-13, 13-SRNP-21873, BOLD:AAA5134; identifiedBy: AJ Fleming; dateldentified: 2017; language: en; institutionCode: CNC; collectionCode: Insects; basisOfRecord: Pinned Specimen

ao. ScientificName: Hyphantrophaga danausophaga; phylum: Arthropoda; class: Insecta; order: Diptera; family: Tachinidae; genus: Hyphantrophaga; specificEpithet: danausophaga; scientificNameAuthorship: Fleming \& Wood, 2018; continent: Central America; country: Costa Rica; countryCode: CR; stateProvince: Guanacaste; county: Sector El Hacha; locality: Area de Conservacion Guanacaste; verbatimLocality: Los 
Inocentes; verbatimElevation: 275 ; verbatimLatitude: 11.0324 ; verbatimLongitude: -85.4993; verbatimCoordinateSystem: Decimal; decimalLatitude: 11.0324; decimalLongitude: -85.4993; samplingProtocol: Reared from the larva of the Nymphalidae, Danaus plexippus; verbatimEventDate: 14-Sep-2013; individualID: DHJPAR0053351; individualCount: 1; sex: male; lifeStage: adult; preparations: pinned; catalogNumber: DHJPAR0053351; occurrenceDetails: http://janzen.sas.upenn.edu; recordedBy: D.H. Janzen, W. Hallwachs \& Lucia Rios; otherCatalogNumbers: ASHYM2705-13, 13-SRNP-21870, BOLD:AAA5134; identifiedBy: AJ Fleming; dateldentified: 2017; language: en; institutionCode: CNC; collectionCode: Insects; basisOfRecord: Pinned Specimen

ap. $\quad$ scientificName: Hyphantrophaga danausophaga; phylum: Arthropoda; class: Insecta; order: Diptera; family: Tachinidae; genus: Hyphantrophaga; specificEpithet: danausophaga; scientificNameAuthorship: Fleming \& Wood, 2018; continent: Central America; country: Costa Rica; countryCode: CR; stateProvince: Guanacaste; county: Sector El Hacha; locality: Area de Conservacion Guanacaste; verbatimLocality: Los Inocentes; verbatimElevation: 275 ; verbatimLatitude: 11.0324; verbatimLongitude: -85.4993; verbatimCoordinateSystem: Decimal; decimalLatitude: 11.0324; decimalLongitude: -85.4993; samplingProtocol: Reared from the larva of the Nymphalidae, Danaus plexippus; verbatimEventDate: 11-Sep-2013; individualID: DHJPAR0053352; individualCount: 1; sex: female; lifeStage: adult; preparations: pinned; catalog Number: DHJPAR0053352; occurrenceDetails: http:// janzen.sas.upenn.edu; recordedBy: D.H. Janzen, W. Hallwachs \& Lucia Rios; otherCatalogNumbers: ASHYM2706-13, 13-SRNP-21869, BOLD:AAA5134; identifiedBy: AJ Fleming; dateldentified: 2017; language: en; institutionCode: CNC; collectionCode: Insects; basisOfRecord: Pinned Specimen

aq. $\quad$ scientificName: Hyphantrophaga danausophaga; phylum: Arthropoda; class: Insecta; order: Diptera; family: Tachinidae; genus: Hyphantrophaga; specificEpithet: danausophaga; scientificNameAuthorship: Fleming \& Wood, 2018; continent: Central America; country: Costa Rica; countryCode: CR; stateProvince: Guanacaste; county: Sector El Hacha; locality: Area de Conservacion Guanacaste; verbatimLocality: Los Inocentes; verbatimElevation: 275 ; verbatimLatitude: 11.0324; verbatimLongitude: -85.4993; verbatimCoordinateSystem: Decimal; decimalLatitude: 11.0324; decimalLongitude: -85.4993; samplingProtocol: Reared from the larva of the Nymphalidae, Danaus plexippus; verbatimEventDate: unavailable, 2013; individualID: DHJPAR0053353; individualCount: 1; sex: male; lifeStage: adult; preparations: pinned; catalogNumber: DHJPAR0053353; occurrenceDetails: http://janzen.sas.upenn.edu; recordedBy: D.H. Janzen, W. Hallwachs \& Lucia Rios; otherCatalogNumbers: ASHYM2707-13, 13-SRNP-21921, BOLD:AAA5134; identifiedBy: AJ Fleming; dateldentified: 2017; language: en; institutionCode: CNC; collectionCode: Insects; basisOfRecord: Pinned Specimen

ar. scientificName: Hyphantrophaga danausophaga; phylum: Arthropoda; class: Insecta; order: Diptera; family: Tachinidae; genus: Hyphantrophaga; specificEpithet: danausophaga; scientificNameAuthorship: Fleming \& Wood, 2018; continent: Central America; country: Costa Rica; countryCode: CR; stateProvince: Guanacaste; county: Sector El Hacha; locality: Area de Conservacion Guanacaste; verbatimLocality: Los Inocentes; verbatimElevation: 275 ; verbatimLatitude: 11.0324; verbatimLongitude: -85.4993; verbatimCoordinateSystem: Decimal; decimalLatitude: 11.0324; decimalLongitude: -85.4993; samplingProtocol: Reared from the larva of the Nymphalidae, Danaus plexippus; verbatimEventDate: unavailable, 2013; individuallD: DHJPAR0053354; individualCount: 1; sex: male; lifeStage: adult; preparations: pinned; 
catalogNumber: DHJPAR0053354; occurrenceDetails: http://janzen.sas.upenn.edu; recordedBy: D.H. Janzen, W. Hallwachs \& Lucia Rios; otherCatalog Numbers: ASHYM2708-13, 13-SRNP-21924, BOLD:AAA5134; identifiedBy: AJ Fleming; dateldentified: 2017; language: en; institutionCode: CNC; collectionCode: Insects; basisOfRecord: Pinned Specimen

as. ScientificName: Hyphantrophaga danausophaga; phylum: Arthropoda; class: Insecta; order: Diptera; family: Tachinidae; genus: Hyphantrophaga; specificEpithet: danausophaga; scientificNameAuthorship: Fleming \& Wood, 2018; continent: Central America; country: Costa Rica; countryCode: CR; stateProvince: Guanacaste; county: Sector El Hacha; locality: Area de Conservacion Guanacaste; verbatimLocality: Los Inocentes; verbatimElevation: 275 ; verbatimLatitude: 11.0324; verbatimLongitude: -85.4993; verbatimCoordinateSystem: Decimal; decimalLatitude: 11.0324; decimalLongitude: -85.4993; samplingProtocol: Reared from the larva of the Nymphalidae, Danaus plexippus; verbatimEventDate: 12-Sep-2013; individualID: DHJPAR0053355; individualCount: 1; sex: female; lifeStage: adult; preparations: pinned; catalogNumber: DHJPAR0053355; occurrenceDetails: http:// janzen.sas.upenn.edu; recordedBy: D.H. Janzen, W. Hallwachs \& Lucia Rios; otherCatalogNumbers: ASHYM2709-13, 13-SRNP-21859, BOLD:AAA5134; identifiedBy: AJ Fleming; dateldentified: 2017; language: en; institutionCode: CNC; collectionCode: Insects; basisOfRecord: Pinned Specimen

at. ScientificName: Hyphantrophaga danausophaga; phylum: Arthropoda; class: Insecta; order: Diptera; family: Tachinidae; genus: Hyphantrophaga; specificEpithet: danausophaga; scientificNameAuthorship: Fleming \& Wood, 2018; continent: Central America; country: Costa Rica; countryCode: CR; stateProvince: Guanacaste; county: Sector El Hacha; locality: Area de Conservacion Guanacaste; verbatimLocality: Los Inocentes; verbatimElevation: 275 ; verbatimLatitude: 11.0324 ; verbatimLongitude: -85.4993; verbatimCoordinateSystem: Decimal; decimalLatitude: 11.0324; decimalLongitude: -85.4993; samplingProtocol: Reared from the larva of the Nymphalidae, Danaus plexippus; verbatimEventDate: 12-Sep-2013; individualID: DHJPAR0053356; individualCount: 1; sex: male; lifeStage: adult; preparations: pinned; catalogNumber: DHJPAR0053356; occurrenceDetails: http://janzen.sas.upenn.edu; recordedBy: D.H. Janzen, W. Hallwachs \& Lucia Rios; otherCatalogNumbers: ASHYM2710-13, 13-SRNP-21854, BOLD:AAA5134; identifiedBy: AJ Fleming; dateldentified: 2017; language: en; institutionCode: CNC; collectionCode: Insects; basisOfRecord: Pinned Specimen

au. ScientificName: Hyphantrophaga danausophaga; phylum: Arthropoda; class: Insecta; order: Diptera; family: Tachinidae; genus: Hyphantrophaga; specificEpithet: danausophaga; scientificNameAuthorship: Fleming \& Wood, 2018; continent: Central America; country: Costa Rica; countryCode: CR; stateProvince: Guanacaste; county: Sector El Hacha; locality: Area de Conservacion Guanacaste; verbatimLocality: Los Inocentes; verbatimElevation: 275 ; verbatimLatitude: 11.0324; verbatimLongitude: -85.4993; verbatimCoordinateSystem: Decimal; decimalLatitude: 11.0324; decimalLongitude: -85.4993; samplingProtocol: Reared from the larva of the Nymphalidae, Danaus plexippus; verbatimEventDate: 14-Oct-2013; individualID: DHJPAR0053359; individualCount: 1; sex: female; lifeStage: adult; preparations: pinned; catalogNumber: DHJPAR0053359; occurrenceDetails: http:// janzen.sas.upenn.edu; recordedBy: D.H. Janzen, W. Hallwachs \& Lucia Rios; otherCatalog Numbers: ASHYM2713-13, 13-SRNP-21995, BOLD:AAA5134; identifiedBy: AJ Fleming; dateldentified: 2017; language: en; institutionCode: CNC; collectionCode: Insects; basisOfRecord: Pinned Specimen 
av. ScientificName: Hyphantrophaga danausophaga; phylum: Arthropoda; class: Insecta; order: Diptera; family: Tachinidae; genus: Hyphantrophaga; specificEpithet: danausophaga; scientificNameAuthorship: Fleming \& Wood, 2018; continent: Central America; country: Costa Rica; countryCode: CR; stateProvince: Guanacaste; county: Sector El Hacha; locality: Area de Conservacion Guanacaste; verbatimLocality: Los Inocentes; verbatimElevation: 275 ; verbatimLatitude: 11.0324; verbatimLongitude: -85.4993; verbatimCoordinateSystem: Decimal; decimalLatitude: 11.0324; decimalLongitude: -85.4993; samplingProtocol: Reared from the larva of the Nymphalidae, Danaus plexippus; verbatimEventDate: 03-Sep-2013; individuallD: DHJPAR0053360; individualCount: 1; sex: female; lifeStage: adult; preparations: pinned; catalogNumber: DHJPAR0053360; occurrenceDetails: http:// janzen.sas.upenn.edu; recordedBy: D.H. Janzen, W. Hallwachs \& Lucia Rios; otherCatalogNumbers: ASHYM2714-13, 13-SRNP-21853, BOLD:AAA5134; identifiedBy: AJ Fleming; dateldentified: 2017; language: en; institutionCode: CNC; collectionCode: Insects; basisOfRecord: Pinned Specimen

aw. ScientificName: Hyphantrophaga danausophaga; phylum: Arthropoda; class: Insecta; order: Diptera; family: Tachinidae; genus: Hyphantrophaga; specificEpithet:

danausophaga; scientificNameAuthorship: Fleming \& Wood, 2018; continent: Central America; country: Costa Rica; countryCode: CR; stateProvince: Guanacaste; county: Sector El Hacha; locality: Area de Conservacion Guanacaste; verbatimLocality: Los Inocentes; verbatimElevation: 275 ; verbatimLatitude: 11.0324; verbatimLongitude: -85.4993; verbatimCoordinateSystem: Decimal; decimalLatitude: 11.0324; decimalLongitude: -85.4993; samplingProtocol: Reared from the larva of the Nymphalidae, Danaus plexippus; verbatimEventDate: 13-Sep-2013; individualID: DHJPAR0053361; individualCount: 1; sex: female; lifeStage: adult; preparations: pinned; catalogNumber: DHJPAR0053361; occurrenceDetails: http:// janzen.sas.upenn.edu; recordedBy: D.H. Janzen, W. Hallwachs \& Lucia Rios; otherCatalogNumbers: ASHYM2715-13, 13-SRNP-21852, BOLD:AAA5134; identifiedBy: AJ Fleming; dateldentified: 2017; language: en; institutionCode: CNC; collectionCode: Insects; basisOfRecord: Pinned Specimen

ax. ScientificName: Hyphantrophaga danausophaga; phylum: Arthropoda; class: Insecta; order: Diptera; family: Tachinidae; genus: Hyphantrophaga; specificEpithet: danausophaga; scientificNameAuthorship: Fleming \& Wood, 2018; continent: Central America; country: Costa Rica; countryCode: CR; stateProvince: Guanacaste; county: Sector El Hacha; locality: Area de Conservacion Guanacaste; verbatimLocality: Los Inocentes; verbatimElevation: 275 ; verbatimLatitude: 11.0324 ; verbatimLongitude: -85.4993; verbatimCoordinateSystem: Decimal; decimalLatitude: 11.0324; decimalLongitude: -85.4993; samplingProtocol: Reared from the larva of the Nymphalidae, Danaus plexippus; verbatimEventDate: 14-Oct-2013; individuallD: DHJPAR0053363; individualCount: 1; sex: female; lifeStage: adult; preparations: pinned; catalogNumber: DHJPAR0053363; occurrenceDetails: http:// janzen.sas.upenn.edu; recordedBy: D.H. Janzen, W. Hallwachs \& Lucia Rios; otherCatalogNumbers: ASHYM2717-13, 13-SRNP-22002, BOLD:AAA5134; identifiedBy: AJ Fleming; dateldentified: 2017; language: en; institutionCode: CNC; collectionCode: Insects; basisOfRecord: Pinned Specimen

ay. $\quad$ scientificName: Hyphantrophaga danausophaga; phylum: Arthropoda; class: Insecta; order: Diptera; family: Tachinidae; genus: Hyphantrophaga; specificEpithet:

danausophaga; scientificNameAuthorship: Fleming \& Wood, 2018; continent: Central America; country: Costa Rica; countryCode: CR; stateProvince: Guanacaste; county: Sector El Hacha; locality: Area de Conservacion Guanacaste; verbatimLocality: Los 
Inocentes; verbatimElevation: 275 ; verbatimLatitude: 11.0324; verbatimLongitude: -85.4993; verbatimCoordinateSystem: Decimal; decimalLatitude: 11.0324; decimalLongitude: -85.4993; samplingProtocol: Reared from the larva of the Nymphalidae, Danaus plexippus; verbatimEventDate: 10-Aug-2013; individualID: DHJPAR0053370; individualCount: 1; sex: male; lifeStage: adult; preparations: pinned; catalogNumber: DHJPAR0053370; occurrenceDetails: http://janzen.sas.upenn.edu; recordedBy: D.H. Janzen, W. Hallwachs \& Elieth Cantillano; otherCatalogNumbers: ASHYM2724-13, 13-SRNP-21248, BOLD:AAA5134; identifiedBy: AJ Fleming; dateldentified: 2017; language: en; institutionCode: CNC; collectionCode: Insects; basisOfRecord: Pinned Specimen

az. $\quad$ scientificName: Hyphantrophaga danausophaga; phylum: Arthropoda; class: Insecta; order: Diptera; family: Tachinidae; genus: Hyphantrophaga; specificEpithet: danausophaga; scientificNameAuthorship: Fleming \& Wood, 2018; continent: Central America; country: Costa Rica; countryCode: CR; stateProvince: Guanacaste; county: Sector El Hacha; locality: Area de Conservacion Guanacaste; verbatimLocality: Los Inocentes; verbatimElevation: 275 ; verbatimLatitude: 11.0324; verbatimLongitude: -85.4993; verbatimCoordinateSystem: Decimal; decimalLatitude: 11.0324; decimalLongitude: -85.4993; samplingProtocol: Reared from the larva of the Nymphalidae, Danaus plexippus; verbatimEventDate: 23-Sep-2013; individuallD: DHJPAR0053371; individualCount: 1; sex: female; lifeStage: adult; preparations: pinned; catalogNumber: DHJPAR0053371; occurrenceDetails: http:// janzen.sas.upenn.edu; recordedBy: D.H. Janzen, W. Hallwachs \& Lucia Rios; otherCatalogNumbers: ASHYM2725-13, 13-SRNP-22004, BOLD:AAA5134; identifiedBy: AJ Fleming; dateldentified: 2017; language: en; institutionCode: CNC; collectionCode: Insects; basisOfRecord: Pinned Specimen

ba. $\quad$ scientificName: Hyphantrophaga danausophaga; phylum: Arthropoda; class: Insecta; order: Diptera; family: Tachinidae; genus: Hyphantrophaga; specificEpithet: danausophaga; scientificNameAuthorship: Fleming \& Wood, 2018; continent: Central America; country: Costa Rica; countryCode: CR; stateProvince: Guanacaste; county: Sector EI Hacha; locality: Area de Conservacion Guanacaste; verbatimLocality: Los Inocentes; verbatimElevation: 275; verbatimLatitude: 11.0324; verbatimLongitude: -85.4993; verbatimCoordinateSystem: Decimal; decimalLatitude: 11.0324; decimalLongitude: -85.4993; samplingProtocol: Reared from the larva of the Nymphalidae, Danaus plexippus; verbatimEventDate: 23-Sep-2013; individualID: DHJPAR0053372; individualCount: 1; sex: female; lifeStage: adult; preparations: pinned; catalogNumber: DHJPAR0053372; occurrenceDetails: http:// janzen.sas.upenn.edu; recordedBy: D.H. Janzen, W. Hallwachs \& Lucia Rios; otherCatalogNumbers: ASHYM2726-13, 13-SRNP-21994, BOLD:AAA5134; identifiedBy: AJ Fleming; dateldentified: 2017; language: en; institutionCode: CNC; collectionCode: Insects; basisOfRecord: Pinned Specimen

bb. $\quad$ scientificName: Hyphantrophaga danausophaga; phylum: Arthropoda; class: Insecta; order: Diptera; family: Tachinidae; genus: Hyphantrophaga; specificEpithet: danausophaga; scientificNameAuthorship: Fleming \& Wood, 2018; continent: Central America; country: Costa Rica; countryCode: CR; stateProvince: Guanacaste; county: Sector El Hacha; locality: Area de Conservacion Guanacaste; verbatimLocality: Los Inocentes; verbatimElevation: 275 ; verbatimLatitude: 11.0324; verbatimLongitude: -85.4993; verbatimCoordinateSystem: Decimal; decimalLatitude: 11.0324; decimalLongitude: -85.4993; samplingProtocol: Reared from the larva of the Nymphalidae, Danaus plexippus; verbatimEventDate: 17-Sep-2013; individualID: DHJPAR0053373; individualCount: 1; sex: female; lifeStage: adult; preparations: 
pinned; catalogNumber: DHJPAR0053373; occurrenceDetails: http:// janzen.sas.upenn.edu; recordedBy: D.H. Janzen, W. Hallwachs \& Lucia Rios; otherCatalogNumbers: ASHYM2727-13, 13-SRNP-21990, BOLD:AAA5134; identifiedBy: AJ Fleming; dateldentified: 2017; language: en; institutionCode: CNC; collectionCode: Insects; basisOfRecord: Pinned Specimen

bc. $\quad$ scientificName: Hyphantrophaga danausophaga; phylum: Arthropoda; class: Insecta; order: Diptera; family: Tachinidae; genus: Hyphantrophaga; specificEpithet: danausophaga; scientificNameAuthorship: Fleming \& Wood, 2018; continent: Central America; country: Costa Rica; countryCode: CR; stateProvince: Guanacaste; county: Sector El Hacha; locality: Area de Conservacion Guanacaste; verbatimLocality: Los Inocentes; verbatimElevation: 275 ; verbatimLatitude: 11.0324; verbatimLongitude: -85.4993; verbatimCoordinateSystem: Decimal; decimalLatitude: 11.0324; decimalLongitude: -85.4993; samplingProtocol: Reared from the larva of the Nymphalidae, Danaus plexippus; verbatimEventDate: 23-Sep-2013; individualID: DHJPAR0053374; individualCount: 1; sex: male; lifeStage: adult; preparations: pinned; catalogNumber: DHJPAR0053374; occurrenceDetails: http://janzen.sas.upenn.edu; recordedBy: D.H. Janzen, W. Hallwachs \& Lucia Rios; otherCatalogNumbers: ASHYM2728-13, 13-SRNP-22022, BOLD:AAA5134; identifiedBy: AJ Fleming; dateldentified: 2017; language: en; institutionCode: CNC; collectionCode: Insects; basisOfRecord: Pinned Specimen

bd. ScientificName: Hyphantrophaga danausophaga; phylum: Arthropoda; class: Insecta; order: Diptera; family: Tachinidae; genus: Hyphantrophaga; specificEpithet: danausophaga; scientificNameAuthorship: Fleming \& Wood, 2018; continent: Central America; country: Costa Rica; countryCode: CR; stateProvince: Guanacaste; county: Sector El Hacha; locality: Area de Conservacion Guanacaste; verbatimLocality: Los Inocentes; verbatimElevation: 275 ; verbatimLatitude: 11.0324; verbatimLongitude: -85.4993; verbatimCoordinateSystem: Decimal; decimalLatitude: 11.0324; decimalLongitude: -85.4993; samplingProtocol: Reared from the larva of the Nymphalidae, Danaus plexippus; verbatimEventDate: 17-Sep-2013; individualID: DHJPAR0053377; individualCount: 1; sex: female; lifeStage: adult; preparations: pinned; catalogNumber: DHJPAR0053377; occurrenceDetails: http:// janzen.sas.upenn.edu; recordedBy: D.H. Janzen, W. Hallwachs \& Lucia Rios; otherCatalogNumbers: ASHYM2731-13, 13-SRNP-21991, BOLD:AAA5134; identifiedBy: AJ Fleming; dateldentified: 2017; language: en; institutionCode: CNC; collectionCode: Insects; basisOfRecord: Pinned Specimen

be. $\quad$ scientificName: Hyphantrophaga danausophaga; phylum: Arthropoda; class: Insecta; order: Diptera; family: Tachinidae; genus: Hyphantrophaga; specificEpithet: danausophaga; scientificNameAuthorship: Fleming \& Wood, 2018; continent: Central America; country: Costa Rica; countryCode: CR; stateProvince: Guanacaste; county: Sector El Hacha; locality: Area de Conservacion Guanacaste; verbatimLocality: Los Inocentes; verbatimElevation: 275 ; verbatimLatitude: 11.0324; verbatimLongitude: -85.4993; verbatimCoordinateSystem: Decimal; decimalLatitude: 11.0324; decimalLongitude: -85.4993; samplingProtocol: Reared from the larva of the Nymphalidae, Danaus plexippus; verbatimEventDate: 11-Sep-2013; individualID: DHJPAR0053379; individualCount: 1; lifeStage: adult; preparations: pinned; catalogNumber: DHJPAR0053379; occurrenceDetails: http://janzen.sas.upenn.edu; recordedBy: D.H. Janzen, W. Hallwachs \& Lucia Rios; otherCatalogNumbers: ASHYM2733-13, 13-SRNP-21866,; identifiedBy: AJ Fleming; dateldentified: 2017; language: en; institutionCode: CNC; collectionCode: Insects; basisOfRecord: Pinned Specimen 
bf. scientificName: Hyphantrophaga danausophaga; phylum: Arthropoda; class: Insecta; order: Diptera; family: Tachinidae; genus: Hyphantrophaga; specificEpithet: danausophaga; scientificNameAuthorship: Fleming \& Wood, 2018; continent: Central America; country: Costa Rica; countryCode: CR; stateProvince: Guanacaste; county: Sector El Hacha; locality: Area de Conservacion Guanacaste; verbatimLocality: Los Inocentes; verbatimElevation: 275 ; verbatimLatitude: 11.0324; verbatimLongitude: -85.4993; verbatimCoordinateSystem: Decimal; decimalLatitude: 11.0324; decimalLongitude: -85.4993; samplingProtocol: Reared from the larva of the Nymphalidae, Danaus plexippus; verbatimEventDate: 11-Sep-2013; individualID: DHJPAR0053380; individualCount: 1; sex: male; lifeStage: adult; preparations: pinned; catalogNumber: DHJPAR0053380; occurrenceDetails: http://janzen.sas.upenn.edu; recordedBy: D.H. Janzen, W. Hallwachs \& Lucia Rios; otherCatalogNumbers: ASHYM2734-13, 13-SRNP-21872, BOLD:AAA5134; identifiedBy: AJ Fleming; dateldentified: 2017; language: en; institutionCode: CNC; collectionCode: Insects; basisOfRecord: Pinned Specimen

bg. ScientificName: Hyphantrophaga danausophaga; phylum: Arthropoda; class: Insecta; order: Diptera; family: Tachinidae; genus: Hyphantrophaga; specificEpithet: danausophaga; scientificNameAuthorship: Fleming \& Wood, 2018; continent: Central America; country: Costa Rica; countryCode: CR; stateProvince: Guanacaste; county: Sector El Hacha; locality: Area de Conservacion Guanacaste; verbatimLocality: Los Inocentes; verbatimElevation: 275 ; verbatimLatitude: 11.0324; verbatimLongitude: -85.4993; verbatimCoordinateSystem: Decimal; decimalLatitude: 11.0324; decimalLongitude: -85.4993; samplingProtocol: Reared from the larva of the Nymphalidae, Danaus plexippus; verbatimEventDate: 13-Sep-2013; individualID: DHJPAR0053383; individualCount: 1; sex: male; lifeStage: adult; preparations: pinned; catalogNumber: DHJPAR0053383; occurrenceDetails: http://janzen.sas.upenn.edu; recordedBy: D.H. Janzen, W. Hallwachs \& Lucia Rios; otherCatalogNumbers: ASHYM2737-13, 13-SRNP-21871, BOLD:AAA5134; identifiedBy: AJ Fleming; dateldentified: 2017; language: en; institutionCode: CNC; collectionCode: Insects; basisOfRecord: Pinned Specimen

bh. ScientificName: Hyphantrophaga danausophaga; phylum: Arthropoda; class: Insecta; order: Diptera; family: Tachinidae; genus: Hyphantrophaga; specificEpithet: danausophaga; scientificNameAuthorship: Fleming \& Wood, 2018; continent: Central America; country: Costa Rica; countryCode: CR; stateProvince: Guanacaste; county: Sector El Hacha; locality: Area de Conservacion Guanacaste; verbatimLocality: Los Inocentes; verbatimElevation: 275 ; verbatimLatitude: 11.0324 ; verbatimLongitude: -85.4993; verbatimCoordinateSystem: Decimal; decimalLatitude: 11.0324; decimalLongitude: -85.4993; samplingProtocol: Reared from the larva of the Nymphalidae, Danaus plexippus; verbatimEventDate: 01-Sep-2013; individuallD: DHJPAR0053384; individualCount: 1; sex: male; lifeStage: adult; preparations: pinned; catalogNumber: DHJPAR0053384; occurrenceDetails: http://janzen.sas.upenn.edu; recordedBy: D.H. Janzen, W. Hallwachs \& Lucia Rios; otherCatalogNumbers: ASHYM2738-13, 13-SRNP-21767, BOLD:AAA5134; identifiedBy: AJ Fleming; dateldentified: 2017; language: en; institutionCode: CNC; collectionCode: Insects; basisOfRecord: Pinned Specimen

bi. ScientificName: Hyphantrophaga danausophaga; phylum: Arthropoda; class: Insecta; order: Diptera; family: Tachinidae; genus: Hyphantrophaga; specificEpithet: danausophaga; scientificNameAuthorship: Fleming \& Wood, 2018; continent: Central America; country: Costa Rica; countryCode: CR; stateProvince: Guanacaste; county: Sector El Hacha; locality: Area de Conservacion Guanacaste; verbatimLocality: Los 
Inocentes; verbatimElevation: 275 ; verbatimLatitude: 11.0324 ; verbatimLongitude: -85.4993; verbatimCoordinateSystem: Decimal; decimalLatitude: 11.0324; decimalLongitude: -85.4993; samplingProtocol: Reared from the larva of the Nymphalidae, Danaus plexippus; verbatimEventDate: 14-Sep-2013; individualID: DHJPAR0053387; individualCount: 1; sex: female; lifeStage: adult; preparations: pinned; catalogNumber: DHJPAR0053387; occurrenceDetails: http:// janzen.sas.upenn.edu; recordedBy: D.H. Janzen, W. Hallwachs \& Lucia Rios; otherCatalogNumbers: ASHYM2741-13, 13-SRNP-21858, BOLD:AAA5134; identifiedBy: AJ Fleming; dateldentified: 2017; language: en; institutionCode: CNC; collectionCode: Insects; basisOfRecord: Pinned Specimen

bj. $\quad$ scientificName: Hyphantrophaga danausophaga; phylum: Arthropoda; class: Insecta; order: Diptera; family: Tachinidae; genus: Hyphantrophaga; specificEpithet: danausophaga; scientificNameAuthorship: Fleming \& Wood, 2018; continent: Central America; country: Costa Rica; countryCode: CR; stateProvince: Guanacaste; county: Sector El Hacha; locality: Area de Conservacion Guanacaste; verbatimLocality: Los Inocentes; verbatimElevation: 275 ; verbatimLatitude: 11.0324; verbatimLongitude: -85.4993; verbatimCoordinateSystem: Decimal; decimalLatitude: 11.0324; decimalLongitude: -85.4993; samplingProtocol: Reared from the larva of the Nymphalidae, Danaus plexippus; verbatimEventDate: 16-Sep-2013; individualID: DHJPAR0053388; individualCount: 1; sex: male; lifeStage: adult; preparations: pinned; catalogNumber: DHJPAR0053388; occurrenceDetails: http://janzen.sas.upenn.edu; recordedBy: D.H. Janzen, W. Hallwachs \& Lucia Rios; otherCatalog Numbers: ASHYM2742-13, 13-SRNP-21922, BOLD:AAA5134; identifiedBy: AJ Fleming; dateldentified: 2017; language: en; institutionCode: CNC; collectionCode: Insects; basisOfRecord: Pinned Specimen

bk. $\quad$ scientificName: Hyphantrophaga danausophaga; phylum: Arthropoda; class: Insecta; order: Diptera; family: Tachinidae; genus: Hyphantrophaga; specificEpithet: danausophaga; scientificNameAuthorship: Fleming \& Wood, 2018; continent: Central America; country: Costa Rica; countryCode: CR; stateProvince: Guanacaste; county: Sector El Hacha; locality: Area de Conservacion Guanacaste; verbatimLocality: Estacion Los Almendros; verbatimElevation: 290; verbatimLatitude: 11.0323; verbatimLongitude: -85.5278; verbatimCoordinateSystem: Decimal; decimalLatitude: 11.0323; decimalLongitude: -85.5278; samplingProtocol: Reared from the larva of the Nymphalidae, Danaus plexippus; verbatimEventDate: 07-Jan-2014; individualID: DHJPAR0054978; individualCount: 1; sex: male; lifeStage: adult; preparations: pinned; catalogNumber: DHJPAR0054978; occurrenceDetails: http://janzen.sas.upenn.edu; recordedBy: D.H. Janzen, W. Hallwachs \& Lucia Rios; otherCatalog Numbers: ASHYH1525-14, 13-SRNP-22908, BOLD:AAA5134; identifiedBy: AJ Fleming; dateldentified: 2017; language: en; institutionCode: CNC; collectionCode: Insects; basisOfRecord: Pinned Specimen

bl. scientificName: Hyphantrophaga danausophaga; phylum: Arthropoda; class: Insecta; order: Diptera; family: Tachinidae; genus: Hyphantrophaga; specificEpithet: danausophaga; scientificNameAuthorship: Fleming \& Wood, 2018; continent: Central America; country: Costa Rica; countryCode: CR; stateProvince: Guanacaste; county: Sector El Hacha; locality: Area de Conservacion Guanacaste; verbatimLocality: Rio Sabalo; verbatimCoordinateSystem: Decimal; samplingProtocol: Reared from the larva of the Nymphalidae, Danaus plexippus; verbatimEventDate: unavailable, 2016; individualID: DHJPAR0057601; individualCount: 1; lifeStage: adult; preparations: pinned; catalogNumber: DHJPAR0057601; occurrenceDetails: http:// janzen.sas.upenn.edu; recordedBy: D.H. Janzen, W. Hallwachs \& Lucia Rios; 
otherCatalogNumbers: ASTAX013-15, 15-SRNP-20226,; identifiedBy: AJ Fleming; dateldentified: 2017; language: en; institutionCode: CNC; collectionCode: Insects; basisOfRecord: Pinned Specimen

bm. $\quad$ scientificName: Hyphantrophaga danausophaga; phylum: Arthropoda; class: Insecta; order: Diptera; family: Tachinidae; genus: Hyphantrophaga; specificEpithet:

danausophaga; scientificNameAuthorship: Fleming \& Wood, 2018; continent: Central America; country: Costa Rica; countryCode: CR; stateProvince: Guanacaste; county: Sector San Cristobal; locality: Area de Conservacion Guanacaste; verbatimLocality: Finca San Gabriel; verbatimElevation: 645; verbatimLatitude: 10.8777; verbatimLongitude: -85.3934; verbatimCoordinateSystem: Decimal; decimalLatitude: 10.8777; decimalLongitude: -85.3934; samplingProtocol: Reared from the larva of the Nymphalidae, Danaus plexippus; verbatimEventDate: 13-Apr-2017; individualID:

DHJPAR0060975; individualCount: 1; lifeStage: adult; preparations: pinned; catalogNumber: DHJPAR0060975; occurrenceDetails: http://janzen.sas.upenn.edu; recordedBy: D.H. Janzen, W. Hallwachs \& Osvaldo Espinoza; otherCatalogNumbers: ACGBA7396-17, 17-SRNP-605, BOLD:AAA5134; identifiedBy: AJ Fleming; dateldentified: 2017; language: en; institutionCode: CNC; collectionCode: Insects; basisOfRecord: Pinned Specimen

bn. $\quad$ scientificName: Hyphantrophaga danausophaga; phylum: Arthropoda; class: Insecta; order: Diptera; family: Tachinidae; genus: Hyphantrophaga; specificEpithet: danausophaga; scientificNameAuthorship: Fleming \& Wood, 2018; continent: Central America; country: Costa Rica; countryCode: CR; stateProvince: Guanacaste; county: Sector San Cristobal; locality: Area de Conservacion Guanacaste; verbatimLocality: Finca San Gabriel; verbatimElevation: 645; verbatimLatitude: 10.8777 ; verbatimLongitude: -85.3934; verbatimCoordinateSystem: Decimal; decimalLatitude: 10.8777; decimalLongitude: -85.3934; samplingProtocol: Reared from the larva of the Nymphalidae, Danaus plexippus; verbatimEventDate: 08-Apr-2017; individuallD: DHJPAR0060977; individualCount: 1; lifeStage: adult; preparations: pinned; catalogNumber: DHJPAR0060977; occurrenceDetails: http://janzen.sas.upenn.edu; recordedBy: D.H. Janzen, W. Hallwachs \& Osvaldo Espinoza; otherCatalog Numbers: ACGBA7398-17, 17-SRNP-602, BOLD:AAA5134; identifiedBy: AJ Fleming; dateldentified: 2017; language: en; institutionCode: CNC; collectionCode: Insects; basisOfRecord: Pinned Specimen

bo. $\quad$ scientificName: Hyphantrophaga danausophaga; phylum: Arthropoda; class: Insecta; order: Diptera; family: Tachinidae; genus: Hyphantrophaga; specificEpithet: danausophaga; scientificNameAuthorship: Fleming \& Wood, 2018; continent: Central America; country: Costa Rica; countryCode: CR; stateProvince: Guanacaste; county: Sector San Cristobal; locality: Area de Conservacion Guanacaste; verbatimLocality: Finca San Gabriel; verbatimElevation: 645; verbatimLatitude: 10.8777 ; verbatimLongitude: -85.3934; verbatimCoordinateSystem: Decimal; samplingProtocol: Reared from the larva of the Nymphalidae, Danaus plexippus; verbatimEventDate: 08Apr-2017; individualID: DHJPAR0060978; individualCount: 1; lifeStage: adult; preparations: pinned; catalogNumber: DHJPAR0060978; occurrenceDetails: http:// janzen.sas.upenn.edu; recordedBy: D.H. Janzen, W. Hallwachs \& Osvaldo Espinoza; otherCatalogNumbers: ACGBA7399-17, 17-SRNP-603, BOLD:AAA5134; identifiedBy: AJ Fleming; dateldentified: 2017; language: en; institutionCode: CNC; collectionCode: Insects; basisOfRecord: Pinned Specimen 


\section{Description}

Male (Fig. 9). Length: 7-11mm. Head (Fig. 9b): vertex 1/4 of head width; two reclinate upper orbital setae; ocellar setae arising beside anterior ocellus; ocellar triangle of dark blackened gold colour, with light gold tomentum around margin; fronto-orbital plate dull grey tomentose with slight gold tinge, gold stronger around vertex; eye densely haired; fronto-orbital plate setulose, setulae not extending below lowest frontal seta; frontoorbital plate shiny silver; parafacial bare; facial ridge bare; pedicel black with a small spot of orange basally, sometimes extending into adjacent region of postpedicel; otherwise concolorous with postpedicel; arista black, very minutely pubescent, gradually tapered apically, beginning on basal 1/3-1/4; palpus ranging from yellow to brown. Thorax (Fig. 9a, c): entirely densely hirsute with short black setulae amongst setae; prosternum setose with 1-3 strong setae surrounded by a brush of weaker setulae; four prominent dorsal vittae, outermost two broken across suture, innermost pair unbroken across suture, not reaching beyond 2nd postsutural dorsocentral seta; postpronotum with five setae arranged in a triangle; chaetotaxy: acrostichal setae 3:3; dorsocentral setae 3:4; intra-alar setae 3:3; supra-alar setae 2:3; katepisternum with three setae, basal seta weakest, arising anterior to suture; lateral scutellar setae $1 / 2$ as long as subapical setae, slightly curving inwards medially; subapical setae subequal in length of basal scutellar setae, the latter arising above plane of remaining marginal scutellar setae; apical scutellar setae ranging from $1 / 2$ as long as lateral scutellar setae to subequal in length but $1 / 2$ the diameter; one pair of discal scutellar setae; scutellum gold tomentose along posterior margin, transitioning to grey tomentose along basal edge. Legs (Fig. 9c): femora and coxae black in ground colour; tibiae yellow in ground colour, densely covered in short black hairs; appearing darkened almost black; fore femur with dense silver tomentum on posterodorsal surface; hind coxa either bare or with a single seta along posterior margin. Wing (Fig. 9a): pale translucent, not distinctly infuscate; vein $\mathrm{R}_{4+5}$ with 2-3 (most often 2) setulae at base. Abdomen (Fig. 9a, c): ground colour brown; middorsal depression on ST1+2 extending almost to margin; median marginal setae absent on ST1+2 and T3; a complete row of marginal setae present on T4; discal setae only on T5; sex patch covering ventral surfaces of T4-T5 and posterior $1 / 3$ of T3; distinct tomentose bands along anterior $2 / 3$ of T3 and T4, broken medially by a dorsocentral stripe; T5 with silver tomentum over its entirety. Terminalia: sternite 5 (Fig. 9f) with a deeply excavated median cleft, smoothly Ushaped, margins covered in dense tomentum. Lateral lobes of sternite rounded apically, with 2-3 strong setae surrounded by many shorter, weaker setulae. Anterior plate of sternite 5 from subequal to slightly longer than apical lobes; unsclerotised "window" appearing blunt convex, with a slightly rectangular base and a wider crown making it appear mushroom-shaped, as wide as median cleft. Cerci in posterior view (Fig. 9d) rectangular and slightly shorter than surstyli, blunt and rounded at apex, completely separate medially, appearing slightly divergent basally, twice as wide as at apex; in lateral view with a slight downward curve in apical 1/3; densely setulose along basal 2/3 dorsally, setulose ventrally along entire length (visible in lateral view). Surstylus in lateral view (Fig. 9e) narrow, almost parallel-sided along its length, ending in a slightly downcurved apex, making the structure appear blade-like; when viewed 
dorsally surstyli appearing to point outward. Pregonite short and wide, well-developed, subequal in length to postgonite, as long as distiphallus, bare and squared off apically. Postgonite slightly narrow, $1 / 3$ as wide as pregonite, sharply pointed and curved at apex. Distiphallus rectangular, only weakly flaring apically with a slender median longitudinal sclerotised reinforcement on its posterior surface and a broad, anterolateral, apically clubbed sclerotised acrophallus on each side, joining the plate of opposite side on anterior surface near apex.

Female. Length: 8-11 mm. As male, differing only by the presence of two pairs of proclinate orbital setae.

\section{Diagnosis}

To date, Hyphantrophaga danausophaga sp. n. can only be distinguished from its closest congener, $H$. morphophaga sp. n., by its host selection and habitat, being found parasitising only medium-sized, naked, ringed Nymphalidae larvae in open, heavily insolated grassland and pasture habitats.

\section{Etymology}

Named after the host genus, Danaus and the Greek "phago" meaning "eating", in reference to its primary host species.

\section{Distribution}

Costa Rica, ACG, Alajuela and Guanacaste Provinces, 90-1150 m elevation.

\section{Ecology}

Hyphantrophaga danausophaga sp. $\mathbf{n}$. has been reared 105 times from three species of Lepidoptera in the family Nymphalidae, Danaus plexippus (Linnaeus, 1758), Danaus gilippus (Cramer, 1775) and Lycorea atergatis (Doubleday, 1847), in cloud forest, rain forest, dry forest and dry-rain lowland intergrade. 


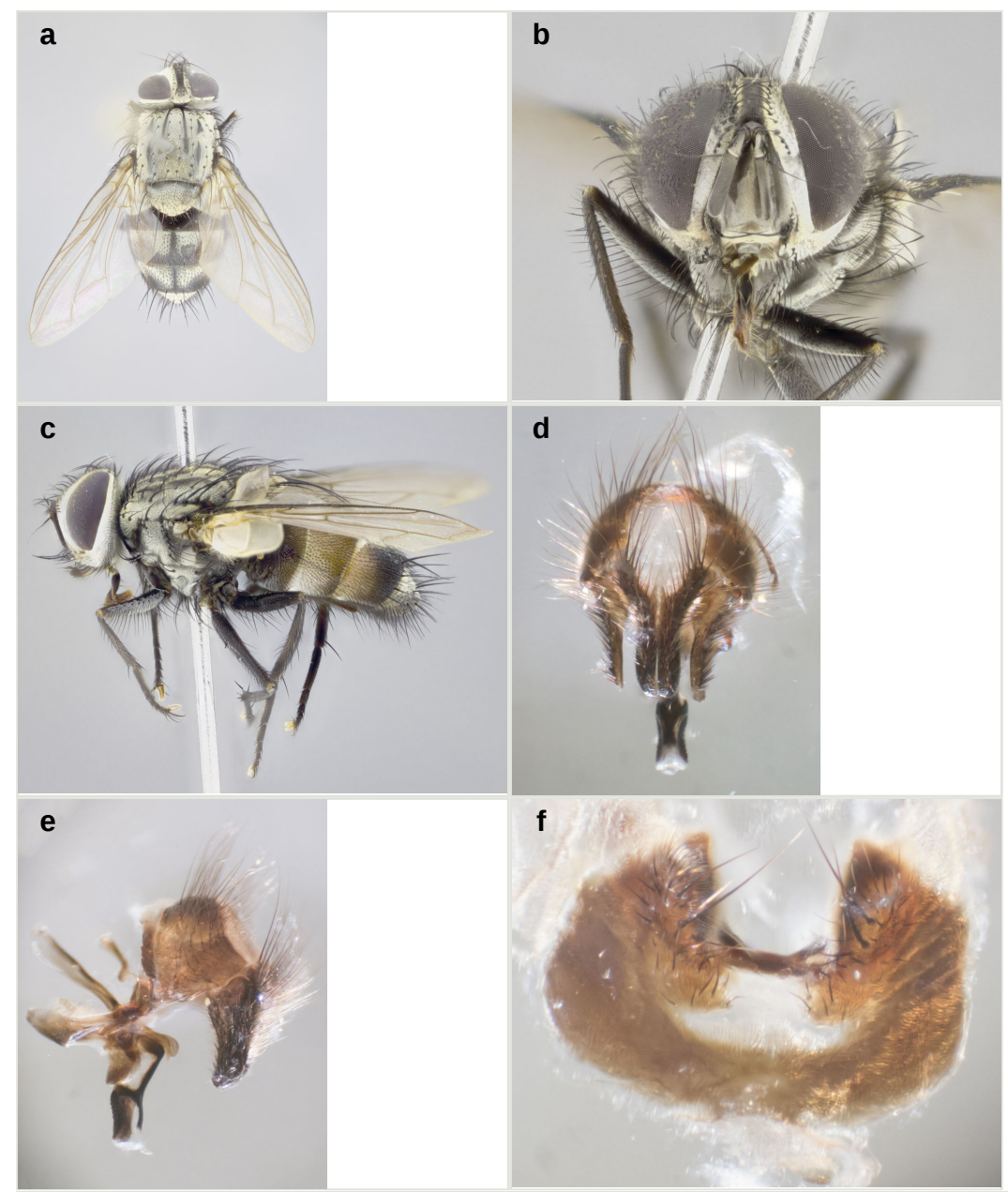

Figure 9.

Hyphantrophaga danausophaga sp. n.; a-c: habitus; holotype male, voucher n. DHJPAR0010000; d-f: terminalia; male paratype, voucher n. DHJPAR0042587.
a: dorsal view doi
b: frontal view doi
c: lateral view doi
d: dorsal view doi
e: lateral view doi
f: sternite 5 , ventral view doi 


\section{Hyphantrophaga diniamartinezae Fleming \& Wood, sp. n.}

- ZooBank urn:Isid:Zoobank.org:act:3D997452-E42A-40CD-80B8-18221259BA18

\section{Materials}

\section{Holotype:}

a. $\quad$ scientificName: Hyphantrophaga diniamartinezae; phylum: Arthropoda; class: Insecta; order: Diptera; family: Tachinidae; genus: Hyphantrophaga; specificEpithet: diniamartinezae; scientificNameAuthorship: Fleming \& Wood, 2018; continent: Central America; country: Costa Rica; countryCode: CR; stateProvince: Guanacaste; county: Sector Pitilla; locality: Area de Conservacion Guanacaste; verbatimLocality: Manguera; verbatimElevation: 470; verbatimLatitude: 10.9959; verbatimLongitude: -85.3984; verbatimCoordinateSystem: Decimal; decimalLatitude: 10.9959; decimalLongitude: -85.3984; samplingProtocol: Reared from the larva of the Sphingidae, Xylophanes ceratomioides; verbatimEventDate: 06-Oct-2011; individualID: DHJPAR0045610; individualCount: 1; sex: male; lifeStage: adult; preparations: pinned; catalogNumber: DHJPAR0045610; occurrenceDetails: http://janzen.sas.upenn.edu; recordedBy: D.H. Janzen, W. Hallwachs \& Ricardo Calero; otherCatalogNumbers: ACGAZ799-11, 11SRNP-71939, BOLD:AAM3713; identifiedBy: AJ Fleming; dateldentified: 2017; language: en; institutionCode: CNC; collectionCode: Insects; basisOfRecord: Pinned Specimen

\section{Paratypes:}

a. ScientificName: Hyphantrophaga diniamartinezae; phylum: Arthropoda; class: Insecta; order: Diptera; family: Tachinidae; genus: Hyphantrophaga; specificEpithet: diniamartinezae; scientificNameAuthorship: Fleming \& Wood, 2018; continent: Central America; country: Costa Rica; countryCode: CR; stateProvince: Alajuela; county: Sector Rincon Rain Forest; locality: Area de Conservacion Guanacaste; verbatimLocality: Quebrada Escondida; verbatimElevation: 420; verbatimLatitude: 10.8993; verbatimLongitude: -85.2749; verbatimCoordinateSystem: Decimal; decimalLatitude: 10.8993; decimalLongitude: -85.2749; samplingProtocol: Reared from the larva of the Sphingidae, Xylophanes chiron; verbatimEventDate: 04-Dec-2000; individualID: DHJPAR0008100; individualCount: 1; sex: female; lifeStage: adult; preparations: pinned; catalogNumber: DHJPAR0008100; occurrenceDetails: http:// janzen.sas.upenn.edu; recordedBy: D.H. Janzen, W. Hallwachs \& Jose Perez; otherCatalog Numbers: ASTAT872-06, 00-SRNP-20702, BOLD:AAM3713; identifiedBy: AJ Fleming; dateldentified: 2017; language: en; institutionCode: CNC; collectionCode: Insects; basisOfRecord: Pinned Specimen

b. $\quad$ scientificName: Hyphantrophaga diniamartinezae; phylum: Arthropoda; class: Insecta; order: Diptera; family: Tachinidae; genus: Hyphantrophaga; specificEpithet: diniamartinezae; scientificNameAuthorship: Fleming \& Wood, 2018; continent: Central America; country: Costa Rica; countryCode: CR; stateProvince: Guanacaste; county: Sector Del Oro; locality: Area de Conservacion Guanacaste; verbatimLocality: Margarita; verbatimElevation: 380 ; verbatimLatitude: 11.0323 ; verbatimLongitude: -85.4395; verbatimCoordinateSystem: Decimal; decimalLatitude: 11.0323; decimalLongitude: -85.4395; samplingProtocol: Reared from the larva of the Sphingidae, Xylophanes porcus; verbatimEventDate: 27-Nov-2007; individualID: DHJPAR0023083; individualCount: 1; sex: female; lifeStage: adult; preparations: pinned; catalogNumber: DHJPAR0023083; occurrenceDetails: http:// janzen.sas.upenn.edu; recordedBy: D.H. Janzen, W. Hallwachs \& Roster Moraga; 
otherCatalog Numbers: ASTAW244-08, 07-SRNP-24104, BOLD:AAM3713; identifiedBy: AJ Fleming; dateldentified: 2017; language: en; institutionCode: CNC; collectionCode: Insects; basisOfRecord: Pinned Specimen

c. ScientificName: Hyphantrophaga diniamartinezae; phylum: Arthropoda; class: Insecta; order: Diptera; family: Tachinidae; genus: Hyphantrophaga; specificEpithet: diniamartinezae; scientificNameAuthorship: Fleming \& Wood, 2018; continent: Central America; country: Costa Rica; countryCode: CR; stateProvince: Alajuela; county: Sector Rincon Rain Forest; locality: Area de Conservacion Guanacaste; verbatimLocality: Quebrada Escondida; verbatimElevation: 420; verbatimLatitude: 10.8993; verbatimLongitude: -85.2749; verbatimCoordinateSystem: Decimal; decimalLatitude: 10.8993; decimalLongitude: -85.2749; samplingProtocol: Reared from the larva of the Sphingidae, Xylophanes chiron; verbatimEventDate: 01-Dec-2000; individualID: DHJPAR0008093; individualCount: 1; sex: male; lifeStage: adult; preparations: pinned; catalogNumber: DHJPAR0008093; occurrenceDetails: http:// janzen.sas.upenn.edu; recordedBy: D.H. Janzen, W. Hallwachs \& Freyci Vargas; otherCatalogNumbers: ASTAT865-06, 00-SRNP-20696, BOLD:AAM3713; identifiedBy: AJ Fleming; dateldentified: 2017; language: en; institutionCode: CNC; collectionCode: Insects; basisOfRecord: Pinned Specimen

\section{Description}

Male (Fig. 10). Length: 7-10 mm. Head (Fig. 10b): vertex 1/5 of head width; two pairs of reclinate upper orbital setae; ocellar setae arising behind anterior ocellus; ocellar triangle pale brassy; fronto-orbital plate pale brassy on upper $80 \%$, densely setulose, setulae not extending below lowest frontal seta; parafacial silver and bare; eye densely haired; facial ridge bare; pedicel black, concolorous with postpedicel; arista black, very minutely pubescent, distinctly thickened on basal 1/3-1/4; palpus yellow and haired apically, narrow and digitiform. Thorax (Fig. 10a, c): dull brassy tomentose dorsally, contrasting with slightly brighter silver tomentose laterally, lateral surfaces with dense dark setulae making it appear darker; thorax with dense dark hairs interspersed amongst setae; four thick dorsal vittae, outermost two broken across suture, innermost pair unbroken, reaching 3rd postsutural dorsocentral seta, both pairs of vittae widening postsuturally and smudging together; postpronotum with 3-4 setae arranged in a triangle; chaetotaxy: acrostichal setae 3:3; dorsocentral setae 3:4; intra-alar setae 34:3; supra-alar setae 2:3; three katepisternal setae; basal scutellar setae subequal in length to subapical scutellar setae; lateral scutellar setae less than $2 / 3$ as long as subapical setae, strongly curving inwards medially; apical scutellar setae subequal in length to lateral scutellar setae, crossed apically; one pair of discal scutellar setae more widely set than apical scutellar setae, but more narrowly than subapical scutellar setae; scutellum very slightly darkened across basal 25\%, remainder concolorous with scutum. Legs (Fig. 10c): black in ground colour; fore femur with dense silver tomentum on posterodorsal surface; hind coxa bare. Wing (Fig. 10a): pale translucent, hyaline; vein $\mathrm{R}_{4+5}$ with only 2-3 setulae at base. Abdomen (Fig. 10a, c): ground colour black dorsally, yellow lateroventrally; middorsal depression on ST1+2 reaching hind margin; median marginal setae present on ST1+2-T3; a complete row of marginal setae present on T4; discal setae only on T5; sex patch covering ventral surfaces of T4-T5; distinct brassy tomentose bands along anterior edge of T3 and T4, broken medially by 
a dorsocentral stripe and covering almost $80 \%$ of tergites; T3 with silver tomentum ventrolaterally over $60 \%$ of surface; 15 with brassy tomentum throughout. Terminalia (Fig. 10d, e, f): sternite 5 (Fig. 10f) with a deeply excavated median cleft, widely Ushaped, margins covered in dense tomentum, unsclerotised window tri-lobed and "fleur-de-lys"-shaped. Lateral lobes of sternite pointed apically, many short, stout setae throughout. Anterior plate of sternite $52 \mathrm{X}$ longer than apical lobes. Cerci in posterior view (Fig. 10d) rounded rectangular-shaped, subequal in length to surstyli, blunt and rounded at apex, completely separate medially, parallel; in lateral view (Fig. 10e) with an evenly rounded downward curve throughout; densely setulose along basal 2/3. Surstylus in lateral view round, tapered and straight along bottom edge; opposite edge evenly rounded, giving it a cleaver-blade appearance; when viewed dorsally, surstyli pointing outward, not strongly convergent. Pregonite short, not well-developed, 0.5 times as long as distiphallus, bare and rounded apically, having the appearance of an upside down boot. Postgonite slightly narrow, 1/3 as wide as pregonite, sharply pointed and curved at apex. Epiphallus well-developed and apically hooked. Distiphallus rectangular with a slender median longitudinal sclerotised reinforcement on its posterior surface and a broad, anterolateral, sclerotised acrophallus on each side, joining on anterior surface near apex.

Female. Length: 8-10 mm. As male, differing only by the presence of two pairs of proclinate orbital setae.

\section{Diagnosis}

Hyphantrophaga diniamartinezae sp. n. can be distinguished from all other Hyphantrophaga species by the following combination of traits: thorax with three postsutural acrostichal setae, four postsutural dorsocentral setae, and three katepisternal setae, legs black, hind coxa bare, tomentum covering less than $50 \%$ of abdominal T3, ventral edge of T3 darkened up to $10 \%$ of tergite and T5 with brassy tomentum throughout.

\section{Etymology}

Hyphantrophaga diniamartinezae sp. $\mathbf{n}$. is named in recognition of Dinia Maria Martinez Cheves' dedication and work in finding and rearing the ACG caterpillars that contained tachinid larvae.

\section{Distribution}

Costa Rica, ACG, Guanacaste Province, 380-420 m elevation.

\section{Ecology}

Hyphantrophaga diniamartinezae sp. $\mathbf{n}$. has been reared two times from two species of Lepidoptera in the family Sphingidae, Xylophanes chiron (Drury, 1773) and Xylophanes porcus (Hübner, 1823), in rain forest and dry-rain lowland intergrades. 


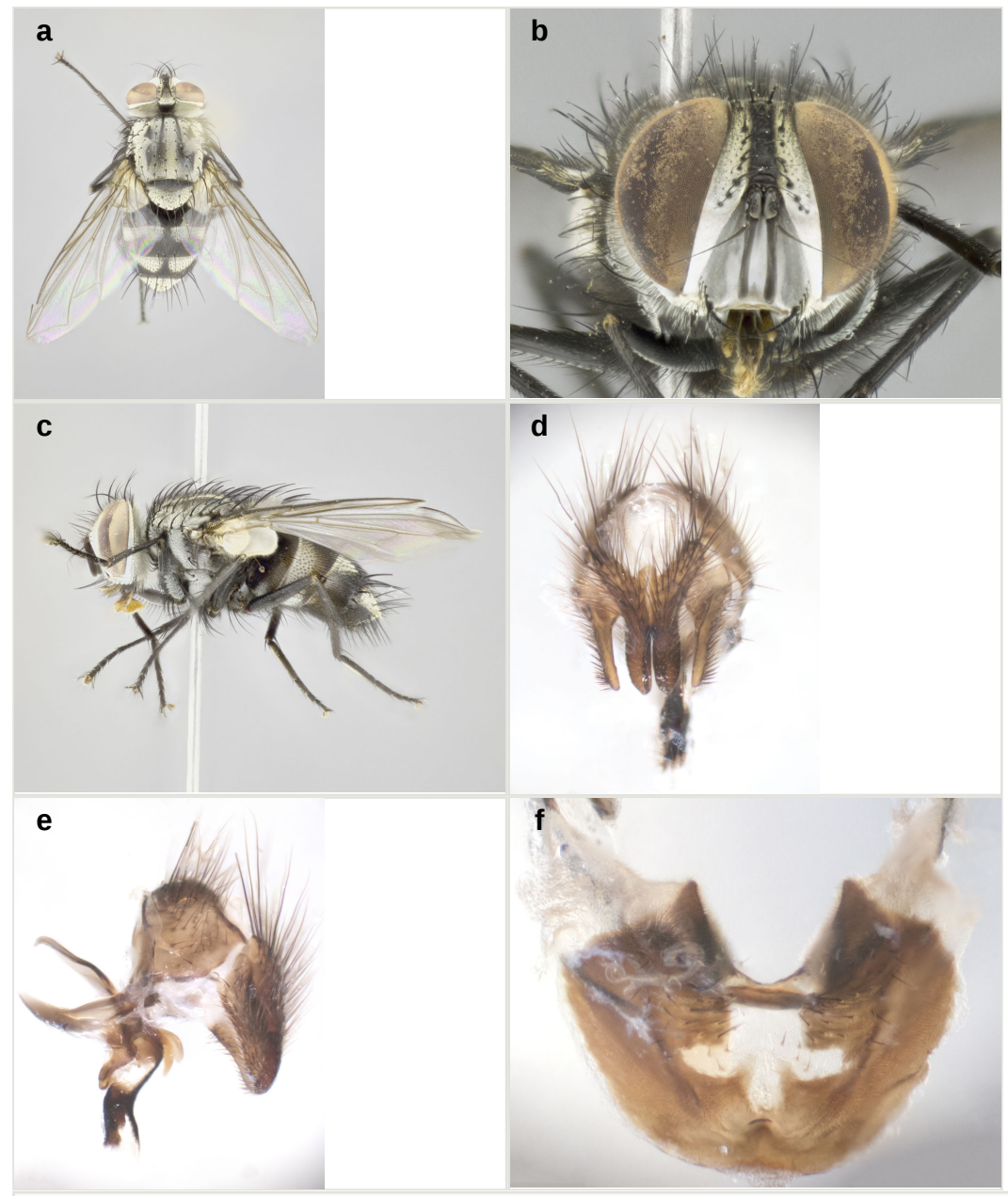

Figure 10.

Hyphantrophaga diniamartinezae sp. n.; a-c: habitus; holotype male, voucher $\mathrm{n}$. DHJPAR0045610; d-f: terminalia; male paratype, voucher $\mathrm{n}$. DHJPAR0008093.
a: dorsal view doi
b: frontal view doi
c: lateral view doi
d: dorsal view doi
e: lateral view doi
f: sternite 5 , ventral view doi 


\section{Hyphantrophaga duniagarciae Fleming \& Wood, sp. n.}

- ZooBank urn:Isid:Zoobank.org:act:1F6DC396-3A43-489B-A7BA-BD1B7FDA7B1B

\section{Material}

Holotype:

a. scientificName: Hyphantrophaga duniagarciae; phylum: Arthropoda; class: Insecta; order: Diptera; family: Tachinidae; genus: Hyphantrophaga; specificEpithet: duniagarciae; scientificNameAuthorship: Fleming \& Wood, 2018; continent: Central America; country: Costa Rica; countryCode: CR; stateProvince: Guanacaste; county: Sector Pocosol; locality: Area de Conservacion Guanacaste; verbatimLocality: Casa Centeno; verbatimElevation: 215; verbatimLatitude: 10.8784; verbatimLongitude: -85.5728; verbatimCoordinateSystem: Decimal; decimalLatitude: 10.8784; decimalLongitude: -85.5728; samplingProtocol: Reared from the larva of the Zygaenidae, Neoilliberis thyesta; verbatimEventDate: 06-Jun-2005; individualID: DHJPAR0011464; individualCount: 1; sex: female; lifeStage: adult; preparations: pinned; catalogNumber: DHJPAR0011464; occurrenceDetails: http:// janzen.sas.upenn.edu; recordedBy: D.H. Janzen, W. Hallwachs \& Guillermo Pereira; otherCatalogNumbers: ASTAQ851-06, 04-SRNP-12099; identifiedBy: AJ Fleming; dateldentified: 2017; language: en; institutionCode: CNC; collectionCode: Insects; basisOfRecord: Pinned Specimen

\section{Description}

Male. Not known at this time.

Female (Fig. 11). Length: $4 \mathrm{~mm}$. Head (Fig. 11b): vertex 1/3 of head width; two pairs of reclinate upper orbital setae and two pairs of proclinate orbital setae; ocellar setae arising behind anterior ocellus; ocellar triangle brilliant silver; fronto-orbital plate pale brassy on upper $50 \%$ coded as silver, remainder dull silver, sparsely setulose, only one or two setulae extending below lowest orbital seta; parafacial silver and bare; eye densely setulose; facial ridge bare; pedicel black, concolorous with postpedicel; arista black, very minutely pubescent, distinctly thickened on basal 1/3-1/4; palpus yellow and haired apically, narrow and digitiform. Thorax (Fig. 11a, c): dull grey tomentose dorsally, appearing almost brassy when viewed under certain angles, contrasting with slightly more silver-grey tomentose laterally; dark setulae on both dorsal and lateral surfaces; four thick dorsal vittae, outermost two broken across suture, innermost pair unbroken, reaching 2nd postsutural dorsocentral seta; postpronotum with three setae arranged in an obtuse triangle; chaetotaxy: acrostichal setae 2:3; dorsocentral setae 3:4; intra-alar setae 2:3; supra-alar setae 2:3; three katepisternal setae, basal seta extremely weak and anterior to suture; basal scutellar setae subequal in length to subapical scutellar setae; lateral scutellar setae less than $2 / 3$ as long as subapical setae, strongly curving inwards medially; apical scutellar setae $1 / 2$ length of lateral scutellar setae, crossed apically; one pair of discal scutellar setae more widely set than subapical scutellar setae; scutellum very slightly darkened across basal $15 \%$, remainder concolorous with scutum. Legs (Fig. 11c): black in ground colour; fore femur with dense silver tomentum on posterodorsal surface; hind coxa setose. Wing (Fig. 
11a): pale translucent, hyaline; vein $\mathrm{R}_{4+5}$ with only one setula at base. Abdomen (Fig. $11 \mathrm{a}, \mathrm{c})$ : ground colour black; middorsal depression on ST1+2 almost reaching hind margin; median marginal setae present on ST1+2-T3; a complete row of marginal setae present on T4-T5; discal setae present on T3-T5; distinct brassy tomentose bands along anterior edge of T3-T5 unbroken medially, covering almost $70 \%$ of tergites; T5 with silver tomentum throughout. Terminalia: not examined.
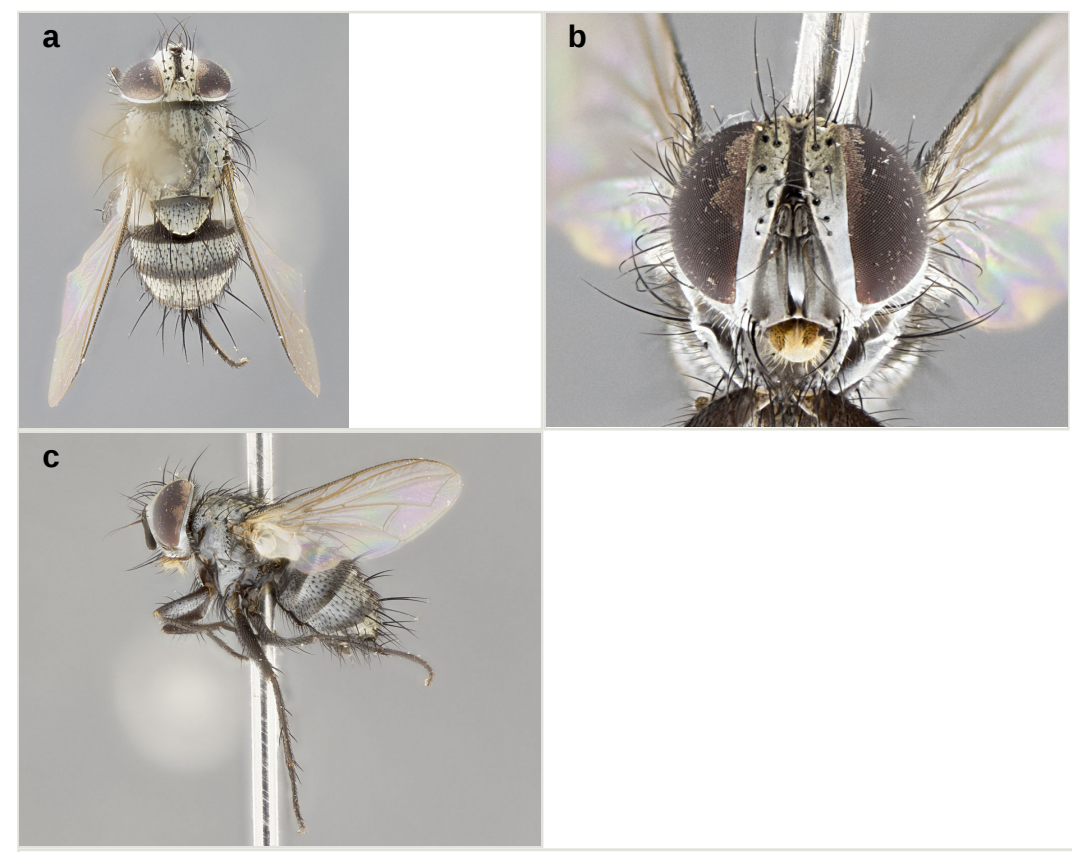

Figure 11.

Hyphantrophaga duniagarciae sp. n.; a-c: habitus; holotype female, voucher $\mathrm{n}$. DHJPAR0011464.
a: dorsal view doi
b: frontal view doi
c: lateral view doi

\section{Diagnosis}

Hyphantrophaga duniagarciae sp. n. can be distinguished from all other Hyphantrophaga species by the following combination of traits: fronto-orbital plate pale brassy on upper $50 \%$ (so pale coded as silver), remainder dull silver, pedicel black, four postsutural dorsocentral setae, thorax entirely silver/grey tomentose, legs black, hind coxa setose, median marginal setae present on ST1+2, discal setae present T3-T5. 


\section{Etymology}

Hyphantrophaga duniagarciae sp. $\mathbf{n}$. is named in recognition of Dunia Garcia Garcia's dedication and work in finding and rearing the ACG caterpillars that contained tachinid larvae.

\section{Distribution}

Costa Rica, ACG, Guanacaste Province, 215 m elevation.

\section{Ecology}

Hyphantrophaga duniagarciae sp. $\mathbf{n}$. has been reared six times from one species of Lepidopterain the family Zygaenidae, Neoilliberis thyesta (Druce, 1884), in dry forest.

\section{Hyphantrophaga edwinapui Fleming \& Wood, sp. $\mathrm{n}$.}

- ZooBank urn:Isid:zoobank.org:act:F8AEEBB3-E9A6-4067-8F75-F5E2496EFE1B

\section{Materials}

Holotype:

a. $\quad$ scientificName: Hyphantrophaga edwinapui; phylum: Arthropoda; class: Insecta; order: Diptera; family: Tachinidae; genus: Hyphantrophaga; specificEpithet: edwinapui; scientificNameAuthorship: Fleming \& Wood, 2017; continent: Central America; country: Costa Rica; countryCode: CR; stateProvince: Guanacaste; county: Sector Santa Rosa; locality: Area de Conservacion Guanacaste; verbatimLocality: Quebrada Cebollines; verbatimElevation: 270; verbatimLatitude: 10.8222; verbatimLongitude: -85.6434; verbatimCoordinateSystem: Decimal; decimalLatitude: 10.8222; decimalLongitude: -85.6434; samplingProtocol: Reared from the larva of the Doidae, Doa Janzen01; verbatimEventDate: 02-Nov-2001; individualID: DHJPAR0007463; individualCount: 1; sex: male; lifeStage: adult; preparations: pinned; catalogNumber: DHJPAR0007463; occurrenceDetails: http://janzen.sas.upenn.edu; recordedBy: D.H. Janzen, W. Hallwachs \& gusaneros; otherCatalogNumbers: ASTAT235-06, 01-SRNP-17538, BOLD:AAC5464; identifiedBy: AJ Fleming; dateldentified: 2017; language: en; institutionCode: CNC; collectionCode: Insects; basisOfRecord: Pinned Specimen

Paratypes:

a. $\quad$ scientificName: Hyphantrophaga edwinapui; phylum: Arthropoda; class: Insecta; order: Diptera; family: Tachinidae; genus: Hyphantrophaga; specificEpithet: edwinapui; scientificNameAuthorship: Fleming \& Wood, 2017; continent: Central America; country: Costa Rica; countryCode: CR; stateProvince: Guanacaste; county: Sector Santa Rosa; locality: Area de Conservacion Guanacaste; verbatimLocality: Quebrada Cebollines; verbatimElevation: 270; verbatimLatitude: 10.8222; verbatimLongitude: -85.6434; verbatimCoordinateSystem: Decimal; decimalLatitude: 10.8222; decimalLongitude: -85.6434; samplingProtocol: Reared from the larva of the Doidae, Doa Janzen01; verbatimEventDate: 08-Nov-2001; individualID: DHJPAR0007465; individualCount: 1; sex: female; lifeStage: adult; preparations: pinned; catalog Number: DHJPAR0007465; occurrenceDetails: http://janzen.sas.upenn.edu; recordedBy: D.H. Janzen, W. Hallwachs \& gusaneros; otherCatalogNumbers: ASTAT237-06, 01-SRNP-17505,; 
identifiedBy: AJ Fleming; dateldentified: 2017; language: en; institutionCode: CNC; collectionCode: Insects; basisOfRecord: Pinned Specimen

b. $\quad$ scientificName: Hyphantrophaga edwinapui; phylum: Arthropoda; class: Insecta; order: Diptera; family: Tachinidae; genus: Hyphantrophaga; specificEpithet: edwinapui; scientificNameAuthorship: Fleming \& Wood, 2017; continent: Central America; country: Costa Rica; countryCode: CR; stateProvince: Guanacaste; county: Sector Santa Rosa; locality: Area de Conservacion Guanacaste; verbatimLocality: Quebrada Cebollines; verbatimElevation: 270; verbatimLatitude: 10.8222; verbatimLongitude: -85.6434; verbatimCoordinateSystem: Decimal; decimalLatitude: 10.8222; decimalLongitude: -85.6434; samplingProtocol: Reared from the larva of the Doidae, Doa Janzen01; verbatimEventDate: 05-Nov-2001; individualID: DHJPAR0007469; individualCount: 1; sex: male; lifeStage: adult; preparations: pinned; catalogNumber: DHJPAR0007469; occurrenceDetails: http://janzen.sas.upenn.edu; recordedBy: D.H. Janzen, W. Hallwachs \& gusaneros; otherCatalogNumbers: ASTAT241-06, 01-SRNP-17485, BOLD:AAC5464; identifiedBy: AJ Fleming; dateldentified: 2017; language: en; institutionCode: CNC; collectionCode: Insects; basisOfRecord: Pinned Specimen

c. scientificName: Hyphantrophaga edwinapui; phylum: Arthropoda; class: Insecta; order: Diptera; family: Tachinidae; genus: Hyphantrophaga; specificEpithet: edwinapui; scientificNameAuthorship: Fleming \& Wood, 2017; continent: Central America; country: Costa Rica; countryCode: CR; stateProvince: Guanacaste; county: Sector Santa Rosa; locality: Area de Conservacion Guanacaste; verbatimLocality: Quebrada Cebollines; verbatimElevation: 270; verbatimLatitude: 10.8222; verbatimLongitude: -85.6434; verbatimCoordinateSystem: Decimal; decimalLatitude: 10.8222; decimalLongitude: -85.6434; samplingProtocol: Reared from the larva of the Doidae, Doa Janzen01; verbatimEventDate: 07-Nov-2001; individualID: DHJPAR0007474; individualCount: 1; sex: male; lifeStage: adult; preparations: pinned; catalogNumber: DHJPAR0007474; occurrenceDetails: http://janzen.sas.upenn.edu; recordedBy: D.H. Janzen, W. Hallwachs \& gusaneros; otherCatalogNumbers: ASTAT246-06, 01-SRNP-17509, BOLD:AAC5464; identifiedBy: AJ Fleming; dateldentified: 2017; language: en; institutionCode: CNC; collectionCode: Insects; basisOfRecord: Pinned Specimen

d. scientificName: Hyphantrophaga edwinapui; phylum: Arthropoda; class: Insecta; order: Diptera; family: Tachinidae; genus: Hyphantrophaga; specificEpithet: edwinapui; scientificNameAuthorship: Fleming \& Wood, 2017; continent: Central America; country: Costa Rica; countryCode: CR; stateProvince: Guanacaste; county: Sector Santa Rosa; locality: Area de Conservacion Guanacaste; verbatimLocality: Quebrada Cebollines; verbatimElevation: 270; verbatimLatitude: 10.8222; verbatimLongitude: -85.6434; verbatimCoordinateSystem: Decimal; decimalLatitude: 10.8222 ; decimalLongitude: -85.6434; samplingProtocol: Reared from the larva of the Doidae, Doa Janzen01; verbatimEventDate: 07-Nov-2001; individualID: DHJPAR0007470; individualCount: 1; sex: male; lifeStage: adult; preparations: pinned; catalogNumber: DHJPAR0007470; occurrenceDetails: http://janzen.sas.upenn.edu; recordedBy: D.H. Janzen, W. Hallwachs \& gusaneros; otherCatalogNumbers: ASTAT242-06, 01-SRNP-17477, BOLD:AAC5464; identifiedBy: AJ Fleming; dateldentified: 2017; language: en; institutionCode: CNC; collectionCode: Insects; basisOfRecord: Pinned Specimen

e. ScientificName: Hyphantrophaga edwinapui; phylum: Arthropoda; class: Insecta; order: Diptera; family: Tachinidae; genus: Hyphantrophaga; specificEpithet: edwinapui; scientificNameAuthorship: Fleming \& Wood, 2017; continent: Central America; country: Costa Rica; countryCode: CR; stateProvince: Guanacaste; county: Sector Santa Rosa; locality: Area de Conservacion Guanacaste; verbatimLocality: Quebrada Cebollines; verbatimElevation: 270; verbatimLatitude: 10.8222; verbatimLongitude: -85.6434; 
verbatimCoordinateSystem: Decimal; decimalLatitude: 10.8222; decimalLongitude: -85.6434; sampling Protocol: Reared from the larva of the Doidae, Doa Janzen01; verbatimEventDate: 07-Nov-2001; individualID: DHJPAR0007472; individualCount: 1; sex: male; lifeStage: adult; preparations: pinned; catalogNumber: DHJPAR0007472; occurrenceDetails: http://janzen.sas.upenn.edu; recordedBy: D.H. Janzen, W. Hallwachs \& gusaneros; otherCatalogNumbers: ASTAT244-06, 01-SRNP-17492, BOLD:AAC5464; identifiedBy: AJ Fleming; dateldentified: 2017; language: en; institutionCode: CNC; collectionCode: Insects; basisOfRecord: Pinned Specimen

f. scientificName: Hyphantrophaga edwinapui; phylum: Arthropoda; class: Insecta; order: Diptera; family: Tachinidae; genus: Hyphantrophaga; specificEpithet: edwinapui; scientificNameAuthorship: Fleming \& Wood, 2017; continent: Central America; country: Costa Rica; countryCode: CR; stateProvince: Guanacaste; county: Sector Santa Rosa; locality: Area de Conservacion Guanacaste; verbatimLocality: Quebrada Cebollines; verbatimElevation: 270; verbatimLatitude: 10.8222; verbatimLongitude: -85.6434; verbatimCoordinateSystem: Decimal; decimalLatitude: 10.8222; decimalLongitude: -85.6434; samplingProtocol: Reared from the larva of the Doidae, Doa Janzen01; verbatimEventDate: 05-Nov-2001; individualID: DHJPAR0007471; individualCount: 1; sex: male; lifeStage: adult; preparations: pinned; catalogNumber: DHJPAR0007471; occurrenceDetails: http://janzen.sas.upenn.edu; recordedBy: D.H. Janzen, W. Hallwachs \& gusaneros; otherCatalogNumbers: ASTAT243-06, 01-SRNP-17476, BOLD:AAC5464; identifiedBy: AJ Fleming; dateldentified: 2017; language: en; institutionCode: CNC; collectionCode: Insects; basisOfRecord: Pinned Specimen

g. ScientificName: Hyphantrophaga edwinapui; phylum: Arthropoda; class: Insecta; order: Diptera; family: Tachinidae; genus: Hyphantrophaga; specificEpithet: edwinapui; scientificNameAuthorship: Fleming \& Wood, 2017; continent: Central America; country: Costa Rica; countryCode: CR; stateProvince: Guanacaste; county: Sector Santa Rosa; locality: Area de Conservacion Guanacaste; verbatimLocality: Quebrada Cebollines; verbatimElevation: 270; verbatimLatitude: 10.8222; verbatimLongitude: -85.6434; verbatimCoordinateSystem: Decimal; decimalLatitude: 10.8222; decimalLongitude: -85.6434; samplingProtocol: Reared from the larva of the Doidae, Doa Janzen01; verbatimEventDate: 07-Nov-2001; individualID: DHJPAR0007467; individualCount: 1; sex: female; lifeStage: adult; preparations: pinned; catalogNumber: DHJPAR0007467; occurrenceDetails: http://janzen.sas.upenn.edu; recordedBy: D.H. Janzen, W. Hallwachs \& gusaneros; otherCatalogNumbers: ASTAT239-06, 01-SRNP-17516, BOLD:AAC5464; identifiedBy: AJ Fleming; dateldentified: 2017; language: en; institutionCode: CNC; collectionCode: Insects; basisOfRecord: Pinned Specimen

h. ScientificName: Hyphantrophaga edwinapui; phylum: Arthropoda; class: Insecta; order: Diptera; family: Tachinidae; genus: Hyphantrophaga; specificEpithet: edwinapui; scientificNameAuthorship: Fleming \& Wood, 2017; continent: Central America; country: Costa Rica; countryCode: CR; stateProvince: Guanacaste; county: Sector Santa Rosa; locality: Area de Conservacion Guanacaste; verbatimLocality: Quebrada Cebollines; verbatimElevation: 270; verbatimLatitude: 10.8222; verbatimLongitude: -85.6434; verbatimCoordinateSystem: Decimal; decimalLatitude: 10.8222; decimalLongitude: -85.6434; samplingProtocol: Reared from the larva of the Doidae, Doa Janzen01; verbatimEventDate: 07-Nov-2001; individualID: DHJPAR0007466; individualCount: 1; sex: female; lifeStage: adult; preparations: pinned; catalogNumber: DHJPAR0007466; occurrenceDetails: http://janzen.sas.upenn.edu; recordedBy: D.H. Janzen, W. Hallwachs \& gusaneros; otherCatalogNumbers: ASTAT238-06, 01-SRNP-17484, BOLD:AAC5464; identifiedBy: AJ Fleming; dateldentified: 2017; language: en; institutionCode: CNC; collectionCode: Insects; basisOfRecord: Pinned Specimen 
i. ScientificName: Hyphantrophaga edwinapui; phylum: Arthropoda; class: Insecta; order: Diptera; family: Tachinidae; genus: Hyphantrophaga; specificEpithet: edwinapui; scientificNameAuthorship: Fleming \& Wood, 2017; continent: Central America; country: Costa Rica; countryCode: CR; stateProvince: Guanacaste; county: Sector Santa Rosa; locality: Area de Conservacion Guanacaste; verbatimLocality: Quebrada Cebollines; verbatimElevation: 270; verbatimLatitude: 10.8222; verbatimLongitude: -85.6434; verbatimCoordinateSystem: Decimal; decimalLatitude: 10.8222; decimalLongitude: -85.6434; samplingProtocol: Reared from the larva of the Doidae, Doa Janzen01; verbatimEventDate: 05-Nov-2001; individualID: DHJPAR0007475; individualCount: 1; sex: male; lifeStage: adult; preparations: pinned; catalogNumber: DHJPAR0007475; occurrenceDetails: http://janzen.sas.upenn.edu; recordedBy: D.H. Janzen, W. Hallwachs \& gusaneros; otherCatalogNumbers: ASTAT247-06, 01-SRNP-17549,; identifiedBy: AJ Fleming; dateldentified: 2017; language: en; institutionCode: CNC; collectionCode: Insects; basisOfRecord: Pinned Specimen

j. ScientificName: Hyphantrophaga edwinapui; phylum: Arthropoda; class: Insecta; order: Diptera; family: Tachinidae; genus: Hyphantrophaga; specificEpithet: edwinapui; scientificNameAuthorship: Fleming \& Wood, 2017; continent: Central America; country: Costa Rica; countryCode: CR; stateProvince: Guanacaste; county: Sector Santa Rosa; locality: Area de Conservacion Guanacaste; verbatimLocality: Quebrada Cebollines; verbatimElevation: 270; verbatimLatitude: 10.8222; verbatimLongitude: -85.6434; verbatimCoordinateSystem: Decimal; decimalLatitude: 10.8222 ; decimalLongitude: -85.6434; samplingProtocol: Reared from the larva of the Doidae, Doa Janzen01; verbatimEventDate: 05-Nov-2001; individualID: DHJPAR0007468; individualCount: 1; sex: female; lifeStage: adult; preparations: pinned; catalogNumber: DHJPAR0007468; occurrenceDetails: http://janzen.sas.upenn.edu; recordedBy: D.H. Janzen, W. Hallwachs \& gusaneros; otherCatalogNumbers: ASTAT240-06, 01-SRNP-17495, BOLD:AAC5464; identifiedBy: AJ Fleming; dateldentified: 2017; language: en; institutionCode: CNC; collectionCode: Insects; basisOfRecord: Pinned Specimen

k. ScientificName: Hyphantrophaga edwinapui; phylum: Arthropoda; class: Insecta; order: Diptera; family: Tachinidae; genus: Hyphantrophaga; specificEpithet: edwinapui; scientificNameAuthorship: Fleming \& Wood, 2017; continent: Central America; country: Costa Rica; countryCode: CR; stateProvince: Guanacaste; county: Sector Santa Rosa; locality: Area de Conservacion Guanacaste; verbatimLocality: Quebrada Cebollines; verbatimElevation: 270; verbatimLatitude: 10.8222; verbatimLongitude: -85.6434; verbatimCoordinateSystem: Decimal; decimalLatitude: 10.8222; decimalLongitude: -85.6434; samplingProtocol: Reared from the larva of the Doidae, Doa Janzen01; verbatimEventDate: 07-Nov-2001; individualID: DHJPAR0007473; individualCount: 1; sex: female; lifeStage: adult; preparations: pinned; catalogNumber: DHJPAR0007473; occurrenceDetails: http://janzen.sas.upenn.edu; recordedBy: D.H. Janzen, W. Hallwachs \& gusaneros; otherCatalog Numbers: ASTAT245-06, 01-SRNP-17536, BOLD:AAC5464; identifiedBy: AJ Fleming; dateldentified: 2017; language: en; institutionCode: CNC; collectionCode: Insects; basisOfRecord: Pinned Specimen

I. scientificName: Hyphantrophaga edwinapui; phylum: Arthropoda; class: Insecta; order: Diptera; family: Tachinidae; genus: Hyphantrophaga; specificEpithet: edwinapui; scientificNameAuthorship: Fleming \& Wood, 2017; continent: Central America; country: Costa Rica; countryCode: CR; stateProvince: Guanacaste; county: Sector Santa Rosa; locality: Area de Conservacion Guanacaste; verbatimLocality: Quebrada Cebollines; verbatimElevation: 270; verbatimLatitude: 10.8222; verbatimLongitude: -85.6434; verbatimCoordinateSystem: Decimal; decimalLatitude: 10.8222 ; decimalLongitude: -85.6434; sampling Protocol: Reared from the larva of the Doidae, Doa Janzen01; 
verbatimEventDate: 02-Nov-2001; individualID: DHJPAR0007464; individualCount: 1; sex: female; lifeStage: adult; preparations: pinned; catalogNumber: DHJPAR0007464; occurrenceDetails: http://janzen.sas.upenn.edu; recordedBy: D.H. Janzen, W. Hallwachs \& gusaneros; otherCatalogNumbers: ASTAT236-06, 01-SRNP-17527, BOLD:AAC5464; identifiedBy: AJ Fleming; dateldentified: 2017; language: en; institutionCode: CNC; collectionCode: Insects; basisOfRecord: Pinned Specimen

\section{Description}

Male (Fig. 12). Length: 7-9 mm. Head (Fig. 12b): vertex 1/5 of head width; two pairs of reclinate upper orbital setae; ocellar setae arising behind anterior ocellus; ocellar triangle silver/dull grey; fronto-orbital plate dull silver/grey and densely setulose throughout, setulae not extending beyond level of lowest frontal seta; parafacial concolorous with fronto-orbital plate and bare; eye densely haired; facial ridge bare; pedicel brownish-black (with only a slight light orange fringe at margin between pedicel and postpedicel), concolorous with postpedicel; arista brown, very minutely pubescent, distinctly thickened on basal 1/3-1/4; palpus dark grey/brown, haired apically, slenderdigitiform. Thorax (Fig. 12a, c): dull brassy tomentose throughout; densely covered on all surfaces, with short black setulae interspersed amongst the setae; four thick dorsal vittae, outermost two broken across suture, innermost pair unbroken, reaching 2nd postsutural dorsocentral seta; postpronotum with 4-5 setae arranged in a triangle; chaetotaxy: acrostichal setae 3:3; dorsocentral setae 3:4; intra-alar setae 3:3; supraalar setae 2:3; three katepisternal setae; basal scutellar setae subequal to subapical scutellar setae, curving inwards medially; lateral scutellar setae approximately $1 / 2$ as long as subapical setae, curving inwards medially, parallel to basal scutellar setae; subapical scutellar setae straight and strongly divergent; apical scutellar setae subequal in length to lateral scutellar setae, crossed apically; one pair of discal scutellar setae set more narrowly than subapical setae; scutellum very lightly gold tomentose across apical 20\%, remainder concolorous with scutum. Legs (Fig. 12c): brilliant yellow in ground colour, covered in dark setulae making them appear darker; fore femur with dense silver tomentum on posterodorsal surface; hind coxa bare. Wing (Fig. 12a): pale translucent, hyaline; vein $\mathrm{R}_{4+5}$ with only 2-3 setulae at base. Abdomen (Fig. 12a, c): ground colour yellowish-brown; middorsal depression on ST1+2 almost reaching hind margin; median marginal setae absent on ST1+2, present on T3; a complete row of marginal setae present on T4; discal setae only on T5; sex patch covering ventral surfaces of T4-T5; entire dorsal surface of tergites distinctly brassy tomentose; T5 with brassy tomentum throughout. Terminalia (Fig. 12d, e, f): sternite 5 (Fig. 12f) with a deeply excavated median cleft, squared-off wide V-shaped, margins covered in dense tomentum. Lateral lobes of sternite rounded triangular apically, 1-2 strong setae surrounded by many shorter, weaker setulae. Anterior plate of sternite 5 twice as long as apical lobes; unsclerotised window anterior to median cleft only slightly tri-lobed, with arms as wide as median cleft. Cerci in posterior view (Fig. 12d) narrow, subrectangular and slightly shorter than surstyli, ending in a rounded tip; completely separate medially, straight, divergent in apical $1 / 3$; in lateral view weakly clubbed along anterior $1 / 3$ and weakly curved at beginning of club; densely setulose dorsally up to a 
tapered tip, apparently bare ventrally (visible in lateral view). Surstylus in lateral view (Fig. 12e) almost parallel-sided along its length, with rounded apices, giving it a spatulate appearance; when viewed dorsally, surstyli appearing not divergent. Pregonite broad and well-developed, slightly bent; basal 2/3 slightly cinched, giving it a very slightly clubbed appearance; apically rounded with a few fine setulae along its edge. Postgonite elongate, equally wide along its length with a slight curve at tip, subequal in length to pregonite. Distiphallus rectangular with a very slight apical flare, a slender median longitudinal sclerotised reinforcement on its posterior surface and a broad, anterolateral, sclerotised acrophallus, joined as a plate on anterior surface near apex.

Female. Length: 5-7 mm. As male, differing only by the presence of two pairs of proclinate orbital setae.

\section{Diagnosis}

Hyphantrophaga edwinapui sp. n. can be distinguished from all other Hyphantrophaga species by the following combination of traits: three katepisternal setae, legs yellow, hind coxa bare, abdominal tergites entirely brassy tomentose and median marginal setae absent from ST1+2.

\section{Etymology}

Hyphantrophaga edwinapui sp. $\mathbf{n}$. is named in recognition of Edwin Jose Apu Fajardo's dedication and work in finding and rearing the ACG caterpillars that contained tachinid larvae.

\section{Distribution}

Costa Rica, ACG, Guanacaste Province, 270 m elevation.

\section{Ecology}

Hyphantrophaga edwinapui sp. $\mathbf{n}$. has been reared 22 times from a single species of Lepidoptera in the family Doidae, Doa Janzen01, in dry forest. 


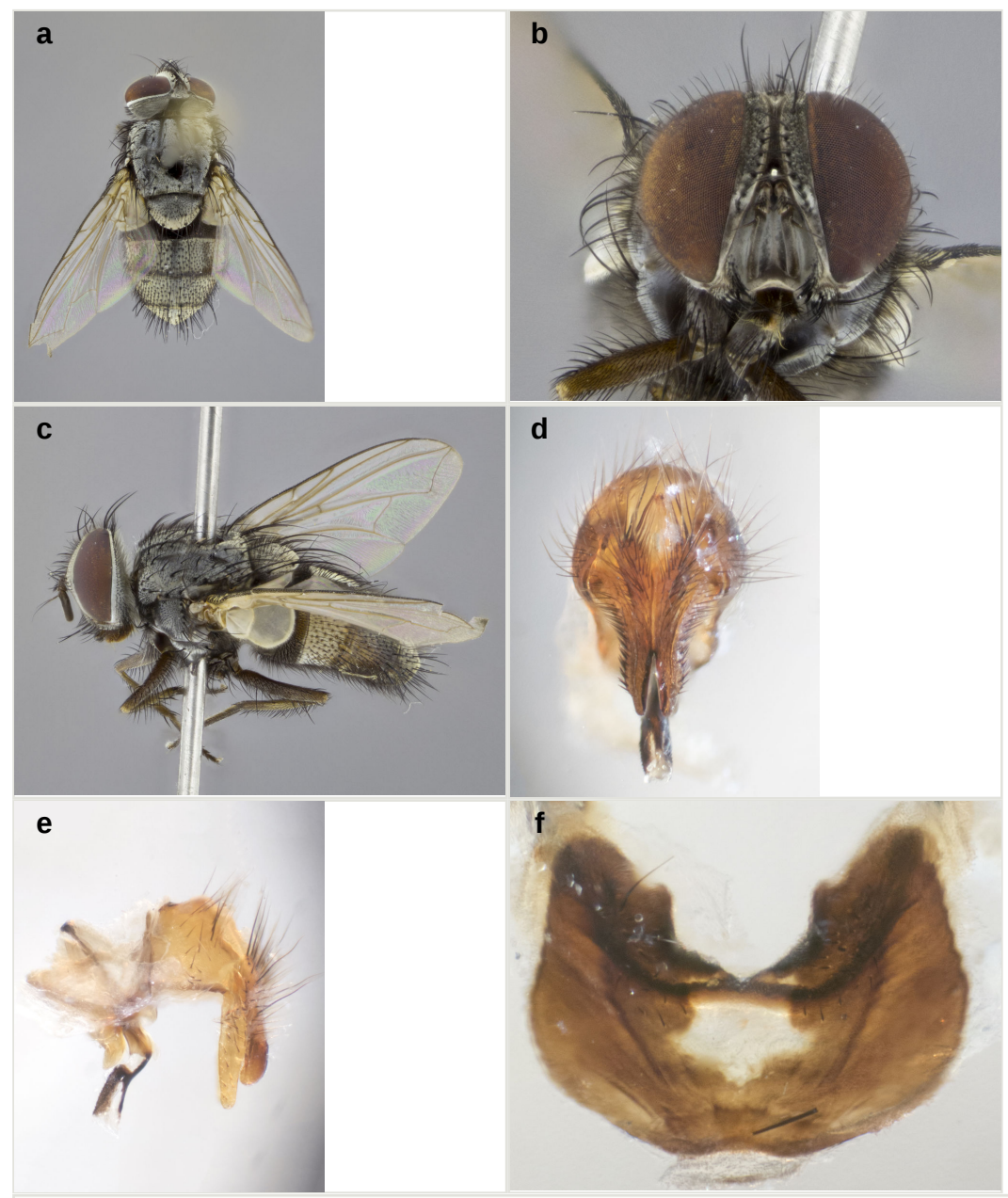

Figure 12.

Hyphantrophaga edwinapui sp. n.; a-c: habitus; holotype male, voucher $\mathrm{n}$. DHJPAR0007463; d-f: terminalia; male paratype, voucher n. DHJPAR007470.

a: dorsal view doi

b: frontal view doi

c: lateral view doi

d: dorsal view doi

e: lateral view doi

f: sternite 5 , ventral view doi 


\section{Hyphantrophaga eldaarayae Fleming \& Wood, sp. n.}

- $\quad$ ZooBank urn:Isid:zoobank.org:act:625985CF-3D13-4FA1-A1BC-27819EA9D933

\section{Materials}

Holotype:

a. $\quad$ scientific Name: Hyphantrophaga eldaarayae; phylum: Arthropoda; class: Insecta; order: Diptera; family: Tachinidae; genus: Hyphantrophaga; specificEpithet: eldaarayae; scientificNameAuthorship: Fleming \& Wood, 2018; continent: Central America; country: Costa Rica; countryCode: CR; stateProvince: Alajuela; county: Sector San Cristobal; locality: Area de Conservacion Guanacaste; verbatimLocality: Rio Blanco Abajo; verbatimElevation: 500; verbatimLatitude: 10.9004; verbatimLongitude: -85.3725 ; verbatimCoordinateSystem: Decimal; decimalLatitude: 10.9004; decimalLongitude: -85.3725; samplingProtocol: Reared from the larva of the Sphingidae, Xylophanes chiron; verbatimEventDate: 28-Nov-2001; individualID: DHJPAR0022963; individualCount: 1; sex: male; lifeStage: adult; preparations: pinned; catalogNumber: DHJPAR0022963; occurrenceDetails: http://janzen.sas.upenn.edu; recordedBy: D.H. Janzen, W. Hallwachs \& Gloria Sihezar; otherCatalogNumbers: ASTAW127-08, 01-SRNP-3991,; identifiedBy: AJ Fleming; dateldentified: 2017; language: en; institutionCode: CNC; collectionCode: Insects; basisOfRecord: Pinned Specimen

\section{Paratypes:}

a. $\quad$ scientificName: Hyphantrophaga eldaarayae; phylum: Arthropoda; class: Insecta; order: Diptera; family: Tachinidae; genus: Hyphantrophaga; specificEpithet: eldaarayae; scientificNameAuthorship: Fleming \& Wood, 2018; continent: Central America; country: Costa Rica; countryCode: CR; stateProvince: Alajuela; county: Sector San Cristobal; locality: Area de Conservacion Guanacaste; verbatimLocality: Rio Blanco Abajo; verbatimElevation: 500; verbatimLatitude: 10.9004; verbatimLongitude: -85.3725 ; verbatimCoordinateSystem: Decimal; decimalLatitude: 10.9004; decimalLongitude: -85.3725; samplingProtocol: Reared from the larva of the Sphingidae, Xylophanes chiron; verbatimEventDate: 26-Jan-2006; individualID: DHJPAR0005525; individualCount: 1; sex: male; lifeStage: adult; preparations: pinned; catalogNumber: DHJPAR0005525; occurrenceDetails: http://janzen.sas.upenn.edu; recordedBy: D.H. Janzen, W. Hallwachs \& Carolina Cano; otherCatalogNumbers: ASTA644-06, 05-SRNP-8070,; identifiedBy: AJ Fleming; dateldentified: 2017; language: en; institutionCode: CNC; collectionCode: Insects; basisOfRecord: Pinned Specimen

b. $\quad$ scientificName: Hyphantrophaga eldaarayae; phylum: Arthropoda; class: Insecta; order: Diptera; family: Tachinidae; genus: Hyphantrophaga; specificEpithet: eldaarayae; scientificNameAuthorship: Fleming \& Wood, 2018; continent: Central America; country: Costa Rica; countryCode: CR; stateProvince: Alajuela; county: Sector Rincon Rain Forest; locality: Area de Conservacion Guanacaste; verbatimLocality: San Lucas; verbatimElevation: 320; verbatimLatitude: 10.9185; verbatimLongitude: -85.3034; verbatimCoordinateSystem: Decimal; decimalLatitude: 10.9185; decimalLongitude: -85.3034; samplingProtocol: Reared from the larva of the Sphingidae, Xylophanes chiron; verbatimEventDate: 02-Aug-2003; individualID: DHJPAR0008059; individualCount: 1; sex: male; lifeStage: adult; preparations: pinned; catalogNumber: DHJPAR0008059; occurrenceDetails: http://janzen.sas.upenn.edu; recordedBy: D.H. Janzen, W. Hallwachs \& Jose Perez; otherCatalogNumbers: 
ASTAT831-06, 03-SRNP-11664, BOLD:AAB0592; identifiedBy: AJ Fleming; dateldentified: 2017; language: en; institutionCode: CNC; collectionCode: Insects; basisOfRecord: Pinned Specimen

c. ScientificName: Hyphantrophaga eldaarayae; phylum: Arthropoda; class: Insecta; order: Diptera; family: Tachinidae; genus: Hyphantrophaga; specificEpithet: eldaarayae; scientificNameAuthorship: Fleming \& Wood, 2018; continent: Central America; country: Costa Rica; countryCode: CR; stateProvince: Alajuela; county: Sector Rincon Rain Forest; locality: Area de Conservacion Guanacaste; verbatimLocality: San Lucas; verbatimElevation: 320; verbatimLatitude: 10.9185; verbatimLongitude: -85.3034; verbatimCoordinateSystem: Decimal; decimalLatitude: 10.9185; decimalLongitude: -85.3034; samplingProtocol: Reared from the larva of the Sphingidae, Xylophanes chiron; verbatimEventDate: 14-Nov-2003; individualID: DHJPAR0008064; individualCount: 1; sex: male; lifeStage: adult; preparations: pinned; catalogNumber: DHJPAR0008064; occurrenceDetails: http://janzen.sas.upenn.edu; recordedBy: D.H. Janzen, W. Hallwachs \& Jose Perez; otherCatalogNumbers: ASTAT836-06, 03-SRNP-31066,; identifiedBy: AJ Fleming; dateldentified: 2017; language: en; institutionCode: CNC; collectionCode: Insects; basisOfRecord: Pinned Specimen

d. $\quad$ scientificName: Hyphantrophaga eldaarayae; phylum: Arthropoda; class: Insecta; order: Diptera; family: Tachinidae; genus: Hyphantrophaga; specificEpithet: eldaarayae; scientificNameAuthorship: Fleming \& Wood, 2018; continent: Central America; country: Costa Rica; countryCode: CR; stateProvince: Alajuela; county: Sector San Cristobal; locality: Area de Conservacion Guanacaste; verbatimLocality: Quebrada Cementerio; verbatimElevation: 700; verbatimLatitude: 10.8712; verbatimLongitude: -85.3875 ; verbatimCoordinateSystem: Decimal; decimalLatitude: 10.8712; decimalLongitude: -85.3875; samplingProtocol: Reared from the larva of the Sphingidae, Xylophanes chiron; verbatimEventDate: 02-Jul-1998; individualID: DHJPAR0008066; individualCount: 1; sex: male; lifeStage: adult; preparations: pinned; catalogNumber: DHJPAR0008066; occurrenceDetails: http://janzen.sas.upenn.edu; recordedBy: D.H. Janzen, W. Hallwachs \& Gloria Sihezar; otherCatalogNumbers: ASTAT838-06, 98-SRNP-6070,; identifiedBy: AJ Fleming; dateldentified: 2017; language: en; institutionCode: CNC; collectionCode: Insects; basisOfRecord: Pinned Specimen

e. $\quad$ scientificName: Hyphantrophaga eldaarayae; phylum: Arthropoda; class: Insecta; order: Diptera; family: Tachinidae; genus: Hyphantrophaga; specificEpithet: eldaarayae; scientificNameAuthorship: Fleming \& Wood, 2018; continent: Central America; country: Costa Rica; countryCode: CR; stateProvince: Guanacaste; county: Sector Pitilla; locality: Area de Conservacion Guanacaste; verbatimLocality: Estacion Pitilla; verbatimElevation: 675; verbatimLatitude: 10.9893; verbatimLongitude: -85.4258; verbatimCoordinateSystem: Decimal; decimalLatitude: 10.9893; decimalLongitude: -85.4258; samplingProtocol: Reared from the larva of the Sphingidae, Xylophanes chiron; verbatimEventDate: 16-Jan-2004; individualID: DHJPAR0008069; individualCount: 1; sex: male; lifeStage: adult; preparations: pinned; catalogNumber: DHJPAR0008069; occurrenceDetails: http://janzen.sas.upenn.edu; recordedBy: D.H. Janzen, W. Hallwachs \& Lucia Rios; otherCatalogNumbers: ASTAT841-06, 03-SRNP-37359, BOLD:AAB0592; identifiedBy: AJ Fleming; dateldentified: 2017; language: en; institutionCode: CNC; collectionCode: Insects; basisOfRecord: Pinned Specimen

f. ScientificName: Hyphantrophaga eldaarayae; phylum: Arthropoda; class: Insecta; order: Diptera; family: Tachinidae; genus: Hyphantrophaga; specificEpithet: 
eldaarayae; scientificNameAuthorship: Fleming \& Wood, 2018; continent: Central America; country: Costa Rica; countryCode: CR; stateProvince: Guanacaste; county: Sector Pitilla; locality: Area de Conservacion Guanacaste; verbatimLocality: Pasmompa; verbatimElevation: 440; verbatimLatitude: 11.0193; verbatimLongitude: -85.41; verbatimCoordinateSystem: Decimal; decimalLatitude: 11.0193; decimalLongitude: -85.41; samplingProtocol: Reared from the larva of the Sphingidae, Xylophanes chiron; verbatimEventDate: 26-Dec-2004; individualID: DHJPAR0008075; individualCount: 1; sex: male; lifeStage: adult; preparations: pinned; catalogNumber: DHJPAR0008075; occurrenceDetails: http://janzen.sas.upenn.edu; recordedBy: D.H. Janzen, W. Hallwachs \& Calixto Moraga; otherCatalogNumbers: ASTAT847-06, 04SRNP-56432, BOLD:AAB0592; identifiedBy: AJ Fleming; dateldentified: 2017; language: en; institutionCode: CNC; collectionCode: Insects; basisOfRecord: Pinned Specimen

g. ScientificName: Hyphantrophaga eldaarayae; phylum: Arthropoda; class: Insecta; order: Diptera; family: Tachinidae; genus: Hyphantrophaga; specificEpithet: eldaarayae; scientificNameAuthorship: Fleming \& Wood, 2018; continent: Central America; country: Costa Rica; countryCode: CR; stateProvince: Guanacaste; county: Sector Santa Rosa; locality: Area de Conservacion Guanacaste; verbatimLocality: Sendero Inga Peluda; verbatimElevation: 280; verbatimLatitude: 10.8733; verbatimLongitude: -85.6067; verbatimCoordinateSystem: Decimal; decimalLatitude: 10.8733; decimalLongitude: -85.6067; samplingProtocol: Reared from the larva of the Sphingidae, Xylophanes chiron; verbatimEventDate: 18-Aug-2003; individualID: DHJPAR0008080; individualCount: 1; sex: male; lifeStage: adult; preparations: pinned; catalogNumber: DHJPAR0008080; occurrenceDetails: http://janzen.sas.upenn.edu; recordedBy: D.H. Janzen, W. Hallwachs \& Guillermo Pereira; otherCatalogNumbers: ASTAT852-06, 03-SRNP-14320,; identifiedBy: AJ Fleming; dateldentified: 2017; language: en; institutionCode: CNC; collectionCode: Insects; basisOfRecord: Pinned Specimen

h. ScientificName: Hyphantrophaga eldaarayae; phylum: Arthropoda; class: Insecta; order: Diptera; family: Tachinidae; genus: Hyphantrophaga; specificEpithet: eldaarayae; scientificNameAuthorship: Fleming \& Wood, 2018; continent: Central America; country: Costa Rica; countryCode: CR; stateProvince: Guanacaste; county: Sector Pitilla; locality: Area de Conservacion Guanacaste; verbatimLocality: Pasmompa; verbatimElevation: 440; verbatimLatitude: 11.0193; verbatimLongitude: -85.41; verbatimCoordinateSystem: Decimal; decimalLatitude: 11.0193; decimalLongitude: -85.41; samplingProtocol: Reared from the larva of the Sphingidae, Xylophanes chiron; verbatimEventDate: 04-Aug-2003; individualID: DHJPAR0008081; individualCount: 1; sex: male; lifeStage: adult; preparations: pinned; catalogNumber: DHJPAR0008081; occurrenceDetails: http://janzen.sas.upenn.edu; recordedBy: D.H. Janzen, W. Hallwachs \& Petrona Rios; otherCatalogNumbers: ASTAT853-06, 03SRNP-20030,; identifiedBy: AJ Fleming; dateldentified: 2017; language: en; institutionCode: CNC; collectionCode: Insects; basisOfRecord: Pinned Specimen

i. $\quad$ scientificName: Hyphantrophaga eldaarayae; phylum: Arthropoda; class: Insecta; order: Diptera; family: Tachinidae; genus: Hyphantrophaga; specificEpithet: eldaarayae; scientificNameAuthorship: Fleming \& Wood, 2018; continent: Central America; country: Costa Rica; countryCode: CR; stateProvince: Alajuela; county: Sector San Cristobal; locality: Area de Conservacion Guanacaste; verbatimLocality: Rio Blanco Abajo; verbatimElevation: 500; verbatimLatitude: 10.9004; verbatimLongitude: -85.3725 ; verbatimCoordinateSystem: Decimal; decimalLatitude: 10.9004; decimalLongitude: -85.3725 ; samplingProtocol: Reared from the larva of the 
Sphingidae, Xylophanes chiron; verbatimEventDate: 03-Oct-2005; individualID: DHJPAR0008082; individualCount: 1; sex: male; lifeStage: adult; preparations: pinned; catalogNumber: DHJPAR0008082; occurrenceDetails: http://janzen.sas.upenn.edu; recordedBy: D.H. Janzen, W. Hallwachs \& Anabelle Cordoba; otherCatalogNumbers: ASTAT854-06, 05-SRNP-5411, BOLD:AAB0592; identifiedBy: AJ Fleming; dateldentified: 2017; language: en; institutionCode: CNC; collectionCode: Insects; basisOfRecord: Pinned Specimen

j. $\quad$ scientificName: Hyphantrophaga eldaarayae; phylum: Arthropoda; class: Insecta; order: Diptera; family: Tachinidae; genus: Hyphantrophaga; specificEpithet: eldaarayae; scientificNameAuthorship: Fleming \& Wood, 2018; continent: Central America; country: Costa Rica; countryCode: CR; stateProvince: Guanacaste; county: Sector Pitilla; locality: Area de Conservacion Guanacaste; verbatimLocality: Pasmompa; verbatimElevation: 440; verbatimLatitude: 11.0193; verbatimLongitude: -85.41; verbatimCoordinateSystem: Decimal; decimalLatitude: 11.0193; decimalLongitude: -85.41; samplingProtocol: Reared from the larva of the Sphingidae, Xylophanes chiron; verbatimEventDate: 20-Aug-2004; individualID: DHJPAR0008083; individualCount: 1; sex: male; lifeStage: adult; preparations: pinned; catalogNumber: DHJPAR0008083; occurrenceDetails: http://janzen.sas.upenn.edu; recordedBy: D.H. Janzen, W. Hallwachs \& Petrona Rios; otherCatalog Numbers: ASTAT855-06, 04SRNP-33892, BOLD:AAB0592; identifiedBy: AJ Fleming; dateldentified: 2017; language: en; institutionCode: CNC; collectionCode: Insects; basisOfRecord: Pinned Specimen

k. ScientificName: Hyphantrophaga eldaarayae; phylum: Arthropoda; class: Insecta; order: Diptera; family: Tachinidae; genus: Hyphantrophaga; specificEpithet: eldaarayae; scientificNameAuthorship: Fleming \& Wood, 2018; continent: Central America; country: Costa Rica; countryCode: CR; stateProvince: Alajuela; county: Sector San Cristobal; locality: Area de Conservacion Guanacaste; verbatimLocality: Rio Blanco Abajo; verbatimElevation: 500; verbatimLatitude: 10.9004; verbatimLongitude: -85.3725 ; verbatimCoordinateSystem: Decimal; decimalLatitude: 10.9004; decimalLongitude: -85.3725 ; samplingProtocol: Reared from the larva of the Sphingidae, Xylophanes chiron; verbatimEventDate: 05-Oct-2003; individualID: DHJPAR0008060; individualCount: 1; sex: female; lifeStage: adult; preparations: pinned; catalogNumber: DHJPAR0008060; occurrenceDetails: http:// janzen.sas.upenn.edu; recordedBy: D.H. Janzen, W. Hallwachs \& Carolina Cano; otherCatalogNumbers: ASTAT832-06, 03-SRNP-8261,; identifiedBy: AJ Fleming; dateldentified: 2017; language: en; institutionCode: CNC; collectionCode: Insects; basisOfRecord: Pinned Specimen

I. scientificName: Hyphantrophaga eldaarayae; phylum: Arthropoda; class: Insecta; order: Diptera; family: Tachinidae; genus: Hyphantrophaga; specificEpithet: eldaarayae; scientificNameAuthorship: Fleming \& Wood, 2018; continent: Central America; country: Costa Rica; countryCode: CR; stateProvince: Alajuela; county: Sector San Cristobal; locality: Area de Conservacion Guanacaste; verbatimLocality: Rio Blanco Abajo; verbatimElevation: 500; verbatimLatitude: 10.9004; verbatimLongitude: -85.3725 ; verbatimCoordinateSystem: Decimal; decimalLatitude: 10.9004; decimalLongitude: -85.3725; samplingProtocol: Reared from the larva of the Sphingidae, Xylophanes chiron; verbatimEventDate: 13-Nov-2001; individualID: DHJPAR0008062; individualCount: 1; sex: female; lifeStage: adult; preparations: pinned; catalogNumber: DHJPAR0008062; occurrenceDetails: http:// janzen.sas.upenn.edu; recordedBy: D.H. Janzen, W. Hallwachs \& Osvaldo Espinoza; otherCatalogNumbers: ASTAT834-06, 01-SRNP-22084,; identifiedBy: AJ Fleming; 
dateldentified: 2017; Ianguage: en; institutionCode: CNC; collectionCode: Insects; basisOfRecord: Pinned Specimen

m. $\quad$ scientificName: Hyphantrophaga eldaarayae; phylum: Arthropoda; class: Insecta; order: Diptera; family: Tachinidae; genus: Hyphantrophaga; specificEpithet: eldaarayae; scientificNameAuthorship: Fleming \& Wood, 2018; continent: Central America; country: Costa Rica; countryCode: CR; stateProvince: Guanacaste; county: Sector El Hacha; locality: Area de Conservacion Guanacaste; verbatimLocality: Sendero Tigre; verbatimElevation: 280 ; verbatimLatitude: 11.0317; verbatimLongitude: -85.5262; verbatimCoordinateSystem: Decimal; decimalLatitude: 11.0317; decimalLongitude: -85.5262; samplingProtocol: Reared from the larva of the Sphingidae, Xylophanes pluto; verbatimEventDate: 04-Sep-2001; individualID: DHJPAR0008063; individualCount: 1; sex: female; lifeStage: adult; preparations: pinned; catalogNumber: DHJPAR0008063; occurrenceDetails: http:// janzen.sas.upenn.edu; recordedBy: D.H. Janzen, W. Hallwachs \& Lucia Rios; otherCatalogNumbers: ASTAT835-06, 01-SRNP-10457,; identifiedBy: AJ Fleming; dateldentified: 2017; language: en; institutionCode: CNC; collectionCode: Insects; basisOfRecord: Pinned Specimen

n. $\quad$ scientificName: Hyphantrophaga eldaarayae; phylum: Arthropoda; class: Insecta; order: Diptera; family: Tachinidae; genus: Hyphantrophaga; specificEpithet: eldaarayae; scientificNameAuthorship: Fleming \& Wood, 2018; continent: Central America; country: Costa Rica; countryCode: CR; stateProvince: Alajuela; county: Sector San Cristobal; locality: Area de Conservacion Guanacaste; verbatimLocality: Rio Blanco Abajo; verbatimElevation: 500; verbatimLatitude: 10.9004; verbatimLongitude: -85.3725 ; verbatimCoordinateSystem: Decimal; decimalLatitude: 10.9004; decimalLongitude: -85.3725 ; samplingProtocol: Reared from the larva of the Sphingidae, Xylophanes chiron; verbatimEventDate: 02-Jun-2002; individualID: DHJPAR0008065; individualCount: 1; sex: female; lifeStage: adult; preparations: pinned; catalogNumber: DHJPAR0008065; occurrenceDetails: http:// janzen.sas.upenn.edu; recordedBy: D.H. Janzen, W. Hallwachs \& Gloria Sihezar; otherCatalog Numbers: ASTAT837-06, 02-SRNP-2965, BOLD:AAB0592; identifiedBy: AJ Fleming; dateldentified: 2017; language: en; institutionCode: CNC; collectionCode: Insects; basisOfRecord: Pinned Specimen

o. ScientificName: Hyphantrophaga eldaarayae; phylum: Arthropoda; class: Insecta; order: Diptera; family: Tachinidae; genus: Hyphantrophaga; specificEpithet: eldaarayae; scientificNameAuthorship: Fleming \& Wood, 2018; continent: Central America; country: Costa Rica; countryCode: CR; stateProvince: Guanacaste; county: Sector Santa Rosa; locality: Area de Conservacion Guanacaste; verbatimLocality: Ojochal; verbatimElevation: 10; verbatimLatitude: 10.7851; verbatimLongitude: -85.6637; verbatimCoordinateSystem: Decimal; decimalLatitude: 10.7851; decimalLongitude: -85.6637; samplingProtocol: Reared from the larva of the Sphingidae, Xylophanes pluto; verbatimEventDate: 23-Nov-1998; individualID: DHJPAR0008067; individualCount: 1; sex: female; lifeStage: adult; preparations: pinned; catalogNumber: DHJPAR0008067; occurrenceDetails: http:// janzen.sas.upenn.edu; recordedBy: D.H. Janzen, W. Hallwachs \& gusaneros; otherCatalogNumbers: ASTAT839-06, 98-SRNP-12655,; identifiedBy: AJ Fleming; dateldentified: 2017; language: en; institutionCode: CNC; collectionCode: Insects; basisOfRecord: Pinned Specimen

p. ScientificName: Hyphantrophaga eldaarayae; phylum: Arthropoda; class: Insecta; order: Diptera; family: Tachinidae; genus: Hyphantrophaga; specificEpithet: eldaarayae; scientificNameAuthorship: Fleming \& Wood, 2018; continent: Central 
America; country: Costa Rica; countryCode: CR; stateProvince: Guanacaste; county: Sector El Hacha; locality: Area de Conservacion Guanacaste; verbatimLocality: Sendero Bejuquilla; verbatimElevation: 280; verbatimLatitude: 11.03; verbatimLongitude: -85.527; verbatimCoordinateSystem: Decimal; decimalLatitude: 11.03; decimalLongitude: -85.527; samplingProtocol: Reared from the larva of the Sphingidae, Xylophanes tyndarus; verbatimEventDate: 23-Dec-1998; individualID: DHJPAR0008068; individualCount: 1; sex: female; lifeStage: adult; preparations: pinned; catalogNumber: DHJPAR0008068; occurrenceDetails: http:// janzen.sas.upenn.edu; recordedBy: D.H. Janzen, W. Hallwachs \& Lucia Rios; otherCatalog Numbers: ASTAT840-06, 98-SRNP-13992,; identifiedBy: AJ Fleming; dateldentified: 2017; language: en; institutionCode: CNC; collectionCode: Insects; basisOfRecord: Pinned Specimen

q. ScientificName: Hyphantrophaga eldaarayae; phylum: Arthropoda; class: Insecta; order: Diptera; family: Tachinidae; genus: Hyphantrophaga; specificEpithet: eldaarayae; scientificNameAuthorship: Fleming \& Wood, 2018; continent: Central America; country: Costa Rica; countryCode: CR; stateProvince: Guanacaste; county: Sector Pitilla; locality: Area de Conservacion Guanacaste; verbatimLocality: Pasmompa; verbatimElevation: 440; verbatimLatitude: 11.0193; verbatimLongitude: -85.41; verbatimCoordinateSystem: Decimal; decimalLatitude: 11.0193; decimalLongitude: -85.41; samplingProtocol: Reared from the larva of the Sphingidae, Xylophanes chiron; verbatimEventDate: 11-Sep-2003; individualID: DHJPAR0008070; individualCount: 1; sex: female; lifeStage: adult; preparations: pinned; catalog Number: DHJPAR0008070; occurrenceDetails: http://janzen.sas.upenn.edu; recordedBy: D.H. Janzen, W. Hallwachs \& Calixto Moraga; otherCatalogNumbers: ASTAT842-06, 03SRNP-20157,; identifiedBy: AJ Fleming; dateldentified: 2017; language: en; institutionCode: CNC; collectionCode: Insects; basisOfRecord: Pinned Specimen

r. scientificName: Hyphantrophaga eldaarayae; phylum: Arthropoda; class: Insecta; order: Diptera; family: Tachinidae; genus: Hyphantrophaga; specificEpithet: eldaarayae; scientificNameAuthorship: Fleming \& Wood, 2018; continent: Central America; country: Costa Rica; countryCode: CR; stateProvince: Alajuela; county: Sector Rincon Rain Forest; locality: Area de Conservacion Guanacaste; verbatimLocality: San Lucas; verbatimElevation: 320; verbatimLatitude: 10.9185; verbatimLongitude: -85.3034; verbatimCoordinateSystem: Decimal; decimalLatitude: 10.9185; decimalLongitude: -85.3034; samplingProtocol: Reared from the larva of the Sphingidae, Xylophanes chiron; verbatimEventDate: 14-Nov-2003; individualID: DHJPAR0008071; individualCount: 1; sex: female; lifeStage: adult; preparations: pinned; catalogNumber: DHJPAR0008071; occurrenceDetails: http:// janzen.sas.upenn.edu; recordedBy: D.H. Janzen, W. Hallwachs \& Jose Perez; otherCatalog Numbers: ASTAT843-06, 03-SRNP-31066, BOLD:AAB0592; identifiedBy: AJ Fleming; dateldentified: 2017; language: en; institutionCode: CNC; collectionCode: Insects; basisOfRecord: Pinned Specimen

s. ScientificName: Hyphantrophaga eldaarayae; phylum: Arthropoda; class: Insecta; order: Diptera; family: Tachinidae; genus: Hyphantrophaga; specificEpithet: eldaarayae; scientificNameAuthorship: Fleming \& Wood, 2018; continent: Central America; country: Costa Rica; countryCode: CR; stateProvince: Alajuela; county: Sector San Cristobal; locality: Area de Conservacion Guanacaste; verbatimLocality: Rio Blanco Abajo; verbatimElevation: 500; verbatimLatitude: 10.9004; verbatimLongitude: -85.3725; verbatimCoordinateSystem: Decimal; decimalLatitude: 10.9004; decimalLongitude: -85.3725 ; samplingProtocol: Reared from the larva of the Sphingidae, Xylophanes chiron; verbatimEventDate: 14-Oct-2002; individualID: 
DHJPAR0008072; individualCount: 1; sex: female; lifeStage: adult; preparations: pinned; catalogNumber: DHJPAR0008072; occurrenceDetails: http:// janzen.sas.upenn.edu; recordedBy: D.H. Janzen, W. Hallwachs \& Carolina Cano; otherCatalog Numbers: ASTAT844-06, 02-SRNP-19190,; identifiedBy: AJ Fleming; dateldentified: 2017; language: en; institutionCode: CNC; collectionCode: Insects; basisOfRecord: Pinned Specimen

t. scientificName: Hyphantrophaga eldaarayae; phylum: Arthropoda; class: Insecta; order: Diptera; family: Tachinidae; genus: Hyphantrophaga; specificEpithet: eldaarayae; scientificNameAuthorship: Fleming \& Wood, 2018; continent: Central America; country: Costa Rica; countryCode: CR; stateProvince: Guanacaste; county: Sector Santa Rosa; locality: Area de Conservacion Guanacaste; verbatimLocality: Sendero Inga Peluda; verbatimElevation: 280; verbatimLatitude: 10.8733; verbatimLongitude: -85.6067; verbatimCoordinateSystem: Decimal; decimalLatitude: 10.8733; decimalLongitude: -85.6067; samplingProtocol: Reared from the larva of the Sphingidae, Xylophanes chiron; verbatimEventDate: 10-Aug-2003; individualID: DHJPAR0008073; individualCount: 1; sex: female; lifeStage: adult; preparations: pinned; catalogNumber: DHJPAR0008073; occurrenceDetails: http:// janzen.sas.upenn.edu; recordedBy: D.H. Janzen, W. Hallwachs \& Guillermo Pereira; otherCatalogNumbers: ASTAT845-06, 03-SRNP-14322,; identifiedBy: AJ Fleming; dateldentified: 2017; language: en; institutionCode: CNC; collectionCode: Insects; basisOfRecord: Pinned Specimen

u. ScientificName: Hyphantrophaga eldaarayae; phylum: Arthropoda; class: Insecta; order: Diptera; family: Tachinidae; genus: Hyphantrophaga; specificEpithet: eldaarayae; scientificNameAuthorship: Fleming \& Wood, 2018; continent: Central America; country: Costa Rica; countryCode: CR; stateProvince: Guanacaste; county: Sector Santa Rosa; locality: Area de Conservacion Guanacaste; verbatimLocality: Sendero Inga Peluda; verbatimElevation: 280; verbatimLatitude: 10.8733; verbatimLongitude: -85.6067; verbatimCoordinateSystem: Decimal; decimalLatitude: 10.8733; decimalLongitude: -85.6067; samplingProtocol: Reared from the larva of the Sphingidae, Xylophanes chiron; verbatimEventDate: 10-Aug-2003; individualID: DHJPAR0008074; individualCount: 1; sex: female; lifeStage: adult; preparations: pinned; catalogNumber: DHJPAR0008074; occurrenceDetails: http:// janzen.sas.upenn.edu; recordedBy: D.H. Janzen, W. Hallwachs \& Mariano Pereira; otherCatalogNumbers: ASTAT846-06, 03-SRNP-14331,; identifiedBy: AJ Fleming; dateldentified: 2017; language: en; institutionCode: CNC; collectionCode: Insects; basisOfRecord: Pinned Specimen

v. scientificName: Hyphantrophaga eldaarayae; phylum: Arthropoda; class: Insecta; order: Diptera; family: Tachinidae; genus: Hyphantrophaga; specificEpithet: eldaarayae; scientificNameAuthorship: Fleming \& Wood, 2018; continent: Central America; country: Costa Rica; countryCode: CR; stateProvince: Guanacaste; county: Sector Santa Rosa; locality: Area de Conservacion Guanacaste; verbatimLocality: Sendero Inga Peluda; verbatimElevation: 280; verbatimLatitude: 10.8733; verbatimLongitude: -85.6067; verbatimCoordinateSystem: Decimal; decimalLatitude: 10.8733; decimalLongitude: -85.6067; samplingProtocol: Reared from the larva of the Sphingidae, Xylophanes chiron; verbatimEventDate: 06-Aug-2003; individualID: DHJPAR0008076; individualCount: 1; sex: female; lifeStage: adult; preparations: pinned; catalogNumber: DHJPAR0008076; occurrenceDetails: http:// janzen.sas.upenn.edu; recordedBy: D.H. Janzen, W. Hallwachs \& Guillermo Pereira; otherCatalogNumbers: ASTAT848-06, 03-SRNP-14312,; identifiedBy: AJ Fleming; 
dateldentified: 2017; language: en; institutionCode: CNC; collectionCode: Insects; basisOfRecord: Pinned Specimen

w. $\quad$ scientificName: Hyphantrophaga eldaarayae; phylum: Arthropoda; class: Insecta; order: Diptera; family: Tachinidae; genus: Hyphantrophaga; specificEpithet: eldaarayae; scientificNameAuthorship: Fleming \& Wood, 2018; continent: Central America; country: Costa Rica; countryCode: CR; stateProvince: Alajuela; county: Sector San Cristobal; locality: Area de Conservacion Guanacaste; verbatimLocality: Sendero Palo Alto; verbatimElevation: 570; verbatimLatitude: 10.8819; verbatimLongitude: -85.3822; verbatimCoordinateSystem: Decimal; decimalLatitude: 10.8819; decimalLongitude: -85.3822; samplingProtocol: Reared from the larva of the Sphingidae, Xylophanes chiron; verbatimEventDate: 11-Jul-2004; individualID: DHJPAR0008077; individualCount: 1; sex: female; lifeStage: adult; preparations: pinned; catalogNumber: DHJPAR0008077; occurrenceDetails: http:// janzen.sas.upenn.edu; recordedBy: D.H. Janzen, W. Hallwachs \& Elda Araya; otherCatalogNumbers: ASTAT849-06, 04-SRNP-2696, BOLD:AAB0592; identifiedBy: AJ Fleming; dateldentified: 2017; language: en; institutionCode: CNC; collectionCode: Insects; basisOfRecord: Pinned Specimen

\section{Other materials:}

a. $\quad$ scientificName: Hyphantrophaga eldaarayae; phylum: Arthropoda; class: Insecta; order: Diptera; family: Tachinidae; genus: Hyphantrophaga; specificEpithet: eldaarayae; scientificNameAuthorship: Fleming \& Wood, 2018; continent: Central America; country: Costa Rica; countryCode: CR; stateProvince: Guanacaste; county: Sector Pitilla; Iocality: Area de Conservacion Guanacaste; verbatimLocality: Estacion Pitilla; verbatimElevation: 675; verbatimLatitude: 10.9893; verbatimLongitude: -85.4258; verbatimCoordinateSystem: Decimal; decimalLatitude: 10.9893; decimalLongitude: -85.4258; samplingProtocol: Reared from the larva of the Sphingidae, Xylophanes chiron; verbatimEventDate: 15-Aug-2004; individualID: DHJPAR0008086; individualCount: 1; sex: male; lifeStage: adult; preparations: pinned; catalogNumber: DHJPAR0008086; occurrenceDetails: http://janzen.sas.upenn.edu; recordedBy: D.H. Janzen, W. Hallwachs \& Calixto Moraga; otherCatalog Numbers: ASTAT858-06, 04-SRNP-33860, BOLD:AAB0592; identifiedBy: AJ Fleming; dateldentified: 2017; language: en; institutionCode: CNC; collectionCode: Insects; basisOfRecord: Pinned Specimen

b. scientificName: Hyphantrophaga eldaarayae; phylum: Arthropoda; class: Insecta; order: Diptera; family: Tachinidae; genus: Hyphantrophaga; specificEpithet: eldaarayae; scientificNameAuthorship: Fleming \& Wood, 2018; continent: Central America; country: Costa Rica; countryCode: CR; stateProvince: Alajuela; county: Sector San Cristobal; locality: Area de Conservacion Guanacaste; verbatimLocality: Finca San Gabriel; verbatimElevation: 645; verbatimLatitude: 10.8777 ; verbatimLongitude: -85.3934; verbatimCoordinateSystem: Decimal; decimalLatitude: 10.8777; decimalLongitude: -85.3934; samplingProtocol: Reared from the larva of the Sphingidae, Xylophanes chiron; verbatimEventDate: 24-Sep-2004; individualID: DHJPAR0008087; individualCount: 1; sex: male; lifeStage: adult; preparations: pinned; catalogNumber: DHJPAR0008087; occurrenceDetails: http://janzen.sas.upenn.edu; recordedBy: D.H. Janzen, W. Hallwachs \& Elda Araya; otherCatalogNumbers: ASTAT859-06, 04-SRNP-4398,; identifiedBy: AJ Fleming; dateldentified: 2017; language: en; institutionCode: CNC; collectionCode: Insects; basisOfRecord: Pinned Specimen 
c. scientificName: Hyphantrophaga eldaarayae; phylum: Arthropoda; class: Insecta; order: Diptera; family: Tachinidae; genus: Hyphantrophaga; specificEpithet: eldaarayae; scientificNameAuthorship: Fleming \& Wood, 2018; continent: Central America; country: Costa Rica; countryCode: CR; stateProvince: Guanacaste; county: Sector El Hacha; locality: Area de Conservacion Guanacaste; verbatimLocality: Sendero Tigre; verbatimElevation: 280 ; verbatimLatitude: 11.0317; verbatimLongitude: -85.5262; verbatimCoordinateSystem: Decimal; decimalLatitude: 11.0317; decimalLongitude: -85.5262; samplingProtocol: Reared from the larva of the Sphingidae, Xylophanes tyndarus; verbatimEventDate: 09-Oct-2000; individualID: DHJPAR0008088; individualCount: 1; sex: male; lifeStage: adult; preparations: pinned; catalogNumber: DHJPAR0008088; occurrenceDetails: http://janzen.sas.upenn.edu; recordedBy: D.H. Janzen, W. Hallwachs \& Lucia Rios; otherCatalogNumbers: ASTAT860-06, 00-SRNP-2879.1,; identifiedBy: AJ Fleming; dateldentified: 2017; language: en; institutionCode: CNC; collectionCode: Insects; basisOfRecord: Pinned Specimen

d. ScientificName: Hyphantrophaga eldaarayae; phylum: Arthropoda; class: Insecta; order: Diptera; family: Tachinidae; genus: Hyphantrophaga; specificEpithet: eldaarayae; scientificNameAuthorship: Fleming \& Wood, 2018; continent: Central America; country: Costa Rica; countryCode: CR; stateProvince: Guanacaste; county: Sector Santa Rosa; locality: Area de Conservacion Guanacaste; verbatimLocality: Bosque Interseccion; verbatimElevation: 15; verbatimLatitude: 10.8005; verbatimLongitude: -85.6492; verbatimCoordinateSystem: Decimal; decimalLatitude: 10.8005; decimalLongitude: -85.6492; samplingProtocol: Reared from the larva of the Sphingidae, Xylophanes pluto; verbatimEventDate: 22-Nov-1998; individualID: DHJPAR0008091; individualCount: 1; sex: male; lifeStage: adult; preparations: pinned; catalog Number: DHJPAR0008091; occurrenceDetails: http://janzen.sas.upenn.edu; recordedBy: D.H. Janzen, W. Hallwachs \& gusaneros; otherCatalogNumbers: ASTAT863-06, 98-SRNP-12367, BOLD:AAB0592; identifiedBy: AJ Fleming; dateldentified: 2017; language: en; institutionCode: CNC; collectionCode: Insects; basisOfRecord: Pinned Specimen

e. $\quad$ scientificName: Hyphantrophaga eldaarayae; phylum: Arthropoda; class: Insecta; order: Diptera; family: Tachinidae; genus: Hyphantrophaga; specificEpithet: eldaarayae; scientificNameAuthorship: Fleming \& Wood, 2018; continent: Central America; country: Costa Rica; countryCode: CR; stateProvince: Guanacaste; county: Sector El Hacha; locality: Area de Conservacion Guanacaste; verbatimLocality: Sendero Tigre; verbatimElevation: 280 ; verbatimLatitude: 11.0317 ; verbatimLongitude: -85.5262; verbatimCoordinateSystem: Decimal; decimalLatitude: 11.0317; decimalLongitude: -85.5262; samplingProtocol: Reared from the larva of the Sphingidae, Xylophanes chiron; verbatimEventDate: 23-Jul-1997; individualID: DHJPAR0008094; individualCount: 1; sex: male; lifeStage: adult; preparations: pinned; catalogNumber: DHJPAR0008094; occurrenceDetails: http://janzen.sas.upenn.edu; recordedBy: D.H. Janzen, W. Hallwachs \& Mariano Pereira; otherCatalogNumbers: ASTAT866-06, 97-SRNP-4527, BOLD:AAB0592; identifiedBy: AJ Fleming; dateldentified: 2017; language: en; institutionCode: CNC; collectionCode: Insects; basisOfRecord: Pinned Specimen

f. ScientificName: Hyphantrophaga eldaarayae; phylum: Arthropoda; class: Insecta; order: Diptera; family: Tachinidae; genus: Hyphantrophaga; specificEpithet: eldaarayae; scientificNameAuthorship: Fleming \& Wood, 2018; continent: Central America; country: Costa Rica; countryCode: CR; stateProvince: Guanacaste; county: Sector Pitilla; locality: Area de Conservacion Guanacaste; verbatimLocality: 
Pasmompa; verbatimElevation: 440; verbatimLatitude: 11.0193; verbatimLongitude: -85.41; verbatimCoordinateSystem: Decimal; decimalLatitude: 11.0193; decimalLongitude: -85.41; samplingProtocol: Reared from the larva of the Sphingidae, Xylophanes maculator; verbatimEventDate: 29-Jan-2001; individualID:

DHJPAR0008096; individualCount: 1; sex: male; lifeStage: adult; preparations: pinned; catalogNumber: DHJPAR0008096; occurrenceDetails: http://janzen.sas.upenn.edu; recordedBy: D.H. Janzen, W. Hallwachs \& Elieth Cantillano; otherCatalog Numbers: ASTAT868-06, 00-SRNP-4448,; identifiedBy: AJ Fleming; dateldentified: 2017; language: en; institutionCode: CNC; collectionCode: Insects; basisOfRecord: Pinned Specimen

g. ScientificName: Hyphantrophaga eldaarayae; phylum: Arthropoda; class: Insecta; order: Diptera; family: Tachinidae; genus: Hyphantrophaga; specificEpithet: eldaarayae; scientificNameAuthorship: Fleming \& Wood, 2018; continent: Central America; country: Costa Rica; countryCode: CR; stateProvince: Guanacaste; county: Sector Santa Rosa; Iocality: Area de Conservacion Guanacaste; verbatimLocality: Bosque Interseccion; verbatimElevation: 15; verbatimLatitude: 10.8005; verbatimLongitude: -85.6492; verbatimCoordinateSystem: Decimal; decimalLatitude: 10.8005; decimalLongitude: -85.6492; samplingProtocol: Reared from the larva of the Sphingidae, Xylophanes pluto; verbatimEventDate: 16-Nov-1998; individualID: DHJPAR0008098; individualCount: 1; sex: male; lifeStage: adult; preparations: pinned; catalogNumber: DHJPAR0008098; occurrenceDetails: http://janzen.sas.upenn.edu; recordedBy: D.H. Janzen, W. Hallwachs \& gusaneros; otherCatalogNumbers: ASTAT870-06, 98-SRNP-12364,; identifiedBy: AJ Fleming; dateldentified: 2017; language: en; institutionCode: CNC; collectionCode: Insects; basisOfRecord: Pinned Specimen

h. ScientificName: Hyphantrophaga eldaarayae; phylum: Arthropoda; class: Insecta; order: Diptera; family: Tachinidae; genus: Hyphantrophaga; specificEpithet: eldaarayae; scientificNameAuthorship: Fleming \& Wood, 2018; continent: Central America; country: Costa Rica; countryCode: CR; stateProvince: Guanacaste; county: Sector El Hacha; locality: Area de Conservacion Guanacaste; verbatimLocality: Sendero Bejuquilla; verbatimElevation: 280; verbatimLatitude: 11.03; verbatimLongitude: -85.527; verbatimCoordinateSystem: Decimal; decimalLatitude 11.03; decimalLongitude: -85.527; samplingProtocol: Reared from the larva of the Sphingidae, Xylophanes chiron; verbatimEventDate: 13-Dec-1998; individualID: DHJPAR0008099; individualCount: 1; sex: male; lifeStage: adult; preparations: pinned; catalogNumber: DHJPAR0008099; occurrenceDetails: http://janzen.sas.upenn.edu; recordedBy: D.H. Janzen, W. Hallwachs \& Lucia Rios; otherCatalogNumbers: ASTAT871-06, 98-SRNP-14084, BOLD:AAB0592; identifiedBy: AJ Fleming; dateldentified: 2017; language: en; institutionCode: CNC; collectionCode: Insects; basisOfRecord: Pinned Specimen

i. ScientificName: Hyphantrophaga eldaarayae; phylum: Arthropoda; class: Insecta; order: Diptera; family: Tachinidae; genus: Hyphantrophaga; specificEpithet: eldaarayae; scientificNameAuthorship: Fleming \& Wood, 2018; continent: Central America; country: Costa Rica; countryCode: CR; stateProvince: Alajuela; county: Sector Rincon Rain Forest; locality: Area de Conservacion Guanacaste; verbatimLocality: San Lucas; verbatimElevation: 320; verbatimLatitude: 10.9185; verbatimLongitude: -85.3034; verbatimCoordinateSystem: Decimal; decimalLatitude: 10.9185; decimalLongitude: -85.3034; samplingProtocol: Reared from the larva of the Sphingidae, Xylophanes chiron; verbatimEventDate: 05-Nov-2003; individualID: DHJPAR0008108; individualCount: 1; sex: male; lifeStage: adult; preparations: pinned; 
catalogNumber: DHJPAR0008108; occurrenceDetails: http://janzen.sas.upenn.edu; recordedBy: D.H. Janzen, W. Hallwachs \& Carolina Cano; otherCatalogNumbers: ASTAT880-06, 03-SRNP-13019.1, BOLD:AAB0592; identifiedBy: AJ Fleming; dateldentified: 2017; language: en; institutionCode: CNC; collectionCode: Insects; basisOfRecord: Pinned Specimen

j. $\quad$ scientificName: Hyphantrophaga eldaarayae; phylum: Arthropoda; class: Insecta; order: Diptera; family: Tachinidae; genus: Hyphantrophaga; specificEpithet: eldaarayae; scientificNameAuthorship: Fleming \& Wood, 2018; continent: Central America; country: Costa Rica; countryCode: CR; stateProvince: Guanacaste; county: Sector Del Oro; locality: Area de Conservacion Guanacaste; verbatimLocality: Margarita; verbatimElevation: 380; verbatimLatitude: 11.0323; verbatimLongitude: -85.4395; verbatimCoordinateSystem: Decimal; decimalLatitude: 11.0323; decimalLongitude: -85.4395; samplingProtocol: Reared from the larva of the Sphingidae, Xylophanes chiron; verbatimEventDate: 09-Jul-2006; individualID: DHJPAR0015261; individualCount: 1; sex: male; lifeStage: adult; preparations: pinned; catalogNumber: DHJPAR0015261; occurrenceDetails: http://janzen.sas.upenn.edu; recordedBy: D.H. Janzen, W. Hallwachs \& Roster Moraga; otherCatalogNumbers: ASTAS016-06, 06-SRNP-21420, BOLD:AAB0592; identifiedBy: AJ Fleming; dateldentified: 2017; language: en; institutionCode: CNC; collectionCode: Insects; basisOfRecord: Pinned Specimen

k. scientificName: Hyphantrophaga eldaarayae; phylum: Arthropoda; class: Insecta; order: Diptera; family: Tachinidae; genus: Hyphantrophaga; specificEpithet: eldaarayae; scientificNameAuthorship: Fleming \& Wood, 2018; continent: Central America; country: Costa Rica; countryCode: CR; stateProvince: Guanacaste; county: Sector Del Oro; locality: Area de Conservacion Guanacaste; verbatimLocality: Puente Mena; verbatimElevation: 280; verbatimLatitude: 11.0456 ; verbatimLongitude: -85.4574; verbatimCoordinateSystem: Decimal; decimalLatitude: 11.0456; decimalLongitude: -85.4574; samplingProtocol: Reared from the larva of the Sphingidae, Xylophanes chiron; verbatimEventDate: 08-Jul-2006; individualID: DHJPAR0015262; individualCount: 1; sex: male; lifeStage: adult; preparations: pinned; catalogNumber: DHJPAR0015262; occurrenceDetails: http://janzen.sas.upenn.edu; recordedBy: D.H. Janzen, W. Hallwachs \& Roster Moraga; otherCatalogNumbers: ASTAS017-06, 06-SRNP-21347, BOLD:AAB0592; identifiedBy: AJ Fleming; dateldentified: 2017; language: en; institutionCode: CNC; collectionCode: Insects; basisOfRecord: Pinned Specimen

I. scientificName: Hyphantrophaga eldaarayae; phylum: Arthropoda; class: Insecta; order: Diptera; family: Tachinidae; genus: Hyphantrophaga; specificEpithet: eldaarayae; scientificNameAuthorship: Fleming \& Wood, 2018; continent: Central America; country: Costa Rica; countryCode: CR; stateProvince: Alajuela; county: Sector Rincon Rain Forest; locality: Area de Conservacion Guanacaste; verbatimLocality: Puente Rio Negro; verbatimElevation: 340; verbatimLatitude: 10.9038; verbatimLongitude: -85.3027; verbatimCoordinateSystem: Decimal; decimalLatitude: 10.9038; decimalLongitude: -85.3027; samplingProtocol: Reared from the larva of the Sphingidae, Xylophanes chiron; verbatimEventDate: 09-Jul-2006; individualID: DHJPAR0015268; individualCount: 1; sex: male; lifeStage: adult; preparations: pinned; catalogNumber: DHJPAR0015268; occurrenceDetails: http:// janzen.sas.upenn.edu; recordedBy: D.H. Janzen, W. Hallwachs \& Minor Carmona; otherCatalogNumbers: ASTAS023-06, 06-SRNP-42101,; identifiedBy: AJ Fleming; dateldentified: 2017; language: en; institutionCode: CNC; collectionCode: Insects; basisOfRecord: Pinned Specimen 
m. scientificName: Hyphantrophaga eldaarayae; phylum: Arthropoda; class: Insecta; order: Diptera; family: Tachinidae; genus: Hyphantrophaga; specificEpithet: eldaarayae; scientificNameAuthorship: Fleming \& Wood, 2018; continent: Central America; country: Costa Rica; countryCode: CR; stateProvince: Alajuela; county: Sector San Cristobal; locality: Area de Conservacion Guanacaste; verbatimLocality: Finca San Gabriel; verbatimElevation: 645; verbatimLatitude: 10.8777 ; verbatimLongitude: -85.3934; verbatimCoordinateSystem: Decimal; decimalLatitude: 10.8777; decimalLongitude: -85.3934; samplingProtocol: Reared from the larva of the Sphingidae, Xylophanes chiron; verbatimEventDate: 18-Oct-2006; individualID: DHJPAR0016114; individualCount: 1; sex: male; lifeStage: adult; preparations: pinned; catalogNumber: DHJPAR0016114; occurrenceDetails: http://janzen.sas.upenn.edu; recordedBy: D.H. Janzen, W. Hallwachs \& Anabelle Cordoba; otherCatalogNumbers: ASTAP143-06, 06-SRNP-7629, BOLD:AAB0592; identifiedBy: AJ Fleming; dateldentified: 2017; language: en; institutionCode: CNC; collectionCode: Insects; basisOfRecord: Pinned Specimen

n. $\quad$ scientificName: Hyphantrophaga eldaarayae; phylum: Arthropoda; class: Insecta; order: Diptera; family: Tachinidae; genus: Hyphantrophaga; specificEpithet: eldaarayae; scientificNameAuthorship: Fleming \& Wood, 2018; continent: Central America; country: Costa Rica; countryCode: CR; stateProvince: Alajuela; county: Brasilia; locality: Area de Conservacion Guanacaste; verbatimLocality: Piedrona; verbatimElevation: 340; verbatimLatitude: 11.0162; verbatimLongitude: -85.359; verbatimCoordinateSystem: Decimal; decimalLatitude: 11.0162; decimalLongitude: -85.359; samplingProtocol: Reared from the larva of the Sphingidae, Xylophanes chiron; verbatimEventDate: 12-Sep-2007; individualID: DHJPAR0022017; individualCount: 1; sex: male; lifeStage: adult; preparations: pinned; catalogNumber: DHJPAR0022017; occurrenceDetails: http://janzen.sas.upenn.edu; recordedBy: D.H. Janzen, W. Hallwachs \& Duvalier Briceno; otherCatalogNumbers: ASTAT1155-07, 07SRNP-65374, BOLD:AAB0592; identifiedBy: AJ Fleming; dateldentified: 2017; language: en; institutionCode: CNC; collectionCode: Insects; basisOfRecord: Pinned Specimen

o. $\quad$ scientificName: Hyphantrophaga eldaarayae; phylum: Arthropoda; class: Insecta; order: Diptera; family: Tachinidae; genus: Hyphantrophaga; specificEpithet: eldaarayae; scientificNameAuthorship: Fleming \& Wood, 2018; continent: Central America; country: Costa Rica; countryCode: CR; stateProvince: Guanacaste; county: Sector Santa Rosa; Iocality: Area de Conservacion Guanacaste; verbatimLocality: Bosque Humedo; verbatimElevation: 290; verbatimLatitude: 10.8514; verbatimLongitude: -85.608; verbatimCoordinateSystem: Decimal; decimalLatitude: 10.8514; decimalLongitude: -85.608; samplingProtocol: Reared from the larva of the Sphingidae, Xylophanes chiron; verbatimEventDate: 24-Jul-1986; individualID: DHJPAR0022961; individualCount: 1; sex: male; lifeStage: adult; preparations: pinned; catalogNumber: DHJPAR0022961; occurrenceDetails: http://janzen.sas.upenn.edu; recordedBy: D.H. Janzen, W. Hallwachs \& gusaneros; otherCatalogNumbers: ASTAW125-08, 86-SRNP-226, BOLD:AAB0592; identifiedBy: AJ Fleming; dateldentified: 2017; language: en; institutionCode: CNC; collectionCode: Insects; basisOfRecord: Pinned Specimen

p. $\quad$ scientificName: Hyphantrophaga eldaarayae; phylum: Arthropoda; class: Insecta; order: Diptera; family: Tachinidae; genus: Hyphantrophaga; specificEpithet: eldaarayae; scientificNameAuthorship: Fleming \& Wood, 2018; continent: Central America; country: Costa Rica; countryCode: CR; stateProvince: Alajuela; county: Sector San Cristobal; locality: Area de Conservacion Guanacaste; verbatimLocality: 
Rio Blanco Abajo; verbatimElevation: 500; verbatimLatitude: 10.9004; verbatimLongitude: -85.3725; verbatimCoordinateSystem: Decimal; decimalLatitude: 10.9004; decimalLongitude: -85.3725 ; samplingProtocol: Reared from the larva of the Sphingidae, Xylophanes chiron; verbatimEventDate: 28-Nov-2001; individuallD: DHJPAR0022962; individualCount: 1; sex: male; lifeStage: adult; preparations: pinned; catalogNumber: DHJPAR0022962; occurrenceDetails: http://janzen.sas.upenn.edu; recordedBy: D.H. Janzen, W. Hallwachs \& Gloria Sihezar; otherCatalogNumbers: ASTAW126-08, 01-SRNP-3991,; identifiedBy: AJ Fleming; dateldentified: 2017; language: en; institutionCode: CNC; collectionCode: Insects; basisOfRecord: Pinned Specimen

q. $\quad$ scientificName: Hyphantrophaga eldaarayae; phylum: Arthropoda; class: Insecta; order: Diptera; family: Tachinidae; genus: Hyphantrophaga; specificEpithet: eldaarayae; scientific NameAuthorship: Fleming \& Wood, 2018; continent: Central America; country: Costa Rica; countryCode: CR; stateProvince: Alajuela; county: Sector San Cristobal; locality: Area de Conservacion Guanacaste; verbatimLocality: Sendero Pinyal; verbatimElevation: 630; verbatimLatitude: 10.8716; verbatimLongitude: -85.3933; verbatimCoordinateSystem: Decimal; decimalLatitude: 10.8716; decimalLongitude: -85.3933; samplingProtocol: Reared from the larva of the Sphingidae, Xylophanes chiron; verbatimEventDate: 30-Jan-2006; individualID: DHJPAR0005524; individualCount: 1; sex: male; lifeStage: adult; preparations: pinned; catalogNumber: DHJPAR0005524; occurrenceDetails: http://janzen.sas.upenn.edu; recordedBy: D.H. Janzen, W. Hallwachs \& Carolina Cano; otherCatalogNumbers: ASTA643-06, 05-SRNP-7881,; identifiedBy: AJ Fleming; dateldentified: 2017; language: en; institutionCode: CNC; collectionCode: Insects; basisOfRecord: Pinned Specimen

r. $\quad$ scientificName: Hyphantrophaga eldaarayae; phylum: Arthropoda; class: Insecta; order: Diptera; family: Tachinidae; genus: Hyphantrophaga; specificEpithet: eldaarayae; scientificNameAuthorship: Fleming \& Wood, 2018; continent: Central America; country: Costa Rica; countryCode: CR; stateProvince: Alajuela; county: Sector San Cristobal; locality: Area de Conservacion Guanacaste; verbatimLocality: Rio Blanco Abajo; verbatimElevation: 500; verbatimLatitude: 10.9004; verbatimLongitude: -85.3725; verbatimCoordinateSystem: Decimal; decimalLatitude: 10.9004; decimalLongitude: -85.3725 ; samplingProtocol: Reared from the larva of the Sphingidae, Xylophanes chiron; verbatimEventDate: 28-Nov-2001; individualID: DHJPAR0022964; individualCount: 1; sex: male; lifeStage: adult; preparations: pinned; catalogNumber: DHJPAR0022964; occurrenceDetails: http://janzen.sas.upenn.edu; recordedBy: D.H. Janzen, W. Hallwachs \& Gloria Sihezar; otherCatalogNumbers: ASTAW128-08, 01-SRNP-3991, BOLD:AAB0592; identifiedBy: AJ Fleming; dateldentified: 2017; language: en; institutionCode: CNC; collectionCode: Insects; basisOfRecord: Pinned Specimen

s. ScientificName: Hyphantrophaga eldaarayae; phylum: Arthropoda; class: Insecta; order: Diptera; family: Tachinidae; genus: Hyphantrophaga; specificEpithet: eldaarayae; scientificNameAuthorship: Fleming \& Wood, 2018; continent: Central America; country: Costa Rica; countryCode: CR; stateProvince: Guanacaste; county: Sector Pitilla; locality: Area de Conservacion Guanacaste; verbatimLocality: Sendero Mismo; verbatimElevation: 680; verbatimLatitude: 10.9876; verbatimLongitude: -85.4197; verbatimCoordinateSystem: Decimal; decimalLatitude: 10.9876; decimalLongitude: -85.4197; samplingProtocol: Reared from the larva of the Sphingidae, Xylophanes chiron; verbatimEventDate: 18-Apr-2008; individualID: DHJPAR0024606; individualCount: 1; sex: male; lifeStage: adult; preparations: pinned; 
catalogNumber: DHJPAR0024606; occurrenceDetails: http://janzen.sas.upenn.edu; recordedBy: D.H. Janzen, W. Hallwachs \& Calixto Moraga; otherCatalog Numbers: ASTAW716-08, 08-SRNP-30750,; identifiedBy: AJ Fleming; dateldentified: 2017; language: en; institutionCode: CNC; collectionCode: Insects; basisOfRecord: Pinned Specimen

t. ScientificName: Hyphantrophaga eldaarayae; phylum: Arthropoda; class: Insecta; order: Diptera; family: Tachinidae; genus: Hyphantrophaga; specificEpithet: eldaarayae; scientificNameAuthorship: Fleming \& Wood, 2018; continent: Central America; country: Costa Rica; countryCode: CR; stateProvince: Guanacaste; county: Sector Pitilla; locality: Area de Conservacion Guanacaste; verbatimLocality: Quebradona; verbatimElevation: 475; verbatimLatitude: 10.991; verbatimLongitude: -85.3954; verbatimCoordinateSystem: Decimal; decimalLatitude: 10.991; decimalLongitude: -85.3954; samplingProtocol: Reared from the larva of the Sphingidae, Xylophanes chiron; verbatimEventDate: 08-Sep-2008; individualID: DHJPAR0029703; individualCount: 1; sex: male; lifeStage: adult; preparations: pinned; catalogNumber: DHJPAR0029703; occurrenceDetails: http://janzen.sas.upenn.edu; recordedBy: D.H. Janzen, W. Hallwachs \& Ronald Siezar; otherCatalogNumbers: ASHYM1124-09, 08-SRNP-71975, BOLD:AAB0592; identifiedBy: AJ Fleming; dateldentified: 2017; language: en; institutionCode: CNC; collectionCode: Insects; basisOfRecord: Pinned Specimen

u. ScientificName: Hyphantrophaga eldaarayae; phylum: Arthropoda; class: Insecta; order: Diptera; family: Tachinidae; genus: Hyphantrophaga; specificEpithet: eldaarayae; scientificNameAuthorship: Fleming \& Wood, 2018; continent: Central America; country: Costa Rica; countryCode: CR; stateProvince: Alajuela; county: Sector San Cristobal; locality: Area de Conservacion Guanacaste; verbatimLocality: Tajo Angeles; verbatimElevation: 540; verbatimLatitude: 10.8647; verbatimLongitude: -85.4153; verbatimCoordinateSystem: Decimal; decimalLatitude: 10.8647; decimalLongitude: -85.4153; samplingProtocol: Reared from the larva of the Sphingidae, Xylophanes chiron; verbatimEventDate: 03-Dec-2007; individualID: DHJPAR0030007; individualCount: 1; sex: male; lifeStage: adult; preparations: pinned; catalogNumber: DHJPAR0030007; occurrenceDetails: http://janzen.sas.upenn.edu; recordedBy: D.H. Janzen, W. Hallwachs \& Carolina Cano; otherCatalog Numbers: ASHYB751-09, 08-SRNP-6017, BOLD:AAB0592; identifiedBy: AJ Fleming; dateldentified: 2017; language: en; institutionCode: CNC; collectionCode: Insects; basisOfRecord: Pinned Specimen

v. ScientificName: Hyphantrophaga eldaarayae; phylum: Arthropoda; class: Insecta; order: Diptera; family: Tachinidae; genus: Hyphantrophaga; specificEpithet: eldaarayae; scientificNameAuthorship: Fleming \& Wood, 2018; continent: Central America; country: Costa Rica; countryCode: CR; stateProvince: Alajuela; county: Sector Rincon Rain Forest; locality: Area de Conservacion Guanacaste; verbatimLocality: San Lucas; verbatimElevation: 320; verbatimLatitude: 10.9185; verbatimLongitude: -85.3034; verbatimCoordinateSystem: Decimal; decimalLatitude: 10.9185; decimalLongitude: -85.3034; samplingProtocol: Reared from the larva of the Sphingidae, Xylophanes porcus; verbatimEventDate: 16-Feb-2009; individualID: DHJPAR0030035; individualCount: 1; sex: male; lifeStage: adult; preparations: pinned; catalogNumber: DHJPAR0030035; occurrenceDetails: http://janzen.sas.upenn.edu; recordedBy: D.H. Janzen, W. Hallwachs \& Anabelle Cordoba; otherCatalog Numbers: ASHYB779-09, 09-SRNP-40036, BOLD:AAB0592; identifiedBy: AJ Fleming; dateldentified: 2017; language: en; institutionCode: CNC; collectionCode: Insects; basisOfRecord: Pinned Specimen 
w. ScientificName: Hyphantrophaga eldaarayae; phylum: Arthropoda; class: Insecta; order: Diptera; family: Tachinidae; genus: Hyphantrophaga; specificEpithet: eldaarayae; scientificNameAuthorship: Fleming \& Wood, 2018; continent: Central America; country: Costa Rica; countryCode: CR; stateProvince: Guanacaste; county: Sector Del Oro; locality: Area de Conservacion Guanacaste; verbatimLocality: Rio Chon; verbatimElevation: 320; verbatimLatitude: 11.0412; verbatimLongitude: -85.4417; verbatimCoordinateSystem: Decimal; decimalLatitude: 11.0412; decimalLongitude: -85.4417; samplingProtocol: Reared from the larva of the Sphingidae, Xylophanes chiron; verbatimEventDate: 31-Jul-2009; individualID: DHJPAR0036488; individualCount: 1; sex: male; lifeStage: adult; preparations: pinned; catalogNumber: DHJPAR0036488; occurrenceDetails: http://janzen.sas.upenn.edu; recordedBy: D.H. Janzen, W. Hallwachs \& Roster Moraga; otherCatalogNumbers: ASHYE1399-09, 09-SRNP-21680, BOLD:AAB0592; identifiedBy: AJ Fleming; dateldentified: 2017; language: en; institutionCode: CNC; collectionCode: Insects; basisOfRecord: Pinned Specimen

x. $\quad$ scientificName: Hyphantrophaga eldaarayae; phylum: Arthropoda; class: Insecta; order: Diptera; family: Tachinidae; genus: Hyphantrophaga; specificEpithet: eldaarayae; scientificNameAuthorship: Fleming \& Wood, 2018; continent: Central America; country: Costa Rica; countryCode: CR; stateProvince: Alajuela; county: Sector Rincon Rain Forest; locality: Area de Conservacion Guanacaste; verbatimLocality: Estacion Llanura; verbatimElevation: 135; verbatimLatitude: 10.9333; verbatimLongitude: -85.2533; verbatimCoordinateSystem: Decimal; decimalLatitude: 10.9333; decimalLongitude: -85.2533; samplingProtocol: Reared from the larva of the Sphingidae, Xylophanes chiron; verbatimEventDate: 21-Sep-2009; individualID: DHJPAR0036559; individualCount: 1; sex: male; lifeStage: adult; preparations: pinned; catalogNumber: DHJPAR0036559; occurrenceDetails: http://janzen.sas.upenn.edu; recordedBy: D.H. Janzen, W. Hallwachs \& Cirilo Umaña; otherCatalogNumbers: ASHYE1470-09, 09-SRNP-75401, BOLD:AAB0592; identifiedBy: AJ Fleming; dateldentified: 2017; language: en; institutionCode: CNC; collectionCode: Insects; basisOfRecord: Pinned Specimen

y. $\quad$ scientificName: Hyphantrophaga eldaarayae; phylum: Arthropoda; class: Insecta; order: Diptera; family: Tachinidae; genus: Hyphantrophaga; specificEpithet: eldaarayae; scientificNameAuthorship: Fleming \& Wood, 2018; continent: Central America; country: Costa Rica; countryCode: CR; stateProvince: Alajuela; county: Sector Rincon Rain Forest; locality: Area de Conservacion Guanacaste; verbatimLocality: Estacion Llanura; verbatimElevation: 135; verbatimLatitude: 10.9333; verbatimLongitude: -85.2533; verbatimCoordinateSystem: Decimal; decimalLatitude: 10.9333; decimalLongitude: -85.2533; samplingProtocol: Reared from the larva of the Sphingidae, Xylophanes chiron; verbatimEventDate: 09-Feb-2010; individualID: DHJPAR0037479; individualCount: 1; sex: male; lifeStage: adult; preparations: pinned; catalogNumber: DHJPAR0037479; occurrenceDetails: http://janzen.sas.upenn.edu; recordedBy: D.H. Janzen, W. Hallwachs \& Cirilo Umaña; otherCatalogNumbers: ASHYC4224-10, 10-SRNP-75042, BOLD:AAB0592; identifiedBy: AJ Fleming; dateldentified: 2017; language: en; institutionCode: CNC; collectionCode: Insects; basisOfRecord: Pinned Specimen

z. $\quad$ scientificName: Hyphantrophaga eldaarayae; phylum: Arthropoda; class: Insecta; order: Diptera; family: Tachinidae; genus: Hyphantrophaga; specificEpithet: eldaarayae; scientificNameAuthorship: Fleming \& Wood, 2018; continent: Central America; country: Costa Rica; countryCode: CR; stateProvince: Alajuela; county: Sector San Cristobal; locality: Area de Conservacion Guanacaste; verbatimLocality: 
Vado Rio Cucaracho; verbatimElevation: 640; verbatimLatitude: 10.8702; verbatimLongitude: -85.3915; verbatimCoordinateSystem: Decimal; decimalLatitude: 10.8702; decimalLongitude: -85.3915 ; samplingProtocol: Reared from the larva of the Sphingidae, Xylophanes chiron; verbatimEventDate: 25-Jul-2007; individualID:

DHJPAR0037657; individualCount: 1; sex: male; lifeStage: adult; preparations: pinned; catalogNumber: DHJPAR0037657; occurrenceDetails: http://janzen.sas.upenn.edu; recordedBy: D.H. Janzen, W. Hallwachs \& Carolina Cano; otherCatalogNumbers: ASHYC4402-10, 07-SRNP-2058, BOLD:AAB0592; identifiedBy: AJ Fleming; dateldentified: 2017; language: en; institutionCode: CNC; collectionCode: Insects; basisOfRecord: Pinned Specimen

aa. $\quad$ scientificName: Hyphantrophaga eldaarayae; phylum: Arthropoda; class: Insecta; order: Diptera; family: Tachinidae; genus: Hyphantrophaga; specificEpithet: eldaarayae; scientific NameAuthorship: Fleming \& Wood, 2018; continent: Central America; country: Costa Rica; countryCode: CR; stateProvince: Guanacaste; county: Sector Pitilla; locality: Area de Conservacion Guanacaste; verbatimLocality: Medrano; verbatimElevation: 380; verbatimLatitude: 11.016; verbatimLongitude: -85.3805; verbatimCoordinateSystem: Decimal; decimalLatitude: 11.016; decimalLongitude: -85.3805; samplingProtocol: Reared from the larva of the Sphingidae, Xylophanes porcusDHJ04; verbatimEventDate: 16-Aug-2010; individualID: DHJPAR0040181; individualCount: 1; sex: male; lifeStage: adult; preparations: pinned; catalogNumber: DHJPAR0040181; occurrenceDetails: http://janzen.sas.upenn.edu; recordedBy: D.H. Janzen, W. Hallwachs \& Dinia Martinez; otherCatalogNumbers: ASHYE2348-11, 10SRNP-72048,; identifiedBy: AJ Fleming; dateldentified: 2017; language: en; institutionCode: CNC; collectionCode: Insects; basisOfRecord: Pinned Specimen ab. ScientificName: Hyphantrophaga eldaarayae; phylum: Arthropoda; class: Insecta; order: Diptera; family: Tachinidae; genus: Hyphantrophaga; specificEpithet: eldaarayae; scientificNameAuthorship: Fleming \& Wood, 2018; continent: Central America; country: Costa Rica; countryCode: CR; stateProvince: Guanacaste; county: Sector Pitilla; locality: Area de Conservacion Guanacaste; verbatimLocality: Medrano; verbatimElevation: 380; verbatimLatitude: 11.016; verbatimLongitude: -85.3805; verbatimCoordinateSystem: Decimal; decimalLatitude: 11.016; decimalLongitude: -85.3805; samplingProtocol: Reared from the larva of the Sphingidae, Xylophanes porcusDHJ04; verbatimEventDate: 13-Aug-2010; individualID: DHJPAR0040182; individualCount: 1; sex: male; lifeStage: adult; preparations: pinned; catalogNumber: DHJPAR0040182; occurrenceDetails: http://janzen.sas.upenn.edu; recordedBy: D.H. Janzen, W. Hallwachs \& Ricardo Calero; otherCatalogNumbers: ASHYE2349-11, 10SRNP-72047,; identifiedBy: AJ Fleming; dateldentified: 2017; language: en; institutionCode: CNC; collectionCode: Insects; basisOfRecord: Pinned Specimen ac. scientificName: Hyphantrophaga eldaarayae; phylum: Arthropoda; class: Insecta; order: Diptera; family: Tachinidae; genus: Hyphantrophaga; specificEpithet: eldaarayae; scientificNameAuthorship: Fleming \& Wood, 2018; continent: Central America; country: Costa Rica; countryCode: CR; stateProvince: Guanacaste; county: Sector Pitilla; locality: Area de Conservacion Guanacaste; verbatimLocality: Manguera; verbatimElevation: 470; verbatimLatitude: 10.9959; verbatimLongitude: -85.3984; verbatimCoordinateSystem: Decimal; decimalLatitude: 10.9959; decimalLongitude: -85.3984; samplingProtocol: Reared from the larva of the Sphingidae, Xylophanes chiron; verbatimEventDate: 21-Nov-2011; individualID: DHJPAR0046641; individualCount: 1; sex: male; lifeStage: adult; preparations: pinned; catalogNumber: DHJPAR0046641; occurrenceDetails: http://janzen.sas.upenn.edu; recordedBy: D.H. Janzen, W. Hallwachs \& Ricardo Calero; otherCatalog Numbers: ACGBA814-12, 11- 
SRNP-72185, BOLD:AAB0592; identifiedBy: AJ Fleming; dateldentified: 2017; language: en; institutionCode: CNC; collectionCode: Insects; basisOfRecord: Pinned Specimen

ad. $\quad$ scientificName: Hyphantrophaga eldaarayae; phylum: Arthropoda; class: Insecta; order: Diptera; family: Tachinidae; genus: Hyphantrophaga; specificEpithet: eldaarayae; scientificNameAuthorship: Fleming \& Wood, 2018; continent: Central America; country: Costa Rica; countryCode: CR; stateProvince: Alajuela; county: Sector Rincon Rain Forest; locality: Area de Conservacion Guanacaste; verbatimLocality: Quebrada Bambu; verbatimElevation: 109; verbatimLatitude: 10.9301; verbatimLongitude: -85.2521; verbatimCoordinateSystem: Decimal; decimalLatitude: 10.9301; decimalLongitude: -85.2521; samplingProtocol: Reared from the larva of the Sphingidae, Xylophanes chiron; verbatimEventDate: 16-Sep-2012; individualID: DHJPAR0050202; individualCount: 1; sex: male; lifeStage: adult; preparations: pinned; catalogNumber: DHJPAR0050202; occurrenceDetails: http:// janzen.sas.upenn.edu; recordedBy: D.H. Janzen, W. Hallwachs \& Cirilo Umaña; otherCatalogNumbers: ACGAZ1516-12, 12-SRNP-76615, BOLD:AAB0592; identifiedBy: AJ Fleming; dateldentified: 2017; language: en; institutionCode: CNC; collectionCode: Insects; basisOfRecord: Pinned Specimen

ae. $\quad$ scientificName: Hyphantrophaga eldaarayae; phylum: Arthropoda; class: Insecta; order: Diptera; family: Tachinidae; genus: Hyphantrophaga; specificEpithet: eldaarayae; scientificNameAuthorship: Fleming \& Wood, 2018; continent: Central America; country: Costa Rica; countryCode: CR; stateProvince: Alajuela; county: Sector Rincon Rain Forest; locality: Area de Conservacion Guanacaste; verbatimLocality: Selva; verbatimElevation: 410; verbatimLatitude: 10.9229; verbatimLongitude: -85.3188; verbatimCoordinateSystem: Decimal; decimalLatitude: 10.9229; decimalLongitude: -85.3188; samplingProtocol: Reared from the larva of the Sphingidae, Xylophanes hannemanni; verbatimEventDate: 04-Apr-2012; individualID: DHJPAR0050239; individualCount: 1; sex: male; lifeStage: adult; preparations: pinned; catalogNumber: DHJPAR0050239; occurrenceDetails: http://janzen.sas.upenn.edu; recordedBy: D.H. Janzen, W. Hallwachs \& Edwin Apu; otherCatalog Numbers: ACGAZ1553-12, 12-SRNP-69449, BOLD:AAB0592; identifiedBy: AJ Fleming; dateldentified: 2017; language: en; institutionCode: CNC; collectionCode: Insects; basisOfRecord: Pinned Specimen

af. ScientificName: Hyphantrophaga eldaarayae; phylum: Arthropoda; class: Insecta; order: Diptera; family: Tachinidae; genus: Hyphantrophaga; specificEpithet: eldaarayae; scientificNameAuthorship: Fleming \& Wood, 2018; continent: Central America; country: Costa Rica; countryCode: CR; stateProvince: Alajuela; county: Sector Rincon Rain Forest; locality: Area de Conservacion Guanacaste; verbatimLocality: Finca Esmeralda; verbatimElevation: 123; verbatimLatitude: 10.9355; verbatimLongitude: -85.2531; verbatimCoordinateSystem: Decimal; decimalLatitude: 10.9355; decimalLongitude: -85.2531; samplingProtocol: Reared from the larva of the Sphingidae, Xylophanes chiron; verbatimEventDate: 04-Jan-2013; individualID: DHJPAR0050493; individualCount: 1; sex: male; lifeStage: adult; preparations: pinned; catalogNumber: DHJPAR0050493; occurrenceDetails: http://janzen.sas.upenn.edu; recordedBy: D.H. Janzen, W. Hallwachs \& Cirilo Umaña; otherCatalogNumbers: ACGBA3085-13, 12-SRNP-77956, BOLD:AAB0592; identifiedBy: AJ Fleming; dateldentified: 2017; language: en; institutionCode: CNC; collectionCode: Insects; basisOfRecord: Pinned Specimen

ag. ScientificName: Hyphantrophaga eldaarayae; phylum: Arthropoda; class: Insecta; order: Diptera; family: Tachinidae; genus: Hyphantrophaga; specificEpithet: 
eldaarayae; scientificNameAuthorship: Fleming \& Wood, 2018; continent: Central America; country: Costa Rica; countryCode: CR; stateProvince: Guanacaste; county: Sector Cacao; locality: Area de Conservacion Guanacaste; verbatimLocality: Estacion Cacao; verbatimElevation: 1150; verbatimLatitude: 10.9269; verbatimLongitude: -85.4682; verbatimCoordinateSystem: Decimal; decimalLatitude: 10.9269; decimalLongitude: -85.4682; samplingProtocol: Reared from the larva of the Sphingidae, Xylophanes crotonis; verbatimEventDate: 19-Dec-2012; individualID: DHJPAR0050533; individualCount: 1; sex: male; lifeStage: adult; preparations: pinned; catalogNumber: DHJPAR0050533; occurrenceDetails: http://janzen.sas.upenn.edu; recordedBy: D.H. Janzen, W. Hallwachs \& Harry Ramirez; otherCatalog Numbers: ACGBA3125-13, 12-SRNP-35511, BOLD:AAB0592; identifiedBy: AJ Fleming; dateldentified: 2017; language: en; institutionCode: CNC; collectionCode: Insects; basisOfRecord: Pinned Specimen

ah. ScientificName: Hyphantrophaga eldaarayae; phylum: Arthropoda; class: Insecta; order: Diptera; family: Tachinidae; genus: Hyphantrophaga; specificEpithet: eldaarayae; scientificNameAuthorship: Fleming \& Wood, 2018; continent: Central America; country: Costa Rica; countryCode: CR; stateProvince: Alajuela; county: Sector Rincon Rain Forest; locality: Area de Conservacion Guanacaste; verbatimLocality: Pochote; verbatimElevation: 150; verbatimLatitude: 10.953; verbatimLongitude: -85.2698; verbatimCoordinateSystem: Decimal; decimalLatitude: 10.953; decimalLongitude: -85.2698; samplingProtocol: Reared from the larva of the Sphingidae, Xylophanes hannemanni; verbatimEventDate: 13-Dec-2012; individualID: DHJPAR0050644; individualCount: 1; sex: male; lifeStage: adult; preparations: pinned; catalogNumber: DHJPAR0050644; occurrenceDetails: http://janzen.sas.upenn.edu; recordedBy: D.H. Janzen, W. Hallwachs \& Keiner Aragon; otherCatalogNumbers: ACGBA3236-13, 12-SRNP-68836,; identifiedBy: AJ Fleming; dateldentified: 2017; language: en; institutionCode: CNC; collectionCode: Insects; basisOfRecord: Pinned Specimen

ai. $\quad$ scientificName: Hyphantrophaga eldaarayae; phylum: Arthropoda; class: Insecta; order: Diptera; family: Tachinidae; genus: Hyphantrophaga; specificEpithet: eldaarayae; scientificNameAuthorship: Fleming \& Wood, 2018; continent: Central America; country: Costa Rica; countryCode: CR; stateProvince: Alajuela; county: Sector Rincon Rain Forest; locality: Area de Conservacion Guanacaste; verbatimLocality: Palomo; verbatimElevation: 96; verbatimLatitude: 10.9619; verbatimLongitude: -85.2804 ; verbatimCoordinateSystem: Decimal; decimalLatitude: 10.9619; decimalLongitude: -85.2804; samplingProtocol: Reared from the larva of the Sphingidae, Xylophanes chiron; verbatimEventDate: 05-Sep-2013; individualID: DHJPAR0053425; individualCount: 1; sex: male; lifeStage: adult; preparations: pinned; catalog Number: DHJPAR0053425; occurrenceDetails: http://janzen.sas.upenn.edu; recordedBy: D.H. Janzen, W. Hallwachs \& Keiner Aragon; otherCatalogNumbers: ASHYM2779-13, 13-SRNP-79150, BOLD:AAB0592; identifiedBy: AJ Fleming; dateldentified: 2017; language: en; institutionCode: CNC; collectionCode: Insects; basisOfRecord: Pinned Specimen

aj. $\quad$ scientificName: Hyphantrophaga eldaarayae; phylum: Arthropoda; class: Insecta; order: Diptera; family: Tachinidae; genus: Hyphantrophaga; specificEpithet: eldaarayae; scientificNameAuthorship: Fleming \& Wood, 2018; continent: Central America; country: Costa Rica; countryCode: CR; stateProvince: Alajuela; county: Sector Rincon Rain Forest; locality: Area de Conservacion Guanacaste; verbatimLocality: Jacobo; verbatimElevation: 461; verbatimLatitude: 10.9408; verbatimLongitude: -85.3177; verbatimCoordinateSystem: Decimal; decimalLatitude: 
10.9408; decimalLongitude: -85.3177; samplingProtocol: Reared from the larva of the Sphingidae, Xylophanes chiron; verbatimEventDate: 21-Sep-2014; individualID: DHJPAR0056176; individualCount: 1; sex: male; lifeStage: adult; preparations: pinned; catalogNumber: DHJPAR0056176; occurrenceDetails: http://janzen.sas.upenn.edu; recordedBy: D.H. Janzen, W. Hallwachs \& Edwin Apu; otherCatalog Numbers: ASHYH2433-14, 14-SRNP-81055,; identifiedBy: AJ Fleming; dateldentified: 2017; language: en; institutionCode: CNC; collectionCode: Insects; basisOfRecord: Pinned Specimen

ak. $\quad$ scientificName: Hyphantrophaga eldaarayae; phylum: Arthropoda; class: Insecta; order: Diptera; family: Tachinidae; genus: Hyphantrophaga; specificEpithet: eldaarayae; scientificNameAuthorship: Fleming \& Wood, 2018; continent: Central America; country: Costa Rica; countryCode: CR; stateProvince: Alajuela; county: Sector Rincon Rain Forest; locality: Area de Conservacion Guanacaste; verbatimLocality: Palomo; verbatimElevation: 96; verbatimLatitude: 10.9619; verbatimLongitude: -85.2804; verbatimCoordinateSystem: Decimal; decimalLatitude: 10.9619; decimalLongitude: -85.2804; samplingProtocol: Reared from the larva of the Sphingidae, Xylophanes chiron; verbatimEventDate: 11-Sep-2014; individualID: DHJPAR0056177; individualCount: 1; sex: male; lifeStage: adult; preparations: pinned; catalogNumber: DHJPAR0056177; occurrenceDetails: http://janzen.sas.upenn.edu; recordedBy: D.H. Janzen, W. Hallwachs \& Keiner Aragon; otherCatalogNumbers: ASHYH2434-14, 14-SRNP-46592, BOLD:AAB0592; identifiedBy: AJ Fleming; dateldentified: 2017; language: en; institutionCode: CNC; collectionCode: Insects; basisOfRecord: Pinned Specimen

al. $\quad$ scientificName: Hyphantrophaga eldaarayae; phylum: Arthropoda; class: Insecta; order: Diptera; family: Tachinidae; genus: Hyphantrophaga; specificEpithet: eldaarayae; scientificNameAuthorship: Fleming \& Wood, 2018; continent: Central America; country: Costa Rica; countryCode: CR; stateProvince: Alajuela; county: Sector Rincon Rain Forest; locality: Area de Conservacion Guanacaste; verbatimLocality: Chayito; verbatimElevation: 470 ; verbatimLatitude: 10.9459; verbatimLongitude: -85.3204; verbatimCoordinateSystem: Decimal; decimalLatitude: 10.9459; decimalLongitude: -85.3204; samplingProtocol: Reared from the larva of the Sphingidae, Xylophanes chiron; verbatimEventDate: 03-Nov-2014; individualID: DHJPAR0057150; individualCount: 1; sex: male; lifeStage: adult; preparations: pinned; catalog Number: DHJPAR0057150; occurrenceDetails: http://janzen.sas.upenn.edu; recordedBy: D.H. Janzen, W. Hallwachs \& Minor Carmona; otherCatalogNumbers: ACGBA5060-15, 14-SRNP-81304, BOLD:AAB0592; identifiedBy: AJ Fleming; dateldentified: 2017; language: en; institutionCode: CNC; collectionCode: Insects; basisOfRecord: Pinned Specimen

am. ScientificName: Hyphantrophaga eldaarayae; phylum: Arthropoda; class: Insecta; order: Diptera; family: Tachinidae; genus: Hyphantrophaga; specificEpithet: eldaarayae; scientificNameAuthorship: Fleming \& Wood, 2018; continent: Central America; country: Costa Rica; countryCode: CR; stateProvince: Alajuela; county: Sector Rincon Rain Forest; locality: Area de Conservacion Guanacaste; verbatimLocality: Jacobo; verbatimElevation: 461; verbatimLatitude: 10.9408; verbatimLongitude: -85.3177; verbatimCoordinateSystem: Decimal; decimalLatitude: 10.9408; decimalLongitude: -85.3177; samplingProtocol: Reared from the larva of the Sphingidae, Xylophanes chiron; verbatimEventDate: 27-Dec-2014; individualID: DHJPAR0057226; individualCount: 1; sex: male; lifeStage: adult; preparations: pinned; catalog Number: DHJPAR0057226; occurrenceDetails: http://janzen.sas.upenn.edu; recordedBy: D.H. Janzen, W. Hallwachs \& Edwin Apu; otherCatalogNumbers: 
ACGBA5136-15, 14-SRNP-81650, BOLD:AAB0592; identifiedBy: AJ Fleming; dateldentified: 2017; language: en; institutionCode: CNC; collectionCode: Insects; basisOfRecord: Pinned Specimen

an. ScientificName: Hyphantrophaga eldaarayae; phylum: Arthropoda; class: Insecta; order: Diptera; family: Tachinidae; genus: Hyphantrophaga; specificEpithet: eldaarayae; scientificNameAuthorship: Fleming \& Wood, 2018; continent: Central America; country: Costa Rica; countryCode: CR; stateProvince: Alajuela; county: Sector Rincon Rain Forest; locality: Area de Conservacion Guanacaste; verbatimLocality: Finca Esmeralda; verbatimElevation: 123; verbatimLatitude: 10.9355; verbatimLongitude: -85.2531; verbatimCoordinateSystem: Decimal; decimalLatitude: 10.9355; decimalLongitude: -85.2531; samplingProtocol: Reared from the larva of the Sphingidae, Xylophanes cthulhu; verbatimEventDate: 06-Feb-2015; individualID: DHJPAR0057245; individualCount: 1; sex: male; lifeStage: adult; preparations: pinned; catalogNumber: DHJPAR0057245; occurrenceDetails: http://janzen.sas.upenn.edu; recordedBy: D.H. Janzen, W. Hallwachs \& Cirilo Umaña; otherCatalogNumbers: ACGBA5155-15, 15-SRNP-75028,; identifiedBy: AJ Fleming; dateldentified: 2017; language: en; institutionCode: CNC; collectionCode: Insects; basisOfRecord: Pinned Specimen

ao. $\quad$ scientificName: Hyphantrophaga eldaarayae; phylum: Arthropoda; class: Insecta; order: Diptera; family: Tachinidae; genus: Hyphantrophaga; specificEpithet: eldaarayae; scientificNameAuthorship: Fleming \& Wood, 2018; continent: Central America; country: Costa Rica; countryCode: CR; stateProvince: Alajuela; county: Sector Rincon Rain Forest; locality: Area de Conservacion Guanacaste; verbatimLocality: Palomo; verbatimElevation: 96; verbatimLatitude: 10.9619; verbatimLongitude: -85.2804; verbatimCoordinateSystem: Decimal; decimalLatitude: 10.9619; decimalLongitude: -85.2804; samplingProtocol: Reared from the larva of the Sphingidae, Xylophanes germen; verbatimEventDate: 28-Oct-2015; individualID: DHJPAR0058314; individualCount: 1; sex: male; lifeStage: adult; preparations: pinned; catalogNumber: DHJPAR0058314; occurrenceDetails: http://janzen.sas.upenn.edu; recordedBy: D.H. Janzen, W. Hallwachs \& Keiner Aragon; otherCatalogNumbers: MHMYN7914-16, 15-SRNP-46229, BOLD:AAB0592; identifiedBy: AJ Fleming; dateldentified: 2017; language: en; institutionCode: CNC; collectionCode: Insects; basisOfRecord: Pinned Specimen

ap. $\quad$ scientificName: Hyphantrophaga eldaarayae; phylum: Arthropoda; class: Insecta; order: Diptera; family: Tachinidae; genus: Hyphantrophaga; specificEpithet: eldaarayae; scientificNameAuthorship: Fleming \& Wood, 2018; continent: Central America; country: Costa Rica; countryCode: CR; stateProvince: Alajuela; county: Sector Rincon Rain Forest; locality: Area de Conservacion Guanacaste; verbatimLocality: Casa Keyner; verbatimElevation: 121; verbatimLatitude: 10.9564; verbatimLongitude: -85.2661; verbatimCoordinateSystem: Decimal; decimalLatitude: 10.9564; decimalLongitude: -85.2661; samplingProtocol: Reared from the larva of the Sphingidae, Xylophanes guianensis; verbatimEventDate: 05-Jan-2016; individualID: DHJPAR0058415; individualCount: 1; sex: male; lifeStage: adult; preparations: pinned; catalogNumber: DHJPAR0058415; occurrenceDetails: http://janzen.sas.upenn.edu; recordedBy: D.H. Janzen, W. Hallwachs \& Keiner Aragon; otherCatalogNumbers: MHMYN8015-16, 15-SRNP-46524, BOLD:AAB0592; identifiedBy: AJ Fleming; dateldentified: 2017; language: en; institutionCode: CNC; collectionCode: Insects; basisOfRecord: Pinned Specimen

aq. ScientificName: Hyphantrophaga eldaarayae; phylum: Arthropoda; class: Insecta; order: Diptera; family: Tachinidae; genus: Hyphantrophaga; specificEpithet: 
eldaarayae; scientificNameAuthorship: Fleming \& Wood, 2018; continent: Central America; country: Costa Rica; countryCode: CR; stateProvince: Guanacaste; county: Sector Del Oro; locality: Area de Conservacion Guanacaste; verbatimLocality: Sendero Puertas; verbatimElevation: 400; verbatimLatitude: 11.0109; verbatimLongitude: -85.4882; verbatimCoordinateSystem: Decimal; decimalLatitude: 11.0109; decimalLongitude: -85.4882; samplingProtocol: Reared from the larva of the Sphingidae, Xylophanes chiron; verbatimEventDate: 12-Aug-2003; individualID: DHJPAR0008079; individualCount: 1; sex: female; lifeStage: adult; preparations: pinned; catalogNumber: DHJPAR0008079; occurrenceDetails: http:// janzen.sas.upenn.edu; recordedBy: D.H. Janzen, W. Hallwachs \& Roster Moraga; otherCatalogNumbers: ASTAT851-06, 03-SRNP-17832,; identifiedBy: AJ Fleming; dateldentified: 2017; language: en; institutionCode: CNC; collectionCode: Insects; basisOfRecord: Pinned Specimen

ar. ScientificName: Hyphantrophaga eldaarayae; phylum: Arthropoda; class: Insecta; order: Diptera; family: Tachinidae; genus: Hyphantrophaga; specificEpithet: eldaarayae; scientificNameAuthorship: Fleming \& Wood, 2018; continent: Central America; country: Costa Rica; countryCode: CR; stateProvince: Guanacaste; county: Sector Santa Rosa; locality: Area de Conservacion Guanacaste; verbatimLocality: Quebrada Puercos; verbatimElevation: 155; verbatimLatitude: 10.8591; verbatimLongitude: -85.5709; verbatimCoordinateSystem: Decimal; decimalLatitude: 10.8591; decimalLongitude: -85.5709; samplingProtocol: Reared from the larva of the Sphingidae, Xylophanes pluto; verbatimEventDate: 19-Oct-2005; individualID: DHJPAR0008085; individualCount: 1; sex: female; lifeStage: adult; preparations: pinned; catalogNumber: DHJPAR0008085; occurrenceDetails: http:// janzen.sas.upenn.edu; recordedBy: D.H. Janzen, W. Hallwachs \& Guillermo Pereira; otherCatalogNumbers: ASTAT857-06, 05-SRNP-61306,; identifiedBy: AJ Fleming; dateldentified: 2017; language: en; institutionCode: CNC; collectionCode: Insects; basisOfRecord: Pinned Specimen

as. $\quad$ scientificName: Hyphantrophaga eldaarayae; phylum: Arthropoda; class: Insecta; order: Diptera; family: Tachinidae; genus: Hyphantrophaga; specificEpithet: eldaarayae; scientificNameAuthorship: Fleming \& Wood, 2018; continent: Central America; country: Costa Rica; countryCode: CR; stateProvince: Guanacaste; county: Sector El Hacha; locality: Area de Conservacion Guanacaste; verbatimLocality: Sendero Tigre; verbatimElevation: 280 ; verbatimLatitude: 11.0317; verbatimLongitude: -85.5262; verbatimCoordinateSystem: Decimal; decimalLatitude: 11.0317; decimalLongitude: -85.5262; samplingProtocol: Reared from the larva of the Sphingidae, Xylophanes tyndarus; verbatimEventDate: 17-Jul-2000; individualID: DHJPAR0008089; individualCount: 1; sex: female; lifeStage: adult; preparations: pinned; catalogNumber: DHJPAR0008089; occurrenceDetails: http:// janzen.sas.upenn.edu; recordedBy: D.H. Janzen, W. Hallwachs \& Lucia Rios; otherCatalogNumbers: ASTAT861-06, 00-SRNP-2778, BOLD:AAB0592; identifiedBy: AJ Fleming; dateldentified: 2017; language: en; institutionCode: CNC; collectionCode: Insects; basisOfRecord: Pinned Specimen

at. ScientificName: Hyphantrophaga eldaarayae; phylum: Arthropoda; class: Insecta; order: Diptera; family: Tachinidae; genus: Hyphantrophaga; specificEpithet: eldaarayae; scientificNameAuthorship: Fleming \& Wood, 2018; continent: Central America; country: Costa Rica; countryCode: CR; stateProvince: Guanacaste; county: Sector El Hacha; locality: Area de Conservacion Guanacaste; verbatimLocality: Sendero Tigre; verbatimElevation: 280; verbatimLatitude: 11.0317; verbatimLongitude: -85.5262; verbatimCoordinateSystem: Decimal; decimalLatitude: 11.0317; 
decimalLongitude: -85.5262; samplingProtocol: Reared from the larva of the Sphingidae, Xylophanes chiron; verbatimEventDate: 23-Jul-1997; individualID: DHJPAR0008090; individualCount: 1; sex: female; lifeStage: adult; preparations: pinned; catalogNumber: DHJPAR0008090; occurrenceDetails: http:// janzen.sas.upenn.edu; recordedBy: D.H. Janzen, W. Hallwachs \& Mariano Pereira; otherCatalogNumbers: ASTAT862-06, 97-SRNP-4524,; identifiedBy: AJ Fleming; dateldentified: 2017; language: en; institutionCode: CNC; collectionCode: Insects; basisOfRecord: Pinned Specimen

au. $\quad$ scientificName: Hyphantrophaga eldaarayae; phylum: Arthropoda; class: Insecta; order: Diptera; family: Tachinidae; genus: Hyphantrophaga; specificEpithet: eldaarayae; scientificNameAuthorship: Fleming \& Wood, 2018; continent: Central America; country: Costa Rica; countryCode: CR; stateProvince: Alajuela; county: Sector Rincon Rain Forest; locality: Area de Conservacion Guanacaste; verbatimLocality: Camino Porvenir; verbatimElevation: 383; verbatimLatitude: 10.9038; verbatimLongitude: -85.2596; verbatimCoordinateSystem: Decimal; decimalLatitude: 10.9038; decimalLongitude: -85.2596; samplingProtocol: Reared from the larva of the Sphingidae, Xylophanes chiron; verbatimEventDate: 13-Feb-2004; individualID: DHJPAR0008092; individualCount: 1; sex: female; lifeStage: adult; preparations: pinned; catalogNumber: DHJPAR0008092; occurrenceDetails: http:// janzen.sas.upenn.edu; recordedBy: D.H. Janzen, W. Hallwachs \& Jose Perez; otherCatalogNumbers: ASTAT864-06, 04-SRNP-40099, BOLD:AAB0592; identifiedBy: AJ Fleming; dateldentified: 2017; language: en; institutionCode: CNC; collectionCode: Insects; basisOfRecord: Pinned Specimen

av. ScientificName: Hyphantrophaga eldaarayae; phylum: Arthropoda; class: Insecta; order: Diptera; family: Tachinidae; genus: Hyphantrophaga; specificEpithet: eldaarayae; scientificNameAuthorship: Fleming \& Wood, 2018; continent: Central America; country: Costa Rica; countryCode: CR; stateProvince: Guanacaste; county: Sector El Hacha; locality: Area de Conservacion Guanacaste; verbatimLocality: Sendero Tigre; verbatimElevation: 280 ; verbatimLatitude: 11.0317; verbatimLongitude: -85.5262; verbatimCoordinateSystem: Decimal; decimalLatitude: 11.0317; decimalLongitude: -85.5262; samplingProtocol: Reared from the larva of the Sphingidae, Xylophanes chiron; verbatimEventDate: 02-Sep-1999; individualID: DHJPAR0008101; individualCount: 1; sex: female; lifeStage: adult; preparations: pinned; catalogNumber: DHJPAR0008101; occurrenceDetails: http:// janzen.sas.upenn.edu; recordedBy: D.H. Janzen, W. Hallwachs \& Lucia Rios; otherCatalogNumbers: ASTAT873-06, 99-SRNP-2043, BOLD:AAB0592; identifiedBy: AJ Fleming; dateldentified: 2017; language: en; institutionCode: CNC; collectionCode: Insects; basisOfRecord: Pinned Specimen

aw. scientificName: Hyphantrophaga eldaarayae; phylum: Arthropoda; class: Insecta; order: Diptera; family: Tachinidae; genus: Hyphantrophaga; specificEpithet: eldaarayae; scientificNameAuthorship: Fleming \& Wood, 2018; continent: Central America; country: Costa Rica; countryCode: CR; stateProvince: Guanacaste; county: Sector El Hacha; locality: Area de Conservacion Guanacaste; verbatimLocality: Sendero Bejuquilla; verbatimElevation: 280; verbatimLatitude: 11.03; verbatimLongitude: -85.527 ; verbatimCoordinateSystem: Decimal; decimalLatitude: 11.03; decimalLongitude: -85.527; samplingProtocol: Reared from the larva of the Sphingidae, Xylophanes chiron; verbatimEventDate: 08-May-1997; individualID: DHJPAR0008102; individualCount: 1; sex: female; lifeStage: adult; preparations: pinned; catalogNumber: DHJPAR0008102; occurrenceDetails: http:// janzen.sas.upenn.edu; recordedBy: D.H. Janzen, W. Hallwachs \& Lucia Rios; 
otherCatalogNumbers: ASTAT874-06, 97-SRNP-4646, BOLD:AAB0592; identifiedBy: AJ Fleming; dateldentified: 2017; language: en; institutionCode: CNC; collectionCode: Insects; basisOfRecord: Pinned Specimen

ax. $\quad$ scientificName: Hyphantrophaga eldaarayae; phylum: Arthropoda; class: Insecta; order: Diptera; family: Tachinidae; genus: Hyphantrophaga; specificEpithet: eldaarayae; scientificNameAuthorship: Fleming \& Wood, 2018; continent: Central America; country: Costa Rica; countryCode: CR; stateProvince: Guanacaste; county: Sector Santa Rosa; locality: Area de Conservacion Guanacaste; verbatimLocality: Bosque Humedo; verbatimElevation: 290; verbatimLatitude: 10.8514; verbatimLongitude: -85.608; verbatimCoordinateSystem: Decimal; decimalLatitude: 10.8514; decimalLongitude: -85.608; samplingProtocol: Reared from the larva of the Sphingidae, Xylophanes anubus; verbatimEventDate: 18-Sep-1991; individualID: DHJPAR0008103; individualCount: 1; sex: female; lifeStage: adult; preparations: pinned; catalogNumber: DHJPAR0008103; occurrenceDetails: http:// janzen.sas.upenn.edu; recordedBy: D.H. Janzen, W. Hallwachs \& gusaneros; otherCatalogNumbers: ASTAT875-06, 91-SRNP-3000, BOLD:AAB0592; identifiedBy: AJ Fleming; dateldentified: 2017; language: en; institutionCode: CNC; collectionCode: Insects; basisOfRecord: Pinned Specimen

ay. $\quad$ scientificName: Hyphantrophaga eldaarayae; phylum: Arthropoda; class: Insecta; order: Diptera; family: Tachinidae; genus: Hyphantrophaga; specificEpithet: eldaarayae; scientificNameAuthorship: Fleming \& Wood, 2018; continent: Central America; country: Costa Rica; countryCode: CR; stateProvince: Guanacaste; county: Sector Cacao; locality: Area de Conservacion Guanacaste; verbatimLocality: Sendero Nayo; verbatimElevation: 1090; verbatimLatitude: 10.9245; verbatimLongitude: -85.4695; verbatimCoordinateSystem: Decimal; decimalLatitude: 10.9245; decimalLongitude: -85.4695; samplingProtocol: Reared from the larva of the Sphingidae, Xylophanes crotonis; verbatimEventDate: 09-Jul-1999; individualID: DHJPAR0008104; individualCount: 1; sex: female; lifeStage: adult; preparations: pinned; catalogNumber: DHJPAR0008104; occurrenceDetails: http:// janzen.sas.upenn.edu; recordedBy: D.H. Janzen, W. Hallwachs \& Mariano Pereira; otherCatalog Numbers: ASTAT876-06, 99-SRNP-1301, BOLD:AAB0592; identifiedBy: AJ Fleming; dateldentified: 2017; language: en; institutionCode: CNC; collectionCode: Insects; basisOfRecord: Pinned Specimen

az. $\quad$ scientificName: Hyphantrophaga eldaarayae; phylum: Arthropoda; class: Insecta; order: Diptera; family: Tachinidae; genus: Hyphantrophaga; specificEpithet: eldaarayae; scientificNameAuthorship: Fleming \& Wood, 2018; continent: Central America; country: Costa Rica; countryCode: CR; stateProvince: Guanacaste; county: Sector El Hacha; locality: Area de Conservacion Guanacaste; verbatimLocality: Sendero Bejuquilla; verbatimElevation: 280; verbatimLatitude: 11.03; verbatimLongitude: -85.527 ; verbatimCoordinateSystem: Decimal; decimalLatitude: 11.03; decimalLongitude: -85.527; samplingProtocol: Reared from the larva of the Sphingidae, Xylophanes chiron; verbatimEventDate: 27-Oct-1998; individualID: DHJPAR0008105; individualCount: 1; sex: female; lifeStage: adult; preparations: pinned; catalogNumber: DHJPAR0008105; occurrenceDetails: http:// janzen.sas.upenn.edu; recordedBy: D.H. Janzen, W. Hallwachs \& Lucia Rios; otherCatalog Numbers: ASTAT877-06, 98-SRNP-13639, BOLD:AAB0592; identifiedBy: AJ Fleming; dateldentified: 2017; language: en; institutionCode: CNC; collectionCode: Insects; basisOfRecord: Pinned Specimen

ba. $\quad$ scientificName: Hyphantrophaga eldaarayae; phylum: Arthropoda; class: Insecta; order: Diptera; family: Tachinidae; genus: Hyphantrophaga; specificEpithet: 
eldaarayae; scientificNameAuthorship: Fleming \& Wood, 2018; continent: Central America; country: Costa Rica; countryCode: CR; stateProvince: Alajuela; county: Sector Rincon Rain Forest; locality: Area de Conservacion Guanacaste; verbatimLocality: San Lucas; verbatimElevation: 320; verbatimLatitude: 10.9185; verbatimLongitude: -85.3034; verbatimCoordinateSystem: Decimal; decimalLatitude: 10.9185; decimalLongitude: -85.3034; samplingProtocol: Reared from the larva of the Sphingidae, Xylophanes chiron; verbatimEventDate: 02-Sep-2003; individualID: DHJPAR0008107; individualCount: 1; sex: female; lifeStage: adult; preparations: pinned; catalogNumber: DHJPAR0008107; occurrenceDetails: http:// janzen.sas.upenn.edu; recordedBy: D.H. Janzen, W. Hallwachs \& Jose Perez; otherCatalog Numbers: ASTAT879-06, 03-SRNP-11998,; identifiedBy: AJ Fleming; dateldentified: 2017; language: en; institutionCode: CNC; collectionCode: Insects; basisOfRecord: Pinned Specimen

bb. $\quad$ scientificName: Hyphantrophaga eldaarayae; phylum: Arthropoda; class: Insecta; order: Diptera; family: Tachinidae; genus: Hyphantrophaga; specificEpithet: eldaarayae; scientificNameAuthorship: Fleming \& Wood, 2018; continent: Central America; country: Costa Rica; countryCode: CR; stateProvince: Guanacaste; county: Sector Santa Rosa; locality: Area de Conservacion Guanacaste; verbatimLocality: Tanquetas; verbatimElevation: 295; verbatimLatitude: 10.8708; verbatimLongitude: -85.6053; verbatimCoordinateSystem: Decimal; decimalLatitude: 10.8708; decimalLongitude: -85.6053; samplingProtocol: Reared from the larva of the Sphingidae, Xylophanes libyaDHJ02; verbatimEventDate: 27-Nov-1998; individualID: DHJPAR0008109; individualCount: 1; sex: female; lifeStage: adult; preparations: pinned; catalogNumber: DHJPAR0008109; occurrenceDetails: http:// janzen.sas.upenn.edu; recordedBy: D.H. Janzen, W. Hallwachs \& gusaneros; otherCatalog Numbers: ASTAT881-06, 98-SRNP-12603, BOLD:AAB0592; identifiedBy: AJ Fleming; dateldentified: 2017; language: en; institutionCode: CNC; collectionCode: Insects; basisOfRecord: Pinned Specimen

bc. $\quad$ scientificName: Hyphantrophaga eldaarayae; phylum: Arthropoda; class: Insecta; order: Diptera; family: Tachinidae; genus: Hyphantrophaga; specificEpithet: eldaarayae; scientificNameAuthorship: Fleming \& Wood, 2018; continent: Central America; country: Costa Rica; countryCode: CR; stateProvince: Guanacaste; county: Sector El Hacha; locality: Area de Conservacion Guanacaste; verbatimLocality: Sendero Bejuquilla; verbatimElevation: 280; verbatimLatitude: 11.03; verbatimLongitude: -85.527 ; verbatimCoordinateSystem: Decimal; decimalLatitude: 11.03; decimalLongitude: -85.527; samplingProtocol: Reared from the larva of the Sphingidae, Xylophanes chiron; verbatimEventDate: 13-Dec-1998; individualID: DHJPAR0008110; individualCount: 1; sex: female; lifeStage: adult; preparations: pinned; catalogNumber: DHJPAR0008110; occurrenceDetails: http:// janzen.sas.upenn.edu; recordedBy: D.H. Janzen, W. Hallwachs \& Lucia Rios; otherCatalog Numbers: ASTAT882-06, 98-SRNP-14083,; identifiedBy: AJ Fleming; dateldentified: 2017; language: en; institutionCode: CNC; collectionCode: Insects; basisOfRecord: Pinned Specimen

bd. $\quad$ scientificName: Hyphantrophaga eldaarayae; phylum: Arthropoda; class: Insecta; order: Diptera; family: Tachinidae; genus: Hyphantrophaga; specificEpithet: eldaarayae; scientificNameAuthorship: Fleming \& Wood, 2018; continent: Central America; country: Costa Rica; countryCode: CR; stateProvince: Guanacaste; county: Sector Del Oro; locality: Area de Conservacion Guanacaste; verbatimLocality: San Antonio; verbatimElevation: 335; verbatimLatitude: 11.0353; verbatimLongitude: -85.4453; verbatimCoordinateSystem: Decimal; decimalLatitude: 11.0353; 
decimalLongitude: -85.4453; samplingProtocol: Reared from the larva of the Sphingidae, Xylophanes chiron; verbatimEventDate: 05-Jul-2006; individuallD: DHJPAR0015010; individualCount: 1; sex: female; lifeStage: adult; preparations: pinned; catalogNumber: DHJPAR0015010; occurrenceDetails: http:// janzen.sas.upenn.edu; recordedBy: D.H. Janzen, W. Hallwachs \& Roster Moraga; otherCatalogNumbers: ASTAV701-06, 06-SRNP-21373,; identifiedBy: AJ Fleming; dateldentified: 2017; language: en; institutionCode: CNC; collectionCode: Insects; basisOfRecord: Pinned Specimen

be. $\quad$ scientificName: Hyphantrophaga eldaarayae; phylum: Arthropoda; class: Insecta; order: Diptera; family: Tachinidae; genus: Hyphantrophaga; specificEpithet: eldaarayae; scientificNameAuthorship: Fleming \& Wood, 2018; continent: Central America; country: Costa Rica; countryCode: CR; stateProvince: Guanacaste; county: Sector Del Oro; locality: Area de Conservacion Guanacaste; verbatimLocality: Margarita; verbatimElevation: 380 ; verbatimLatitude: 11.0323 ; verbatimLongitude: -85.4395; verbatimCoordinateSystem: Decimal; decimalLatitude: 11.0323; decimalLongitude: -85.4395; samplingProtocol: Reared from the larva of the Sphingidae, Xylophanes chiron; verbatimEventDate: 10-Jul-2006; individualID: DHJPAR0015260; individualCount: 1; sex: female; lifeStage: adult; preparations: pinned; catalogNumber: DHJPAR0015260; occurrenceDetails: http:// janzen.sas.upenn.edu; recordedBy: D.H. Janzen, W. Hallwachs \& Roster Moraga; otherCatalogNumbers: ASTAS015-06, 06-SRNP-21415, BOLD:AAB0592; identifiedBy: AJ Fleming; dateldentified: 2017; language: en; institutionCode: CNC; collectionCode: Insects; basisOfRecord: Pinned Specimen

bf. $\quad$ scientificName: Hyphantrophaga eldaarayae; phylum: Arthropoda; class: Insecta; order: Diptera; family: Tachinidae; genus: Hyphantrophaga; specificEpithet: eldaarayae; scientificNameAuthorship: Fleming \& Wood, 2018; continent: Central America; country: Costa Rica; countryCode: CR; stateProvince: Alajuela; county: Sector San Cristobal; locality: Area de Conservacion Guanacaste; verbatimLocality: Vado Rio Cucaracho; verbatimElevation: 640; verbatimLatitude: 10.8702; verbatimLongitude: -85.3915; verbatimCoordinateSystem: Decimal; decimalLatitude: 10.8702; decimalLongitude: -85.3915 ; samplingProtocol: Reared from the larva of the Sphingidae, Xylophanes jocasta; verbatimEventDate: 16-Aug-2006; individualID: DHJPAR0010343; individualCount: 1; sex: female; lifeStage: adult; preparations: pinned; catalogNumber: DHJPAR0010343; occurrenceDetails: http:// janzen.sas.upenn.edu; recordedBy: D.H. Janzen, W. Hallwachs \& Carolina Cano; otherCatalogNumbers: ASTAS174-06, 06-SRNP-5715, BOLD:AAB0592; identifiedBy: AJ Fleming; dateldentified: 2017; language: en; institutionCode: CNC; collectionCode: Insects; basisOfRecord: Pinned Specimen

bg. ScientificName: Hyphantrophaga eldaarayae; phylum: Arthropoda; class: Insecta; order: Diptera; family: Tachinidae; genus: Hyphantrophaga; specificEpithet: eldaarayae; scientificNameAuthorship: Fleming \& Wood, 2018; continent: Central America; country: Costa Rica; countryCode: CR; stateProvince: Alajuela; county: Sector Rincon Rain Forest; locality: Area de Conservacion Guanacaste; verbatimLocality: Puente Rio Negro; verbatimElevation: 340 ; verbatimLatitude: 10.9038; verbatimLongitude: -85.3027; verbatimCoordinateSystem: Decimal; decimalLatitude: 10.9038; decimalLongitude: -85.3027; samplingProtocol: Reared from the larva of the Sphingidae, Xylophanes chiron; verbatimEventDate: 22-Oct-2006; individualID: DHJPAR0016596; individualCount: 1; sex: female; lifeStage: adult; preparations: pinned; catalogNumber: DHJPAR0016596; occurrenceDetails: http:// janzen.sas.upenn.edu; recordedBy: D.H. Janzen, W. Hallwachs \& Jose Perez; 
otherCatalog Numbers: ASTAP800-07, 06-SRNP-43632, BOLD:AAB0592; identifiedBy: AJ Fleming; dateldentified: 2017; language: en; institutionCode: CNC; collectionCode: Insects; basisOfRecord: Pinned Specimen

bh. $\quad$ scientificName: Hyphantrophaga eldaarayae; phylum: Arthropoda; class: Insecta; order: Diptera; family: Tachinidae; genus: Hyphantrophaga; specificEpithet: eldaarayae; scientificNameAuthorship: Fleming \& Wood, 2018; continent: Central America; country: Costa Rica; countryCode: CR; stateProvince: Alajuela; county: Brasilia; locality: Area de Conservacion Guanacaste; verbatimLocality: Piedrona; verbatimElevation: 340; verbatimLatitude: 11.0162; verbatimLongitude: -85.359; verbatimCoordinateSystem: Decimal; decimalLatitude: 11.0162; decimalLongitude: -85.359; sampling Protocol: Reared from the larva of the Sphingidae, Xylophanes chiron; verbatimEventDate: 05-Sep-2007; individualID: DHJPAR0022019; individualCount: 1; sex: female; lifeStage: adult; preparations: pinned; catalogNumber: DHJPAR0022019; occurrenceDetails: http://janzen.sas.upenn.edu; recordedBy: D.H. Janzen, W. Hallwachs \& Duvalier Briceno; otherCatalog Numbers: ASTAT1157-07, 07SRNP-65316, BOLD:AAB0592; identifiedBy: AJ Fleming; dateldentified: 2017; language: en; institutionCode: CNC; collectionCode: Insects; basisOfRecord: Pinned Specimen

bi. $\quad$ scientificName: Hyphantrophaga eldaarayae; phylum: Arthropoda; class: Insecta; order: Diptera; family: Tachinidae; genus: Hyphantrophaga; specificEpithet: eldaarayae; scientificNameAuthorship: Fleming \& Wood, 2018; continent: Central America; country: Costa Rica; countryCode: CR; stateProvince: Guanacaste; county: Sector Santa Rosa; locality: Area de Conservacion Guanacaste; verbatimLocality: Bosque Humedo; verbatimElevation: 290; verbatimLatitude: 10.8514; verbatimLongitude: -85.608; verbatimCoordinateSystem: Decimal; decimalLatitude: 10.8514; decimalLongitude: -85.608; samplingProtocol: Reared from the larva of the Sphingidae, Xylophanes chiron; verbatimEventDate: 24-Jul-1986; individualID: DHJPAR0022960; individualCount: 1; sex: female; lifeStage: adult; preparations: pinned; catalogNumber: DHJPAR0022960; occurrenceDetails: http:// janzen.sas.upenn.edu; recordedBy: D.H. Janzen, W. Hallwachs \& gusaneros; otherCatalogNumbers: ASTAW124-08, 86-SRNP-226,; identifiedBy: AJ Fleming; dateldentified: 2017; language: en; institutionCode: CNC; collectionCode: Insects; basisOfRecord: Pinned Specimen

bj. $\quad$ scientificName: Hyphantrophaga eldaarayae; phylum: Arthropoda; class: Insecta; order: Diptera; family: Tachinidae; genus: Hyphantrophaga; specificEpithet: eldaarayae; scientificNameAuthorship: Fleming \& Wood, 2018; continent: Central America; country: Costa Rica; countryCode: CR; stateProvince: Alajuela; county: Sector San Cristobal; locality: Area de Conservacion Guanacaste; verbatimLocality: Rio Blanco Abajo; verbatimElevation: 500; verbatimLatitude: 10.9004; verbatimLongitude: -85.3725 ; verbatimCoordinateSystem: Decimal; decimalLatitude: 10.9004; decimalLongitude: -85.3725; samplingProtocol: Reared from the larva of the Sphingidae, Xylophanes chiron; verbatimEventDate: 14-Dec-2001; individualID: DHJPAR0022965; individualCount: 1; sex: female; lifeStage: adult; preparations: pinned; catalogNumber: DHJPAR0022965; occurrenceDetails: http:// janzen.sas.upenn.edu; recordedBy: D.H. Janzen, W. Hallwachs \& Osvaldo Espinoza; otherCatalog Numbers: ASTAW129-08, 01-SRNP-22178, BOLD:AAB0592; identifiedBy: AJ Fleming; dateldentified: 2017; language: en; institutionCode: CNC; collectionCode: Insects; basisOfRecord: Pinned Specimen

bk. ScientificName: Hyphantrophaga eldaarayae; phylum: Arthropoda; class: Insecta; order: Diptera; family: Tachinidae; genus: Hyphantrophaga; specificEpithet: 
eldaarayae; scientificNameAuthorship: Fleming \& Wood, 2018; continent: Central America; country: Costa Rica; countryCode: CR; stateProvince: Alajuela; county: Sector San Cristobal; locality: Area de Conservacion Guanacaste; verbatimLocality: Rio Blanco Abajo; verbatimElevation: 500; verbatimLatitude: 10.9004; verbatimLongitude: -85.3725 ; verbatimCoordinateSystem: Decimal; decimalLatitude: 10.9004; decimalLongitude: -85.3725; samplingProtocol: Reared from the larva of the Sphingidae, Xylophanes chiron; verbatimEventDate: 14-Dec-2001; individualID: DHJPAR0022966; individualCount: 1; sex: female; lifeStage: adult; preparations: pinned; catalogNumber: DHJPAR0022966; occurrenceDetails: http:// janzen.sas.upenn.edu; recordedBy: D.H. Janzen, W. Hallwachs \& Osvaldo Espinoza; otherCatalog Numbers: ASTAW130-08, 01-SRNP-22178, BOLD:AAB0592; identifiedBy: AJ Fleming; dateldentified: 2017; language: en; institutionCode: CNC; collectionCode: Insects; basisOfRecord: Pinned Specimen

bl. $\quad$ scientificName: Hyphantrophaga eldaarayae; phylum: Arthropoda; class: Insecta; order: Diptera; family: Tachinidae; genus: Hyphantrophaga; specificEpithet: eldaarayae; scientificNameAuthorship: Fleming \& Wood, 2018; continent: Central America; country: Costa Rica; countryCode: CR; stateProvince: Guanacaste; county: Sector Santa Rosa; locality: Area de Conservacion Guanacaste; verbatimLocality: Bosque San Emilio; verbatimElevation: 300; verbatimLatitude: 10.8439; verbatimLongitude: -85.6138; verbatimCoordinateSystem: Decimal; decimalLatitude: 10.8439; decimalLongitude: -85.6138; samplingProtocol: Reared from the larva of the Sphingidae, Xylophanes chiron; verbatimEventDate: 12-Jun-2008; individualID: DHJPAR0027792; individualCount: 1; sex: female; lifeStage: adult; preparations: pinned; catalogNumber: DHJPAR0027792; occurrenceDetails: http:// janzen.sas.upenn.edu; recordedBy: D.H. Janzen, W. Hallwachs \& Lucia Vargas; otherCatalogNumbers: ASHYE029-08, 08-SRNP-13448, BOLD:AAB0592; identifiedBy: AJ Fleming; dateldentified: 2017; language: en; institutionCode: CNC; collectionCode: Insects; basisOfRecord: Pinned Specimen

bm. $\quad$ scientificName: Hyphantrophaga eldaarayae; phylum: Arthropoda; class: Insecta; order: Diptera; family: Tachinidae; genus: Hyphantrophaga; specificEpithet: eldaarayae; scientificNameAuthorship: Fleming \& Wood, 2018; continent: Central America; country: Costa Rica; countryCode: CR; stateProvince: Alajuela; county: Sector Rincon Rain Forest; locality: Area de Conservacion Guanacaste; verbatimLocality: Estacion Llanura; verbatimElevation: 135; verbatimLatitude: 10.9333; verbatimLongitude: -85.2533 ; verbatimCoordinateSystem: Decimal; decimalLatitude: 10.9333; decimalLongitude: -85.2533; samplingProtocol: Reared from the larva of the Sphingidae, Xylophanes chiron; verbatimEventDate: 13-Feb-2010; individualID: DHJPAR0037476; individualCount: 1; sex: female; lifeStage: adult; preparations: pinned; catalogNumber: DHJPAR0037476; occurrenceDetails: http:// janzen.sas.upenn.edu; recordedBy: D.H. Janzen, W. Hallwachs \& Cirilo Umaña; otherCatalog Numbers: ASHYC4221-10, 10-SRNP-75043, BOLD:AAB0592; identifiedBy: AJ Fleming; dateldentified: 2017; language: en; institutionCode: CNC; collectionCode: Insects; basisOfRecord: Pinned Specimen

bn. $\quad$ scientificName: Hyphantrophaga eldaarayae; phylum: Arthropoda; class: Insecta; order: Diptera; family: Tachinidae; genus: Hyphantrophaga; specificEpithet: eldaarayae; scientificNameAuthorship: Fleming \& Wood, 2018; continent: Central America; country: Costa Rica; countryCode: CR; stateProvince: Alajuela; county: Sector San Cristobal; locality: Area de Conservacion Guanacaste; verbatimLocality: Vado Rio Cucaracho; verbatimElevation: 640; verbatimLatitude: 10.8702; verbatimLongitude: -85.3915; verbatimCoordinateSystem: Decimal; decimalLatitude: 
10.8702; decimalLongitude: -85.3915; samplingProtocol: Reared from the larva of the Sphingidae, Xylophanes chiron; verbatimEventDate: 25-Jul-2007; individuallD: DHJPAR0037658; individualCount: 1; sex: female; lifeStage: adult; preparations: pinned; catalogNumber: DHJPAR0037658; occurrenceDetails: http:// janzen.sas.upenn.edu; recordedBy: D.H. Janzen, W. Hallwachs \& Carolina Cano; otherCatalogNumbers: ASHYC4403-10, 07-SRNP-2058,; identifiedBy: AJ Fleming; dateldentified: 2017; language: en; institutionCode: CNC; collectionCode: Insects; basisOfRecord: Pinned Specimen

bo. $\quad$ scientificName: Hyphantrophaga eldaarayae; phylum: Arthropoda; class: Insecta; order: Diptera; family: Tachinidae; genus: Hyphantrophaga; specificEpithet: eldaarayae; scientificNameAuthorship: Fleming \& Wood, 2018; continent: Central America; country: Costa Rica; countryCode: CR; stateProvince: Guanacaste; county: Sector Mundo Nuevo; locality: Area de Conservacion Guanacaste; verbatimLocality: Quebrada Tibio Perla; verbatimElevation: 330; verbatimLatitude: 10.7626; verbatimLongitude: -85.4298; verbatimCoordinateSystem: Decimal; decimalLatitude: 10.7626; decimalLongitude: -85.4298; samplingProtocol: Reared from the larva of the Sphingidae, Xylophanes pluto; verbatimEventDate: 31-Jan-2012; individualID: DHJPAR0046567; individualCount: 1; sex: female; lifeStage: adult; preparations: pinned; catalogNumber: DHJPAR0046567; occurrenceDetails: http:// janzen.sas.upenn.edu; recordedBy: D.H. Janzen, W. Hallwachs \& Jose Cortez; otherCatalogNumbers: ACGBA740-12, 11-SRNP-57619,; identifiedBy: AJ Fleming; dateldentified: 2017; language: en; institutionCode: CNC; collectionCode: Insects; basisOfRecord: Pinned Specimen

bp. $\quad$ scientificName: Hyphantrophaga eldaarayae; phylum: Arthropoda; class: Insecta; order: Diptera; family: Tachinidae; genus: Hyphantrophaga; specificEpithet: eldaarayae; scientificNameAuthorship: Fleming \& Wood, 2018; continent: Central America; country: Costa Rica; countryCode: CR; stateProvince: Alajuela; county: Sector Rincon Rain Forest; locality: Area de Conservacion Guanacaste; verbatimLocality: San Lucas; verbatimElevation: 320; verbatimLatitude: 10.9185; verbatimLongitude: -85.3034; verbatimCoordinateSystem: Decimal; decimalLatitude: 10.9185; decimalLongitude: -85.3034; samplingProtocol: Reared from the larva of the Sphingidae, Xylophanes chiron; verbatimEventDate: 26-Mar-2012; individualID: DHJPAR0048429; individualCount: 1; sex: female; lifeStage: adult; preparations: pinned; catalogNumber: DHJPAR0048429; occurrenceDetails: http:// janzen.sas.upenn.edu; recordedBy: D.H. Janzen, W. Hallwachs \& Anabelle Cordoba; otherCatalogNumbers: ACGBA1971-12, 12-SRNP-40735, BOLD:AAB0592; identifiedBy: AJ Fleming; dateldentified: 2017; language: en; institutionCode: CNC; collectionCode: Insects; basisOfRecord: Pinned Specimen

bq. ScientificName: Hyphantrophaga eldaarayae; phylum: Arthropoda; class: Insecta; order: Diptera; family: Tachinidae; genus: Hyphantrophaga; specificEpithet: eldaarayae; scientificNameAuthorship: Fleming \& Wood, 2018; continent: Central America; country: Costa Rica; countryCode: CR; stateProvince: Guanacaste; county: Sector Pitilla; locality: Area de Conservacion Guanacaste; verbatimLocality: Medrano; verbatimElevation: 380; verbatimLatitude: 11.016; verbatimLongitude: -85.3805; verbatimCoordinateSystem: Decimal; decimalLatitude: 11.016; decimalLongitude: -85.3805; samplingProtocol: Reared from the larva of the Sphingidae, Xylophanes porcusDHJ03; verbatimEventDate: 13-Sep-2012; individualID: DHJPAR0050186; individualCount: 1; sex: female; lifeStage: adult; preparations: pinned; catalogNumber: DHJPAR0050186; occurrenceDetails: http://janzen.sas.upenn.edu; recordedBy: D.H. Janzen, W. Hallwachs \& Ricardo Calero; otherCatalogNumbers: ACGAZ1500-12, 12- 
SRNP-71976, BOLD:AAB0592; identifiedBy: AJ Fleming; dateldentified: 2017; language: en; institutionCode: CNC; collectionCode: Insects; basisOfRecord: Pinned Specimen

br. $\quad$ scientificName: Hyphantrophaga eldaarayae; phylum: Arthropoda; class: Insecta; order: Diptera; family: Tachinidae; genus: Hyphantrophaga; specificEpithet: eldaarayae; scientificNameAuthorship: Fleming \& Wood, 2018; continent: Central America; country: Costa Rica; countryCode: CR; stateProvince: Alajuela; county: Sector Rincon Rain Forest; locality: Area de Conservacion Guanacaste; verbatimLocality: Malaguenya; verbatimElevation: 221; verbatimLatitude: 10.9555; verbatimLongitude: -85.2838; verbatimCoordinateSystem: Decimal; decimalLatitude: 10.9555; decimalLongitude: -85.2838; samplingProtocol: Reared from the larva of the Sphingidae, Xylophanes guianensis; verbatimEventDate: 20-Dec-2012; individuallD: DHJPAR0050631; individualCount: 1; sex: female; lifeStage: adult; preparations: pinned; catalogNumber: DHJPAR0050631; occurrenceDetails: http:// janzen.sas.upenn.edu; recordedBy: D.H. Janzen, W. Hallwachs \& Keiner Aragon; otherCatalogNumbers: ACGBA3223-13, 12-SRNP-68832, BOLD:AAB0592; identifiedBy: AJ Fleming; dateldentified: 2017; language: en; institutionCode: CNC; collectionCode: Insects; basisOfRecord: Pinned Specimen

bs. $\quad$ scientificName: Hyphantrophaga eldaarayae; phylum: Arthropoda; class: Insecta; order: Diptera; family: Tachinidae; genus: Hyphantrophaga; specificEpithet: eldaarayae; scientificNameAuthorship: Fleming \& Wood, 2018; continent: Central America; country: Costa Rica; countryCode: CR; stateProvince: Alajuela; county: Sector Rincon Rain Forest; locality: Area de Conservacion Guanacaste; verbatimLocality: Finca Esmeralda; verbatimElevation: 123; verbatimLatitude: 10.9355; verbatimLongitude: -85.2531; verbatimCoordinateSystem: Decimal; decimalLatitude: 10.9355; decimalLongitude: -85.2531; samplingProtocol: Reared from the larva of the Sphingidae, Xylophanes chiron; verbatimEventDate: 21-Sep-2013; individualID: DHJPAR0053416; individualCount: 1; sex: female; lifeStage: adult; preparations: pinned; catalogNumber: DHJPAR0053416; occurrenceDetails: http:// janzen.sas.upenn.edu; recordedBy: D.H. Janzen, W. Hallwachs \& Cirilo Umaña; otherCatalogNumbers: ASHYM2770-13, 13-SRNP-77374, BOLD:AAB0592; identifiedBy: AJ Fleming; dateldentified: 2017; language: en; institutionCode: CNC; collectionCode: Insects; basisOfRecord: Pinned Specimen

bt. $\quad$ scientificName: Hyphantrophaga eldaarayae; phylum: Arthropoda; class: Insecta; order: Diptera; family: Tachinidae; genus: Hyphantrophaga; specificEpithet: eldaarayae; scientificNameAuthorship: Fleming \& Wood, 2018; continent: Central America; country: Costa Rica; countryCode: CR; stateProvince: Alajuela; county: Sector Rincon Rain Forest; locality: Area de Conservacion Guanacaste; verbatimLocality: Sendero Venado; verbatimElevation: 420; verbatimLatitude: 10.8968; verbatimLongitude: -85.27 ; verbatimCoordinateSystem: Decimal; decimalLatitude: 10.8968; decimalLongitude: -85.27; samplingProtocol: Reared from the larva of the Sphingidae, Xylophanes chiron; verbatimEventDate: 26-Sep-2014; individualID: DHJPAR0056192; individualCount: 1; sex: female; lifeStage: adult; preparations: pinned; catalogNumber: DHJPAR0056192; occurrenceDetails: http:// janzen.sas.upenn.edu; recordedBy: D.H. Janzen, W. Hallwachs \& Pablo Umaña Calderon; otherCatalogNumbers: ASHYH2449-14, 14-SRNP-43786,; identifiedBy: AJ Fleming; dateldentified: 2017; language: en; institutionCode: CNC; collectionCode: Insects; basisOfRecord: Pinned Specimen

bu. $\quad$ scientificName: Hyphantrophaga eldaarayae; phylum: Arthropoda; class: Insecta; order: Diptera; family: Tachinidae; genus: Hyphantrophaga; specificEpithet: 
eldaarayae; scientificNameAuthorship: Fleming \& Wood, 2018; continent: Central America; country: Costa Rica; countryCode: CR; stateProvince: Guanacaste; county: Sector Pitilla; locality: Area de Conservacion Guanacaste; verbatimLocality: Pasmompa; verbatimElevation: 440; verbatimLatitude: 11.0193; verbatimLongitude: -85.41; verbatimCoordinateSystem: Decimal; decimalLatitude: 11.0193; decimalLongitude: -85.41; samplingProtocol: Reared from the larva of the Sphingidae, Xylophanes loelia; verbatimEventDate: 20-Feb-2015; individualID: DHJPAR0057035; individualCount: 1; sex: female; lifeStage: adult; preparations: pinned; catalogNumber: DHJPAR0057035; occurrenceDetails: http://janzen.sas.upenn.edu; recordedBy: D.H. Janzen, W. Hallwachs \& Manuel Rios; otherCatalogNumbers: ACGBA4945-15, 15SRNP-30018, BOLD:AAB0592; identifiedBy: AJ Fleming; dateldentified: 2017; language: en; institutionCode: CNC; collectionCode: Insects; basisOfRecord: Pinned Specimen

bv. ScientificName: Hyphantrophaga eldaarayae; phylum: Arthropoda; class: Insecta; order: Diptera; family: Tachinidae; genus: Hyphantrophaga; specificEpithet: eldaarayae; scientificNameAuthorship: Fleming \& Wood, 2018; continent: Central America; country: Costa Rica; countryCode: CR; stateProvince: Alajuela; county: Sector Rincon Rain Forest; locality: Area de Conservacion Guanacaste; verbatimLocality: Finca Esmeralda; verbatimElevation: 123; verbatimLatitude: 10.9355; verbatimLongitude: -85.2531; verbatimCoordinateSystem: Decimal; decimalLatitude: 10.9355; decimalLongitude: -85.2531; samplingProtocol: Reared from the larva of the Sphingidae, Xylophanes chiron; verbatimEventDate: 03-Feb-2015; individualID: DHJPAR0057248; individualCount: 1; sex: female; lifeStage: adult; preparations: pinned; catalogNumber: DHJPAR0057248; occurrenceDetails: http:// janzen.sas.upenn.edu; recordedBy: D.H. Janzen, W. Hallwachs \& Cirilo Umaña; otherCatalogNumbers: ACGBA5158-15, 15-SRNP-75070, BOLD:AAB0592; identifiedBy: AJ Fleming; dateldentified: 2017; language: en; institutionCode: CNC; collectionCode: Insects; basisOfRecord: Pinned Specimen

bw. $\quad$ scientificName: Hyphantrophaga eldaarayae; phylum: Arthropoda; class: Insecta; order: Diptera; family: Tachinidae; genus: Hyphantrophaga; specificEpithet: eldaarayae; scientificNameAuthorship: Fleming \& Wood, 2018; continent: Central America; country: Costa Rica; countryCode: CR; stateProvince: Alajuela; county: Sector Rincon Rain Forest; locality: Area de Conservacion Guanacaste; verbatimLocality: San Lucas; verbatimElevation: 320; verbatimLatitude: 10.9185; verbatimLongitude: -85.3034 ; verbatimCoordinateSystem: Decimal; decimalLatitude: 10.9185; decimalLongitude: -85.3034; samplingProtocol: Reared from the larva of the Sphingidae, Xylophanes chiron; verbatimEventDate: 18-Aug-2003; individualID: DHJPAR0008078; individualCount: 1; lifeStage: adult; preparations: pinned; catalogNumber: DHJPAR0008078; occurrenceDetails: http://janzen.sas.upenn.edu; recordedBy: D.H. Janzen, W. Hallwachs \& Minor Carmona; otherCatalogNumbers: ASTAT850-06, 03-SRNP-11723, BOLD:AAB0592; identifiedBy: AJ Fleming; dateldentified: 2017; Ianguage: en; institutionCode: CNC; collectionCode: Insects; basisOfRecord: Pinned Specimen

bx. $\quad$ scientificName: Hyphantrophaga eldaarayae; phylum: Arthropoda; class: Insecta; order: Diptera; family: Tachinidae; genus: Hyphantrophaga; specificEpithet: eldaarayae; scientificNameAuthorship: Fleming \& Wood, 2018; continent: Central America; country: Costa Rica; countryCode: CR; stateProvince: Guanacaste; county: Sector El Hacha; locality: Area de Conservacion Guanacaste; verbatimLocality: Sendero Bejuquilla; verbatimElevation: 280; verbatimLatitude: 11.03; verbatimLongitude: -85.527; verbatimCoordinateSystem: Decimal; decimalLatitude: 
11.03; decimalLongitude: -85.527; samplingProtocol: Reared from the larva of the Sphingidae, Xylophanes chiron; verbatimEventDate: 12-Mar-1998; individualID: DHJPAR0008106; individualCount: 1; lifeStage: adult; preparations: pinned; catalogNumber: DHJPAR0008106; occurrenceDetails: http://janzen.sas.upenn.edu; recordedBy: D.H. Janzen, W. Hallwachs \& Lucia Rios; otherCatalogNumbers: ASTAT878-06, 98-SRNP-13986, BOLD:AAB0592; identifiedBy: AJ Fleming; dateldentified: 2017; language: en; institutionCode: CNC; collectionCode: Insects; basisOfRecord: Pinned Specimen

by. $\quad$ scientificName: Hyphantrophaga eldaarayae; phylum: Arthropoda; class: Insecta; order: Diptera; family: Tachinidae; genus: Hyphantrophaga; specificEpithet: eldaarayae; scientificNameAuthorship: Fleming \& Wood, 2018; continent: Central America; country: Costa Rica; countryCode: CR; stateProvince: Alajuela; county: Sector Rincon Rain Forest; locality: Area de Conservacion Guanacaste; verbatimLocality: Finca Aurita; verbatimElevation: 460; verbatimLatitude: 10.8841; verbatimLongitude: -85.2573 ; verbatimCoordinateSystem: Decimal; decimalLatitude: 10.8841; decimalLongitude: -85.2573; samplingProtocol: Reared from the larva of the Sphingidae, Xylophanes chiron; verbatimEventDate: 17-Mar-2006; individualID: DHJPAR0006956; individualCount: 1; lifeStage: adult; preparations: pinned; catalogNumber: DHJPAR0006956; occurrenceDetails: http://janzen.sas.upenn.edu; recordedBy: D.H. Janzen, W. Hallwachs \& Minor Carmona; otherCatalogNumbers: ASTAV198-06, 06-SRNP-40507,; identifiedBy: AJ Fleming; dateldentified: 2017; language: en; institutionCode: CNC; collectionCode: Insects; basisOfRecord: Pinned Specimen

bz. $\quad$ scientificName: Hyphantrophaga eldaarayae; phylum: Arthropoda; class: Insecta; order: Diptera; family: Tachinidae; genus: Hyphantrophaga; specificEpithet: eldaarayae; scientificNameAuthorship: Fleming \& Wood, 2018; continent: Central America; country: Costa Rica; countryCode: CR; stateProvince: Alajuela; county: Sector San Cristobal; locality: Area de Conservacion Guanacaste; verbatimLocality: Vado Rio Cucaracho; verbatimElevation: 640; verbatimLatitude: 10.8702; verbatimLongitude: -85.3915; verbatimCoordinateSystem: Decimal; decimalLatitude: 10.8702; decimalLongitude: -85.3915 ; samplingProtocol: Reared from the larva of the Sphingidae, Xylophanes chiron; verbatimEventDate: 25-Jul-2007; individualID: DHJPAR0019719; individualCount: 1; lifeStage: adult; preparations: pinned; catalogNumber: DHJPAR0019719; occurrenceDetails: http://janzen.sas.upenn.edu; recordedBy: D.H. Janzen, W. Hallwachs \& Carolina Cano; otherCatalogNumbers: ASTAB267-07, 07-SRNP-2058,; identifiedBy: AJ Fleming; dateldentified: 2017; language: en; institutionCode: CNC; collectionCode: Insects; basisOfRecord: Pinned Specimen

ca. ScientificName: Hyphantrophaga eldaarayae; phylum: Arthropoda; class: Insecta; order: Diptera; family: Tachinidae; genus: Hyphantrophaga; specificEpithet: eldaarayae; scientificNameAuthorship: Fleming \& Wood, 2018; continent: Central America; country: Costa Rica; countryCode: CR; stateProvince: Alajuela; county: Buenos Aires; locality: Area de Conservacion Guanacaste; verbatimLocality: Finca Tomate; verbatimElevation: 360 ; verbatimLatitude: 10.9035 ; verbatimLongitude: -85.3092; verbatimCoordinateSystem: Decimal; decimalLatitude: 10.9035; decimalLongitude: -85.3092; samplingProtocol: Reared from the larva of the Sphingidae, Xylophanes chiron; verbatimEventDate: 19-Oct-2008; individualID: DHJPAR0029647; individualCount: 1; lifeStage: adult; preparations: pinned; catalogNumber: DHJPAR0029647; occurrenceDetails: http://janzen.sas.upenn.edu; recordedBy: D.H. Janzen, W. Hallwachs \& Elda Araya; otherCatalogNumbers: 
ASHYM1068-09, 08-SRNP-5331,; identifiedBy: AJ Fleming; dateldentified: 2017; language: en; institutionCode: CNC; collectionCode: Insects; basisOfRecord: Pinned Specimen

cb. $\quad$ scientificName: Hyphantrophaga eldaarayae; phylum: Arthropoda; class: Insecta; order: Diptera; family: Tachinidae; genus: Hyphantrophaga; specificEpithet: eldaarayae; scientificNameAuthorship: Fleming \& Wood, 2018; continent: Central America; country: Costa Rica; countryCode: CR; stateProvince: Alajuela; county: Sector San Cristobal; locality: Area de Conservacion Guanacaste; verbatimLocality: Tajo Angeles; verbatimElevation: 540; verbatimLatitude: 10.8647; verbatimLongitude: -85.4153; verbatimCoordinateSystem: Decimal; decimalLatitude: 10.8647; decimalLongitude: -85.4153; samplingProtocol: Reared from the larva of the Sphingidae, Xylophanes chiron; verbatimEventDate: 26-Nov-2010; individuallD: DHJPAR0040932; individualCount: 1; lifeStage: adult; preparations: pinned; catalogNumber: DHJPAR0040932; occurrenceDetails: http://janzen.sas.upenn.edu; recordedBy: D.H. Janzen, W. Hallwachs \& Gloria Sihezar; otherCatalogNumbers: ASHYF847-11, 10-SRNP-6265, BOLD:AAB0592; identifiedBy: AJ Fleming; dateldentified: 2017; language: en; institutionCode: CNC; collectionCode: Insects; basisOfRecord: Pinned Specimen

cc. ScientificName: Hyphantrophaga eldaarayae; phylum: Arthropoda; class: Insecta; order: Diptera; family: Tachinidae; genus: Hyphantrophaga; specificEpithet: eldaarayae; scientificNameAuthorship: Fleming \& Wood, 2018; continent: Central America; country: Costa Rica; countryCode: CR; stateProvince: Alajuela; county: Sector San Cristobal; locality: Area de Conservacion Guanacaste; verbatimLocality: Tajo Angeles; verbatimElevation: 540; verbatimLatitude: 10.8647; verbatimLongitude: -85.4153; verbatimCoordinateSystem: Decimal; decimalLatitude: 10.8647 ; decimalLongitude: -85.4153; samplingProtocol: Reared from the larva of the Sphingidae, Xylophanes chiron; verbatimEventDate: 26-Nov-2010; individualID: DHJPAR0042146; individualCount: 1; lifeStage: adult; preparations: pinned; catalogNumber: DHJPAR0042146; occurrenceDetails: http://janzen.sas.upenn.edu; recordedBy: D.H. Janzen, W. Hallwachs \& Gloria Sihezar; otherCatalogNumbers: ASHYF2034-11, 10-SRNP-6265, BOLD:AAB0592; identifiedBy: AJ Fleming; dateldentified: 2017; language: en; institutionCode: CNC; collectionCode: Insects; basisOfRecord: Pinned Specimen

cd. $\quad$ scientificName: Hyphantrophaga eldaarayae; phylum: Arthropoda; class: Insecta; order: Diptera; family: Tachinidae; genus: Hyphantrophaga; specificEpithet: eldaarayae; scientificNameAuthorship: Fleming \& Wood, 2018; continent: Central America; country: Costa Rica; countryCode: CR; stateProvince: Guanacaste; county: Sector Pitilla; locality: Area de Conservacion Guanacaste; verbatimLocality: Sendero Naciente; verbatimElevation: 700 ; verbatimLatitude: 10.9871; verbatimLongitude: -85.4282; verbatimCoordinateSystem: Decimal; decimalLatitude: 10.9871; decimalLongitude: -85.4282; samplingProtocol: Reared from the larva of the Sphingidae, Xylophanes germen; verbatimEventDate: 04-Apr-2014; individualID: DHJPAR0055641; individualCount: 1; lifeStage: adult; preparations: pinned; catalogNumber: DHJPAR0055641; occurrenceDetails: http://janzen.sas.upenn.edu; recordedBy: D.H. Janzen, W. Hallwachs \& Manuel Rios; otherCatalogNumbers: ASHYH2188-14, 14-SRNP-30344, BOLD:AAB0592; identifiedBy: AJ Fleming; dateldentified: 2017; language: en; institutionCode: CNC; collectionCode: Insects; basisOfRecord: Pinned Specimen

ce. $\quad$ scientificName: Hyphantrophaga eldaarayae; phylum: Arthropoda; class: Insecta; order: Diptera; family: Tachinidae; genus: Hyphantrophaga; specificEpithet: 
eldaarayae; scientificNameAuthorship: Fleming \& Wood, 2018; continent: Central America; country: Costa Rica; countryCode: CR; stateProvince: Guanacaste; county: Sector Pitilla; locality: Area de Conservacion Guanacaste; verbatimLocality: Medrano;

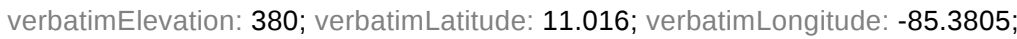
verbatimCoordinateSystem: Decimal; decimalLatitude: 11.016; decimalLongitude: -85.3805; samplingProtocol: Reared from the larva of the Sphingidae, Xylophanes chiron; verbatimEventDate: 21-Aug-2017; individualID: DHJPAR0061283; individualCount: 1; lifeStage: adult; preparations: pinned; catalogNumber: DHJPAR0061283; occurrenceDetails: http://janzen.sas.upenn.edu; recordedBy: D.H. Janzen, W. Hallwachs \& Ricardo Calero; otherCatalog Numbers: ACGBA7666-17, 17SRNP-72587, BOLD:AAB0592; identifiedBy: AJ Fleming; dateldentified: 2017; language: en; institutionCode: CNC; collectionCode: Insects; basisOfRecord: Pinned Specimen

\section{Description}

Male (Fig. 13). Length: 9-12 mm. Head (Fig. 13b): vertex 1/5 of head width; two pairs of reclinate upper orbital setae; ocellar setae arising behind anterior ocellus; ocellar triangle pale brassy; fronto-orbital plate pale brassy on upper $80 \%$, densely setulose, setulae not extending below lowest frontal seta; parafacial silver and bare; eye densely haired; facial ridge bare; pedicel black, concolorous with postpedicel; arista black, very minutely pubescent, distinctly thickened on basal 1/3-1/4; palpus yellow and haired apically, narrow and digitiform, apically pointed. Thorax (Fig. 13a, c): dull brassy tomentose dorsally, contrasting with slightly brighter silver tomentose laterally, lateral surfaces with dense dark setulae making it appear darker; four thick dorsal vittae, outermost two broken across suture, innermost pair unbroken, reaching 3rd postsutural dorsocentral seta, both pairs of vittae not widening postsuturally; postpronotum with 35 setae arranged in a triangle; chaetotaxy: acrostichal setae $3: 3$; dorsocentral setae 3:4; intra-alar setae 3-4:3; supra-alar setae 2:3; three katepisternal setae; basal scutellar setae as long as subapical scutellar setae, curving slightly inwards medially; lateral scutellar setae less than $1 / 2$ as long as subapical setae, curving inwards medially; apical scutellar setae subequal in length to lateral scutellar setae, crossed apically, angled slightly above plane of remaining marginal scutellar setae; one pair of discal scutellar setae more widely set than apical setae; scutellum very slightly darkened across basal 15\%, shaped as two adjacent crescents, remainder concolorous with scutum. Legs (Fig. 13c): black in ground colour; fore femur with dense silver tomentum on posterodorsal surface; hind coxa bare. Wing (Fig. 13a): pale translucent, hyaline, not distinctly infuscate; vein $\mathrm{R}_{4+5}$ with only 2-3 setulae at base. Abdomen (Fig. 13a, c): ground colour black dorsally, yellow lateroventrally; mid-dorsal depression on ST1+2 reaching hind margin; median marginal setae present on ST1+2T3; a complete row of marginal setae present on T4; discal setae only on T5; sex patch covering ventral surfaces of T4-T5; distinct brassy tomentose bands along anterior edge of T3 and T4, broken medially by a dorsocentral stripe and covering almost $80 \%$ of tergites; T3 with silver tomentum ventrolaterally over $80 \%$ of surface; T5 with brassy tomentum throughout. Terminalia: sternite 5 (Fig. 13f) with a deeply excavated median cleft, widely U-shaped, margins covered in dense tomentum. Lateral lobes of sternite 
rounded apically, many stout setae confined to lobe margins. Anterior plate of sternite 5 subequal to length of apical lobes; unsclerotised "window" anterior to median cleft reduced to a narrow strip as wide as median cleft. Cerci in posterior view (Fig. 13d) spatulate and rounded apically, slightly longer than surstyli, completely separate medially, slightly divergent; in lateral view with an evenly rounded downward curve throughout; densely setulose along basal 2/3. Surstylus in lateral view (Fig. 13e) round, tapered along its length and straight along bottom edge, opposite edge evenly rounded giving it a cleaver blade appearance; when viewed dorsally, surstyli pointing outwards and not strongly convergent. Pregonite short, not well developed, 1/2 as long as distiphallus, bare and rounded apically, having the appearance of an upside-down boot. Postgonite slightly narrow, $1 / 3$ as wide as pregonite, sharply pointed and curved at apex. Epiphallus well-developed and apically hooked. Distiphallus rectangular with a slender median longitudinal sclerotised reinforcement on its posterior surface and a broad, anterolateral, sclerotised acrophallus on each side, joining on anterior surface near apex.

Female. Length: 8-11 mm. As male, differing only by the presence of two pairs of proclinate orbital setae.

\section{Diagnosis}

Hyphantrophaga eldaarayae sp. n. can be distinguished from all other Hyphantrophaga species by the following combination of traits: thorax with three postsutural acrostichal setae, four postsutural dorsocentral setae, and three katepisternal setae, legs black, hind coxa bare, silver tomentum covering more than $80 \%$ of ventrolateral surfaces of $\mathrm{T} 3$, with silver tomentum reaching edge of tergite ventrally.

\section{Etymology}

Hyphantrophaga eldaarayae sp. $\mathbf{n}$. is named in recognition of Elda Araya Martinez's dedication and work in finding and rearing the ACG caterpillars that contained tachinid larvae.

\section{Distribution}

Costa Rica, ACG, Guanacaste Province, 380-420 m elevation.

\section{Ecology}

Hyphantrophaga eldaarayae sp. $\mathbf{n}$. has been reared 116 times from 15 species of Lepidoptera in the family Sphingidae, Xylophanes chiron (Drury, 1773), Xylophanes anubus (Cramer, 1777), Xylophanes tyndarus (Boisduval, 1875), Xylophanes libyaDHJ02, Xylophanes pluto (Fabricius, 1777), Xylophanes crotonis (Walker, 1856), Xylophanes maculator (Boisduval, 1875), Xylophanes jocasta (Druce, 1888), Xylophanes guianensis (Rothschild, 1894), Xylophanes hannemanni Closs, 1917, 
Xylophanes porcusDHJ03, Xylophanes germen (Schaus, 1890), Xylophanes cthulhu Haxaire \& Vaglia, 2008, Xylophanes loelia (Druce, 1878) and Xylophanes porcus (Hübner, 1823); in rain forest and dry-rain lowland intergrades.

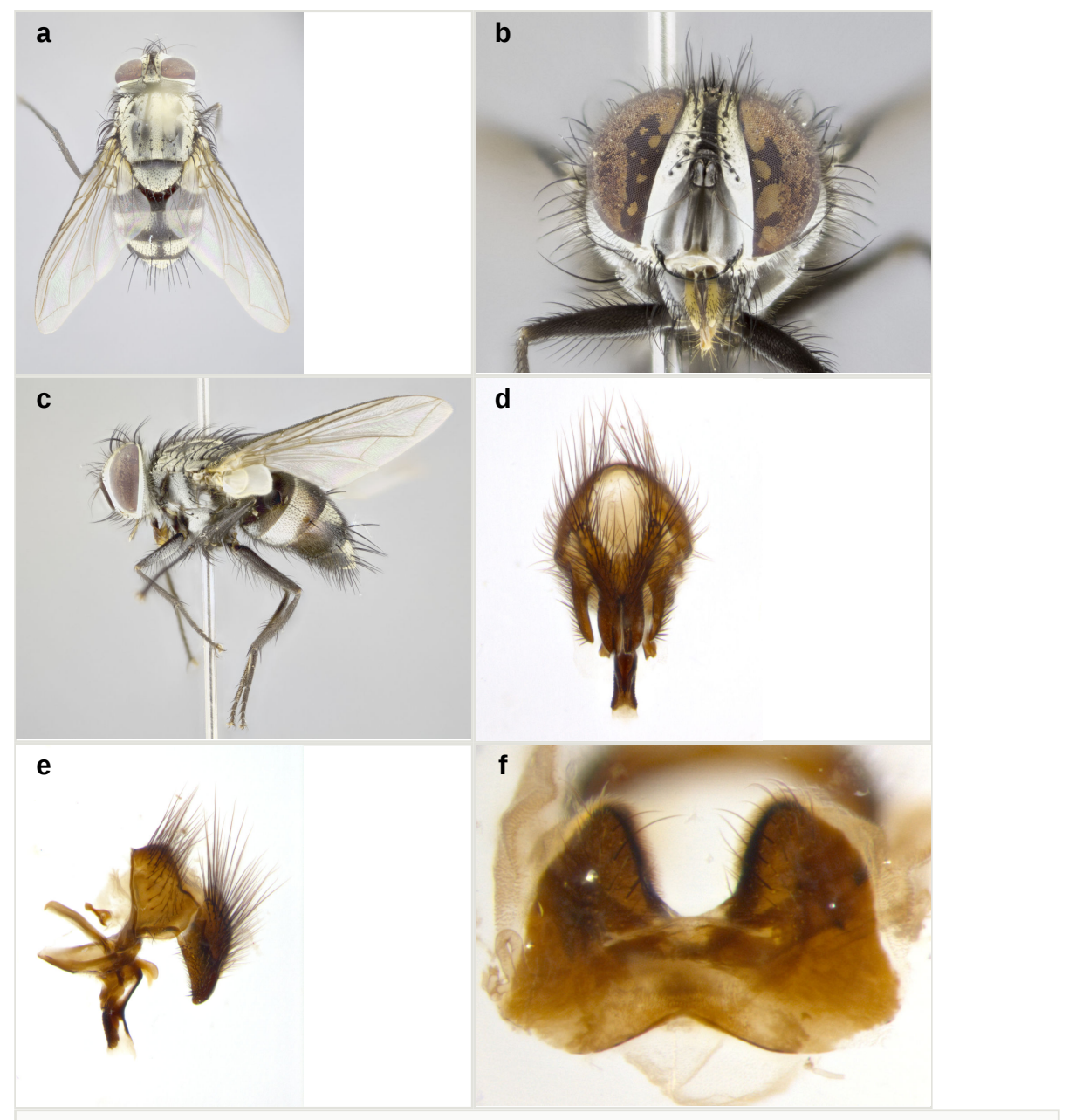

Figure 13.

Hyphantrophaga eldaarayae sp. n.; a-c: habitus; holotype male, voucher $\mathrm{n}$. DHJPAR0022963; d-f: terminalia; male paratype, voucher n. DHJPAR0008106.
a: dorsal view doi
b: frontal view doi
c: lateral view doi
d: dorsal view doi
e: lateral view doi
f: sternite 5 , ventral view doi 


\section{Hyphantrophaga eliethcantillanoae Fleming \& Wood, sp. $\mathrm{n}$.}

- ZooBank urn:Isid:zoobank.org:act:2DB211E5-CB8B-4821-9DF6-4341A2E13252

\section{Materials}

\section{Holotype:}

a. $\quad$ scientificName: Hyphantrophaga eliethcantillanoae; phylum: Arthropoda; class: Insecta; order: Diptera; family: Tachinidae; genus: Hyphantrophaga; specific Epithet: eliethcantillanoae; scientificNameAuthorship: Fleming \& Wood, 2017; continent: Central America; country: Costa Rica; countryCode: CR; stateProvince: Guanacaste; county: Sector Mundo Nuevo; locality: Area de Conservacion Guanacaste; verbatimLocality: Quebrada Tibio Perla; verbatimElevation: 330; verbatimLatitude: 10.7626; verbatimLongitude: -85.4298; verbatimCoordinateSystem: Decimal; decimalLatitude: 10.7626; decimalLongitude: -85.4298; samplingProtocol: Reared from the larva of the Crambidae, Omiodes cuniculalis; verbatimEventDate: 02-Sep-2006; individualID: DHJPAR0015985; individualCount: 1; sex: male; lifeStage: adult; preparations: pinned; catalogNumber: DHJPAR0015985; occurrenceDetails: http:// janzen.sas.upenn.edu; recordedBy: D.H. Janzen, W. Hallwachs \& Mariano Pereira; otherCatalogNumbers: ASTAP014-06, 06-SRNP-57540, BOLD:AAA1698; identifiedBy: AJ Fleming; dateldentified: 2017; language: en; institutionCode: CNC; collectionCode: Insects; basisOfRecord: Pinned Specimen

\section{Paratypes:}

a. ScientificName: Hyphantrophaga eliethcantillanoae; phylum: Arthropoda; class: Insecta; order: Diptera; family: Tachinidae; genus: Hyphantrophaga; specificEpithet: eliethcantillanoae; scientificNameAuthorship: Fleming \& Wood, 2017; continent: Central America; country: Costa Rica; countryCode: CR; stateProvince: Guanacaste; county: Sector Mundo Nuevo; locality: Area de Conservacion Guanacaste; verbatimLocality: Quebrada Tibio Perla; verbatimElevation: 330; verbatimLatitude: 10.7626; verbatimLongitude: -85.4298; verbatimCoordinateSystem: Decimal; decimalLatitude: 10.7626; decimalLongitude: -85.4298; samplingProtocol: Reared from the larva of the Crambidae, Omiodes cuniculalis; verbatimEventDate: 31-Aug-2006; individualID: DHJPAR0016011; individualCount: 1; sex: male; lifeStage: adult; preparations: pinned; catalogNumber: DHJPAR0016011; occurrenceDetails: http:// janzen.sas.upenn.edu; recordedBy: D.H. Janzen, W. Hallwachs \& Mariano Pereira; otherCatalogNumbers: ASTAP040-06, 06-SRNP-57589, BOLD:AAA1698; identifiedBy: AJ Fleming; dateldentified: 2017; language: en; institutionCode: CNC; collectionCode: Insects; basisOfRecord: Pinned Specimen

b. ScientificName: Hyphantrophaga eliethcantillanoae; phylum: Arthropoda; class: Insecta; order: Diptera; family: Tachinidae; genus: Hyphantrophaga; specificEpithet: eliethcantillanoae; scientificNameAuthorship: Fleming \& Wood, 2017; continent: Central America; country: Costa Rica; countryCode: CR; stateProvince: Guanacaste; county: Sector Pitilla; locality: Area de Conservacion Guanacaste; verbatimLocality: Quebradona; verbatimElevation: 475; verbatimLatitude: 10.991; verbatimLongitude: -85.3954; verbatimCoordinateSystem: Decimal; decimalLatitude: 10.991; decimalLongitude: -85.3954; samplingProtocol: Reared from the larva of the Crambidae, Omiodes fulvicauda; verbatimEventDate: 12-Apr-2012; individualID: DHJPAR0048627; individualCount: 1; sex: male; lifeStage: adult; preparations: pinned; catalogNumber: DHJPAR0048627; occurrenceDetails: http://janzen.sas.upenn.edu; recordedBy: D.H. Janzen, W. Hallwachs \& Freddy Quesada; otherCatalogNumbers: 
ACGBA2169-12, 12-SRNP-70590,; identifiedBy: AJ Fleming; dateldentified: 2017; language: en; institutionCode: CNC; collectionCode: Insects; basisOfRecord: Pinned Specimen

c. ScientificName: Hyphantrophaga eliethcantillanoae; phylum: Arthropoda; class: Insecta; order: Diptera; family: Tachinidae; genus: Hyphantrophaga; specificEpithet: eliethcantillanoae; scientificNameAuthorship: Fleming \& Wood, 2017; continent: Central America; country: Costa Rica; countryCode: CR; stateProvince: Guanacaste; county: Sector Mundo Nuevo; locality: Area de Conservacion Guanacaste; verbatimLocality: Quebrada Tibio Perla; verbatimElevation: 330; verbatimLatitude: 10.7626; verbatimLongitude: -85.4298; verbatimCoordinateSystem: Decimal; decimalLatitude: 10.7626; decimalLongitude: -85.4298; samplingProtocol: Reared from the larva of the Crambidae, Omiodes cuniculalis; verbatimEventDate: 02-Sep-2006; individualID: DHJPAR0015991; individualCount: 1; sex: female; lifeStage: adult; preparations: pinned; catalogNumber: DHJPAR0015991; occurrenceDetails: http:// janzen.sas.upenn.edu; recordedBy: D.H. Janzen, W. Hallwachs \& Mariano Pereira; otherCatalogNumbers: ASTAP020-06, 06-SRNP-57542, BOLD:AAA1698; identifiedBy: AJ Fleming; dateldentified: 2017; language: en; institutionCode: CNC; collectionCode: Insects; basisOfRecord: Pinned Specimen

d. scientificName: Hyphantrophaga eliethcantillanoae; phylum: Arthropoda; class: Insecta; order: Diptera; family: Tachinidae; genus: Hyphantrophaga; specificEpithet: eliethcantillanoae; scientificNameAuthorship: Fleming \& Wood, 2017; continent: Central America; country: Costa Rica; countryCode: CR; stateProvince: Guanacaste; county: Sector Mundo Nuevo; locality: Area de Conservacion Guanacaste; verbatimLocality: Quebrada Tibio Perla; verbatimElevation: 330; verbatimLatitude: 10.7626; verbatimLongitude: -85.4298; verbatimCoordinateSystem: Decimal; decimalLatitude: 10.7626; decimalLongitude: -85.4298; samplingProtocol: Reared from the larva of the Crambidae, Omiodes cuniculalis; verbatimEventDate: 02-Sep-2006; individualID: DHJPAR0015979; individualCount: 1; sex: female; lifeStage: adult; preparations: pinned; catalogNumber: DHJPAR0015979; occurrenceDetails: http:// janzen.sas.upenn.edu; recordedBy: D.H. Janzen, W. Hallwachs \& Mariano Pereira; otherCatalogNumbers: ASTAP008-06, 06-SRNP-57601, BOLD:AAA1698; identifiedBy: AJ Fleming; dateldentified: 2017; language: en; institutionCode: CNC; collectionCode: Insects; basisOfRecord: Pinned Specimen

e. ScientificName: Hyphantrophaga eliethcantillanoae; phylum: Arthropoda; class: Insecta; order: Diptera; family: Tachinidae; genus: Hyphantrophaga; specificEpithet: eliethcantillanoae; scientificNameAuthorship: Fleming \& Wood, 2017; continent: Central America; country: Costa Rica; countryCode: CR; stateProvince: Guanacaste; county: Sector Mundo Nuevo; locality: Area de Conservacion Guanacaste; verbatimLocality: Quebrada Tibio Perla; verbatimElevation: 330; verbatimLatitude: 10.7626; verbatimLongitude: -85.4298; verbatimCoordinateSystem: Decimal; decimalLatitude: 10.7626; decimalLongitude: -85.4298; samplingProtocol: Reared from the larva of the Crambidae, Omiodes cuniculalis; verbatimEventDate: 01-Sep-2006; individualID: DHJPAR0016002; individualCount: 1; sex: female; lifeStage: adult; preparations: pinned; catalogNumber: DHJPAR0016002; occurrenceDetails: http:// janzen.sas.upenn.edu; recordedBy: D.H. Janzen, W. Hallwachs \& Mariano Pereira; otherCatalog Numbers: ASTAP031-06, 06-SRNP-57575, BOLD:AAA1698; identifiedBy: AJ Fleming; dateldentified: 2017; language: en; institutionCode: CNC; collectionCode: Insects; basisOfRecord: Pinned Specimen 


\section{Description}

Male (Fig. 14). Length: 5-9 mm. Head (Fig. 14b): vertex 1/4 of head width; two reclinate upper orbital setae; ocellar setae arising behind anterior ocellus; ocellar triangle gold, concolorous with fronto-orbital plate; fronto-orbital plate gold over $75 \%$ of surface, densely setulose, setulae not extending beyond lowest frontal seta; parafacial shiny silver and bare; facial ridge bare; pedicel black, concolorous with postpedicel; arista black, very minutely pubescent, distinctly thickened on basal 1/3-1/4; palpus yellow and haired. Thorax (Fig. 14a, c): thorax gold tomentose dorsally, grey tomentose laterally; four narrow yet prominent dorsal vittae, outermost two broken across suture, innermost pair unbroken, reaching just beyond 1st postsutural dorsocentral seta; postpronotum with $3 \mathbf{- 5}$ setae arranged in a triangle; chaetotaxy: acrostichal setae 4:3; dorsocentral setae 3:4; intra-alar setae 3:3; supra-alar setae 2:3; three katepisternal setae; basal scutellar setae subequal in length to subapical scutellar setae; lateral scutellar setae $4 / 5$ length of basal setae; subapical setae straight and divergent; apical scutellar short and weak, 1/3 length of subapical scutellar setae, erect and crossed midway along length; one pair of discal scutellar setae more widely set than apical scutellar setae, but only slightly narrower than subapical scutellar setae; scutellum concolorous with scutum. Legs (Fig. 14c): black throughout; fore femur with dense silver tomentum on posterodorsal surface; hind coxa setose. Wing (Fig. 14a): clear translucent, not infuscate; vein $\mathrm{R}_{4+5}$ with two setulae at base. Abdomen (Fig. 14a, c): ground colour black; middorsal depression on ST1+2 reaching hind margin; abdominal tomentum present as gold bands spanning anterior $70 \%$ of T3-T4; T5 entirely gold tomentose; median marginal setae present on ST1+2 and T3; a complete row of marginal setae present on T4; discal setae present on T3-T5; sex patch absent. Terminalia (Fig. 14d, e, f): anterior margin of sternite 5 (Fig. 14f) with a slight curved medial depression, posterior margin with a deeply excavated median cleft, smoothly $U$ shaped; posterior lobes of sternite rounded, toothed apically and completely devoid of any setae; anterior plate of sternite $51 / 5$ as long as apical lobes, slightly translucent throughout, not as sclerotised as lobes. Unsclerotised "window" anterior to median cleft umbonate, central portion of "window" $2 \mathrm{X}$ as long as median cleft, "arms" extending $2 \mathrm{X}$ as wide as median cleft. Cerci (Fig. 14d) in posterior view subrectangular and slightly shorter than surstyli, blunt and rounded off towards apex, completely separate medially, diverging slightly at tips; in lateral view (Fig. 14e) strongly tapered along anterior 1/3 and weakly curved at beginning of taper; densely setulose dorsally up to taper point, apparently bare ventrally (visible in lateral view). Surstylus in lateral view slightly swollen basally, otherwise almost parallel-sided with rounded ends; when viewed dorsally, surstyli appearing weakly divergent and slightly open at tips. Pregonite broad and well-developed, slightly bent; basal $2 / 3$ slightly cinched, giving it a very slightly clubbed appearance, apically rounded, devoid of setulae. Postgonite elongate and slender, horn-shaped, subequal in length to pregonite. Distiphallus sail-shaped, apically flared, with a slender median longitudinal sclerotised reinforcement on its posterior surface and a broad, anterolateral, sclerotised acrophallus on each side, joining the plate of opposite side on anterior surface near apex. 


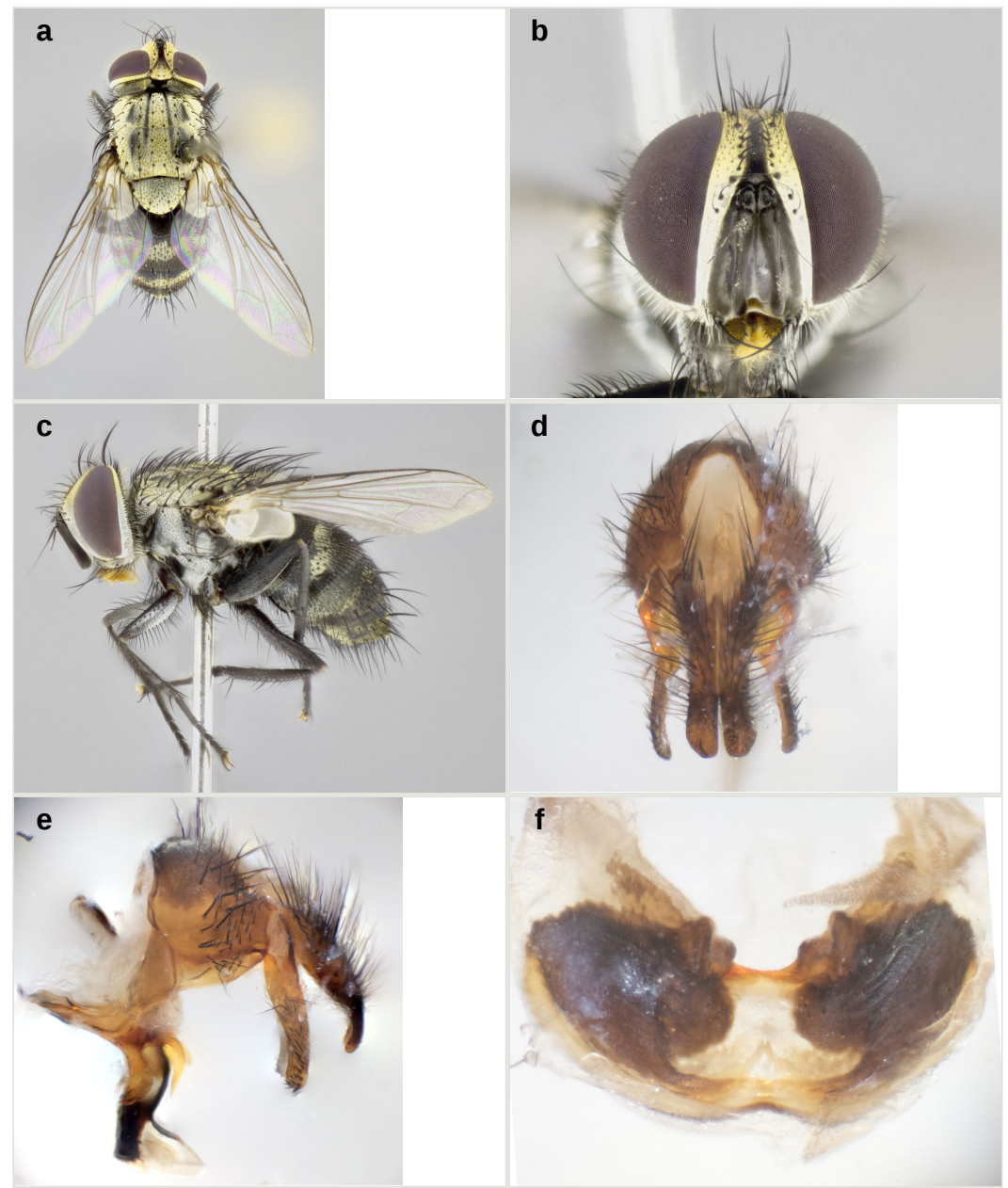

Figure 14.

Hyphantrophaga eliethcantillanoae sp. $\mathbf{n}$.; a-c: habitus; holotype male, voucher $\mathrm{n}$. DHJPAR0015985; d-f: terminalia; male paratype, voucher n. DHJPAR0048627.
a: dorsal view doi
b: frontal view doi
c: lateral view doi
d: dorsal view doi
e: lateral view doi
f: sternite 5 , ventral view doi 
Female. Length: 6-9 mm. As male, differing only by the presence of two pairs of proclinate orbital setae.

\section{Diagnosis}

Hyphantrophaga eliethcantillanoae sp. n. can be distinguished from all other Hyphantrophaga species by the following combination of traits: pedicel and arista concolorous black, four postsutural dorsocentral setae, four thoracic vittae with inner pair solid across suture, hind coxa setose, discal setae present only on T3-T5 and T5 entirely gold tomentose.

\section{Etymology}

Hyphantrophaga eliethcantillanoae sp. $\mathbf{n}$. is named in recognition of Elieth Cantillano Espinoza's dedication and work in finding and rearing the ACG caterpillars that contained tachinid larvae.

\section{Distribution}

Costa Rica, ACG, Alajuela and Guanacaste Provinces, 10-470 m elevation.

\section{Ecology}

Hyphantrophaga eliethcantillanoae sp. $\mathbf{n}$. has been reared 386 times from two species of Lepidoptera in the family Crambidae, Omiodes cuniculalis Guenée, 1854 and Omiodes fulvicauda (Hampson, 1898); in rain forest, dry forest and dry-rain lowland intergrade.

\section{Hyphantrophaga gilberthampiei Fleming \& Wood, sp. n.}

- ZooBank urn:Isid:zoobank.org:act:7E9DB836-E9BD-446B-8AFA-3F0CD45325F7

\section{Material}

Holotype:

a. ScientificName: Hyphantrophaga gilberthampiei; phylum: Arthropoda; class: Insecta; order: Diptera; family: Tachinidae; genus: Hyphantrophaga; specificEpithet: gilberthampiei; scientificNameAuthorship: Fleming \& Wood, 2017; continent: Central America; country: Costa Rica; countryCode: CR; stateProvince: Guanacaste; county: Sector Pitilla; locality: Area de Conservacion Guanacaste; verbatimLocality: Sendero Orosilito; verbatimElevation: 900; verbatimLatitude: 10.98332; verbatimLongitude: -85.43623; verbatimCoordinateSystem: Decimal; decimalLatitude: 10.98332; decimalLongitude: -85.43623; samplingProtocol: Reared from the larva of the Hesperiidae, Cynea megalops; verbatimEventDate: 10-Mar-2016; individualID: DHJPAR0059246; individualCount: 1; sex: female; lifeStage: adult; preparations: pinned; catalogNumber: DHJPAR0059246; occurrenceDetails: http:// janzen.sas.upenn.edu; recordedBy: D.H. Janzen, W. Hallwachs \& Manuel Rios; otherCatalogNumbers: ACGBA5663-16, 16-SRNP-30146, BOLD:ADE0400; 
identifiedBy: AJ Fleming; dateldentified: 2017; language: en; institutionCode: CNC; collectionCode: Insects; basisOfRecord: Pinned Specimen

\section{Description}

Male. Not known at this time.

Female (Fig. 15). Length: 8-11 mm . Head (Fig. 15b): vertex 1/4 of head width; two reclinate upper orbital setae and two pairs of proclinate orbital setae; ocellar setae arising beside anterior ocellus; ocellar triangle gold; fronto-orbital plate gold throughout, sparse short setulae interspersed amongst frontal setae; parafacial shiny silver and bare; facial ridge bare; eye with short sparse ommatrichia up to $2 X$ as long as one ommatidium; pedicel black, concolorous with postpedicel; arista brown, very minutely pubescent, distinctly thickened on basal 1/3-1/4; palpus orange, sparsely haired and oar-shaped. Thorax (Fig. 15a, c): brilliant gold tomentose dorsally, contrasting with silver grey laterally; with black setulae along both dorsal and lateral surfaces; thoracic vittae fused into two prominent stripes spanning entire thorax, unbroken along suture; postpronotum with five setae arranged in a triangle; chaetotaxy: acrostichal setae 3:3; dorsocentral setae 3:4; intra-alar setae 2:3; supra-alar setae 2:3; two katepisternal setae; basal scutellar setae $1 / 2$ as long as subapical scutellar setae and slightly curving inwards medially; lateral scutellar setae subequal to basal scutellar setae; apical scutellar setae 1/3 length of subapical setae and straight; one pair of discal scutellar setae; scutellum concolorous with scutum, black over $50 \%$ of its surface with gold tomentum only along outer margin. Legs (Fig. 15c): black in ground colour; fore femur with dense silver tomentum on posterodorsal surface; hind coxa bare. Wing (Fig. 15a): pale translucent, hyaline, not distinctly infuscate; vein $\mathrm{R}_{4+5}$ with only $2-3$ setulae at base. Abdomen (Fig. 15a, c): ground colour black; middorsal depression on ST1+2 reaching hind margin; median marginal setae present on T3; a complete row of marginal setae present on T4; discal setae only on T5; light silver tomentose bands along anterior edge extending over $20 \%$ of T3 and T4, broken medially by a dorsocentral stripe; T5 with silver tomentum covering anterior half. Terminalia: not examined.

\section{Diagnosis}

Hyphantrophaga gilberthampiei sp. n. can be distinguished from all other Hyphantrophaga species by the following combination of traits: legs entirely black; dorsum of thorax gold tomentose with four dorsal vittae fused into two prominent dark lines extending across thoracic suture; scutellum black over $50 \%$ of its surface, with gold tomentum only along margin; ground colour of abdomen black, T4 with a light dusting of silver tomentum covering approximately $20 \%$ of dorsal surface of tergite; whole abdomen with a velvet black texture and colour. 


\section{Etymology}

Hyphantrophaga gilberthampiei sp. $\mathbf{n}$. is named in recognition of Gilberth Ampie Cruz's dedication and work in finding and rearing the ACG caterpillars that contained tachinid larvae.

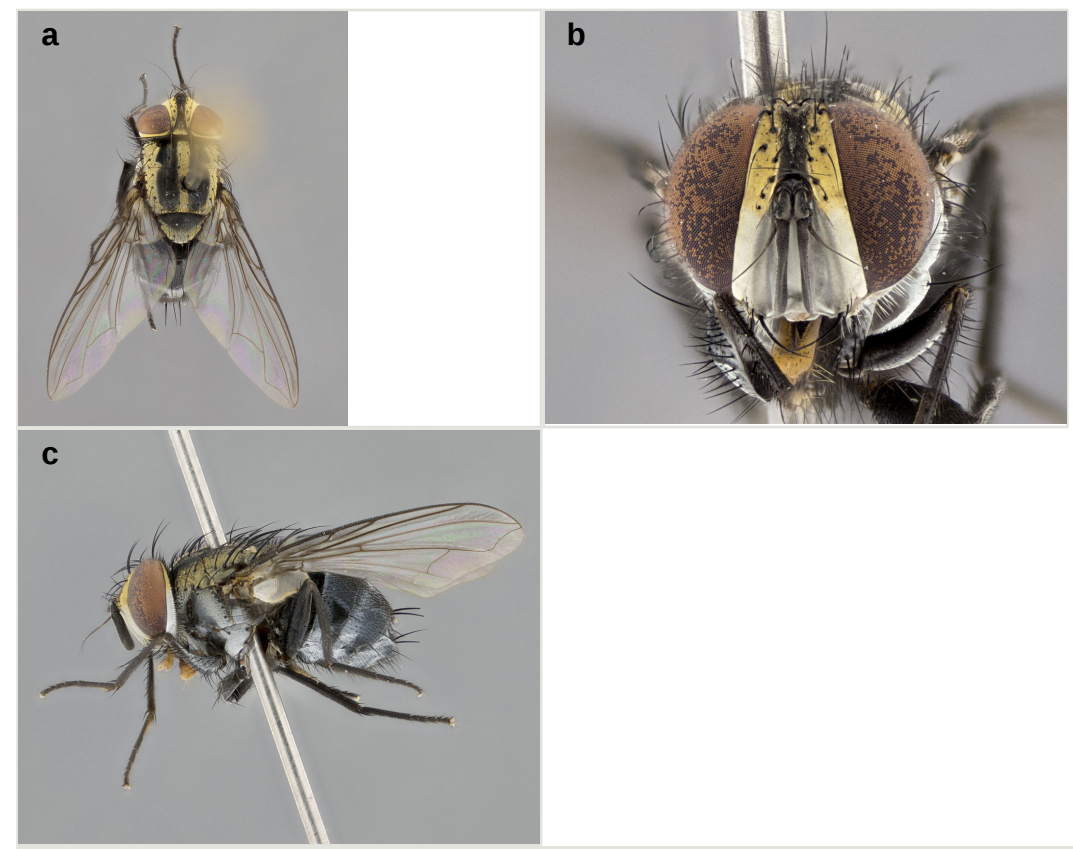

Figure 15.

Hyphantrophaga gillberthampiei sp. n.; a-c: habitus; holotype female, voucher n. DHJPAR0059246.
a: dorsal view doi
b: frontal view doi
c: lateral view doi

\section{Distribution}

Costa Rica, ACG, Guanacaste Province, 900 m elevation.

\section{Ecology}

Hyphantrophaga gilberthampiei sp. $\mathbf{n}$. has been reared once from one species of Lepidoptera in the family Hesperiidae, Cynea megalops (Godman, 1900), in dry forest. 


\section{Hyphantrophaga guillermopereirai Fleming \& Wood, sp. $\mathrm{n}$.}

- ZooBank urn:Isid:Zoobank.org:act:D6D2B422-709C-4B56-A412-6720D7F9AE6D

\section{Materials}

Holotype:

a. scientificName: Hyphantrophaga guillermopereirai; phylum: Arthropoda; class: Insecta; order: Diptera; family: Tachinidae; genus: Hyphantrophaga; specificEpithet: guillermopereirai; scientificNameAuthorship: Fleming \& Wood, 2017; continent: Central America; country: Costa Rica; countryCode: CR; stateProvince: Guanacaste; county: Potrerillos; locality: Area de Conservacion Guanacaste; verbatimLocality: Rio Azufrado; verbatimElevation: 95; verbatimLatitude: 10.8122; verbatimLongitude: -85.5444; verbatimCoordinateSystem: Decimal; decimalLatitude: 10.8122; decimalLongitude: -85.5444; samplingProtocol: Reared from the larva of the Nymphalidae, Agraulis vanillae; verbatimEventDate: 22-Aug-2000; individualID: DHJPAR0007280; individualCount: 1; sex: male; lifeStage: adult; preparations: pinned; catalogNumber: DHJPAR0007280; occurrenceDetails: http://janzen.sas.upenn.edu; recordedBy: D.H. Janzen, W. Hallwachs \& Manuel Pereira; otherCatalogNumbers: ASTAT052-06, 00SRNP-16230, BOLD:AAA1953; identifiedBy: AJ Fleming; dateldentified: 2017; language: en; institutionCode: CNC; collectionCode: Insects; basisOfRecord: Pinned Specimen

\section{Paratypes:}

a. ScientificName: Hyphantrophaga guillermopereirai; phylum: Arthropoda; class: Insecta; order: Diptera; family: Tachinidae; genus: Hyphantrophaga; specificEpithet: guillermopereirai; scientificNameAuthorship: Fleming \& Wood, 2017; continent: Central America; country: Costa Rica; countryCode: CR; stateProvince: Guanacaste; county: Sector Santa Rosa; locality: Area de Conservacion Guanacaste; verbatimLocality: Bosque Humedo; verbatimElevation: 290; verbatimLatitude: 10.8514; verbatimLongitude: -85.608; verbatimCoordinateSystem: Decimal; decimalLatitude: 10.8514; decimalLongitude: -85.608; samplingProtocol: Reared from the larva of the Hesperiidae, Polyctor cleta; verbatimEventDate: 30-Nov-1992; individualID:

DHJPAR0007277; individualCount: 1; sex: male; lifeStage: adult; preparations: pinned; catalogNumber: DHJPAR0007277; occurrenceDetails: http://janzen.sas.upenn.edu; recordedBy: D.H. Janzen, W. Hallwachs \& gusaneros; otherCatalog Numbers: ASTAT049-06, 92-SRNP-5826, BOLD:AAA1953; identifiedBy: AJ Fleming; dateldentified: 2017; language: en; institutionCode: CNC; collectionCode: Insects; basisOfRecord: Pinned Specimen

b. $\quad$ scientificName: Hyphantrophaga guillermopereirai; phylum: Arthropoda; class: Insecta; order: Diptera; family: Tachinidae; genus: Hyphantrophaga; specificEpithet: guillermopereirai; scientificNameAuthorship: Fleming \& Wood, 2017; continent: Central America; country: Costa Rica; countryCode: CR; stateProvince: Guanacaste; county: Sector El Hacha; locality: Area de Conservacion Guanacaste; verbatimLocality: Estacion Los Almendros; verbatimElevation: 290; verbatimLatitude: 11.0323; verbatimLongitude: -85.5278; verbatimCoordinateSystem: Decimal; decimalLatitude: 11.0323; decimalLongitude: -85.5278; samplingProtocol: Reared from the larva of the Erebidae, Renodes curviluna; verbatimEventDate: 12-Apr-2012; individualID: DHJPAR0048680; individualCount: 1; sex: male; lifeStage: adult; preparations: pinned; catalogNumber: DHJPAR0048680; occurrenceDetails: http://janzen.sas.upenn.edu; recordedBy: D.H. Janzen, W. Hallwachs \& Lucia Rios; otherCatalog Numbers: 
ACGBA2222-12, 12-SRNP-20677, BOLD:AAA1953; identifiedBy: AJ Fleming; dateldentified: 2017; language: en; institutionCode: CNC; collectionCode: Insects; basisOfRecord: Pinned Specimen

c. scientificName: Hyphantrophaga guillermopereirai; phylum: Arthropoda; class: Insecta; order: Diptera; family: Tachinidae; genus: Hyphantrophaga; specificEpithet: guillermopereirai; scientificNameAuthorship: Fleming \& Wood, 2017; continent: Central America; country: Costa Rica; countryCode: CR; stateProvince: Guanacaste; county: Sector Santa Rosa; locality: Area de Conservacion Guanacaste; verbatimLocality: Bosque Humedo; verbatimElevation: 290; verbatimLatitude: 10.8514; verbatimLongitude: -85.608; verbatimCoordinateSystem: Decimal; decimalLatitude: 10.8514; decimalLongitude: -85.608; samplingProtocol: Reared from the larva of the Erebidae, Glaucostola romula; verbatimEventDate: 12-Jan-1993; individualID:

DHJPAR0007303; individualCount: 1; sex: male; lifeStage: adult; preparations: pinned; catalogNumber: DHJPAR0007303; occurrenceDetails: http://janzen.sas.upenn.edu; recordedBy: D.H. Janzen, W. Hallwachs \& gusaneros; otherCatalogNumbers: ASTAT075-06, 93-SRNP-7336, BOLD:AAA1953; identifiedBy: AJ Fleming; dateldentified: 2017; language: en; institutionCode: CNC; collectionCode: Insects; basisOfRecord: Pinned Specimen

d. scientificName: Hyphantrophaga guillermopereirai; phylum: Arthropoda; class: Insecta; order: Diptera; family: Tachinidae; genus: Hyphantrophaga; specificEpithet: guillermopereirai; scientificNameAuthorship: Fleming \& Wood, 2017; continent: Central America; country: Costa Rica; countryCode: CR; stateProvince: Guanacaste; county: Sector Mundo Nuevo; locality: Area de Conservacion Guanacaste; verbatimLocality: Sendero Mora; verbatimElevation: 480; verbatimLatitude: 10.7683; verbatimLongitude: -85.4257; verbatimCoordinateSystem: Decimal; decimalLatitude: 10.7683; decimalLongitude: -85.4257; samplingProtocol: Reared from the larva of the Hesperiidae, Chiomara georgina; verbatimEventDate: 15-Jan-2006; individualID: DHJPAR0007029; individualCount: 1; sex: female; lifeStage: adult; preparations: pinned; catalogNumber: DHJPAR0007029; occurrenceDetails: http:// janzen.sas.upenn.edu; recordedBy: D.H. Janzen, W. Hallwachs \& Jose Cortez; otherCatalogNumbers: ASTAV271-06, 05-SRNP-66375, BOLD:AAA1953; identifiedBy: AJ Fleming; dateldentified: 2017; language: en; institutionCode: CNC; collectionCode: Insects; basisOfRecord: Pinned Specimen

e. $\quad$ scientificName: Hyphantrophaga guillermopereirai; phylum: Arthropoda; class: Insecta; order: Diptera; family: Tachinidae; genus: Hyphantrophaga; specificEpithet: guillermopereirai; scientificNameAuthorship: Fleming \& Wood, 2017; continent: Central America; country: Costa Rica; countryCode: CR; stateProvince: Guanacaste; county: Sector El Hacha; locality: Area de Conservacion Guanacaste; verbatimLocality: Sendero Bejuquilla; verbatimElevation: 280; verbatimLatitude: 11.03; verbatimLongitude: $\mathbf{8 5 . 5 2 7}$; verbatimCoordinateSystem: Decimal; decimalLatitude: 11.03; decimalLongitude: -85.527; samplingProtocol: Reared from the larva of the Hesperiidae, Tosta gorgus; verbatimEventDate: 14-Feb-2004; individualID: DHJPAR0007244; individualCount: 1; sex: female; lifeStage: adult; preparations: pinned; catalogNumber: DHJPAR0007244; occurrenceDetails: http:// janzen.sas.upenn.edu; recordedBy: D.H. Janzen, W. Hallwachs \& Elieth Cantillano; otherCatalog Numbers: ASTAT016-06, 04-SRNP-20122, BOLD:AAA1953; identifiedBy: AJ Fleming; dateldentified: 2017; language: en; institutionCode: CNC; collectionCode: Insects; basisOfRecord: Pinned Specimen 


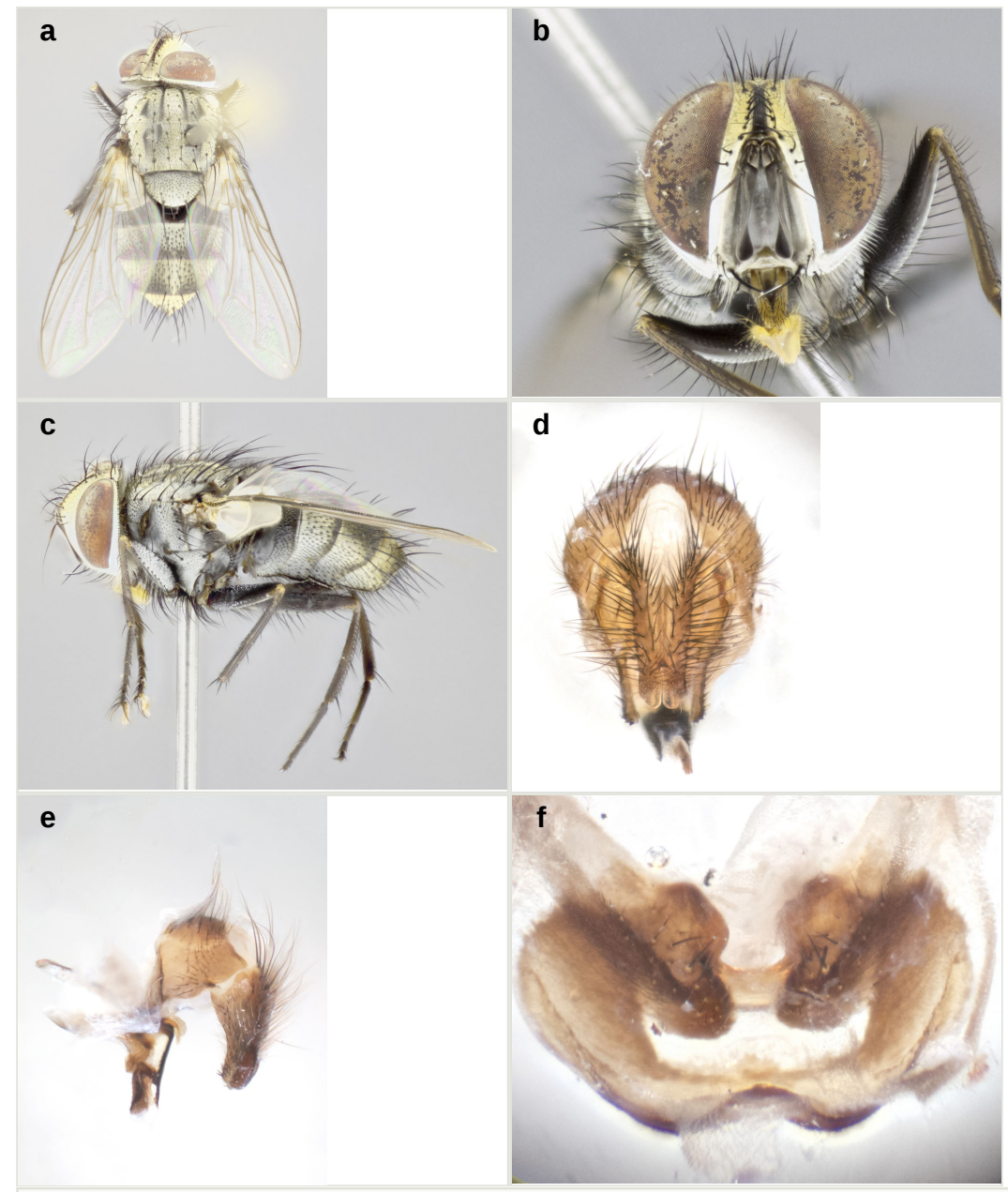

Figure 16.

Hyphantrophaga guillermopereirai sp. n.; a-c: habitus; holotype male, voucher $\mathrm{n}$. DHJPAR0007280; d-f: terminalia; male paratype, voucher n. DHJPAR0048680.
a: dorsal view doi
b: frontal view doi
c: lateral view doi
d: dorsal view doi
e: lateral view doi
f: sternite 5 , ventral view doi

\section{Description}

Male (Fig. 16). Length: 5-8 mm. Head (Fig. 16b): vertex 1/5 of head width; two reclinate upper orbital setae; ocellar setae arising beside anterior ocellus; ocellar triangle gold, concolorous with fronto-orbital plate; fronto-orbital plate gold over $75 \%$ of surface, sparsely setulose, setulae not extending beyond lowest frontal seta; parafacial 
shiny silver and bare; facial ridge bare; pedicel dark brown, slightly lighter than postpedicel; arista brown, very minutely pubescent, distinctly thickened on basal 1/31/4; palpus yellow and haired. Thorax (Fig. 16a, c): pale brassy-gold tomentose dorsally, gray tomentose laterally; four prominent dorsal vittae, outermost two broken across suture, innermost pair slightly broken across suture, not reaching beyond 2nd postsutural dorsocentral seta; postpronotum with three setae arranged in a triangle; chaetotaxy: acrostichal setae 3:3; dorsocentral setae 3-4:4; intra-alar setae 2-3:3; supra-alar setae 2:3; three katepisternal setae, basal seta extremely weak and anterior to suture; basal scutellar setae 3/4 length of subapical scutellar setae; lateral scutellar setae $2 / 3$ as long as basal scutellar setae, lateral scutellar setae $1 / 2$ as thick as both basal and subapical scutellar setae; subapical scutellar setae straight and divergent, $2 \mathrm{X}$ as thick as lateral scutellar setae; apical scutellar setae $1 / 3$ basal scutellar setae crossed apically; one pair of discal scutellar setae more widely set than subapical scutellar setae; scutellum concolorous with scutum. Legs (Fig. 16c): yellow in ground colour with dense covering of black hairs, making them appear darker; fore femur with dense silver tomentum on posterodorsal surface; hind coxa setose. Wing (Fig. 16a): pale translucent; vein $\mathrm{R}_{4+5}$ with 2-3 setulae at base. Abdomen (Fig. 16a, c): ground colour black; mid-dorsal depression on ST1+2 not reaching hind margin; marginal setae ST1+2-T5: median marginal setae present on ST1+2 and T3; a complete row of marginal setae present on T4 and T5; discal setae on T3-T5; sex patch absent; distinct brassy-silver tomentose bands along anterior 2/3 of T3 and T4; T5 with brassy-silver tomentum covering entire tergite. Terminalia (Fig. 16d, e, f): anterior margin of sternite 5 (Fig. 16f) with a shallow curved medial depression, posterior margin with a deeply excavated median cleft, rectangular-shaped; posterior lobes of sternite rounded, toothed apically and completely devoid of setae, inner margins of lobes sinusoidal undulated; unsclerotised "window" anterior to median cleft convex, arced, "arms" extending $3 \mathrm{X}$ as wide as median cleft. Cerci (Fig. 16d) in posterior view subrectangular and slightly shorter than surstyli, blunt and rounded off towards apices, completely separate medially, straight, not obviously divergent from each other; in lateral view (Fig. 16e) strongly tapered along apical $1 / 3$ and weakly curved at beginning of taper; densely setulose dorsally up to taper point, apparently bare ventrally (visible in lateral view). Surstyli in lateral view slightly swollen basally, otherwise almost parallel sided, with rounded apices; when viewed dorsally, surstyli appearing not divergent. Pregonite broad and well-developed, slightly bent; basal $2 / 3$ slightly cinched, giving it a very slightly clubbed appearance, apically rounded, few fine setulae along its edge. Postgonite elongate, parallel-sided along its length, with a slight curve at tip, subequal in length to pregonite. Distiphallus rectangular with a very slight apical flare, with a slender median longitudinal sclerotised reinforcement on its posterior surface and a broad, anterolateral, sclerotised acrophallus, joined as a plate on anterior surface near apex.

Female. Length $6-8 \mathrm{~mm}$. As male, differing only by the presence of two pairs of proclinate orbital setae. 


\section{Diagnosis}

Hyphantrophaga guillermopereirai sp. n. can be distinguished from all other Hyphantrophaga species by the following combination of traits: fronto-orbital plate gold over most of its surface and bare, except at level of vertex, pedicel and arista brown (slightly lighter than postpedicel), thorax with both silver and gold tomentum, four postsutural dorsocentral setae, hind coxa setose, marginal setae present on ST1+2-T5 and discal setae present on T3-T5.

\section{Etymology}

Hyphantrophaga guillermopereirai sp. $\mathbf{n}$. is named in recognition of Guillermo Pereira Espinoza's dedication and work in finding and rearing the ACG caterpillars that contained tachinid larvae.

\section{Distribution}

Costa Rica, ACG, Alajuela and Guanacaste Provinces, 10-752 m elevation.

\section{Ecology}

Hyphantrophaga guillermopereirai sp. $\mathbf{n}$. has been reared 93 times from 56 species of Lepidoptera spanning across 10 families (Lepidoptera: Crambidae; Erebidae; Geometridae; Hesperiidae; Noctuidae; Notodontidae; Nolidae; Nymphalidae; Riodinidae; Saturniidae), in rain forest, dry forest and dry-rain lowland intergrade.

\section{Hyphantrophaga hazelcambroneroae Fleming \& Wood, sp. n.}

- ZooBank urn:Isid:zoobank.org:act:3C8894B8-AAB7-46E2-BF26-AEAC3C3A0169

\section{Materials}

Holotype:

a. ScientificName: Hyphantrophaga hazelcambroneroae; phylum: Arthropoda; class: Insecta; order: Diptera; family: Tachinidae; genus: Hyphantrophaga; specificEpithet: hazelcambroneroae; scientificNameAuthorship: Fleming \& Wood, 2017; continent: Central America; country: Costa Rica; countryCode: CR; stateProvince: Guanacaste; county: Sector Santa Rosa; locality: Area de Conservacion Guanacaste; verbatimLocality: Bosque San Emilio; verbatimElevation: 300; verbatimLatitude: 10.8439; verbatimLongitude: -85.6138; verbatimCoordinateSystem: Decimal; decimalLatitude: 10.8439; decimalLongitude: -85.6138; samplingProtocol: Reared from the larva of the Crambidae, Hoterodes ausonia; verbatimEventDate: 11-Jul-1993; individualID: DHJPAR0007278; individualCount: 1; sex: male; lifeStage: adult; preparations: pinned; catalogNumber: DHJPAR0007278; occurrenceDetails: http:// janzen.sas.upenn.edu; recordedBy: D.H. Janzen, W. Hallwachs and gusaneros; otherCatalog Numbers: ASTAT050-06, 93-SRNP-6894,; identifiedBy: AJ Fleming; dateldentified: 2017; language: en; institutionCode: CNC; collectionCode: Insects; basisOfRecord: Pinned Specimen 


\section{Paratypes:}

a. ScientificName: Hyphantrophaga hazelcambroneroae; phylum: Arthropoda; class: Insecta; order: Diptera; family: Tachinidae; genus: Hyphantrophaga; specificEpithet: hazelcambroneroae; scientificNameAuthorship: Fleming \& Wood, 2017; continent: Central America; country: Costa Rica; countryCode: CR; stateProvince: Guanacaste; county: Sector Santa Rosa; locality: Area de Conservacion Guanacaste; verbatimLocality: Area Administrativa; verbatimElevation: 295; verbatimLatitude: 10.8376; verbatimLongitude: -85.6187; verbatimCoordinateSystem: Decimal; decimalLatitude: 10.8376; decimalLongitude: -85.6187; samplingProtocol: Reared from the larva of the Crambidae, Portentomorpha xanthialis; verbatimEventDate: 20 Aug-2013; individualID: DHJPAR0052525; individualCount: 1; sex: male; lifeStage: adult; preparations: pinned; catalogNumber: DHJPAR0052525; occurrenceDetails: htt p://janzen.sas.upenn.edu; recordedBy: D.H. Janzen, W. Hallwachs and Guillermo Pereira; otherCatalog Numbers: ASHYM1879-13, 13-SRNP-19665, BOLD:ACJ4813; identifiedBy: AJ Fleming; dateldentified: 2017; language: en; institutionCode: CNC; collectionCode: Insects; basisOfRecord: Pinned Specimen

b. ScientificName: Hyphantrophaga hazelcambroneroae; phylum: Arthropoda; class: Insecta; order: Diptera; family: Tachinidae; genus: Hyphantrophaga; specificEpithet: hazelcambroneroae; scientificNameAuthorship: Fleming \& Wood, 2017; continent: Central America; country: Costa Rica; countryCode: CR; stateProvince: Guanacaste; county: Sector Santa Rosa; locality: Area de Conservacion Guanacaste; verbatimLocality: Area Administrativa; verbatimElevation: 295; verbatimLatitude: 10.8376; verbatimLongitude: -85.6187; verbatimCoordinateSystem: Decimal; decimalLatitude: 10.8376; decimalLongitude: -85.6187; samplingProtocol: Reared from the larva of the Crambidae, Portentomorpha xanthialis; verbatimEventDate: 14Aug-2013; individualID: DHJPAR0052653; individualCount: 1; sex: male; lifeStage: adult; preparations: pinned; catalogNumber: DHJPAR0052653; occurrenceDetails: htt p://janzen.sas.upenn.edu; recordedBy: D.H. Janzen, W. Hallwachs and Johan Vargas; otherCatalog Numbers: ASHYM2007-13, 13-SRNP-19649, BOLD:ACJ4813; identifiedBy: AJ Fleming; dateldentified: 2017; language: en; institutionCode: CNC; collectionCode: Insects; basisOfRecord: Pinned Specimen

c. ScientificName: Hyphantrophaga hazelcambroneroae; phylum: Arthropoda; class: Insecta; order: Diptera; family: Tachinidae; genus: Hyphantrophaga; specificEpithet: hazelcambroneroae; scientificNameAuthorship: Fleming \& Wood, 2017; continent: Central America; country: Costa Rica; countryCode: CR; stateProvince: Guanacaste; county: Sector Santa Rosa; locality: Area de Conservacion Guanacaste; verbatimLocality: Area Administrativa; verbatimElevation: 295; verbatimLatitude: 10.8376; verbatimLongitude: -85.6187; verbatimCoordinateSystem: Decimal; decimalLatitude: 10.8376; decimalLongitude: -85.6187; samplingProtocol: Reared from the larva of the Crambidae, Portentomorpha xanthialis; verbatimEventDate: 20 Aug-2013; individualID: DHJPAR0052531; individualCount: 1; sex: male; lifeStage: adult; preparations: pinned; catalogNumber: DHJPAR0052531; occurrenceDetails: htt p://janzen.sas.upenn.edu; recordedBy: D.H. Janzen, W. Hallwachs and Guillermo Pereira; otherCatalogNumbers: ASHYM1885-13, 13-SRNP-19667, BOLD:ACJ4813; identifiedBy: AJ Fleming; dateldentified: 2017; language: en; institutionCode: CNC; collectionCode: Insects; basisOfRecord: Pinned Specimen

d. ScientificName: Hyphantrophaga hazelcambroneroae; phylum: Arthropoda; class: Insecta; order: Diptera; family: Tachinidae; genus: Hyphantrophaga; specificEpithet: hazelcambroneroae; scientificNameAuthorship: Fleming \& Wood, 2017; continent: Central America; country: Costa Rica; countryCode: CR; stateProvince: Guanacaste; 


\begin{abstract}
county: Sector Santa Rosa; locality: Area de Conservacion Guanacaste; verbatimLocality: Area Administrativa; verbatimElevation: 295; verbatimLatitude: 10.8376; verbatimLongitude: -85.6187; verbatimCoordinateSystem: Decimal; decimalLatitude: 10.8376; decimalLongitude: -85.6187; samplingProtocol: Reared from the larva of the Crambidae, Portentomorpha xanthialis; verbatimEventDate: 20Aug-2013; individualID: DHJPAR0052540; individualCount: 1; sex: male; lifeStage: adult; preparations: pinned; catalogNumber: DHJPAR0052540; occurrenceDetails: htt p://janzen.sas.upenn.edu; recordedBy: D.H. Janzen, W. Hallwachs and Guillermo Pereira; otherCatalogNumbers: ASHYM1894-13, 13-SRNP-19685, BOLD:ACJ4813; identifiedBy: AJ Fleming; dateldentified: 2017; language: en; institutionCode: CNC; collectionCode: Insects; basisOfRecord: Pinned Specimen
\end{abstract}

\title{
Description
}

Male (Fig. 17). Length: 5-8 mm. Head (Fig. 17b): vertex 1/4 of head width; two strong reclinate upper orbital setae, these not in line with frontal row (one aberrant male displaying one proclinate orbital seta in addition to the two reclinate pairs, was initially confused as female); ocellar setae arising behind anterior ocellus; ocellar triangle gold, concolorous with upper half of fronto-orbital plate; fronto-orbital plate gold over $50 \%$ of surface, sparsely setulose, setulae not extending beyond lowest frontal seta; parafacial shiny silver and bare; facial ridge haired up to $1 / 2$ its surface, these being strong and widely spaced; pedicel dark brown, slightly lighter than postpedicel; arista brown, very minutely pubescent, distinctly thickened on basal 1/3-1/4; palpus yellow and haired, not clubbed. Thorax (Fig. 17a, c): brassy-gold tomentose dorsally, grey tomentose laterally; four prominent dorsal vittae, outermost two broken across suture, innermost pair slightly broken across suture, not reaching beyond 2nd postsutural dorsocentral seta; postpronotum with three setae arranged in a triangle; chaetotaxy: acrostichal setae 3:3; dorsocentral setae 3:4 (second postsutural seta often reduced and weaker than the other three); intra-alar setae 2:3; supra-alar setae 2:3; two katepisternal setae; lateral scutellar setae $2 / 3$ as long as basal scutellar setae, lateral scutellar setae $1 / 2$ as thick as both basal and subapical scutellar setae; subapical scutellar setae strongest and longest of marginal scutellar setae, strongly divergent; apical scutellar setae shorter than basal scutellar setae crossed apically; one pair of discal scutellar setae; scutellum concolorous with scutum, very slightly darker than thorax along basal $50 \%$. Legs (Fig. 17c): reddish-black in ground colour with dense covering of black hairs, making them appear darker; fore femur with dense silver tomentum on posterodorsal surface; hind coxa bare. Wing (Fig. 17a): pale translucent; vein $\mathrm{R}_{4+5}$ with $2-3$ setulae at base. Abdomen (Fig. 17a, c): ground colour black; middorsal depression on ST1+2 reaching hind margin; median marginal setae present on ST1+2 and T3; a complete row of marginal setae present on T4; discal setae on T3-T5; sex patch absent; distinct brassy-gold tomentose bands dorsally along anterior $2 / 3$ of T3 and T4; T5 with brassygold tomentum covering entire tergite; T3-T5 silver tomentose ventrally. Terminalia: anterior margin of sternite 5 (Fig. 17f) with a deep curved medial depression, posterior margin with a deeply excavated median cleft, two pyramid-shaped lobes; posterior lobes of sternite slightly pointed apically, 1-2 longer setae surrounded by shorter setulae, inner margins of cleft straight with a slight undulation, round cylindrical basally; 
unsclerotised "window" anterior to median cleft convex, with slender "arms" extending $3 \mathrm{X}$ as wide as median cleft with a short rectangular stalk. Cerci (Fig. 17d) in posterior view almost rectangular and subequal in length to surstyli, blunt and rounded off towards apices, separate medially on anterior $1 / 3$, straight and slightly divergent when viewed dorsally, remaining 2/3 fused medially; in lateral view (Fig. 17e), almost parallelsided along entire length, anterior $1 / 3$ with a strong downward curve; densely setulose dorsally up to curve point, apparently bare ventrally (visible in lateral view). Surstyli in lateral view parallel-sided along their entire length, with rounded apices, strongly curved along anterior 1/3; when viewed dorsally, surstyli appearing not divergent. Pregonite broad and well-developed, slightly bent; basal 2/3 slightly cinched, giving it a very slightly clubbed appearance, apically rounded, few fine setulae along its edge. Postgonite elongate, parallel-sided along its length, with a slight curve at tip, subequal in length to pregonite. Distiphallus sail-shaped, with a very strong apical flare, a slender median longitudinal sclerotised reinforcement on its posterior surface and a broad, anterolateral, sclerotised acrophallus, joined as a plate on anterior surface near apex.

Female. Unknown at this time.

\section{Diagnosis}

Hyphantrophaga hazelcambroneroae sp. n. can be distinguished from all other Hyphantrophaga species by the following combination of traits: facial ridge with setae along half its length, four dorsocentral setae, two katepisternal setae, hind coxa bare, both abdomen and thorax brassy tomentose throughout, abdominal ground color black and median marginal setae present on ST1+2.

\section{Etymology}

Hyphantrophaga hazelcambroneroae sp. $\mathbf{n}$. is named in recognition of Hazel Cambronero Romero's dedication and work in finding and rearing the ACG caterpillars that contained tachinid larvae.

\section{Distribution}

Costa Rica, ACG, Guanacaste Province, 295-300 m elevation.

\section{Ecology}

Hyphantrophaga hazelcambroneroae sp. n. has been reared five times from two species of Lepidoptera in the family Crambidae, Hoterodes ausonia Cramer, 1777 and Portentomorpha xanthialis (Guenée, 1854), in dry forest. 

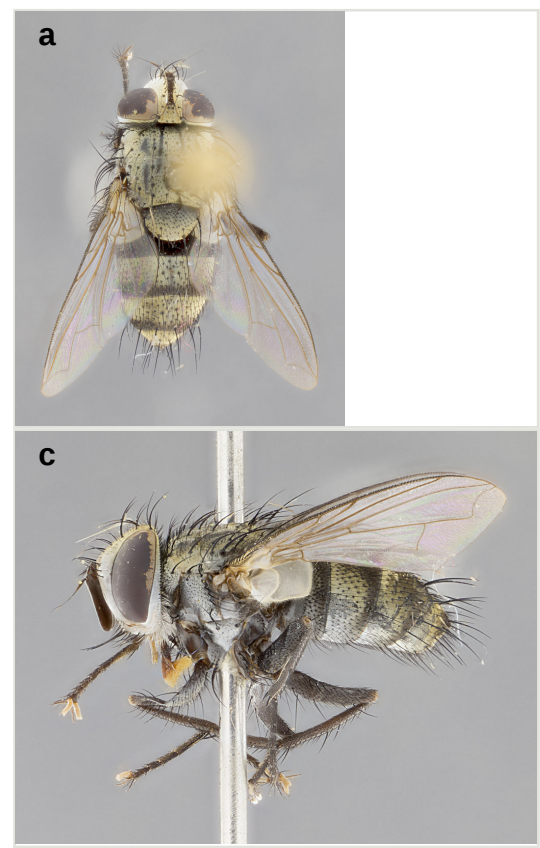

e

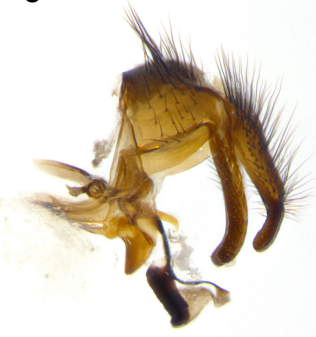

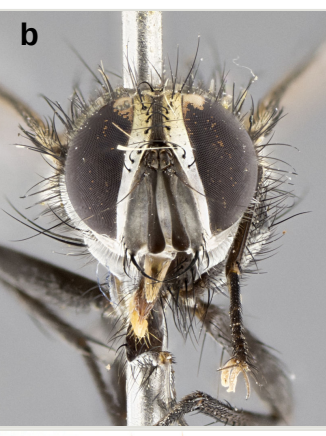

d

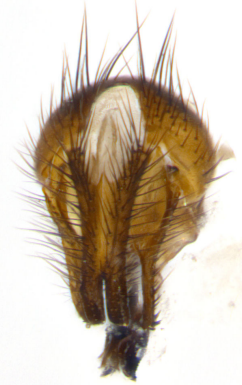

\section{f}

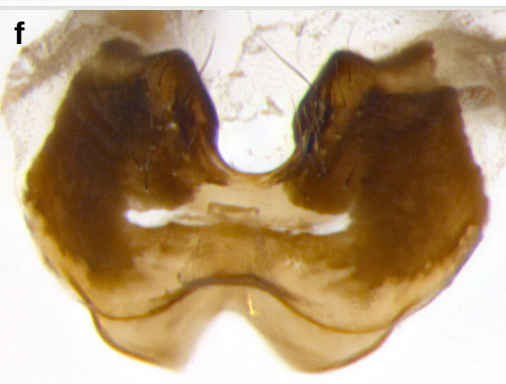

Figure 17.

Hyphantrophaga hazelcambroneroae sp. n.; a-c: habitus; holotype male, voucher n. DHJPAR0007278; d-f: terminalia; male paratype, voucher $n$. DHJPAR0052653.

\section{Hyphantrophaga luciariosae Fleming \& Wood, sp. n.}

- ZooBank urn:Isid:zoobank.org:act:1DBE5C00-14E2-41B9-B9CF-7E7ED59CD497

\section{Materials}

\section{Holotype:}

a. ScientificName: Hyphantrophaga luciariosae; phylum: Arthropoda; class: Insecta; order: Diptera; family: Tachinidae; genus: Hyphantrophaga; specificEpithet: Iuciariosae; scientificNameAuthorship: Fleming \& Wood, 2018; continent: Central America; country: Costa Rica; countryCode: CR; stateProvince: Guanacaste; county: Sector Orosi; Iocality: Area de Conservacion Guanacaste; verbatimLocality: Sendero Orosi; verbatimElevation: 620; verbatimLatitude: 10.9937; verbatimLongitude: -85.4639; 
verbatimCoordinateSystem: Decimal; decimalLatitude: 10.9937; decimalLongitude: -85.4639; samplingProtocol: Reared from the larva of the Sphingidae, Xylophanes juanita; verbatimEventDate: 26-Jul-2001; individualID: DHJPAR0008095; individualCount: 1; sex: male; lifeStage: adult; preparations: pinned; catalogNumber: DHJPAR0008095; occurrenceDetails: http://janzen.sas.upenn.edu; recordedBy: D.H. Janzen, W. Hallwachs \& Roster Moraga; otherCatalogNumbers: ASTAT867-06, 01SRNP-9682, BOLD:AAF3892; identifiedBy: AJ Fleming; dateldentified: 2017; language: en; institutionCode: CNC; collectionCode: Insects; basisOfRecord: Pinned Specimen

\section{Paratypes:}

a. ScientificName: Hyphantrophaga luciariosae; phylum: Arthropoda; class: Insecta; order: Diptera; family: Tachinidae; genus: Hyphantrophaga; specificEpithet: Iuciariosae; scientificNameAuthorship: Fleming \& Wood, 2018; continent: Central America; country: Costa Rica; countryCode: CR; stateProvince: Guanacaste; county: Sector Orosi; locality: Area de Conservacion Guanacaste; verbatimLocality: Sendero Orosi; verbatimElevation: 620; verbatimLatitude: 10.9937; verbatimLongitude: -85.4639; verbatimCoordinateSystem: Decimal; decimalLatitude: 10.9937; decimalLongitude: -85.4639; samplingProtocol: Reared from the larva of the Sphingidae, Xylophanes juanita; verbatimEventDate: 29-Jul-2001; individualID: DHJPAR0008061; individualCount: 1; sex: female; lifeStage: adult; preparations: pinned; catalogNumber: DHJPAR0008061; occurrenceDetails: http://janzen.sas.upenn.edu; recordedBy: D.H. Janzen, W. Hallwachs \& Roster Moraga; otherCatalogNumbers: ASTAT833-06, 01SRNP-9654, BOLD:AAF3892; identifiedBy: AJ Fleming; dateldentified: 2017; language: en; institutionCode: CNC; collectionCode: Insects; basisOfRecord: Pinned Specimen

b. $\quad$ scientificName: Hyphantrophaga luciariosae; phylum: Arthropoda; class: Insecta; order: Diptera; family: Tachinidae; genus: Hyphantrophaga; specificEpithet: luciariosae; scientificNameAuthorship: Fleming \& Wood, 2018; continent: Central America; country: Costa Rica; countryCode: CR; stateProvince: Guanacaste; county: Sector Orosi; Iocality: Area de Conservacion Guanacaste; verbatimLocality: Sendero Orosi; verbatimElevation: 620; verbatimLatitude: 10.9937; verbatimLongitude: -85.4639; verbatimCoordinateSystem: Decimal; decimalLatitude: 10.9937; decimalLongitude: -85.4639; samplingProtocol: Reared from the larva of the Sphingidae, Xylophanes juanita; verbatimEventDate: 16-Jul-2001; individualID: DHJPAR0008097; individualCount: 1; sex: male; lifeStage: adult; preparations: pinned; catalogNumber: DHJPAR0008097; occurrenceDetails: http://janzen.sas.upenn.edu; recordedBy: D.H. Janzen, W. Hallwachs \& Roster Moraga; otherCatalogNumbers: ASTAT869-06, 01SRNP-9680, BOLD:AAF3892; identifiedBy: AJ Fleming; dateldentified: 2017; language: en; institutionCode: CNC; collectionCode: Insects; basisOfRecord: Pinned Specimen

c. scientificName: Hyphantrophaga luciariosae; phylum: Arthropoda; class: Insecta; order: Diptera; family: Tachinidae; genus: Hyphantrophaga; specificEpithet: Iuciariosae; scientificNameAuthorship: Fleming \& Wood, 2018; continent: Central America; country: Costa Rica; countryCode: CR; stateProvince: Guanacaste; county: Sector Rincon Rain Forest; locality: Area de Conservacion Guanacaste; verbatimLocality: Malaguenya; verbatimElevation: 221; verbatimLatitude: 10.9555; verbatimLongitude: -85.2838; verbatimCoordinateSystem: Decimal; decimalLatitude: 10.9555 ; decimalLongitude: -85.2838; samplingProtocol: Reared from the larva of the Sphingidae, Xylophanes adalia; verbatimEventDate: 04-Feb-2016; individualID: DHJPAR0058396; 
individualCount: 1; sex: male; lifeStage: adult; preparations: pinned; catalogNumber: DHJPAR0058396; occurrenceDetails: http://janzen.sas.upenn.edu; recordedBy: D.H. Janzen, W. Hallwachs \& Kemberly Villalobos; otherCatalogNumbers: MHMYN7996-16, 16-SRNP-45023, BOLD:AAF3892; identifiedBy: AJ Fleming; dateldentified: 2017; language: en; institutionCode: CNC; collectionCode: Insects; basisOfRecord: Pinned Specimen

d. ScientificName: Hyphantrophaga luciariosae; phylum: Arthropoda; class: Insecta; order: Diptera; family: Tachinidae; genus: Hyphantrophaga; specificEpithet: Iuciariosae; scientificNameAuthorship: Fleming \& Wood, 2018; continent: Central America; country: Costa Rica; countryCode: CR; stateProvince: Guanacaste; county: Sector Pitilla; locality: Area de Conservacion Guanacaste; verbatimLocality: Sendero Naciente; verbatimElevation: 700; verbatimLatitude: 10.9871; verbatimLongitude: -85.4282; verbatimCoordinateSystem: Decimal; decimalLatitude: 10.9871; decimalLongitude: -85.4282; samplingProtocol: Reared from the larva of the Sphingidae, Xylophanes adalia; verbatimEventDate: Jan-19-2014; individualID: DHJPAR0054189; individualCount: 1; sex: male; lifeStage: adult; preparations: pinned; catalogNumber: DHJPAR0054189; occurrenceDetails: http://janzen.sas.upenn.edu; recordedBy: D.H. Janzen, W. Hallwachs \& Freddy Quesada; identifiedBy: AJ Fleming; dateldentified: 2017; language: en; institutionCode: CNC; collectionCode: Insects; basisOfRecord: Pinned Specimen

\section{Description}

Male (Fig. 18). Length: 7-10 mm. Head (Fig. 18b): vertex 1/4 of head width; two reclinate upper orbital setae; ocellar setae arising beside anterior ocellus; ocellar triangle slightly gold, changing to concolorous with fronto-orbital plate; fronto-orbital plate dark brassy-silver over $90 \%$ of surface, densely setulose, setulae not extending beyond lowest frontal seta; parafacial dull silver and bare; facial ridge bare; pedicel dark brown, slightly lighter than postpedicel; arista brown, very minutely pubescent, distinctly thickened on basal 1/3-1/4; palpus yellow, haired and oar-shaped. Thorax (Fig. 18a, c): pale brassy-gold tomentose dorsally, grey tomentose laterally; four prominent dorsal vittae, outermost two broken across suture, innermost pair unbroken across suture, reaching 3rd postsutural dorsocentral seta; postpronotum with five setae arranged in a triangle; chaetotaxy: acrostichal setae 3:3; dorsocentral setae 3:4; intraalar setae 3:3; supra-alar setae 2:3; two katepisternal setae; basal scutellar setae as long as scutellar setae; two pairs of lateral scutellar setae, $1 / 2$ as long as basal scutellar setae; subapical scutellar setae straight and divergent, $2 \mathrm{X}$ as thick as lateral scutellar setae; apical scutellar setae $1 / 3$ basal scutellar setae, slightly shorter than lateral scutellar setae, crossed apically; one pair of discal scutellar setae more widely set than subapical scutellar setae; scutellum darkened over basal $1 / 3$, remainder concolorous with scutum. Legs (Fig. 18c): reddish-brown ground colour; fore femur with dense silver tomentum on posterodorsal surface; hind coxa setose. Wing (Fig. 18a): pale translucent, hyaline, not distinctly infuscate; veins reddish; vein $R_{4+5}$ with only 2-3 setulae at base. Abdomen (Fig. 18a, c): ground colour reddish-brown; middorsal depression on ST1+2 almost reaching hind margin; median marginal setae present on ST1+2-T3; a complete row of marginal setae present on T4; discal setae only on T5; sex patch covering ventrolateral surfaces of T4-T5; distinct brassy 
tomentose bands along anterior edge of T3 and T4, broken medially by a dorsocentral stripe and covering almost $80 \%$ of tergites; T5 with brassy tomentum throughout. Terminalia: sternite 5 (Fig. 18f) with a deeply excavated median cleft, slightly rounded V-shaped, margins covered in dense tomentum. Lateral lobes of sternite rounded apically, with 2-3 strong setae surrounded by many shorter, weaker setulae. Anterior plate of sternite 5 from subequal to slightly shorter than apical lobes, unsclerotised "window" weak, only very slightly unsclerotised, as wide as median cleft and flat. Cerci in posterior view (Fig. 18d) subrectangular, duck-billed and slightly longer than surstyli, blunt and rounded at apex, completely separate medially, appearing slightly divergent; in lateral view with a strong downward curve in apical 1/3; densely setulose along basal $2 / 3$, setulose ventrally along entire length. Surstylus in lateral view (Fig. 18e) almost parallel-sided along its entire length, ending in a slightly downcurved apex, making the structure appear slightly bladelike; surstylus appearing to be fused with epandrium; when viewed dorsally, surstyli appearing to point inward and strongly convergent, laterally covered in short stout setulae. Pregonite short and stout, well-developed, 1/3 times as long as distiphallus, squared-off apically, with a few short marginal setulae. Postgonite slightly narrow, $1 / 3$ as wide as pregonite, sharply pointed and curved at apex. Distiphallus rectangular, with a very slight apical flare, a slender median longitudinal sclerotised reinforcement on its posterior surface and a broad, anterolateral, sclerotised acrophallus, joined as a plate on anterior surface near apex.

Female. Length: 8-11 mm. As male, differing only by the presence of two pairs of proclinate orbital setae.

\section{Diagnosis}

Hyphantrophaga luciariosae sp. n. can be distinguished from all other Hyphantrophaga species by the following combination of traits: thorax with four postsutural dorsocentral setae and two katepisternal setae, hind coxa setose, median marginal setae present on ST1+2, discal setae absent from T3 and T4.

\section{Etymology}

Hyphantrophaga luciariosae sp. $\mathbf{n}$. is named in recognition of Lucia Rios Castro's dedication and work in finding and rearing the ACG caterpillars that contained tachinid larvae.

\section{Distribution}

Costa Rica, ACG, Guanacaste Province, 10-480 m elevation.

\section{Ecology}

Hyphantrophaga luciariosae sp. $\mathbf{n}$. has been reared four times from two species of Lepidoptera in the family Sphingidae, Xylophanes juanita Rothschild \& Jordan, 1903 and Xylophanes adalia (Druce, 1881), in dry forest and dry-rain lowland intergrades. 

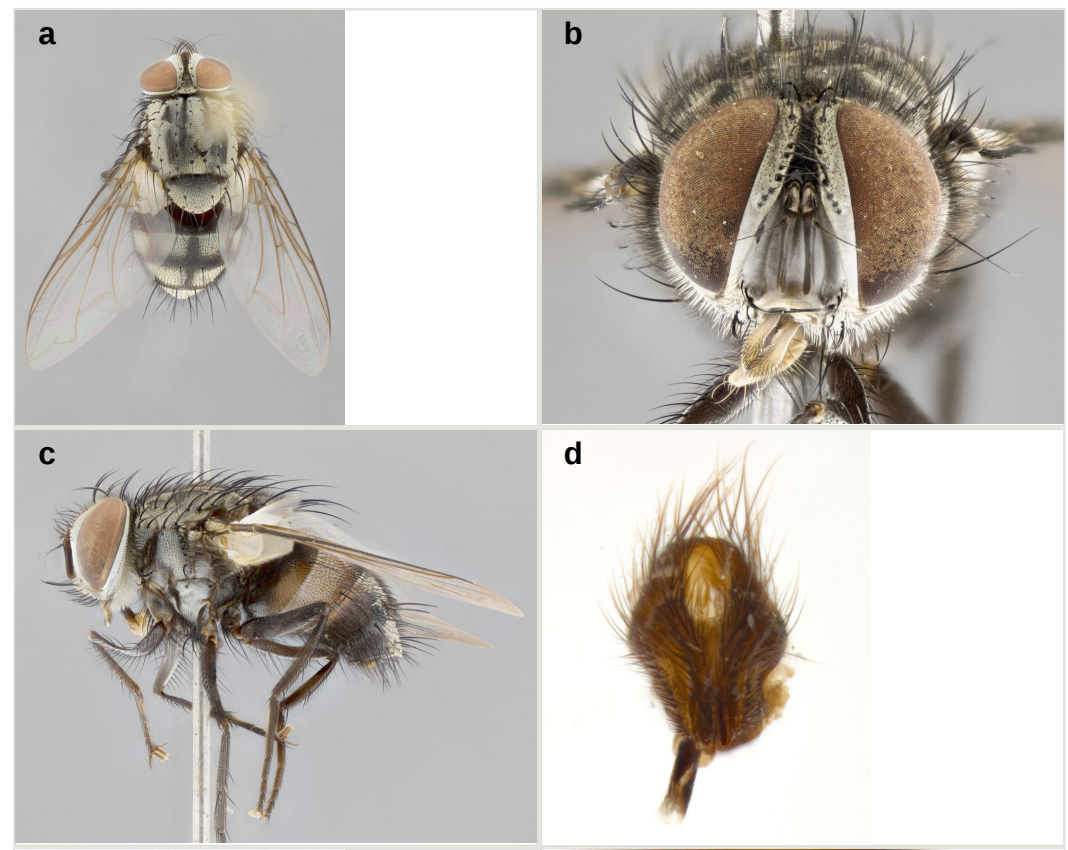

d
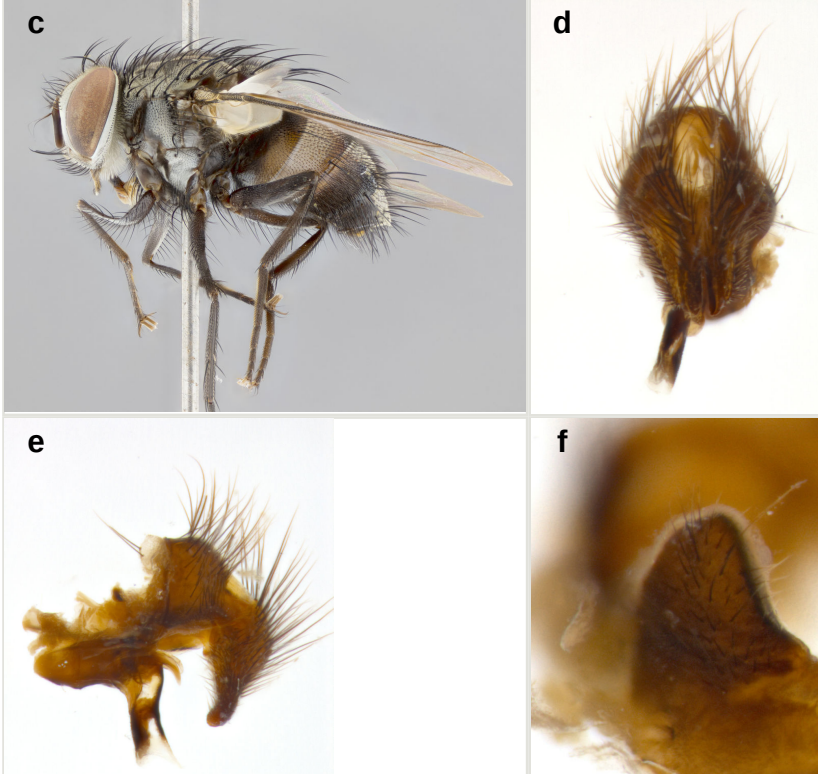

f

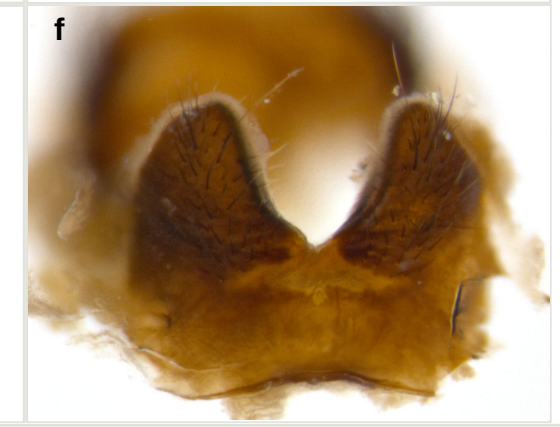

Figure 18.

Hyphantrophaga luciariosae sp. n.; a-c: habitus; holotype male, voucher $\mathrm{n}$. DHJPAR0008095; d-f: terminalia; male paratype, voucher n. DHJPAR0008097.
a: dorsal view doi
b: frontal view doi
c: lateral view doi
d: dorsal view doi
e: lateral view doi
f: sternite 5 , ventral view doi 


\section{Hyphantrophaga manuelriosi Fleming \& Wood, sp. $\mathrm{n}$.}

- ZooBank urn:Isid:zoobank.org:act:5ED9EBB0-36C2-4227-81CD-3712EA8697BB

\section{Materials}

Holotype:

a. $\quad$ scientificName: Hyphantrophaga manuelriosi; phylum: Arthropoda; class: Insecta; order: Diptera; family: Tachinidae; genus: Hyphantrophaga; specificEpithet: manuelriosi; scientificNameAuthorship: Fleming \& Wood, 2018; continent: Central America; country: Costa Rica; countryCode: CR; stateProvince: Guanacaste; county: Sector Santa Rosa; locality: Area de Conservacion Guanacaste; verbatimLocality: Cafetal; verbatimElevation: 280 ; verbatimLatitude: 10.8583; verbatimLongitude: -85.6109; verbatimCoordinateSystem: Decimal; decimalLatitude: 10.8583; decimalLongitude: -85.6109; samplingProtocol: Reared from the larva of the Crambidae, Syllepte belialis; verbatimEventDate: 06-Jul-1994; individualID: DHJPAR0007340; individualCount: 1; sex: male; lifeStage: adult; preparations: pinned; catalogNumber: DHJPAR0007340; occurrenceDetails: http://janzen.sas.upenn.edu; recordedBy: D.H. Janzen, W. Hallwachs \& gusaneros; otherCatalog Numbers: ASTAT112-06, 94-SRNP-2057, BOLD:AAA8930; identifiedBy: AJ Fleming; dateldentified: 2017; language: en; institutionCode: CNC; collectionCode: Insects; basisOfRecord: Pinned Specimen

\section{Paratypes:}

a. $\quad$ scientificName: Hyphantrophaga manuelriosi; phylum: Arthropoda; class: Insecta; order: Diptera; family: Tachinidae; genus: Hyphantrophaga; specificEpithet: manuelriosi; scientificNameAuthorship: Fleming \& Wood, 2018; continent: Central America; country: Costa Rica; countryCode: CR; stateProvince: Guanacaste; county: Sector Santa Rosa; locality: Area de Conservacion Guanacaste; verbatimLocality: Sendero Natural; verbatimElevation: 290; verbatimLatitude: 10.8357; verbatimLongitude: -85.6125 ; verbatimCoordinateSystem: Decimal; decimalLatitude: 10.8357; decimalLongitude: -85.6125; samplingProtocol: Reared from the larva of the Crambidae, Syllepte belialis; verbatimEventDate: 10-May-2002; individualID:

DHJPAR0007331; individualCount: 1; sex: male; lifeStage: adult; preparations: pinned; catalogNumber: DHJPAR0007331; occurrenceDetails: http://janzen.sas.upenn.edu; recordedBy: D.H. Janzen, W. Hallwachs \& gusaneros; otherCatalogNumbers: ASTAT103-06, 01-SRNP-13266, BOLD:AAA8930; identifiedBy: AJ Fleming; dateldentified: 2017; language: en; institutionCode: CNC; collectionCode: Insects; basisOfRecord: Pinned Specimen

b. $\quad$ scientificName: Hyphantrophaga manuelriosi; phylum: Arthropoda; class: Insecta; order: Diptera; family: Tachinidae; genus: Hyphantrophaga; specificEpithet: manuelriosi; scientificNameAuthorship: Fleming \& Wood, 2018; continent: Central America; country: Costa Rica; countryCode: CR; stateProvince: Guanacaste; county: Sector Santa Rosa; locality: Area de Conservacion Guanacaste; verbatimLocality: Sendero Natural; verbatimElevation: 290; verbatimLatitude: 10.8357; verbatimLongitude: -85.6125; verbatimCoordinateSystem: Decimal; decimalLatitude: 10.8357; decimalLongitude: -85.6125 ; samplingProtocol: Reared from the larva of the Crambidae, Syllepte belialis; verbatimEventDate: 01-May-2002; individualID: DHJPAR0007332; individualCount: 1; sex: male; lifeStage: adult; preparations: pinned; catalogNumber: DHJPAR0007332; occurrenceDetails: http://janzen.sas.upenn.edu; recordedBy: D.H. Janzen, W. Hallwachs \& gusaneros; otherCatalog Numbers: 
ASTAT104-06, 01-SRNP-13278, BOLD:AAA8930; identifiedBy: AJ Fleming; dateldentified: 2017; language: en; institutionCode: CNC; collectionCode: Insects; basisOfRecord: Pinned Specimen

c. ScientificName: Hyphantrophaga manuelriosi; phylum: Arthropoda; class: Insecta; order: Diptera; family: Tachinidae; genus: Hyphantrophaga; specificEpithet: manuelriosi; scientificNameAuthorship: Fleming \& Wood, 2018; continent: Central America; country: Costa Rica; countryCode: CR; stateProvince: Guanacaste; county: Sector Santa Rosa; locality: Area de Conservacion Guanacaste; verbatimLocality: Sendero Natural; verbatimElevation: 290; verbatimLatitude: 10.8357; verbatimLongitude: -85.6125; verbatimCoordinateSystem: Decimal; decimalLatitude: 10.8357; decimalLongitude: -85.6125; samplingProtocol: Reared from the larva of the Crambidae, Syllepte belialis; verbatimEventDate: 01-May-2002; individualID:

DHJPAR0007333; individualCount: 1; sex: male; lifeStage: adult; preparations: pinned; catalogNumber: DHJPAR0007333; occurrenceDetails: http://janzen.sas.upenn.edu; recordedBy: D.H. Janzen, W. Hallwachs \& gusaneros; otherCatalog Numbers: ASTAT105-06, 01-SRNP-13253, BOLD:AAA8930; identifiedBy: AJ Fleming; dateldentified: 2017; language: en; institutionCode: CNC; collectionCode: Insects; basisOfRecord: Pinned Specimen

d. $\quad$ scientificName: Hyphantrophaga manuelriosi; phylum: Arthropoda; class: Insecta; order: Diptera; family: Tachinidae; genus: Hyphantrophaga; specificEpithet: manuelriosi; scientificNameAuthorship: Fleming \& Wood, 2018; continent: Central America; country: Costa Rica; countryCode: CR; stateProvince: Guanacaste; county: Sector Santa Rosa; locality: Area de Conservacion Guanacaste; verbatimLocality: Bosque Humedo; verbatimElevation: 290; verbatimLatitude: 10.8514; verbatimLongitude: -85.608 ; verbatimCoordinateSystem: Decimal; decimalLatitude: 10.8514; decimalLongitude: -85.608; samplingProtocol: Reared from the larva of the Crambidae, Syllepte belialis; verbatimEventDate: 28-May-1993; individualID: DHJPAR0007334; individualCount: 1; sex: male; lifeStage: adult; preparations: pinned; catalogNumber: DHJPAR0007334; occurrenceDetails: http://janzen.sas.upenn.edu; recordedBy: D.H. Janzen, W. Hallwachs \& gusaneros; otherCatalogNumbers: ASTAT106-06, 93-SRNP-69, BOLD:AAA8930; identifiedBy: AJ Fleming; dateldentified: 2017; language: en; institutionCode: CNC; collectionCode: Insects; basisOfRecord: Pinned Specimen

e. $\quad$ scientificName: Hyphantrophaga manuelriosi; phylum: Arthropoda; class: Insecta; order: Diptera; family: Tachinidae; genus: Hyphantrophaga; specificEpithet: manuelriosi; scientificNameAuthorship: Fleming \& Wood, 2018; continent: Central America; country: Costa Rica; countryCode: CR; stateProvince: Guanacaste; county: Sector Santa Rosa; locality: Area de Conservacion Guanacaste; verbatimLocality: Cafetal; verbatimElevation: 280 ; verbatimLatitude: 10.8583 ; verbatimLongitude: -85.6109; verbatimCoordinateSystem: Decimal; decimalLatitude: 10.8583; decimalLongitude: -85.6109; samplingProtocol: Reared from the larva of the Crambidae, Syllepte belialis; verbatimEventDate: 06-Aug-1994; individualID: DHJPAR0007336; individualCount: 1; sex: male; lifeStage: adult; preparations: pinned; catalogNumber: DHJPAR0007336; occurrenceDetails: http://janzen.sas.upenn.edu; recordedBy: D.H. Janzen, W. Hallwachs \& gusaneros; otherCatalogNumbers: ASTAT108-06, 94-SRNP-2061, BOLD:AAA8930; identifiedBy: AJ Fleming; dateldentified: 2017; language: en; institutionCode: CNC; collectionCode: Insects; basisOfRecord: Pinned Specimen

f. ScientificName: Hyphantrophaga manuelriosi; phylum: Arthropoda; class: Insecta; order: Diptera; family: Tachinidae; genus: Hyphantrophaga; specificEpithet: 
manuelriosi; scientificNameAuthorship: Fleming \& Wood, 2018; continent: Central America; country: Costa Rica; countryCode: CR; stateProvince: Guanacaste; county: Sector Santa Rosa; locality: Area de Conservacion Guanacaste; verbatimLocality: Bosque Humedo; verbatimElevation: 290; verbatimLatitude: 10.8514; verbatimLongitude: -85.608; verbatimCoordinateSystem: Decimal; decimalLatitude: 10.8514; decimalLongitude: -85.608; samplingProtocol: Reared from the larva of the Crambidae, Syllepte belialis; verbatimEventDate: 06-May-1993; individualID: DHJPAR0007337; individualCount: 1; sex: female; lifeStage: adult; preparations: pinned; catalogNumber: DHJPAR0007337; occurrenceDetails: http:// janzen.sas.upenn.edu; recordedBy: D.H. Janzen, W. Hallwachs \& gusaneros; otherCatalog Numbers: ASTAT109-06, 93-SRNP-71, BOLD:AAA8930; identifiedBy: AJ Fleming; dateldentified: 2017; language: en; institutionCode: CNC; collectionCode: Insects; basisOfRecord: Pinned Specimen

g. ScientificName: Hyphantrophaga manuelriosi; phylum: Arthropoda; class: Insecta; order: Diptera; family: Tachinidae; genus: Hyphantrophaga; specificEpithet: manuelriosi; scientificNameAuthorship: Fleming \& Wood, 2018; continent: Central America; country: Costa Rica; countryCode: CR; stateProvince: Guanacaste; county: Sector Santa Rosa; locality: Area de Conservacion Guanacaste; verbatimLocality: Sendero Natural; verbatimElevation: 290; verbatimLatitude: 10.8357; verbatimLongitude: -85.6125; verbatimCoordinateSystem: Decimal; decimalLatitude: 10.8357; decimalLongitude: -85.6125; samplingProtocol: Reared from the larva of the Crambidae, Syllepte belialis; verbatimEventDate: 02-May-2002; individualID:

DHJPAR0007338; individualCount: 1; sex: male; lifeStage: adult; preparations: pinned; catalogNumber: DHJPAR0007338; occurrenceDetails: http://janzen.sas.upenn.edu; recordedBy: D.H. Janzen, W. Hallwachs \& gusaneros; otherCatalog Numbers: ASTAT110-06, 01-SRNP-13021, BOLD:AAA8930; identifiedBy: AJ Fleming; dateldentified: 2017; language: en; institutionCode: CNC; collectionCode: Insects; basisOfRecord: Pinned Specimen

h. ScientificName: Hyphantrophaga manuelriosi; phylum: Arthropoda; class: Insecta; order: Diptera; family: Tachinidae; genus: Hyphantrophaga; specificEpithet: manuelriosi; scientificNameAuthorship: Fleming \& Wood, 2018; continent: Central America; country: Costa Rica; countryCode: CR; stateProvince: Guanacaste; county: Sector Santa Rosa; locality: Area de Conservacion Guanacaste; verbatimLocality: Cafetal; verbatimElevation: 280 ; verbatimLatitude: 10.8583 ; verbatimLongitude: -85.6109; verbatimCoordinateSystem: Decimal; decimalLatitude: 10.8583; decimalLongitude: -85.6109; samplingProtocol: Reared from the larva of the Crambidae, Syllepte belialis; verbatimEventDate: 17-Mar-1989; individualID: DHJPAR0007339; individualCount: 1; sex: male; lifeStage: adult; preparations: pinned; catalogNumber: DHJPAR0007339; occurrenceDetails: http://janzen.sas.upenn.edu; recordedBy: D.H. Janzen, W. Hallwachs \& gusaneros; otherCatalog Numbers: ASTAT111-06, 88-SRNP-58.2, BOLD:AAA8930; identifiedBy: AJ Fleming; dateldentified: 2017; language: en; institutionCode: CNC; collectionCode: Insects; basisOfRecord: Pinned Specimen

i. ScientificName: Hyphantrophaga manuelriosi; phylum: Arthropoda; class: Insecta; order: Diptera; family: Tachinidae; genus: Hyphantrophaga; specificEpithet: manuelriosi; scientificNameAuthorship: Fleming \& Wood, 2018; continent: Central America; country: Costa Rica; countryCode: CR; stateProvince: Guanacaste; county: Sector Santa Rosa; locality: Area de Conservacion Guanacaste; verbatimLocality: Sendero Natural; verbatimElevation: 290; verbatimLatitude: 10.8357; verbatimLongitude: -85.6125; verbatimCoordinateSystem: Decimal; decimalLatitude: 
10.8357; decimalLongitude: -85.6125; samplingProtocol: Reared from the larva of the Crambidae, Syllepte belialis; verbatimEventDate: 28-Apr-2002; individualID:

DHJPAR0007330; individualCount: 1; sex: male; lifeStage: adult; preparations: pinned; catalogNumber: DHJPAR0007330; occurrenceDetails: http://janzen.sas.upenn.edu; recordedBy: D.H. Janzen, W. Hallwachs \& gusaneros; otherCatalogNumbers: ASTAT102-06, 01-SRNP-13276, BOLD:AAA8930; identifiedBy: AJ Fleming; dateldentified: 2017; language: en; institutionCode: CNC; collectionCode: Insects; basisOfRecord: Pinned Specimen

j. $\quad$ scientificName: Hyphantrophaga manuelriosi; phylum: Arthropoda; class: Insecta; order: Diptera; family: Tachinidae; genus: Hyphantrophaga; specificEpithet: manuelriosi; scientificNameAuthorship: Fleming \& Wood, 2018; continent: Central America; country: Costa Rica; countryCode: CR; stateProvince: Guanacaste; county: Sector Santa Rosa; locality: Area de Conservacion Guanacaste; verbatimLocality: Cafetal; verbatimElevation: 280 ; verbatimLatitude: 10.8583 ; verbatimLongitude: -85.6109; verbatimCoordinateSystem: Decimal; decimalLatitude: 10.8583; decimalLongitude: -85.6109; samplingProtocol: Reared from the larva of the Crambidae, Syllepte belialis; verbatimEventDate: 24-Apr-1995; individualID: DHJPAR0007341; individualCount: 1; sex: female; lifeStage: adult; preparations: pinned; catalogNumber: DHJPAR0007341; occurrenceDetails: http:// janzen.sas.upenn.edu; recordedBy: D.H. Janzen, W. Hallwachs \& gusaneros; otherCatalogNumbers: ASTAT113-06, 94-SRNP-1892, BOLD:AAA8930; identifiedBy: AJ Fleming; dateldentified: 2017; language: en; institutionCode: CNC; collectionCode: Insects; basisOfRecord: Pinned Specimen

k. $\quad$ scientificName: Hyphantrophaga manuelriosi; phylum: Arthropoda; class: Insecta; order: Diptera; family: Tachinidae; genus: Hyphantrophaga; specificEpithet: manuelriosi; scientificNameAuthorship: Fleming \& Wood, 2018; continent: Central America; country: Costa Rica; countryCode: CR; stateProvince: Guanacaste; county: Sector Santa Rosa; locality: Area de Conservacion Guanacaste; verbatimLocality: Cafetal; verbatimElevation: 280 ; verbatimLatitude: 10.8583; verbatimLongitude: -85.6109; verbatimCoordinateSystem: Decimal; decimalLatitude: 10.8583; decimalLongitude: -85.6109; samplingProtocol: Reared from the larva of the Crambidae, Syllepte belialis; verbatimEventDate: 06-Sep-1994; individualID: DHJPAR0007344; individualCount: 1; sex: male; lifeStage: adult; preparations: pinned; catalog Number: DHJPAR0007344; occurrenceDetails: http://janzen.sas.upenn.edu; recordedBy: D.H. Janzen, W. Hallwachs \& gusaneros; otherCatalogNumbers: ASTAT116-06, 94-SRNP-2054, BOLD:AAA8930; identifiedBy: AJ Fleming; dateldentified: 2017; language: en; institutionCode: CNC; collectionCode: Insects; basisOfRecord: Pinned Specimen

I. scientificName: Hyphantrophaga manuelriosi; phylum: Arthropoda; class: Insecta; order: Diptera; family: Tachinidae; genus: Hyphantrophaga; specificEpithet: manuelriosi; scientificNameAuthorship: Fleming \& Wood, 2018; continent: Central America; country: Costa Rica; countryCode: CR; stateProvince: Guanacaste; county: Sector Santa Rosa; locality: Area de Conservacion Guanacaste; verbatimLocality: Cafetal; verbatimElevation: 280 ; verbatimLatitude: 10.8583; verbatimLongitude: -85.6109; verbatimCoordinateSystem: Decimal; decimalLatitude: 10.8583; decimalLongitude: -85.6109; samplingProtocol: Reared from the larva of the Crambidae, Syllepte belialis; verbatimEventDate: 16-Apr-1995; individuallD: DHJPAR0007345; individualCount: 1; sex: male; lifeStage: adult; preparations: pinned; catalogNumber: DHJPAR0007345; occurrenceDetails: http://janzen.sas.upenn.edu; recordedBy: D.H. Janzen, W. Hallwachs \& gusaneros; otherCatalogNumbers: 
ASTAT117-06, 94-SRNP-1890, BOLD:AAA8930; identifiedBy: AJ Fleming; dateldentified: 2017; language: en; institutionCode: CNC; collectionCode: Insects; basisOfRecord: Pinned Specimen

m. $\quad$ scientificName: Hyphantrophaga manuelriosi; phylum: Arthropoda; class: Insecta; order: Diptera; family: Tachinidae; genus: Hyphantrophaga; specificEpithet: manuelriosi; scientificNameAuthorship: Fleming \& Wood, 2018; continent: Central America; country: Costa Rica; countryCode: CR; stateProvince: Guanacaste; county: Sector Santa Rosa; locality: Area de Conservacion Guanacaste; verbatimLocality: Mirador Naranjo; verbatimElevation: 240; verbatimLatitude: 10.8064; verbatimLongitude: -85.6433; verbatimCoordinateSystem: Decimal; decimalLatitude: 10.8064; decimalLongitude: -85.6433; samplingProtocol: Reared from the larva of the Crambidae, Syllepte belialis; verbatimEventDate: 25-Apr-2005; individualID:

DHJPAR0007346; individualCount: 1; sex: male; lifeStage: adult; preparations: pinned; catalogNumber: DHJPAR0007346; occurrenceDetails: http://janzen.sas.upenn.edu; recordedBy: D.H. Janzen, W. Hallwachs \& Freddy Quesada; otherCatalog Numbers: ASTAT118-06, 04-SRNP-10778, BOLD:AAA8930; identifiedBy: AJ Fleming; dateldentified: 2017; language: en; institutionCode: CNC; collectionCode: Insects; basisOfRecord: Pinned Specimen

n. $\quad$ scientificName: Hyphantrophaga manuelriosi; phylum: Arthropoda; class: Insecta; order: Diptera; family: Tachinidae; genus: Hyphantrophaga; specificEpithet: manuelriosi; scientificNameAuthorship: Fleming \& Wood, 2018; continent: Central America; country: Costa Rica; countryCode: CR; stateProvince: Guanacaste; county: Sector Santa Rosa; locality: Area de Conservacion Guanacaste; verbatimLocality: Cafetal; verbatimElevation: 280 ; verbatimLatitude: 10.8583; verbatimLongitude: -85.6109; verbatimCoordinateSystem: Decimal; decimalLatitude: 10.8583; decimalLongitude: -85.6109; samplingProtocol: Reared from the larva of the Crambidae, Syllepte belialis; verbatimEventDate: 19-Apr-1995; individualID: DHJPAR0007347; individualCount: 1; sex: male; lifeStage: adult; preparations: pinned; catalogNumber: DHJPAR0007347; occurrenceDetails: http://janzen.sas.upenn.edu; recordedBy: D.H. Janzen, W. Hallwachs \& gusaneros; otherCatalogNumbers: ASTAT119-06, 94-SRNP-2059, BOLD:AAA8930; identifiedBy: AJ Fleming; dateldentified: 2017; language: en; institutionCode: CNC; collectionCode: Insects; basisOfRecord: Pinned Specimen

o. $\quad$ scientificName: Hyphantrophaga manuelriosi; phylum: Arthropoda; class: Insecta; order: Diptera; family: Tachinidae; genus: Hyphantrophaga; specificEpithet: manuelriosi; scientificNameAuthorship: Fleming \& Wood, 2018; continent: Central America; country: Costa Rica; countryCode: CR; stateProvince: Guanacaste; county: Sector Santa Rosa; locality: Area de Conservacion Guanacaste; verbatimLocality: Cafetal; verbatimElevation: 280 ; verbatimLatitude: 10.8583; verbatimLongitude: -85.6109; verbatimCoordinateSystem: Decimal; decimalLatitude: 10.8583; decimalLongitude: -85.6109; samplingProtocol: Reared from the larva of the Crambidae, Syllepte belialis; verbatimEventDate: 04-Aug-1995; individualID: DHJPAR0007348; individualCount: 1; sex: female; lifeStage: adult; preparations: pinned; catalogNumber: DHJPAR0007348; occurrenceDetails: http:// janzen.sas.upenn.edu; recordedBy: D.H. Janzen, W. Hallwachs \& gusaneros; otherCatalogNumbers: ASTAT120-06, 94-SRNP-2044, BOLD:AAA8930; identifiedBy: AJ Fleming; dateldentified: 2017; language: en; institutionCode: CNC; collectionCode: Insects; basisOfRecord: Pinned Specimen

p. $\quad$ scientificName: Hyphantrophaga manuelriosi; phylum: Arthropoda; class: Insecta; order: Diptera; family: Tachinidae; genus: Hyphantrophaga; specificEpithet: 
manuelriosi; scientificNameAuthorship: Fleming \& Wood, 2018; continent: Central America; country: Costa Rica; countryCode: CR; stateProvince: Guanacaste; county: Sector Mundo Nuevo; locality: Area de Conservacion Guanacaste; verbatimLocality: Sendero Mora; verbatimElevation: 480; verbatimLatitude: 10.7683; verbatimLongitude: -85.4257; verbatimCoordinateSystem: Decimal; decimalLatitude: 10.7683; decimalLongitude: -85.4257; samplingProtocol: Reared from the larva of the Crambidae, Syllepte belialis; verbatimEventDate: 28-May-2007; individualID: DHJPAR0019677; individualCount: 1; sex: male; lifeStage: adult; preparations: pinned; catalogNumber: DHJPAR0019677; occurrenceDetails: http://janzen.sas.upenn.edu; recordedBy: D.H. Janzen, W. Hallwachs \& Jose Alberto Sanchez; otherCatalog Numbers: ASTAB225-07, 07-SRNP-56154, BOLD:AAA8930; identifiedBy: AJ Fleming; dateldentified: 2017; language: en; institutionCode: CNC; collectionCode: Insects; basisOfRecord: Pinned Specimen

q. $\quad$ scientificName: Hyphantrophaga manuelriosi; phylum: Arthropoda; class: Insecta; order: Diptera; family: Tachinidae; genus: Hyphantrophaga; specificEpithet: manuelriosi; scientificNameAuthorship: Fleming \& Wood, 2018; continent: Central America; country: Costa Rica; countryCode: CR; stateProvince: Guanacaste; county: Sector Mundo Nuevo; locality: Area de Conservacion Guanacaste; verbatimLocality: Sendero Mora; verbatimElevation: 480; verbatimLatitude: 10.7683; verbatimLongitude: -85.4257; verbatimCoordinateSystem: Decimal; decimalLatitude: 10.7683; decimalLongitude: -85.4257; samplingProtocol: Reared from the larva of the Crambidae, Syllepte belialis; verbatimEventDate: 25-May-2007; individuallID: DHJPAR0019679; individualCount: 1; sex: male; lifeStage: adult; preparations: pinned; catalogNumber: DHJPAR0019679; occurrenceDetails: http://janzen.sas.upenn.edu; recordedBy: D.H. Janzen, W. Hallwachs \& Jose Alberto Sanchez; otherCatalog Numbers: ASTAB227-07, 07-SRNP-56157, BOLD:AAA8930; identifiedBy: AJ Fleming; dateldentified: 2017; language: en; institutionCode: CNC; collectionCode: Insects; basisOfRecord: Pinned Specimen

r. $\quad$ scientificName: Hyphantrophaga manuelriosi; phylum: Arthropoda; class: Insecta; order: Diptera; family: Tachinidae; genus: Hyphantrophaga; specificEpithet: manuelriosi; scientificNameAuthorship: Fleming \& Wood, 2018; continent: Central America; country: Costa Rica; countryCode: CR; stateProvince: Guanacaste; county: Sector Santa Rosa; locality: Area de Conservacion Guanacaste; verbatimLocality: Sendero Natural; verbatimElevation: 290; verbatimLatitude: 10.8357; verbatimLongitude: -85.6125 ; verbatimCoordinateSystem: Decimal; decimalLatitude: 10.8357; decimalLongitude: -85.6125; samplingProtocol: Reared from the larva of the Crambidae, Syllepte belialis; verbatimEventDate: 09-Apr-2009; individualID:

DHJPAR0030460; individualCount: 1; sex: male; lifeStage: adult; preparations: pinned; catalogNumber: DHJPAR0030460; occurrenceDetails: http://janzen.sas.upenn.edu; recordedBy: D.H. Janzen, W. Hallwachs \& Lucia Vargas; otherCatalogNumbers: ASHYB1203-09, 08-SRNP-12948, BOLD:AAA8930; identifiedBy: AJ Fleming; dateldentified: 2017; language: en; institutionCode: CNC; collectionCode: Insects; basisOfRecord: Pinned Specimen

s. $\quad$ scientificName: Hyphantrophaga manuelriosi; phylum: Arthropoda; class: Insecta; order: Diptera; family: Tachinidae; genus: Hyphantrophaga; specificEpithet: manuelriosi; scientificNameAuthorship: Fleming \& Wood, 2018; continent: Central America; country: Costa Rica; countryCode: CR; stateProvince: Guanacaste; county: Sector Mundo Nuevo; locality: Area de Conservacion Guanacaste; verbatimLocality: Estacion La Perla; verbatimElevation: 325; verbatimLatitude: 10.7674; verbatimLongitude: -85.4331; verbatimCoordinateSystem: Decimal; decimalLatitude: 
10.7674; decimalLongitude: -85.4331; samplingProtocol: Reared from the larva of the Crambidae, Syllepte belialis; verbatimEventDate: 13-Jun-2010; individualID: DHJPAR0039306; individualCount: 1; sex: female; lifeStage: adult; preparations: pinned; catalogNumber: DHJPAR0039306; occurrenceDetails: http:// janzen.sas.upenn.edu; recordedBy: D.H. Janzen, W. Hallwachs \& Mariano Pereira; otherCatalogNumbers: ASTAV869-10, 10-SRNP-55351, BOLD:AAA8930; identifiedBy: AJ Fleming; dateldentified: 2017; language: en; institutionCode: CNC; collectionCode: Insects; basisOfRecord: Pinned Specimen

t. scientificName: Hyphantrophaga manuelriosi; phylum: Arthropoda; class: Insecta; order: Diptera; family: Tachinidae; genus: Hyphantrophaga; specificEpithet: manuelriosi; scientificNameAuthorship: Fleming \& Wood, 2018; continent: Central America; country: Costa Rica; countryCode: CR; stateProvince: Guanacaste; county: Sector Mundo Nuevo; locality: Area de Conservacion Guanacaste; verbatimLocality: Sendero Aguacate; verbatimElevation: 335 ; verbatimLatitude: 10.769 ; verbatimLongitude: -85.4346 ; verbatimCoordinateSystem: Decimal; decimalLatitude: 10.769; decimalLongitude: -85.4346; samplingProtocol: Reared from the larva of the Crambidae, Syllepte belialis; verbatimEventDate: 11-Sep-2014; individualID: DHJPAR0056209; individualCount: 1; sex: male; lifeStage: adult; preparations: pinned; catalogNumber: DHJPAR0056209; occurrenceDetails: http://janzen.sas.upenn.edu; recordedBy: D.H. Janzen, W. Hallwachs \& Jose Cortez; otherCatalogNumbers: ASHYH2466-14, 14-SRNP-55897, BOLD:AAA8930; identifiedBy: AJ Fleming; dateldentified: 2017; language: en; institutionCode: CNC; collectionCode: Insects; basisOfRecord: Pinned Specimen

u. $\quad$ scientificName: Hyphantrophaga manuelriosi; phylum: Arthropoda; class: Insecta; order: Diptera; family: Tachinidae; genus: Hyphantrophaga; specificEpithet: manuelriosi; scientificNameAuthorship: Fleming \& Wood, 2018; continent: Central America; country: Costa Rica; countryCode: CR; stateProvince: Guanacaste; county: Sector Mundo Nuevo; locality: Area de Conservacion Guanacaste; verbatimLocality: Sendero Aguacate; verbatimElevation: 335; verbatimLatitude: 10.769; verbatimLongitude: -85.4346; verbatimCoordinateSystem: Decimal; decimalLatitude: 10.769; decimalLongitude: -85.4346; samplingProtocol: Reared from the larva of the Crambidae, Syllepte belialis; verbatimEventDate: 10-Sep-2014; individualID: DHJPAR0056220; individualCount: 1; sex: female; lifeStage: adult; preparations: pinned; catalogNumber: DHJPAR0056220; occurrenceDetails: http:// janzen.sas.upenn.edu; recordedBy: D.H. Janzen, W. Hallwachs \& Jose Cortez; otherCatalogNumbers: ASHYH2477-14, 14-SRNP-55899, BOLD:AAA8930; identifiedBy: AJ Fleming; dateldentified: 2017; language: en; institutionCode: CNC; collectionCode: Insects; basisOfRecord: Pinned Specimen

v. ScientificName: Hyphantrophaga manuelriosi; phylum: Arthropoda; class: Insecta; order: Diptera; family: Tachinidae; genus: Hyphantrophaga; specificEpithet: manuelriosi; scientificNameAuthorship: Fleming \& Wood, 2018; continent: Central America; country: Costa Rica; countryCode: CR; stateProvince: Guanacaste; county: Sector Mundo Nuevo; locality: Area de Conservacion Guanacaste; verbatimLocality: Punta Plancha; verbatimElevation: 420; verbatimLatitude: 10.7416 ; verbatimLongitude: -85.4273; verbatimCoordinateSystem: Decimal; decimalLatitude: 10.7416; decimalLongitude: -85.4273; samplingProtocol: Reared from the larva of the Crambidae, Syllepte belialis; verbatimEventDate: 05-Aug-2014; individualID: DHJPAR0055853; individualCount: 1; sex: female; lifeStage: adult; preparations: pinned; catalogNumber: DHJPAR0055853; occurrenceDetails: http:// janzen.sas.upenn.edu; recordedBy: D.H. Janzen, W. Hallwachs \& Jose Cortez; 
otherCatalog Numbers: ASHYH2585-14, 14-SRNP-55853, BOLD:AAA8930; identifiedBy: AJ Fleming; dateldentified: 2017; language: en; institutionCode: CNC; collectionCode: Insects; basisOfRecord: Pinned Specimen

w. $\quad$ scientificName: Hyphantrophaga manuelriosi; phylum: Arthropoda; class: Insecta; order: Diptera; family: Tachinidae; genus: Hyphantrophaga; specificEpithet: manuelriosi; scientificNameAuthorship: Fleming \& Wood, 2018; continent: Central America; country: Costa Rica; countryCode: CR; stateProvince: Guanacaste; county: Sector Mundo Nuevo; locality: Area de Conservacion Guanacaste; verbatimLocality: Sendero Aguacate; verbatimElevation: 335; verbatimLatitude: 10.769; verbatimLongitude: -85.4346; verbatimCoordinateSystem: Decimal; decimalLatitude: 10.769; decimalLongitude: -85.4346 ; samplingProtocol: Reared from the larva of the Crambidae, Syllepte belialis; verbatimEventDate: 26-Jul-2014; individualID:

DHJPAR0055855; individualCount: 1; sex: male; lifeStage: adult; preparations: pinned; catalogNumber: DHJPAR0055855; occurrenceDetails: http://janzen.sas.upenn.edu; recordedBy: D.H. Janzen, W. Hallwachs \& Jose Cortez; otherCatalogNumbers: ASHYH2587-14, 14-SRNP-55890, BOLD:AAA8930; identifiedBy: AJ Fleming; dateldentified: 2017; language: en; institutionCode: CNC; collectionCode: Insects; basisOfRecord: Pinned Specimen

x. scientificName: Hyphantrophaga manuelriosi; phylum: Arthropoda; class: Insecta; order: Diptera; family: Tachinidae; genus: Hyphantrophaga; specificEpithet: manuelriosi; scientificNameAuthorship: Fleming \& Wood, 2018; continent: Central America; country: Costa Rica; countryCode: CR; stateProvince: Guanacaste; county: Sector Mundo Nuevo; locality: Area de Conservacion Guanacaste; verbatimLocality: Sendero Aguacate; verbatimElevation: 335 ; verbatimLatitude: 10.769 ; verbatimLongitude: -85.4346 ; verbatimCoordinateSystem: Decimal; decimalLatitude: 10.769; decimalLongitude: -85.4346; samplingProtocol: Reared from the larva of the Crambidae, Syllepte belialis; verbatimEventDate: 15-Jul-2014; individualID: DHJPAR0055857; individualCount: 1; sex: male; lifeStage: adult; preparations: pinned; catalogNumber: DHJPAR0055857; occurrenceDetails: http://janzen.sas.upenn.edu; recordedBy: D.H. Janzen, W. Hallwachs \& Jose Cortez; otherCatalogNumbers: ASHYH2589-14, 14-SRNP-55898, BOLD:AAA8930; identifiedBy: AJ Fleming; dateldentified: 2017; language: en; institutionCode: CNC; collectionCode: Insects; basisOfRecord: Pinned Specimen

\section{Other materials:}

a. ScientificName: Hyphantrophaga manuelriosi; phylum: Arthropoda; class: Insecta; order: Diptera; family: Tachinidae; genus: Hyphantrophaga; specificEpithet: manuelriosi; scientificNameAuthorship: Fleming \& Wood, 2018; continent: Central America; country: Costa Rica; countryCode: CR; stateProvince: Guanacaste; county: Sector Santa Rosa; locality: Area de Conservacion Guanacaste; verbatimLocality: Sendero Natural; verbatimElevation: 290; verbatimLatitude: 10.8357; verbatimLongitude: -85.6125; verbatimCoordinateSystem: Decimal; decimalLatitude: 10.8357; decimalLongitude: -85.6125; samplingProtocol: Reared from the larva of the Crambidae, Syllepte belialis; verbatimEventDate: 09-Apr-2009; individualID: DHJPAR0034334; individualCount: 1; lifeStage: adult; preparations: pinned; catalogNumber: DHJPAR0034334; occurrenceDetails: http://janzen.sas.upenn.edu; recordedBy: D.H. Janzen, W. Hallwachs \& Lucia Vargas; otherCatalogNumbers: ASHYB1627-09, 08-SRNP-12941, BOLD:AAA8930; identifiedBy: AJ Fleming; dateldentified: 2017; language: en; institutionCode: CNC; collectionCode: Insects; basisOfRecord: Pinned Specimen 
b. $\quad$ scientificName: Hyphantrophaga manuelriosi; phylum: Arthropoda; class: Insecta; order: Diptera; family: Tachinidae; genus: Hyphantrophaga; specificEpithet: manuelriosi; scientificNameAuthorship: Fleming \& Wood, 2018; continent: Central America; country: Costa Rica; countryCode: CR; stateProvince: Guanacaste; county: Sector Santa Rosa; locality: Area de Conservacion Guanacaste; verbatimLocality: Area Administrativa; verbatimElevation: 295; verbatimLatitude: 10.8376; verbatimLongitude: -85.6187; verbatimCoordinateSystem: Decimal; decimalLatitude: 10.8376; decimalLongitude: -85.6187; samplingProtocol: Reared from the larva of the Crambidae, Syllepte belialis; verbatimEventDate: 17-May-2009; individualID: DHJPAR0035565; individualCount: 1; lifeStage: adult; preparations: pinned; catalogNumber: DHJPAR0035565; occurrenceDetails: http://janzen.sas.upenn.edu; recordedBy: D.H. Janzen, W. Hallwachs \& Lucia Vargas; otherCatalogNumbers: ASHYC1770-09, 08-SRNP-12897, BOLD:AAA8930; identifiedBy: AJ Fleming; dateldentified: 2017; language: en; institutionCode: CNC; collectionCode: Insects; basisOfRecord: Pinned Specimen

c. scientificName: Hyphantrophaga manuelriosi; phylum: Arthropoda; class: Insecta; order: Diptera; family: Tachinidae; genus: Hyphantrophaga; specificEpithet: manuelriosi; scientificNameAuthorship: Fleming \& Wood, 2018; continent: Central America; country: Costa Rica; countryCode: CR; stateProvince: Guanacaste; county: Sector Santa Rosa; locality: Area de Conservacion Guanacaste; verbatimLocality: Area Administrativa; verbatimElevation: 295; verbatimLatitude: 10.8376; verbatimLongitude: -85.6187; verbatimCoordinateSystem: Decimal; decimalLatitude: 10.8376; decimalLongitude: -85.6187 ; samplingProtocol: Reared from the larva of the Crambidae, Syllepte belialis; verbatimEventDate: 29-Apr-2009; individualID: DHJPAR0035567; individualCount: 1; lifeStage: adult; preparations: pinned; catalogNumber: DHJPAR0035567; occurrenceDetails: http://janzen.sas.upenn.edu; recordedBy: D.H. Janzen, W. Hallwachs \& Lucia Vargas; otherCatalogNumbers: ASHYC1772-09, 08-SRNP-12900, BOLD:AAA8930; identifiedBy: AJ Fleming; dateldentified: 2017; language: en; institutionCode: CNC; collectionCode: Insects; basisOfRecord: Pinned Specimen

d. ScientificName: Hyphantrophaga manuelriosi; phylum: Arthropoda; class: Insecta; order: Diptera; family: Tachinidae; genus: Hyphantrophaga; specificEpithet: manuelriosi; scientificNameAuthorship: Fleming \& Wood, 2018; continent: Central America; country: Costa Rica; countryCode: CR; stateProvince: Guanacaste; county: Sector Santa Rosa; locality: Area de Conservacion Guanacaste; verbatimLocality: Area Administrativa; verbatimElevation: 295; verbatimLatitude: 10.8376; verbatimLongitude: -85.6187; verbatimCoordinateSystem: Decimal; decimalLatitude: 10.8376; decimalLongitude: -85.6187; samplingProtocol: Reared from the larva of the Crambidae, Syllepte belialis; verbatimEventDate: 14-Apr-2009; individualID: DHJPAR0035568; individualCount: 1; lifeStage: adult; preparations: pinned; catalog Number: DHJPAR0035568; occurrenceDetails: http://janzen.sas.upenn.edu; recordedBy: D.H. Janzen, W. Hallwachs \& Lucia Vargas; otherCatalogNumbers: ASHYC1773-09, 08-SRNP-12905, BOLD:AAA8930; identifiedBy: AJ Fleming; dateldentified: 2017; language: en; institutionCode: CNC; collectionCode: Insects; basisOfRecord: Pinned Specimen

e. ScientificName: Hyphantrophaga manuelriosi; phylum: Arthropoda; class: Insecta; order: Diptera; family: Tachinidae; genus: Hyphantrophaga; specificEpithet: manuelriosi; scientificNameAuthorship: Fleming \& Wood, 2018; continent: Central America; country: Costa Rica; countryCode: CR; stateProvince: Guanacaste; county: Sector Santa Rosa; locality: Area de Conservacion Guanacaste; verbatimLocality: 
Sendero Natural; verbatimElevation: 290; verbatimLatitude: 10.8357 ; verbatimLongitude: -85.6125; verbatimCoordinateSystem: Decimal; decimalLatitude: 10.8357; decimalLongitude: -85.6125 ; samplingProtocol: Reared from the larva of the Crambidae, Syllepte belialis; verbatimEventDate: 14-May-2009; individualID: DHJPAR0035570; individualCount: 1; lifeStage: adult; preparations: pinned; catalogNumber: DHJPAR0035570; occurrenceDetails: http://janzen.sas.upenn.edu; recordedBy: D.H. Janzen, W. Hallwachs \& Lucia Vargas; otherCatalogNumbers: ASHYC1775-09, 08-SRNP-12940, BOLD:AAA8930; identifiedBy: AJ Fleming; dateldentified: 2017; language: en; institutionCode: CNC; collectionCode: Insects; basisOfRecord: Pinned Specimen

f. scientificName: Hyphantrophaga manuelriosi; phylum: Arthropoda; class: Insecta; order: Diptera; family: Tachinidae; genus: Hyphantrophaga; specificEpithet: manuelriosi; scientificNameAuthorship: Fleming \& Wood, 2018; continent: Central America; country: Costa Rica; countryCode: CR; stateProvince: Guanacaste; county: Sector Santa Rosa; locality: Area de Conservacion Guanacaste; verbatimLocality: Sendero Natural; verbatimElevation: 290; verbatimLatitude: 10.8357 ; verbatimLongitude: -85.6125; verbatimCoordinateSystem: Decimal; decimalLatitude: 10.8357; decimalLongitude: -85.6125; samplingProtocol: Reared from the larva of the Crambidae, Syllepte belialis; verbatimEventDate: 12-May-2009; individualID: DHJPAR0035571; individualCount: 1; lifeStage: adult; preparations: pinned; catalogNumber: DHJPAR0035571; occurrenceDetails: http://janzen.sas.upenn.edu; recordedBy: D.H. Janzen, W. Hallwachs \& Guillermo Pereira; otherCatalogNumbers: ASHYC1776-09, 08-SRNP-13153, BOLD:AAA8930; identifiedBy: AJ Fleming; dateldentified: 2017; language: en; institutionCode: CNC; collectionCode: Insects; basisOfRecord: Pinned Specimen

g. ScientificName: Hyphantrophaga manuelriosi; phylum: Arthropoda; class: Insecta; order: Diptera; family: Tachinidae; genus: Hyphantrophaga; specificEpithet: manuelriosi; scientificNameAuthorship: Fleming \& Wood, 2018; continent: Central America; country: Costa Rica; country Code: CR; stateProvince: Guanacaste; county: Sector Santa Rosa; locality: Area de Conservacion Guanacaste; verbatimLocality: Sendero Natural; verbatimElevation: 290; verbatimLatitude: 10.8357; verbatimLongitude: -85.6125; verbatimCoordinateSystem: Decimal; decimalLatitude: 10.8357; decimalLongitude: -85.6125; samplingProtocol: Reared from the larva of the Crambidae, Syllepte belialis; verbatimEventDate: 09-May-2009; individualID: DHJPAR0035572; individualCount: 1; lifeStage: adult; preparations: pinned; catalogNumber: DHJPAR0035572; occurrenceDetails: http://janzen.sas.upenn.edu; recordedBy: D.H. Janzen, W. Hallwachs \& Guillermo Pereira; otherCatalogNumbers: ASHYC1777-09, 08-SRNP-13170, BOLD:AAA8930; identifiedBy: AJ Fleming; dateldentified: 2017; language: en; institutionCode: CNC; collectionCode: Insects; basisOfRecord: Pinned Specimen

h. ScientificName: Hyphantrophaga manuelriosi; phylum: Arthropoda; class: Insecta; order: Diptera; family: Tachinidae; genus: Hyphantrophaga; specificEpithet: manuelriosi; scientificNameAuthorship: Fleming \& Wood, 2018; continent: Central America; country: Costa Rica; countryCode: CR; stateProvince: Guanacaste; county: Sector Santa Rosa; locality: Area de Conservacion Guanacaste; verbatimLocality: Area Administrativa; verbatimElevation: 295; verbatimLatitude: 10.8376; verbatimLongitude: -85.6187; verbatimCoordinateSystem: Decimal; decimalLatitude: 10.8376; decimalLongitude: -85.6187; samplingProtocol: Reared from the larva of the Crambidae, Syllepte belialis; verbatimEventDate: 14-May-2009; individualID: DHJPAR0035573; individualCount: 1; lifeStage: adult; preparations: pinned; 
catalogNumber: DHJPAR0035573; occurrenceDetails: http://janzen.sas.upenn.edu; recordedBy: D.H. Janzen, W. Hallwachs \& Lucia Vargas; otherCatalogNumbers: ASHYC1778-09, 08-SRNP-12902, BOLD:AAA8930; identifiedBy: AJ Fleming; dateldentified: 2017; language: en; institutionCode: CNC; collectionCode: Insects; basisOfRecord: Pinned Specimen

i. $\quad$ scientificName: Hyphantrophaga manuelriosi; phylum: Arthropoda; class: Insecta; order: Diptera; family: Tachinidae; genus: Hyphantrophaga; specificEpithet: manuelriosi; scientificNameAuthorship: Fleming \& Wood, 2018; continent: Central America; country: Costa Rica; countryCode: CR; stateProvince: Guanacaste; county: Sector Santa Rosa; locality: Area de Conservacion Guanacaste; verbatimLocality: Area Administrativa; verbatimElevation: 295; verbatimLatitude: 10.8376; verbatimLongitude: -85.6187; verbatimCoordinateSystem: Decimal; decimalLatitude: 10.8376; decimalLongitude: -85.6187; samplingProtocol: Reared from the larva of the Crambidae, Syllepte belialis; verbatimEventDate: 14-Apr-2009; individualID: DHJPAR0035576; individualCount: 1; lifeStage: adult; preparations: pinned; catalogNumber: DHJPAR0035576; occurrenceDetails: http://janzen.sas.upenn.edu; recordedBy: D.H. Janzen, W. Hallwachs \& Lucia Vargas; otherCatalogNumbers: ASHYC1781-09, 08-SRNP-12906, BOLD:AAA8930; identifiedBy: AJ Fleming; dateldentified: 2017; language: en; institutionCode: CNC; collectionCode: Insects; basisOfRecord: Pinned Specimen

j. $\quad$ scientificName: Hyphantrophaga manuelriosi; phylum: Arthropoda; class: Insecta; order: Diptera; family: Tachinidae; genus: Hyphantrophaga; specificEpithet: manuelriosi; scientificNameAuthorship: Fleming \& Wood, 2018; continent: Central America; country: Costa Rica; countryCode: CR; stateProvince: Guanacaste; county: Sector Santa Rosa; locality: Area de Conservacion Guanacaste; verbatimLocality: Area Administrativa; verbatimElevation: 295; verbatimLatitude: 10.8376; verbatimLongitude: -85.6187; verbatimCoordinateSystem: Decimal; decimalLatitude: 10.8376; decimalLongitude: -85.6187; samplingProtocol: Reared from the larva of the Crambidae, Syllepte belialis; verbatimEventDate: 17-May-2009; individualID: DHJPAR0035577; individualCount: 1; lifeStage: adult; preparations: pinned; catalogNumber: DHJPAR0035577; occurrenceDetails: http://janzen.sas.upenn.edu; recordedBy: D.H. Janzen, W. Hallwachs \& Lucia Vargas; otherCatalogNumbers: ASHYC1782-09, 08-SRNP-12912, BOLD:AAA8930; identifiedBy: AJ Fleming; dateldentified: 2017; language: en; institutionCode: CNC; collectionCode: Insects; basisOfRecord: Pinned Specimen

k. $\quad$ scientificName: Hyphantrophaga manuelriosi; phylum: Arthropoda; class: Insecta; order: Diptera; family: Tachinidae; genus: Hyphantrophaga; specificEpithet: manuelriosi; scientificNameAuthorship: Fleming \& Wood, 2018; continent: Central America; country: Costa Rica; countryCode: CR; stateProvince: Guanacaste; county: Sector Santa Rosa; locality: Area de Conservacion Guanacaste; verbatimLocality: Area Administrativa; verbatimElevation: 295; verbatimLatitude: 10.8376; verbatimLongitude: -85.6187; verbatimCoordinateSystem: Decimal; decimalLatitude: 10.8376; decimalLongitude: -85.6187; samplingProtocol: Reared from the larva of the Crambidae, Syllepte belialis; verbatimEventDate: 17-Apr-2009; individualID: DHJPAR0035578; individualCount: 1; lifeStage: adult; preparations: pinned; catalogNumber: DHJPAR0035578; occurrenceDetails: http://janzen.sas.upenn.edu; recordedBy: D.H. Janzen, W. Hallwachs \& Lucia Vargas; otherCatalogNumbers: ASHYC1783-09, 08-SRNP-12915, BOLD:AAA8930; identifiedBy: AJ Fleming; dateldentified: 2017; language: en; institutionCode: CNC; collectionCode: Insects; basisOfRecord: Pinned Specimen 
I. scientificName: Hyphantrophaga manuelriosi; phylum: Arthropoda; class: Insecta; order: Diptera; family: Tachinidae; genus: Hyphantrophaga; specificEpithet: manuelriosi; scientificNameAuthorship: Fleming \& Wood, 2018; continent: Central America; country: Costa Rica; countryCode: CR; stateProvince: Guanacaste; county: Sector Santa Rosa; locality: Area de Conservacion Guanacaste; verbatimLocality: Area Administrativa; verbatimElevation: 295; verbatimLatitude: 10.8376; verbatimLongitude: -85.6187; verbatimCoordinateSystem: Decimal; decimalLatitude: 10.8376; decimalLongitude: -85.6187; samplingProtocol: Reared from the larva of the Crambidae, Syllepte belialis; verbatimEventDate: 14-May-2009; individualID: DHJPAR0036440; individualCount: 1; lifeStage: adult; preparations: pinned; catalogNumber: DHJPAR0036440; occurrenceDetails: http://janzen.sas.upenn.edu; recordedBy: D.H. Janzen, W. Hallwachs \& Lucia Vargas; otherCatalogNumbers: ASHYD1631-09, 08-SRNP-12914, BOLD:AAA8930; identifiedBy: AJ Fleming; dateldentified: 2017; language: en; institutionCode: CNC; collectionCode: Insects; basisOfRecord: Pinned Specimen

m. scientificName: Hyphantrophaga manuelriosi; phylum: Arthropoda; class: Insecta; order: Diptera; family: Tachinidae; genus: Hyphantrophaga; specificEpithet: manuelriosi; scientificNameAuthorship: Fleming \& Wood, 2018; continent: Central America; country: Costa Rica; countryCode: CR; stateProvince: Guanacaste; county: Sector Santa Rosa; locality: Area de Conservacion Guanacaste; verbatimLocality: Sendero Natural; verbatimElevation: 290; verbatimLatitude: 10.8357; verbatimLongitude: -85.6125; verbatimCoordinateSystem: Decimal; decimalLatitude: 10.8357; decimalLongitude: -85.6125 ; samplingProtocol: Reared from the larva of the Crambidae, Syllepte belialis; verbatimEventDate: 09-May-2009; individualID: DHJPAR0036445; individualCount: 1; lifeStage: adult; preparations: pinned; catalogNumber: DHJPAR0036445; occurrenceDetails: http://janzen.sas.upenn.edu; recordedBy: D.H. Janzen, W. Hallwachs \& Guillermo Pereira; otherCatalogNumbers: ASHYD1636-09, 08-SRNP-13159, BOLD:AAA8930; identifiedBy: AJ Fleming; dateldentified: 2017; language: en; institutionCode: CNC; collectionCode: Insects; basisOfRecord: Pinned Specimen

n. ScientificName: Hyphantrophaga manuelriosi; phylum: Arthropoda; class: Insecta; order: Diptera; family: Tachinidae; genus: Hyphantrophaga; specificEpithet: manuelriosi; scientificNameAuthorship: Fleming \& Wood, 2018; continent: Central America; country: Costa Rica; countryCode: CR; stateProvince: Guanacaste; county: Sector Santa Rosa; locality: Area de Conservacion Guanacaste; verbatimLocality: Sendero Natural; verbatimElevation: 290; verbatimLatitude: 10.8357; verbatimLongitude: -85.6125; verbatimCoordinateSystem: Decimal; decimalLatitude: 10.8357; decimalLongitude: -85.6125 ; samplingProtocol: Reared from the larva of the Crambidae, Syllepte belialis; verbatimEventDate: 24-Apr-2009; individualID: DHJPAR0036447; individualCount: 1; lifeStage: adult; preparations: pinned; catalogNumber: DHJPAR0036447; occurrenceDetails: http://janzen.sas.upenn.edu; recordedBy: D.H. Janzen, W. Hallwachs \& Guillermo Pereira; otherCatalogNumbers: ASHYD1638-09, 08-SRNP-13162, BOLD:AAA8930; identifiedBy: AJ Fleming; dateldentified: 2017; language: en; institutionCode: CNC; collectionCode: Insects; basisOfRecord: Pinned Specimen

o. ScientificName: Hyphantrophaga manuelriosi; phylum: Arthropoda; class: Insecta; order: Diptera; family: Tachinidae; genus: Hyphantrophaga; specificEpithet: manuelriosi; scientificNameAuthorship: Fleming \& Wood, 2018; continent: Central America; country: Costa Rica; countryCode: CR; stateProvince: Guanacaste; county: Sector Santa Rosa; locality: Area de Conservacion Guanacaste; verbatimLocality: 
Area Administrativa; verbatimElevation: 295; verbatimLatitude: 10.8376; verbatimLongitude: -85.6187; verbatimCoordinateSystem: Decimal; decimalLatitude: 10.8376; decimalLongitude: -85.6187; samplingProtocol: Reared from the larva of the Crambidae, Syllepte belialis; verbatimEventDate: 17-May-2009; individualID: DHJPAR0036448; individualCount: 1; lifeStage: adult; preparations: pinned; catalogNumber: DHJPAR0036448; occurrenceDetails: http://janzen.sas.upenn.edu; recordedBy: D.H. Janzen, W. Hallwachs \& Lucia Vargas; otherCatalogNumbers: ASHYD1639-09, 08-SRNP-12928, BOLD:AAA8930; identifiedBy: AJ Fleming; dateldentified: 2017; language: en; institutionCode: CNC; collectionCode: Insects; basisOfRecord: Pinned Specimen

p. $\quad$ scientificName: Hyphantrophaga manuelriosi; phylum: Arthropoda; class: Insecta; order: Diptera; family: Tachinidae; genus: Hyphantrophaga; specificEpithet: manuelriosi; scientificNameAuthorship: Fleming \& Wood, 2018; continent: Central America; country: Costa Rica; countryCode: CR; stateProvince: Guanacaste; county: Sector Santa Rosa; locality: Area de Conservacion Guanacaste; verbatimLocality: Area Administrativa; verbatimElevation: 295; verbatimLatitude: 10.8376; verbatimLongitude: -85.6187; verbatimCoordinateSystem: Decimal; decimalLatitude: 10.8376; decimalLongitude: -85.6187; samplingProtocol: Reared from the larva of the Crambidae, Syllepte belialis; verbatimEventDate: 15-Apr-2009; individualID: DHJPAR0036449; individualCount: 1; lifeStage: adult; preparations: pinned; catalogNumber: DHJPAR0036449; occurrenceDetails: http://janzen.sas.upenn.edu; recordedBy: D.H. Janzen, W. Hallwachs \& Lucia Vargas; otherCatalogNumbers: ASHYD1640-09, 08-SRNP-12916, BOLD:AAA8930; identifiedBy: AJ Fleming; dateldentified: 2017; language: en; institutionCode: CNC; collectionCode: Insects; basisOfRecord: Pinned Specimen

q. $\quad$ scientificName: Hyphantrophaga manuelriosi; phylum: Arthropoda; class: Insecta; order: Diptera; family: Tachinidae; genus: Hyphantrophaga; specificEpithet: manuelriosi; scientificNameAuthorship: Fleming \& Wood, 2018; continent: Central America; country: Costa Rica; countryCode: CR; stateProvince: Guanacaste; county: Sector Santa Rosa; locality: Area de Conservacion Guanacaste; verbatimLocality: Area Administrativa; verbatimElevation: 295; verbatimLatitude: 10.8376; verbatimLongitude: -85.6187; verbatimCoordinateSystem: Decimal; decimalLatitude: 10.8376; decimalLongitude: -85.6187 ; samplingProtocol: Reared from the larva of the Crambidae, Syllepte belialis; verbatimEventDate: 21-Apr-2009; individualID: DHJPAR0036450; individualCount: 1; lifeStage: adult; preparations: pinned; catalogNumber: DHJPAR0036450; occurrenceDetails: http://janzen.sas.upenn.edu; recordedBy: D.H. Janzen, W. Hallwachs \& Lucia Vargas; otherCatalogNumbers: ASHYD1641-09, 08-SRNP-12929, BOLD:AAA8930; identifiedBy: AJ Fleming; dateldentified: 2017; language: en; institutionCode: CNC; collectionCode: Insects; basisOfRecord: Pinned Specimen

r. ScientificName: Hyphantrophaga manuelriosi; phylum: Arthropoda; class: Insecta; order: Diptera; family: Tachinidae; genus: Hyphantrophaga; specificEpithet: manuelriosi; scientificNameAuthorship: Fleming \& Wood, 2018; continent: Central America; country: Costa Rica; countryCode: CR; stateProvince: Guanacaste; county: Sector Santa Rosa; locality: Area de Conservacion Guanacaste; verbatimLocality: Sendero Natural; verbatimElevation: 290; verbatimLatitude: 10.8357; verbatimLongitude: -85.6125; verbatimCoordinateSystem: Decimal; decimalLatitude: 10.8357; decimalLongitude: -85.6125; samplingProtocol: Reared from the larva of the Crambidae, Syllepte belialis; verbatimEventDate: 24-Apr-2009; individualID: DHJPAR0036452; individualCount: 1; lifeStage: adult; preparations: pinned; 
catalogNumber: DHJPAR0036452; occurrenceDetails: http://janzen.sas.upenn.edu; recordedBy: D.H. Janzen, W. Hallwachs \& Guillermo Pereira; otherCatalogNumbers: ASHYD1643-09, 08-SRNP-13158, BOLD:AAA8930; identifiedBy: AJ Fleming; dateldentified: 2017; language: en; institutionCode: CNC; collectionCode: Insects; basisOfRecord: Pinned Specimen

s. $\quad$ scientificName: Hyphantrophaga manuelriosi; phylum: Arthropoda; class: Insecta; order: Diptera; family: Tachinidae; genus: Hyphantrophaga; specificEpithet: manuelriosi; scientificNameAuthorship: Fleming \& Wood, 2018; continent: Central America; country: Costa Rica; countryCode: CR; stateProvince: Guanacaste; county: Sector Santa Rosa; locality: Area de Conservacion Guanacaste; verbatimLocality: Sendero Natural; verbatimElevation: 290; verbatimLatitude: 10.8357; verbatimLongitude: -85.6125; verbatimCoordinateSystem: Decimal; decimalLatitude: 10.8357; decimalLongitude: -85.6125; samplingProtocol: Reared from the larva of the Crambidae, Syllepte belialis; verbatimEventDate: 24-Apr-2009; individualID: DHJPAR0036453; individualCount: 1; lifeStage: adult; preparations: pinned; catalogNumber: DHJPAR0036453; occurrenceDetails: http://janzen.sas.upenn.edu; recordedBy: D.H. Janzen, W. Hallwachs \& Guillermo Pereira; otherCatalogNumbers: ASHYD1644-09, 08-SRNP-13157, BOLD:AAA8930; identifiedBy: AJ Fleming; dateldentified: 2017; language: en; institutionCode: CNC; collectionCode: Insects; basisOfRecord: Pinned Specimen

\section{Description}

Male (Fig. 19). Length: 5-8 mm. Head (Fig. 19b): vertex 1/3 of head width; two pairs of reclinate upper orbital setae; ocellar setae arising beside anterior ocellus; ocellar triangle silver (slight gold tinge present but overall concolorous with fronto-orbital plate); eye densely haired; parafacial bare; fronto-orbital plate sparsely setulose, hairs not extending below upper 1/3; fronto-orbital plate shiny silver; facial ridge bare; pedicel light brown-orange, lighter than black postpedicel; arista brown, very minutely pubescent, distinctly thickened on basal 1/3-1/4. Thorax (Fig. 19a, c): pale grey tomentose dorsally and laterally; four prominent dorsal vittae broken across suture, innermost pair not reaching 2nd postsutural dorsocentral seta; postpronotum with three setae arranged in a triangle; chaetotaxy: acrostichal setae 3:3; dorsocentral setae 3:4; intra-alar setae 2:3; supra-alar setae 2:3; three katepisternal setae; basal scutellar setae as long as scutellar setae; two pairs of lateral scutellar setae $2 / 3$ as long as basal scutellar setae; subapical scutellar setae straight and divergent, $2 \mathrm{X}$ as thick as lateral scutellar setae; apical scutellar setae 1/4 length of basal scutellar setae crossed apically; one pair of discal scutellar setae set as widely apart as subapical scutellar setae; scutellum concolorous with scutum. Legs (Fig. 19c): yellow in ground colour, densely covered in black hairs making them appear almost black; fore femur with dense silver tomentum on posterodorsal surface; hind coxa setose. Wing (Fig. 19a, c): pale translucent, hyaline, not distinctly infuscate; vein $R_{4+5}$ with only $2-3$ setulae at base. Abdomen (Fig. 19a, c): ground colour black; mid-dorsal depression on ST1+2 almost reaching hind margin; entire abdomen covered in silver tomentum with brown tones towards tergal edges; median marginal setae present on ST1+2-T3; a complete row of marginal setae present on T4; discal setae only on T5; sex patch absent. Terminalia: sternite 5 (Fig. 19f) with a deeply excavated median cleft, slightly rounded 
V-shaped, margins covered in dense tomentum. Lateral lobes of sternite rounded apically, with 2-3 strong setae surrounded by many shorter, weaker setulae. Anterior plate of sternite 5 from subequal to slightly shorter than apical lobes, unsclerotised "window" weak, only very slightly unsclerotised, as wide as median cleft and flat. Cerci in posterior view (Fig. 19d) subrectangular, duck-billed and slightly longer than surstyli, blunt and rounded at apex, slightly divergent over apical 1/3; basal 2/3 fused; in lateral view rounded at tip, making them appear slightly downturned; densely setulose along basal $2 / 3$, setulose ventrally along entire length. Surstylus in lateral view (Fig. 19e) almost parallel-sided along its entire length, ending in a slightly downcurved apex, making the structure appear slightly blade-like; surstylus appearing to be fused with epandrium; when viewed dorsally, surstyli appearing to point inwards and strongly convergent, laterally covered in short stout setulae. Pregonite short and stout, welldeveloped, 0.3 times as long as distiphallus, squared-off apically, with a few short marginal setulae. Postgonite slightly narrow, 1/3 as wide as pregonite, sharply pointed and curved at apex. Distiphallus rectangular, with a very slight apical flare, a slender median longitudinal sclerotised reinforcement on its posterior surface and a broad, anterolateral, sclerotised acrophallus, joined as a plate on anterior surface near apex.

Female. Length: 5-7 mm. As male, differing only by the presence of two pairs of proclinate orbital setae.

\section{Diagnosis}

Hyphantrophaga manuelriosi sp. n. can be distinguished from all other Hyphantrophaga species by the following combination of traits: ocellar triangle silver, fronto-orbital plate setulose throughout, pedicel light brown-orange, three katepisternal setae, hind coxa setose, median marginal setae present on ST1+2, discal setae absent from T3 and T4, sex patch absent, abdominal tomentum silver with brown tones at tergal edges.

\section{Etymology}

Hyphantrophaga manuelriosi sp. $\mathbf{n}$. is named in recognition of Manuel Rios Castro's dedication and work in finding and rearing the ACG caterpillars that contained tachinid larvae.

\section{Distribution}

Costa Rica, ACG, Guanacaste Province, 10-480 m elevation.

\section{Ecology}

Hyphantrophaga manuelriosi sp. $\mathbf{n}$. has been reared 58 times from one species of Lepidoptera in the family Crambidae, Syllepte belialis (Walker, 1859), in dry forest and dry-rain lowland intergrades. 

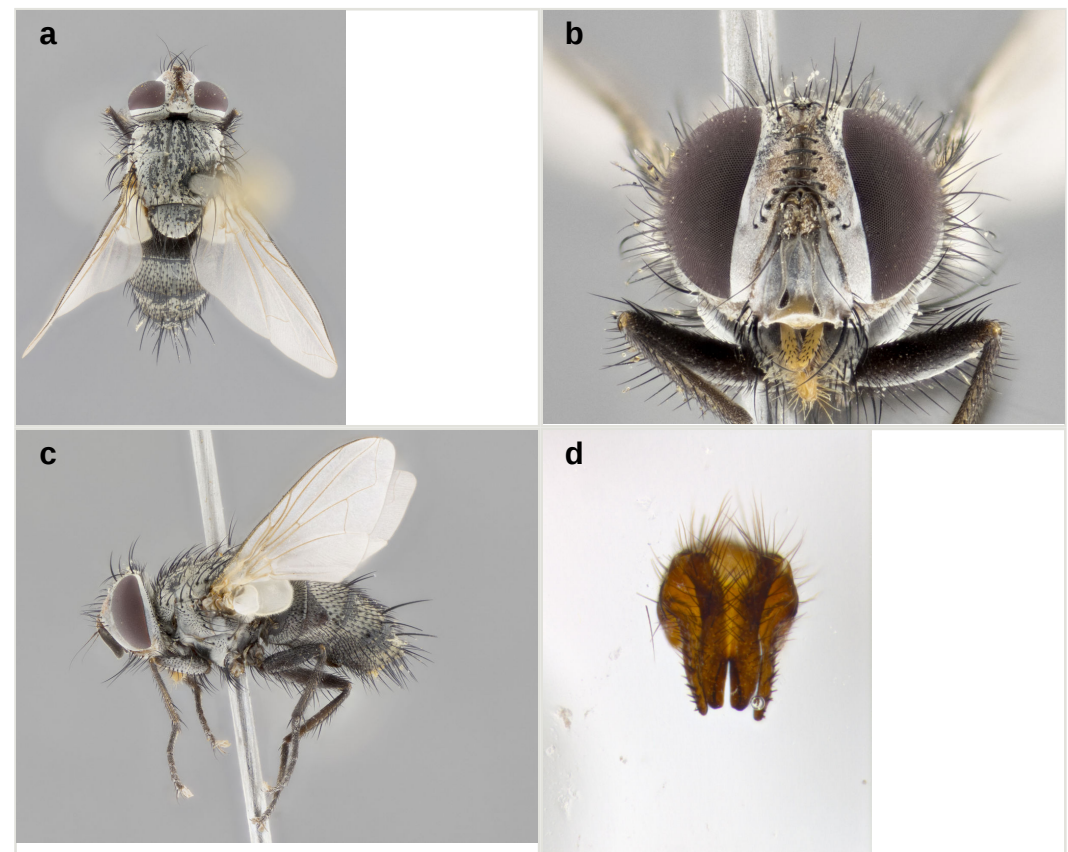

d

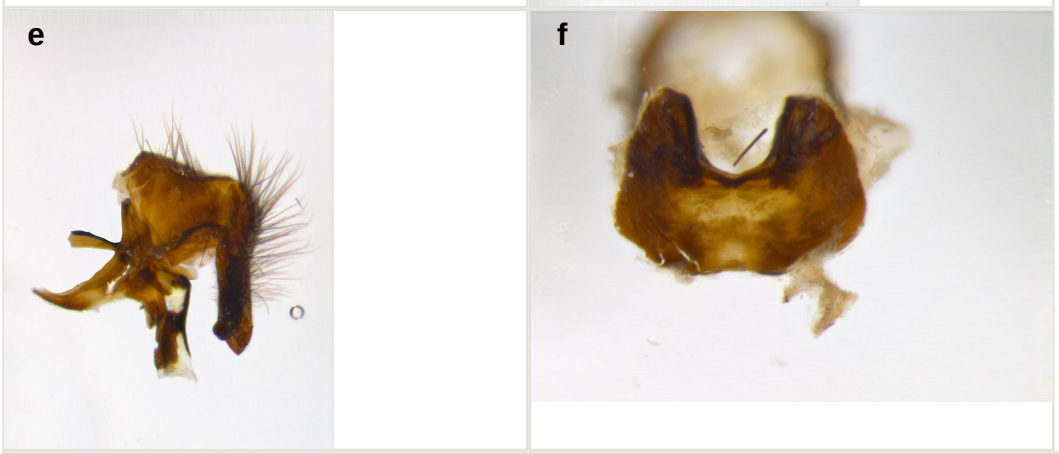

Figure 19.

Hyphantrophaga manuelriosi sp. n.; a-c: habitus; holotype male, voucher $\mathrm{n}$. DHJPAR0007340; d-f: terminalia; male paratype, voucher n. 01-SRNP-13278.
a: dorsal view doi
b: frontal view doi
c: lateral view doi
d: dorsal view doi
e: lateral view doi
f: sternite 5 , ventral view doi 


\section{Hyphantrophaga morphophaga Fleming \& Wood, sp. n.}

- ZooBank urn:Isid:Zoobank.org:act:F3E79C06-F806-4C34-A03B-58D7163280E8

\section{Materials}

\section{Holotype:}

a. $\quad$ scientificName: Hyphantrophaga morphophaga; phylum: Arthropoda; class: Insecta; order: Diptera; family: Tachinidae; genus: Hyphantrophaga; specificEpithet: morphophaga; scientificNameAuthorship: Fleming \& Wood, 2018; continent: Central America; country: Costa Rica; countryCode: CR; stateProvince: Guanacaste; county: Sector Pailas; locality: Area de Conservacion Guanacaste; verbatimLocality: Gemelos; verbatimElevation: 1276; verbatimLatitude: 10.7693; verbatimLongitude: -85.3466; verbatimCoordinateSystem: Decimal; decimalLatitude: 10.7693; decimalLongitude: -85.3466; samplingProtocol: Reared from the larva of the Nymphalidae, Morpho catalina; verbatimEventDate: 07-Jan-1995; individualID: DHJPAR0007354; individualCount: 1; sex: male; lifeStage: adult; preparations: pinned; catalogNumber: DHJPAR0007354; occurrenceDetails: http://janzen.sas.upenn.edu; recordedBy: D.H. Janzen, W. Hallwachs \& gusaneros; otherCatalogNumbers: ASTAT126-06, 95SRNP-4612, BOLD:AAA5134; identifiedBy: AJ Fleming; dateldentified: 2017; language: en; institutionCode: CNC; collectionCode: Insects; basisOfRecord: Pinned Specimen

\section{Paratypes:}

a. ScientificName: Hyphantrophaga morphophaga; phylum: Arthropoda; class: Insecta; order: Diptera; family: Tachinidae; genus: Hyphantrophaga; specificEpithet: morphophaga; scientificNameAuthorship: Fleming \& Wood, 2018; continent: Central America; country: Costa Rica; countryCode: CR; stateProvince: Guanacaste; county: Sector Cacao; locality: Area de Conservacion Guanacaste; verbatimLocality: Estacion Gongora; verbatimElevation: 570; verbatimLatitude: 10.887 ; verbatimLongitude: -85.4744; verbatimCoordinateSystem: Decimal; decimalLatitude: 10.887; decimalLongitude: -85.4744; samplingProtocol: Reared from the larva of the Nymphalidae, Morpho helenor; verbatimEventDate: 15-Nov-2005; individualID: DHJPAR0005457; individualCount: 1; sex: male; lifeStage: adult; preparations: pinned; catalogNumber: DHJPAR0005457; occurrenceDetails: http://janzen.sas.upenn.edu; recordedBy: D.H. Janzen, W. Hallwachs \& Manuel Pereira; otherCatalogNumbers: ASTA577-06, 05-SRNP-47682,; identifiedBy: AJ Fleming; dateldentified: 2017; language: en; institutionCode: CNC; collectionCode: Insects; basisOfRecord: Pinned Specimen

b. $\quad$ scientificName: Hyphantrophaga morphophaga; phylum: Arthropoda; class: Insecta; order: Diptera; family: Tachinidae; genus: Hyphantrophaga; specificEpithet: morphophaga; scientificNameAuthorship: Fleming \& Wood, 2018; continent: Central America; country: Costa Rica; countryCode: CR; stateProvince: Guanacaste; county: Sector Santa Rosa; locality: Area de Conservacion Guanacaste; verbatimLocality: Bosque San Emilio; verbatimElevation: 300; verbatimLatitude: 10.8439; verbatimLongitude: -85.6138; verbatimCoordinateSystem: Decimal; decimalLatitude: 10.8439; decimalLongitude: -85.6138; samplingProtocol: Reared from the larva of the Nymphalidae, Morpho helenor; verbatimEventDate: Unavailable, 1984; individualID: DHJPAR0007350; individualCount: 1; sex: male; lifeStage: adult; preparations: pinned; catalogNumber: DHJPAR0007350; occurrenceDetails: http://janzen.sas.upenn.edu; recordedBy: D.H. Janzen, W. Hallwachs \& Daniel H. Janzen; otherCatalog Numbers: 
ASTAT122-06, 84-SRNP-837, BOLD:AAA5134; identifiedBy: AJ Fleming; dateldentified: 2017; language: en; institutionCode: CNC; collectionCode: Insects; basisOfRecord: Pinned Specimen

c. ScientificName: Hyphantrophaga morphophaga; phylum: Arthropoda; class: Insecta; order: Diptera; family: Tachinidae; genus: Hyphantrophaga; specificEpithet: morphophaga; scientificNameAuthorship: Fleming \& Wood, 2018; continent: Central America; country: Costa Rica; countryCode: CR; stateProvince: Guanacaste; county: Sector Cacao; locality: Area de Conservacion Guanacaste; verbatimLocality: Estacion Cacao; verbatimElevation: 1150; verbatimLatitude: 10.9269; verbatimLongitude: -85.4682; verbatimCoordinateSystem: Decimal; decimalLatitude: 10.9269; decimalLongitude: -85.4682; samplingProtocol: Reared from the larva of the Nymphalidae, Morpho helenor; verbatimEventDate: 27-Jan-1998; individualID: DHJPAR0007351; individualCount: 1; sex: male; lifeStage: adult; preparations: pinned; catalogNumber: DHJPAR0007351; occurrenceDetails: http://janzen.sas.upenn.edu; recordedBy: D.H. Janzen, W. Hallwachs \& Ruth Franco; otherCatalog Numbers: ASTAT123-06, 97-SRNP-11139, BOLD:AAA5134; identifiedBy: AJ Fleming; dateldentified: 2017; language: en; institutionCode: CNC; collectionCode: Insects; basisOfRecord: Pinned Specimen

d. $\quad$ scientificName: Hyphantrophaga morphophaga; phylum: Arthropoda; class: Insecta; order: Diptera; family: Tachinidae; genus: Hyphantrophaga; specificEpithet: morphophaga; scientificNameAuthorship: Fleming \& Wood, 2018; continent: Central America; country: Costa Rica; countryCode: CR; stateProvince: Guanacaste; county: Sector Cacao; locality: Area de Conservacion Guanacaste; verbatimLocality: Sendero Arenales; verbatimElevation: 1080; verbatimLatitude: 10.9247; verbatimLongitude: -85.4674; verbatimCoordinateSystem: Decimal; decimalLatitude: 10.9247; decimalLongitude: -85.4674; samplingProtocol: Reared from the larva of the Nymphalidae, Morpho helenor; verbatimEventDate: 08-Sep-2002; individualID: DHJPAR0007352; individualCount: 1; sex: male; lifeStage: adult; preparations: pinned; catalogNumber: DHJPAR0007352; occurrenceDetails: http://janzen.sas.upenn.edu; recordedBy: D.H. Janzen, W. Hallwachs \& Harry Ramirez; otherCatalogNumbers: ASTAT124-06, 02-SRNP-23408, BOLD:AAA5134; identifiedBy: AJ Fleming; dateldentified: 2017; language: en; institutionCode: CNC; collectionCode: Insects; basisOfRecord: Pinned Specimen

e. $\quad$ scientificName: Hyphantrophaga morphophaga; phylum: Arthropoda; class: Insecta; order: Diptera; family: Tachinidae; genus: Hyphantrophaga; specificEpithet: morphophaga; scientificNameAuthorship: Fleming \& Wood, 2018; continent: Central America; country: Costa Rica; countryCode: CR; stateProvince: Guanacaste; county: Sector Pailas; locality: Area de Conservacion Guanacaste; verbatimLocality: Gemelos; verbatimElevation: 1276; verbatimLatitude: 10.7693; verbatimLongitude: -85.3466; verbatimCoordinateSystem: Decimal; decimalLatitude: 10.7693; decimalLongitude: -85.3466; samplingProtocol: Reared from the larva of the Nymphalidae, Morpho catalina; verbatimEventDate: 07-Jan-1995; individualID: DHJPAR0007353; individualCount: 1; sex: male; lifeStage: adult; preparations: pinned; catalogNumber: DHJPAR0007353; occurrenceDetails: http://janzen.sas.upenn.edu; recordedBy: D.H. Janzen, W. Hallwachs \& gusaneros; otherCatalogNumbers: ASTAT125-06, 95SRNP-4613, BOLD:AAA5134; identifiedBy: AJ Fleming; dateldentified: 2017; language: en; institutionCode: CNC; collectionCode: Insects; basisOfRecord: Pinned Specimen

f. ScientificName: Hyphantrophaga morphophaga; phylum: Arthropoda; class: Insecta; order: Diptera; family: Tachinidae; genus: Hyphantrophaga; specificEpithet: 
morphophaga; scientificNameAuthorship: Fleming \& Wood, 2018; continent: Central America; country: Costa Rica; countryCode: CR; stateProvince: Guanacaste; county: Sector Cacao; locality: Area de Conservacion Guanacaste; verbatimLocality: Sendero Abajo; verbatimElevation: 1020; verbatimLatitude: 10.9255; verbatimLongitude: -85.4716; verbatimCoordinateSystem: Decimal; decimalLatitude: 10.9255; decimalLongitude: -85.4716; samplingProtocol: Reared from the larva of the Nymphalidae, Morpho catalina; verbatimEventDate: 25-Feb-2004; individualID: DHJPAR0007357; individualCount: 1; sex: male; lifeStage: adult; preparations: pinned; catalogNumber: DHJPAR0007357; occurrenceDetails: http://janzen.sas.upenn.edu; recordedBy: D.H. Janzen, W. Hallwachs \& Mariano Pereira; otherCatalogNumbers: ASTAT129-06, 04-SRNP-35010, BOLD:AAA5134; identifiedBy: AJ Fleming; dateldentified: 2017; language: en; institutionCode: CNC; collectionCode: Insects; basisOfRecord: Pinned Specimen

g. scientificName: Hyphantrophaga morphophaga; phylum: Arthropoda; class: Insecta; order: Diptera; family: Tachinidae; genus: Hyphantrophaga; specificEpithet: morphophaga; scientificNameAuthorship: Fleming \& Wood, 2018; continent: Central America; country: Costa Rica; countryCode: CR; stateProvince: Guanacaste; county: Sector Del Oro; locality: Area de Conservacion Guanacaste; verbatimLocality: Quebrada Romero; verbatimElevation: 490; verbatimLatitude: 11.0052; verbatimLongitude: -85.474; verbatimCoordinateSystem: Decimal; decimalLatitude: 11.0052; decimalLongitude: -85.474; samplingProtocol: Reared from the larva of the Nymphalidae, Morpho helenor; verbatimEventDate: 22-Oct-2005; individualID: DHJPAR0007361; individualCount: 1; sex: male; lifeStage: adult; preparations: pinned; catalogNumber: DHJPAR0007361; occurrenceDetails: http://janzen.sas.upenn.edu; recordedBy: D.H. Janzen, W. Hallwachs \& Elieth Cantillano; otherCatalogNumbers: ASTAT133-06, 05-SRNP-23929, BOLD:AAA5134; identifiedBy: AJ Fleming; dateldentified: 2017; language: en; institutionCode: CNC; collectionCode: Insects; basisOfRecord: Pinned Specimen

h. scientificName: Hyphantrophaga morphophaga; phylum: Arthropoda; class: Insecta; order: Diptera; family: Tachinidae; genus: Hyphantrophaga; specificEpithet: morphophaga; scientificNameAuthorship: Fleming \& Wood, 2018; continent: Central America; country: Costa Rica; countryCode: CR; stateProvince: Guanacaste; county: Sector Del Oro; locality: Area de Conservacion Guanacaste; verbatimLocality: Quebrada Trigal; verbatimElevation: 290; verbatimLatitude: 11.0268; verbatimLongitude: -85.4955 ; verbatimCoordinateSystem: Decimal; decimalLatitude: 11.0268; decimalLongitude: -85.4955; samplingProtocol: Reared from the larva of the Nymphalidae, Morpho helenor; verbatimEventDate: 25-Oct-2005; individualID:

DHJPAR0007364; individualCount: 1; sex: male; lifeStage: adult; preparations: pinned; catalog Number: DHJPAR0007364; occurrenceDetails: http://janzen.sas.upenn.edu; recordedBy: D.H. Janzen, W. Hallwachs \& Lucia Rios; otherCatalogNumbers: ASTAT136-06, 05-SRNP-24089, BOLD:AAA5134; identifiedBy: AJ Fleming; dateldentified: 2017; language: en; institutionCode: CNC; collectionCode: Insects; basisOfRecord: Pinned Specimen

i. $\quad$ scientificName: Hyphantrophaga morphophaga; phylum: Arthropoda; class: Insecta; order: Diptera; family: Tachinidae; genus: Hyphantrophaga; specificEpithet: morphophaga; scientificNameAuthorship: Fleming \& Wood, 2018; continent: Central America; country: Costa Rica; countryCode: CR; stateProvince: Guanacaste; county: Sector Cacao; locality: Area de Conservacion Guanacaste; verbatimLocality: Sendero Circular; verbatimElevation: 1185; verbatimLatitude: 10.9271; verbatimLongitude: -85.4668; verbatimCoordinateSystem: Decimal; decimalLatitude: 10.9271; 
decimalLongitude: -85.4668; samplingProtocol: Reared from the larva of the Nymphalidae, Morpho helenor; verbatimEventDate: 29-Dec-2002; individualID: DHJPAR0007366; individualCount: 1; sex: male; lifeStage: adult; preparations: pinned; catalogNumber: DHJPAR0007366; occurrenceDetails: http://janzen.sas.upenn.edu; recordedBy: D.H. Janzen, W. Hallwachs \& Freddy Quesada; otherCatalog Numbers: ASTAT138-06, 02-SRNP-24447, BOLD:AAA5134; identifiedBy: AJ Fleming; dateldentified: 2017; language: en; institutionCode: CNC; collectionCode: Insects; basisOfRecord: Pinned Specimen

j. $\quad$ scientificName: Hyphantrophaga morphophaga; phylum: Arthropoda; class: Insecta; order: Diptera; family: Tachinidae; genus: Hyphantrophaga; specificEpithet: morphophaga; scientificNameAuthorship: Fleming \& Wood, 2018; continent: Central America; country: Costa Rica; countryCode: CR; stateProvince: Guanacaste; county: Sector Del Oro; locality: Area de Conservacion Guanacaste; verbatimLocality: Quebrada Raiz; verbatimElevation: 280; verbatimLatitude: 11.0287; verbatimLongitude: -85.4867; verbatimCoordinateSystem: Decimal; decimalLatitude: 11.0287; decimalLongitude: -85.4867; samplingProtocol: Reared from the larva of the Nymphalidae, Morpho helenor; verbatimEventDate: 20-Oct-2002; individualID: DHJPAR0007368; individualCount: 1; sex: male; lifeStage: adult; preparations: pinned; catalogNumber: DHJPAR0007368; occurrenceDetails: http://janzen.sas.upenn.edu; recordedBy: D.H. Janzen, W. Hallwachs \& Manuel Pereira; otherCatalogNumbers: ASTAT140-06, 02-SRNP-29899, BOLD:AAA5134; identifiedBy: AJ Fleming; dateldentified: 2017; language: en; institutionCode: CNC; collectionCode: Insects; basisOfRecord: Pinned Specimen

k. ScientificName: Hyphantrophaga morphophaga; phylum: Arthropoda; class: Insecta; order: Diptera; family: Tachinidae; genus: Hyphantrophaga; specificEpithet: morphophaga; scientificNameAuthorship: Fleming \& Wood, 2018; continent: Central America; country: Costa Rica; countryCode: CR; stateProvince: Guanacaste; county: Sector Del Oro; locality: Area de Conservacion Guanacaste; verbatimLocality: Quebrada Salazar; verbatimElevation: 560; verbatimLatitude: 11.0022; verbatimLongitude: -85.4634; verbatimCoordinateSystem: Decimal; decimalLatitude: 11.0022; decimalLongitude: -85.4634; samplingProtocol: Reared from the larva of the Nymphalidae, Morpho helenor; verbatimEventDate: 02-Oct-2007; individualID: DHJPAR0021984; individualCount: 1; sex: male; lifeStage: adult; preparations: pinned; catalog Number: DHJPAR0021984; occurrenceDetails: http://janzen.sas.upenn.edu; recordedBy: D.H. Janzen, W. Hallwachs \& Lucia Rios; otherCatalogNumbers: ASTAT1122-07, 07-SRNP-23642, BOLD:AAA5134; identifiedBy: AJ Fleming; dateldentified: 2017; language: en; institutionCode: CNC; collectionCode: Insects; basisOfRecord: Pinned Specimen

I. ScientificName: Hyphantrophaga morphophaga; phylum: Arthropoda; class: Insecta; order: Diptera; family: Tachinidae; genus: Hyphantrophaga; specificEpithet: morphophaga; scientificNameAuthorship: Fleming \& Wood, 2018; continent: Central America; country: Costa Rica; countryCode: CR; stateProvince: Guanacaste; county: Sector Del Oro; locality: Area de Conservacion Guanacaste; verbatimLocality: Quebrada Trigal; verbatimElevation: 290; verbatimLatitude: 11.0268; verbatimLongitude: -85.4955; verbatimCoordinateSystem: Decimal; decimalLatitude: 11.0268; decimalLongitude: -85.4955; samplingProtocol: Reared from the larva of the Nymphalidae, Morpho helenor; verbatimEventDate: 02-Aug-2004; individualID: DHJPAR0006269; individualCount: 1; sex: female; lifeStage: adult; preparations: pinned; catalogNumber: DHJPAR0006269; occurrenceDetails: http:// janzen.sas.upenn.edu; recordedBy: D.H. Janzen, W. Hallwachs \& Lucia Rios; 
otherCatalogNumbers: ASTAI697-06, 04-SRNP-22553, BOLD:AAA5134; identifiedBy: AJ Fleming; dateldentified: 2017; language: en; institutionCode: CNC; collectionCode Insects; basisOfRecord: Pinned Specimen

m. ScientificName: Hyphantrophaga morphophaga; phylum: Arthropoda; class: Insecta; order: Diptera; family: Tachinidae; genus: Hyphantrophaga; specificEpithet: morphophaga; scientificNameAuthorship: Fleming \& Wood, 2018; continent: Central America; country: Costa Rica; countryCode: CR; stateProvince: Guanacaste; county: Sector Del Oro; locality: Area de Conservacion Guanacaste; verbatimLocality: Quebrada Raiz; verbatimElevation: 280; verbatimLatitude: 11.0287; verbatimLongitude: -85.4867; verbatimCoordinateSystem: Decimal; decimalLatitude: 11.0287; decimalLongitude: -85.4867; samplingProtocol: Reared from the larva of the Nymphalidae, Morpho helenor; verbatimEventDate: 30-Jul-2016; individuallD:

DHJPAR0059547; individualCount: 1; sex: female; lifeStage: adult; preparations: pinned; catalogNumber: DHJPAR0059547; occurrenceDetails: http:// janzen.sas.upenn.edu; recordedBy: D.H. Janzen, W. Hallwachs \& Lucia Rios; otherCatalog Numbers: ACGBA5964-16, 16-SRNP-20807, BOLD:AAA5134; identifiedBy: AJ Fleming; dateldentified: 2017; language: en; institutionCode: CNC; collectionCode: Insects; basisOfRecord: Pinned Specimen

n. ScientificName: Hyphantrophaga morphophaga; phylum: Arthropoda; class: Insecta; order: Diptera; family: Tachinidae; genus: Hyphantrophaga; specificEpithet: morphophaga; scientificNameAuthorship: Fleming \& Wood, 2018; continent: Central America; country: Costa Rica; countryCode: CR; stateProvince: Guanacaste; county: Sector Del Oro; locality: Area de Conservacion Guanacaste; verbatimLocality: Quebrada Trigal; verbatimElevation: 290; verbatimLatitude: 11.0268; verbatimLongitude: -85.4955; verbatimCoordinateSystem: Decimal; decimalLatitude: 11.0268; decimalLongitude: -85.4955; samplingProtocol: Reared from the larva of the Nymphalidae, Morpho helenor; verbatimEventDate: 17-Nov-2005; individualID: DHJPAR0005521; individualCount: 1; sex: female; lifeStage: adult; preparations: pinned; catalogNumber: DHJPAR0005521; occurrenceDetails: http:// janzen.sas.upenn.edu; recordedBy: D.H. Janzen, W. Hallwachs \& Elieth Cantillano; otherCatalogNumbers: ASTA640-06, 05-SRNP-24423, BOLD:AAA5134; identifiedBy: AJ Fleming; dateldentified: 2017; language: en; institutionCode: CNC; collectionCode: Insects; basisOfRecord: Pinned Specimen

o. ScientificName: Hyphantrophaga morphophaga; phylum: Arthropoda; class: Insecta; order: Diptera; family: Tachinidae; genus: Hyphantrophaga; specificEpithet: morphophaga; scientificNameAuthorship: Fleming \& Wood, 2018; continent: Central America; country: Costa Rica; countryCode: CR; stateProvince: Guanacaste; county: Sector El Hacha; locality: Area de Conservacion Guanacaste; verbatimLocality: Finca Araya; verbatimElevation: 295; verbatimLatitude: 11.0154; verbatimLongitude: -85.5113; verbatimCoordinateSystem: Decimal; decimalLatitude: 11.0154; decimalLongitude: -85.5113; samplingProtocol: Reared from the larva of the Nymphalidae, Morpho helenor; verbatimEventDate: 14-Nov-2002; individualID: DHJPAR0007365; individualCount: 1; sex: female; lifeStage: adult; preparations: pinned; catalogNumber: DHJPAR0007365; occurrenceDetails: http:// janzen.sas.upenn.edu; recordedBy: D.H. Janzen, W. Hallwachs \& Manuel Pereira; otherCatalogNumbers: ASTAT137-06, 02-SRNP-30058, BOLD:AAA5134; identifiedBy: AJ Fleming; dateldentified: 2017; language: en; institutionCode: CNC; collectionCode: Insects; basisOfRecord: Pinned Specimen

p. $\quad$ scientificName: Hyphantrophaga morphophaga; phylum: Arthropoda; class: Insecta; order: Diptera; family: Tachinidae; genus: Hyphantrophaga; specificEpithet: 
morphophaga; scientificNameAuthorship: Fleming \& Wood, 2018; continent: Central America; country: Costa Rica; countryCode: CR; stateProvince: Guanacaste; county: Sector Santa Elena; locality: Area de Conservacion Guanacaste; verbatimLocality: Casa Potrero Grande; verbatimElevation: 17; verbatimLatitude: 10.8492 ; verbatimLongitude: -85.7731; verbatimCoordinateSystem: Decimal; decimalLatitude: 10.8492; decimalLongitude: -85.7731; samplingProtocol: Reared from the larva of the Nymphalidae, Morpho helenor; verbatimEventDate: 31-Oct-2004; individualID: DHJPAR0011459; individualCount: 1; sex: female; lifeStage: adult; preparations: pinned; catalogNumber: DHJPAR0011459; occurrenceDetails: http:// janzen.sas.upenn.edu; recordedBy: D.H. Janzen, W. Hallwachs \& Guillermo Pereira; otherCatalogNumbers: ASTAQ846-06, 04-SRNP-14345,; identifiedBy: AJ Fleming; dateldentified: 2017; language: en; institutionCode: CNC; collectionCode: Insects; basisOfRecord: Pinned Specimen

q. $\quad$ scientificName: Hyphantrophaga morphophaga; phylum: Arthropoda; class: Insecta; order: Diptera; family: Tachinidae; genus: Hyphantrophaga; specificEpithet: morphophaga; scientificNameAuthorship: Fleming \& Wood, 2018; continent: Central America; country: Costa Rica; countryCode: CR; stateProvince: Guanacaste; county: Sector Mundo Nuevo; locality: Area de Conservacion Guanacaste; verbatimLocality: Vado Miramonte; verbatimElevation: 305; verbatimLatitude: 10.7718 ; verbatimLongitude: -85.434; verbatimCoordinateSystem: Decimal; decimalLatitude: 10.7718; decimalLongitude: -85.434; samplingProtocol: Reared from the larva of the Nymphalidae, Morpho helenor; verbatimEventDate: 27-Dec-2007; individualID: DHJPAR0023229; individualCount: 1; sex: female; lifeStage: adult; preparations: pinned; catalogNumber: DHJPAR0023229; occurrenceDetails: http:// janzen.sas.upenn.edu; recordedBy: D.H. Janzen, W. Hallwachs \& Jose Cortez; otherCatalogNumbers: ASTAW390-08, 07-SRNP-60840, BOLD:AAA5134; identifiedBy: AJ Fleming; dateldentified: 2017; language: en; institutionCode: CNC; collectionCode: Insects; basisOfRecord: Pinned Specimen

r. $\quad$ scientificName: Hyphantrophaga morphophaga; phylum: Arthropoda; class: Insecta; order: Diptera; family: Tachinidae; genus: Hyphantrophaga; specificEpithet: morphophaga; scientificNameAuthorship: Fleming \& Wood, 2018; continent: Central America; country: Costa Rica; countryCode: CR; stateProvince: Guanacaste; county: Sector El Hacha; locality: Area de Conservacion Guanacaste; verbatimLocality: Finca Araya; verbatimElevation: 295; verbatimLatitude: 11.0154; verbatimLongitude: -85.5113; verbatimCoordinateSystem: Decimal; decimalLatitude: 11.0154; decimalLongitude: -85.5113; samplingProtocol: Reared from the larva of the Nymphalidae, Morpho helenor; verbatimEventDate: 24-Oct-2002; individualID: DHJPAR0006278; individualCount: 1; sex: female; lifeStage: adult; preparations: pinned; catalogNumber: DHJPAR0006278; occurrenceDetails: http:// janzen.sas.upenn.edu; recordedBy: D.H. Janzen, W. Hallwachs \& Manuel Pereira; otherCatalogNumbers: ASTAI706-06, 02-SRNP-29986, BOLD:AAA5134; identifiedBy: AJ Fleming; dateldentified: 2017; language: en; institutionCode: CNC; collectionCode: Insects; basisOfRecord: Pinned Specimen

s. $\quad$ scientificName: Hyphantrophaga morphophaga; phylum: Arthropoda; class: Insecta; order: Diptera; family: Tachinidae; genus: Hyphantrophaga; specificEpithet: morphophaga; scientificNameAuthorship: Fleming \& Wood, 2018; continent: Central America; country: Costa Rica; countryCode: CR; stateProvince: Guanacaste; county: Sector Cacao; locality: Area de Conservacion Guanacaste; verbatimLocality: Sendero Segundo; verbatimElevation: 1180; verbatimLatitude: 10.9268; verbatimLongitude: -85.4533; verbatimCoordinateSystem: Decimal; decimalLatitude: 10.9268; 
decimalLongitude: -85.4533; samplingProtocol: Reared from the larva of the Nymphalidae, Morpho helenor; verbatimEventDate: 25-Jul-2004; individualID: DHJPAR0006289; individualCount: 1; sex: female; lifeStage: adult; preparations: pinned; catalogNumber: DHJPAR0006289; occurrenceDetails: http:// janzen.sas.upenn.edu; recordedBy: D.H. Janzen, W. Hallwachs \& Harry Ramirez; otherCatalog Numbers: ASTAI717-06, 04-SRNP-35577, BOLD:AAA5134; identifiedBy: AJ Fleming; dateldentified: 2017; language: en; institutionCode: CNC; collectionCode: Insects; basisOfRecord: Pinned Specimen

t. $\quad$ scientificName: Hyphantrophaga morphophaga; phylum: Arthropoda; class: Insecta; order: Diptera; family: Tachinidae; genus: Hyphantrophaga; specificEpithet: morphophaga; scientificNameAuthorship: Fleming \& Wood, 2018; continent: Central America; country: Costa Rica; countryCode: CR; stateProvince: Guanacaste; county: Sector Cacao; locality: Area de Conservacion Guanacaste; verbatimLocality: Estacion Gongora; verbatimElevation: 570; verbatimLatitude: 10.887 ; verbatimLongitude: -85.4744; verbatimCoordinateSystem: Decimal; decimalLatitude: 10.887 ; decimalLongitude: -85.4744; samplingProtocol: Reared from the larva of the Nymphalidae, Morpho helenor; verbatimEventDate: 02-Nov-2005; individualID: DHJPAR0005474; individualCount: 1; sex: female; lifeStage: adult; preparations: pinned; catalogNumber: DHJPAR0005474; occurrenceDetails: http:// janzen.sas.upenn.edu; recordedBy: D.H. Janzen, W. Hallwachs \& Manuel Pereira; otherCatalogNumbers: ASTA594-06, 05-SRNP-47688, BOLD:AAA5134; identifiedBy: AJ Fleming; dateldentified: 2017; language: en; institutionCode: CNC; collectionCode: Insects; basisOfRecord: Pinned Specimen

u. $\quad$ scientificName: Hyphantrophaga morphophaga; phylum: Arthropoda; class: Insecta; order: Diptera; family: Tachinidae; genus: Hyphantrophaga; specificEpithet: morphophaga; scientificNameAuthorship: Fleming \& Wood, 2018; continent: Central America; country: Costa Rica; countryCode: CR; stateProvince: Guanacaste; county: Sector Cacao; locality: Area de Conservacion Guanacaste; verbatimLocality: Sendero Arenales; verbatimElevation: 1080 ; verbatimLatitude: 10.9247 ; verbatimLongitude: -85.4674; verbatimCoordinateSystem: Decimal; decimalLatitude: 10.9247; decimalLongitude: -85.4674; samplingProtocol: Reared from the larva of the Nymphalidae, Morpho catalina; verbatimEventDate: 22-Mar-2002; individualID: DHJPAR0007356; individualCount: 1; sex: female; lifeStage: adult; preparations: pinned; catalogNumber: DHJPAR0007356; occurrenceDetails: http:// janzen.sas.upenn.edu; recordedBy: D.H. Janzen, W. Hallwachs \& Harry Ramirez; otherCatalogNumbers: ASTAT128-06, 02-SRNP-8033, BOLD:AAA5134; identifiedBy: AJ Fleming; dateldentified: 2017; language: en; institutionCode: CNC; collectionCode: Insects; basisOfRecord: Pinned Specimen

v. ScientificName: Hyphantrophaga morphophaga; phylum: Arthropoda; class: Insecta; order: Diptera; family: Tachinidae; genus: Hyphantrophaga; specificEpithet: morphophaga; scientificNameAuthorship: Fleming \& Wood, 2018; continent: Central America; country: Costa Rica; countryCode: CR; stateProvince: Guanacaste; county: Sector Del Oro; locality: Area de Conservacion Guanacaste; verbatimLocality: Quebrada Romero; verbatimElevation: 490; verbatimLatitude: 11.0052; verbatimLongitude: -85.474; verbatimCoordinateSystem: Decimal; decimalLatitude: 11.0052; decimalLongitude: -85.474; samplingProtocol: Reared from the larva of the Nymphalidae, Morpho helenor; verbatimEventDate: 22-Oct-2005; individualID: DHJPAR0007358; individualCount: 1; sex: female; lifeStage: adult; preparations: pinned; catalogNumber: DHJPAR0007358; occurrenceDetails: http:// janzen.sas.upenn.edu; recordedBy: D.H. Janzen, W. Hallwachs \& Elieth Cantillano; 
otherCatalog Numbers: ASTAT130-06, 05-SRNP-23929, BOLD:AAA5134; identifiedBy: AJ Fleming; dateldentified: 2017; language: en; institutionCode: CNC; collectionCode: Insects; basisOfRecord: Pinned Specimen

w. ScientificName: Hyphantrophaga morphophaga; phylum: Arthropoda; class: Insecta; order: Diptera; family: Tachinidae; genus: Hyphantrophaga; specificEpithet: morphophaga; scientificNameAuthorship: Fleming \& Wood, 2018; continent: Central America; country: Costa Rica; countryCode: CR; stateProvince: Guanacaste; county: Sector Santa Elena; locality: Area de Conservacion Guanacaste; verbatimLocality: Quebrada Megalopyge; verbatimElevation: 20; verbatimLatitude: 10.8471; verbatimLongitude: -85.7714; verbatimCoordinateSystem: Decimal; decimalLatitude: 10.8471; decimalLongitude: -85.7714; samplingProtocol: Reared from the larva of the Nymphalidae, Morpho helenor; verbatimEventDate: 13-Mar-2003; individualID: DHJPAR0007359; individualCount: 1; sex: female; lifeStage: adult; preparations: pinned; catalogNumber: DHJPAR0007359; occurrenceDetails: http:// janzen.sas.upenn.edu; recordedBy: D.H. Janzen, W. Hallwachs \& Dunia Garcia; otherCatalogNumbers: ASTAT131-06, 03-SRNP-392, BOLD:AAA5134; identifiedBy: AJ Fleming; dateldentified: 2017; language: en; institutionCode: CNC; collectionCode: Insects; basisOfRecord: Pinned Specimen

x. $\quad$ scientificName: Hyphantrophaga morphophaga; phylum: Arthropoda; class: Insecta; order: Diptera; family: Tachinidae; genus: Hyphantrophaga; specificEpithet: morphophaga; scientificNameAuthorship: Fleming \& Wood, 2018; continent: Central America; country: Costa Rica; countryCode: CR; stateProvince: Guanacaste; county: Sector El Hacha; locality: Area de Conservacion Guanacaste; verbatimLocality: Finca Araya; verbatimElevation: 295; verbatimLatitude: 11.0154; verbatimLongitude: -85.5113; verbatimCoordinateSystem: Decimal; decimalLatitude: 11.0154; decimalLongitude: -85.5113; samplingProtocol: Reared from the larva of the Nymphalidae, Morpho helenor; verbatimEventDate: 04-Nov-2002; individualID: DHJPAR0007360; individualCount: 1; sex: female; lifeStage: adult; preparations: pinned; catalogNumber: DHJPAR0007360; occurrenceDetails: http:// janzen.sas.upenn.edu; recordedBy: D.H. Janzen, W. Hallwachs \& Ruth Franco; otherCatalogNumbers: ASTAT132-06, 02-SRNP-30652, BOLD:AAA5134; identifiedBy: AJ Fleming; dateldentified: 2017; language: en; institutionCode: CNC; collectionCode: Insects; basisOfRecord: Pinned Specimen

y. $\quad$ scientificName: Hyphantrophaga morphophaga; phylum: Arthropoda; class: Insecta; order: Diptera; family: Tachinidae; genus: Hyphantrophaga; specificEpithet: morphophaga; scientificNameAuthorship: Fleming \& Wood, 2018; continent: Central America; country: Costa Rica; countryCode: CR; stateProvince: Guanacaste; county: Sector Cacao; locality: Area de Conservacion Guanacaste; verbatimLocality: Sendero Abajo; verbatimElevation: 1020; verbatimLatitude: 10.9255; verbatimLongitude: -85.4716; verbatimCoordinateSystem: Decimal; decimalLatitude: 10.9255; decimalLongitude: -85.4716; samplingProtocol: Reared from the larva of the Nymphalidae, Morpho helenor; verbatimEventDate: 06-Aug-2005; individualID: DHJPAR0007362; individualCount: 1; sex: female; lifeStage: adult; preparations: pinned; catalogNumber: DHJPAR0007362; occurrenceDetails: http:// janzen.sas.upenn.edu; recordedBy: D.H. Janzen, W. Hallwachs \& Harry Ramirez; otherCatalog Numbers: ASTAT134-06, 05-SRNP-35404, BOLD:AAA5134; identifiedBy: AJ Fleming; dateldentified: 2017; language: en; institutionCode: CNC; collectionCode: Insects; basisOfRecord: Pinned Specimen

z. $\quad$ scientificName: Hyphantrophaga morphophaga; phylum: Arthropoda; class: Insecta; order: Diptera; family: Tachinidae; genus: Hyphantrophaga; specificEpithet: 
morphophaga; scientificNameAuthorship: Fleming \& Wood, 2018; continent: Central America; country: Costa Rica; countryCode: CR; stateProvince: Guanacaste; county: Sector Del Oro; locality: Area de Conservacion Guanacaste; verbatimLocality: Quebrada Romero; verbatimElevation: 490; verbatimLatitude: 11.0052; verbatimLongitude: -85.474; verbatimCoordinateSystem: Decimal; decimalLatitude: 11.0052; decimalLongitude: -85.474; samplingProtocol: Reared from the larva of the Nymphalidae, Morpho helenor; verbatimEventDate: 27-Oct-2005; individualID: DHJPAR0007363; individualCount: 1; sex: female; lifeStage: adult; preparations: pinned; catalogNumber: DHJPAR0007363; occurrenceDetails: http:// janzen.sas.upenn.edu; recordedBy: D.H. Janzen, W. Hallwachs \& Elieth Cantillano; otherCatalog Numbers: ASTAT135-06, 05-SRNP-23927, BOLD:AAA5134; identifiedBy: AJ Fleming; dateldentified: 2017; language: en; institutionCode: CNC; collectionCode: Insects; basisOfRecord: Pinned Specimen

aa. scientificName: Hyphantrophaga morphophaga; phylum: Arthropoda; class: Insecta; order: Diptera; family: Tachinidae; genus: Hyphantrophaga; specificEpithet: morphophaga; scientificNameAuthorship: Fleming \& Wood, 2018; continent: Central America; country: Costa Rica; countryCode: CR; stateProvince: Guanacaste; county: Sector El Hacha; locality: Area de Conservacion Guanacaste; verbatimLocality: Finca Araya; verbatimElevation: 295; verbatimLatitude: 11.0154; verbatimLongitude: -85.5113; verbatimCoordinateSystem: Decimal; decimalLatitude: 11.0154; decimalLongitude: -85.5113; samplingProtocol: Reared from the larva of the Nymphalidae, Morpho helenor; verbatimEventDate: 22-Nov-2002; individualID: DHJPAR0007367; individualCount: 1; sex: female; lifeStage: adult; preparations: pinned; catalogNumber: DHJPAR0007367; occurrenceDetails: http:// janzen.sas.upenn.edu; recordedBy: D.H. Janzen, W. Hallwachs \& Lucia Rios; otherCatalog Numbers: ASTAT139-06, 02-SRNP-31434, BOLD:AAA5134; identifiedBy: AJ Fleming; dateldentified: 2017; language: en; institutionCode: CNC; collectionCode: Insects; basisOfRecord: Pinned Specimen

\section{Other materials:}

a. $\quad$ scientificName: Hyphantrophaga morphophaga; phylum: Arthropoda; class: Insecta; order: Diptera; family: Tachinidae; genus: Hyphantrophaga; specificEpithet: morphophaga; scientificNameAuthorship: Fleming \& Wood, 2018; continent: Central America; country: Costa Rica; countryCode: CR; stateProvince: Alajuela; county: Sector San Cristobal; locality: Area de Conservacion Guanacaste; verbatimLocality: Quebrada Garcia; verbatimElevation: 495; verbatimLatitude: 10.8607; verbatimLongitude: -85.4256; verbatimCoordinateSystem: Decimal; decimalLatitude: 10.8607; decimalLongitude: -85.4256; samplingProtocol: Reared from the larva of the Nymphalidae, Morpho helenor; verbatimEventDate: 20-Oct-2008; individualID: DHJPAR0029643; individualCount: 1; sex: male; lifeStage: adult; preparations: pinned; catalogNumber: DHJPAR0029643; occurrenceDetails: http://janzen.sas.upenn.edu; recordedBy: D.H. Janzen, W. Hallwachs \& Elda Araya; otherCatalogNumbers: ASHYM1064-09, 08-SRNP-5341, BOLD:AAA5134; identifiedBy: AJ Fleming; dateldentified: 2017; language: en; institutionCode: CNC; collectionCode: Insects; basisOfRecord: Pinned Specimen

b. $\quad$ scientificName: Hyphantrophaga morphophaga; phylum: Arthropoda; class: Insecta; order: Diptera; family: Tachinidae; genus: Hyphantrophaga; specificEpithet: morphophaga; scientificNameAuthorship: Fleming \& Wood, 2018; continent: Central America; country: Costa Rica; countryCode: CR; stateProvince: Guanacaste; county: Sector Mundo Nuevo; locality: Area de Conservacion Guanacaste; verbatimLocality: 
Vado Zanja Tapada; verbatimElevation: 550; verbatimLatitude: 10.7648; verbatimLongitude: -85.3845; verbatimCoordinateSystem: Decimal; decimalLatitude: 10.7648; decimalLongitude: -85.3845 ; samplingProtocol: Reared from the larva of the Nymphalidae, Morpho helenor; verbatimEventDate: 02-Jan-2009; individualID:

DHJPAR0030122; individualCount: 1; sex: male; lifeStage: adult; preparations: pinned; catalogNumber: DHJPAR0030122; occurrenceDetails: http://janzen.sas.upenn.edu; recordedBy: D.H. Janzen, W. Hallwachs \& Jose Cortez; otherCatalogNumbers: ASHYB866-09, 08-SRNP-58574, BOLD:AAA5134; identifiedBy: AJ Fleming; dateldentified: 2017; language: en; institutionCode: CNC; collectionCode: Insects; basisOfRecord: Pinned Specimen

c. scientificName: Hyphantrophaga morphophaga; phylum: Arthropoda; class: Insecta; order: Diptera; family: Tachinidae; genus: Hyphantrophaga; specificEpithet: morphophaga; scientificNameAuthorship: Fleming \& Wood, 2018; continent: Central America; country: Costa Rica; countryCode: CR; stateProvince: Guanacaste; county: Sector Del Oro; locality: Area de Conservacion Guanacaste; verbatimLocality: Quebrada Trigal; verbatimElevation: 290; verbatimLatitude: 11.0268; verbatimLongitude: -85.4955 ; verbatimCoordinateSystem: Decimal; decimalLatitude: 11.0268; decimalLongitude: -85.4955; samplingProtocol: Reared from the larva of the Nymphalidae, Morpho helenor; verbatimEventDate: 24-Dec-2012; individualID: DHJPAR0050520; individualCount: 1; sex: male; lifeStage: adult; preparations: pinned; catalogNumber: DHJPAR0050520; occurrenceDetails: http://janzen.sas.upenn.edu; recordedBy: D.H. Janzen, W. Hallwachs \& Lucia Rios; otherCatalogNumbers: ACGBA3112-13, 12-SRNP-21848, BOLD:AAA5134; identifiedBy: AJ Fleming; dateldentified: 2017; language: en; institutionCode: CNC; collectionCode: Insects; basisOfRecord: Pinned Specimen

d. ScientificName: Hyphantrophaga morphophaga; phylum: Arthropoda; class: Insecta; order: Diptera; family: Tachinidae; genus: Hyphantrophaga; specificEpithet: morphophaga; scientificNameAuthorship: Fleming \& Wood, 2018; continent: Central America; country: Costa Rica; countryCode: CR; stateProvince: Guanacaste; county: Sector Cacao; locality: Area de Conservacion Guanacaste; verbatimLocality: Sendero Abajo; verbatimElevation: 1020; verbatimLatitude: 10.9255; verbatimLongitude: -85.4716; verbatimCoordinateSystem: Decimal; decimalLatitude: 10.9255; decimalLongitude: -85.4716; samplingProtocol: Reared from the larva of the Nymphalidae, Morpho catalina; verbatimEventDate: 13-Sep-2001; individualID: DHJPAR0007355; individualCount: 1; sex: male; lifeStage: adult; preparations: pinned; catalogNumber: DHJPAR0007355; occurrenceDetails: http://janzen.sas.upenn.edu; recordedBy: D.H. Janzen, W. Hallwachs \& Harry Ramirez; otherCatalog Numbers: ASTAT127-06, 01-SRNP-7320,; identifiedBy: AJ Fleming; dateldentified: 2017; language: en; institutionCode: CNC; collectionCode: Insects; basisOfRecord: Pinned Specimen

e. ScientificName: Hyphantrophaga morphophaga; phylum: Arthropoda; class: Insecta; order: Diptera; family: Tachinidae; genus: Hyphantrophaga; specificEpithet: morphophaga; scientific NameAuthorship: Fleming \& Wood, 2018; continent: Central America; country: Costa Rica; countryCode: CR; stateProvince: Guanacaste; county: Sector Del Oro; locality: Area de Conservacion Guanacaste; verbatimLocality: Finca Araya; verbatimElevation: 295; verbatimLatitude: 11.0154; verbatimLongitude: -85.5113; verbatimCoordinateSystem: Decimal; decimalLatitude: 11.0154; decimalLongitude: -85.5113; samplingProtocol: Reared from the larva of the Nymphalidae, Morpho helenor; verbatimEventDate: 27-Oct-2014; individualID: DHJPAR0057239; individualCount: 1; sex: male; lifeStage: adult; preparations: pinned; 
catalogNumber: DHJPAR0057239; occurrenceDetails: http://janzen.sas.upenn.edu; recordedBy: D.H. Janzen, W. Hallwachs \& Manuel Rios; otherCatalog Numbers: ACGBA5149-15, 14-SRNP-20990, BOLD:AAA5134; identifiedBy: AJ Fleming; dateldentified: 2017; language: en; institutionCode: CNC; collectionCode: Insects; basisOfRecord: Pinned Specimen

f. ScientificName: Hyphantrophaga morphophaga; phylum: Arthropoda; class: Insecta; order: Diptera; family: Tachinidae; genus: Hyphantrophaga; specificEpithet: morphophaga; scientificNameAuthorship: Fleming \& Wood, 2018; continent: Central America; country: Costa Rica; countryCode: CR; stateProvince: Guanacaste; county: Sector Santa Elena; locality: Area de Conservacion Guanacaste; verbatimLocality: Casa Potrero Grande; verbatimElevation: 17; verbatimLatitude: 10.8492; verbatimLongitude: -85.7731; verbatimCoordinateSystem: Decimal; decimalLatitude: 10.8492; decimalLongitude: -85.7731; samplingProtocol: Reared from the larva of the Nymphalidae, Morpho helenor; verbatimEventDate: 11-Nov-2004; individualID:

DHJPAR0007370; individualCount: 1; sex: male; lifeStage: adult; preparations: pinned; catalogNumber: DHJPAR0007370; occurrenceDetails: http://janzen.sas.upenn.edu; recordedBy: D.H. Janzen, W. Hallwachs \& Freddy Quesada; otherCatalogNumbers: ASTAT142-06, 04-SRNP-14946, BOLD:AAA5134; identifiedBy: AJ Fleming; dateldentified: 2017; language: en; institutionCode: CNC; collectionCode: Insects; basisOfRecord: Pinned Specimen

g. scientificName: Hyphantrophaga morphophaga; phylum: Arthropoda; class: Insecta; order: Diptera; family: Tachinidae; genus: Hyphantrophaga; specificEpithet: morphophaga; scientificNameAuthorship: Fleming \& Wood, 2018; continent: Central America; country: Costa Rica; countryCode: CR; stateProvince: Guanacaste; county: Sector Cacao; locality: Area de Conservacion Guanacaste; verbatimLocality: Quebrada Otilio; verbatimElevation: 550; verbatimLatitude: 10.89; verbatimLongitude: -85.4797; verbatimCoordinateSystem: Decimal; decimalLatitude: 10.89 ; decimalLongitude: -85.4797; samplingProtocol: Reared from the larva of the Nymphalidae, Morpho helenor; verbatimEventDate: 23-Nov-2004; individualID: DHJPAR0007371; individualCount: 1; sex: male; lifeStage: adult; preparations: pinned; catalogNumber: DHJPAR0007371; occurrenceDetails: http://janzen.sas.upenn.edu; recordedBy: D.H. Janzen, W. Hallwachs \& Manuel Pereira; otherCatalogNumbers: ASTAT143-06, 04-SRNP-49474, BOLD:AAA5134; identifiedBy: AJ Fleming; dateldentified: 2017; language: en; institutionCode: CNC; collectionCode: Insects; basisOfRecord: Pinned Specimen

h. $\quad$ scientificName: Hyphantrophaga morphophaga; phylum: Arthropoda; class: Insecta; order: Diptera; family: Tachinidae; genus: Hyphantrophaga; specificEpithet: morphophaga; scientificNameAuthorship: Fleming \& Wood, 2018; continent: Central America; country: Costa Rica; countryCode: CR; stateProvince: Guanacaste; county: Sector Cacao; locality: Area de Conservacion Guanacaste; verbatimLocality: Sendero Abajo; verbatimElevation: 1020; verbatimLatitude: 10.9255; verbatimLongitude: -85.4716; verbatimCoordinateSystem: Decimal; decimalLatitude: 10.9255; decimalLongitude: -85.4716; samplingProtocol: Reared from the larva of the Nymphalidae, Morpho helenor; verbatimEventDate: 17-Dec-2004; individualID: DHJPAR0007373; individualCount: 1; sex: male; lifeStage: adult; preparations: pinned; catalogNumber: DHJPAR0007373; occurrenceDetails: http://janzen.sas.upenn.edu; recordedBy: D.H. Janzen, W. Hallwachs \& Mariano Pereira; otherCatalogNumbers: ASTAT145-06, 04-SRNP-35959, BOLD:AAA5134; identifiedBy: AJ Fleming; dateldentified: 2017; language: en; institutionCode: CNC; collectionCode: Insects; basisOfRecord: Pinned Specimen 
i. $\quad$ scientificName: Hyphantrophaga morphophaga; phylum: Arthropoda; class: Insecta; order: Diptera; family: Tachinidae; genus: Hyphantrophaga; specificEpithet: morphophaga; scientificNameAuthorship: Fleming \& Wood, 2018; continent: Central America; country: Costa Rica; countryCode: CR; stateProvince: Guanacaste; county: Sector Santa Elena; locality: Area de Conservacion Guanacaste; verbatimLocality: Casa Potrero Grande; verbatimElevation: 17 ; verbatimLatitude: 10.8492 ; verbatimLongitude: -85.7731; verbatimCoordinateSystem: Decimal; decimalLatitude: 10.8492; decimalLongitude: -85.7731; samplingProtocol: Reared from the larva of the Nymphalidae, Morpho helenor; verbatimEventDate: 05-Oct-2004; individualID: DHJPAR0007377; individualCount: 1; sex: male; lifeStage: adult; preparations: pinned; catalogNumber: DHJPAR0007377; occurrenceDetails: http://janzen.sas.upenn.edu; recordedBy: D.H. Janzen, W. Hallwachs \& Freddy Quesada; otherCatalogNumbers: ASTAT149-06, 04-SRNP-14354, BOLD:AAA5134; identifiedBy: AJ Fleming; dateldentified: 2017; language: en; institutionCode: CNC; collectionCode: Insects; basisOfRecord: Pinned Specimen

j. $\quad$ scientificName: Hyphantrophaga morphophaga; phylum: Arthropoda; class: Insecta; order: Diptera; family: Tachinidae; genus: Hyphantrophaga; specificEpithet: morphophaga; scientificNameAuthorship: Fleming \& Wood, 2018; continent: Central America; country: Costa Rica; countryCode: CR; stateProvince: Guanacaste; county: Sector Santa Rosa; locality: Area de Conservacion Guanacaste; verbatimLocality: Bosque Humedo; verbatimElevation: 290; verbatimLatitude: 10.8514; verbatimLongitude: -85.608; verbatimCoordinateSystem: Decimal; decimalLatitude: 10.8514; decimalLongitude: -85.608; samplingProtocol: Reared from the larva of the Nymphalidae, Morpho helenor; verbatimEventDate: 28-Dec-2003; individualID: DHJPAR0007383; individualCount: 1; sex: male; lifeStage: adult; preparations: pinned; catalog Number: DHJPAR0007383; occurrenceDetails: http://janzen.sas.upenn.edu; recordedBy: D.H. Janzen, W. Hallwachs \& Ruth Franco; otherCatalogNumbers: ASTAT155-06, 03-SRNP-27782, BOLD:AAA5134; identifiedBy: AJ Fleming; dateldentified: 2017; language: en; institutionCode: CNC; collectionCode: Insects; basisOfRecord: Pinned Specimen

k. scientificName: Hyphantrophaga morphophaga; phylum: Arthropoda; class: Insecta; order: Diptera; family: Tachinidae; genus: Hyphantrophaga; specificEpithet: morphophaga; scientific NameAuthorship: Fleming \& Wood, 2018; continent: Central America; country: Costa Rica; countryCode: CR; stateProvince: Guanacaste; county: Sector Cacao; locality: Area de Conservacion Guanacaste; verbatimLocality: Sendero Segundo; verbatimElevation: 1180 ; verbatimLatitude: 10.9268 ; verbatimLongitude: -85.4533; verbatimCoordinateSystem: Decimal; decimalLatitude: 10.9268; decimalLongitude: -85.4533; samplingProtocol: Reared from the larva of the Nymphalidae, Morpho helenor; verbatimEventDate: 06-Mar-2004; individuallD: DHJPAR0007384; individualCount: 1; sex: male; lifeStage: adult; preparations: pinned; catalogNumber: DHJPAR0007384; occurrenceDetails: http://janzen.sas.upenn.edu; recordedBy: D.H. Janzen, W. Hallwachs \& Dunia Garcia; otherCatalogNumbers: ASTAT156-06, 04-SRNP-35102, BOLD:AAA5134; identifiedBy: AJ Fleming; dateldentified: 2017; language: en; institutionCode: CNC; collectionCode: Insects; basisOfRecord: Pinned Specimen

I. $\quad$ scientificName: Hyphantrophaga morphophaga; phylum: Arthropoda; class: Insecta; order: Diptera; family: Tachinidae; genus: Hyphantrophaga; specificEpithet: morphophaga; scientificNameAuthorship: Fleming \& Wood, 2018; continent: Central America; country: Costa Rica; countryCode: CR; stateProvince: Guanacaste; county: Sector Cacao; locality: Area de Conservacion Guanacaste; verbatimLocality: Estacion 
Cacao; verbatimElevation: 1150; verbatimLatitude: 10.9269; verbatimLongitude: -85.4682; verbatimCoordinateSystem: Decimal; decimalLatitude: 10.9269; decimalLongitude: -85.4682; samplingProtocol: Reared from the larva of the Nymphalidae, Morpho helenor; verbatimEventDate: 23-Nov-2003; individualID: DHJPAR0007385; individualCount: 1; sex: male; lifeStage: adult; preparations: pinned; catalogNumber: DHJPAR0007385; occurrenceDetails: http://janzen.sas.upenn.edu; recordedBy: D.H. Janzen, W. Hallwachs \& Harry Ramirez; otherCatalog Numbers: ASTAT157-06, 03-SRNP-22589,; identifiedBy: AJ Fleming; dateldentified: 2017; language: en; institutionCode: CNC; collectionCode: Insects; basisOfRecord: Pinned Specimen

m. scientificName: Hyphantrophaga morphophaga; phylum: Arthropoda; class: Insecta; order: Diptera; family: Tachinidae; genus: Hyphantrophaga; specificEpithet: morphophaga; scientificNameAuthorship: Fleming \& Wood, 2018; continent: Central America; country: Costa Rica; countryCode: CR; stateProvince: Guanacaste; county: Sector Cacao; locality: Area de Conservacion Guanacaste; verbatimLocality: Cuesta Caimito; verbatimElevation: 640; verbatimLatitude: 10.8908; verbatimLongitude: -85.4719; verbatimCoordinateSystem: Decimal; decimalLatitude: 10.8908; decimalLongitude: -85.4719; samplingProtocol: Reared from the larva of the Nymphalidae, Morpho helenor; verbatimEventDate: 10-Dec-2002; individualID: DHJPAR0007386; individualCount: 1; sex: male; lifeStage: adult; preparations: pinned; catalogNumber: DHJPAR0007386; occurrenceDetails: http://janzen.sas.upenn.edu; recordedBy: D.H. Janzen, W. Hallwachs \& Freddy Quesada; otherCatalogNumbers: ASTAT158-06, 02-SRNP-24360, BOLD:AAA5134; identifiedBy: AJ Fleming; dateldentified: 2017; language: en; institutionCode: CNC; collectionCode: Insects; basisOfRecord: Pinned Specimen

n. ScientificName: Hyphantrophaga morphophaga; phylum: Arthropoda; class: Insecta; order: Diptera; family: Tachinidae; genus: Hyphantrophaga; specificEpithet: morphophaga; scientificNameAuthorship: Fleming \& Wood, 2018; continent: Central America; country: Costa Rica; countryCode: CR; stateProvince: Guanacaste; county: Sector Cacao; locality: Area de Conservacion Guanacaste; verbatimLocality: Sendero Arenales; verbatimElevation: 1080; verbatimLatitude: 10.9247; verbatimLongitude: -85.4674; verbatimCoordinateSystem: Decimal; decimalLatitude: 10.9247; decimalLongitude: -85.4674; samplingProtocol: Reared from the larva of the Nymphalidae, Morpho helenor; verbatimEventDate: 04-Dec-2003; individualID: DHJPAR0007389; individualCount: 1; sex: male; lifeStage: adult; preparations: pinned; catalogNumber: DHJPAR0007389; occurrenceDetails: http://janzen.sas.upenn.edu; recordedBy: D.H. Janzen, W. Hallwachs \& Harry Ramirez; otherCatalog Numbers: ASTAT161-06, 03-SRNP-23510, BOLD:AAA5134; identifiedBy: AJ Fleming; dateldentified: 2017; language: en; institutionCode: CNC; collectionCode: Insects; basisOfRecord: Pinned Specimen

o. ScientificName: Hyphantrophaga morphophaga; phylum: Arthropoda; class: Insecta; order: Diptera; family: Tachinidae; genus: Hyphantrophaga; specificEpithet: morphophaga; scientificNameAuthorship: Fleming \& Wood, 2018; continent: Central America; country: Costa Rica; countryCode: CR; stateProvince: Guanacaste; county: Sector Del Oro; locality: Area de Conservacion Guanacaste; verbatimLocality: Quebrada Trigal; verbatimElevation: 290; verbatimLatitude: 11.0268; verbatimLongitude: -85.4955; verbatimCoordinateSystem: Decimal; decimalLatitude: 11.0268; decimalLongitude: -85.4955; samplingProtocol: Reared from the larva of the Nymphalidae, Morpho helenor; verbatimEventDate: 21-Oct-2003; individualID: DHJPAR0007391; individualCount: 1; sex: male; lifeStage: adult; preparations: pinned; 
catalogNumber: DHJPAR0007391; occurrenceDetails: http://janzen.sas.upenn.edu; recordedBy: D.H. Janzen, W. Hallwachs \& Dunia Garcia; otherCatalogNumbers: ASTAT163-06, 03-SRNP-19784, BOLD:AAA5134; identifiedBy: AJ Fleming; dateldentified: 2017; language: en; institutionCode: CNC; collectionCode: Insects; basisOfRecord: Pinned Specimen

p. ScientificName: Hyphantrophaga morphophaga; phylum: Arthropoda; class: Insecta; order: Diptera; family: Tachinidae; genus: Hyphantrophaga; specificEpithet: morphophaga; scientificNameAuthorship: Fleming \& Wood, 2018; continent: Central America; country: Costa Rica; countryCode: CR; stateProvince: Guanacaste; county: Sector Cacao; locality: Area de Conservacion Guanacaste; verbatimLocality: Sendero Ponderosa; verbatimElevation: 1060; verbatimLatitude: 10.9146; verbatimLongitude: -85.4626; verbatimCoordinateSystem: Decimal; decimalLatitude: 10.9146; decimalLongitude: -85.4626; samplingProtocol: Reared from the larva of the Nymphalidae, Morpho helenor; verbatimEventDate: 01-Apr-2003; individualID: DHJPAR0007392; individualCount: 1; sex: male; lifeStage: adult; preparations: pinned; catalogNumber: DHJPAR0007392; occurrenceDetails: http://janzen.sas.upenn.edu; recordedBy: D.H. Janzen, W. Hallwachs \& Mariano Pereira; otherCatalogNumbers: ASTAT164-06, 03-SRNP-3015, BOLD:AAA5134; identifiedBy: AJ Fleming; dateldentified: 2017; language: en; institutionCode: CNC; collectionCode: Insects; basisOfRecord: Pinned Specimen

q. $\quad$ scientificName: Hyphantrophaga morphophaga; phylum: Arthropoda; class: Insecta; order: Diptera; family: Tachinidae; genus: Hyphantrophaga; specificEpithet: morphophaga; scientificNameAuthorship: Fleming \& Wood, 2018; continent: Central America; country: Costa Rica; countryCode: CR; stateProvince: Guanacaste; county: Sector Cacao; locality: Area de Conservacion Guanacaste; verbatimLocality: Sendero Abajo; verbatimElevation: 1020; verbatimLatitude: 10.9255 ; verbatimLongitude: -85.4716; verbatimCoordinateSystem: Decimal; decimalLatitude: 10.9255; decimalLongitude: -85.4716; samplingProtocol: Reared from the larva of the Nymphalidae, Morpho catalina; verbatimEventDate: 13-Sep-2001; individualID: DHJPAR0007395; individualCount: 1; sex: male; lifeStage: adult; preparations: pinned; catalogNumber: DHJPAR0007395; occurrenceDetails: http://janzen.sas.upenn.edu; recordedBy: D.H. Janzen, W. Hallwachs \& Harry Ramirez; otherCatalogNumbers: ASTAT167-06, 01-SRNP-7320,; identifiedBy: AJ Fleming; dateldentified: 2017; language: en; institutionCode: CNC; collectionCode: Insects; basisOfRecord: Pinned Specimen

r. ScientificName: Hyphantrophaga morphophaga; phylum: Arthropoda; class: Insecta; order: Diptera; family: Tachinidae; genus: Hyphantrophaga; specificEpithet: morphophaga; scientificNameAuthorship: Fleming \& Wood, 2018; continent: Central America; country: Costa Rica; countryCode: CR; stateProvince: Guanacaste; county: Sector Cacao; locality: Area de Conservacion Guanacaste; verbatimLocality: Sendero Circular; verbatimElevation: 1185; verbatimLatitude: 10.9271; verbatimLongitude: -85.4668; verbatimCoordinateSystem: Decimal; decimalLatitude: 10.9271; decimalLongitude: -85.4668; samplingProtocol: Reared from the larva of the Nymphalidae, Morpho helenor; verbatimEventDate: 01-Jun-1999; individualID: DHJPAR0007399; individualCount: 1; sex: male; lifeStage: adult; preparations: pinned; catalogNumber: DHJPAR0007399; occurrenceDetails: http://janzen.sas.upenn.edu; recordedBy: D.H. Janzen, W. Hallwachs \& Mariano Pereira; otherCatalogNumbers: ASTAT171-06, 98-SRNP-15802, BOLD:AAA5134; identifiedBy: AJ Fleming; dateldentified: 2017; language: en; institutionCode: CNC; collectionCode: Insects; basisOfRecord: Pinned Specimen 
s. $\quad$ scientificName: Hyphantrophaga morphophaga; phylum: Arthropoda; class: Insecta; order: Diptera; family: Tachinidae; genus: Hyphantrophaga; specificEpithet: morphophaga; scientificNameAuthorship: Fleming \& Wood, 2018; continent: Central America; country: Costa Rica; countryCode: CR; stateProvince: Guanacaste; county: Sector Cacao; locality: Area de Conservacion Guanacaste; verbatimLocality: Sendero Arenales; verbatimElevation: 1080; verbatimLatitude: 10.9247 ; verbatimLongitude: -85.4674; verbatimCoordinateSystem: Decimal; decimalLatitude: 10.9247; decimalLongitude: -85.4674; samplingProtocol: Reared from the larva of the Nymphalidae, Morpho helenor; verbatimEventDate: 09-Nov-2002; individualID: DHJPAR0007405; individualCount: 1; sex: male; lifeStage: adult; preparations: pinned; catalogNumber: DHJPAR0007405; occurrenceDetails: http://janzen.sas.upenn.edu; recordedBy: D.H. Janzen, W. Hallwachs \& Mariano Pereira; otherCatalogNumbers: ASTAT177-06, 02-SRNP-23914, BOLD:AAA5134; identifiedBy: AJ Fleming; dateldentified: 2017; language: en; institutionCode: CNC; collectionCode: Insects; basisOfRecord: Pinned Specimen

t. scientificName: Hyphantrophaga morphophaga; phylum: Arthropoda; class: Insecta; order: Diptera; family: Tachinidae; genus: Hyphantrophaga; specificEpithet: morphophaga; scientificNameAuthorship: Fleming \& Wood, 2018; continent: Central America; country: Costa Rica; countryCode: CR; stateProvince: Guanacaste; county: Sector Cacao; locality: Area de Conservacion Guanacaste; verbatimLocality: Sendero Arenales; verbatimElevation: 1080 ; verbatimLatitude: 10.9247 ; verbatimLongitude: -85.4674; verbatimCoordinateSystem: Decimal; decimalLatitude: 10.9247; decimalLongitude: -85.4674; samplingProtocol: Reared from the larva of the Nymphalidae, Morpho helenor; verbatimEventDate: 09-Nov-2002; individualID: DHJPAR0007406; individualCount: 1; sex: male; lifeStage: adult; preparations: pinned; catalogNumber: DHJPAR0007406; occurrenceDetails: http://janzen.sas.upenn.edu; recordedBy: D.H. Janzen, W. Hallwachs \& Mariano Pereira; otherCatalogNumbers: ASTAT178-06, 02-SRNP-23915, BOLD:AAA5134; identifiedBy: AJ Fleming; dateldentified: 2017; language: en; institutionCode: CNC; collectionCode: Insects; basisOfRecord: Pinned Specimen

u. $\quad$ scientificName: Hyphantrophaga morphophaga; phylum: Arthropoda; class: Insecta; order: Diptera; family: Tachinidae; genus: Hyphantrophaga; specificEpithet: morphophaga; scientificNameAuthorship: Fleming \& Wood, 2018; continent: Central America; country: Costa Rica; countryCode: CR; stateProvince: Guanacaste; county: Sector Cacao; locality: Area de Conservacion Guanacaste; verbatimLocality: Sendero Nayo; verbatimElevation: 1090; verbatimLatitude: 10.9245; verbatimLongitude: -85.4695; verbatimCoordinateSystem: Decimal; decimalLatitude: 10.9245; decimalLongitude: -85.4695; samplingProtocol: Reared from the larva of the Nymphalidae, Morpho helenor; verbatimEventDate: 19-Dec-2002; individualID: DHJPAR0007407; individualCount: 1; sex: male; lifeStage: adult; preparations: pinned; catalogNumber: DHJPAR0007407; occurrenceDetails: http://janzen.sas.upenn.edu; recordedBy: D.H. Janzen, W. Hallwachs \& Freddy Quesada; otherCatalog Numbers: ASTAT179-06, 02-SRNP-24432, BOLD:AAA5134; identifiedBy: AJ Fleming; dateldentified: 2017; language: en; institutionCode: CNC; collectionCode: Insects; basisOfRecord: Pinned Specimen

v. ScientificName: Hyphantrophaga morphophaga; phylum: Arthropoda; class: Insecta; order: Diptera; family: Tachinidae; genus: Hyphantrophaga; specificEpithet: morphophaga; scientificNameAuthorship: Fleming \& Wood, 2018; continent: Central America; country: Costa Rica; countryCode: CR; stateProvince: Guanacaste; county: Sector Del Oro; locality: Area de Conservacion Guanacaste; verbatimLocality: 
Quebrada Raiz; verbatimElevation: 280; verbatimLatitude: 11.0287;

verbatimLongitude: -85.4867; verbatimCoordinateSystem: Decimal; decimalLatitude: 11.0287; decimalLongitude: -85.4867; samplingProtocol: Reared from the larva of the Nymphalidae, Morpho helenor; verbatimEventDate: 20-Oct-2002; individualID:

DHJPAR0007408; individualCount: 1; sex: male; lifeStage: adult; preparations: pinned; catalogNumber: DHJPAR0007408; occurrenceDetails: http://janzen.sas.upenn.edu; recordedBy: D.H. Janzen, W. Hallwachs \& Lucia Rios; otherCatalogNumbers: ASTAT180-06, 02-SRNP-29406, BOLD:AAA5134; identifiedBy: AJ Fleming; dateldentified: 2017; language: en; institutionCode: CNC; collectionCode: Insects; basisOfRecord: Pinned Specimen

w. scientificName: Hyphantrophaga morphophaga; phylum: Arthropoda; class: Insecta; order: Diptera; family: Tachinidae; genus: Hyphantrophaga; specificEpithet: morphophaga; scientificNameAuthorship: Fleming \& Wood, 2018; continent: Central America; country: Costa Rica; countryCode: CR; stateProvince: Guanacaste; county: Sector Cacao; locality: Area de Conservacion Guanacaste; verbatimLocality: Gongora Bananal; verbatimElevation: 600; verbatimLatitude: 10.8892; verbatimLongitude: -85.4761; verbatimCoordinateSystem: Decimal; decimalLatitude: 10.8892; decimalLongitude: -85.4761; samplingProtocol: Reared from the larva of the Nymphalidae, Morpho helenor; verbatimEventDate: 08-Feb-1999; individualID: DHJPAR0007409; individualCount: 1; sex: male; lifeStage: adult; preparations: pinned; catalogNumber: DHJPAR0007409; occurrenceDetails: http://janzen.sas.upenn.edu; recordedBy: D.H. Janzen, W. Hallwachs \& Bienvenida Chavarria; otherCatalogNumbers: ASTAT181-06, 99-SRNP-947, BOLD:AAA5134; identifiedBy: AJ Fleming; dateldentified: 2017; language: en; institutionCode: CNC; collectionCode: Insects; basisOfRecord: Pinned Specimen

x. $\quad$ scientificName: Hyphantrophaga morphophaga; phylum: Arthropoda; class: Insecta; order: Diptera; family: Tachinidae; genus: Hyphantrophaga; specificEpithet: morphophaga; scientificNameAuthorship: Fleming \& Wood, 2018; continent: Central America; country: Costa Rica; countryCode: CR; stateProvince: Guanacaste; county: Sector Cacao; locality: Area de Conservacion Guanacaste; verbatimLocality: Sendero Circular; verbatimElevation: 1185; verbatimLatitude: 10.9271; verbatimLongitude: -85.4668; verbatimCoordinateSystem: Decimal; decimalLatitude: 10.9271; decimalLongitude: -85.4668; samplingProtocol: Reared from the larva of the Nymphalidae, Morpho helenor; verbatimEventDate: 08-Mar-1999; individualID: DHJPAR0007410; individualCount: 1; sex: male; lifeStage: adult; preparations: pinned; catalogNumber: DHJPAR0007410; occurrenceDetails: http://janzen.sas.upenn.edu; recordedBy: D.H. Janzen, W. Hallwachs \& Mariano Pereira; otherCatalogNumbers: ASTAT182-06, 99-SRNP-863, BOLD:AAA5134; identifiedBy: AJ Fleming; dateldentified: 2017; language: en; institutionCode: CNC; collectionCode: Insects; basisOfRecord: Pinned Specimen

y. $\quad$ scientificName: Hyphantrophaga morphophaga; phylum: Arthropoda; class: Insecta; order: Diptera; family: Tachinidae; genus: Hyphantrophaga; specificEpithet: morphophaga; scientific NameAuthorship: Fleming \& Wood, 2018; continent: Central America; country: Costa Rica; countryCode: CR; stateProvince: Guanacaste; county: Sector Cacao; locality: Area de Conservacion Guanacaste; verbatimLocality: Estacion Gongora; verbatimElevation: 570; verbatimLatitude: 10.887; verbatimLongitude: -85.4744; verbatimCoordinateSystem: Decimal; decimalLatitude: 10.887; decimalLongitude: -85.4744; samplingProtocol: Reared from the larva of the Nymphalidae, Morpho helenor; verbatimEventDate: 11-Nov-1996; individualID: DHJPAR0007411; individualCount: 1; sex: male; lifeStage: adult; preparations: pinned; 
catalogNumber: DHJPAR0007411; occurrenceDetails: http://janzen.sas.upenn.edu; recordedBy: D.H. Janzen, W. Hallwachs \& gusaneros; otherCatalog Numbers: ASTAT183-06, 96-SRNP-11101, BOLD:AAA5134; identifiedBy: AJ Fleming; dateldentified: 2017; language: en; institutionCode: CNC; collectionCode: Insects; basisOfRecord: Pinned Specimen

z. $\quad$ scientificName: Hyphantrophaga morphophaga; phylum: Arthropoda; class: Insecta; order: Diptera; family: Tachinidae; genus: Hyphantrophaga; specificEpithet: morphophaga; scientificNameAuthorship: Fleming \& Wood, 2018; continent: Central America; country: Costa Rica; countryCode: CR; stateProvince: Guanacaste; county: Sector Pitilla; locality: Area de Conservacion Guanacaste; verbatimLocality: Sendero Evangelista; verbatimElevation: 660; verbatimLatitude: 10.9868; verbatimLongitude: -85.4208; verbatimCoordinateSystem: Decimal; decimalLatitude: 10.9868; decimalLongitude: -85.4208; samplingProtocol: Reared from the larva of the Nymphalidae, Morpho amathonte; verbatimEventDate: 12-Aug-2004; individualID: DHJPAR0011460; individualCount: 1; sex: male; lifeStage: adult; preparations: pinned; catalogNumber: DHJPAR0011460; occurrenceDetails: http://janzen.sas.upenn.edu; recordedBy: D.H. Janzen, W. Hallwachs \& Calixto Moraga; otherCatalogNumbers: ASTAQ847-06, 04-SRNP-32851, BOLD:AAA5134; identifiedBy: AJ Fleming; dateldentified: 2017; language: en; institutionCode: CNC; collectionCode: Insects; basisOfRecord: Pinned Specimen

aa. ScientificName: Hyphantrophaga morphophaga; phylum: Arthropoda; class: Insecta; order: Diptera; family: Tachinidae; genus: Hyphantrophaga; specificEpithet: morphophaga; scientificNameAuthorship: Fleming \& Wood, 2018; continent: Central America; country: Costa Rica; countryCode: CR; stateProvince: Guanacaste; county: Sector Cacao; locality: Area de Conservacion Guanacaste; verbatimLocality: Gongora Bananal; verbatimElevation: 600; verbatimLatitude: 10.8892; verbatimLongitude: -85.4761; verbatimCoordinateSystem: Decimal; decimalLatitude: 10.8892; decimalLongitude: -85.4761; samplingProtocol: Reared from the larva of the Nymphalidae, Morpho helenor; verbatimEventDate: 22-Oct-2004; individuallD: DHJPAR0011462; individualCount: 1; sex: male; lifeStage: adult; preparations: pinned; catalogNumber: DHJPAR0011462; occurrenceDetails: http://janzen.sas.upenn.edu; recordedBy: D.H. Janzen, W. Hallwachs \& Dunia Garcia; otherCatalog Numbers: ASTAQ849-06, 04-SRNP-48895, BOLD:AAA5134; identifiedBy: AJ Fleming; dateldentified: 2017; language: en; institutionCode: CNC; collectionCode: Insects; basisOfRecord: Pinned Specimen

ab. $\quad$ scientificName: Hyphantrophaga morphophaga; phylum: Arthropoda; class: Insecta; order: Diptera; family: Tachinidae; genus: Hyphantrophaga; specificEpithet: morphophaga; scientificNameAuthorship: Fleming \& Wood, 2018; continent: Central America; country: Costa Rica; countryCode: CR; stateProvince: Guanacaste; county: Sector Cacao; locality: Area de Conservacion Guanacaste; verbatimLocality: Estacion Cacao; verbatimElevation: 1150; verbatimLatitude: 10.9269; verbatimLongitude: -85.4682; verbatimCoordinateSystem: Decimal; decimalLatitude: 10.9269; decimalLongitude: -85.4682; samplingProtocol: Reared from the larva of the Nymphalidae, Morpho helenor; verbatimEventDate: 28-Nov-2006; individualID: DHJPAR0016681; individualCount: 1; sex: male; lifeStage: adult; preparations: pinned; catalogNumber: DHJPAR0016681; occurrenceDetails: http://janzen.sas.upenn.edu; recordedBy: D.H. Janzen, W. Hallwachs \& Manuel Pereira; otherCatalogNumbers: ASTAP986-07, 06-SRNP-36610, BOLD:AAA5134; identifiedBy: AJ Fleming; dateldentified: 2017; language: en; institutionCode: CNC; collectionCode: Insects; basisOfRecord: Pinned Specimen 
ac. scientificName: Hyphantrophaga morphophaga; phylum: Arthropoda; class: Insecta; order: Diptera; family: Tachinidae; genus: Hyphantrophaga; specificEpithet: morphophaga; scientificNameAuthorship: Fleming \& Wood, 2018; continent: Central America; country: Costa Rica; countryCode: CR; stateProvince: Guanacaste; county: Sector Cacao; locality: Area de Conservacion Guanacaste; verbatimLocality: Sendero Arenales; verbatimElevation: 1080; verbatimLatitude: 10.9247 ; verbatimLongitude: -85.4674; verbatimCoordinateSystem: Decimal; decimalLatitude: 10.9247; decimalLongitude: -85.4674; samplingProtocol: Reared from the larva of the Nymphalidae, Morpho helenor; verbatimEventDate: 27-Dec-2006; individualID: DHJPAR0016682; individualCount: 1; sex: male; lifeStage: adult; preparations: pinned; catalogNumber: DHJPAR0016682; occurrenceDetails: http://janzen.sas.upenn.edu; recordedBy: D.H. Janzen, W. Hallwachs \& Manuel Pereira; otherCatalogNumbers: ASTAP987-07, 06-SRNP-36868, BOLD:AAA5134; identifiedBy: AJ Fleming; dateldentified: 2017; language: en; institutionCode: CNC; collectionCode: Insects; basisOfRecord: Pinned Specimen

ad. $\quad$ scientificName: Hyphantrophaga morphophaga; phylum: Arthropoda; class: Insecta; order: Diptera; family: Tachinidae; genus: Hyphantrophaga; specificEpithet: morphophaga; scientificNameAuthorship: Fleming \& Wood, 2018; continent: Central America; country: Costa Rica; countryCode: CR; stateProvince: Guanacaste; county: Sector Cacao; locality: Area de Conservacion Guanacaste; verbatimLocality: Sendero Derrumbe; verbatimElevation: 1220 ; verbatimLatitude: 10.9292; verbatimLongitude: -85.4643; verbatimCoordinateSystem: Decimal; decimalLatitude: 10.9292; decimalLongitude: -85.4643 ; samplingProtocol: Reared from the larva of the Nymphalidae, Morpho helenor; verbatimEventDate: 17-Aug-2007; individualID: DHJPAR0019956; individualCount: 1; sex: male; lifeStage: adult; preparations: pinned; catalogNumber: DHJPAR0019956; occurrenceDetails: http://janzen.sas.upenn.edu; recordedBy: D.H. Janzen, W. Hallwachs \& Manuel Pereira; otherCatalogNumbers: ASTA1239-07, 07-SRNP-36054, BOLD:AAA5134; identifiedBy: AJ Fleming; dateldentified: 2017; language: en; institutionCode: CNC; collectionCode: Insects; basisOfRecord: Pinned Specimen

ae. $\quad$ scientificName: Hyphantrophaga morphophaga; phylum: Arthropoda; class: Insecta; order: Diptera; family: Tachinidae; genus: Hyphantrophaga; specificEpithet: morphophaga; scientificNameAuthorship: Fleming \& Wood, 2018; continent: Central America; country: Costa Rica; countryCode: CR; stateProvince: Guanacaste; county: Sector Cacao; locality: Area de Conservacion Guanacaste; verbatimLocality: Sendero Abajo; verbatimElevation: 1020; verbatimLatitude: 10.9255; verbatimLongitude: -85.4716; verbatimCoordinateSystem: Decimal; decimalLatitude: 10.9255; decimalLongitude: -85.4716; samplingProtocol: Reared from the larva of the Nymphalidae, Morpho helenor; verbatimEventDate: 23-Dec-2004; individualID: DHJPAR0007369; individualCount: 1; sex: female; lifeStage: adult; preparations: pinned; catalogNumber: DHJPAR0007369; occurrenceDetails: http:// janzen.sas.upenn.edu; recordedBy: D.H. Janzen, W. Hallwachs \& Manuel Pereira; otherCatalog Numbers: ASTAT141-06, 04-SRNP-35956, BOLD:AAA5134; identifiedBy: AJ Fleming; dateldentified: 2017; language: en; institutionCode: CNC; collectionCode: Insects; basisOfRecord: Pinned Specimen

af. ScientificName: Hyphantrophaga morphophaga; phylum: Arthropoda; class: Insecta; order: Diptera; family: Tachinidae; genus: Hyphantrophaga; specificEpithet: morphophaga; scientificNameAuthorship: Fleming \& Wood, 2018; continent: Central America; country: Costa Rica; countryCode: CR; stateProvince: Guanacaste; county: Sector Cacao; locality: Area de Conservacion Guanacaste; verbatimLocality: Gongora 
Bananal; verbatimElevation: 600; verbatimLatitude: 10.8892; verbatimLongitude: -85.4761; verbatimCoordinateSystem: Decimal; decimalLatitude: 10.8892; decimalLongitude: -85.4761; samplingProtocol: Reared from the larva of the Nymphalidae, Morpho helenor; verbatimEventDate: 22-Oct-2004; individualID: DHJPAR0007372; individualCount: 1; sex: female; lifeStage: adult; preparations: pinned; catalogNumber: DHJPAR0007372; occurrenceDetails: http:// janzen.sas.upenn.edu; recordedBy: D.H. Janzen, W. Hallwachs \& Dunia Garcia; otherCatalog Numbers: ASTAT144-06, 04-SRNP-48895, BOLD:AAA5134; identifiedBy: AJ Fleming; dateldentified: 2017; language: en; institutionCode: CNC; collectionCode: Insects; basisOfRecord: Pinned Specimen

ag. $\quad$ scientificName: Hyphantrophaga morphophaga; phylum: Arthropoda; class: Insecta; order: Diptera; family: Tachinidae; genus: Hyphantrophaga; specificEpithet: morphophaga; scientificNameAuthorship: Fleming \& Wood, 2018; continent: Central America; country: Costa Rica; countryCode: CR; stateProvince: Guanacaste; county: Sector Cacao; locality: Area de Conservacion Guanacaste; verbatimLocality: Gongora Bananal; verbatimElevation: 600; verbatimLatitude: 10.8892; verbatimLongitude: -85.4761; verbatimCoordinateSystem: Decimal; decimalLatitude: 10.8892; decimalLongitude: -85.4761; samplingProtocol: Reared from the larva of the Nymphalidae, Morpho helenor; verbatimEventDate: 30-Oct-2005; individualID: DHJPAR0007375; individualCount: 1; sex: female; lifeStage: adult; preparations: pinned; catalogNumber: DHJPAR0007375; occurrenceDetails: http:// janzen.sas.upenn.edu; recordedBy: D.H. Janzen, W. Hallwachs \& Harry Ramirez; otherCatalog Numbers: ASTAT147-06, 05-SRNP-48498, BOLD:AAA5134; identifiedBy: AJ Fleming; dateldentified: 2017; language: en; institutionCode: CNC; collectionCode: Insects; basisOfRecord: Pinned Specimen

ah. ScientificName: Hyphantrophaga morphophaga; phylum: Arthropoda; class: Insecta; order: Diptera; family: Tachinidae; genus: Hyphantrophaga; specificEpithet: morphophaga; scientificNameAuthorship: Fleming \& Wood, 2018; continent: Central America; country: Costa Rica; countryCode: CR; stateProvince: Guanacaste; county: Sector Cacao; locality: Area de Conservacion Guanacaste; verbatimLocality: Estacion Gongora; verbatimElevation: 570; verbatimLatitude: 10.887; verbatimLongitude: -85.4744; verbatimCoordinateSystem: Decimal; decimalLatitude: 10.887; decimalLongitude: -85.4744; samplingProtocol: Reared from the larva of the Nymphalidae, Morpho helenor; verbatimEventDate: 28-Oct-2005; individualID: DHJPAR0007376; individualCount: 1; sex: female; lifeStage: adult; preparations: pinned; catalogNumber: DHJPAR0007376; occurrenceDetails: http:// janzen.sas.upenn.edu; recordedBy: D.H. Janzen, W. Hallwachs \& Dunia Garcia; otherCatalog Numbers: ASTAT148-06, 05-SRNP-47741, BOLD:AAA5134; identifiedBy: AJ Fleming; dateldentified: 2017; language: en; institutionCode: CNC; collectionCode: Insects; basisOfRecord: Pinned Specimen

ai. $\quad$ scientificName: Hyphantrophaga morphophaga; phylum: Arthropoda; class: Insecta; order: Diptera; family: Tachinidae; genus: Hyphantrophaga; specificEpithet: morphophaga; scientificNameAuthorship: Fleming \& Wood, 2018; continent: Central America; country: Costa Rica; countryCode: CR; stateProvince: Guanacaste; county: Sector Cacao; locality: Area de Conservacion Guanacaste; verbatimLocality: Gongora Bananal; verbatimElevation: 600; verbatimLatitude: 10.8892; verbatimLongitude: -85.4761; verbatimCoordinateSystem: Decimal; decimalLatitude: 10.8892; decimalLongitude: -85.4761; samplingProtocol: Reared from the larva of the Nymphalidae, Morpho helenor; verbatimEventDate: 19-Oct-2004; individualID: DHJPAR0007378; individualCount: 1; sex: female; lifeStage: adult; preparations: 
pinned; catalogNumber: DHJPAR0007378; occurrenceDetails: http:// janzen.sas.upenn.edu; recordedBy: D.H. Janzen, W. Hallwachs \& Dunia Garcia; otherCatalogNumbers: ASTAT150-06, 04-SRNP-48894, BOLD:AAA5134; identifiedBy: AJ Fleming; dateldentified: 2017; language: en; institutionCode: CNC; collectionCode: Insects; basisOfRecord: Pinned Specimen

aj. ScientificName: Hyphantrophaga morphophaga; phylum: Arthropoda; class: Insecta; order: Diptera; family: Tachinidae; genus: Hyphantrophaga; specificEpithet: morphophaga; scientificNameAuthorship: Fleming \& Wood, 2018; continent: Central America; country: Costa Rica; countryCode: CR; stateProvince: Guanacaste; county: Sector Cacao; locality: Area de Conservacion Guanacaste; verbatimLocality: Cuesta Caimito; verbatimElevation: 640; verbatimLatitude: 10.8908; verbatimLongitude: -85.4719; verbatimCoordinateSystem: Decimal; decimalLatitude: 10.8908; decimalLongitude: -85.4719; samplingProtocol: Reared from the larva of the Nymphalidae, Morpho helenor; verbatimEventDate: 10-Dec-2002; individualID: DHJPAR0007379; individualCount: 1; sex: female; lifeStage: adult; preparations: pinned; catalogNumber: DHJPAR0007379; occurrenceDetails: http:// janzen.sas.upenn.edu; recordedBy: D.H. Janzen, W. Hallwachs \& Freddy Quesada; otherCatalogNumbers: ASTAT151-06, 02-SRNP-24360, BOLD:AAA5134; identifiedBy: AJ Fleming; dateldentified: 2017; language: en; institutionCode: CNC; collectionCode: Insects; basisOfRecord: Pinned Specimen

ak. ScientificName: Hyphantrophaga morphophaga; phylum: Arthropoda; class: Insecta; order: Diptera; family: Tachinidae; genus: Hyphantrophaga; specificEpithet: morphophaga; scientificNameAuthorship: Fleming \& Wood, 2018; continent: Central America; country: Costa Rica; countryCode: CR; stateProvince: Guanacaste; county: Sector Cacao; locality: Area de Conservacion Guanacaste; verbatimLocality: Sendero Arenales; verbatimElevation: 1080; verbatimLatitude: 10.9247; verbatimLongitude: -85.4674; verbatimCoordinateSystem: Decimal; decimalLatitude: 10.9247; decimalLongitude: -85.4674; samplingProtocol: Reared from the larva of the Nymphalidae, Morpho helenor; verbatimEventDate: 24-Mar-2004; individualID: DHJPAR0007380; individualCount: 1; sex: female; lifeStage: adult; preparations: pinned; catalogNumber: DHJPAR0007380; occurrenceDetails: http:// janzen.sas.upenn.edu; recordedBy: D.H. Janzen, W. Hallwachs \& Dunia Garcia; otherCatalog Numbers: ASTAT152-06, 03-SRNP-23856, BOLD:AAA5134; identifiedBy: AJ Fleming; dateldentified: 2017; language: en; institutionCode: CNC; collectionCode: Insects; basisOfRecord: Pinned Specimen

al. $\quad$ scientificName: Hyphantrophaga morphophaga; phylum: Arthropoda; class: Insecta; order: Diptera; family: Tachinidae; genus: Hyphantrophaga; specificEpithet: morphophaga; scientificNameAuthorship: Fleming \& Wood, 2018; continent: Central America; country: Costa Rica; countryCode: CR; stateProvince: Guanacaste; county: Sector Cacao; locality: Area de Conservacion Guanacaste; verbatimLocality: Sendero Derrumbe; verbatimElevation: 1220; verbatimLatitude: 10.9292; verbatimLongitude: -85.4643; verbatimCoordinateSystem: Decimal; decimalLatitude: 10.9292; decimalLongitude: -85.4643; samplingProtocol: Reared from the larva of the Nymphalidae, Morpho helenor; verbatimEventDate: 05-Dec-2002; individualID: DHJPAR0007381; individualCount: 1; sex: female; lifeStage: adult; preparations: pinned; catalogNumber: DHJPAR0007381; occurrenceDetails: http:// janzen.sas.upenn.edu; recordedBy: D.H. Janzen, W. Hallwachs \& Harry Ramirez; otherCatalog Numbers: ASTAT153-06, 02-SRNP-24376, BOLD:AAA5134; identifiedBy: AJ Fleming; dateldentified: 2017; language: en; institutionCode: CNC; collectionCode: Insects; basisOfRecord: Pinned Specimen 
am. ScientificName: Hyphantrophaga morphophaga; phylum: Arthropoda; class: Insecta; order: Diptera; family: Tachinidae; genus: Hyphantrophaga; specificEpithet: morphophaga; scientificNameAuthorship: Fleming \& Wood, 2018; continent: Central America; country: Costa Rica; countryCode: CR; stateProvince: Guanacaste; county: Sector Cacao; locality: Area de Conservacion Guanacaste; verbatimLocality: Sendero Arenales; verbatimElevation: 1080; verbatimLatitude: 10.9247; verbatimLongitude: -85.4674; verbatimCoordinateSystem: Decimal; decimalLatitude: 10.9247; decimalLongitude: -85.4674; samplingProtocol: Reared from the larva of the Nymphalidae, Morpho helenor; verbatimEventDate: 15-Jan-1998; individualID: DHJPAR0007387; individualCount: 1; sex: female; lifeStage: adult; preparations: pinned; catalogNumber: DHJPAR0007387; occurrenceDetails: http:// janzen.sas.upenn.edu; recordedBy: D.H. Janzen, W. Hallwachs \& Roster Moraga; otherCatalogNumbers: ASTAT159-06, 97-SRNP-11061, BOLD:AAA5134; identifiedBy: AJ Fleming; dateldentified: 2017; language: en; institutionCode: CNC; collectionCode: Insects; basisOfRecord: Pinned Specimen

an. ScientificName: Hyphantrophaga morphophaga; phylum: Arthropoda; class: Insecta; order: Diptera; family: Tachinidae; genus: Hyphantrophaga; specificEpithet: morphophaga; scientificNameAuthorship: Fleming \& Wood, 2018; continent: Central America; country: Costa Rica; countryCode: CR; stateProvince: Guanacaste; county: Sector Cacao; locality: Area de Conservacion Guanacaste; verbatimLocality: Sendero Arenales; verbatimElevation: 1080; verbatimLatitude: 10.9247; verbatimLongitude: -85.4674; verbatimCoordinateSystem: Decimal; decimalLatitude: 10.9247; decimalLongitude: -85.4674; samplingProtocol: Reared from the larva of the Nymphalidae, Morpho helenor; verbatimEventDate: 18-Nov-2003; individualID: DHJPAR0007388; individualCount: 1; sex: female; lifeStage: adult; preparations: pinned; catalogNumber: DHJPAR0007388; occurrenceDetails: http:// janzen.sas.upenn.edu; recordedBy: D.H. Janzen, W. Hallwachs \& Harry Ramirez; otherCatalogNumbers: ASTAT160-06, 03-SRNP-23698, BOLD:AAA5134; identifiedBy: AJ Fleming; dateldentified: 2017; language: en; institutionCode: CNC; collectionCode: Insects; basisOfRecord: Pinned Specimen

ao. ScientificName: Hyphantrophaga morphophaga; phylum: Arthropoda; class: Insecta; order: Diptera; family: Tachinidae; genus: Hyphantrophaga; specificEpithet: morphophaga; scientificNameAuthorship: Fleming \& Wood, 2018; continent: Central America; country: Costa Rica; countryCode: CR; stateProvince: Guanacaste; county: Sector Cacao; locality: Area de Conservacion Guanacaste; verbatimLocality: Sendero Ponderosa; verbatimElevation: 1060; verbatimLatitude: 10.9146; verbatimLongitude: -85.4626; verbatimCoordinateSystem: Decimal; decimalLatitude: 10.9146; decimalLongitude: -85.4626; samplingProtocol: Reared from the larva of the Nymphalidae, Morpho helenor; verbatimEventDate: 09-Dec-2003; individualID DHJPAR0007390; individualCount: 1; sex: female; lifeStage: adult; preparations: pinned; catalogNumber: DHJPAR0007390; occurrenceDetails: http:// janzen.sas.upenn.edu; recordedBy: D.H. Janzen, W. Hallwachs \& Harry Ramirez; otherCatalog Numbers: ASTAT162-06, 03-SRNP-23364, BOLD:AAA5134; identifiedBy: AJ Fleming; dateldentified: 2017; language: en; institutionCode: CNC; collectionCode: Insects; basisOfRecord: Pinned Specimen

ap. ScientificName: Hyphantrophaga morphophaga; phylum: Arthropoda; class: Insecta; order: Diptera; family: Tachinidae; genus: Hyphantrophaga; specificEpithet: morphophaga; scientificNameAuthorship: Fleming \& Wood, 2018; continent: Central America; country: Costa Rica; countryCode: CR; stateProvince: Guanacaste; county: Sector Cacao; locality: Area de Conservacion Guanacaste; verbatimLocality: Sendero 
Nayo; verbatimElevation: 1090; verbatimLatitude: 10.9245; verbatimLongitude: -85.4695; verbatimCoordinateSystem: Decimal; decimalLatitude: 10.9245; decimalLongitude: -85.4695; samplingProtocol: Reared from the larva of the Nymphalidae, Morpho helenor; verbatimEventDate: 20-May-2003; individualID: DHJPAR0007393; individualCount: 1; sex: female; lifeStage: adult; preparations: pinned; catalogNumber: DHJPAR0007393; occurrenceDetails: http:// janzen.sas.upenn.edu; recordedBy: D.H. Janzen, W. Hallwachs \& Freddy Quesada; otherCatalog Numbers: ASTAT165-06, 03-SRNP-3816, BOLD:AAA5134; identifiedBy: AJ Fleming; dateldentified: 2017; language: en; institutionCode: CNC; collectionCode: Insects; basisOfRecord: Pinned Specimen

aq. $\quad$ scientificName: Hyphantrophaga morphophaga; phylum: Arthropoda; class: Insecta; order: Diptera; family: Tachinidae; genus: Hyphantrophaga; specificEpithet: morphophaga; scientificNameAuthorship: Fleming \& Wood, 2018; continent: Central America; country: Costa Rica; countryCode: CR; stateProvince: Guanacaste; county: Sector Cacao; locality: Area de Conservacion Guanacaste; verbatimLocality: Sendero Arenales; verbatimElevation: 1080 ; verbatimLatitude: 10.9247 ; verbatimLongitude: -85.4674; verbatimCoordinateSystem: Decimal; decimalLatitude: 10.9247; decimalLongitude: -85.4674; samplingProtocol: Reared from the larva of the Nymphalidae, Morpho helenor; verbatimEventDate: 07-Aug-2001; individualID: DHJPAR0007394; individualCount: 1; sex: female; lifeStage: adult; preparations: pinned; catalogNumber: DHJPAR0007394; occurrenceDetails: http:// janzen.sas.upenn.edu; recordedBy: D.H. Janzen, W. Hallwachs \& Mariano Pereira; otherCatalogNumbers: ASTAT166-06, 01-SRNP-6970, BOLD:AAA5134; identifiedBy: AJ Fleming; dateldentified: 2017; language: en; institutionCode: CNC; collectionCode: Insects; basisOfRecord: Pinned Specimen

ar. $\quad$ scientificName: Hyphantrophaga morphophaga; phylum: Arthropoda; class: Insecta; order: Diptera; family: Tachinidae; genus: Hyphantrophaga; specificEpithet: morphophaga; scientificNameAuthorship: Fleming \& Wood, 2018; continent: Central America; country: Costa Rica; countryCode: CR; stateProvince: Guanacaste; county: Sector Cacao; locality: Area de Conservacion Guanacaste; verbatimLocality: Sendero Salto; verbatimElevation: 1000; verbatimLatitude: 10.9302; verbatimLongitude: -85.4694; verbatimCoordinateSystem: Decimal; decimalLatitude: 10.9302; decimalLongitude: -85.4694; samplingProtocol: Reared from the larva of the Nymphalidae, Morpho helenor; verbatimEventDate: 16-Nov-1998; individualID: DHJPAR0007396; individualCount: 1; sex: female; lifeStage: adult; preparations: pinned; catalogNumber: DHJPAR0007396; occurrenceDetails: http:// janzen.sas.upenn.edu; recordedBy: D.H. Janzen, W. Hallwachs \& Harry Ramirez; otherCatalog Numbers: ASTAT168-06, 98-SRNP-15571, BOLD:AAA5134; identifiedBy: AJ Fleming; dateldentified: 2017; language: en; institutionCode: CNC; collectionCode: Insects; basisOfRecord: Pinned Specimen

as. $\quad$ scientificName: Hyphantrophaga morphophaga; phylum: Arthropoda; class: Insecta; order: Diptera; family: Tachinidae; genus: Hyphantrophaga; specificEpithet: morphophaga; scientificNameAuthorship: Fleming \& Wood, 2018; continent: Central America; country: Costa Rica; countryCode: CR; stateProvince: Guanacaste; county: Sector Cacao; locality: Area de Conservacion Guanacaste; verbatimLocality: Sendero Toma Agua; verbatimElevation: 1140; verbatimLatitude: 10.9285 ; verbatimLongitude: -85.4668; verbatimCoordinateSystem: Decimal; decimalLatitude: 10.9285; decimalLongitude: -85.4668; samplingProtocol: Reared from the larva of the Nymphalidae, Morpho helenor; verbatimEventDate: 15-Dec-1998; individualID: DHJPAR0007397; individualCount: 1; sex: female; lifeStage: adult; preparations: 
pinned; catalogNumber: DHJPAR0007397; occurrenceDetails: http:// janzen.sas.upenn.edu; recordedBy: D.H. Janzen, W. Hallwachs \& Mariano Pereira; otherCatalogNumbers: ASTAT169-06, 98-SRNP-15774, BOLD:AAA5134; identifiedBy: AJ Fleming; dateldentified: 2017; language: en; institutionCode: CNC; collectionCode: Insects; basisOfRecord: Pinned Specimen

at. ScientificName: Hyphantrophaga morphophaga; phylum: Arthropoda; class: Insecta; order: Diptera; family: Tachinidae; genus: Hyphantrophaga; specificEpithet: morphophaga; scientificNameAuthorship: Fleming \& Wood, 2018; continent: Central America; country: Costa Rica; countryCode: CR; stateProvince: Guanacaste; county: Sector Cacao; locality: Area de Conservacion Guanacaste; verbatimLocality: Sendero Arenales; verbatimElevation: 1080; verbatimLatitude: 10.9247; verbatimLongitude: -85.4674; verbatimCoordinateSystem: Decimal; decimalLatitude: 10.9247; decimalLongitude: -85.4674; samplingProtocol: Reared from the larva of the Nymphalidae, Morpho helenor; verbatimEventDate: 20-Jan-1999; individualID: DHJPAR0007398; individualCount: 1; sex: female; lifeStage: adult; preparations: pinned; catalogNumber: DHJPAR0007398; occurrenceDetails: http:// janzen.sas.upenn.edu; recordedBy: D.H. Janzen, W. Hallwachs \& Harry Ramirez; otherCatalog Numbers: ASTAT170-06, 98-SRNP-15738, BOLD:AAA5134; identifiedBy: AJ Fleming; dateldentified: 2017; language: en; institutionCode: CNC; collectionCode: Insects; basisOfRecord: Pinned Specimen

au. ScientificName: Hyphantrophaga morphophaga; phylum: Arthropoda; class: Insecta; order: Diptera; family: Tachinidae; genus: Hyphantrophaga; specificEpithet: morphophaga; scientificNameAuthorship: Fleming \& Wood, 2018; continent: Central America; country: Costa Rica; countryCode: CR; stateProvince: Guanacaste; county: Sector Cacao; locality: Area de Conservacion Guanacaste; verbatimLocality: Sendero Derrumbe; verbatimElevation: 1220 ; verbatimLatitude: 10.9292; verbatimLongitude: -85.4643; verbatimCoordinateSystem: Decimal; decimalLatitude: 10.9292; decimalLongitude: -85.4643; samplingProtocol: Reared from the larva of the Nymphalidae, Morpho helenor; verbatimEventDate: 20-Aug-2002; individualID: DHJPAR0007400; individualCount: 1; sex: female; lifeStage: adult; preparations: pinned; catalogNumber: DHJPAR0007400; occurrenceDetails: http:// janzen.sas.upenn.edu; recordedBy: D.H. Janzen, W. Hallwachs \& Freddy Quesada; otherCatalogNumbers: ASTAT172-06, 02-SRNP-23313,; identifiedBy: AJ Fleming; dateldentified: 2017; language: en; institutionCode: CNC; collectionCode: Insects; basisOfRecord: Pinned Specimen

av. scientificName: Hyphantrophaga morphophaga; phylum: Arthropoda; class: Insecta; order: Diptera; family: Tachinidae; genus: Hyphantrophaga; specificEpithet: morphophaga; scientificNameAuthorship: Fleming \& Wood, 2018; continent: Central America; country: Costa Rica; countryCode: CR; stateProvince: Guanacaste; county: Sector Cacao; locality: Area de Conservacion Guanacaste; verbatimLocality: Sendero Arenales; verbatimElevation: 1080; verbatimLatitude: 10.9247; verbatimLongitude: -85.4674; verbatimCoordinateSystem: Decimal; decimalLatitude: 10.9247; decimalLongitude: -85.4674; samplingProtocol: Reared from the larva of the Nymphalidae, Morpho helenor; verbatimEventDate: 24-Feb-1999; individualID: DHJPAR0007401; individualCount: 1; sex: female; lifeStage: adult; preparations: pinned; catalogNumber: DHJPAR0007401; occurrenceDetails: http:// janzen.sas.upenn.edu; recordedBy: D.H. Janzen, W. Hallwachs \& Mariano Pereira; otherCatalogNumbers: ASTAT173-06, 98-SRNP-15851, BOLD:AAA5134; identifiedBy: AJ Fleming; dateldentified: 2017; language: en; institutionCode: CNC; collectionCode: Insects; basisOfRecord: Pinned Specimen 
aw. scientificName: Hyphantrophaga morphophaga; phylum: Arthropoda; class: Insecta; order: Diptera; family: Tachinidae; genus: Hyphantrophaga; specificEpithet: morphophaga; scientificNameAuthorship: Fleming \& Wood, 2018; continent: Central America; country: Costa Rica; countryCode: CR; stateProvince: Guanacaste; county: Sector Cacao; locality: Area de Conservacion Guanacaste; verbatimLocality: Sendero Arenales; verbatimElevation: 1080; verbatimLatitude: 10.9247 ; verbatimLongitude: -85.4674; verbatimCoordinateSystem: Decimal; decimalLatitude: 10.9247; decimalLongitude: -85.4674; samplingProtocol: Reared from the larva of the Nymphalidae, Morpho helenor; verbatimEventDate: 06-Feb-1999; individualID: DHJPAR0007402; individualCount: 1; sex: female; lifeStage: adult; preparations: pinned; catalogNumber: DHJPAR0007402; occurrenceDetails: http:// janzen.sas.upenn.edu; recordedBy: D.H. Janzen, W. Hallwachs \& Mariano Pereira; otherCatalogNumbers: ASTAT174-06, 99-SRNP-568, BOLD:AAA5134; identifiedBy: AJ Fleming; dateldentified: 2017; language: en; institutionCode: CNC; collectionCode: Insects; basisOfRecord: Pinned Specimen

ax. $\quad$ scientificName: Hyphantrophaga morphophaga; phylum: Arthropoda; class: Insecta; order: Diptera; family: Tachinidae; genus: Hyphantrophaga; specificEpithet: morphophaga; scientificNameAuthorship: Fleming \& Wood, 2018; continent: Central America; country: Costa Rica; countryCode: CR; stateProvince: Guanacaste; county: Sector Del Oro; locality: Area de Conservacion Guanacaste; verbatimLocality: Puente Mena; verbatimElevation: 280 ; verbatimLatitude: 11.0456 ; verbatimLongitude: -85.4574; verbatimCoordinateSystem: Decimal; decimalLatitude: 11.0456; decimalLongitude: -85.4574; samplingProtocol: Reared from the larva of the Nymphalidae, Morpho helenor; verbatimEventDate: 15-Nov-2002; individualID: DHJPAR0007403; individualCount: 1; sex: female; lifeStage: adult; preparations: pinned; catalogNumber: DHJPAR0007403; occurrenceDetails: http:// janzen.sas.upenn.edu; recordedBy: D.H. Janzen, W. Hallwachs \& Lucia Rios; otherCatalog Numbers: ASTAT175-06, 02-SRNP-30889, BOLD:AAA5134; identifiedBy: AJ Fleming; dateldentified: 2017; language: en; institutionCode: CNC; collectionCode: Insects; basisOfRecord: Pinned Specimen

ay. $\quad$ scientificName: Hyphantrophaga morphophaga; phylum: Arthropoda; class: Insecta; order: Diptera; family: Tachinidae; genus: Hyphantrophaga; specificEpithet: morphophaga; scientific NameAuthorship: Fleming \& Wood, 2018; continent: Central America; country: Costa Rica; countryCode: CR; stateProvince: Guanacaste; county: Sector Santa Rosa; locality: Area de Conservacion Guanacaste; verbatimLocality: Bosque San Emilio; verbatimElevation: 300; verbatimLatitude: 10.8439; verbatimLongitude: -85.6138; verbatimCoordinateSystem: Decimal; decimalLatitude: 10.8439; decimalLongitude: -85.6138; samplingProtocol: Reared from the larva of the Nymphalidae, Morpho helenor; verbatimEventDate: 11-May-1996; individualID: DHJPAR0007404; individualCount: 1; sex: female; lifeStage: adult; preparations: pinned; catalogNumber: DHJPAR0007404; occurrenceDetails: http:// janzen.sas.upenn.edu; recordedBy: D.H. Janzen, W. Hallwachs \& gusaneros; otherCatalogNumbers: ASTAT176-06, 96-SRNP-11367,; identifiedBy: AJ Fleming; dateldentified: 2017; language: en; institutionCode: CNC; collectionCode: Insects; basisOfRecord: Pinned Specimen

az. ScientificName: Hyphantrophaga morphophaga; phylum: Arthropoda; class: Insecta; order: Diptera; family: Tachinidae; genus: Hyphantrophaga; specificEpithet: morphophaga; scientificNameAuthorship: Fleming \& Wood, 2018; continent: Central America; country: Costa Rica; countryCode: CR; stateProvince: Guanacaste; county: Sector Mundo Nuevo; locality: Area de Conservacion Guanacaste; verbatimLocality: 
Sendero Mora; verbatimElevation: 480; verbatimLatitude: 10.7683; verbatimLongitude: -85.4257; verbatimCoordinateSystem: Decimal; decimalLatitude: 10.7683; decimalLongitude: -85.4257; samplingProtocol: Reared from the larva of the Nymphalidae, Morpho helenor; verbatimEventDate: 17-Aug-2007; individualID: DHJPAR0019953; individualCount: 1; sex: female; lifeStage: adult; preparations: pinned; catalogNumber: DHJPAR0019953; occurrenceDetails: http:// janzen.sas.upenn.edu; recordedBy: D.H. Janzen, W. Hallwachs \& Jose Cortez; otherCatalog Numbers: ASTA1236-07, 07-SRNP-58222, BOLD:AAA5134; identifiedBy: AJ Fleming; dateldentified: 2017; language: en; institutionCode: CNC; collectionCode: Insects; basisOfRecord: Pinned Specimen

ba. $\quad$ scientificName: Hyphantrophaga morphophaga; phylum: Arthropoda; class: Insecta; order: Diptera; family: Tachinidae; genus: Hyphantrophaga; specificEpithet: morphophaga; scientificNameAuthorship: Fleming \& Wood, 2018; continent: Central America; country: Costa Rica; countryCode: CR; stateProvince: Guanacaste; county: Sector Mundo Nuevo; locality: Area de Conservacion Guanacaste; verbatimLocality: Quebrada Tibio Perla; verbatimElevation: 330; verbatimLatitude: 10.7626; verbatimLongitude: -85.4298; verbatimCoordinateSystem: Decimal; decimalLatitude: 10.7626; decimalLongitude: -85.4298; samplingProtocol: Reared from the larva of the Nymphalidae, Morpho helenor; verbatimEventDate: 29-Jul-2007; individualID: DHJPAR0020952; individualCount: 1; sex: female; lifeStage: adult; preparations: pinned; catalogNumber: DHJPAR0020952; occurrenceDetails: http:// janzen.sas.upenn.edu; recordedBy: D.H. Janzen, W. Hallwachs \& Jose Alberto Sanchez; otherCatalogNumbers: ASTA1295-07, 07-SRNP-57297, BOLD:AAA5134; identifiedBy: AJ Fleming; dateldentified: 2017; language: en; institutionCode: CNC; collectionCode: Insects; basisOfRecord: Pinned Specimen

bb. $\quad$ scientificName: Hyphantrophaga morphophaga; phylum: Arthropoda; class: Insecta; order: Diptera; family: Tachinidae; genus: Hyphantrophaga; specificEpithet: morphophaga; scientificNameAuthorship: Fleming \& Wood, 2018; continent: Central America; country: Costa Rica; countryCode: CR; stateProvince: Guanacaste; county: Sector Cacao; locality: Area de Conservacion Guanacaste; verbatimLocality: Sendero Abajo; verbatimElevation: 1020; verbatimLatitude: 10.9255; verbatimLongitude: -85.4716; verbatimCoordinateSystem: Decimal; decimalLatitude: 10.9255; decimalLongitude: -85.4716; samplingProtocol: Reared from the larva of the Nymphalidae, Morpho helenor; verbatimEventDate: 17-Dec-2004; individualID: DHJPAR0007374; individualCount: 1; lifeStage: adult; preparations: pinned; catalogNumber: DHJPAR0007374; occurrenceDetails: http://janzen.sas.upenn.edu; recordedBy: D.H. Janzen, W. Hallwachs \& Mariano Pereira; otherCatalogNumbers: ASTAT146-06, 04-SRNP-35960, BOLD:AAA5134; identifiedBy: AJ Fleming; dateldentified: 2017; language: en; institutionCode: CNC; collectionCode: Insects; basisOfRecord: Pinned Specimen

bc. $\quad$ scientificName: Hyphantrophaga morphophaga; phylum: Arthropoda; class: Insecta; order: Diptera; family: Tachinidae; genus: Hyphantrophaga; specificEpithet: morphophaga; scientificNameAuthorship: Fleming \& Wood, 2018; continent: Central America; country: Costa Rica; countryCode: CR; stateProvince: Guanacaste; county: Sector Cacao; locality: Area de Conservacion Guanacaste; verbatimLocality: Quebrada Otilio; verbatimElevation: 550; verbatimLatitude: 10.89; verbatimLongitude: -85.4797; verbatimCoordinateSystem: Decimal; decimalLatitude: 10.89 ; decimalLongitude: -85.4797; samplingProtocol: Reared from the larva of the Nymphalidae, Morpho helenor; verbatimEventDate: 09-Oct-2007; individualID: DHJPAR0021975; individualCount: 1; lifeStage: adult; preparations: pinned; 
catalogNumber: DHJPAR0021975; occurrenceDetails: http://janzen.sas.upenn.edu; recordedBy: D.H. Janzen, W. Hallwachs \& Dunia Garcia; otherCatalogNumbers: ASTAT1113-07, 07-SRNP-46175, BOLD:AAA5134; identifiedBy: AJ Fleming; dateldentified: 2017; language: en; institutionCode: CNC; collectionCode: Insects; basisOfRecord: Pinned Specimen

bd. ScientificName: Hyphantrophaga morphophaga; phylum: Arthropoda; class: Insecta; order: Diptera; family: Tachinidae; genus: Hyphantrophaga; specificEpithet: morphophaga; scientificNameAuthorship: Fleming \& Wood, 2018; continent: Central America; country: Costa Rica; countryCode: CR; stateProvince: Guanacaste; county: Sector Mundo Nuevo; locality: Area de Conservacion Guanacaste; verbatimLocality: Sendero Mora; verbatimElevation: 480; verbatimLatitude: 10.7683; verbatimLongitude: -85.4257; verbatimCoordinateSystem: Decimal; decimalLatitude: 10.7683; decimalLongitude: -85.4257; samplingProtocol: Reared from the larva of the Nymphalidae, Morpho helenor; verbatimEventDate: 17-Aug-2007; individualID: DHJPAR0022286; individualCount: 1; lifeStage: adult; preparations: pinned; catalogNumber: DHJPAR0022286; occurrenceDetails: http://janzen.sas.upenn.edu; recordedBy: D.H. Janzen, W. Hallwachs \& Jose Cortez; otherCatalogNumbers: ASMGI562-08, 07-SRNP-58222,; identifiedBy: AJ Fleming; dateldentified: 2017; language: en; institutionCode: CNC; collectionCode: Insects; basisOfRecord: Pinned Specimen

be. $\quad$ scientificName: Hyphantrophaga morphophaga; phylum: Arthropoda; class: Insecta; order: Diptera; family: Tachinidae; genus: Hyphantrophaga; specificEpithet: morphophaga; scientificNameAuthorship: Fleming \& Wood, 2018; continent: Central America; country: Costa Rica; countryCode: CR; stateProvince: Guanacaste; county: Sector Cacao; locality: Area de Conservacion Guanacaste; verbatimLocality: Sendero Derrumbe; verbatimElevation: 1220 ; verbatimLatitude: 10.9292; verbatimLongitude: -85.4643; verbatimCoordinateSystem: Decimal; decimalLatitude: 10.9292; decimalLongitude: -85.4643; samplingProtocol: Reared from the larva of the Nymphalidae, Morpho helenor; verbatimEventDate: 14-Aug-2008; individualID: DHJPAR0027845; individualCount: 1; lifeStage: adult; preparations: pinned; catalogNumber: DHJPAR0027845; occurrenceDetails: http://janzen.sas.upenn.edu; recordedBy: D.H. Janzen, W. Hallwachs \& Harry Ramirez; otherCatalogNumbers: ASHYE082-08, 08-SRNP-35746, BOLD:AAA5134; identifiedBy: AJ Fleming; dateldentified: 2017; language: en; institutionCode: CNC; collectionCode: Insects; basisOfRecord: Pinned Specimen

bf. $\quad$ scientificName: Hyphantrophaga morphophaga; phylum: Arthropoda; class: Insecta; order: Diptera; family: Tachinidae; genus: Hyphantrophaga; specificEpithet: morphophaga; scientificNameAuthorship: Fleming \& Wood, 2018; continent: Central America; country: Costa Rica; countryCode: CR; stateProvince: Guanacaste; county: Sector Del Oro; locality: Area de Conservacion Guanacaste; verbatimLocality: Finca Araya; verbatimElevation: 295; verbatimLatitude: 11.0154; verbatimLongitude: -85.5113; verbatimCoordinateSystem: Decimal; decimalLatitude: 11.0154; decimalLongitude: -85.5113; samplingProtocol: Reared from the larva of the Nymphalidae, Morpho helenor; verbatimEventDate: 21-Oct-2014; individualID: DHJPAR0057238; individualCount: 1; lifeStage: adult; preparations: pinned; catalogNumber: DHJPAR0057238; occurrenceDetails: http://janzen.sas.upenn.edu; recordedBy: D.H. Janzen, W. Hallwachs \& Lucia Rios; otherCatalog Numbers: ACGBA5148-15, 14-SRNP-20989,; identifiedBy: AJ Fleming; dateldentified: 2017; language: en; institutionCode: CNC; collectionCode: Insects; basisOfRecord: Pinned Specimen 
bg. scientificName: Hyphantrophaga morphophaga; phylum: Arthropoda; class: Insecta; order: Diptera; family: Tachinidae; genus: Hyphantrophaga; specificEpithet: morphophaga; scientificNameAuthorship: Fleming \& Wood, 2018; continent: Central America; country: Costa Rica; countryCode: CR; stateProvince: Guanacaste; county: Pailas Dos; locality: Area de Conservacion Guanacaste; verbatimLocality: PL12-1; verbatimElevation: 828; verbatimLatitude: 10.7642; verbatimLongitude: -85.335; verbatimCoordinateSystem: Decimal; decimalLatitude: 10.7642; decimalLongitude: -85.335; samplingProtocol: Reared from the larva of the Malaise trap PL12-1, Malaise trap PL12-1; verbatimEventDate: 05-Dec-2013; individualID: BIOUG28650-B08; individualCount: 1; lifeStage: adult; preparations: pinned; catalogNumber: BIOUG28650-B08; occurrenceDetails: http://janzen.sas.upenn.edu; recordedBy: D.H. Janzen, W. Hallwachs \& D.Janzen, W.Hallwachs; otherCatalogNumbers: JICAJ024-16, GMP\#06717, BOLD:AAA5134; identifiedBy: AJ Fleming; dateldentified: 2017; language: en; institutionCode: CNC; collectionCode: Insects; basisOfRecord: Pinned Specimen

bh. $\quad$ scientificName: Hyphantrophaga morphophaga; phylum: Arthropoda; class: Insecta; order: Diptera; family: Tachinidae; genus: Hyphantrophaga; specificEpithet: morphophaga; scientificNameAuthorship: Fleming \& Wood, 2018; continent: Central America; country: Costa Rica; countryCode: CR; stateProvince: Guanacaste; county: Sector Del Oro; locality: Area de Conservacion Guanacaste; verbatimLocality: Sendero Puertas; verbatimElevation: 400; verbatimLatitude: 11.0109; verbatimLongitude: -85.4882; verbatimCoordinateSystem: Decimal; decimalLatitude: 11.0109; decimalLongitude: -85.4882; samplingProtocol: Reared from the larva of the Nymphalidae, Morpho helenor; verbatimEventDate: 16-Oct-2016; individualID: DHJPAR0059908; individualCount: 1; lifeStage: adult; preparations: pinned; catalogNumber: DHJPAR0059908; occurrenceDetails: http://janzen.sas.upenn.edu; recordedBy: D.H. Janzen, W. Hallwachs \& Lucia Rios; otherCatalogNumbers: ACGBA6329-16, 16-SRNP-21403, BOLD:AAA5134; identifiedBy: AJ Fleming; dateldentified: 2017; language: en; institutionCode: CNC; collectionCode: Insects; basisOfRecord: Pinned Specimen

bi. ScientificName: Hyphantrophaga morphophaga; phylum: Arthropoda; class: Insecta; order: Diptera; family: Tachinidae; genus: Hyphantrophaga; specificEpithet: morphophaga; scientificNameAuthorship: Fleming \& Wood, 2018; continent: Central America; country: Costa Rica; countryCode: CR; stateProvince: Guanacaste; county: Sector Del Oro; locality: Area de Conservacion Guanacaste; verbatimLocality: Sendero Puertas; verbatimElevation: 400; verbatimLatitude: 11.0109; verbatimLongitude: -85.4882; verbatimCoordinateSystem: Decimal; decimalLatitude: 11.0109; decimalLongitude: -85.4882; samplingProtocol: Reared from the larva of the Nymphalidae, Morpho helenor; verbatimEventDate: 03-Nov-2016; individualID: DHJPAR0060314; individualCount: 1; lifeStage: adult; preparations: pinned; catalogNumber: DHJPAR0060314; occurrenceDetails: http://janzen.sas.upenn.edu; recordedBy: D.H. Janzen, W. Hallwachs \& Lucia Rios; otherCatalogNumbers: ACGBA6735-17, 16-SRNP-21438, BOLD:AAA5134; identifiedBy: AJ Fleming; dateldentified: 2017; language: en; institutionCode: CNC; collectionCode: Insects; basisOfRecord: Pinned Specimen

\section{Description}

Male (Fig. 20). Length: 7-11 mm. Head (Fig. 20b): vertex 1/4 of head width; two reclinate upper orbital setae; ocellar setae arising beside anterior ocellus; ocellar 
triangle dark blackened gold with light gold tomentum around margin; fronto-orbital plate dull grey tomentose with slight gold tinge, stronger around vertex; eye densely haired; fronto-orbital plate setulose, setulae not extending below lowest frontal seta; fronto-orbital plate shiny silver; parafacial bare; facial ridge bare; pedicel black with a small spot of orange basally, sometimes extending into adjacent region of postpedicel; otherwise concolorous with postpedicel; arista black, very minutely pubescent, gradually tapered apically beginning on basal $1 / 3-1 / 4$; palpus ranging from yellow to brown. Thorax (Fig. 20a, c): entire thorax densely hirsute with short black setulae amongst setae; prosternum setose with 1-3 strong setae surrounded by a brush of weaker setulae; four prominent dorsal vittae, outermost pair broken across suture, innermost pair unbroken across suture, not reaching beyond 2nd postsutural dorsocentral seta; postpronotum with five setae arranged in a triangle; chaetotaxy: acrostichal setae 4:3; dorsocentral setae 4:4; intra-alar setae 3:3; supra-alar setae 2:3; katepisternum with three setae, basal seta weakest, arising anterior to suture; lateral scutellar setae $1 / 2$ as long as subapical setae, slightly curving inwards medially; subapical setae subequal in length to basal scutellar setae, the latter arising above plane of remaining marginal scutellar setae; apical scutellar setae ranging from $1 / 2$ as long as lateral scutellar setae to subequal in length but $1 / 2$ the diameter; one pair of discal scutellar setae; scutellum gold tomentose along apical margin, transitioning to grey tomentose basally. Legs (Fig. 20c): femora and coxae black in ground colour; tibiae yellow in ground colour, densely covered in short black hairs, appearing darkened, almost black; fore femur with dense silver tomentum on posterodorsal surface; hind coxa either bare or with a single seta along posterior margin. Wing (Fig. 20a): pale translucent, not distinctly infuscate; vein $R_{4+5}$ with 2-3 (most often 2) setulae at base. Abdomen (Fig. 20a, c): ground colour brown; mid-dorsal depression on ST1 +2 extending almost to margin; median marginal setae absent on ST1+2 and T3; a complete row of marginal setae present on T4; discal setae only on T5; sex patch covering ventral surfaces of T4-T5, the posterior $1 / 3$ of T3; distinct tomentose bands along anterior $2 / 3$ of T3 and T4, broken medially by a dorsocentral stripe; T5 with silver tomentum over its entirety. Terminalia (Fig. 20d, e, f): sternite 5 (Fig. 20f) with a deeply excavated median cleft, smoothly U-shaped, margins covered in dense tomentum. Lateral lobes of sternite rounded apically, with 2-3 strong setae surrounded by many shorter, weaker setulae. Anterior plate of sternite 5 from subequal to slightly longer than apical lobes; unsclerotised "window" appearing blunt umbonate with a slightly rectangular base and a wider crown making it appear mushroom-shaped, as wide as median cleft. Cerci in posterior view (Fig. 20d) rectangular and slightly shorter than surstyli, blunt and rounded at apex, completely separate medially appearing slightly divergent basally, twice as wide as apex; in lateral view with a slight downward curve in apical $1 / 3$; densely setulose along basal $2 / 3$, setulose ventrally along entire length (visible in lateral view). Surstylus in lateral view (Fig. 20e) narrow, almost parallel-sided along its entire length, ending in a slightly downcurved apex, making the structure appear blade-like; when viewed dorsally, surstyli appearing to point outwards. Pregonite short and wide, well-developed, subequal in length to postgonite, as long as distiphallus, bare and squared-off apically. Postgonite slightly narrow $1 / 3$ as wide as pregonite, sharply pointed and curved at apex. Distiphallus rectangular, only weakly 
flaring apically with a slender, median longitudinal sclerotised reinforcement on its posterior surface and a broad, anterolateral, apically clubbed sclerotised acrophallus on each side, joining the plate of opposite side on anterior surface near apex.

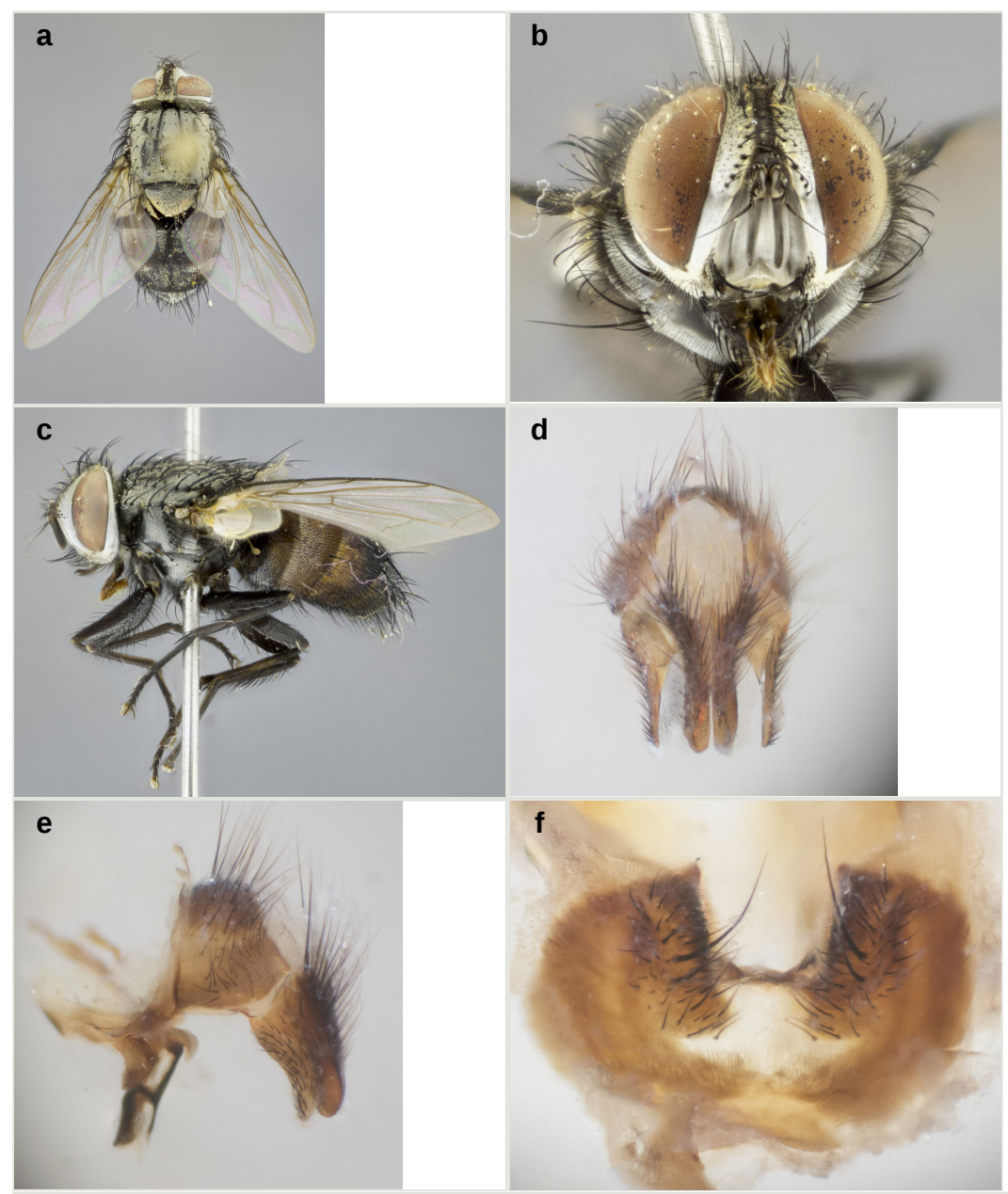

Figure 20.

Hyphantrophaga morphophaga sp. n.; a-c: habitus; holotype male, voucher $\mathrm{n}$. DHJPAR0007354; d-f: terminalia; male paratype, voucher n. DHJPAR0021984.
a: dorsal view doi
b: frontal view doi
c: lateral view doi
d: dorsal view doi
e: lateral view doi
f: sternite 5 , ventral view doi

Female. Length: 8-11 mm. As male, differing only by the presence of two pairs of proclinate orbital setae. 


\section{Diagnosis}

To date Hyphantrophaga morphophaga sp. n. can only be distinguished from its closest congener $H$. danausophaga sp. $\mathbf{n}$. by its host selection and habitat, being found parasitising only large, hairy, non-ringed cryptic Nymphalidae larvae in densely shaded habitat within the forest.

\section{Etymology}

Named after the genus Morpho and the Greek "phago" meaning "eating", with reference to its primary hosts.

\section{Distribution}

Costa Rica, ACG, Alajuela and Guanacaste Provinces, 17-1276 m elevation.

\section{Ecology}

Hyphantrophaga morphophaga sp. $\mathbf{n}$. has been reared 130 times from three species of Lepidoptera in the family Nymphalidae, Morpho amathonte (Deyrolle, 1860), Morpho polyphemus catalina (Corea and Chacon, 1984) and Morpho helenor (Catalina, 1776), in cloud forest, rain forest, dry forest and dry-rain lowland intergrade.

\section{Hyphantrophaga myersi (Aldrich, 1933)}

\section{Materials}

Holotype:

a. ScientificName: Hyphantrophaga myersi; phylum: Arthropoda; class: Insecta; order: Diptera; family: Tachinidae; genus: Prophryno; specific Epithet: myersi; scientificNameAuthorship: (Aldrich, 1933); continent: South America; country: Guyana (as British Guiana); stateProvince: Pakaraima (as Pakeraima); verbatimLocality: Upper Ireng River; samplingProtocol: Reared from the larva of the Selenis suere Cramer [sic]; eventDate: March, 1931; individualCount: 1; sex: male; preparations: pinned; recordedBy: J.G. Myers; otherCatalogNumbers: Cat. No. 49789 U. S. N. M.; identifiedBy: M. Wood; language: en; institutionCode: BMNH; collectionCode: Insects; basisOfRecord: Pinned Specimen

\section{Other materials:}

a. $\quad$ scientificName: Hyphantrophaga myersi; phylum: Arthropoda; class: Insecta; order: Diptera; family: Tachinidae; genus: Hyphantrophaga; specificEpithet: myersi; scientificNameAuthorship: (Aldrich, 1933); continent: Central America; country: Costa Rica; countryCode: CR; stateProvince: Alajuela; county: Sector San Cristobal; locality: Area de Conservacion Guanacaste; verbatimLocality: Estacion San Cristobal; verbatimElevation: 640; verbatimLatitude: 10.871; verbatimLongitude: -85.3914; verbatimCoordinateSystem: Decimal; decimalLatitude: 10.871; decimalLongitude: -85.3914; samplingProtocol: Reared from the larva of the Crambidae, Portentomorpha xanthialis; verbatimEventDate: 30-Jul-1998; individualID: DHJPAR0007460; individualCount: 1; lifeStage: adult; preparations: pinned; catalogNumber: 
DHJPAR0007460; occurrenceDetails: http://janzen.sas.upenn.edu; recordedBy: D.H. Janzen, W. Hallwachs \& Osvaldo Espinoza; otherCatalog Numbers: ASTAT232-06, 98SRNP-6859, BOLD:AAA9858; identifiedBy: AJ Fleming; dateldentified: 2017; language: en; institutionCode: CNC; collectionCode: Insects; basisOfRecord: Pinned Specimen

b. $\quad$ scientificName: Hyphantrophaga myersi; phylum: Arthropoda; class: Insecta; order: Diptera; family: Tachinidae; genus: Hyphantrophaga; specificEpithet: myersi; scientificNameAuthorship: (Aldrich, 1933); continent: Central America; country: Costa Rica; countryCode: CR; stateProvince: Guanacaste; county: Sector Horizontes; locality: Area de Conservacion Guanacaste; verbatimLocality: Bejuco; verbatimElevation: 180; verbatimLatitude: 10.7671; verbatimLongitude: -85.5966; verbatimCoordinateSystem: Decimal; decimalLatitude: 10.7671 ; decimalLongitude: -85.5966; sampling Protocol: Reared from the larva of the Crambidae, Portentomorpha xanthialis; verbatimEventDate: 24-Jul-2003; individualID: DHJPAR0007445; individualCount: 1; lifeStage: adult; preparations: pinned; catalogNumber: DHJPAR0007445; occurrenceDetails: http://janzen.sas.upenn.edu; recordedBy: D.H. Janzen, W. Hallwachs \& Guillermo Pereira; otherCatalog Numbers: ASTAT217-06, 03SRNP-13817, BOLD:AAA9858; identifiedBy: AJ Fleming; dateldentified: 2017; language: en; institutionCode: CNC; collectionCode: Insects; basisOfRecord: Pinned Specimen

c. ScientificName: Hyphantrophaga myersi; phylum: Arthropoda; class: Insecta; order: Diptera; family: Tachinidae; genus: Hyphantrophaga; specificEpithet: myersi; scientificNameAuthorship: (Aldrich, 1933); continent: Central America; country: Costa Rica; countryCode: CR; stateProvince: Guanacaste; county: Sector Horizontes; locality: Area de Conservacion Guanacaste; verbatimLocality: Bejuco; verbatimElevation: 180; verbatimLatitude: 10.7671; verbatimLongitude: -85.5966; verbatimCoordinateSystem: Decimal; decimalLatitude: 10.7671; decimalLongitude: -85.5966; samplingProtocol: Reared from the larva of the Crambidae, Portentomorpha xanthialis; verbatimEventDate: 26-Jul-2003; individualID: DHJPAR0007447; individualCount: 1; lifeStage: adult; preparations: pinned; catalogNumber: DHJPAR0007447; occurrenceDetails: http://janzen.sas.upenn.edu; recordedBy: D.H. Janzen, W. Hallwachs \& Jose Cortez; otherCatalogNumbers: ASTAT219-06, 03SRNP-13919, BOLD:AAA9858; identifiedBy: AJ Fleming; dateldentified: 2017; language: en; institutionCode: CNC; collectionCode: Insects; basisOfRecord: Pinned Specimen

d. ScientificName: Hyphantrophaga myersi; phylum: Arthropoda; class: Insecta; order: Diptera; family: Tachinidae; genus: Hyphantrophaga; specificEpithet: myersi; scientificNameAuthorship: (Aldrich, 1933); continent: Central America; country: Costa Rica; countryCode: CR; stateProvince: Alajuela; county: Sector San Cristobal; locality: Area de Conservacion Guanacaste; verbatimLocality: Estacion San Cristobal; verbatimElevation: 640; verbatimLatitude: 10.871; verbatimLongitude: -85.3914; verbatimCoordinateSystem: Decimal; decimalLatitude: 10.871; decimalLongitude: -85.3914; samplingProtocol: Reared from the larva of the Crambidae, Hoterodes ausonia; verbatimEventDate: 28-Jul-1998; individualID: DHJPAR0007461; individualCount: 1; lifeStage: adult; preparations: pinned; catalogNumber: DHJPAR0007461; occurrenceDetails: http://janzen.sas.upenn.edu; recordedBy: D.H. Janzen, W. Hallwachs \& Osvaldo Espinoza; otherCatalogNumbers: ASTAT233-06, 98SRNP-6878, BOLD:AAA9858; identifiedBy: AJ Fleming; dateldentified: 2017; language: en; institutionCode: CNC; collectionCode: Insects; basisOfRecord: Pinned Specimen 
e. $\quad$ scientificName: Hyphantrophaga myersi; phylum: Arthropoda; class: Insecta; order: Diptera; family: Tachinidae; genus: Hyphantrophaga; specificEpithet: myersi; scientificNameAuthorship: (Aldrich, 1933); continent: Central America; country: Costa Rica; countryCode: CR; stateProvince: Guanacaste; county: Sector Horizontes; locality: Area de Conservacion Guanacaste; verbatimLocality: Bejuco; verbatimElevation: 180; verbatimLatitude: 10.7671; verbatimLongitude: -85.5966; verbatimCoordinateSystem: Decimal; decimalLatitude: 10.7671; decimalLongitude: -85.5966; samplingProtocol: Reared from the larva of the Crambidae, Portentomorpha xanthialis; verbatimEventDate: 26-Jul-2003; individualID: DHJPAR0007450; individualCount: 1; lifeStage: adult; preparations: pinned; catalogNumber: DHJPAR0007450; occurrenceDetails: http://janzen.sas.upenn.edu; recordedBy: D.H. Janzen, W. Hallwachs \& Guillermo Pereira; otherCatalog Numbers: ASTAT222-06, 03SRNP-13852, BOLD:AAA9858; identifiedBy: AJ Fleming; dateldentified: 2017; language: en; institutionCode: CNC; collectionCode: Insects; basisOfRecord: Pinned Specimen

\section{Description}

Male (Fig. 21). Length: 5-7 mm. Head (Fig. 21b): vertex $1 / 3$ of head width; two reclinate upper orbital setae; ocellar setae arising slightly behind anterior ocellus; ocellar triangle gold, concolorous with fronto-orbital plate; fronto-orbital plate entirely gold and sparsely setulose throughout, setulae not reaching below lowest frontal seta; parafacial shiny silver and bare; facial ridge setose along its entire length; eye with short sparse ommatrichia up to $2 \mathrm{X}$ as long as one ommatidium; pedicel light brown; postpedicel black; arista brown, bare, distinctly thickened on basal 1/3-1/4; palpus dark amber yellow and haired. Thorax (Fig. 21a, c): pale brassy-silver tomentose dorsally, grey tomentose laterally; four prominent dorsal vittae, outermost two slightly broken across suture, innermost pair unbroken across suture, reaching 2nd postsutural dorsocentral seta; postpronotum with 3-4 setae arranged in a triangle; chaetotaxy: acrostichal setae 3:3; dorsocentral setae 3:4; intra-alar setae 3-4:3; supra-alar setae 2:3; three katepisternal setae; lateral scutellar setae shorter than subapical scutellar setae; apical scutellar setae curving inwards medially and slightly upturned so as to rise above the plane of remaining scutellar setae; one pair of discal scutellar setae; scutellum slightly darker and grey along basal 3/4, apically concolorous with scutum. Legs (Fig. 21c): femora and coxae black in ground colour; tibiae yellow in ground colour, densely covered in short black hairs, appearing darkened, almost black; fore femur with dense silver tomentum on posterodorsal surface; hind coxa with a single seta along posterior margin. Wing (Fig. 21a): pale translucent, hyaline, not distinctly infuscate; vein $\mathrm{R}_{4+5}$ with only two setulae at base. Abdomen (Fig. 21a, c): ground colour black; mid-dorsal depression on ST1+2 extending only halfway across tergite; median marginal setae absent on ST1+2 and weak on T3; T4-T5 with a complete row of marginal setae; discal setae absent on all tergites; sex patch covering ventral surfaces of T4-T5; dorsal surface of abdomen covered in brassy-silver tomentum on ST1+2-T4; T5 with brilliant gold tomentum over its entirety. Terminalia: sternite 5 (Fig. 21f) with a deeply excavated median cleft, rounded U-shaped, inner margins covered in dense tomentum. Lateral lobes of sternite rounded apically, with 2-3 strong setae 
surrounded by 2-3 shorter, weaker setulae. Anterior plate of sternite 5 from subequal to slightly longer than apical lobes, unsclerotised "window" rectangular, 1/2 as wide as median cleft and slightly convex. Cerci in posterior view (Fig. 21d) slightly rectangular, shorter than surstyli, blunt and rounded at apex, fused along basal $2 / 3$, separating medially along apical $1 / 3$; in lateral view with a strong downward curve in apical 1/3; densely setulose along basal 2/3 dorsally, setulose ventrally along entire length (visible in lateral view). Surstylus in lateral view (Fig. 21e) almost parallel-sided along its length, rounded at tip; surstylus appearing to be fused with epandrium; when viewed dorsally, surstyli appearing slender and straight, not strongly convergent. Pregonite broad and well-developed, bent medially, apically rounded, devoid of setulae. Postgonite elongate, slender, very slightly hooked at tip, subequal in length to pregonite. Distiphallus vaguely cone-shaped, could be seen as rectangular, weakly flaring apically, with a slender median longitudinal sclerotised reinforcement on its posterior surface and a broad, anterolateral, sclerotised acrophallus on each side, joining the plate of opposite side on anterior surface near apex.

Female. Length: 5-7 mm. As male, differing only by the presence of two pairs of proclinate orbital setae.

\section{Diagnosis}

Hyphantrophaga myersi (Aldrich) can be distinguished from all other Hyphantrophaga species by the following combination of traits: facial ridge with stout setae along entire length and sternite 5 distinctly golden tomentose.

\section{Distribution}

Neotropical species recorded from South America: Guyana, Venezuela; Lesser Antilles: Trinidad \& Tobago; and Mesoamerica: Costa Rica, ACG (Provinces of Alajuela and Guanacaste), 180-640 m elevation.

\section{Ecology}

Hyphantrophaga myersi has been reared 43 times within the ACG inventory from eight species of Lepidoptera, six in the family Erebidae (Renodes curviluna (Druce, 1890), Anomis illita Guenée, 1852, Anomis luridula Guenée, 1852, Eulepidotis folium (Schaus, 1911), Helia sueroides (Guenée, 1852) and Dysschema lycaste (Klug, 1836)); and two in the family Geometridae (Semiothisa sp. and geoJanzen01 Janzen17), in rain forest, dry forest and dry-rain lowland intergrade. 


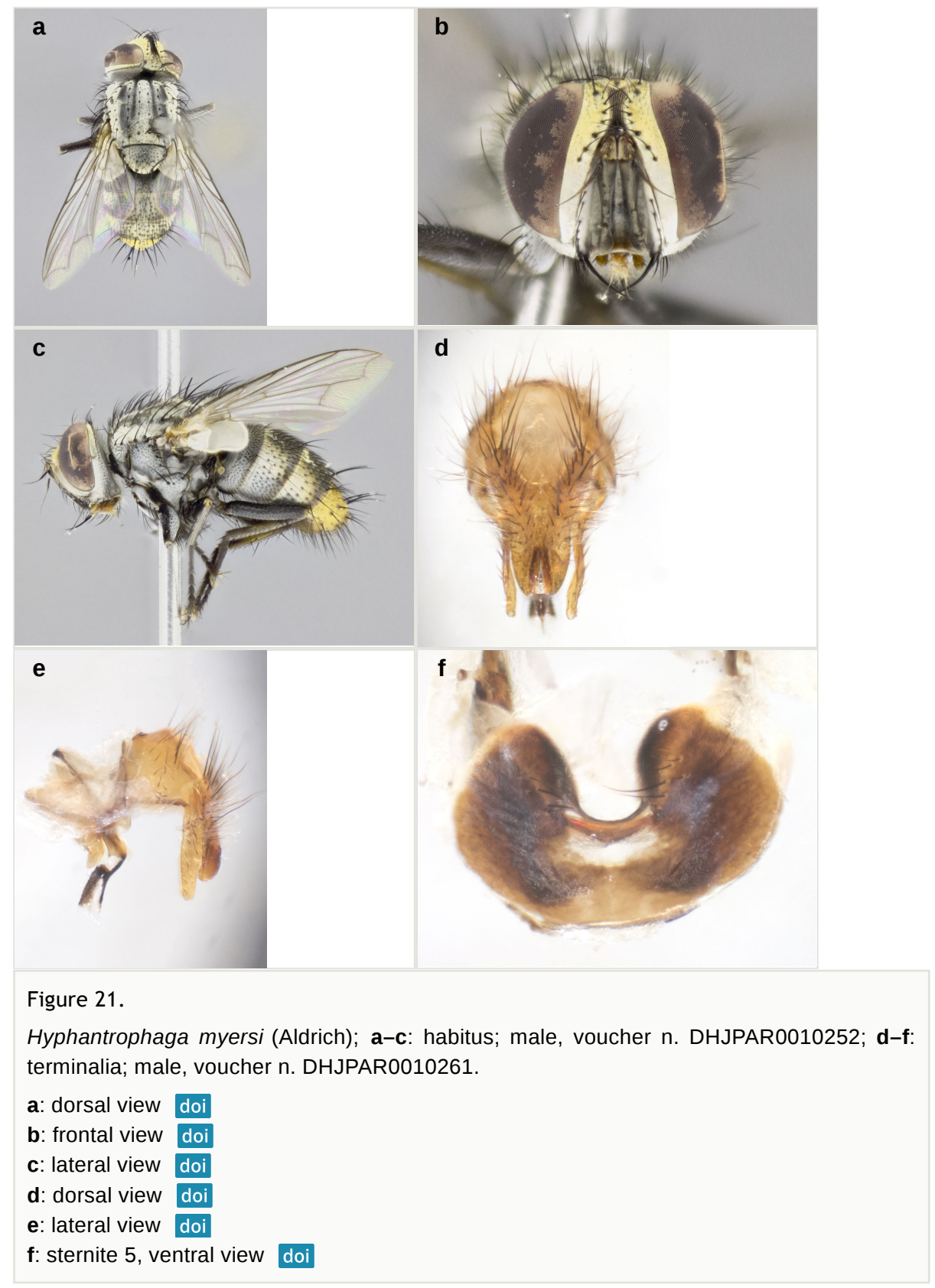




\section{Hyphantrophaga nigricauda Fleming \& Wood, sp. $\mathrm{n}$.}

- ZooBank urn:Isid:zoobank.org:act:E866E684-19A6-4A47-A070-0935DFA78F75

\section{Materials}

Holotype:

a. scientificName: Hyphantrophaga nigricauda; phylum: Arthropoda; class: Insecta; order: Diptera; family: Tachinidae; genus: Hyphantrophaga; specificEpithet: nigricauda; scientificNameAuthorship: Fleming \& Wood, 2017; continent: Central America; country: Costa Rica; countryCode: CR; stateProvince: Guanacaste; county: Sector Pitilla; locality: Area de Conservacion Guanacaste; verbatimLocality: Leonel; verbatimElevation: 510; verbatimLatitude: 10.9964; verbatimLongitude: -85.4019; verbatimCoordinateSystem: Decimal; decimalLatitude: 10.9964; decimalLongitude: -85.4019; samplingProtocol: Reared from the larva of the Geometridae, Cyclomia disparilis; verbatimEventDate: 28-Jun-2008; individualID: DHJPAR0027908; individualCount: 1; sex: male; lifeStage: adult; preparations: pinned; catalogNumber: DHJPAR0027908; occurrenceDetails: http://janzen.sas.upenn.edu; recordedBy: D.H. Janzen, W. Hallwachs \& Leonel Siezar; otherCatalogNumbers: ASHYE145-08, 08SRNP-70578, BOLD:AAA1929; identifiedBy: AJ Fleming; dateldentified: 2017; language: en; institutionCode: CNC; collectionCode: Insects; basisOfRecord: Pinned Specimen

\section{Paratypes:}

a. ScientificName: Hyphantrophaga nigricauda; phylum: Arthropoda; class: Insecta; order: Diptera; family: Tachinidae; genus: Hyphantrophaga; specificEpithet: nigricauda; scientificNameAuthorship: Fleming \& Wood, 2017; continent: Central America; country: Costa Rica; countryCode: CR; stateProvince: Guanacaste; county: Sector Pitilla; locality: Area de Conservacion Guanacaste; verbatimLocality: Pasmompa; verbatimElevation: 440; verbatimLatitude: 11.0193; verbatimLongitude: -85.41; verbatimCoordinateSystem: Decimal; decimalLatitude: 11.0193; decimalLongitude: -85.41; samplingProtocol: Reared from the larva of the Geometridae, Cyclomia disparilis; verbatimEventDate: 20-Jul-2005; individualID: DHJPAR0007291; individualCount: 1; sex: female; lifeStage: adult; preparations: pinned; catalogNumber: DHJPAR0007291; occurrenceDetails: http://janzen.sas.upenn.edu; recordedBy: D.H. Janzen, W. Hallwachs \& Calixto Moraga; otherCatalog Numbers: ASTAT063-06, 05SRNP-32268, BOLD:AAA1929; identifiedBy: AJ Fleming; dateldentified: 2017; language: en; institutionCode: CNC; collectionCode: Insects; basisOfRecord: Pinned Specimen

b. scientificName: Hyphantrophaga nigricauda; phylum: Arthropoda; class: Insecta; order: Diptera; family: Tachinidae; genus: Hyphantrophaga; specificEpithet: nigricauda; scientificNameAuthorship: Fleming \& Wood, 2017; continent: Central America; country: Costa Rica; countryCode: CR; stateProvince: Guanacaste; county: Sector Pitilla; locality: Area de Conservacion Guanacaste; verbatimLocality: Sendero Laguna; verbatimElevation: 680; verbatimLatitude: 10.9888; verbatimLongitude: -85.4234; verbatimCoordinateSystem: Decimal; decimalLatitude: 10.9888; decimalLongitude: -85.4234; sampling Protocol: Reared from the larva of the Crambidae, Eulepte Solis15; verbatimEventDate: 20-May-2011; individualID: DHJPAR0042693; individualCount: 1; sex: female; lifeStage: adult; preparations: pinned; catalogNumber: DHJPAR0042693; occurrenceDetails: http://janzen.sas.upenn.edu; recordedBy: D.H. Janzen, W. Hallwachs \& Freddy Quesada; otherCatalogNumbers: ASHYH451-11, 11- 
SRNP-31067, BOLD:AAA1929; identifiedBy: AJ Fleming; dateldentified: 2017; language: en; institutionCode: CNC; collectionCode: Insects; basisOfRecord: Pinned Specimen

c. scientificName: Hyphantrophaga nigricauda; phylum: Arthropoda; class: Insecta; order: Diptera; family: Tachinidae; genus: Hyphantrophaga; specificEpithet: nigricauda; scientificNameAuthorship: Fleming \& Wood, 2017; continent: Central America; country: Costa Rica; countryCode: CR; stateProvince: Guanacaste; county: Sector Pitilla; locality: Area de Conservacion Guanacaste; verbatimLocality: Pasmompa; verbatimElevation: 440; verbatimLatitude: 11.0193; verbatimLongitude: -85.41; verbatimCoordinateSystem: Decimal; decimalLatitude: 11.0193; decimalLongitude: -85.41; samplingProtocol: Reared from the larva of the Geometridae, Cyclomia disparilis; verbatimEventDate: 20-Jul-2005; individualID: DHJPAR0007293; individualCount: 1; sex: female; lifeStage: adult; preparations: pinned; catalog Number: DHJPAR0007293; occurrenceDetails: http://janzen.sas.upenn.edu; recordedBy: D.H. Janzen, W. Hallwachs \& Calixto Moraga; otherCatalogNumbers: ASTAT065-06, 05SRNP-32270, BOLD:AAA1929; identifiedBy: AJ Fleming; dateldentified: 2017; language: en; institutionCode: CNC; collectionCode: Insects; basisOfRecord: Pinned Specimen

d. ScientificName: Hyphantrophaga nigricauda; phylum: Arthropoda; class: Insecta; order: Diptera; family: Tachinidae; genus: Hyphantrophaga; specificEpithet: nigricauda; scientificNameAuthorship: Fleming \& Wood, 2017; continent: Central America; country: Costa Rica; countryCode: CR; stateProvince: Guanacaste; county: Sector Pitilla; locality: Area de Conservacion Guanacaste; verbatimLocality: Pasmompa; verbatimElevation: 440; verbatimLatitude: 11.0193; verbatimLongitude: -85.41; verbatimCoordinateSystem: Decimal; decimalLatitude: 11.0193; decimalLongitude: -85.41; samplingProtocol: Reared from the larva of the Geometridae, Cyclomia disparilis; verbatimEventDate: 16-Jul-2005; individualID: DHJPAR0007290; individualCount: 1; sex: male; lifeStage: adult; preparations: pinned; catalogNumber: DHJPAR0007290; occurrenceDetails: http://janzen.sas.upenn.edu; recordedBy: D.H. Janzen, W. Hallwachs \& Calixto Moraga; otherCatalog Numbers: ASTAT062-06, 05SRNP-32248, BOLD:AAA1929; identifiedBy: AJ Fleming; dateldentified: 2017; language: en; institutionCode: CNC; collectionCode: Insects; basisOfRecord: Pinned Specimen

e. $\quad$ scientificName: Hyphantrophaga nigricauda; phylum: Arthropoda; class: Insecta; order: Diptera; family: Tachinidae; genus: Hyphantrophaga; specificEpithet: nigricauda; scientificNameAuthorship: Fleming \& Wood, 2017; continent: Central America; country: Costa Rica; countryCode: CR; stateProvince: Guanacaste; county: Sector Santa Rosa; locality: Area de Conservacion Guanacaste; verbatimLocality: Camino Borrachos; verbatimElevation: 295; verbatimLatitude: 10.8429; verbatimLongitude: -85.6161; verbatimCoordinateSystem: Decimal; decimalLatitude: 10.8429 ; decimalLongitude: -85.6161; samplingProtocol: Reared from the larva of the Crambidae, Conchylodes platinalis; verbatimEventDate: 01-Aug-2009; individualID: DHJPAR0036438; individualCount: 1; sex: female; lifeStage: adult; preparations: pinned; catalogNumber: DHJPAR0036438; occurrenceDetails: http://janzen.sas.upenn.edu; recordedBy: D.H. Janzen, W. Hallwachs \& Guillermo Pereira; otherCatalogNumbers: ASHYD1629-09, 09-SRNP-14037, BOLD:AAA1929; identifiedBy: AJ Fleming; dateldentified: 2017; language: en; institutionCode: CNC; collectionCode: Insects; basisOfRecord: Pinned Specimen

f. ScientificName: Hyphantrophaga nigricauda; phylum: Arthropoda; class: Insecta; order: Diptera; family: Tachinidae; genus: Hyphantrophaga; specificEpithet: nigricauda; 
scientificNameAuthorship: Fleming \& Wood, 2017; continent: Central America; country: Costa Rica; countryCode: CR; stateProvince: Alajuela; county: Sector Rincon Rain Forest; locality: Area de Conservacion Guanacaste; verbatimLocality: Sendero Juntas; verbatimElevation: 400; verbatimLatitude: 10.9066; verbatimLongitude: -85.2878; verbatimCoordinateSystem: Decimal; decimalLatitude: 10.9066; decimalLongitude: -85.2878; samplingProtocol: Reared from the larva of the Geometridae, Cyclomia disparilis; verbatimEventDate: 01-Jul-2009; individualID: DHJPAR0035694; individualCount: 1; sex: female; lifeStage: adult; preparations: pinned; catalogNumber: DHJPAR0035694; occurrenceDetails: http://janzen.sas.upenn.edu; recordedBy: D.H. Janzen, W. Hallwachs \& Jose Perez; otherCatalog Numbers: ASHYD1075-09, 09SRNP-41180, BOLD:AAA1929; identifiedBy: AJ Fleming; dateldentified: 2017; language: en; institutionCode: CNC; collectionCode: Insects; basisOfRecord: Pinned Specimen

g. ScientificName: Hyphantrophaga nigricauda; phylum: Arthropoda; class: Insecta; order: Diptera; family: Tachinidae; genus: Hyphantrophaga; specificEpithet: nigricauda; scientificNameAuthorship: Fleming \& Wood, 2017; continent: Central America; country: Costa Rica; countryCode: CR; stateProvince: Alajuela; county: Sector Rincon Rain Forest; locality: Area de Conservacion Guanacaste; verbatimLocality: Sendero Guaca; verbatimElevation: 400; verbatimLatitude: 10.9061; verbatimLongitude: -85.2828; verbatimCoordinateSystem: Decimal; decimalLatitude: 10.9061; decimalLongitude: -85.2828; samplingProtocol: Reared from the larva of the Crambidae, Pleuroptya Solis01; verbatimEventDate: 31-Aug-2006; individualID: DHJPAR0010356; individualCount: 1; sex: female; lifeStage: adult; preparations: pinned; catalogNumber: DHJPAR0010356; occurrenceDetails: http://janzen.sas.upenn.edu; recordedBy: D.H. Janzen, W. Hallwachs \& Minor Carmona; otherCatalogNumbers: ASTAS187-06, 06SRNP-42821, BOLD:AAA1929; identifiedBy: AJ Fleming; dateldentified: 2017; language: en; institutionCode: CNC; collectionCode: Insects; basisOfRecord: Pinned Specimen

h. ScientificName: Hyphantrophaga nigricauda; phylum: Arthropoda; class: Insecta; order: Diptera; family: Tachinidae; genus: Hyphantrophaga; specificEpithet: nigricauda; scientificNameAuthorship: Fleming \& Wood, 2017; continent: Central America; country: Costa Rica; countryCode: CR; stateProvince: Alajuela; county: Sector Rincon Rain Forest; locality: Area de Conservacion Guanacaste; verbatimLocality: Quebrada Escondida; verbatimElevation: 420; verbatimLatitude: 10.8993; verbatimLongitude: -85.2749; verbatimCoordinateSystem: Decimal; decimalLatitude: 10.8993; decimalLongitude: -85.2749; samplingProtocol: Reared from the larva of the Geometridae, Cyclomia disparilis; verbatimEventDate: 19-Aug-2001; individualID: DHJPAR0011461; individualCount: 1; sex: male; lifeStage: adult; preparations: pinned; catalog Number: DHJPAR0011461; occurrenceDetails: http://janzen.sas.upenn.edu; recordedBy: D.H. Janzen, W. Hallwachs \& Jose Perez; otherCatalogNumbers: ASTAQ848-06, 01-SRNP-5433, BOLD:AAA1929; identifiedBy: AJ Fleming; dateldentified: 2017; language: en; institutionCode: CNC; collectionCode: Insects; basisOfRecord: Pinned Specimen

i. scientificName: Hyphantrophaga nigricauda; phylum: Arthropoda; class: Insecta; order: Diptera; family: Tachinidae; genus: Hyphantrophaga; specificEpithet: nigricauda; scientificNameAuthorship: Fleming \& Wood, 2017; continent: Central America; country: Costa Rica; countryCode: CR; stateProvince: Alajuela; county: Sector Rincon Rain Forest; locality: Area de Conservacion Guanacaste; verbatimLocality: Jacobo; verbatimElevation: 461; verbatimLatitude: 10.9408; verbatimLongitude: -85.3177; verbatimCoordinateSystem: Decimal; decimalLatitude: 10.9408; decimalLongitude: 
-85.3177; samplingProtocol: Reared from the larva of the Geometridae, Cyclomia disparilis; verbatimEventDate: 07-Mar-2011; individualID: DHJPAR0042303; individualCount: 1; sex: male; lifeStage: adult; preparations: pinned; catalogNumber: DHJPAR0042303; occurrenceDetails: http://janzen.sas.upenn.edu; recordedBy: D.H. Janzen, W. Hallwachs \& Edwin Apu; otherCatalogNumbers: ASHYH067-11, 11SRNP-69487, BOLD:AAA1929; identifiedBy: AJ Fleming; dateldentified: 2017; language: en; institutionCode: CNC; collectionCode: Insects; basisOfRecord: Pinned Specimen

j. ScientificName: Hyphantrophaga nigricauda; phylum: Arthropoda; class: Insecta; order: Diptera; family: Tachinidae; genus: Hyphantrophaga; specificEpithet: nigricauda; scientificNameAuthorship: Fleming \& Wood, 2017; continent: Central America; country: Costa Rica; countryCode: CR; stateProvince: Guanacaste; county: Sector Pitilla; locality: Area de Conservacion Guanacaste; verbatimLocality: Pasmompa; verbatimElevation: 440; verbatimLatitude: 11.0193; verbatimLongitude: -85.41; verbatimCoordinateSystem: Decimal; decimalLatitude: 11.0193; decimalLongitude: -85.41; samplingProtocol: Reared from the larva of the Geometridae, Cyclomia disparilis; verbatimEventDate: 17-Jul-2005; individualID: DHJPAR0007294; individualCount: 1; sex: female; lifeStage: adult; preparations: pinned; catalogNumber: DHJPAR0007294; occurrenceDetails: http://janzen.sas.upenn.edu; recordedBy: D.H. Janzen, W. Hallwachs \& Calixto Moraga; otherCatalogNumbers: ASTAT066-06, 05SRNP-32262, BOLD:AAA1929; identifiedBy: AJ Fleming; dateldentified: 2017; language: en; institutionCode: CNC; collectionCode: Insects; basisOfRecord: Pinned Specimen

k. ScientificName: Hyphantrophaga nigricauda; phylum: Arthropoda; class: Insecta; order: Diptera; family: Tachinidae; genus: Hyphantrophaga; specificEpithet: nigricauda; scientificNameAuthorship: Fleming \& Wood, 2017; continent: Central America; country: Costa Rica; countryCode: CR; stateProvince: Guanacaste; county: Sector Del Oro; locality: Area de Conservacion Guanacaste; verbatimLocality: Monte Cristo; verbatimElevation: 525; verbatimLatitude: 11.0137; verbatimLongitude: -85.4253; verbatimCoordinateSystem: Decimal; decimalLatitude: 11.0137; decimalLongitude: -85.4253; sampling Protocol: Reared from the larva of the Geometridae, Cyclomia disparilis; verbatimEventDate: 17-Jul-2008; individualID: DHJPAR0027944; individualCount: 1; sex: female; lifeStage: adult; preparations: pinned; catalogNumber: DHJPAR0027944; occurrenceDetails: http://janzen.sas.upenn.edu; recordedBy: D.H. Janzen, W. Hallwachs \& Roster Moraga; otherCatalogNumbers: ASHYE181-08, 08SRNP-21716, BOLD:AAA1929; identifiedBy: AJ Fleming; dateldentified: 2017; language: en; institutionCode: CNC; collectionCode: Insects; basisOfRecord: Pinned Specimen

I. scientificName: Hyphantrophaga nigricauda; phylum: Arthropoda; class: Insecta; order: Diptera; family: Tachinidae; genus: Hyphantrophaga; specificEpithet: nigricauda; scientificNameAuthorship: Fleming \& Wood, 2017; continent: Central America; country: Costa Rica; countryCode: CR; stateProvince: Alajuela; county: Sector Rincon Rain Forest; locality: Area de Conservacion Guanacaste; verbatimLocality: Jacobo; verbatimElevation: 461; verbatimLatitude: 10.9408; verbatimLongitude: -85.3177; verbatimCoordinateSystem: Decimal; decimalLatitude: 10.9408; decimalLongitude: -85.3177; samplingProtocol: Reared from the larva of the Geometridae, Cyclomia disparilis; verbatimEventDate: 13-Jul-2009; individualID: DHJPAR0035815; individualCount: 1; sex: male; lifeStage: adult; preparations: pinned; catalogNumber: DHJPAR0035815; occurrenceDetails: http://janzen.sas.upenn.edu; recordedBy: D.H. Janzen, W. Hallwachs \& Noe Castillo; otherCatalogNumbers: ASHYD1196-09, 09- 
SRNP-69335, BOLD:AAA1929; identifiedBy: AJ Fleming; dateldentified: 2017; language: en; institutionCode: CNC; collectionCode: Insects; basisOfRecord: Pinned Specimen

m. ScientificName: Hyphantrophaga nigricauda; phylum: Arthropoda; class: Insecta; order: Diptera; family: Tachinidae; genus: Hyphantrophaga; specificEpithet: nigricauda; scientificNameAuthorship: Fleming \& Wood, 2017; continent: Central America; country: Costa Rica; countryCode: CR; stateProvince: Guanacaste; county: Sector Pitilla; locality: Area de Conservacion Guanacaste; verbatimLocality: Pasmompa; verbatimElevation: 440; verbatimLatitude: 11.0193; verbatimLongitude: -85.41; verbatimCoordinateSystem: Decimal; decimalLatitude: 11.0193; decimalLongitude: -85.41; samplingProtocol: Reared from the larva of the Geometridae, Cyclomia disparilis; verbatimEventDate: 14-Jul-2005; individualID: DHJPAR0007295; individualCount: 1; sex: female; lifeStage: adult; preparations: pinned; catalogNumber: DHJPAR0007295; occurrenceDetails: http://janzen.sas.upenn.edu; recordedBy: D.H. Janzen, W. Hallwachs \& Calixto Moraga; otherCatalogNumbers: ASTAT067-06, 05SRNP-32276, BOLD:AAA1929; identifiedBy: AJ Fleming; dateldentified: 2017; language: en; institutionCode: CNC; collectionCode: Insects; basisOfRecord: Pinned Specimen

n. scientificName: Hyphantrophaga nigricauda; phylum: Arthropoda; class: Insecta; order: Diptera; family: Tachinidae; genus: Hyphantrophaga; specificEpithet: nigricauda; scientificNameAuthorship: Fleming \& Wood, 2017; continent: Central America; country: Costa Rica; countryCode: CR; stateProvince: Guanacaste; county: Sector Pitilla; locality: Area de Conservacion Guanacaste; verbatimLocality: Sendero Laguna; verbatimElevation: 680; verbatimLatitude: 10.9888; verbatimLongitude: -85.4234; verbatimCoordinateSystem: Decimal; decimalLatitude: 10.9888; decimalLongitude: -85.4234; samplingProtocol: Reared from the larva of the Crambidae, Eulepte Solis15; verbatimEventDate: 19-May-2011; individualID: DHJPAR0042702; individualCount: 1; sex: male; lifeStage: adult; preparations: pinned; catalogNumber: DHJPAR0042702; occurrenceDetails: http://janzen.sas.upenn.edu; recordedBy: D.H. Janzen, W. Hallwachs \& Freddy Quesada; otherCatalogNumbers: ASHYH460-11, 11SRNP-31063, BOLD:AAA1929; identifiedBy: AJ Fleming; dateldentified: 2017; language: en; institutionCode: CNC; collectionCode: Insects; basisOfRecord: Pinned Specimen

o. scientificName: Hyphantrophaga nigricauda; phylum: Arthropoda; class: Insecta; order: Diptera; family: Tachinidae; genus: Hyphantrophaga; specificEpithet: nigricauda; scientificNameAuthorship: Fleming \& Wood, 2017; continent: Central America; country: Costa Rica; countryCode: CR; stateProvince: Guanacaste; county: Sector Pitilla; Iocality: Area de Conservacion Guanacaste; verbatimLocality: Sendero Mismo; verbatimElevation: 680; verbatimLatitude: 10.9876; verbatimLongitude: -85.4197; verbatimCoordinateSystem: Decimal; decimalLatitude: 10.9876; decimalLongitude: -85.4197; samplingProtocol: Reared from the larva of the Thyrididae, Dysodia Janzen10; verbatimEventDate: 24-Sep-2009; individualID: DHJPAR0036606; individualCount: 1; sex: male; lifeStage: adult; preparations: pinned; catalogNumber: DHJPAR0036606; occurrenceDetails: http://janzen.sas.upenn.edu; recordedBy: D.H. Janzen, W. Hallwachs \& Calixto Moraga; otherCatalogNumbers: ASHYE1517-09, 09SRNP-30852, BOLD:AAA1929; identifiedBy: AJ Fleming; dateldentified: 2017; language: en; institutionCode: CNC; collectionCode: Insects; basisOfRecord: Pinned Specimen

p. scientificName: Hyphantrophaga nigricauda; phylum: Arthropoda; class: Insecta; order: Diptera; family: Tachinidae; genus: Hyphantrophaga; specificEpithet: nigricauda; 
scientificNameAuthorship: Fleming \& Wood, 2017; continent: Central America; country: Costa Rica; countryCode: CR; stateProvince: Guanacaste; county: Sector Pitilla; locality: Area de Conservacion Guanacaste; verbatimLocality: Sendero Laguna; verbatimElevation: 680; verbatimLatitude: 10.9888; verbatimLongitude: -85.4234; verbatimCoordinateSystem: Decimal; decimalLatitude: 10.9888; decimalLongitude: -85.4234; samplingProtocol: Reared from the larva of the Crambidae, Eulepte Solis15; verbatimEventDate: 16-May-2011; individualID: DHJPAR0042558; individualCount: 1; sex: female; lifeStage: adult; preparations: pinned; catalogNumber: DHJPAR0042558; occurrenceDetails: http://janzen.sas.upenn.edu; recordedBy: D.H. Janzen, W. Hallwachs \& Freddy Quesada; otherCatalogNumbers: ASHYH316-11, 11SRNP-31057, BOLD:AAA1929; identifiedBy: AJ Fleming; dateldentified: 2017; language: en; institutionCode: CNC; collectionCode: Insects; basisOfRecord: Pinned Specimen

q. $\quad$ scientificName: Hyphantrophaga nigricauda; phylum: Arthropoda; class: Insecta; order: Diptera; family: Tachinidae; genus: Hyphantrophaga; specificEpithet: nigricauda; scientificNameAuthorship: Fleming \& Wood, 2017; continent: Central America; country: Costa Rica; countryCode: CR; stateProvince: Guanacaste; county: Sector Del Oro; locality: Area de Conservacion Guanacaste; verbatimLocality: Monte Cristo; verbatimElevation: 525; verbatimLatitude: 11.0137; verbatimLongitude: -85.4253; verbatimCoordinateSystem: Decimal; decimalLatitude: 11.0137; decimalLongitude: -85.4253; samplingProtocol: Reared from the larva of the Geometridae, Cyclomia disparilis; verbatimEventDate: 01-Jul-2005; individualID: DHJPAR0007289; individualCount: 1; sex: male; lifeStage: adult; preparations: pinned; catalog Number: DHJPAR0007289; occurrenceDetails: http://janzen.sas.upenn.edu; recordedBy: D.H. Janzen, W. Hallwachs \& Lucia Rios; otherCatalogNumbers: ASTAT061-06, 05SRNP-22093, BOLD:AAA1929; identifiedBy: AJ Fleming; dateldentified: 2017; language: en; institutionCode: CNC; collectionCode: Insects; basisOfRecord: Pinned Specimen

r. ScientificName: Hyphantrophaga nigricauda; phylum: Arthropoda; class: Insecta; order: Diptera; family: Tachinidae; genus: Hyphantrophaga; specificEpithet: nigricauda; scientificNameAuthorship: Fleming \& Wood, 2017; continent: Central America; country: Costa Rica; countryCode: CR; stateProvince: Guanacaste; county: Sector Pitilla; locality: Area de Conservacion Guanacaste; verbatimLocality: Sendero Laguna; verbatimElevation: 680; verbatimLatitude: 10.9888; verbatimLongitude: -85.4234; verbatimCoordinateSystem: Decimal; decimalLatitude: 10.9888; decimalLongitude: -85.4234; samplingProtocol: Reared from the larva of the Crambidae, Eulepte Solis15; verbatimEventDate: 18-May-2011; individualID: DHJPAR0045600; individualCount: 1; sex: male; lifeStage: adult; preparations: pinned; catalogNumber: DHJPAR0045600; occurrenceDetails: http://janzen.sas.upenn.edu; recordedBy: D.H. Janzen, W. Hallwachs \& Freddy Quesada; otherCatalogNumbers: ACGAZ789-11, 11SRNP-31069, BOLD:AAA1929; identifiedBy: AJ Fleming; dateldentified: 2017; language: en; institutionCode: CNC; collectionCode: Insects; basisOfRecord: Pinned Specimen

s. ScientificName: Hyphantrophaga nigricauda; phylum: Arthropoda; class: Insecta; order: Diptera; family: Tachinidae; genus: Hyphantrophaga; specificEpithet: nigricauda; scientificNameAuthorship: Fleming \& Wood, 2017; continent: Central America; country: Costa Rica; countryCode: CR; stateProvince: Guanacaste; county: Sector Pitilla; locality: Area de Conservacion Guanacaste; verbatimLocality: Pasmompa; verbatimElevation: 440; verbatimLatitude: 11.0193; verbatimLongitude: -85.41; verbatimCoordinateSystem: Decimal; decimalLatitude: 11.0193; decimalLongitude: 
-85.41; samplingProtocol: Reared from the larva of the Geometridae, Cyclomia disparilis; verbatimEventDate: 16-Jul-2005; individualID: DHJPAR0007292; individualCount: 1; sex: female; lifeStage: adult; preparations: pinned; catalogNumber: DHJPAR0007292; occurrenceDetails: http://janzen.sas.upenn.edu; recordedBy: D.H. Janzen, W. Hallwachs \& Calixto Moraga; otherCatalogNumbers: ASTAT064-06, 05SRNP-32272, BOLD:AAA1929; identifiedBy: AJ Fleming; dateldentified: 2017; language: en; institutionCode: CNC; collectionCode: Insects; basisOfRecord: Pinned Specimen

t. ScientificName: Hyphantrophaga nigricauda; phylum: Arthropoda; class: Insecta; order: Diptera; family: Tachinidae; genus: Hyphantrophaga; specificEpithet: nigricauda; scientificNameAuthorship: Fleming \& Wood, 2017; continent: Central America; country: Costa Rica; countryCode: CR; stateProvince: Guanacaste; county: Sector Del Oro; locality: Area de Conservacion Guanacaste; verbatimLocality: Puente Mena; verbatimElevation: 280; verbatimLatitude: 11.0456; verbatimLongitude: -85.4574; verbatimCoordinateSystem: Decimal; decimalLatitude: 11.0456; decimalLongitude: -85.4574; samplingProtocol: Reared from the larva of the Geometridae, Cyclomia disparilis; verbatimEventDate: 23-Jul-2009; individualID: DHJPAR0037607; individualCount: 1; sex: male; lifeStage: adult; preparations: pinned; catalogNumber: DHJPAR0037607; occurrenceDetails: http://janzen.sas.upenn.edu; recordedBy: D.H. Janzen, W. Hallwachs \& Elieth Cantillano; otherCatalogNumbers: ASHYC4352-10, 09SRNP-21421, BOLD:AAA1929; identifiedBy: AJ Fleming; dateldentified: 2017; language: en; institutionCode: CNC; collectionCode: Insects; basisOfRecord: Pinned Specimen

u. $\quad$ scientificName: Hyphantrophaga nigricauda; phylum: Arthropoda; class: Insecta; order: Diptera; family: Tachinidae; genus: Hyphantrophaga; specificEpithet: nigricauda; scientificNameAuthorship: Fleming \& Wood, 2017; continent: Central America; country: Costa Rica; countryCode: CR; stateProvince: Guanacaste; county: Sector Del Oro; locality: Area de Conservacion Guanacaste; verbatimLocality: Monte Cristo; verbatimElevation: 525; verbatimLatitude: 11.0137; verbatimLongitude: -85.4253; verbatimCoordinateSystem: Decimal; decimalLatitude: 11.0137; decimalLongitude: -85.4253; samplingProtocol: Reared from the larva of the Geometridae, Cyclomia disparilis; verbatimEventDate: 28-Jun-2005; individualID: DHJPAR0007313; individualCount: 1; sex: male; lifeStage: adult; preparations: pinned; catalogNumber: DHJPAR0007313; occurrenceDetails: http://janzen.sas.upenn.edu; recordedBy: D.H. Janzen, W. Hallwachs \& Elieth Cantillano; otherCatalog Numbers: ASTAT085-06, 05SRNP-22072, BOLD:AAA1929; identifiedBy: AJ Fleming; dateldentified: 2017; language: en; institutionCode: CNC; collectionCode: Insects; basisOfRecord: Pinned Specimen

\section{Description}

Male (Fig. 22). Length: 4-7 mm. Head (Fig. 22b): vertex 1/5 of head width; two reclinate upper orbital setae; ocellar setae arising beside anterior ocellus; ocellar triangle gold, concolorous with fronto-orbital plate; fronto-orbital plate gold over $90 \%$ of surface, sparsely setulose, setulae not extending below lowest frontal seta; parafacial shiny silver and bare; facial ridge bare; pedicel black, concolorous with postpedicel; arista black, very minutely pubescent, distinctly thickened on basal $1 / 3-1 / 4$; palpus yellow and haired. Thorax (Fig. 22a, c): gold tomentose dorsally, grey tomentose laterally; four prominent dorsal vittae, outermost two broken across suture, innermost 
pair unbroken across suture, reaching 3rd postsutural dorsocentral seta; postpronotum with 3-4 setae arranged in a triangle; chaetotaxy: acrostichal setae 3:3; dorsocentral setae 3:4; intra-alar setae 2:3; supra-alar setae 2:3; three katepisternal setae, basal seta extremely weak and anterior to suture; lateral scutellar setae subequal in length to apical setae, curving inwards medially; lateral scutellar setae subequal in length to basal setae; subapical setae strongest and longest of marginal scutellar setae, these not strongly divergent; apical scutellar setae short and relatively weak, erect and crossed midway; one pair of discal scutellar setae; scutellum with a darkened crescent across basal 30\%, remainder concolorous with scutum. Legs (Fig. 22c): black throughout; hind coxa setose. Wing (Fig. 22a): pale grey translucent, slightly infuscate, veins dark amber brown; vein $\mathrm{R}_{4+5}$ with two setulae at base. Abdomen (Fig. 22a, c): ground colour black; mid-dorsal depression on ST1+2 reaching hind margin; abdominal tomentum present as gold bands spanning anterior 50\% of T3-T4; T5 gold tomentose with black apex; median marginal setae present on ST1+2-T3; a complete row of marginal setae present on T4; discal setae present on T3-T5; sex patch absent. Terminalia (Fig. 22d, e, f): anterior margin of sternite 5 (Fig. 22f) with a slight curved medial depression, posterior edge with a deeply excavated median cleft, smoothly Ushaped; posterior lobes of sternite rounded apically and completely devoid of setae; apical plate shorter than posterior lobes. Unsclerotised "window" anterior to median cleft with central rectangular gap as wide as median cleft, branched on either side, with two thin arms reaching around base of posterior lobes. Cerci (Fig. 22d) in posterior view subrectangular and slightly shorter than surstyli, blunt and squared off towards apex, completely separate medially, diverging at tips; in lateral view (Fig. 22e) with a strong, almost 90 degree bend $1 / 3$ along their length apically; densely setulose almost to apex dorsally, setulose ventrally along basal 2/3 (visible in lateral view). Surstylus in lateral view almost parallel-sided along its length, ending in a slightly downcurved apex, making the structure appear blade-like; surstylus appearing fused with epandrium; when viewed dorsally, surstyli appearing strongly divergent and wide open. Pregonite broad and well-developed, bent basally, apical 2/3 cinched, giving it a petiolate appearance, apically rounded, devoid of setulae. Postgonite elongate, slender, very slightly hooked at tip, subequal in length to pregonite. Distiphallus cone-shaped, apically flared, with a slender median longitudinal sclerotised reinforcement on its posterior surface and a broad, anterolateral, sclerotised acrophallus on each side, joining the plate of opposite side on anterior surface near apex; approximately two times as long as basiphallus.

Female. Length: 5-7 mm. As male, differing only by the presence of two pairs of proclinate orbital setae.

\section{Diagnosis}

Hyphantrophaga nigricauda sp. n. can be distinguished from all other Hyphantrophaga species by the following combination of traits: pedicel and arista concolorous, dark brown/black, four postsutural dorsocentral setae, four thoracic vittae, inner pair solid 
across suture, hind coxa setose, discal setae present on T3-T5 and T5 gold tomentose with a black apex.

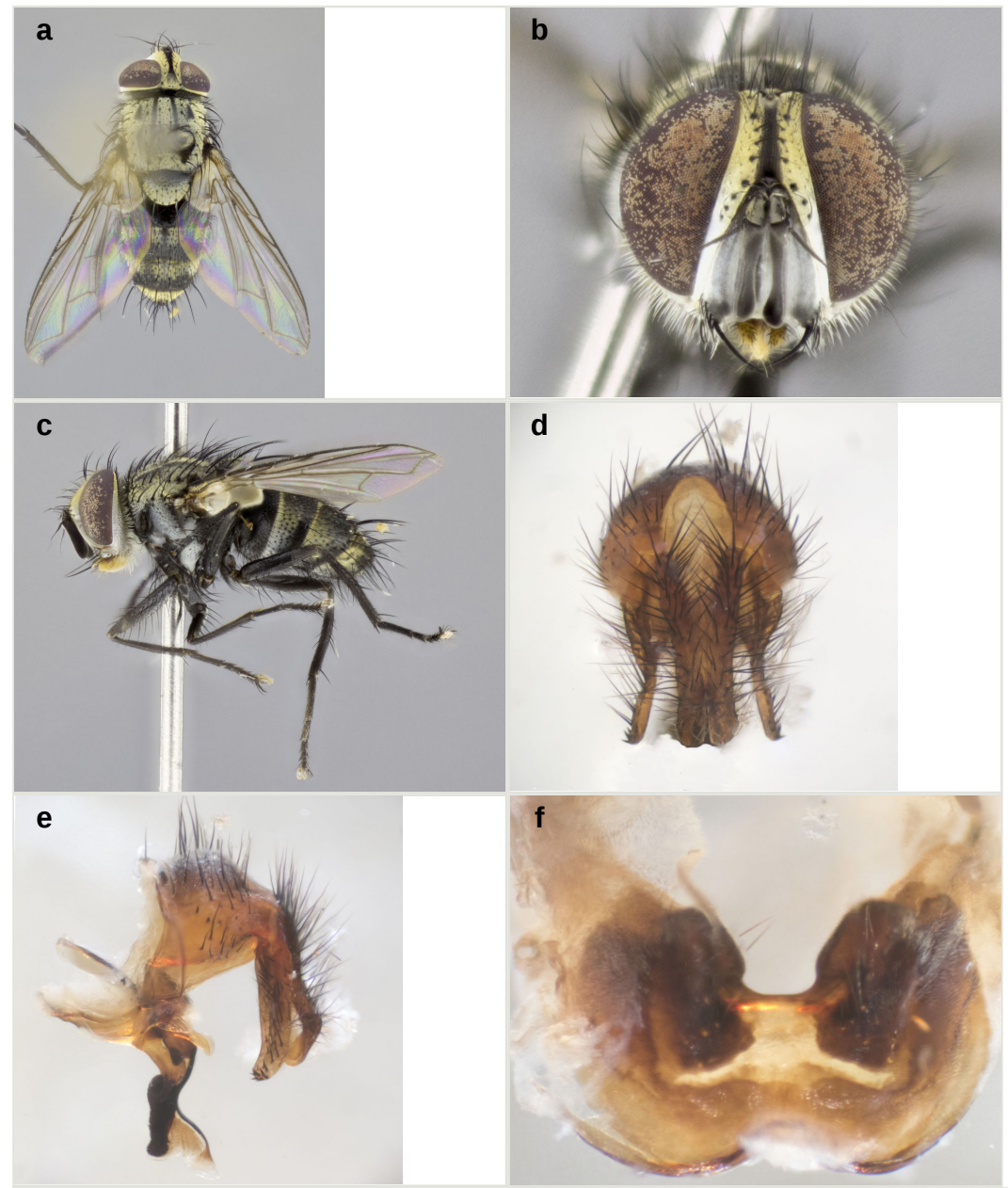

Figure 22.

Hyphantrophaga nigricauda sp. $\mathbf{n}$.; a-c: habitus; holotype male, voucher $\mathrm{n}$. DHJPAR0027908; d-f: terminalia; male paratype, voucher n. DHJPAR0042693.
a: dorsal view doi
b: frontal view doi
c: lateral view doi
d: dorsal view doi
e: lateral view doi
f: sternite 5 , ventral view doi 


\section{Etymology}

From the Latin adjective "nigrum" meaning black and the Latin noun "cauda" meaning tail, in reference to the apically black $\mathrm{T5}$.

\section{Distribution}

Costa Rica, ACG, Alajuela and Guanacaste Provinces, 280-680 m elevation.

\section{Ecology}

Hyphantrophaga nigricauda sp. $\mathbf{n}$. has been reared 22 times from five species of Lepidoptera in the families Geometridae, (Cyclomia disparilis Schaus, 1911; Crambidae: Pleuroptya Solis02, Eulepte Solis15 and Conchylodes platinalis (Guenée, 1854)); and Thyrididae, (Dysodia Janzen10); in rain forest and dry-rain lowland intergrade.

\section{Hyphantrophaga osvaldoespinozai Fleming \& Wood, sp. n.}

- ZooBank urn:Isid:zoobank.org:act:E78E0490-A791-42D4-8874-1D62E818C94E

\section{Materials}

Holotype:

a. $\quad$ scientificName: Hyphantrophaga osvaldoespinozai; phylum: Arthropoda; class: Insecta; order: Diptera; family: Tachinidae; genus: Hyphantrophaga; specificEpithet: osvaldoespinozai; scientificNameAuthorship: Fleming \& Wood, 2017; continent: Central America; country: Costa Rica; countryCode: CR; stateProvince: Guanacaste; county: Camino Bahia Hachal; locality: Area de Conservacion Guanacaste; verbatimLocality: Camino Bahia Hachal; verbatimElevation: 5; verbatimLatitude: 10.758; verbatimLongitude: -85.6086; verbatimCoordinateSystem: Decimal; decimalLatitude: 10.758; decimalLongitude: -85.6086; samplingProtocol: Reared from the larva of the Noctuidae, Diastema morata; verbatimEventDate: 26-Jul-2006; individualID: DHJPAR0010389; individualCount: 1; sex: male; lifeStage: adult; preparations: pinned; catalogNumber: DHJPAR0010389; occurrenceDetails: http:// janzen.sas.upenn.edu; recordedBy: D.H. Janzen, W. Hallwachs \& Eilyn Camacho; otherCatalogNumbers: ASTAS220-06, 06-SRNP-16192, BOLD:AAD2740; identifiedBy: AJ Fleming; dateldentified: 2017; language: en; institutionCode: CNC; collectionCode: Insects; basisOfRecord: Pinned Specimen

\section{Paratypes:}

a. scientificName: Hyphantrophaga osvaldoespinozai; phylum: Arthropoda; class: Insecta; order: Diptera; family: Tachinidae; genus: Hyphantrophaga; specificEpithet: osvaldoespinozai; scientificNameAuthorship: Fleming \& Wood, 2017; continent: Central America; country: Costa Rica; countryCode: CR; stateProvince: Guanacaste; county: Presa; locality: Area de Conservacion Guanacaste; verbatimLocality: Presa; verbatimElevation: 180; verbatimLatitude: 10.758; verbatimLongitude: -85.6086; verbatimCoordinateSystem: Decimal; decimalLatitude: 10.758 ; decimalLongitude: -85.6086; samplingProtocol: Reared from the larva of the Noctuidae, Diastema tigris; verbatimEventDate: 15-Dec-1997; individualID: DHJPAR0007478; individualCount: 1; 
sex: female; lifeStage: adult; preparations: pinned; catalogNumber: DHJPAR0007478; occurrenceDetails: http://janzen.sas.upenn.edu; recordedBy: D.H. Janzen, W. Hallwachs \& gusaneros; otherCatalog Numbers: ASTAT250-06, 97-SRNP-10178, BOLD:AAD2740; identifiedBy: AJ Fleming; dateldentified: 2017; language: en; institutionCode: CNC; collectionCode: Insects; basisOfRecord: Pinned Specimen

b. scientificName: Hyphantrophaga osvaldoespinozai; phylum: Arthropoda; class: Insecta; order: Diptera; family: Tachinidae; genus: Hyphantrophaga; specificEpithet: osvaldoespinozai; scientificNameAuthorship: Fleming \& Wood, 2017; continent: Central America; country: Costa Rica; countryCode: CR; stateProvince: Guanacaste; county: Bejuco; locality: Area de Conservacion Guanacaste; verbatimLocality: Bejuco;

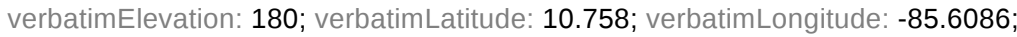
verbatimCoordinateSystem: Decimal; decimalLatitude: 10.758; decimalLongitude: -85.6086; samplingProtocol: Reared from the larva of the Noctuidae, Diastema tigris; verbatimEventDate: 20-Nov-1997; individualID: DHJPAR0007476; individualCount: 1; sex: female; lifeStage: adult; preparations: pinned; catalogNumber: DHJPAR0007476; occurrenceDetails: http://janzen.sas.upenn.edu; recordedBy: D.H. Janzen, W. Hallwachs \& gusaneros; otherCatalog Numbers: ASTAT248-06, 97-SRNP-9721, BOLD:AAD2740; identifiedBy: AJ Fleming; dateldentified: 2017; language: en; institutionCode: CNC; collectionCode: Insects; basisOfRecord: Pinned Specimen

c. scientificName: Hyphantrophaga osvaldoespinozai; phylum: Arthropoda; class: Insecta; order: Diptera; family: Tachinidae; genus: Hyphantrophaga; specificEpithet: osvaldoespinozai; scientificNameAuthorship: Fleming \& Wood, 2017; continent: Central America; country: Costa Rica; countryCode: CR; stateProvince: Guanacaste; county: Estacion Junquillal; locality: Area de Conservacion Guanacaste; verbatimLocality: Estacion Junquillal; verbatimElevation: 5; verbatimLatitude: 10.758; verbatimLongitude: -85.6086; verbatimCoordinateSystem: Decimal; decimalLatitude: 10.758; decimalLongitude: -85.6086; samplingProtocol: Reared from the larva of the Noctuidae, Diastema morata; verbatimEventDate: 07-Jul-2006; individualID:

DHJPAR0010244; individualCount: 1; sex: male; lifeStage: adult; preparations: pinned; catalogNumber: DHJPAR0010244; occurrenceDetails: http://janzen.sas.upenn.edu; recordedBy: D.H. Janzen, W. Hallwachs \& Dionis Rivera; otherCatalogNumbers: ASTAS075-06, 06-SRNP-16266, BOLD:AAD2740; identifiedBy: AJ Fleming; dateldentified: 2017; language: en; institutionCode: CNC; collectionCode: Insects; basisOfRecord: Pinned Specimen

d. scientificName: Hyphantrophaga osvaldoespinozai; phylum: Arthropoda; class: Insecta; order: Diptera; family: Tachinidae; genus: Hyphantrophaga; specificEpithet: osvaldoespinozai; scientificNameAuthorship: Fleming \& Wood, 2017; continent: Central America; country: Costa Rica; countryCode: CR; stateProvince: Guanacaste; county: Finca Jenny; locality: Area de Conservacion Guanacaste; verbatimLocality: Finca Jenny; verbatimElevation: 205; verbatimLatitude: 10.758; verbatimLongitude: -85.6086; verbatimCoordinateSystem: Decimal; decimalLatitude: 10.758; decimalLongitude: -85.6086; samplingProtocol: Reared from the larva of the Noctuidae, Diastema tigris; verbatimEventDate: 06-Nov-2004; individualID: DHJPAR0007479; individualCount: 1; sex: female; lifeStage: adult; preparations: pinned; catalogNumber: DHJPAR0007479; occurrenceDetails: http:// janzen.sas.upenn.edu; recordedBy: D.H. Janzen, W. Hallwachs \& Roberto Espinosa; otherCatalogNumbers: ASTAT251-06, 04-SRNP-14794, BOLD:AAD2740; identifiedBy: AJ Fleming; dateldentified: 2017; language: en; institutionCode: CNC; collectionCode: Insects; basisOfRecord: Pinned Specimen 
e. ScientificName: Hyphantrophaga osvaldoespinozai; phylum: Arthropoda; class: Insecta; order: Diptera; family: Tachinidae; genus: Hyphantrophaga; specificEpithet: osvaldoespinozai; scientificNameAuthorship: Fleming \& Wood, 2017; continent: Central America; country: Costa Rica; countryCode: CR; stateProvince: Guanacaste; county: Camino Bahia Hachal; locality: Area de Conservacion Guanacaste; verbatimLocality: Camino Bahia Hachal; verbatimElevation: 5; verbatimLatitude: 10.758; verbatimLongitude: -85.6086; verbatimCoordinateSystem: Decimal; decimalLatitude: 10.758; decimalLongitude: -85.6086; samplingProtocol: Reared from the larva of the Noctuidae, Diastema morata; verbatimEventDate: 12-Jul-2006; individualID: DHJPAR0010306; individualCount: 1; sex: male; lifeStage: adult; preparations: pinned; catalogNumber: DHJPAR0010306; occurrenceDetails: http:// janzen.sas.upenn.edu; recordedBy: D.H. Janzen, W. Hallwachs \& Guillermo Pereira; otherCatalogNumbers: ASTAS137-06, 06-SRNP-16246, BOLD:AAD2740; identifiedBy: AJ Fleming; dateldentified: 2017; language: en; institutionCode: CNC; collectionCode: Insects; basisOfRecord: Pinned Specimen

f. ScientificName: Hyphantrophaga osvaldoespinozai; phylum: Arthropoda; class: Insecta; order: Diptera; family: Tachinidae; genus: Hyphantrophaga; specific Epithet: osvaldoespinozai; scientificNameAuthorship: Fleming \& Wood, 2017; continent: Central America; country: Costa Rica; countryCode: CR; stateProvince: Guanacaste; county: Bejuco; locality: Area de Conservacion Guanacaste; verbatimLocality: Bejuco; verbatimElevation: 180; verbatimLatitude: 10.758; verbatimLongitude: -85.6086; verbatimCoordinateSystem: Decimal; decimalLatitude: 10.758; decimalLongitude: -85.6086; samplingProtocol: Reared from the larva of the Noctuidae, Diastema tigris; verbatimEventDate: 14-Nov-1997; individualID: DHJPAR0007477; individualCount: 1; sex: female; lifeStage: adult; preparations: pinned; catalogNumber: DHJPAR0007477; occurrenceDetails: http://janzen.sas.upenn.edu; recordedBy: D.H. Janzen, W. Hallwachs \& gusaneros; otherCatalogNumbers: ASTAT249-06, 97-SRNP-9719, BOLD:AAD2740; identifiedBy: AJ Fleming; dateldentified: 2017; language: en; institutionCode: CNC; collectionCode: Insects; basisOfRecord: Pinned Specimen

g. scientificName: Hyphantrophaga osvaldoespinozai; phylum: Arthropoda; class: Insecta; order: Diptera; family: Tachinidae; genus: Hyphantrophaga; specificEpithet: osvaldoespinozai; scientificNameAuthorship: Fleming \& Wood, 2017; continent: Central America; country: Costa Rica; countryCode: CR; stateProvince: Guanacaste; county: Tanquetas; locality: Area de Conservacion Guanacaste; verbatimLocality: Tanquetas; verbatimElevation: 295 ; verbatimLatitude: 10.758 ; verbatimLongitude: -85.6086; verbatimCoordinateSystem: Decimal; decimalLatitude: 10.758; decimalLongitude: -85.6086; samplingProtocol: Reared from the larva of the Noctuidae, Diastema tigris; verbatimEventDate: 07-Aug-1994; individualID: DHJPAR0022959; individualCount: 1; sex: female; lifeStage: adult; preparations: pinned; catalogNumber: DHJPAR0022959; occurrenceDetails: http:// janzen.sas.upenn.edu; recordedBy: D.H. Janzen, W. Hallwachs \& gusaneros; otherCatalog Numbers: ASTAW123-08, 94-SRNP-3920, BOLD:AAD2740; identifiedBy: AJ Fleming; dateldentified: 2017; language: en; institutionCode: CNC; collectionCode: Insects; basisOfRecord: Pinned Specimen

h. ScientificName: Hyphantrophaga osvaldoespinozai; phylum: Arthropoda; class: Insecta; order: Diptera; family: Tachinidae; genus: Hyphantrophaga; specificEpithet: osvaldoespinozai; scientificNameAuthorship: Fleming \& Wood, 2017; continent: Central America; country: Costa Rica; countryCode: CR; stateProvince: Guanacaste; county: Bosque San Emilio; locality: Area de Conservacion Guanacaste; verbatimLocality: Bosque San Emilio; verbatimElevation: 300; verbatimLatitude: 
10.758; verbatimLongitude: -85.6086; verbatimCoordinateSystem: Decimal; decimalLatitude: 10.758; decimalLongitude: -85.6086; samplingProtocol: Reared from the larva of the Noctuidae, Diastema tigris; verbatimEventDate: 10-Nov-1987; individualID: DHJPAR0007274; individualCount: 1; sex: female; lifeStage: adult; preparations: pinned; catalogNumber: DHJPAR0007274; occurrenceDetails: http:// janzen.sas.upenn.edu; recordedBy: D.H. Janzen, W. Hallwachs \& gusaneros; otherCatalogNumbers: ASTAT046-06, 87-SRNP-654,; identifiedBy: AJ Fleming; dateldentified: 2017; language: en; institutionCode: CNC; collectionCode: Insects; basisOfRecord: Pinned Specimen

\section{Description}

Male (Fig. 23). Length: 6-7 mm. Head (Fig. 23b): vertex 1/5 of head width; one pair of reclinate upper orbital setae; ocellar setae arising behind anterior ocellus; ocellar triangle silver (slight gold tinge present but overall concolorous with fronto-orbital plate); fronto-orbital plate shiny silver and sparsely setulose, setulae not extending below lowest frontal seta; parafacial silver and bare; facial ridge bare; eye densely haired; pedicel brownish-black with some orange, concolorous with postpedicel; arista brown, very minutely pubescent, distinctly thickened on basal 1/3-1/4; palpus yellow, slender and haired apically. Thorax (Fig. 23a, c): dull brassy-grey tomentose dorsally, grey tomentose laterally; densely covered in black setulae along anterior and lateral surfaces, dorsally with dense dark setulae interspersed amongst macrosetae; four prominent dorsal vittae, outermost two broken across suture, innermost pair unbroken across suture, extending to 2nd postsutural dorsocentral seta; postpronotum with three setae arranged in a triangle; chaetotaxy: acrostichal setae 3:3; dorsocentral setae 3:4; intra-alar setae 2:3; supra-alar setae 2:3; 2-3 katepisternal setae, basal seta, when present, extremely weak and anterior to suture; lateral scutellar setae less than $1 / 2$ as long as subapical setae, curving inwards medially, as long as subapical setae; apical scutellar setae subequal in length to basal scutellar setae crossed apically; one pair of discal scutellar setae more widely set than apical setae; scutellum concolorous with scutum. Legs (Fig. 23c): dark reddish-brown to black in ground colour; fore femur with dense silver tomentum on posterodorsal surface; hind coxa setose. Wing (Fig. 23a, c): pale translucent, hyaline, not distinctly infuscate; vein $R_{4+5}$ with only $2-3$ setulae at base. Abdomen (Fig. 23a, c): ground colour black; mid-dorsal depression on ST1+2 reaching hind margin; median marginal setae on ST1+2 weak to absent, present on T3; a complete row of marginal setae present on T4; discal setae present on T3-T5, although these can at times appear weak, they are still distinct from abdominal setulae; sex patch absent; distinct brassy-grey tomentum covering all but posterior margin of T3; T3-T4 with darkened patches dorsolaterally, these more concolorous with ST1+2; T5 with brassy-grey tomentum throughout. Terminalia (Fig. 23d, e, f): sternite 5 (Fig. 23f) with a deeply excavated median cleft, smoothly U-shaped, with two smaller lobes inside median cleft. Lateral lobes of sternite pointed apically, with 3-4 strong setae surrounded by many shorter, weaker setulae. Anterior plate of sternite 5 from subequal to slightly shorter than apical lobes, unsclerotised window narrow and rectangular, $2 \mathrm{X}$ as wide as median cleft. Cerci in posterior view (Fig. 23d) subrectangular and slightly shorter than surstyli, blunt and squared at apex, completely separate medially, slightly 
divergent apically; in lateral view with a strong downward curve in apical $1 / 3$, slightly lobed at tip; densely setulose along basal 2/3 dorsally, setulose ventrally along entire length (visible in lateral view). Surstylus in lateral view (Fig. 23e) almost parallel sided along its length, ending in a slightly downcurved apex, making the structure appear blade-like; surstylus slightly fused with epandrium; when viewed dorsally, surstyli appearing to point outwards, not strongly convergent. Pregonite short, not welldeveloped, 0.3 times as long as distiphallus, bare and squared. Postgonite slightly narrow, 1/3 as wide as pregonite, sharply pointed and curved at apex. Distiphallus sailshaped, apically flared, with a slender median longitudinal sclerotised reinforcement on its posterior surface and a broad, anterolateral, sclerotised acrophallus on each side, joining the plate of opposite side on anterior surface near apex.

Female. Length: 6-8 mm. As male, differing only by the presence of two pairs of proclinate orbital setae.

\section{Diagnosis}

Hyphantrophaga osvaldoespinozai sp. n. can be distinguished from all other Hyphantrophaga species by the following combination of traits: pedicel concolorous with postpedicel, three katepisternal setae, hind coxa setose, tomentum on T5 matching rest of abdominal tomentum, median marginal setae absent from ST1+2 and discal setae present on T3-T5 (can be weak but distinct).

\section{Etymology}

Hyphantrophaga osvaldoespinozai sp. $\mathbf{n}$. is named in recognition of Osvaldo Espinoza Obando's dedication and work in finding and rearing the ACG caterpillars that contained tachinid larvae.

\section{Distribution}

Costa Rica, ACG, Guanacaste Province, 5-300 m elevation.

\section{Ecology}

Hyphantrophaga osvaldoespinozai sp. $\mathbf{n}$. has been reared nine times from two species of Lepidoptera in the family Noctuidae, Diastema tigris Guenée, 1852 and Diastema morata Schaus, 1894, in dry forest. 


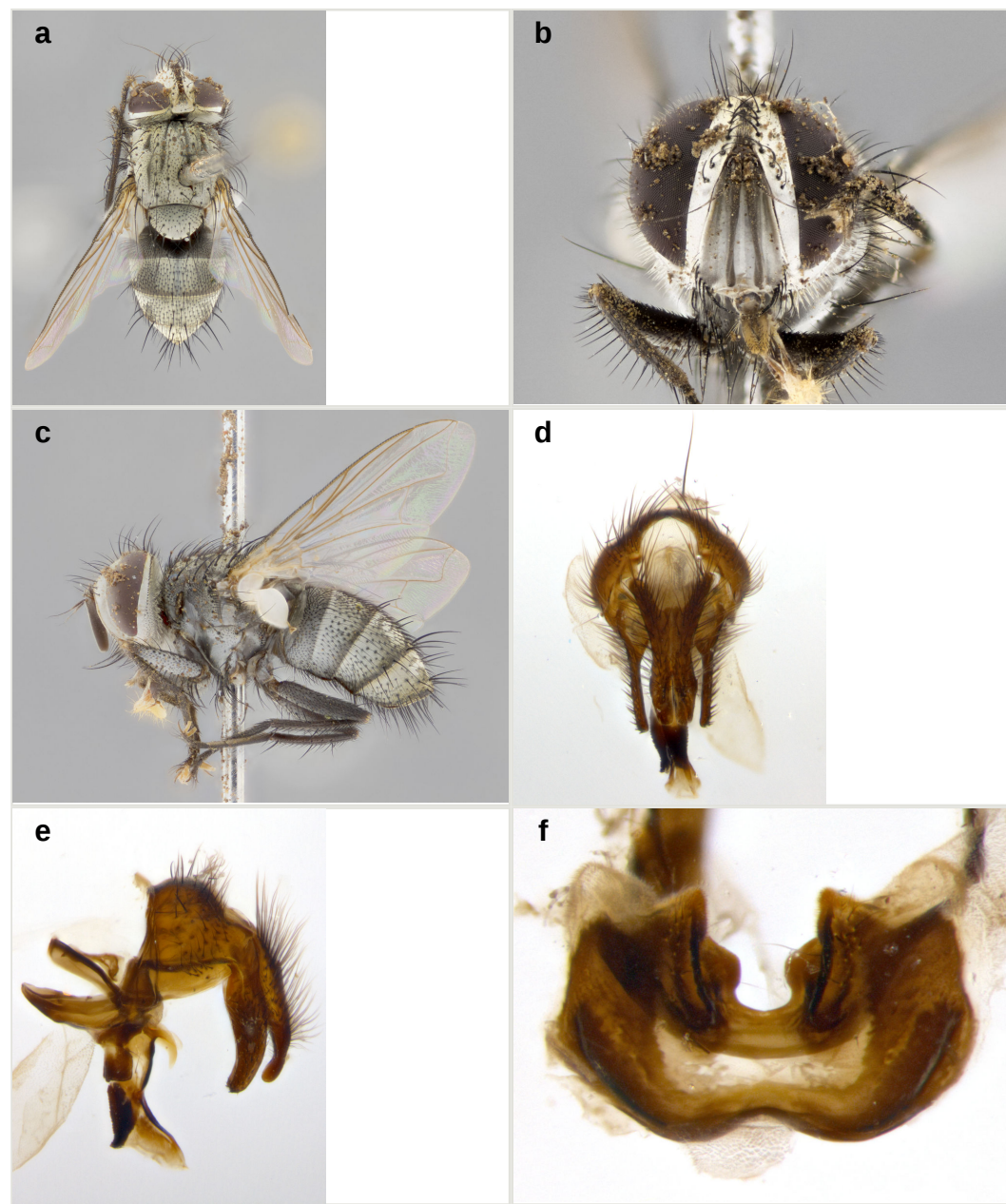

Figure 23.

Hyphantrophaga osvaldoespinozai sp. n.; a-c: habitus; holotype male, voucher $\mathrm{n}$. DHJPAR0010389; d-f: terminalia; male paratype, voucher n. DHJPAR0010306.

a: dorsal view doi

b: frontal view doi

c: lateral view doi

d: dorsal view doi

e: lateral view doi

f: sternite 5 , ventral view doi 


\section{Hyphantrophaga pabloumanai Fleming \& Wood, sp. n.}

- ZooBank urn:Isid:Zoobank.org:act:95D2B9E9-61E4-41E8-B8DD-A7052C5E741B

\section{Material}

Holotype:

a. $\quad$ scientificName: Hyphantrophaga pabloumanai; phylum: Arthropoda; class: Insecta; order: Diptera; family: Tachinidae; genus: Hyphantrophaga; specificEpithet: pabloumanai; scientificNameAuthorship: Fleming \& Wood, 2017; continent: Central America; country: Costa Rica; countryCode: CR; stateProvince: Guanacaste; county: Sector Santa Rosa; locality: Area de Conservacion Guanacaste; verbatimLocality: Area Administrativa; verbatimElevation: 295; verbatimLatitude: 10.8376; verbatimLongitude: -85.6187 ; verbatimCoordinateSystem: Decimal; decimalLatitude: 10.8376; decimalLongitude: -85.6187; samplingProtocol: Reared from the larva of the Apatelodidae, Colabata Iybia; verbatimEventDate: 05-Oct-2003; individualID: DHJPAR0007490; individualCount: 1; sex: male; lifeStage: adult; preparations: pinned; catalogNumber: DHJPAR0007490; occurrenceDetails: http://janzen.sas.upenn.edu; recordedBy: D.H. Janzen, W. Hallwachs \& Ruth Franco; otherCatalog Numbers: ASTAT262-06, 03-SRNP-14280,; identifiedBy: AJ Fleming; dateldentified: 2017; language: en; institutionCode: CNC; collectionCode: Insects; basisOfRecord: Pinned Specimen

\section{Description}

Male (Fig. 24). Length: 6-7 mm. Head (Fig. 24b): vertex 1/4 of head width; two pairs of reclinate upper orbital setae; ocellar setae arising beside to slightly behind anterior ocellus; ocellar triangle pale brassy-silver (slight gold tinge present but overall concolorous with fronto-orbital plate); fronto-orbital plate pale brassy-silver over $75 \%$, changing to brilliant silver adjacent to parafacial, densely setulose, setulae not extending below lowest frontal seta; parafacial silver and bare; facial ridge bare; eye densely haired; pedicel orange, lighter than dark brown/black postpedicel; arista brown, very minutely pubescent, distinctly thickened on basal 1/3-1/4; palpus yellow/orange, slender and haired apically. Thorax (Fig. 24a, c): dull brassy-grey tomentose dorsally, grey tomentose laterally; densely covered in black setulae along anterior and lateral surfaces, dorsally with dense dark setulae interspersed amongst setae; four prominent dorsal vittae, outermost two broken across suture, innermost pair unbroken across suture, extending to 2 nd postsutural dorsocentral seta; postpronotum with 4-5 setae arranged in a triangle; chaetotaxy: acrostichal setae 3:3; dorsocentral setae 3:4; intraalar setae 2:3; supra-alar setae 2:3; three katepisternal setae, basal seta weak and anterior to suture; lateral scutellar setae less than $1 / 2$ as long as subapical setae; basal scutellar setae curving inwards medially, as long as subapical setae; apical scutellar setae short, crossed apically; one pair of discal scutellar setae more widely set than apical setae; scutellum concolorous with scutum. Legs (Fig. 24c): dark reddish-brown to black in ground colour; fore femur with dense silver tomentum on posterodorsal surface; hind coxa bare. Wing (Fig. 24a, c): pale translucent, hyaline, not distinctly infuscate; vein $\mathrm{R}_{4+5}$ with only two setulae at base. Abdomen (Fig. 24a, c): ground colour reddish-brown; mid-dorsal depression on ST1+2 reaching hind margin; median 
marginal setae present on ST1+2-T3; a complete row of marginal setae present on T4; discal setae only T5; sex patch present on T4-T5; distinct tomentose bands along anterior 2/3 of T3 and T4, broken medially by a dorsocentral stripe; T5 with silver tomentum over its entire dorsal surface; T3-T4 with darkened patches dorsolaterally, these more concolorous with ST1+2. Terminalia: not examined.

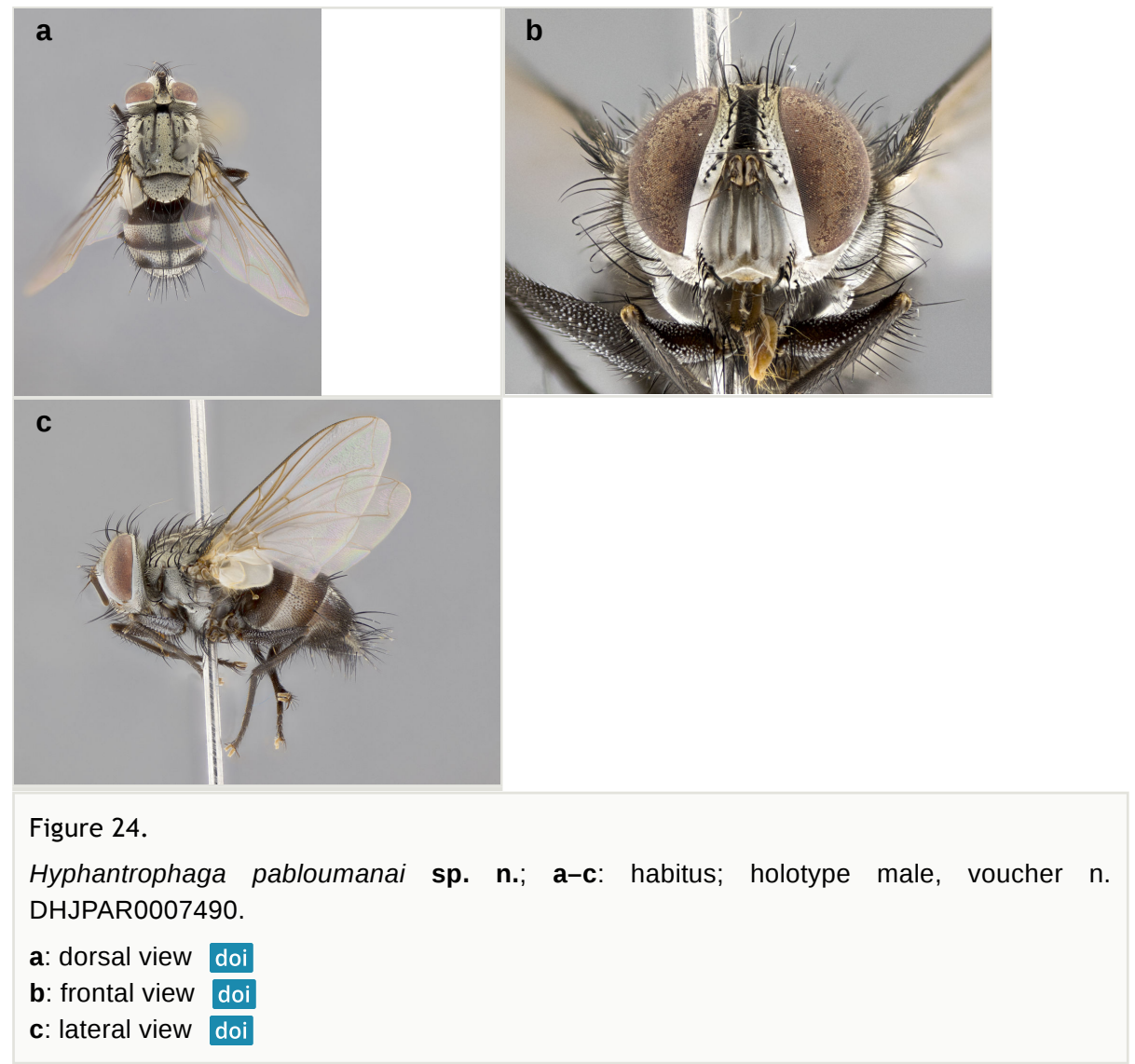

Female. Not known at this time.

\section{Diagnosis}

Hyphantrophaga pabloumanai sp. n. can be distinguished from all other Hyphantrophaga species by the following combination of traits: fronto-orbital plate silver tomentose, pedicel orange, four postsutural dorsocentral setae, legs reddishblack, hind coxa bare, median marginal setae present on ST1+2, discal setae only on T5. 


\section{Etymology}

Hyphantrophaga pabloumanai sp. $\mathbf{n}$. is named in recognition of Pablo José Umaña Calderon's dedication and work in finding and rearing the ACG caterpillars that contained tachinid larvae.

\section{Distribution}

Costa Rica, ACG, Guanacaste province, 295 m elevation.

\section{Ecology}

Hyphantrophaga pabloumanai sp. $\mathbf{n}$. has been reared once from one species of Lepidoptera in the family Apatelodidae, Colabata lybia Druce, 1898, in dry forest.

\section{Hyphantrophaga similis Fleming \& Wood, sp. $\mathrm{n}$.}

- ZooBank urn:Isid:zoobank.org:act:535A05E1-BDB4-4D26-A724-36B865E3ADDE

\section{Materials}

\section{Holotype:}

a. scientificName: Hyphantrophaga similis; phylum: Arthropoda; class: Insecta; order: Diptera; family: Tachinidae; genus: Hyphantrophaga; specificEpithet: similis; scientificNameAuthorship: Fleming \& Wood, 2017; continent: Central America; country: Costa Rica; countryCode: CR; stateProvince: Alajuela; county: Sector Rincon Rain Forest; locality: Area de Conservacion Guanacaste; verbatimLocality: Sendero Anonas; verbatimElevation: 405; verbatimLatitude: 10.9053; verbatimLongitude: -85.2788; verbatimCoordinateSystem: Decimal; decimalLatitude: 10.9053; decimalLongitude: -85.2788; samplingProtocol: Reared from the larva of the Erebidae, Letis buteoDHJ02; verbatimEventDate: 23-Aug-2003; individualID: DHJPAR0007325; individualCount: 1; sex: male; lifeStage: adult; preparations: pinned; catalogNumber: DHJPAR0007325; occurrenceDetails: http://janzen.sas.upenn.edu; recordedBy: D.H. Janzen, W. Hallwachs \& Jose Perez; otherCatalogNumbers: ASTAT097-06, 03SRNP-11568, BOLD:AAE1435; identifiedBy: AJ Fleming; dateldentified: 2017; language: en; institutionCode: CNC; collectionCode: Insects; basisOfRecord: Pinned Specimen

\section{Paratypes:}

a. scientificName: Hyphantrophaga similis; phylum: Arthropoda; class: Insecta; order: Diptera; family: Tachinidae; genus: Hyphantrophaga; specificEpithet: similis; scientificNameAuthorship: Fleming \& Wood, 2017; continent: Central America; country: Costa Rica; countryCode: CR; stateProvince: Alajuela; county: Sector Rincon Rain Forest; locality: Area de Conservacion Guanacaste; verbatimLocality: Sendero Rincon; verbatimElevation: 430; verbatimLatitude: 10.8962; verbatimLongitude: -85.2777; decimalLatitude: 10.8962; decimalLongitude: -85.2777; samplingProtocol: Reared from the larva of the Erebidae, Letis buteoDHJ01; verbatimEventDate: 28-Nov-2000; individualID: DHJPAR0007327; individualCount: 1; sex: female; lifeStage: adult; preparations: pinned; catalogNumber: DHJPAR0007327; occurrenceDetails: http:// janzen.sas.upenn.edu; recordedBy: D.H. Janzen, W. Hallwachs \& Freyci Vargas; 
otherCatalogNumbers: ASTAT099-06, 00-SRNP-20556, BOLD:AAE1435; identifiedBy: M. Wood; dateldentified: 2017; language: en; institutionCode: CNC; collectionCode: Insects; basisOfRecord: Pinned Specimen

b. ScientificName: Hyphantrophaga similis; phylum: Arthropoda; class: Insecta; order: Diptera; family: Tachinidae; genus: Hyphantrophaga; specificEpithet: similis; scientificNameAuthorship: Fleming \& Wood, 2017; continent: Central America; country: Costa Rica; countryCode: CR; stateProvince: Alajuela; county: Sector Rincon Rain Forest; locality: Area de Conservacion Guanacaste; verbatimLocality: Camino Rio Francia; verbatimElevation: 410; verbatimLatitude: 10.9043; verbatimLongitude: -85.2865; verbatimCoordinateSystem: Decimal; decimalLatitude: 10.9043; decimalLongitude: -85.2865; samplingProtocol: Reared from the larva of the Erebidae, Letis buteoDHJ01; verbatimEventDate: 11-Dec-2000; individualID: DHJPAR0007328; individualCount: 1; sex: male; lifeStage: adult; preparations: pinned; catalogNumber: DHJPAR0007328; occurrenceDetails: http://janzen.sas.upenn.edu; recordedBy: D.H. Janzen, W. Hallwachs \& Freyci Vargas; otherCatalogNumbers: ASTAT100-06, 00SRNP-20740, BOLD:AAE1435; identifiedBy: AJ Fleming; dateldentified: 2017; language: en; institutionCode: CNC; collectionCode: Insects; basisOfRecord: Pinned Specimen

c. ScientificName: Hyphantrophaga similis; phylum: Arthropoda; class: Insecta; order: Diptera; family: Tachinidae; genus: Hyphantrophaga; specificEpithet: similis; scientificNameAuthorship: Fleming \& Wood, 2017; continent: Central America; country: Costa Rica; countryCode: CR; stateProvince: Guanacaste; county: Sector Del Oro; Iocality: Area de Conservacion Guanacaste; verbatimLocality: Margarita; verbatimElevation: 380; verbatimLatitude: 11.0323; verbatimLongitude: -85.4395; verbatimCoordinateSystem: Decimal; decimalLatitude: 11.0323; decimalLongitude: -85.4395; samplingProtocol: Reared from the larva of the Erebidae, Letis mycerina; verbatimEventDate: 28-Jul-2005; individualID: DHJPAR0007329; individualCount: 1; sex: male; lifeStage: adult; preparations: pinned; catalogNumber: DHJPAR0007329; occurrenceDetails: http://janzen.sas.upenn.edu; recordedBy: D.H. Janzen, W. Hallwachs \& Elieth Cantillano; otherCatalogNumbers: ASTAT101-06, 05-SRNP-22366, BOLD:AAE1435; identifiedBy: AJ Fleming; dateldentified: 2017; language: en; institutionCode: CNC; collectionCode: Insects; basisOfRecord: Pinned Specimen

d. scientificName: Hyphantrophaga similis; phylum: Arthropoda; class: Insecta; order: Diptera; family: Tachinidae; genus: Hyphantrophaga; specificEpithet: similis; scientificNameAuthorship: Fleming \& Wood, 2017; continent: Central America; country: Costa Rica; countryCode: CR; stateProvince: Alajuela; county: Brasilia; locality: Area de Conservacion Guanacaste; verbatimLocality: Gallinazo; verbatimElevation: 360; verbatimLatitude: 11.0183; verbatimLongitude: -85.372; verbatimCoordinateSystem: Decimal; decimalLatitude: 11.0183; decimalLongitude: -85.372; samplingProtocol: Reared from the larva of the Erebidae, Letis buteoDHJ01; verbatimEventDate: 25Sep-2011; individualID: DHJPAR0045663; individualCount: 1; sex: male; lifeStage: adult; preparations: pinned; catalogNumber: DHJPAR0045663; occurrenceDetails: htt p://janzen.sas.upenn.edu; recordedBy: D.H. Janzen, W. Hallwachs \& Duvalier Briceno; otherCatalogNumbers: ACGAZ852-11, 11-SRNP-65874, BOLD:AAE1435; identifiedBy: AJ Fleming; dateldentified: 2017; language: en; institutionCode: CNC; collectionCode: Insects; basisOfRecord: Pinned Specimen

e. ScientificName: Hyphantrophaga similis; phylum: Arthropoda; class: Insecta; order: Diptera; family: Tachinidae; genus: Hyphantrophaga; specificEpithet: similis; scientificNameAuthorship: Fleming \& Wood, 2017; continent: Central America; country: Costa Rica; countryCode: CR; stateProvince: Alajuela; county: Sector Rincon Rain 


\begin{abstract}
Forest; locality: Area de Conservacion Guanacaste; verbatimLocality: Sendero Rincon; verbatimElevation: 430; verbatimLatitude: 10.8962; verbatimLongitude: -85.2777; verbatimCoordinateSystem: Decimal; decimalLatitude: 10.8962; decimalLongitude: -85.2777; samplingProtocol: Reared from the larva of the Erebidae, Letis buteoDHJ01; verbatimEventDate: 23-Oct-2000; individualID: DHJPAR0007326; individualCount: 1; sex: female; lifeStage: adult; preparations: pinned; catalogNumber: DHJPAR0007326; occurrenceDetails: http://janzen.sas.upenn.edu; recordedBy: D.H. Janzen, W. Hallwachs \& Freyci Vargas; otherCatalogNumbers: ASTAT098-06, 00-SRNP-14698, BOLD:AAE1435; identifiedBy: AJ Fleming; dateldentified: 2017; language: en; institutionCode: CNC; collectionCode: Insects; basisOfRecord: Pinned Specimen
\end{abstract}

\title{
Description
}

Male (Fig. 25). Length: 8-12 mm. Head (Fig. 25b): vertex 1/4 of head width; two reclinate upper orbital setae; ocellar setae arising beside anterior ocellus; ocellar triangle silver, concolorous with fronto-orbital plate; fronto-orbital plate silver with a slight gold tinge over frontal setae, setulose, setulae not extending below lowest frontal seta; frontal setae extending up to base of postpedicel; parafacial silver and bare; facial ridge bare; eye densely haired; pedicel black, concolorous with postpedicel; arista brown, very minutely pubescent, distinctly thickened on basal 1/3-1/4. Thorax (Fig. 25a, c): dull brassy-grey tomentose dorsally, grey tomentose laterally; four prominent dorsal vittae, outermost two broken across suture, innermost pair unbroken across suture, extending to 3rd postsutural dorsocentral seta; postpronotum with five setae arranged in a triangle; chaetotaxy: acrostichal setae 3:3; dorsocentral setae 4:4; intraalar setae 3:3; supra-alar setae 2:3; two katepisternal setae; lateral scutellar setae shorter than subapical setae, curving inwards medially; apical scutellar setae subequal in length to basal scutellar setae crossed apically; one pair of discal scutellar setae; scutellum black over basal 20\%, remainder concolorous with scutum. Legs (Fig. 25c): black in ground colour; fore femur with dense silver tomentum on posterodorsal surface; hind coxa bare. Wing (Fig. 25a): pale translucent, hyaline, not distinctly infuscate; vein $\mathrm{R}_{4+5}$ with only 2-3 setulae at base. Abdomen (Fig. 25a, c): ground colour black; mid-dorsal depression on ST1+2 almost reaching hind margin; median marginal setae present on T3; a complete row of marginal setae present on T4; discal setae only on T5; sex patch covering ventral surfaces of T4-T5 and posterior 1/3 of T3; distinct tomentose bands along anterior edge of T3 and T4, broken medially by a dorsocentral stripe; T5 with silver tomentum covering anterior half. Terminalia (Fig. 25d, e, f): sternite 5 (Fig. 25f) with a deeply excavated median cleft, smoothly Ushaped, margins covered in dense tomentum. Lateral lobes of sternite rounded apically, with 2-3 strong setae surrounded by many shorter, weaker setulae. Anterior plate of sternite 5 from subequal to slightly longer than apical lobes; unsclerotised "window" ovoid to slightly rectangular, as wide as median cleft. Cerci in posterior view (Fig. 25d) triangular and slightly shorter than surstyli, blunt and rounded at apex, completely separate medially, appearing slightly divergent; in lateral view with a strong downward curve in apical 1/3; densely setulose along basal 2/3 dorsally, setulose ventrally along entire length (visible in lateral view). Surstylus in lateral view (Fig. 25e) almost parallel-sided along its length, ending in a slightly downcurved apex, making the 
structure appear blade-like; when viewed dorsally, surstyli appearing to point outwards, not strongly convergent. Pregonite short, not well-developed, $1 / 2$ as long as distiphallus, bare and squared-off apically. Postgonite slightly narrow, 1/3 as wide as pregonite, sharply pointed and curved at apex. Distiphallus vaguely cone-shaped (could be seen as rectangular), only weakly flaring apically with a slender arrowshaped median longitudinal sclerotised reinforcement on its posterior surface and a broad, anterolateral, apically clubbed sclerotised acrophallus on each side, joining the plate of opposite side on anterior surface near apex.

Female. Length: 8-11 mm. As male, differing only by the presence of two pairs of proclinate orbital setae.

\section{Diagnosis}

Hyphantrophaga similis sp. n. can be distinguished from all other Hyphantrophaga species by the following combination of traits: parafacial, fronto-orbital plate and ocellar triangle all silver, frontal setae extending up to base of postpedicel. Differs from its most similar congener $H$. niveifacies by the colour of the wings and the presence of only two katepisternal setae.

\section{Etymology}

From the Latin adjective "similis", meaning similar or resembling, in reference to its similarity to the Brazilian species $H$. niveifacies (Macquart, 1851).

\section{Distribution}

Costa Rica, ACG, Alajuela and Guanacaste Provinces, 320-430 m elevation.

\section{Ecology}

Hyphantrophaga similis sp. $\mathbf{n}$. has been reared seven times from three species of Lepidoptera in the family Erebidae, Letis buteoDHJ01, Letis buteoDHJ02 and Letis mycerina (Cramer, 1777), in rain forest and dry-rain lowland intergrade. 


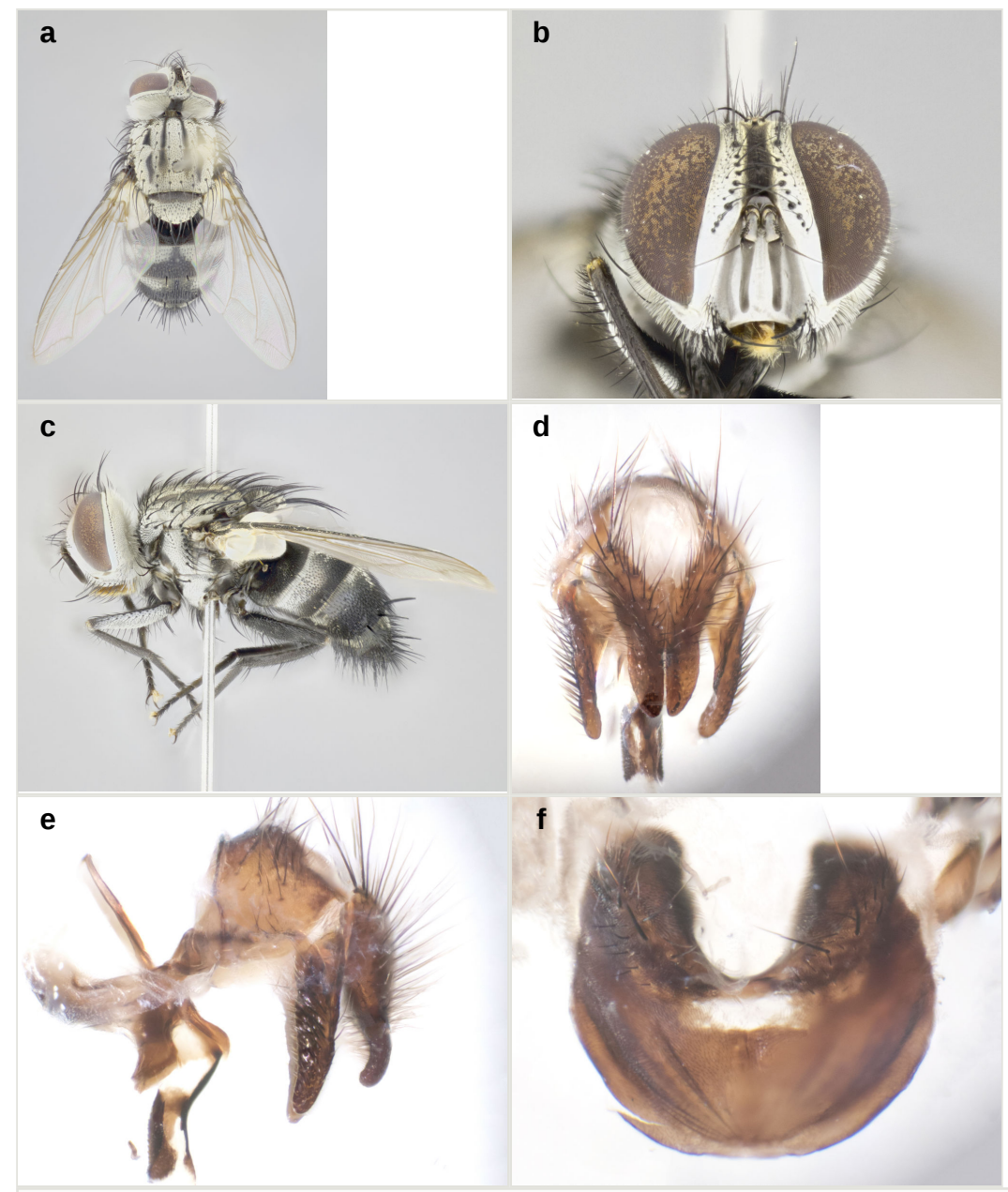

Figure 25.

Hyphantrophaga similis sp. n.; a-c: habitus; holotype male, voucher n. DHJPAR0007325; d-f: terminalia; male paratype, voucher n. 05-SRNP-22366.
a: dorsal view doi
b: frontal view doi
c: lateral view doi
d: dorsal view doi
e: lateral view doi
f: sternite 5 , ventral view doi 


\section{Hyphantrophaga virilis (Aldrich \& Webber, 1924)}

\section{Materials}

a. ScientificName: Hyphantrophaga virilis; phylum: Arthropoda; class: Insecta; order: Diptera; family: Tachinidae; genus: Hyphantrophaga; specificEpithet: virilis; scientificNameAuthorship: (Aldrich \& Webber, 1924); continent: Central America; country: Costa Rica; countryCode: CR; stateProvince: Guanacaste; county: Sector Mundo Nuevo; locality: Area de Conservacion Guanacaste; verbatimLocality: Vado Miramonte; verbatimElevation: 305; verbatimLatitude: 10.7718; verbatimLongitude: -85.434; verbatimCoordinateSystem: Decimal; decimalLatitude: 10.7718; decimalLongitude: -85.434; samplingProtocol: Reared from the larva of the Hesperiidae, Saliana fusta; verbatimEventDate: 02-Jul-2005; individualID: DHJPAR0003295; individualCount: 1; sex: male; lifeStage: adult; preparations: pinned; catalogNumber: DHJPAR0003295; occurrenceDetails: http://janzen.sas.upenn.edu; recordedBy: D.H. Janzen, W. Hallwachs \& Mariano Pereira; otherCatalogNumbers: ASTA202-05, 05-SRNP-56212, BOLD:AAA1577; identifiedBy: AJ Fleming; dateldentified: 2017; language: en; institutionCode: CNC; collectionCode: Insects; basisOfRecord: Pinned Specimen

b. scientificName: Hyphantrophaga virilis; phylum: Arthropoda; class: Insecta; order: Diptera; family: Tachinidae; genus: Hyphantrophaga; specificEpithet: virilis; scientificNameAuthorship: (Aldrich \& Webber, 1924); continent: Central America; country: Costa Rica; countryCode: CR; stateProvince: Guanacaste; county: Sector Del Oro; locality: Area de Conservacion Guanacaste; verbatimLocality: Bosque Aguirre; verbatimElevation: 620; verbatimLatitude: 11.0006; verbatimLongitude: -85.438; verbatimCoordinateSystem: Decimal; decimalLatitude: 11.0006; decimalLongitude: -85.438; samplingProtocol: Reared from the larva of the Erebidae, Antiblemma Poole05; verbatimEventDate: 24-Dec-2004; individualID: DHJPAR0003299; individualCount: 1; sex: male; lifeStage: adult; preparations: pinned; catalogNumber: DHJPAR0003299; occurrenceDetails: http://janzen.sas.upenn.edu; recordedBy: D.H. Janzen, W. Hallwachs \& Roster Moraga; otherCatalogNumbers: ASTA206-05, 04SRNP-26847, BOLD:AAA1577; identifiedBy: AJ Fleming; dateldentified: 2017; language: en; institutionCode: CNC; collectionCode: Insects; basisOfRecord: Pinned Specimen

c. ScientificName: Hyphantrophaga virilis; phylum: Arthropoda; class: Insecta; order: Diptera; family: Tachinidae; genus: Hyphantrophaga; specificEpithet: virilis; scientificNameAuthorship: (Aldrich \& Webber, 1924); continent: Central America; country: Costa Rica; countryCode: CR; stateProvince: Guanacaste; county: Sector Santa Rosa; locality: Area de Conservacion Guanacaste; verbatimLocality: Area Administrativa; verbatimElevation: 295; verbatimLatitude: 10.8376; verbatimLongitude: -85.6187; verbatimCoordinateSystem: Decimal; decimalLatitude: 10.8376; decimalLongitude: -85.6187; samplingProtocol: Reared from the larva of the Megalopygidae, Norape nigrovenosa; verbatimEventDate: 20-Dec-2004; individualID: DHJPAR0003301; individualCount: 1; sex: male; lifeStage: adult; preparations: pinned; catalogNumber: DHJPAR0003301; occurrenceDetails: http://janzen.sas.upenn.edu; recordedBy: D.H. Janzen, W. Hallwachs \& Freddy Quesada; otherCatalogNumbers: ASTA208-05, 04-SRNP-15774, BOLD:AAA1577; identifiedBy: AJ Fleming; dateldentified: 2017; language: en; institutionCode: CNC; collectionCode: Insects; basisOfRecord: Pinned Specimen

d. ScientificName: Hyphantrophaga virilis; phylum: Arthropoda; class: Insecta; order: Diptera; family: Tachinidae; genus: Hyphantrophaga; specificEpithet: virilis; 
scientificNameAuthorship: (Aldrich \& Webber, 1924); continent: Central America; country: Costa Rica; countryCode: CR; stateProvince: Guanacaste; county: Sector Santa Rosa; locality: Area de Conservacion Guanacaste; verbatimLocality: Bosque San Emilio; verbatimElevation: 300; verbatimLatitude: 10.8439; verbatimLongitude: -85.6138; verbatimCoordinateSystem: Decimal; decimalLatitude: 10.8439; decimalLongitude: -85.6138; samplingProtocol: Reared from the larva of the Hesperiidae, Mysoria ambigua; verbatimEventDate: 27-Nov-2004; individualID: DHJPAR0003302; individualCount: 1; sex: male; lifeStage: adult; preparations: pinned; catalogNumber: DHJPAR0003302; occurrenceDetails: http://janzen.sas.upenn.edu; recordedBy: D.H. Janzen, W. Hallwachs \& Noemi Espinosa; otherCatalogNumbers: ASTA209-05, 04-SRNP-15262, BOLD:AAA1577; identifiedBy: AJ Fleming; dateldentified: 2017; language: en; institutionCode: CNC; collectionCode: Insects; basisOfRecord: Pinned Specimen

e. ScientificName: Hyphantrophaga virilis; phylum: Arthropoda; class: Insecta; order: Diptera; family: Tachinidae; genus: Hyphantrophaga; specificEpithet: virilis; scientific NameAuthorship: (Aldrich \& Webber, 1924); continent: Central America; country: Costa Rica; countryCode: CR; stateProvince: Guanacaste; county: Potrerillos; locality: Area de Conservacion Guanacaste; verbatimLocality: Rio Azufrado; verbatimElevation: 95; verbatimLatitude: 10.8122; verbatimLongitude: -85.5444; verbatimCoordinateSystem: Decimal; decimalLatitude: 10.8122; decimalLongitude: -85.5444; samplingProtocol: Reared from the larva of the Notodontidae, Calledema plusia; verbatimEventDate: 07-Dec-2004; individualID: DHJPAR0003303; individualCount: 1; sex: male; lifeStage: adult; preparations: pinned; catalogNumber: DHJPAR0003303; occurrenceDetails: http://janzen.sas.upenn.edu; recordedBy: D.H. Janzen, W. Hallwachs \& Ruth Franco; otherCatalogNumbers: ASTA210-05, 04SRNP-15402, BOLD:AAA1577; identifiedBy: AJ Fleming; dateldentified: 2017; language: en; institutionCode: CNC; collectionCode: Insects; basisOfRecord: Pinned Specimen

f. ScientificName: Hyphantrophaga virilis; phylum: Arthropoda; class: Insecta; order: Diptera; family: Tachinidae; genus: Hyphantrophaga; specificEpithet: virilis; scientificNameAuthorship: (Aldrich \& Webber, 1924); continent: Central America; country: Costa Rica; countryCode: CR; stateProvince: Guanacaste; county: Sector Santa Rosa; locality: Area de Conservacion Guanacaste; verbatimLocality: Bosque Humedo; verbatimElevation: 290; verbatimLatitude: 10.8514; verbatimLongitude: -85.608; verbatimCoordinateSystem: Decimal; decimalLatitude: 10.8514; decimalLongitude: -85.608; samplingProtocol: Reared from the larva of the Hesperiidae, Nascus Burns01; verbatimEventDate: 18-Nov-2004; individualID: DHJPAR0003309; individualCount: 1; sex: male; lifeStage: adult; preparations: pinned; catalogNumber: DHJPAR0003309; occurrenceDetails: http://janzen.sas.upenn.edu; recordedBy: D.H. Janzen, W. Hallwachs \& Ruth Franco; otherCatalogNumbers: ASTA216-05, 04-SRNP-14653, BOLD:AAA1577; identifiedBy: AJ Fleming; dateldentified: 2017; language: en; institutionCode: CNC; collectionCode: Insects; basisOfRecord: Pinned Specimen

g. ScientificName: Hyphantrophaga virilis; phylum: Arthropoda; class: Insecta; order: Diptera; family: Tachinidae; genus: Hyphantrophaga; specificEpithet: virilis; scientificNameAuthorship: (Aldrich \& Webber, 1924); continent: Central America; country: Costa Rica; countryCode: CR; stateProvince: Guanacaste; county: Sector Cacao; locality: Area de Conservacion Guanacaste; verbatimLocality: Sendero Guayabal; verbatimElevation: 500; verbatimLatitude: 10.8857; verbatimLongitude: -85.4818; verbatimCoordinateSystem: Decimal; decimalLatitude: 10.8857; 
decimalLongitude: -85.4818; samplingProtocol: Reared from the larva of the Erebidae, Pachydota saduca; verbatimEventDate: 15-Nov-2004; individualID: DHJPAR0003310; individualCount: 1; sex: male; lifeStage: adult; preparations: pinned; catalogNumber: DHJPAR0003310; occurrenceDetails: http://janzen.sas.upenn.edu; recordedBy: D.H. Janzen, W. Hallwachs \& Harry Ramirez; otherCatalogNumbers: ASTA217-05, 04SRNP-49374, BOLD:AAA1577; identifiedBy: AJ Fleming; dateldentified: 2017; language: en; institutionCode: CNC; collectionCode: Insects; basisOfRecord: Pinned Specimen

h. $\quad$ scientificName: Hyphantrophaga virilis; phylum: Arthropoda; class: Insecta; order: Diptera; family: Tachinidae; genus: Hyphantrophaga; specificEpithet: virilis; scientificNameAuthorship: (Aldrich \& Webber, 1924); continent: Central America; country: Costa Rica; countryCode: CR; stateProvince: Alajuela; county: Sector San Cristobal; locality: Area de Conservacion Guanacaste; verbatimLocality: Puente Palma; verbatimElevation: 460; verbatimLatitude: 10.9163; verbatimLongitude: -85.3787; verbatimCoordinateSystem: Decimal; decimalLatitude: 10.9163; decimalLongitude: -85.3787; samplingProtocol: Reared from the larva of the Hesperiidae, Polyctor polyctor; verbatimEventDate: 03-Jan-2005; individualID: DHJPAR0003315; individualCount: 1; sex: male; lifeStage: adult; preparations: pinned; catalogNumber: DHJPAR0003315; occurrenceDetails: http://janzen.sas.upenn.edu; recordedBy: D.H. Janzen, W. Hallwachs \& Gloria Sihezar; otherCatalogNumbers: ASTA222-05, 04-SRNP-61141, BOLD:AAA1577; identifiedBy: AJ Fleming; dateldentified: 2017; language: en; institutionCode: CNC; collectionCode: Insects; basisOfRecord: Pinned Specimen

i. scientificName: Hyphantrophaga virilis; phylum: Arthropoda; class: Insecta; order: Diptera; family: Tachinidae; genus: Hyphantrophaga; specificEpithet: virilis; scientificNameAuthorship: (Aldrich \& Webber, 1924); continent: Central America; country: Costa Rica; countryCode: CR; stateProvince: Guanacaste; county: Sector Del Oro; locality: Area de Conservacion Guanacaste; verbatimLocality: Bosque Aguirre; verbatimElevation: 620; verbatimLatitude: 11.0006; verbatimLongitude: -85.438; verbatimCoordinateSystem: Decimal; decimalLatitude: 11.0006 ; decimalLongitude: -85.438; samplingProtocol: Reared from the larva of the Crambidae, Syllepte amandoDHJ02; verbatimEventDate: 22-Jan-2005; individualID: DHJPAR0003316; individualCount: 1; sex: male; lifeStage: adult; preparations: pinned; catalogNumber: DHJPAR0003316; occurrenceDetails: http://janzen.sas.upenn.edu; recordedBy: D.H. Janzen, W. Hallwachs \& Roster Moraga; otherCatalog Numbers: ASTA223-05, 04SRNP-26832, BOLD:AAA1577; identifiedBy: AJ Fleming; dateldentified: 2017; language: en; institutionCode: CNC; collectionCode: Insects; basisOfRecord: Pinned Specimen

j. $\quad$ scientificName: Hyphantrophaga virilis; phylum: Arthropoda; class: Insecta; order; Diptera; family: Tachinidae; genus: Hyphantrophaga; specificEpithet: virilis; scientificNameAuthorship: (Aldrich \& Webber, 1924); continent: Central America; country: Costa Rica; countryCode: CR; stateProvince: Guanacaste; county: Sector Mundo Nuevo; locality: Area de Conservacion Guanacaste; verbatimLocality: Vado Agria; verbatimElevation: 560; verbatimLatitude: 10.7588; verbatimLongitude: -85.3754; verbatimCoordinateSystem: Decimal; decimalLatitude: 10.7588; decimalLongitude: -85.3754; samplingProtocol: Reared from the larva of the Notodontidae, Elasmia mandela; verbatimEventDate: 23-Aug-2005; individualID: DHJPAR0003321; individualCount: 1; sex: male; lifeStage: adult; preparations: pinned; catalog Number: DHJPAR0003321; occurrenceDetails: http://janzen.sas.upenn.edu; recordedBy: D.H. Janzen, W. Hallwachs \& Jose Alberto Sanchez; 
otherCatalog Numbers: ASTA228-05, 05-SRNP-58573, BOLD:AAA1577; identifiedBy: AJ Fleming; dateldentified: 2017; language: en; institutionCode: CNC; collectionCode: Insects; basisOfRecord: Pinned Specimen

k. $\quad$ scientificName: Hyphantrophaga virilis; phylum: Arthropoda; class: Insecta; order: Diptera; family: Tachinidae; genus: Hyphantrophaga; specificEpithet: virilis; scientificNameAuthorship: (Aldrich \& Webber, 1924); continent: Central America; country: Costa Rica; countryCode: CR; stateProvince: Guanacaste; county: Sector Mundo Nuevo; locality: Area de Conservacion Guanacaste; verbatimLocality: Mamones; verbatimElevation: 365 ; verbatimLatitude: 10.7707; verbatimLongitude: -85.4287; verbatimCoordinateSystem: Decimal; decimalLatitude: 10.7707; decimalLongitude: -85.4287; samplingProtocol: Reared from the larva of the Notodontidae, Elasmia mandela; verbatimEventDate: 13-Aug-2005; individualID: DHJPAR0003322; individualCount: 1; sex: male; lifeStage: adult; preparations: pinned; catalogNumber: DHJPAR0003322; occurrenceDetails: http://janzen.sas.upenn.edu; recordedBy: D.H. Janzen, W. Hallwachs \& Mariano Pereira; otherCatalogNumbers: ASTA229-05, 05-SRNP-58078, BOLD:AAA1577; identifiedBy: AJ Fleming; dateldentified: 2017; language: en; institutionCode: CNC; collectionCode: Insects; basisOfRecord: Pinned Specimen

I. scientificName: Hyphantrophaga virilis; phylum: Arthropoda; class: Insecta; order: Diptera; family: Tachinidae; genus: Hyphantrophaga; specificEpithet: virilis; scientificNameAuthorship: (Aldrich \& Webber, 1924); continent: Central America; country: Costa Rica; countryCode: CR; stateProvince: Guanacaste; county: Sector Santa Rosa; locality: Area de Conservacion Guanacaste; verbatimLocality: Bosque Humedo; verbatimElevation: 290; verbatimLatitude: 10.8514; verbatimLongitude: -85.608; verbatimCoordinateSystem: Decimal; decimalLatitude: 10.8514; decimalLongitude: -85.608; samplingProtocol: Reared from the larva of the Hesperiidae, Nascus Burns01; verbatimEventDate: 11-Nov-2004; individualID: DHJPAR0003324; individualCount: 1; sex: male; lifeStage: adult; preparations: pinned; catalogNumber: DHJPAR0003324; occurrenceDetails: http://janzen.sas.upenn.edu; recordedBy: D.H. Janzen, W. Hallwachs \& Ruth Franco; otherCatalogNumbers: ASTA231-05, 04-SRNP-14651, BOLD:AAA1577; identifiedBy: AJ Fleming; dateldentified: 2017; language: en; institutionCode: CNC; collectionCode: Insects; basisOfRecord: Pinned Specimen

m. $\quad$ scientificName: Hyphantrophaga virilis; phylum: Arthropoda; class: Insecta; order: Diptera; family: Tachinidae; genus: Hyphantrophaga; specificEpithet: virilis; scientificNameAuthorship: (Aldrich \& Webber, 1924); continent: Central America; country: Costa Rica; countryCode: CR; stateProvince: Guanacaste; county: Sector Cacao; locality: Area de Conservacion Guanacaste; verbatimLocality: Sendero Ponderosa; verbatimElevation: 1060; verbatimLatitude: 10.9146; verbatimLongitude: -85.4626; verbatimCoordinateSystem: Decimal; decimalLatitude: 10.9146; decimalLongitude: -85.4626; samplingProtocol: Reared from the larva of the Erebidae, Pachydota drucei; verbatimEventDate: 06-Mar-2004; individualID: DHJPAR0003330; individualCount: 1; sex: male; lifeStage: adult; preparations: pinned; catalogNumber: DHJPAR0003330; occurrenceDetails: http://janzen.sas.upenn.edu; recordedBy: D.H. Janzen, W. Hallwachs \& Mariano Pereira; otherCatalogNumbers: ASTA237-05, 04SRNP-35033, BOLD:AAA1577; identifiedBy: AJ Fleming; dateldentified: 2017; language: en; institutionCode: CNC; collectionCode: Insects; basisOfRecord: Pinned Specimen

n. ScientificName: Hyphantrophaga virilis; phylum: Arthropoda; class: Insecta; order: Diptera; family: Tachinidae; genus: Hyphantrophaga; specificEpithet: virilis; 
scientificNameAuthorship: (Aldrich \& Webber, 1924); continent: Central America; country: Costa Rica; countryCode: CR; stateProvince: Guanacaste; county: Sector Del Oro; locality: Area de Conservacion Guanacaste; verbatimLocality: Canyon Rio Mena; verbatimElevation: 560; verbatimLatitude: 10.9962; verbatimLongitude: -85.4556; verbatimCoordinateSystem: Decimal; decimalLatitude: 10.9962; decimalLongitude: -85.4556; samplingProtocol: Reared from the larva of the Hesperiidae, Polyctor polyctor; verbatimEventDate: 17-Feb-2004; individualID: DHJPAR0003332; individualCount: 1; sex: male; lifeStage: adult; preparations: pinned; catalogNumber: DHJPAR0003332; occurrenceDetails: http://janzen.sas.upenn.edu; recordedBy: D.H. Janzen, W. Hallwachs \& Lucia Rios; otherCatalogNumbers: ASTA239-05, 04SRNP-20238, BOLD:AAA1577; identifiedBy: AJ Fleming; dateldentified: 2017; language: en; institutionCode: CNC; collectionCode: Insects; basisOfRecord: Pinned Specimen

o. scientificName: Hyphantrophaga virilis; phylum: Arthropoda; class: Insecta; order: Diptera; family: Tachinidae; genus: Hyphantrophaga; specificEpithet: virilis; scientificNameAuthorship: (Aldrich \& Webber, 1924); continent: Central America; country: Costa Rica; countryCode: CR; stateProvince: Guanacaste; county: Sector Cacao; locality: Area de Conservacion Guanacaste; verbatimLocality: Casa Fran; verbatimElevation: 1140; verbatimLatitude: 10.9366; verbatimLongitude: -85.4669; verbatimCoordinateSystem: Decimal; decimalLatitude: 10.9366; decimalLongitude: -85.4669; samplingProtocol: Reared from the larva of the Nymphalidae, Mesotaenia barnesi; verbatimEventDate: 14-May-2003; individualID: DHJPAR0003333; individualCount: 1; sex: male; lifeStage: adult; preparations: pinned; catalogNumber: DHJPAR0003333; occurrenceDetails: http://janzen.sas.upenn.edu; recordedBy: D.H. Janzen, W. Hallwachs \& Mariano Pereira; otherCatalogNumbers: ASTA240-05, 03SRNP-3649, BOLD:AAA1577; identifiedBy: AJ Fleming; dateldentified: 2017; language: en; institutionCode: CNC; collectionCode: Insects; basisOfRecord: Pinned Specimen

p. scientificName: Hyphantrophaga virilis; phylum: Arthropoda; class: Insecta; order: Diptera; family: Tachinidae; genus: Hyphantrophaga; specificEpithet: virilis; scientificNameAuthorship: (Aldrich \& Webber, 1924); continent: Central America; country: Costa Rica; countryCode: CR; stateProvince: Guanacaste; county: Sector Cacao; locality: Area de Conservacion Guanacaste; verbatimLocality: Sendero Maritza; verbatimElevation: 760; verbatimLatitude: 10.9364; verbatimLongitude: -85.4776; verbatimCoordinateSystem: Decimal; decimalLatitude: 10.9364; decimalLongitude: -85.4776; samplingProtocol: Reared from the larva of the Geometridae, geometrid 01-SRNP-6789; verbatimEventDate: 18-Apr-2003; individualID: DHJPAR0003336; individualCount: 1; sex: male; lifeStage: adult; preparations: pinned; catalogNumber: DHJPAR0003336; occurrenceDetails: http:// janzen.sas.upenn.edu; recordedBy: D.H. Janzen, W. Hallwachs \& Freddy Quesada; otherCatalogNumbers: ASTA243-05, 03-SRNP-3459, BOLD:AAA1577; identifiedBy: AJ Fleming; dateldentified: 2017; language: en; institutionCode: CNC; collectionCode: Insects; basisOfRecord: Pinned Specimen

q. ScientificName: Hyphantrophaga virilis; phylum: Arthropoda; class: Insecta; order: Diptera; family: Tachinidae; genus: Hyphantrophaga; specificEpithet: virilis; scientificNameAuthorship: (Aldrich \& Webber, 1924); continent: Central America; country: Costa Rica; countryCode: CR; stateProvince: Guanacaste; county: Sector Cacao; locality: Area de Conservacion Guanacaste; verbatimLocality: Sendero Abajo; verbatimElevation: 1020; verbatimLatitude: 10.9255; verbatimLongitude: -85.4716; verbatimCoordinateSystem: Decimal; decimalLatitude: 10.9255; decimalLongitude: 
-85.4716; samplingProtocol: Reared from the larva of the Pyralidae, Omphalocera cariosa; verbatimEventDate: 01-Aug-2002; individualID: DHJPAR0003339; individualCount: 1; sex: male; lifeStage: adult; preparations: pinned; catalogNumber: DHJPAR0003339; occurrenceDetails: http://janzen.sas.upenn.edu; recordedBy: D.H. Janzen, W. Hallwachs \& Freddy Quesada; otherCatalog Numbers: ASTA246-05, 02SRNP-9335,; identifiedBy: AJ Fleming; dateldentified: 2017; language: en; institutionCode: CNC; collectionCode: Insects; basisOfRecord: Pinned Specimen

r. scientificName: Hyphantrophaga virilis; phylum: Arthropoda; class: Insecta; order: Diptera; family: Tachinidae; genus: Hyphantrophaga; specificEpithet: virilis; scientificNameAuthorship: (Aldrich \& Webber, 1924); country: Costa Rica; countryCode: CR; stateProvince: Guanacaste; county: Sector Santa Rosa; locality: Area de Conservacion Guanacaste; verbatimLocality: Area Administrativa; verbatimElevation: 295; verbatimLatitude: 10.8376; verbatimLongitude: -85.6187; verbatimCoordinateSystem: Decimal; decimalLatitude: 10.8376 ; decimalLongitude: -85.6187; samplingProtocol: Reared from the larva of the Hesperiidae, Mysoria ambigua; verbatimEventDate: 08-Nov-2003; individualID: DHJPAR0005680; individualCount: 1; sex: male; lifeStage: adult; preparations: pinned; catalogNumber: DHJPAR0005680; occurrenceDetails: http://janzen.sas.upenn.edu; recordedBy: D.H. Janzen, W. Hallwachs \& Ruth Franco; otherCatalogNumbers: ASTAI108-06, 03SRNP-25554, BOLD:AAA1577; language: en; institutionCode: CNC; collectionCode: Insects; basisOfRecord: Pinned Specimen

s. ScientificName: Hyphantrophaga virilis; phylum: Arthropoda; class: Insecta; order: Diptera; family: Tachinidae; genus: Hyphantrophaga; specificEpithet: virilis; scientificNameAuthorship: (Aldrich \& Webber, 1924); country: Costa Rica; countryCode: CR; stateProvince: Guanacaste; county: Sector Cacao; locality: Area de Conservacion Guanacaste; verbatimLocality: Cuesta Caimito; verbatimElevation: 640; verbatimLatitude: 10.8908; verbatimLongitude: -85.4719 ; verbatimCoordinateSystem: Decimal; decimalLatitude: 10.8908; decimalLongitude: -85.4719; samplingProtocol: Reared from the larva of the Noctuidae, Dyops cuprescens; verbatimEventDate: 13Sep-2005; individualID: DHJPAR0006292; individualCount: 1; sex: male; lifeStage: adult; preparations: pinned; catalogNumber: DHJPAR0006292; occurrenceDetails: htt p://janzen.sas.upenn.edu; recordedBy: D.H. Janzen, W. Hallwachs \& Manuel Pereira; otherCatalogNumbers: ASTAI720-06, 05-SRNP-47173, BOLD:AAA1577; language: en; institutionCode: CNC; collectionCode: Insects; basisOfRecord: Pinned Specimen

t. ScientificName: Hyphantrophaga virilis; phylum: Arthropoda; class: Insecta; order: Diptera; family: Tachinidae; genus: Hyphantrophaga; specificEpithet: virilis; scientificNameAuthorship: (Aldrich \& Webber, 1924); country: Costa Rica; countryCode: CR; stateProvince: Guanacaste; county: Sector Santa Rosa; locality: Area de Conservacion Guanacaste; verbatimLocality: Cafetal; verbatimElevation: 280; verbatimLatitude: 10.8583; verbatimLongitude: -85.6109; verbatimCoordinateSystem: Decimal; decimalLatitude: 10.8583; decimalLongitude: -85.6109; samplingProtocol: Reared from the larva of the Hesperiidae, Saliana fusta; verbatimEventDate: 22Oct-2003; individualID: DHJPAR0006364; individualCount: 1; sex: male; lifeStage: adult; preparations: pinned; catalogNumber: DHJPAR0006364; occurrenceDetails: htt p://janzen.sas.upenn.edu; recordedBy: D.H. Janzen, W. Hallwachs \& Ruth Franco; otherCatalogNumbers: ASTAI792-06, 03-SRNP-25976, BOLD:AAA1577; language: en; institutionCode: CNC; collectionCode: Insects; basisOfRecord: Pinned Specimen

u. scientificName: Hyphantrophaga virilis; phylum: Arthropoda; class: Insecta; order: Diptera; family: Tachinidae; genus: Hyphantrophaga; specificEpithet: virilis; scientificNameAuthorship: (Aldrich \& Webber, 1924); country: Costa Rica; 
countryCode: CR; stateProvince: Guanacaste; county: Sector Santa Rosa; locality: Area de Conservacion Guanacaste; verbatimLocality: Cafetal; verbatimElevation: 280; verbatimLatitude: 10.8583; verbatimLongitude: -85.6109; verbatimCoordinateSystem: Decimal; decimalLatitude: 10.8583; decimalLongitude: -85.6109; samplingProtocol: Reared from the larva of the Notodontidae, Tachuda plumipesICG02; verbatimEventDate: 29-Nov-2001; individualID: DHJPAR0006383; individualCount: 1; sex: male; lifeStage: adult; preparations: pinned; catalogNumber: DHJPAR0006383; occurrenceDetails: http://janzen.sas.upenn.edu; recordedBy: D.H. Janzen, W. Hallwachs \& Guillermo Pereira; otherCatalog Numbers: ASTAI811-06, 01SRNP-18100, BOLD:AAA1577; language: en; institutionCode: CNC; collectionCode: Insects; basisOfRecord: Pinned Specimen

v. ScientificName: Hyphantrophaga virilis; phylum: Arthropoda; class: Insecta; order: Diptera; family: Tachinidae; genus: Hyphantrophaga; specificEpithet: virilis; scientificNameAuthorship: (Aldrich \& Webber, 1924); country: Costa Rica; countryCode: CR; stateProvince: Guanacaste; county: Sector Mundo Nuevo; locality: Area de Conservacion Guanacaste; verbatimLocality: Estacion La Perla;

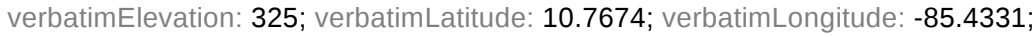
verbatimCoordinateSystem: Decimal; decimalLatitude: 10.7674; decimalLongitude: -85.4331; samplingProtocol: Reared from the larva of the Hesperiidae, Mysoria ambigua; verbatimEventDate: 23-Nov-2005; individualID: DHJPAR0005545; individualCount: 1; sex: male; lifeStage: adult; preparations: pinned; catalogNumber: DHJPAR0005545; occurrenceDetails: http://janzen.sas.upenn.edu; recordedBy: D.H. Janzen, W. Hallwachs \& Mariano Pereira; otherCatalog Numbers: ASTA664-06, 05SRNP-65176, BOLD:AAA1577; language: en; institutionCode: CNC; collectionCode: Insects; basisOfRecord: Pinned Specimen

w. ScientificName: Hyphantrophaga virilis; phylum: Arthropoda; class: Insecta; order: Diptera; family: Tachinidae; genus: Hyphantrophaga; specificEpithet: virilis; scientificNameAuthorship: (Aldrich \& Webber, 1924); country: Costa Rica; countryCode: CR; stateProvince: Guanacaste; county: Sector Santa Rosa; locality: Area de Conservacion Guanacaste; verbatimLocality: Bosque Humedo;

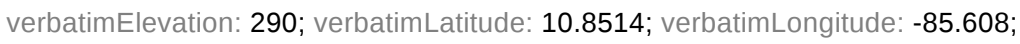
verbatimCoordinateSystem: Decimal; decimalLatitude: 10.8514; decimalLongitude: -85.608; samplingProtocol: Reared from the larva of the Hesperiidae, Gindanes brontinus; verbatimEventDate: 23-Jan-2005; individualID: DHJPAR0006621; individualCount: 1; sex: male; lifeStage: adult; preparations: pinned; catalogNumber: DHJPAR0006621; occurrenceDetails: http://janzen.sas.upenn.edu; recordedBy: D.H. Janzen, W. Hallwachs \& Guillermo Pereira; otherCatalogNumbers: ASTA799-06, 05SRNP-64402, BOLD:AAA1577; language: en; institutionCode: CNC; collectionCode: Insects; basisOfRecord: Pinned Specimen

x. ScientificName: Hyphantrophaga virilis; phylum: Arthropoda; class: Insecta; order: Diptera; family: Tachinidae; genus: Hyphantrophaga; specificEpithet: virilis; scientificNameAuthorship: (Aldrich \& Webber, 1924); country: Costa Rica; countryCode: CR; stateProvince: Guanacaste; county: Sector Mundo Nuevo; locality: Area de Conservacion Guanacaste; verbatimLocality: Vado Miramonte; verbatimElevation: 305; verbatimLatitude: 10.7718; verbatimLongitude: $\mathbf{- 8 5 . 4 3 4}$; verbatimCoordinateSystem: Decimal; decimalLatitude: 10.7718; decimalLongitude: -85.434; samplingProtocol: Reared from the larva of the Depressariidae, Anadasmus Janzen25; verbatimEventDate: 16-Dec-2005; individualID: DHJPAR0006688; individualCount: 1; sex: male; lifeStage: adult; preparations: pinned; catalogNumber: DHJPAR0006688; occurrenceDetails: http://janzen.sas.upenn.edu; recordedBy: D.H. 
Janzen, W. Hallwachs \& Mariano Pereira; otherCatalogNumbers: ASTA866-06, 05SRNP-65604,; language: en; institutionCode: CNC; collectionCode: Insects; basisOfRecord: Pinned Specimen

y. $\quad$ scientificName: Hyphantrophaga virilis; phylum: Arthropoda; class: Insecta; order: Diptera; family: Tachinidae; genus: Hyphantrophaga; specificEpithet: virilis; scientificNameAuthorship: (Aldrich \& Webber, 1924); country: Costa Rica; countryCode: CR; stateProvince: Guanacaste; county: Sector Pitilla; locality: Area de Conservacion Guanacaste; verbatimLocality: Loaiciga; verbatimElevation: 445; verbatimLatitude: 11.0198; verbatimLongitude: -85.4134 ; verbatim CoordinateSystem: Decimal; decimalLatitude: 11.0198; decimalLongitude: -85.4134; samplingProtocol: Reared from the larva of the Hesperiidae, Xenophanes tryxus; verbatimEventDate: 15Mar-2006; individualID: DHJPAR0007064; individualCount: 1; sex: male; lifeStage: adult; preparations: pinned; catalogNumber: DHJPAR0007064; occurrenceDetails: htt p://janzen.sas.upenn.edu; recordedBy: D.H. Janzen, W. Hallwachs \& Manuel Rios; otherCatalogNumbers: ASTAV306-06, 06-SRNP-30587,; language: en; institutionCode: CNC; collectionCode: Insects; basisOfRecord: Pinned Specimen

z. ScientificName: Hyphantrophaga virilis; phylum: Arthropoda; class: Insecta; order: Diptera; family: Tachinidae; genus: Hyphantrophaga; specificEpithet: virilis; scientificNameAuthorship: (Aldrich \& Webber, 1924); country: Costa Rica; countryCode: CR; stateProvince: Guanacaste; county: Sector Mundo Nuevo; locality: Area de Conservacion Guanacaste; verbatimLocality: Mamones; verbatimElevation: 365; verbatimLatitude: 10.7707 ; verbatimLongitude: -85.4287 ; verbatimCoordinateSystem: Decimal; decimalLatitude: 10.7707 ; decimalLongitude: -85.4287; samplingProtocol: Reared from the larva of the Bombycidae, Epia muscosa; verbatimEventDate: 10-Feb-2006; individualID: DHJPAR0007090; individualCount: 1; sex: male; lifeStage: adult; preparations: pinned; catalogNumber: DHJPAR0007090; occurrenceDetails: http://janzen.sas.upenn.edu; recordedBy: D.H. Janzen, W. Hallwachs \& Jose Alberto Sanchez; otherCatalogNumbers: ASTAV332-06, 06SRNP-55067,; language: en; institutionCode: CNC; collectionCode: Insects; basisOfRecord: Pinned Specimen

aa. scientificName: Hyphantrophaga virilis; phylum: Arthropoda; class: Insecta; order: Diptera; family: Tachinidae; genus: Hyphantrophaga; specificEpithet: virilis; scientificNameAuthorship: (Aldrich \& Webber, 1924); country: Costa Rica; countryCode: CR; stateProvince: Guanacaste; county: Sector Mundo Nuevo; locality: Area de Conservacion Guanacaste; verbatimLocality: Mamones; verbatimElevation: 365; verbatimLatitude: 10.7707 ; verbatimLongitude: -85.4287; verbatimCoordinateSystem: Decimal; decimalLatitude: 10.7707 ; decimalLongitude: -85.4287; samplingProtocol: Reared from the larva of the Nymphalidae, Colobura dirce; verbatimEventDate: 10-Feb-2006; individualID: DHJPAR0007095; individualCount: 1; sex: male; lifeStage: adult; preparations: pinned; catalogNumber: DHJPAR0007095; occurrenceDetails: http://janzen.sas.upenn.edu; recordedBy: D.H. Janzen, W. Hallwachs \& Jose Alberto Sanchez; otherCatalogNumbers: ASTAV337-06, 06-SRNP-55244,; language: en; institutionCode: CNC; collectionCode: Insects; basisOfRecord: Pinned Specimen

ab. ScientificName: Hyphantrophaga virilis; phylum: Arthropoda; class: Insecta; order: Diptera; family: Tachinidae; genus: Hyphantrophaga; specificEpithet: virilis; scientificNameAuthorship: (Aldrich \& Webber, 1924); country: Costa Rica; countryCode: CR; stateProvince: Alajuela; county: Sector San Cristobal; locality: Area de Conservacion Guanacaste; verbatimLocality: Rio Blanco Abajo; verbatimElevation: 500; verbatimLatitude: 10.9004; verbatimLongitude: -85.3725; 
verbatimCoordinateSystem: Decimal; decimalLatitude: 10.9004; decimalLongitude: -85.3725; sampling Protocol: Reared from the larva of the Hesperiidae, Talides sinois; verbatimEventDate: 26-Mar-2006; individualID: DHJPAR0007124; individualCount: 1; sex: male; lifeStage: adult; preparations: pinned; catalogNumber: DHJPAR0007124; occurrenceDetails: http://janzen.sas.upenn.edu; recordedBy: D.H. Janzen, W. Hallwachs \& Carolina Cano; otherCatalogNumbers: ASTAV366-06, 06-SRNP-1423, BOLD:AAA1577; language: en; institutionCode: CNC; collectionCode: Insects; basisOfRecord: Pinned Specimen

ac. scientificName: Hyphantrophaga virilis; phylum: Arthropoda; class: Insecta; order: Diptera; family: Tachinidae; genus: Hyphantrophaga; specificEpithet: virilis; scientificNameAuthorship: (Aldrich \& Webber, 1924); country: Costa Rica; countryCode: CR; stateProvince: Alajuela; county: Sector San Cristobal; locality: Area de Conservacion Guanacaste; verbatimLocality: Sendero Huerta; verbatimElevation: 527; verbatimLatitude: 10.9305; verbatimLongitude: -85.3722 ;

verbatimCoordinateSystem: Decimal; decimalLatitude: 10.9305; decimalLongitude: -85.3722; samplingProtocol: Reared from the larva of the Crambidae, Pantographa suffusalis; verbatimEventDate: 22-Feb-2006; individualID: DHJPAR0007150; individualCount: 1; sex: male; lifeStage: adult; preparations: pinned; catalogNumber: DHJPAR0007150; occurrenceDetails: http://janzen.sas.upenn.edu; recordedBy: D.H. Janzen, W. Hallwachs \& Gloria Sihezar; otherCatalogNumbers: ASTAV392-06, 06SRNP-81, BOLD:AAA1577; language: en; institutionCode: CNC; collectionCode: Insects; basisOfRecord: Pinned Specimen

ad. $\quad$ scientificName: Hyphantrophaga virilis; phylum: Arthropoda; class: Insecta; order: Diptera; family: Tachinidae; genus: Hyphantrophaga; specificEpithet: virilis; scientificNameAuthorship: (Aldrich \& Webber, 1924); country: Costa Rica; countryCode: CR; stateProvince: Guanacaste; county: Sector Santa Rosa; locality: Area de Conservacion Guanacaste; verbatimLocality: Cuesta Canyon Tigre; verbatimElevation: 270 ; verbatimLatitude: 10.817 ; verbatimLongitude: -85.6437; verbatimCoordinateSystem: Decimal; decimalLatitude: 10.817 ; decimalLongitude: -85.6437; samplingProtocol: Reared from the larva of the Noctuidae, Euscirrhopterus poeyi; verbatimEventDate: 18-Jun-2006; individualID: DHJPAR0010315; individualCount: 1; sex: male; lifeStage: adult; preparations: pinned; catalogNumber: DHJPAR0010315; occurrenceDetails: http://janzen.sas.upenn.edu; recordedBy: D.H. Janzen, W. Hallwachs \& Lucia Vargas; otherCatalogNumbers: ASTAS146-06, 06SRNP-13968, BOLD:AAA1577; language: en; institutionCode: CNC; collectionCode: Insects; basisOfRecord: Pinned Specimen

ae. ScientificName: Hyphantrophaga virilis; phylum: Arthropoda; class: Insecta; order: Diptera; family: Tachinidae; genus: Hyphantrophaga; specificEpithet: virilis; scientificNameAuthorship: (Aldrich \& Webber, 1924); country: Costa Rica; countryCode: CR; stateProvince: Guanacaste; county: Sector Cacao; locality: Area de Conservacion Guanacaste; verbatimLocality: Quebrada Otilio; verbatimElevation: 550; verbatimLatitude: 10.89 ; verbatimLongitude: -85.4797 ; verbatimCoordinateSystem: Decimal; decimalLatitude: 10.89; decimalLongitude: -85.4797; samplingProtocol: Reared from the larva of the Erebidae, Phyprosopus parthenope; verbatimEventDate: 21-Jul-2006; individualID: DHJPAR0010448; individualCount: 1; sex: male; lifeStage: adult; preparations: pinned; catalogNumber: DHJPAR0010448; occurrenceDetails: htt p://janzen.sas.upenn.edu; recordedBy: D.H. Janzen, W. Hallwachs \& Harry Ramirez; otherCatalogNumbers: ASTAS279-06, 06-SRNP-45690, BOLD:AAA1577; language: en; institutionCode: CNC; collectionCode: Insects; basisOfRecord: Pinned Specimen 
af. scientificName: Hyphantrophaga virilis; phylum: Arthropoda; class: Insecta; order: Diptera; family: Tachinidae; genus: Hyphantrophaga; specificEpithet: virilis; scientificNameAuthorship: (Aldrich \& Webber, 1924); country: Costa Rica; countryCode: CR; stateProvince: Guanacaste; county: Sector Mundo Nuevo; locality: Area de Conservacion Guanacaste; verbatimLocality: Estacion La Perla; verbatimElevation: 325; verbatimLatitude: 10.7674; verbatimLongitude: -85.4331; verbatimCoordinateSystem: Decimal; decimalLatitude: 10.7674; decimalLongitude: -85.4331; samplingProtocol: Reared from the larva of the Nymphalidae, Colobura dirce; verbatimEventDate: 29-Jun-2006; individualID: DHJPAR0010497; individualCount: 1; sex: male; lifeStage: adult; preparations: pinned; catalogNumber: DHJPAR0010497; occurrenceDetails: http://janzen.sas.upenn.edu; recordedBy: D.H. Janzen, W. Hallwachs \& Jose Alberto Sanchez; otherCatalogNumbers: ASTAS328-06, 06-SRNP-56269, BOLD:AAA1577; language: en; institutionCode: CNC; collectionCode: Insects; basisOfRecord: Pinned Specimen

ag. scientificName: Hyphantrophaga virilis; phylum: Arthropoda; class: Insecta; order: Diptera; family: Tachinidae; genus: Hyphantrophaga; specificEpithet: virilis; scientificNameAuthorship: (Aldrich \& Webber, 1924); country: Costa Rica; countryCode: CR; stateProvince: Guanacaste; county: Sector Mundo Nuevo; locality: Area de Conservacion Guanacaste; verbatimLocality: Estacion La Perla; verbatimElevation: 325; verbatimLatitude: 10.7674; verbatimLongitude: -85.4331; verbatimCoordinateSystem: Decimal; decimalLatitude: 10.7674 ; decimalLongitude: -85.4331; samplingProtocol: Reared from the larva of the Nymphalidae, Myscelia pattenia; verbatimEventDate: 28-Jun-2006; individualID: DHJPAR0010500; individualCount: 1; sex: male; lifeStage: adult; preparations: pinned; catalogNumber: DHJPAR0010500; occurrenceDetails: http://janzen.sas.upenn.edu; recordedBy: D.H. Janzen, W. Hallwachs \& Mariano Pereira; otherCatalogNumbers: ASTAS331-06, 06SRNP-56178,; language: en; institutionCode: CNC; collectionCode: Insects; basisOfRecord: Pinned Specimen

ah. ScientificName: Hyphantrophaga virilis; phylum: Arthropoda; class: Insecta; order: Diptera; family: Tachinidae; genus: Hyphantrophaga; specificEpithet: virilis; scientificNameAuthorship: (Aldrich \& Webber, 1924); country: Costa Rica; countryCode: CR; stateProvince: Guanacaste; county: Sector Mundo Nuevo; locality: Area de Conservacion Guanacaste; verbatimLocality: Vado Lonchocarpus; verbatimElevation: 490; verbatimLatitude: 10.7623; verbatimLongitude: -85.4; verbatimCoordinateSystem: Decimal; decimalLatitude: 10.7623; decimalLongitude: -85.4; samplingProtocol: Reared from the larva of the Nymphalidae, Mechanitis isthmia; verbatimEventDate: 09-Sep-2006; individualID: DHJPAR0015986; individualCount: 1; sex: male; lifeStage: adult; preparations: pinned; catalogNumber: DHJPAR0015986; occurrenceDetails: http://janzen.sas.upenn.edu; recordedBy: D.H. Janzen, W. Hallwachs \& Mariano Pereira; otherCatalogNumbers: ASTAP015-06, 06SRNP-57962, BOLD:AAA1577; language: en; institutionCode: CNC; collectionCode: Insects; basisOfRecord: Pinned Specimen

ai. $\quad$ scientificName: Hyphantrophaga virilis; phylum: Arthropoda; class: Insecta; order: Diptera; family: Tachinidae; genus: Hyphantrophaga; specificEpithet: virilis; scientificNameAuthorship: (Aldrich \& Webber, 1924); country: Costa Rica; countryCode: CR; stateProvince: Guanacaste; county: Sector Mundo Nuevo; locality: Area de Conservacion Guanacaste; verbatimLocality: Porton Rivas; verbatimElevation: 570; verbatimLatitude: 10.7586; verbatimLongitude: -85.3727; verbatimCoordinateSystem: Decimal; decimalLatitude: 10.7586; decimalLongitude: -85.3727; samplingProtocol: Reared from the larva of the Notodontidae, Boriza tonac; 
verbatimEventDate: 10-Sep-2006; individualID: DHJPAR0016037; individualCount: 1; sex: male; lifeStage: adult; preparations: pinned; catalogNumber: DHJPAR0016037; occurrenceDetails: http://janzen.sas.upenn.edu; recordedBy: D.H. Janzen, W. Hallwachs \& Jose Alberto Sanchez; otherCatalogNumbers: ASTAP066-06, 06SRNP-57772, BOLD:AAA1577; language: en; institutionCode: CNC; collectionCode: Insects; basisOfRecord: Pinned Specimen

aj. $\quad$ scientificName: Hyphantrophaga virilis; phylum: Arthropoda; class: Insecta; order: Diptera; family: Tachinidae; genus: Hyphantrophaga; specificEpithet: virilis; scientificNameAuthorship: (Aldrich \& Webber, 1924); country: Costa Rica; countryCode: CR; stateProvince: Guanacaste; county: Sector Mundo Nuevo; locality: Area de Conservacion Guanacaste; verbatimLocality: Mamones; verbatimElevation: 365; verbatimLatitude: 10.7707 ; verbatimLongitude: -85.4287 ; verbatimCoordinateSystem: Decimal; decimalLatitude: 10.7707 ; decimalLongitude: -85.4287; samplingProtocol: Reared from the larva of the Erebidae, Anomis editrix; verbatimEventDate: 26-Sep-2006; individualID: DHJPAR0016080; individualCount: 1; sex: male; lifeStage: adult; preparations: pinned; catalogNumber: DHJPAR0016080; occurrenceDetails: http://janzen.sas.upenn.edu; recordedBy: D.H. Janzen, W. Hallwachs \& Jose Alberto Sanchez; otherCatalogNumbers: ASTAP109-06, 06SRNP-58018, BOLD:AAA1577; language: en; institutionCode: CNC; collectionCode: Insects; basisOfRecord: Pinned Specimen

ak. scientificName: Hyphantrophaga virilis; phylum: Arthropoda; class: Insecta; order: Diptera; family: Tachinidae; genus: Hyphantrophaga; specificEpithet: virilis; scientificNameAuthorship: (Aldrich \& Webber, 1924); country: Costa Rica; countryCode: CR; stateProvince: Guanacaste; county: Sector Cacao; locality: Area de Conservacion Guanacaste; verbatimLocality: Casa Fran; verbatimElevation: 1140; verbatimLatitude: 10.9366; verbatimLongitude: -85.4669 ; verbatimCoordinateSystem: Decimal; decimalLatitude: 10.9366; decimalLongitude: -85.4669; samplingProtocol: Reared from the larva of the Hesperiidae, Ridens biolleyi; verbatimEventDate: 06Sep-2006; individualID: DHJPAR0016324; individualCount: 1; sex: male; lifeStage: adult; preparations: pinned; catalogNumber: DHJPAR0016324; occurrenceDetails: htt p://janzen.sas.upenn.edu; recordedBy: D.H. Janzen, W. Hallwachs \& Manuel Pereira; otherCatalogNumbers: ASTAP353-06, 06-SRNP-35176, BOLD:AAA1577; language: en; institutionCode: CNC; collectionCode: Insects; basisOfRecord: Pinned Specimen

al. $\quad$ scientificName: Hyphantrophaga virilis; phylum: Arthropoda; class: Insecta; order: Diptera; family: Tachinidae; genus: Hyphantrophaga; specificEpithet: virilis; scientificNameAuthorship: (Aldrich \& Webber, 1924); country: Costa Rica; countryCode: CR; stateProvince: Guanacaste; county: Sector Cacao; locality: Area de Conservacion Guanacaste; verbatimLocality: Sendero Pajarito; verbatimElevation: 600; verbatimLatitude: 10.8899; verbatimLongitude: -85.4743 ; verbatimCoordinateSystem: Decimal; decimalLatitude: 10.8899 ; decimalLongitude: -85.4743; samplingProtocol: Reared from the larva of the Hesperiidae, Pseudonascus paulliniae; verbatimEventDate: 27-Oct-2006; individualID: DHJPAR0016347; individualCount: 1; sex: male; lifeStage: adult; preparations: pinned; catalogNumber: DHJPAR0016347; occurrenceDetails: http://janzen.sas.upenn.edu; recordedBy: D.H. Janzen, W. Hallwachs \& Manuel Pereira; otherCatalogNumbers: ASTAP376-06, 06SRNP-47353, BOLD:AAA1577; language: en; institutionCode: CNC; collectionCode: Insects; basisOfRecord: Pinned Specimen

am. scientificName: Hyphantrophaga virilis; phylum: Arthropoda; class: Insecta; order: Diptera; family: Tachinidae; genus: Hyphantrophaga; specificEpithet: virilis; scientificNameAuthorship: (Aldrich \& Webber, 1924); country: Costa Rica; 
countryCode: CR; stateProvince: Guanacaste; county: Sector Pitilla; locality: Area de Conservacion Guanacaste; verbatimLocality: Loaiciga; verbatimElevation: 445; verbatimLatitude: 11.0198; verbatimLongitude: -85.4134 ; verbatimCoordinateSystem: Decimal; decimalLatitude: 11.0198; decimalLongitude: -85.4134; samplingProtocol: Reared from the larva of the Hesperiidae, Justinia Burns01; verbatimEventDate: 10Feb-2007; individualID: DHJPAR0017045; individualCount: 1; sex: male; lifeStage: adult; preparations: pinned; catalogNumber: DHJPAR0017045; occurrenceDetails: $\underline{\text { htt }}$ p://janzen.sas.upenn.edu; recordedBy: D.H. Janzen, W. Hallwachs \& Calixto Moraga; otherCatalog Numbers: ASTAP483-07, 06-SRNP-65757, BOLD:AAA1577; language: en; institutionCode: CNC; collectionCode: Insects; basisOfRecord: Pinned Specimen

an. scientificName: Hyphantrophaga virilis; phylum: Arthropoda; class: Insecta; order: Diptera; family: Tachinidae; genus: Hyphantrophaga; specificEpithet: virilis; scientificNameAuthorship: (Aldrich \& Webber, 1924); country: Costa Rica; countryCode: CR; stateProvince: Guanacaste; county: Sector Del Oro; locality: Area de Conservacion Guanacaste; verbatimLocality: Bosque Aguirre; verbatimElevation: 620; verbatimLatitude: 11.0006; verbatimLongitude: -85.438 ; verbatimCoordinateSystem: Decimal; decimalLatitude: 11.0006; decimalLongitude: -85.438; sampling Protocol: Reared from the larva of the Hesperiidae, Cynea anthracinus; verbatimEventDate: 12-Mar-2007; individualID: DHJPAR0017185; individualCount: 1; sex: male; lifeStage: adult; preparations: pinned; catalogNumber: DHJPAR0017185; occurrenceDetails: http://janzen.sas.upenn.edu; recordedBy: D.H. Janzen, W. Hallwachs \& Roster Moraga; otherCatalogNumbers: ASTAP623-07, 07SRNP-20244, BOLD:AAA1577; language: en; institutionCode: CNC; collectionCode: Insects; basisOfRecord: Pinned Specimen

ao. ScientificName: Hyphantrophaga virilis; phylum: Arthropoda; class: Insecta; order: Diptera; family: Tachinidae; genus: Hyphantrophaga; specificEpithet: virilis; scientificNameAuthorship: (Aldrich \& Webber, 1924); country: Costa Rica; countryCode: CR; stateProvince: Alajuela; county: Sector San Cristobal; locality: Area de Conservacion Guanacaste; verbatimLocality: Puente Palma; verbatimElevation: 460; verbatimLatitude: 10.9163; verbatimLongitude: -85.3787 ; verbatimCoordinateSystem: Decimal; decimalLatitude: 10.9163 ; decimalLongitude: -85.3787; samplingProtocol: Reared from the larva of the Crambidae, Omiodes humeralis; verbatimEventDate: 01-Jan-2007; individualID: DHJPAR0016486; individualCount: 1; sex: male; lifeStage: adult; preparations: pinned; catalogNumber: DHJPAR0016486; occurrenceDetails: http://janzen.sas.upenn.edu; recordedBy: D.H. Janzen, W. Hallwachs \& Anabelle Cordoba; otherCatalogNumbers: ASTAP690-07, 06SRNP-9688, BOLD:AAA1577; language: en; institutionCode: CNC; collectionCode: Insects; basisOfRecord: Pinned Specimen

ap. ScientificName: Hyphantrophaga virilis; phylum: Arthropoda; class: Insecta; order: Diptera; family: Tachinidae; genus: Hyphantrophaga; specificEpithet: virilis; scientificNameAuthorship: (Aldrich \& Webber, 1924); country: Costa Rica; countryCode: CR; stateProvince: Guanacaste; county: Sector Mundo Nuevo; locality: Area de Conservacion Guanacaste; verbatimLocality: Porton Rivas; verbatimElevation: 570; verbatimLatitude: 10.7586; verbatimLongitude: -85.3727;

verbatimCoordinateSystem: Decimal; decimalLatitude: 10.7586 ; decimalLongitude: -85.3727; samplingProtocol: Reared from the larva of the Lasiocampidae, Euglyphis jessiehillae; verbatimEventDate: 22-Dec-2006; individualID: DHJPAR0016522; individualCount: 1; sex: male; lifeStage: adult; preparations: pinned; catalogNumber: DHJPAR0016522; occurrenceDetails: http://janzen.sas.upenn.edu; recordedBy: D.H. Janzen, W. Hallwachs \& Jose Cortez; otherCatalogNumbers: ASTAP726-07, 06- 
SRNP-59750, BOLD:AAA1577; language: en; institutionCode: CNC; collectionCode: Insects; basisOfRecord: Pinned Specimen

aq. ScientificName: Hyphantrophaga virilis; phylum: Arthropoda; class: Insecta; order: Diptera; family: Tachinidae; genus: Hyphantrophaga; specificEpithet: virilis; scientificNameAuthorship: (Aldrich \& Webber, 1924); country: Costa Rica; countryCode: CR; stateProvince: Guanacaste; county: Sector Mundo Nuevo; locality: Area de Conservacion Guanacaste; verbatimLocality: Porton Rivas; verbatimElevation: 570; verbatimLatitude: 10.7586; verbatimLongitude: -85.3727; verbatimCoordinateSystem: Decimal; decimalLatitude: 10.7586; decimalLongitude: -85.3727; samplingProtocol: Reared from the larva of the Hesperiidae, Carrhenes canescens; verbatimEventDate: 08-Nov-2006; individualID: DHJPAR0016776; individualCount: 1; sex: male; lifeStage: adult; preparations: pinned; catalogNumber: DHJPAR0016776; occurrenceDetails: http://janzen.sas.upenn.edu; recordedBy: D.H. Janzen, W. Hallwachs \& Jose Alberto Sanchez; otherCatalogNumbers: ASTAP886-07, 06-SRNP-58934, BOLD:AAA1577; language: en; institutionCode: CNC;

collectionCode: Insects; basisOfRecord: Pinned Specimen

ar. scientificName: Hyphantrophaga virilis; phylum: Arthropoda; class: Insecta; order: Diptera; family: Tachinidae; genus: Hyphantrophaga; specificEpithet: virilis; scientificNameAuthorship: (Aldrich \& Webber, 1924); country: Costa Rica; countryCode: CR; stateProvince: Guanacaste; county: Sector Mundo Nuevo; locality: Area de Conservacion Guanacaste; verbatimLocality: Vado Lonchocarpus;

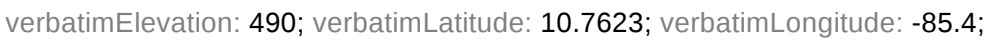
verbatimCoordinateSystem: Decimal; decimalLatitude: 10.7623; decimalLongitude: -85.4; samplingProtocol: Reared from the larva of the Pyralidae, Accinctapubes albifasciataDHJ01; verbatimEventDate: 31-Oct-2006; individualID: DHJPAR0016817; individualCount: 1; sex: male; lifeStage: adult; preparations: pinned; catalogNumber: DHJPAR0016817; occurrenceDetails: http://janzen.sas.upenn.edu; recordedBy: D.H. Janzen, W. Hallwachs \& Jose Alberto Sanchez; otherCatalogNumbers: ASTAP927-07, 06-SRNP-58813, BOLD:AAA1577; language: en; institutionCode: CNC; collectionCode: Insects; basisOfRecord: Pinned Specimen

as. ScientificName: Hyphantrophaga virilis; phylum: Arthropoda; class: Insecta; order: Diptera; family: Tachinidae; genus: Hyphantrophaga; specificEpithet: virilis; scientificNameAuthorship: (Aldrich \& Webber, 1924); country: Costa Rica; countryCode: CR; stateProvince: Guanacaste; county: Sector Mundo Nuevo; locality: Area de Conservacion Guanacaste; verbatimLocality: Vado Lonchocarpus; verbatimElevation: 490; verbatimLatitude: 10.7623; verbatimLongitude: -85.4; verbatimCoordinateSystem: Decimal; decimalLatitude: 10.7623; decimalLongitude: -85.4; samplingProtocol: Reared from the larva of the Pyralidae, Accinctapubes albifasciataDHJ01; verbatimEventDate: 28-Oct-2006; individualID: DHJPAR0016690; individualCount: 1; sex: male; lifeStage: adult; preparations: pinned; catalogNumber: DHJPAR0016690; occurrenceDetails: http://janzen.sas.upenn.edu; recordedBy: D.H. Janzen, W. Hallwachs \& Jose Alberto Sanchez; otherCatalogNumbers: ASTAP995-07, 06-SRNP-58842, BOLD:AAA1577; language: en; institutionCode: CNC; collectionCode: Insects; basisOfRecord: Pinned Specimen

at. ScientificName: Hyphantrophaga virilis; phylum: Arthropoda; class: Insecta; order: Diptera; family: Tachinidae; genus: Hyphantrophaga; specificEpithet: virilis; scientificNameAuthorship: (Aldrich \& Webber, 1924); country: Costa Rica; countryCode: CR; stateProvince: Guanacaste; county: Sector Santa Rosa; locality: Area de Conservacion Guanacaste; verbatimLocality: Bosque San Emilio; verbatimElevation: 300; verbatimLatitude: 10.8439; verbatimLongitude: $\mathbf{- 8 5 . 6 1 3 8}$; 
verbatimCoordinateSystem: Decimal; decimalLatitude: 10.8439; decimalLongitude: -85.6138; samplingProtocol: Reared from the larva of the Notodontidae, Elasmia mandela; verbatimEventDate: 30-Aug-2007; individualID: DHJPAR0021850; individualCount: 1; sex: male; lifeStage: adult; preparations: pinned; catalogNumber: DHJPAR0021850; occurrenceDetails: http://janzen.sas.upenn.edu; recordedBy: D.H. Janzen, W. Hallwachs \& Lucia Vargas; otherCatalogNumbers: ASTAT988-07, 07SRNP-14584, BOLD:AAA1577; language: en; institutionCode: CNC; collectionCode: Insects; basisOfRecord: Pinned Specimen

au. scientificName: Hyphantrophaga virilis; phylum: Arthropoda; class: Insecta; order: Diptera; family: Tachinidae; genus: Hyphantrophaga; specificEpithet: virilis; scientificNameAuthorship: (Aldrich \& Webber, 1924); country: Costa Rica; countryCode: CR; stateProvince: Guanacaste; county: Sector Mundo Nuevo; locality: Area de Conservacion Guanacaste; verbatimLocality: Quebrada Tibio Perla; verbatimElevation: 330; verbatimLatitude: 10.7626; verbatimLongitude: -85.4298; verbatimCoordinateSystem: Decimal; decimalLatitude: 10.7626 ; decimalLongitude: -85.4298; samplingProtocol: Reared from the larva of the Nymphalidae, Siderone galanthis; verbatimEventDate: 12-Sep-2007; individualID: DHJPAR0021852; individualCount: 1; sex: male; lifeStage: adult; preparations: pinned; catalogNumber: DHJPAR0021852; occurrenceDetails: http://janzen.sas.upenn.edu; recordedBy: D.H. Janzen, W. Hallwachs \& Jose Alberto Sanchez; otherCatalogNumbers: ASTAT990-07, 07-SRNP-58979, BOLD:AAA1577; language: en; institutionCode: CNC; collectionCode: Insects; basisOfRecord: Pinned Specimen

av. scientificName: Hyphantrophaga virilis; phylum: Arthropoda; class: Insecta; order: Diptera; family: Tachinidae; genus: Hyphantrophaga; specificEpithet: virilis; scientificNameAuthorship: (Aldrich \& Webber, 1924); country: Costa Rica; countryCode: CR; stateProvince: Guanacaste; county: Sector Mundo Nuevo; locality: Area de Conservacion Guanacaste; verbatimLocality: Quebrada Tibio Perla; verbatimElevation: 330; verbatimLatitude: 10.7626; verbatimLongitude: -85.4298; verbatimCoordinateSystem: Decimal; decimalLatitude: 10.7626 ; decimalLongitude: -85.4298; samplingProtocol: Reared from the larva of the Notodontidae, Ianassa rusticaDHJ05; verbatimEventDate: 09-Sep-2007; individualID: DHJPAR0021863; individualCount: 1; sex: male; lifeStage: adult; preparations: pinned; catalogNumber: DHJPAR0021863; occurrenceDetails: http://janzen.sas.upenn.edu; recordedBy: D.H. Janzen, W. Hallwachs \& Jose Alberto Sanchez; otherCatalogNumbers: ASTAT1001-07, 07-SRNP-58945, BOLD:AAA1577; language: en; institutionCode: CNC; collectionCode: Insects; basisOfRecord: Pinned Specimen

aw. scientificName: Hyphantrophaga virilis; phylum: Arthropoda; class: Insecta; order: Diptera; family: Tachinidae; genus: Hyphantrophaga; specificEpithet: virilis; scientificNameAuthorship: (Aldrich \& Webber, 1924); country: Costa Rica; countryCode: CR; stateProvince: Guanacaste; county: Potrerillos; locality: Area de Conservacion Guanacaste; verbatimLocality: Rio Azufrado; verbatimElevation: 95; verbatimLatitude: 10.8122; verbatimLongitude: -85.5444 ; verbatimCoordinateSystem: Decimal; decimalLatitude: 10.8122; decimalLongitude: -85.5444; samplingProtocol: Reared from the larva of the Geometridae, Opisthoxia uncinata; verbatimEventDate: 06-Sep-2007; individualID: DHJPAR0021952; individualCount: 1; sex: male; lifeStage: adult; preparations: pinned; catalogNumber: DHJPAR0021952; occurrenceDetails: htt p://janzen.sas.upenn.edu; recordedBy: D.H. Janzen, W. Hallwachs \& Johan Vargas; otherCatalog Numbers: ASTAT1090-07, 07-SRNP-14986, BOLD:AAA1577; language: en; institutionCode: CNC; collectionCode: Insects; basisOfRecord: Pinned Specimen 
ax. scientificName: Hyphantrophaga virilis; phylum: Arthropoda; class: Insecta; order: Diptera; family: Tachinidae; genus: Hyphantrophaga; specificEpithet: virilis; scientificNameAuthorship: (Aldrich \& Webber, 1924); country: Costa Rica; countryCode: CR; stateProvince: Guanacaste; county: Sector Horizontes; locality: Area de Conservacion Guanacaste; verbatimLocality: Vado Esperanza; verbatimElevation: 85; verbatimLatitude: 10.7894; verbatimLongitude: -85.551; verbatimCoordinateSystem: Decimal; decimalLatitude: 10.7894; decimalLongitude: -85.551; samplingProtocol: Reared from the larva of the Pieridae, Ganyra josephina; verbatimEventDate: 10-Sep-2007; individualID: DHJPAR0021959; individualCount: 1; sex: male; lifeStage: adult; preparations: pinned; catalogNumber: DHJPAR0021959; occurrenceDetails: http://janzen.sas.upenn.edu; recordedBy: D.H. Janzen, W. Hallwachs \& Johan Vargas; otherCatalogNumbers: ASTAT1097-07, 07-SRNP-15034, BOLD:AAA1577; language: en; institutionCode: CNC; collectionCode: Insects; basisOfRecord: Pinned Specimen

ay. ScientificName: Hyphantrophaga virilis; phylum: Arthropoda; class: Insecta; order: Diptera; family: Tachinidae; genus: Hyphantrophaga; specificEpithet: virilis; scientificNameAuthorship: (Aldrich \& Webber, 1924); country: Costa Rica; countryCode: CR; stateProvince: Guanacaste; county: Sector Orosi; locality: Area de Conservacion Guanacaste; verbatimLocality: Quebrada Las Yeguitas; verbatimElevation: 560; verbatimLatitude: 10.9616; verbatimLongitude: -85.4958; verbatimCoordinateSystem: Decimal; decimalLatitude: 10.9616; decimalLongitude: -85.4958; samplingProtocol: Reared from the larva of the Notodontidae, Ianassa druceiDHJ04; verbatimEventDate: 13-Sep-2007; individualID: DHJPAR0021988; individualCount: 1; sex: male; lifeStage: adult; preparations: pinned; catalogNumber: DHJPAR0021988; occurrenceDetails: http://janzen.sas.upenn.edu; recordedBy: D.H. Janzen, W. Hallwachs \& Roster Moraga; otherCatalogNumbers: ASTAT1126-07, 07SRNP-23254, BOLD:AAA1577; language: en; institutionCode: CNC; collectionCode: Insects; basisOfRecord: Pinned Specimen

az. ScientificName: Hyphantrophaga virilis; phylum: Arthropoda; class: Insecta; order: Diptera; family: Tachinidae; genus: Hyphantrophaga; specificEpithet: virilis; scientificNameAuthorship: (Aldrich \& Webber, 1924); country: Costa Rica; countryCode: CR; stateProvince: Guanacaste; county: Sector Santa Rosa; locality: Area de Conservacion Guanacaste; verbatimLocality: Sendero Natural; verbatimElevation: 290; verbatimLatitude: 10.8357; verbatimLongitude: -85.6125; verbatimCoordinateSystem: Decimal; decimalLatitude: 10.8357; decimalLongitude: -85.6125; samplingProtocol: Reared from the larva of the Geometridae, see description; verbatimEventDate: 19-Aug-2008; individualID: DHJPAR0029753; individualCount: 1; sex: male; lifeStage: adult; preparations: pinned; catalogNumber: DHJPAR0029753; occurrenceDetails: http://janzen.sas.upenn.edu; recordedBy: D.H. Janzen, W. Hallwachs \& Guillermo Pereira; otherCatalog Numbers: ASHYM1174-09, 08-SRNP-14629, BOLD:AAA1577; language: en; institutionCode: CNC; collectionCode: Insects; basisOfRecord: Pinned Specimen

ba. ScientificName: Hyphantrophaga virilis; phylum: Arthropoda; class: Insecta; order; Diptera; family: Tachinidae; genus: Hyphantrophaga; specificEpithet: virilis; scientificNameAuthorship: (Aldrich \& Webber, 1924); country: Costa Rica; countryCode: CR; stateProvince: Guanacaste; county: Sector Santa Rosa; locality: Area de Conservacion Guanacaste; verbatimLocality: Sendero Natural; verbatimElevation: 290; verbatimLatitude: 10.8357; verbatimLongitude: -85.6125; verbatimCoordinateSystem: Decimal; decimalLatitude: 10.8357 ; decimalLongitude: -85.6125; samplingProtocol: Reared from the larva of the Notodontidae, Boriza tonac; 
verbatimEventDate: 23-Aug-2008; individualID: DHJPAR0029762; individualCount: 1; sex: male; lifeStage: adult; preparations: pinned; catalogNumber: DHJPAR0029762; occurrenceDetails: http://janzen.sas.upenn.edu; recordedBy: D.H. Janzen, W. Hallwachs \& Guillermo Pereira; otherCatalogNumbers: ASHYM1183-09, 08SRNP-14503, BOLD:AAA1577; language: en; institutionCode: CNC; collectionCode: Insects; basisOfRecord: Pinned Specimen

bb. ScientificName: Hyphantrophaga virilis; phylum: Arthropoda; class: Insecta; order: Diptera; family: Tachinidae; genus: Hyphantrophaga; specificEpithet: virilis; scientificNameAuthorship: (Aldrich \& Webber, 1924); country: Costa Rica; countryCode: CR; stateProvince: Guanacaste; county: Sector Santa Rosa; locality: Area de Conservacion Guanacaste; verbatimLocality: Bosque San Emilio; verbatimElevation: 300; verbatimLatitude: 10.8439; verbatimLongitude: -85.6138; verbatimCoordinateSystem: Decimal; decimalLatitude: 10.8439 ; decimalLongitude: -85.6138; samplingProtocol: Reared from the larva of the Notodontidae, Elasmia mandela; verbatimEventDate: 24-Aug-2008; individualID: DHJPAR0029767; individualCount: 1; sex: male; lifeStage: adult; preparations: pinned; catalogNumber: DHJPAR0029767; occurrenceDetails: http://janzen.sas.upenn.edu; recordedBy: D.H. Janzen, W. Hallwachs \& Guillermo Pereira; otherCatalogNumbers: ASHYM1188-09, 08-SRNP-14562, BOLD:AAA1577; language: en; institutionCode: CNC; collectionCode: Insects; basisOfRecord: Pinned Specimen

bc. ScientificName: Hyphantrophaga virilis; phylum: Arthropoda; class: Insecta; order: Diptera; family: Tachinidae; genus: Hyphantrophaga; specificEpithet: virilis; scientificNameAuthorship: (Aldrich \& Webber, 1924); country: Costa Rica; countryCode: CR; stateProvince: Guanacaste; county: Sector Santa Rosa; locality: Area de Conservacion Guanacaste; verbatimLocality: Bosque Humedo; verbatimElevation: 290; verbatimLatitude: 10.8514; verbatimLongitude: -85.608; verbatimCoordinateSystem: Decimal; decimalLatitude: 10.8514; decimalLongitude: -85.608; samplingProtocol: Reared from the larva of the Nymphalidae, Mechanitis isthmia; verbatimEventDate: 16-Jan-2009; individualID: DHJPAR0030074; individualCount: 1; sex: male; lifeStage: adult; preparations: pinned; catalogNumber: DHJPAR0030074; occurrenceDetails: http://janzen.sas.upenn.edu; recordedBy: D.H. Janzen, W. Hallwachs \& Johan Vargas; otherCatalogNumbers: ASHYB818-09, 08SRNP-16975, BOLD:AAA1577; language: en; institutionCode: CNC; collectionCode: Insects; basisOfRecord: Pinned Specimen

bd. ScientificName: Hyphantrophaga virilis; phylum: Arthropoda; class: Insecta; order: Diptera; family: Tachinidae; genus: Hyphantrophaga; specificEpithet: virilis; scientificNameAuthorship: (Aldrich \& Webber, 1924); country: Costa Rica; countryCode: CR; stateProvince: Guanacaste; county: Sector Santa Rosa; locality: Area de Conservacion Guanacaste; verbatimLocality: Cafetal; verbatimElevation: 280; verbatimLatitude: 10.8583; verbatimLongitude: -85.6109; verbatimCoordinateSystem: Decimal; decimalLatitude: 10.8583; decimalLongitude: -85.6109; samplingProtocol: Reared from the larva of the Hesperiidae, Celaenorrhinus eligius; verbatimEventDate: 31-Dec-2008; individualID: DHJPAR0030078; individualCount: 1; sex: male; lifeStage: adult; preparations: pinned; catalogNumber: DHJPAR0030078; occurrenceDetails: htt p://janzen.sas.upenn.edu; recordedBy: D.H. Janzen, W. Hallwachs \& Lucia Vargas; otherCatalogNumbers: ASHYB822-09, 08-SRNP-16895, BOLD:AAA1577; language: en; institutionCode: CNC; collectionCode: Insects; basisOfRecord: Pinned Specimen

be. scientificName: Hyphantrophaga virilis; phylum: Arthropoda; class: Insecta; order: Diptera; family: Tachinidae; genus: Hyphantrophaga; specificEpithet: virilis; scientificNameAuthorship: (Aldrich \& Webber, 1924); country: Costa Rica; 
countryCode: CR; stateProvince: Guanacaste; county: Sector Santa Rosa; locality: Area de Conservacion Guanacaste; verbatimLocality: Bosque San Emilio; verbatimElevation: 300; verbatimLatitude: 10.8439; verbatimLongitude: $\mathbf{8 5 . 6 1 3 8 ;}$ verbatimCoordinateSystem: Decimal; decimalLatitude: 10.8439; decimalLongitude: -85.6138; samplingProtocol: Reared from the larva of the Hesperiidae, Mysoria ambigua; verbatimEventDate: 10-Dec-2008; individualID: DHJPAR0030080; individualCount: 1; sex: male; lifeStage: adult; preparations: pinned; catalogNumber: DHJPAR0030080; occurrenceDetails: http://janzen.sas.upenn.edu; recordedBy: D.H. Janzen, W. Hallwachs \& Guillermo Pereira; otherCatalogNumbers: ASHYB824-09, 08SRNP-16271, BOLD:AAA1577; language: en; institutionCode: CNC; collectionCode: Insects; basisOfRecord: Pinned Specimen

bf. ScientificName: Hyphantrophaga virilis; phylum: Arthropoda; class: Insecta; order: Diptera; family: Tachinidae; genus: Hyphantrophaga; specificEpithet: virilis; scientificNameAuthorship: (Aldrich \& Webber, 1924); country: Costa Rica; countryCode: CR; stateProvince: Guanacaste; county: Sector Del Oro; locality: Area de Conservacion Guanacaste; verbatimLocality: Monte Cristo; verbatimElevation: 525; verbatimLatitude: 11.0137; verbatimLongitude: -85.4253; verbatimCoordinateSystem: Decimal; decimalLatitude: 11.0137; decimalLongitude: -85.4253; samplingProtocol: Reared from the larva of the Hesperiidae, Cynea anthracinus; verbatimEventDate: 14Dec-2010; individualID: DHJPAR0040981; individualCount: 1; sex: male; lifeStage: adult; preparations: pinned; catalogNumber: DHJPAR0040981; occurrenceDetails: htt p://janzen.sas.upenn.edu; recordedBy: D.H. Janzen, W. Hallwachs \& Roster Moraga; otherCatalogNumbers: ASHYF896-11, 10-SRNP-22425, BOLD:AAA1577; language: en; institutionCode: CNC; collectionCode: Insects; basisOfRecord: Pinned Specimen

bg. ScientificName: Hyphantrophaga virilis; phylum: Arthropoda; class: Insecta; order: Diptera; family: Tachinidae; genus: Hyphantrophaga; specificEpithet: virilis; scientificNameAuthorship: (Aldrich \& Webber, 1924); country: Costa Rica; countryCode: CR; stateProvince: Guanacaste; county: Sector Mundo Nuevo; locality: Area de Conservacion Guanacaste; verbatimLocality: Punta Plancha;

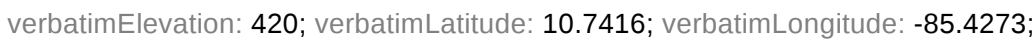
verbatimCoordinateSystem: Decimal; decimalLatitude: 10.7416; decimalLongitude: -85.4273; samplingProtocol: Reared from the larva of the Pyralidae, Carthara abruptaDHJ02; verbatimEventDate: 16-Nov-2010; individualID: DHJPAR0040990; individualCount: 1; sex: male; lifeStage: adult; preparations: pinned; catalogNumber: DHJPAR0040990; occurrenceDetails: http://janzen.sas.upenn.edu; recordedBy: D.H. Janzen, W. Hallwachs \& Mariano Pereira; otherCatalogNumbers: ASHYF905-11, 10SRNP-56976, BOLD:AAA1577; language: en; institutionCode: CNC; collectionCode: Insects; basisOfRecord: Pinned Specimen

bh. ScientificName: Hyphantrophaga virilis; phylum: Arthropoda; class: Insecta; order: Diptera; family: Tachinidae; genus: Hyphantrophaga; specificEpithet: virilis; scientificNameAuthorship: (Aldrich \& Webber, 1924); country: Costa Rica; countryCode: CR; stateProvince: Guanacaste; county: Sector Mundo Nuevo; locality: Area de Conservacion Guanacaste; verbatimLocality: Quebrada Tibio Perla;

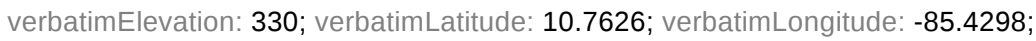
verbatimCoordinateSystem: Decimal; decimalLatitude: 10.7626; decimalLongitude: -85.4298; samplingProtocol: Reared from the larva of the Nolidae, Iscadia purpurascens; verbatimEventDate: 01-Sep-2011; individualID: DHJPAR0046535; individualCount: 1; sex: male; lifeStage: adult; preparations: pinned; catalogNumber: DHJPAR0046535; occurrenceDetails: http://janzen.sas.upenn.edu; recordedBy: D.H. Janzen, W. Hallwachs \& Mariano Pereira; otherCatalogNumbers: ACGBA708-12, 11- 
SRNP-56306, BOLD:AAA1577; language: en; institutionCode: CNC; collectionCode: Insects; basisOfRecord: Pinned Specimen

bi. $\quad$ scientificName: Hyphantrophaga virilis; phylum: Arthropoda; class: Insecta; order: Diptera; family: Tachinidae; genus: Hyphantrophaga; specificEpithet: virilis; scientificNameAuthorship: (Aldrich \& Webber, 1924); country: Costa Rica; countryCode: CR; stateProvince: Guanacaste; county: Sector Santa Rosa; locality: Area de Conservacion Guanacaste; verbatimLocality: Finca Jenny; verbatimElevation: 205; verbatimLatitude: 10.8633; verbatimLongitude: -85.5744; verbatimCoordinateSystem: Decimal; decimalLatitude: 10.8633 ; decimalLongitude: -85.5744; samplingProtocol: Reared from the larva of the Notodontidae, Tachuda plumipesICG02; verbatimEventDate: 25-Sep-2005; individualID: DHJPAR0006302; individualCount: 1; sex: female; lifeStage: adult; preparations: pinned; catalogNumber: DHJPAR0006302; occurrenceDetails: http://janzen.sas.upenn.edu; recordedBy: D.H. Janzen, W. Hallwachs \& Freddy Quesada; otherCatalogNumbers: ASTAI730-06, 05SRNP-60214, BOLD:AAA1577; language: en; institutionCode: CNC; collectionCode: Insects; basisOfRecord: Pinned Specimen

bj. $\quad$ scientificName: Hyphantrophaga virilis; phylum: Arthropoda; class: Insecta; order: Diptera; family: Tachinidae; genus: Hyphantrophaga; specificEpithet: virilis; scientificNameAuthorship: (Aldrich \& Webber, 1924); country: Costa Rica; countryCode: CR; stateProvince: Guanacaste; county: Sector Cacao; locality: Area de Conservacion Guanacaste; verbatimLocality: Cuesta Caimito; verbatimElevation: 640; verbatimLatitude: 10.8908; verbatimLongitude: -85.4719 ; verbatimCoordinateSystem: Decimal; decimalLatitude: 10.8908; decimalLongitude: -85.4719; samplingProtocol: Reared from the larva of the Noctuidae, Dyops cuprescens; verbatimEventDate: 13Dec-2002; individualID: DHJPAR0006309; individualCount: 1; sex: female; lifeStage: adult; preparations: pinned; catalogNumber: DHJPAR0006309; occurrenceDetails: htt p://janzen.sas.upenn.edu; recordedBy: D.H. Janzen, W. Hallwachs \& Harry Ramirez; otherCatalogNumbers: ASTAI737-06, 02-SRNP-24305, BOLD:AAA1577; language: en; institutionCode: CNC; collectionCode: Insects; basisOfRecord: Pinned Specimen

bk. ScientificName: Hyphantrophaga virilis; phylum: Arthropoda; class: Insecta; order: Diptera; family: Tachinidae; genus: Hyphantrophaga; specificEpithet: virilis; scientificNameAuthorship: (Aldrich \& Webber, 1924); country: Costa Rica; countryCode: CR; stateProvince: Guanacaste; county: Sector Santa Rosa; locality: Area de Conservacion Guanacaste; verbatimLocality: Area Administrativa; verbatimElevation: 295; verbatimLatitude: 10.8376; verbatimLongitude: -85.6187; verbatimCoordinateSystem: Decimal; decimalLatitude: 10.8376; decimalLongitude: -85.6187; samplingProtocol: Reared from the larva of the Notodontidae, Sericochroa felderi; verbatimEventDate: 12-Aug-2003; individualID: DHJPAR0006322; individualCount: 1; sex: female; lifeStage: adult; preparations: pinned; catalogNumber: DHJPAR0006322; occurrenceDetails: http://janzen.sas.upenn.edu; recordedBy: D.H. Janzen, W. Hallwachs \& Ruth Franco; otherCatalog Numbers: ASTAI750-06, 03SRNP-14269,; language: en; institutionCode: CNC; collectionCode: Insects; basisOfRecord: Pinned Specimen

bl. scientificName: Hyphantrophaga virilis; phylum: Arthropoda; class: Insecta; order: Diptera; family: Tachinidae; genus: Hyphantrophaga; specificEpithet: virilis; scientificNameAuthorship: (Aldrich \& Webber, 1924); country: Costa Rica; countryCode: CR; stateProvince: Guanacaste; county: Sector Mundo Nuevo; locality: Area de Conservacion Guanacaste; verbatimLocality: Quebrada Tibio Perla; verbatimElevation: 330; verbatimLatitude: 10.7626; verbatimLongitude: -85.4298; verbatimCoordinateSystem: Decimal; decimalLatitude: 10.7626; decimalLongitude: 
-85.4298; samplingProtocol: Reared from the larva of the Noctuidae, Neotuerta sabulosa; verbatimEventDate: 06-Jun-2007; individualID: DHJPAR0019577; individualCount: 1; sex: female; lifeStage: adult; preparations: pinned; catalogNumber: DHJPAR0019577; occurrenceDetails: http://janzen.sas.upenn.edu; recordedBy: D.H. Janzen, W. Hallwachs \& Mariano Pereira; otherCatalog Numbers: ASTAB125-07, 07SRNP-55882, BOLD:AAA1577; language: en; institutionCode: CNC; collectionCode: Insects; basisOfRecord: Pinned Specimen

bm. ScientificName: Hyphantrophaga virilis; phylum: Arthropoda; class: Insecta; order: Diptera; family: Tachinidae; genus: Hyphantrophaga; specificEpithet: virilis; scientificNameAuthorship: (Aldrich \& Webber, 1924); country: Costa Rica; countryCode: CR; stateProvince: Guanacaste; county: Sector Mundo Nuevo; locality: Area de Conservacion Guanacaste; verbatimLocality: Quebrada Tibio Perla; verbatimElevation: 330; verbatimLatitude: 10.7626; verbatimLongitude: -85.4298; verbatimCoordinateSystem: Decimal; decimalLatitude: 10.7626; decimalLongitude: -85.4298; samplingProtocol: Reared from the larva of the Riodinidae, Melanis sanguinea; verbatimEventDate: 20-Jun-2007; individualID: DHJPAR0019602; individualCount: 1; sex: female; lifeStage: adult; preparations: pinned; catalogNumber: DHJPAR0019602; occurrenceDetails: http://janzen.sas.upenn.edu; recordedBy: D.H. Janzen, W. Hallwachs \& Jose Alberto Sanchez; otherCatalogNumbers: ASTAB150-07, 07-SRNP-56934, BOLD:AAA1577; language: en; institutionCode: CNC; collectionCode: Insects; basisOfRecord: Pinned Specimen

bn. ScientificName: Hyphantrophaga virilis; phylum: Arthropoda; class: Insecta; order: Diptera; family: Tachinidae; genus: Hyphantrophaga; specificEpithet: virilis; scientificNameAuthorship: (Aldrich \& Webber, 1924); country: Costa Rica; countryCode: CR; stateProvince: Guanacaste; county: Sector Santa Rosa; locality: Area de Conservacion Guanacaste; verbatimLocality: Rio Azufrado; verbatimElevation: 95; verbatimLatitude: 10.8122; verbatimLongitude: -85.5444; verbatimCoordinateSystem: Decimal; decimalLatitude: 10.8122; decimalLongitude: -85.5444; samplingProtocol: Reared from the larva of the Notodontidae, Calledema plusia; verbatimEventDate: 23-Nov-2007; individualID: DHJPAR0023210; individualCount: 1; sex: female; lifeStage: adult; preparations: pinned; catalogNumber: DHJPAR0023210; occurrenceDetails: http://janzen.sas.upenn.edu; recordedBy: D.H. Janzen, W. Hallwachs \& Guillermo Pereira; otherCatalogNumbers: ASTAW371-08, 07SRNP-16103, BOLD:AAA1577; language: en; institutionCode: CNC; collectionCode: Insects; basisOfRecord: Pinned Specimen

bo. ScientificName: Hyphantrophaga virilis; phylum: Arthropoda; class: Insecta; order: Diptera; family: Tachinidae; genus: Hyphantrophaga; specificEpithet: virilis; scientificNameAuthorship: (Aldrich \& Webber, 1924); country: Costa Rica; countryCode: CR; stateProvince: Guanacaste; county: Sector Mundo Nuevo; locality: Area de Conservacion Guanacaste; verbatimLocality: Sendero Aguacate;

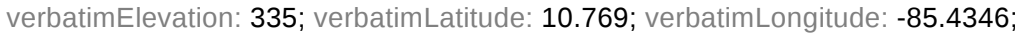
verbatimCoordinateSystem: Decimal; decimalLatitude: 10.769; decimalLongitude: -85.4346; samplingProtocol: Reared from the larva of the Hesperiidae, Mysoria ambigua; verbatimEventDate: 26-Nov-2007; individualID: DHJPAR0023223; individualCount: 1; sex: female; lifeStage: adult; preparations: pinned; catalog Number: DHJPAR0023223; occurrenceDetails: http://janzen.sas.upenn.edu; recordedBy: D.H. Janzen, W. Hallwachs \& Jose Alberto Sanchez; otherCatalog Numbers: ASTAW384-08, 07-SRNP-60273, BOLD:AAA1577; language: en; institutionCode: CNC; collectionCode: Insects; basisOfRecord: Pinned Specimen 
bp. $\quad$ scientificName: Hyphantrophaga virilis; phylum: Arthropoda; class: Insecta; order: Diptera; family: Tachinidae; genus: Hyphantrophaga; specificEpithet: virilis; scientificNameAuthorship: (Aldrich \& Webber, 1924); country: Costa Rica; countryCode: CR; stateProvince: Guanacaste; county: Sector Del Oro; locality: Area de Conservacion Guanacaste; verbatimLocality: Bosque Aguirre; verbatimElevation: 620; verbatimLatitude: 11.0006; verbatimLongitude: -85.438; verbatimCoordinateSystem: Decimal; decimalLatitude: 11.0006; decimalLongitude: -85.438; samplingProtocol: Reared from the larva of the Pieridae, Eurema xanthochlora; verbatimEventDate: 05-Feb-2008; individualID: DHJPAR0023617; individualCount: 1; sex: female; lifeStage: adult; preparations: pinned; catalogNumber: DHJPAR0023617; occurrenceDetails: http://janzen.sas.upenn.edu; recordedBy: D.H. Janzen, W. Hallwachs \& Lucia Rios; otherCatalogNumbers: ASTAW474-08, 08SRNP-20258, BOLD:AAA1577; language: en; institutionCode: CNC; collectionCode: Insects; basisOfRecord: Pinned Specimen

bq. $\quad$ scientificName: Hyphantrophaga virilis; phylum: Arthropoda; class: Insecta; order: Diptera; family: Tachinidae; genus: Hyphantrophaga; specificEpithet: virilis; scientificNameAuthorship: (Aldrich \& Webber, 1924); country: Costa Rica; countryCode: CR; stateProvince: Guanacaste; county: Sector Del Oro; locality: Area de Conservacion Guanacaste; verbatimLocality: Bosque Aguirre; verbatimElevation: 620; verbatimLatitude: 11.0006; verbatimLongitude: -85.438; verbatimCoordinateSystem: Decimal; decimalLatitude: 11.0006; decimalLongitude: -85.438; samplingProtocol: Reared from the larva of the Pieridae, Eurema xanthochlora; verbatimEventDate: 05-Feb-2008; individualID: DHJPAR0023619; individualCount: 1; sex: female; lifeStage: adult; preparations: pinned; catalogNumber: DHJPAR0023619; occurrenceDetails: http://janzen.sas.upenn.edu; recordedBy: D.H. Janzen, W. Hallwachs \& Lucia Rios; otherCatalogNumbers: ASTAW476-08, 08SRNP-20268, BOLD:AAA1577; language: en; institutionCode: CNC; collectionCode: Insects; basisOfRecord: Pinned Specimen

br. $\quad$ scientificName: Hyphantrophaga virilis; phylum: Arthropoda; class: Insecta; order: Diptera; family: Tachinidae; genus: Hyphantrophaga; specificEpithet: virilis; scientificNameAuthorship: (Aldrich \& Webber, 1924); country: Costa Rica; countryCode: CR; stateProvince: Guanacaste; county: Sector Del Oro; locality: Area de Conservacion Guanacaste; verbatimLocality: Bosque Aguirre; verbatimElevation: 620; verbatimLatitude: 11.0006; verbatimLongitude: -85.438; verbatimCoordinateSystem: Decimal; decimalLatitude: 11.0006; decimalLongitude: -85.438; samplingProtocol: Reared from the larva of the Pieridae, Eurema xanthochlora; verbatimEventDate: 05-Feb-2008; individualID: DHJPAR0023620; individualCount: 1; sex: female; lifeStage: adult; preparations: pinned; catalogNumber: DHJPAR0023620; occurrenceDetails: http://janzen.sas.upenn.edu; recordedBy: D.H. Janzen, W. Hallwachs \& Lucia Rios; otherCatalogNumbers: ASTAW477-08, 08SRNP-20264, BOLD:AAA1577; language: en; institutionCode: CNC; collectionCode: Insects; basisOfRecord: Pinned Specimen

bs. $\quad$ scientificName: Hyphantrophaga virilis; phylum: Arthropoda; class: Insecta; order: Diptera; family: Tachinidae; genus: Hyphantrophaga; specificEpithet: virilis; scientificNameAuthorship: (Aldrich \& Webber, 1924); country: Costa Rica; countryCode: CR; stateProvince: Guanacaste; county: Sector Del Oro; locality: Area de Conservacion Guanacaste; verbatimLocality: Bosque Aguirre; verbatimElevation: 620; verbatimLatitude: 11.0006; verbatimLongitude: -85.438; verbatimCoordinateSystem: Decimal; decimalLatitude: 11.0006; decimalLongitude: -85.438; samplingProtocol: Reared from the larva of the Hesperiidae, Astraptes inflatio; 
verbatimEventDate: 09-Mar-2008; individualID: DHJPAR0023634; individualCount: 1; sex: female; lifeStage: adult; preparations: pinned; catalogNumber: DHJPAR0023634; occurrenceDetails: http://janzen.sas.upenn.edu; recordedBy: D.H. Janzen, W. Hallwachs \& Lucia Rios; otherCatalogNumbers: ASTAW491-08, 08-SRNP-20524, BOLD:AAA1577; language: en; institutionCode: CNC; collectionCode: Insects; basisOfRecord: Pinned Specimen

bt. $\quad$ scientificName: Hyphantrophaga virilis; phylum: Arthropoda; class: Insecta; order: Diptera; family: Tachinidae; genus: Hyphantrophaga; specificEpithet: virilis; scientificNameAuthorship: (Aldrich \& Webber, 1924); country: Costa Rica; countryCode: CR; stateProvince: Guanacaste; county: Sector Cacao; locality: Area de Conservacion Guanacaste; verbatimLocality: Sendero Pajarito; verbatimElevation: 600; verbatimLatitude: 10.8899; verbatimLongitude: -85.4743 ; verbatimCoordinateSystem: Decimal; decimalLatitude: 10.8899 ; decimalLongitude: -85.4743; samplingProtocol: Reared from the larva of the Lasiocampidae, Euglyphis jessiehillae; verbatimEventDate: 13-Mar-2008; individualID: DHJPAR0023639; individualCount: 1; sex: female; lifeStage: adult; preparations: pinned; catalogNumber: DHJPAR0023639; occurrenceDetails: http://janzen.sas.upenn.edu; recordedBy: D.H. Janzen, W. Hallwachs \& Harry Ramirez; otherCatalogNumbers: ASTAW496-08, 08SRNP-45029, BOLD:AAA1577; language: en; institutionCode: CNC; collectionCode: Insects; basisOfRecord: Pinned Specimen

bu. $\quad$ scientificName: Hyphantrophaga virilis; phylum: Arthropoda; class: Insecta; order: Diptera; family: Tachinidae; genus: Hyphantrophaga; specificEpithet: virilis; scientificNameAuthorship: (Aldrich \& Webber, 1924); country: Costa Rica; countryCode: CR; stateProvince: Alajuela; county: Sector San Cristobal; locality: Area de Conservacion Guanacaste; verbatimLocality: Finca San Gabriel; verbatimElevation: 645; verbatimLatitude: 10.8777; verbatimLongitude: -85.3934 ; verbatimCoordinateSystem: Decimal; decimalLatitude: 10.8777 ; decimalLongitude: -85.3934; samplingProtocol: Reared from the larva of the Pieridae, Dismorphia praxinoe; verbatimEventDate: 15-Jan-2008; individualID: DHJPAR0023660; individualCount: 1; sex: female; lifeStage: adult; preparations: pinned; catalog Number: DHJPAR0023660; occurrenceDetails: http://janzen.sas.upenn.edu; recordedBy: D.H. Janzen, W. Hallwachs \& Anabelle Cordoba; otherCatalogNumbers: ASTAW517-08, 07SRNP-5016, BOLD:AAA1577; language: en; institutionCode: CNC; collectionCode: Insects; basisOfRecord: Pinned Specimen

bv. $\quad$ scientificName: Hyphantrophaga virilis; phylum: Arthropoda; class: Insecta; order: Diptera; family: Tachinidae; genus: Hyphantrophaga; specificEpithet: virilis; scientificNameAuthorship: (Aldrich \& Webber, 1924); country: Costa Rica; countryCode: CR; stateProvince: Alajuela; county: Sector San Cristobal; locality: Area de Conservacion Guanacaste; verbatimLocality: Puente Palma; verbatimElevation: 460; verbatimLatitude: 10.9163; verbatimLongitude: -85.3787; verbatimCoordinateSystem: Decimal; decimalLatitude: 10.9163 ; decimalLongitude: -85.3787; samplingProtocol: Reared from the larva of the Hesperiidae, Polyctor cleta; verbatimEventDate: 14-Mar-2008; individualID: DHJPAR0024460; individualCount: 1; sex: female; lifeStage: adult; preparations: pinned; catalogNumber: DHJPAR0024460; occurrence Details: http://janzen.sas.upenn.edu; recordedBy: D.H. Janzen, W.

Hallwachs \& Anabelle Cordoba; otherCatalog Numbers: ASTAW570-08, 08-SRNP-351, BOLD:AAA1577; language: en; institutionCode: CNC; collectionCode: Insects; basisOfRecord: Pinned Specimen

bw. $\quad$ scientificName: Hyphantrophaga virilis; phylum: Arthropoda; class: Insecta; order: Diptera; family: Tachinidae; genus: Hyphantrophaga; specificEpithet: virilis; 
scientificNameAuthorship: (Aldrich \& Webber, 1924); country: Costa Rica; countryCode: CR; stateProvince: Alajuela; county: Sector San Cristobal; locality: Area de Conservacion Guanacaste; verbatimLocality: Vado Rio Cucaracho; verbatimElevation: 640; verbatimLatitude: 10.8702; verbatimLongitude: -85.3915; verbatimCoordinateSystem: Decimal; decimalLatitude: 10.8702; decimalLongitude: -85.3915; samplingProtocol: Reared from the larva of the Notodontidae, Tithraustes noctilucesICG02; verbatimEventDate: 21-Mar-2008; individualID: DHJPAR0024470; individualCount: 1; sex: female; lifeStage: adult; preparations: pinned; catalogNumber: DHJPAR0024470; occurrenceDetails: http://janzen.sas.upenn.edu; recordedBy: D.H. Janzen, W. Hallwachs \& Gloria Sihezar; otherCatalogNumbers: ASTAW580-08, 08SRNP-767, BOLD:AAA1577; language: en; institutionCode: CNC; collectionCode: Insects; basisOfRecord: Pinned Specimen

bx. $\quad$ scientificName: Hyphantrophaga virilis; phylum: Arthropoda; class: Insecta; order: Diptera; family: Tachinidae; genus: Hyphantrophaga; specificEpithet: virilis; scientificNameAuthorship: (Aldrich \& Webber, 1924); country: Costa Rica; countryCode: CR; stateProvince: Alajuela; county: Sector San Cristobal; locality: Area de Conservacion Guanacaste; verbatimLocality: Finca San Gabriel; verbatimElevation: 645; verbatimLatitude: 10.8777; verbatimLongitude: -85.3934 ; verbatimCoordinateSystem: Decimal; decimalLatitude: 10.8777 ; decimalLongitude: -85.3934; samplingProtocol: Reared from the larva of the Tortricidae, Anacrusis nephrodes; verbatimEventDate: 20-Mar-2008; individualID: DHJPAR0024472; individualCount: 1; sex: female; lifeStage: adult; preparations: pinned; catalogNumber: DHJPAR0024472; occurrenceDetails: http://janzen.sas.upenn.edu; recordedBy: D.H. Janzen, W. Hallwachs \& Elda Araya; otherCatalogNumbers: ASTAW582-08, 08SRNP-508, BOLD:AAA1577; language: en; institutionCode: CNC; collectionCode: Insects; basisOfRecord: Pinned Specimen

by. $\quad$ scientificName: Hyphantrophaga virilis; phylum: Arthropoda; class: Insecta; order: Diptera; family: Tachinidae; genus: Hyphantrophaga; specificEpithet: virilis; scientificNameAuthorship: (Aldrich \& Webber, 1924); country: Costa Rica; countryCode: CR; stateProvince: Alajuela; county: Sector San Cristobal; locality: Area de Conservacion Guanacaste; verbatimLocality: Puente Palma; verbatimElevation: 460; verbatimLatitude: 10.9163; verbatimLongitude: -85.3787; verbatimCoordinateSystem: Decimal; decimalLatitude: 10.9163 ; decimalLongitude: -85.3787; samplingProtocol: Reared from the larva of the Hesperiidae, Polyctor cleta; verbatimEventDate: 06-Mar-2008; individualID: DHJPAR0024475; individualCount: 1; sex: female; lifeStage: adult; preparations: pinned; catalogNumber: DHJPAR0024475; occurrenceDetails: http://janzen.sas.upenn.edu; recordedBy: D.H. Janzen, W.

Hallwachs \& Anabelle Cordoba; otherCatalogNumbers: ASTAW585-08, 08-SRNP-349, BOLD:AAA1577; language: en; institutionCode: CNC; collectionCode: Insects; basisOfRecord: Pinned Specimen

bz. ScientificName: Hyphantrophaga virilis; phylum: Arthropoda; class: Insecta; order: Diptera; family: Tachinidae; genus: Hyphantrophaga; specificEpithet: virilis; scientificNameAuthorship: (Aldrich \& Webber, 1924); country: Costa Rica; countryCode: CR; stateProvince: Guanacaste; county: Sector Cacao; locality: Area de Conservacion Guanacaste; verbatimLocality: Sendero Derrumbe; verbatimElevation: 1220; verbatimLatitude: 10.9292; verbatimLongitude: -85.4643; verbatimCoordinateSystem: Decimal; decimalLatitude: 10.9292; decimalLongitude: -85.4643; samplingProtocol: Reared from the larva of the Geometridae, Carpela Janzen01; verbatimEventDate: 10-May-2008; individualID: DHJPAR0024561; individualCount: 1; sex: female; lifeStage: adult; preparations: pinned; catalogNumber: 
DHJPAR0024561; occurrenceDetails: http://janzen.sas.upenn.edu; recordedBy: D.H. Janzen, W. Hallwachs \& Manuel Pereira; otherCatalogNumbers: ASTAW671-08, 08SRNP-35348, BOLD:AAA1577; language: en; institutionCode: CNC; collectionCode: Insects; basisOfRecord: Pinned Specimen

ca. ScientificName: Hyphantrophaga virilis; phylum: Arthropoda; class: Insecta; order: Diptera; family: Tachinidae; genus: Hyphantrophaga; specificEpithet: virilis; scientificNameAuthorship: (Aldrich \& Webber, 1924); country: Costa Rica; countryCode: CR; stateProvince: Guanacaste; county: Sector Santa Rosa; locality: Area de Conservacion Guanacaste; verbatimLocality: Sendero Natural; verbatimElevation: 290; verbatimLatitude: 10.8357; verbatimLongitude: -85.6125; verbatimCoordinateSystem: Decimal; decimalLatitude: 10.8357; decimalLongitude: -85.6125; samplingProtocol: Reared from the larva of the Notodontidae, Elasmia mandela; verbatimEventDate: 22-Aug-2008; individualID: DHJPAR0029756; individualCount: 1; sex: female; lifeStage: adult; preparations: pinned; catalogNumber: DHJPAR0029756; occurrenceDetails: http://janzen.sas.upenn.edu; recordedBy: D.H. Janzen, W. Hallwachs \& Guillermo Pereira; otherCatalogNumbers: ASHYM1177-09, 08-SRNP-14508,; language: en; institutionCode: CNC; collectionCode: Insects; basisOfRecord: Pinned Specimen

cb. $\quad$ scientificName: Hyphantrophaga virilis; phylum: Arthropoda; class: Insecta; order: Diptera; family: Tachinidae; genus: Hyphantrophaga; specificEpithet: virilis; scientificNameAuthorship: (Aldrich \& Webber, 1924); country: Costa Rica; countryCode: CR; stateProvince: Guanacaste; county: Sector Santa Rosa; locality: Area de Conservacion Guanacaste; verbatimLocality: Bosque San Emilio; verbatimElevation: 300; verbatimLatitude: 10.8439; verbatimLongitude: -85.6138; verbatimCoordinateSystem: Decimal; decimalLatitude: 10.8439; decimalLongitude: -85.6138; samplingProtocol: Reared from the larva of the Notodontidae, Elasmia mandela; verbatimEventDate: 23-Aug-2008; individualID: DHJPAR0029758; individualCount: 1; sex: female; lifeStage: adult; preparations: pinned; catalogNumber: DHJPAR0029758; occurrenceDetails: http://janzen.sas.upenn.edu; recordedBy: D.H. Janzen, W. Hallwachs \& Guillermo Pereira; otherCatalog Numbers: ASHYM1179-09, 08-SRNP-14565, BOLD:AAA1577; language: en; institutionCode: CNC; collectionCode: Insects; basisOfRecord: Pinned Specimen

cc. ScientificName: Hyphantrophaga virilis; phylum: Arthropoda; class: Insecta; order: Diptera; family: Tachinidae; genus: Hyphantrophaga; specificEpithet: virilis; scientificNameAuthorship: (Aldrich \& Webber, 1924); country: Costa Rica; countryCode: CR; stateProvince: Guanacaste; county: Sector Santa Rosa; locality: Area de Conservacion Guanacaste; verbatimLocality: Bosque San Emilio; verbatimElevation: 300; verbatimLatitude: 10.8439; verbatimLongitude: -85.6138; verbatimCoordinateSystem: Decimal; decimalLatitude: 10.8439 ; decimalLongitude: -85.6138; samplingProtocol: Reared from the larva of the Notodontidae, Elasmia mandela; verbatimEventDate: 23-Aug-2008; individualID: DHJPAR0029760; individualCount: 1; sex: female; lifeStage: adult; preparations: pinned; catalogNumber: DHJPAR0029760; occurrenceDetails: http://janzen.sas.upenn.edu; recordedBy: D.H. Janzen, W. Hallwachs \& Guillermo Pereira; otherCatalogNumbers: ASHYM1181-09, 08-SRNP-14561, BOLD:AAA1577; language: en; institutionCode: CNC; collectionCode: Insects; basisOfRecord: Pinned Specimen

cd. $\quad$ scientificName: Hyphantrophaga virilis; phylum: Arthropoda; class: Insecta; order: Diptera; family: Tachinidae; genus: Hyphantrophaga; specificEpithet: virilis; scientificNameAuthorship: (Aldrich \& Webber, 1924); country: Costa Rica; countryCode: CR; stateProvince: Guanacaste; county: Sector Santa Rosa; locality: 
Area de Conservacion Guanacaste; verbatimLocality: Bosque San Emilio; verbatimElevation: 300; verbatimLatitude: 10.8439; verbatimLongitude: -85.6138; verbatimCoordinateSystem: Decimal; decimalLatitude: 10.8439; decimalLongitude: -85.6138; samplingProtocol: Reared from the larva of the Notodontidae, Elasmia mandela; verbatimEventDate: 24-Aug-2008; individualID: DHJPAR0029763; individualCount: 1; sex: female; lifeStage: adult; preparations: pinned; catalogNumber: DHJPAR0029763; occurrenceDetails: http://janzen.sas.upenn.edu; recordedBy: D.H. Janzen, W. Hallwachs \& Guillermo Pereira; otherCatalogNumbers: ASHYM1184-09, 08-SRNP-14574, BOLD:AAA1577; language: en; institutionCode: CNC; collectionCode: Insects; basisOfRecord: Pinned Specimen

ce. scientificName: Hyphantrophaga virilis; phylum: Arthropoda; class: Insecta; order: Diptera; family: Tachinidae; genus: Hyphantrophaga; specificEpithet: virilis; scientificNameAuthorship: (Aldrich \& Webber, 1924); country: Costa Rica; countryCode: CR; stateProvince: Guanacaste; county: Sector Santa Rosa; locality: Area de Conservacion Guanacaste; verbatimLocality: Bosque San Emilio; verbatimElevation: 300; verbatimLatitude: 10.8439; verbatimLongitude: -85.6138; verbatimCoordinateSystem: Decimal; decimalLatitude: 10.8439; decimalLongitude: -85.6138; samplingProtocol: Reared from the larva of the Notodontidae, Elasmia mandela; verbatimEventDate: 24-Aug-2008; individualID: DHJPAR0029764; individualCount: 1; sex: female; lifeStage: adult; preparations: pinned; catalogNumber: DHJPAR0029764; occurrenceDetails: http://janzen.sas.upenn.edu; recordedBy: D.H. Janzen, W. Hallwachs \& Guillermo Pereira; otherCatalogNumbers: ASHYM1185-09, 08-SRNP-14572, BOLD:AAA1577; language: en; institutionCode: CNC; collectionCode: Insects; basisOfRecord: Pinned Specimen

cf. ScientificName: Hyphantrophaga virilis; phylum: Arthropoda; class: Insecta; order: Diptera; family: Tachinidae; genus: Hyphantrophaga; specificEpithet: virilis; scientificNameAuthorship: (Aldrich \& Webber, 1924); country: Costa Rica; countryCode: CR; stateProvince: Guanacaste; county: Sector Santa Rosa; locality: Area de Conservacion Guanacaste; verbatimLocality: Bosque San Emilio; verbatimElevation: 300; verbatimLatitude: 10.8439; verbatimLongitude: -85.6138; verbatimCoordinateSystem: Decimal; decimalLatitude: 10.8439 ; decimalLongitude: -85.6138; samplingProtocol: Reared from the larva of the Notodontidae, Elasmia mandela; verbatimEventDate: 24-Aug-2008; individualID: DHJPAR0029765; individualCount: 1; sex: female; lifeStage: adult; preparations: pinned; catalogNumber: DHJPAR0029765; occurrenceDetails: http://janzen.sas.upenn.edu; recordedBy: D.H. Janzen, W. Hallwachs \& Guillermo Pereira; otherCatalog Numbers: ASHYM1186-09, 08-SRNP-14584, BOLD:AAA1577; language: en; institutionCode: CNC; collectionCode: Insects; basisOfRecord: Pinned Specimen

cg. ScientificName: Hyphantrophaga virilis; phylum: Arthropoda; class: Insecta; order: Diptera; family: Tachinidae; genus: Hyphantrophaga; specificEpithet: virilis; scientificNameAuthorship: (Aldrich \& Webber, 1924); country: Costa Rica; countryCode: CR; stateProvince: Guanacaste; county: Sector Santa Rosa; locality: Area de Conservacion Guanacaste; verbatimLocality: Bosque San Emilio; verbatimElevation: 300; verbatimLatitude: 10.8439; verbatimLongitude: -85.6138; verbatimCoordinateSystem: Decimal; decimalLatitude: 10.8439 ; decimalLongitude: -85.6138; samplingProtocol: Reared from the larva of the Notodontidae, Boriza tonac; verbatimEventDate: 24-Aug-2008; individualID: DHJPAR0029769; individualCount: 1; sex: female; lifeStage: adult; preparations: pinned; catalogNumber: DHJPAR0029769; occurrenceDetails: http://janzen.sas.upenn.edu; recordedBy: D.H. Janzen, W. Hallwachs \& Guillermo Pereira; otherCatalogNumbers: ASHYM1190-09, 08- 
SRNP-14439, BOLD:AAA1577; language: en; institutionCode: CNC; collectionCode: Insects; basisOfRecord: Pinned Specimen

ch. ScientificName: Hyphantrophaga virilis; phylum: Arthropoda; class: Insecta; order: Diptera; family: Tachinidae; genus: Hyphantrophaga; specificEpithet: virilis; scientificNameAuthorship: (Aldrich \& Webber, 1924); country: Costa Rica; countryCode: CR; stateProvince: Guanacaste; county: Sector Santa Rosa; locality: Area de Conservacion Guanacaste; verbatimLocality: Sendero Natural; verbatimElevation: 290; verbatimLatitude: 10.8357; verbatimLongitude: -85.6125; verbatimCoordinateSystem: Decimal; decimalLatitude: 10.8357; decimalLongitude: -85.6125; samplingProtocol: Reared from the larva of the Notodontidae, Boriza tonac; verbatimEventDate: 24-Aug-2008; individualID: DHJPAR0029770; individualCount: 1; sex: female; lifeStage: adult; preparations: pinned; catalogNumber: DHJPAR0029770; occurrenceDetails: http://janzen.sas.upenn.edu; recordedBy: D.H. Janzen, W. Hallwachs \& Guillermo Pereira; otherCatalogNumbers: ASHYM1191-09, 08SRNP-14594, BOLD:AAA1577; language: en; institutionCode: CNC; collectionCode: Insects; basisOfRecord: Pinned Specimen

ci. $\quad$ scientificName: Hyphantrophaga virilis; phylum: Arthropoda; class: Insecta; order: Diptera; family: Tachinidae; genus: Hyphantrophaga; specificEpithet: virilis; scientificNameAuthorship: (Aldrich \& Webber, 1924); country: Costa Rica; countryCode: CR; stateProvince: Guanacaste; county: Sector Santa Rosa; locality: Area de Conservacion Guanacaste; verbatimLocality: Bosque San Emilio; verbatimElevation: 300; verbatimLatitude: 10.8439; verbatimLongitude: -85.6138; verbatimCoordinateSystem: Decimal; decimalLatitude: 10.8439; decimalLongitude: -85.6138; samplingProtocol: Reared from the larva of the Notodontidae, Elasmia mandela; verbatimEventDate: 24-Aug-2008; individualID: DHJPAR0029773; individualCount: 1; sex: female; lifeStage: adult; preparations: pinned; catalogNumber DHJPAR0029773; occurrenceDetails: http://janzen.sas.upenn.edu; recordedBy: D.H. Janzen, W. Hallwachs \& Guillermo Pereira; otherCatalogNumbers: ASHYM1194-09, 08-SRNP-14576, BOLD:AAA1577; language: en; institutionCode: CNC; collectionCode: Insects; basisOfRecord: Pinned Specimen

cj. ScientificName: Hyphantrophaga virilis; phylum: Arthropoda; class: Insecta; order: Diptera; family: Tachinidae; genus: Hyphantrophaga; specificEpithet: virilis; scientificNameAuthorship: (Aldrich \& Webber, 1924); country: Costa Rica; countryCode: CR; stateProvince: Guanacaste; county: Sector Santa Rosa; locality: Area de Conservacion Guanacaste; verbatimLocality: Sendero Natural; verbatimElevation: 290; verbatimLatitude: 10.8357; verbatimLongitude: -85.6125; verbatimCoordinateSystem: Decimal; decimalLatitude: 10.8357 ; decimalLongitude: -85.6125; samplingProtocol: Reared from the larva of the Notodontidae, Boriza tonac; verbatimEventDate: 25-Aug-2008; individualID: DHJPAR0029774; individualCount: 1; sex: female; lifeStage: adult; preparations: pinned; catalogNumber: DHJPAR0029774; occurrenceDetails: http://janzen.sas.upenn.edu; recordedBy: D.H. Janzen, W. Hallwachs \& Guillermo Pereira; otherCatalogNumbers: ASHYM1195-09, 08SRNP-14600, BOLD:AAA1577; language: en; institutionCode: CNC; collectionCode: Insects; basisOfRecord: Pinned Specimen

ck. ScientificName: Hyphantrophaga virilis; phylum: Arthropoda; class: Insecta; order: Diptera; family: Tachinidae; genus: Hyphantrophaga; specificEpithet: virilis; scientificNameAuthorship: (Aldrich \& Webber, 1924); country: Costa Rica; countryCode: CR; stateProvince: Guanacaste; county: Sector Santa Rosa; locality: Area de Conservacion Guanacaste; verbatimLocality: Bosque San Emilio; verbatimElevation: 300; verbatimLatitude: 10.8439; verbatimLongitude: -85.6138; 
verbatimCoordinateSystem: Decimal; decimalLatitude: 10.8439; decimalLongitude: -85.6138; samplingProtocol: Reared from the larva of the Notodontidae, Boriza tonac; verbatimEventDate: 26-Aug-2008; individualID: DHJPAR0029778; individualCount: 1; sex: female; lifeStage: adult; preparations: pinned; catalogNumber: DHJPAR0029778; occurrenceDetails: http://janzen.sas.upenn.edu; recordedBy: D.H. Janzen, W.

Hallwachs \& Johan Vargas; otherCatalogNumbers: ASHYM1199-09, 08-SRNP-14553, BOLD:AAA1577; language: en; institutionCode: CNC; collectionCode: Insects; basisOfRecord: Pinned Specimen

cl. $\quad$ scientificName: Hyphantrophaga virilis; phylum: Arthropoda; class: Insecta; order: Diptera; family: Tachinidae; genus: Hyphantrophaga; specificEpithet: virilis; scientificNameAuthorship: (Aldrich \& Webber, 1924); country: Costa Rica; countryCode: CR; stateProvince: Guanacaste; county: Sector Santa Rosa; locality: Area de Conservacion Guanacaste; verbatimLocality: Sendero Natural; verbatimElevation: 290; verbatimLatitude: 10.8357; verbatimLongitude: -85.6125; verbatimCoordinateSystem: Decimal; decimalLatitude: 10.8357 ; decimalLongitude: -85.6125; samplingProtocol: Reared from the larva of the Notodontidae, Boriza tonac; verbatimEventDate: 26-Aug-2008; individualID: DHJPAR0029779; individualCount: 1; sex: female; lifeStage: adult; preparations: pinned; catalog Number: DHJPAR0029779; occurrenceDetails: http://janzen.sas.upenn.edu; recordedBy: D.H. Janzen, W. Hallwachs \& Guillermo Pereira; otherCatalogNumbers: ASHYM1200-09, 08SRNP-14617, BOLD:AAA1577; language: en; institutionCode: CNC; collectionCode: Insects; basisOfRecord: Pinned Specimen

cm. ScientificName: Hyphantrophaga virilis; phylum: Arthropoda; class: Insecta; order: Diptera; family: Tachinidae; genus: Hyphantrophaga; specificEpithet: virilis; scientificNameAuthorship: (Aldrich \& Webber, 1924); country: Costa Rica; countryCode: CR; stateProvince: Guanacaste; county: Sector Santa Rosa; locality: Area de Conservacion Guanacaste; verbatimLocality: Bosque San Emilio; verbatimElevation: 300; verbatimLatitude: 10.8439; verbatimLongitude: -85.6138; verbatimCoordinateSystem: Decimal; decimalLatitude: 10.8439; decimalLongitude: -85.6138; samplingProtocol: Reared from the larva of the Notodontidae, Boriza tonac; verbatimEventDate: 27-Aug-2008; individualID: DHJPAR0029784; individualCount: 1; sex: female; lifeStage: adult; preparations: pinned; catalogNumber: DHJPAR0029784; occurrenceDetails: http://janzen.sas.upenn.edu; recordedBy: D.H. Janzen, W. Hallwachs \& Guillermo Pereira; otherCatalogNumbers: ASHYM1205-09, 08SRNP-14461, BOLD:AAA1577; language: en; institutionCode: CNC; collectionCode: Insects; basisOfRecord: Pinned Specimen

cn. ScientificName: Hyphantrophaga virilis; phylum: Arthropoda; class: Insecta; order; Diptera; family: Tachinidae; genus: Hyphantrophaga; specificEpithet: virilis; scientificNameAuthorship: (Aldrich \& Webber, 1924); country: Costa Rica; countryCode: CR; stateProvince: Guanacaste; county: Sector Santa Rosa; locality: Area de Conservacion Guanacaste; verbatimLocality: Sendero Natural; verbatimElevation: 290; verbatimLatitude: 10.8357; verbatimLongitude: -85.6125; verbatimCoordinateSystem: Decimal; decimalLatitude: 10.8357; decimalLongitude: -85.6125; samplingProtocol: Reared from the larva of the Notodontidae, Boriza tonac; verbatimEventDate: 29-Aug-2008; individualID: DHJPAR0029786; individualCount: 1; sex: female; lifeStage: adult; preparations: pinned; catalogNumber: DHJPAR0029786; occurrenceDetails: http://janzen.sas.upenn.edu; recordedBy: D.H. Janzen, W. Hallwachs \& Guillermo Pereira; otherCatalogNumbers: ASHYM1207-09, 08SRNP-14614, BOLD:AAA1577; language: en; institutionCode: CNC; collectionCode: Insects; basisOfRecord: Pinned Specimen 
co. ScientificName: Hyphantrophaga virilis; phylum: Arthropoda; class: Insecta; order: Diptera; family: Tachinidae; genus: Hyphantrophaga; specificEpithet: virilis; scientificNameAuthorship: (Aldrich \& Webber, 1924); country: Costa Rica; countryCode: CR; stateProvince: Guanacaste; county: Sector Santa Rosa; locality: Area de Conservacion Guanacaste; verbatimLocality: Bosque San Emilio; verbatimElevation: 300; verbatimLatitude: 10.8439; verbatimLongitude: -85.6138 ; verbatimCoordinateSystem: Decimal; decimalLatitude: 10.8439; decimalLongitude: -85.6138; samplingProtocol: Reared from the larva of the Notodontidae, Boriza tonac; verbatimEventDate: 29-Aug-2008; individualID: DHJPAR0029787; individualCount: 1; sex: female; lifeStage: adult; preparations: pinned; catalogNumber: DHJPAR0029787; occurrenceDetails: http://janzen.sas.upenn.edu; recordedBy: D.H. Janzen, W. Hallwachs \& Guillermo Pereira; otherCatalogNumbers: ASHYM1208-09, 08SRNP-14441, BOLD:AAA1577; language: en; institutionCode: CNC; collectionCode: Insects; basisOfRecord: Pinned Specimen

cp. ScientificName: Hyphantrophaga virilis; phylum: Arthropoda; class: Insecta; order: Diptera; family: Tachinidae; genus: Hyphantrophaga; specificEpithet: virilis; scientificNameAuthorship: (Aldrich \& Webber, 1924); country: Costa Rica; countryCode: CR; stateProvince: Guanacaste; county: Sector Santa Rosa; locality: Area de Conservacion Guanacaste; verbatimLocality: Bosque San Emilio;

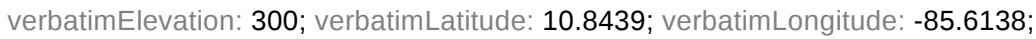
verbatimCoordinateSystem: Decimal; decimalLatitude: 10.8439; decimalLongitude: -85.6138; samplingProtocol: Reared from the larva of the Notodontidae, Boriza tonac; verbatimEventDate: 31-Aug-2008; individualID: DHJPAR0029790; individualCount: 1; sex: female; lifeStage: adult; preparations: pinned; catalogNumber: DHJPAR0029790; occurrenceDetails: http://janzen.sas.upenn.edu; recordedBy: D.H. Janzen, W. Hallwachs \& Guillermo Pereira; otherCatalogNumbers: ASHYM1211-09, 08SRNP-14456, BOLD:AAA1577; language: en; institutionCode: CNC; collectionCode: Insects; basisOfRecord: Pinned Specimen

cq. ScientificName: Hyphantrophaga virilis; phylum: Arthropoda; class: Insecta; order: Diptera; family: Tachinidae; genus: Hyphantrophaga; specificEpithet: virilis; scientificNameAuthorship: (Aldrich \& Webber, 1924); country: Costa Rica; countryCode: CR; stateProvince: Guanacaste; county: Sector Santa Rosa; locality: Area de Conservacion Guanacaste; verbatimLocality: Bosque San Emilio; verbatimElevation: 300; verbatimLatitude: 10.8439; verbatimLongitude: -85.6138; verbatimCoordinateSystem: Decimal; decimalLatitude: 10.8439; decimalLongitude: -85.6138; samplingProtocol: Reared from the larva of the Megalopygidae, Megalopygidae 82-SRNP-848; individualID: DHJPAR0029794; individualCount: 1; sex: female; lifeStage: adult; preparations: pinned; catalogNumber: DHJPAR0029794; occurrenceDetails: http://janzen.sas.upenn.edu; recordedBy: D.H. Janzen, W. Hallwachs \& Lucia Vargas; otherCatalogNumbers: ASHYM1215-09, 08-SRNP-14753, BOLD:AAA1577; language: en; institutionCode: CNC; collectionCode: Insects; basisOfRecord: Pinned Specimen

cr. ScientificName: Hyphantrophaga virilis; phylum: Arthropoda; class: Insecta; order: Diptera; family: Tachinidae; genus: Hyphantrophaga; specificEpithet: virilis; scientificNameAuthorship: (Aldrich \& Webber, 1924); country: Costa Rica; countryCode: CR; stateProvince: Guanacaste; county: Sector Santa Rosa; locality: Area de Conservacion Guanacaste; verbatimLocality: Bosque Humedo; verbatimElevation: 290; verbatimLatitude: 10.8514; verbatimLongitude: -85.608; verbatimCoordinateSystem: Decimal; decimalLatitude: 10.8514; decimalLongitude: -85.608; samplingProtocol: Reared from the larva of the Nymphalidae, Mechanitis 
isthmia; verbatimEventDate: 16-Jan-2009; individualID: DHJPAR0030064; individualCount: 1; sex: female; lifeStage: adult; preparations: pinned; catalogNumber: DHJPAR0030064; occurrenceDetails: http://janzen.sas.upenn.edu; recordedBy: D.H. Janzen, W. Hallwachs \& Johan Vargas; otherCatalogNumbers: ASHYB808-09, 08SRNP-16977, BOLD:AAA1577; language: en; institutionCode: CNC; collectionCode: Insects; basisOfRecord: Pinned Specimen

cs. $\quad$ scientificName: Hyphantrophaga virilis; phylum: Arthropoda; class: Insecta; order: Diptera; family: Tachinidae; genus: Hyphantrophaga; specificEpithet: virilis; scientificNameAuthorship: (Aldrich \& Webber, 1924); country: Costa Rica; countryCode: CR; stateProvince: Guanacaste; county: Sector Santa Rosa; locality: Area de Conservacion Guanacaste; verbatimLocality: Bosque Humedo; verbatimElevation: 290; verbatimLatitude: 10.8514; verbatimLongitude: -85.608; verbatimCoordinateSystem: Decimal; decimalLatitude: 10.8514; decimalLongitude: -85.608; samplingProtocol: Reared from the larva of the Nolidae, Iscadia Poole05; verbatimEventDate: 14-Jan-2009; individualID: DHJPAR0030073; individualCount: 1; sex: female; lifeStage: adult; preparations: pinned; catalog Number: DHJPAR0030073; occurrenceDetails: http://janzen.sas.upenn.edu; recordedBy: D.H. Janzen, W. Hallwachs \& Johan Vargas; otherCatalogNumbers: ASHYB817-09, 08-SRNP-16963, BOLD:AAA1577; language: en; institutionCode: CNC; collectionCode: Insects; basisOfRecord: Pinned Specimen

ct. $\quad$ scientificName: Hyphantrophaga virilis; phylum: Arthropoda; class: Insecta; order: Diptera; family: Tachinidae; genus: Hyphantrophaga; specificEpithet: virilis; scientificNameAuthorship: (Aldrich \& Webber, 1924); country: Costa Rica; countryCode: CR; stateProvince: Alajuela; county: Sector San Cristobal; locality: Area de Conservacion Guanacaste; verbatimLocality: Quebrada Garcia; verbatimElevation: 495; verbatimLatitude: 10.8607; verbatimLongitude: -85.4256 ; verbatimCoordinateSystem: Decimal; decimalLatitude: 10.8607; decimalLongitude: -85.4256; samplingProtocol: Reared from the larva of the Tortricidae, Anacrusis nephrodes; verbatimEventDate: 23-Feb-2009; individualID: DHJPAR0030464; individualCount: 1; sex: female; lifeStage: adult; preparations: pinned; catalog Number: DHJPAR0030464; occurrenceDetails: http://janzen.sas.upenn.edu; recordedBy: D.H. Janzen, W. Hallwachs \& Elda Araya; otherCatalogNumbers: ASHYB1207-09, 09SRNP-112, BOLD:AAA1577; language: en; institutionCode: CNC; collectionCode: Insects; basisOfRecord: Pinned Specimen

cu. $\quad$ scientificName: Hyphantrophaga virilis; phylum: Arthropoda; class: Insecta; order: Diptera; family: Tachinidae; genus: Hyphantrophaga; specificEpithet: virilis; scientificNameAuthorship: (Aldrich \& Webber, 1924); country: Costa Rica; countryCode: CR; stateProvince: Guanacaste; county: Sector Cacao; locality: Area de Conservacion Guanacaste; verbatimLocality: Sendero Arenales; verbatimElevation: 1080; verbatimLatitude: 10.9247; verbatimLongitude: -85.4674 ; verbatimCoordinateSystem: Decimal; decimalLatitude: 10.9247 ; decimalLongitude: -85.4674; samplingProtocol: Reared from the larva of the Geometridae, geometrid 02-8481; verbatimEventDate: 22-May-2009; individualID: DHJPAR0034373;

individualCount: 1; sex: female; lifeStage: adult; preparations: pinned; catalogNumber: DHJPAR0034373; occurrenceDetails: http://janzen.sas.upenn.edu; recordedBy: D.H. Janzen, W. Hallwachs \& Manuel Pereira; otherCatalogNumbers: ASHYC1025-09, 09SRNP-35335, BOLD:AAA1577; language: en; institutionCode: CNC; collectionCode: Insects; basisOfRecord: Pinned Specimen

cv. ScientificName: Hyphantrophaga virilis; phylum: Arthropoda; class: Insecta; order: Diptera; family: Tachinidae; genus: Hyphantrophaga; specificEpithet: virilis; 
scientificNameAuthorship: (Aldrich \& Webber, 1924); country: Costa Rica; countryCode: CR; stateProvince: Guanacaste; county: Sector Pailas; locality: Area de Conservacion Guanacaste; verbatimLocality: Gemelos; verbatimElevation: 1276; verbatimLatitude: 10.7693; verbatimLongitude: -85.3466; verbatimCoordinateSystem: Decimal; decimalLatitude: 10.7693; decimalLongitude: -85.3466; samplingProtocol: Reared from the larva of the Crambidae, same as 06-21595; verbatimEventDate: 05Jul-2009; individualID: DHJPAR0035671; individualCount: 1; sex: female; lifeStage: adult; preparations: pinned; catalogNumber: DHJPAR0035671; occurrenceDetails: htt p://janzen.sas.upenn.edu; recordedBy: D.H. Janzen, W. Hallwachs \& Daniel M.Acuna; otherCatalogNumbers: ASHYD1052-09, 09-SRNP-56316, BOLD:AAA1577; language: en; institutionCode: CNC; collectionCode: Insects; basisOfRecord: Pinned Specimen

cw. ScientificName: Hyphantrophaga virilis; phylum: Arthropoda; class: Insecta; order: Diptera; family: Tachinidae; genus: Hyphantrophaga; specificEpithet: virilis; scientificNameAuthorship: (Aldrich \& Webber, 1924); country: Costa Rica; countryCode: CR; stateProvince: Guanacaste; county: Sector Santa Rosa; locality: Area de Conservacion Guanacaste; verbatimLocality: Area Administrativa; verbatimElevation: 295; verbatimLatitude: 10.8376; verbatimLongitude: -85.6187; verbatimCoordinateSystem: Decimal; decimalLatitude: 10.8376; decimalLongitude: -85.6187; samplingProtocol: Reared from the larva of the Nymphalidae, Lycorea atergatis; verbatimEventDate: 02-Sep-2009; individualID: DHJPAR0035675; individualCount: 1; sex: female; lifeStage: adult; preparations: pinned; catalogNumber: DHJPAR0035675; occurrenceDetails: http://janzen.sas.upenn.edu; recordedBy: D.H. Janzen, W. Hallwachs \& Winnie Hallwachs; otherCatalog Numbers: ASHYD1056-09, 09-SRNP-14232, BOLD:AAA1577; language: en; institutionCode: CNC; collectionCode: Insects; basisOfRecord: Pinned Specimen

cx. ScientificName: Hyphantrophaga virilis; phylum: Arthropoda; class: Insecta; order: Diptera; family: Tachinidae; genus: Hyphantrophaga; specificEpithet: virilis; scientificNameAuthorship: (Aldrich \& Webber, 1924); country: Costa Rica; countryCode: CR; stateProvince: Guanacaste; county: Sector Cacao; locality: Area de Conservacion Guanacaste; verbatimLocality: Sendero Arenales; verbatimElevation: 1080; verbatimLatitude: 10.9247; verbatimLongitude: -85.4674 ; verbatimCoordinateSystem: Decimal; decimalLatitude: 10.9247 ; decimalLongitude: -85.4674; samplingProtocol: Reared from the larva of the Geometridae, geometrid 02-8481; verbatimEventDate: 30-May-2009; individualID: DHJPAR0036643; individualCount: 1; sex: female; lifeStage: adult; preparations: pinned; catalog Number: DHJPAR0036643; occurrenceDetails: http://janzen.sas.upenn.edu; recordedBy: D.H. Janzen, W. Hallwachs \& Manuel Pereira; otherCatalogNumbers: ASHYE1554-09, 09SRNP-35330, BOLD:AAA1577; language: en; institutionCode: CNC; collectionCode: Insects; basisOfRecord: Pinned Specimen

cy. $\quad$ scientificName: Hyphantrophaga virilis; phylum: Arthropoda; class: Insecta; order: Diptera; family: Tachinidae; genus: Hyphantrophaga; specificEpithet: virilis; scientificNameAuthorship: (Aldrich \& Webber, 1924); country: Costa Rica; countryCode: CR; stateProvince: Guanacaste; county: Sector Cacao; locality: Area de Conservacion Guanacaste; verbatimLocality: Sendero Nayo; verbatimElevation: 1090; verbatimLatitude: 10.9245 ; verbatimLongitude: -85.4695 ; verbatimCoordinateSystem: Decimal; decimalLatitude: 10.9245; decimalLongitude: -85.4695; samplingProtocol: Reared from the larva of the Geometridae, Macaria pernicata; verbatimEventDate: 26 Jun-2009; individualID: DHJPAR0036649; individualCount: 1; sex: female; lifeStage: adult; preparations: pinned; catalogNumber: DHJPAR0036649; occurrenceDetails: $\underline{\text { htt }}$ p://janzen.sas.upenn.edu; recordedBy: D.H. Janzen, W. Hallwachs \& Dunia Garcia; 
otherCatalog Numbers: ASHYE1560-09, 09-SRNP-35968, BOLD:AAA1577; language: en; institutionCode: CNC; collectionCode: Insects; basisOfRecord: Pinned Specimen

Cz. scientificName: Hyphantrophaga virilis; phylum: Arthropoda; class: Insecta; order: Diptera; family: Tachinidae; genus: Hyphantrophaga; specificEpithet: virilis; scientificNameAuthorship: (Aldrich \& Webber, 1924); country: Costa Rica; countryCode: CR; stateProvince: Guanacaste; county: Sector Cacao; locality: Area de Conservacion Guanacaste; verbatimLocality: Sendero Nayo; verbatimElevation: 1090; verbatimLatitude: 10.9245; verbatimLongitude: -85.4695 ; verbatimCoordinateSystem: Decimal; decimalLatitude: 10.9245 ; decimalLongitude: -85.4695; samplingProtocol: Reared from the larva of the Geometridae, Macaria pernicata; verbatimEventDate: 26Jun-2009; individualID: DHJPAR0036650; individualCount: 1; sex: female; lifeStage: adult; preparations: pinned; catalogNumber: DHJPAR0036650; occurrenceDetails: $\underline{\mathrm{htt}}$ p://janzen.sas.upenn.edu; recordedBy: D.H. Janzen, W. Hallwachs \& Dunia Garcia; otherCatalog Numbers: ASHYE1561-09, 09-SRNP-35962, BOLD:AAA1577; language: en; institutionCode: CNC; collectionCode: Insects; basisOfRecord: Pinned Specimen

da. scientificName: Hyphantrophaga virilis; phylum: Arthropoda; class: Insecta; order: Diptera; family: Tachinidae; genus: Hyphantrophaga; specificEpithet: virilis; scientificNameAuthorship: (Aldrich \& Webber, 1924); country: Costa Rica; countryCode: CR; stateProvince: Guanacaste; county: Sector Cacao; locality: Area de Conservacion Guanacaste; verbatimLocality: Sendero Nayo; verbatimElevation: 1090; verbatimLatitude: 10.9245; verbatimLongitude: -85.4695 ; verbatimCoordinateSystem: Decimal; decimalLatitude: 10.9245; decimalLongitude: -85.4695; samplingProtocol: Reared from the larva of the Geometridae, Macaria pernicata; verbatimEventDate: $27-$ Jun-2009; individualID: DHJPAR0036653; individualCount: 1; sex: female; lifeStage: adult; preparations: pinned; catalogNumber: DHJPAR0036653; occurrenceDetails: htt p://janzen.sas.upenn.edu; recordedBy: D.H. Janzen, W. Hallwachs \& Dunia Garcia; otherCatalogNumbers: ASHYE1564-09, 09-SRNP-35956, BOLD:AAA1577; language: en; institutionCode: CNC; collectionCode: Insects; basisOfRecord: Pinned Specimen

db. ScientificName: Hyphantrophaga virilis; phylum: Arthropoda; class: Insecta; order: Diptera; family: Tachinidae; genus: Hyphantrophaga; specificEpithet: virilis; scientificNameAuthorship: (Aldrich \& Webber, 1924); country: Costa Rica; countryCode: CR; stateProvince: Guanacaste; county: Sector Cacao; locality: Area de Conservacion Guanacaste; verbatimLocality: Sendero Nayo; verbatimElevation: 1090; verbatimLatitude: 10.9245 ; verbatimLongitude: -85.4695 ; verbatimCoordinateSystem: Decimal; decimalLatitude: 10.9245; decimalLongitude: -85.4695; samplingProtocol: Reared from the larva of the Geometridae, Macaria pernicata; verbatimEventDate: 28 Jun-2009; individualID: DHJPAR0036656; individualCount: 1; sex: female; lifeStage: adult; preparations: pinned; catalogNumber: DHJPAR0036656; occurrenceDetails: htt p://janzen.sas.upenn.edu; recordedBy: D.H. Janzen, W. Hallwachs \& Manuel Pereira; otherCatalog Numbers: ASHYE1567-09, 09-SRNP-35922, BOLD:AAA1577; language: en; institutionCode: CNC; collectionCode: Insects; basisOfRecord: Pinned Specimen

dc. scientificName: Hyphantrophaga virilis; phylum: Arthropoda; class: Insecta; order: Diptera; family: Tachinidae; genus: Hyphantrophaga; specificEpithet: virilis; scientificNameAuthorship: (Aldrich \& Webber, 1924); country: Costa Rica; countryCode: CR; stateProvince: Guanacaste; county: Sector Cacao; locality: Area de Conservacion Guanacaste; verbatimLocality: Sendero Nayo; verbatimElevation: 1090; verbatimLatitude: 10.9245 ; verbatimLongitude: -85.4695 ; verbatimCoordinateSystem: Decimal; decimalLatitude: 10.9245; decimalLongitude: -85.4695; samplingProtocol: Reared from the larva of the Geometridae, Macaria pernicata; verbatimEventDate: 26 Jun-2009; individualID: DHJPAR0036657; individualCount: 1; sex: female; lifeStage: 
adult; preparations: pinned; catalogNumber: DHJPAR0036657; occurrenceDetails: $\underline{\text { htt }}$ p://janzen.sas.upenn.edu; recordedBy: D.H. Janzen, W. Hallwachs \& Dunia Garcia; otherCatalog Numbers: ASHYE1568-09, 09-SRNP-35951, BOLD:AAA1577; language: en; institutionCode: CNC; collectionCode: Insects; basisOfRecord: Pinned Specimen

dd. scientificName: Hyphantrophaga virilis; phylum: Arthropoda; class: Insecta; order: Diptera; family: Tachinidae; genus: Hyphantrophaga; specificEpithet: virilis; scientificNameAuthorship: (Aldrich \& Webber, 1924); country: Costa Rica; countryCode: CR; stateProvince: Guanacaste; county: Sector Cacao; locality: Area de Conservacion Guanacaste; verbatimLocality: Sendero Nayo; verbatimElevation: 1090; verbatimLatitude: 10.9245 ; verbatimLongitude: -85.4695 ; verbatimCoordinateSystem: Decimal; decimalLatitude: 10.9245; decimalLongitude: -85.4695; samplingProtocol: Reared from the larva of the Geometridae, Macaria pernicata; verbatimEventDate: 30 Jun-2009; individualID: DHJPAR0036658; individualCount: 1; sex: female; lifeStage: adult; preparations: pinned; catalogNumber: DHJPAR0036658; occurrenceDetails: htt p://janzen.sas.upenn.edu; recordedBy: D.H. Janzen, W. Hallwachs \& Manuel Pereira; otherCatalogNumbers: ASHYE1569-09, 09-SRNP-35939, BOLD:AAA1577; language: en; institutionCode: CNC; collectionCode: Insects; basisOfRecord: Pinned Specimen

de. $\quad$ scientificName: Hyphantrophaga virilis; phylum: Arthropoda; class: Insecta; order: Diptera; family: Tachinidae; genus: Hyphantrophaga; specificEpithet: virilis; scientificNameAuthorship: (Aldrich \& Webber, 1924); country: Costa Rica; countryCode: CR; stateProvince: Guanacaste; county: Sector Cacao; locality: Area de Conservacion Guanacaste; verbatimLocality: Sendero Nayo; verbatimElevation: 1090; verbatimLatitude: 10.9245; verbatimLongitude: -85.4695 ; verbatimCoordinateSystem: Decimal; decimalLatitude: 10.9245; decimalLongitude: -85.4695; samplingProtocol: Reared from the larva of the Geometridae, Macaria pernicata; verbatimEventDate: 26Jun-2009; individualID: DHJPAR0036659; individualCount: 1; sex: female; lifeStage: adult; preparations: pinned; catalogNumber: DHJPAR0036659; occurrenceDetails: htt p://janzen.sas.upenn.edu; recordedBy: D.H. Janzen, W. Hallwachs \& Dunia Garcia; otherCatalogNumbers: ASHYE1570-09, 09-SRNP-35952, BOLD:AAA1577; language: en; institutionCode: CNC; collectionCode: Insects; basisOfRecord: Pinned Specimen

df. scientificName: Hyphantrophaga virilis; phylum: Arthropoda; class: Insecta; order: Diptera; family: Tachinidae; genus: Hyphantrophaga; specificEpithet: virilis; scientificNameAuthorship: (Aldrich \& Webber, 1924); country: Costa Rica; countryCode: CR; stateProvince: Guanacaste; county: Sector Mundo Nuevo; locality: Area de Conservacion Guanacaste; verbatimLocality: Estacion La Perla; verbatimElevation: 325 ; verbatimLatitude: 10.7674; verbatimLongitude: -85.4331; verbatimCoordinateSystem: Decimal; decimalLatitude: 10.7674 ; decimalLongitude: -85.4331; samplingProtocol: Reared from the larva of the Crambidae, Syllepte belialis; verbatimEventDate: 17-Jun-2010; individualID: DHJPAR0039307; individualCount: 1; sex: female; lifeStage: adult; preparations: pinned; catalogNumber: DHJPAR0039307; occurrenceDetails: http://janzen.sas.upenn.edu; recordedBy: D.H. Janzen, W. Hallwachs \& Mariano Pereira; otherCatalogNumbers: ASTAV870-10, 10-SRNP-55350, BOLD:AAA1577; language: en; institutionCode: CNC; collectionCode: Insects; basisOfRecord: Pinned Specimen

\section{Description}

Male (Fig. 26). Length: 6-10 mm. Head (Fig. 26b): vertex 1/5 of head width; two reclinate upper orbital setae; ocellar setae arising beside anterior ocellus; ocellar 
triangle gold, concolorous with fronto-orbital plate; fronto-orbital plate gold over $80 \%$ of surface, sparsely setulose, setulae not extending beyond lowest frontal seta; parafacial shiny silver and bare; facial ridge bare; eye with short sparse ommatrichia up to $2 \mathrm{X}$ as long as one ommatidium; pedicel black, concolorous with postpedicel; arista brown, very minutely pubescent, distinctly thickened on basal 1/3-1/4; palpus yellow and haired. Thorax (Fig. 26a, c): brilliant gold tomentose dorsally, grey tomentose laterally; four prominent dorsal vittae, outermost two broken across suture, innermost pair unbroken across suture, not reaching 2nd postsutural dorsocentral seta; postpronotum with three setae arranged in a triangle; chaetotaxy: acrostichal setae 3:3; dorsocentral setae 3:3; intra-alar setae 2:3; supra-alar setae 2:3; two katepisternal setae; lateral scutellar setae $2 / 3$ as long as basal scutellar setae, lateral scutellar setae $1 / 2$ as thick as both basal and subapical scutellar setae; subapical scutellar setae subequal to basal scutellar setae; apical scutellar setae 1/2 length of subapical scutellar setae, convergent but not crossed apically; 1-2 pairs of discal scutellar setae; scutellum with two dark grey bilateral crescents along basal 10\%, remainder concolorous with scutum. Legs (Fig. 26c): yellow in ground colour with dense covering of black hairs, making them appear darker; fore femur with dense silver tomentum on posterodorsal surface; hind coxa setose. Wing (Fig. 26a): pale translucent, veins yellow; vein $R_{4+5}$ with 1-2 setulae at base. Abdomen (Fig. 26a, c): ground colour black; mid-dorsal depression on ST1+2 reaching hind margin; median marginal setae present on ST1+2-T3; a complete row of marginal setae present on T4; discal setae present on T3-T5; sex patch present on T4; ST1+2 black; distinct gold tomentose bands along anterior 9/10 of T3-T5; T3 silver tomentose ventrally; T5 gold tomentose ventrally. Terminalia (Fig. 26d, e, f): anterior margin of sternite 5 (Fig. 26f) with a shallow curved medial depression, posterior margin with a deeply excavated pointed U-shaped medial cleft; posterior lobes of sternite rounded triangular apically, with many short setulae; unsclerotised "window" absent. Cerci in posterior view (Fig. 26d) long and slender, together appearing as a narrow isosceles triangle and very slightly longer than surstyli, blunt at apex, completely separate medially and straight, not strongly divergent from each other; in lateral view strongly tapered along anterior 1/3 with a strong basal hump; densely setulose along dorsal 4/5, apparently bare ventrally. Surstylus in lateral view (Fig. 26e) almost parallel-sided along its length, with rounded apices, blade-like; when viewed dorsally, surstyli appearing slender and straight, not divergent, entirely covered in short, stout spines. Pregonite broad and well-developed, slightly bent; basal 2/3 slightly cinched, giving it a very slightly clubbed appearance, apically rounded and bare. Postgonite elongate, equallly wide along its length, 1/2 as wide as pregonite, with a slight curve at tip, subequal in length to pregonite. Distiphallus rectangular, with a very slight apical flare, a slender median longitudinal sclerotised reinforcement on its posterior surface and a broad, anterolateral, sclerotised acrophallus, joined as a plate on anterior surface near apex. 


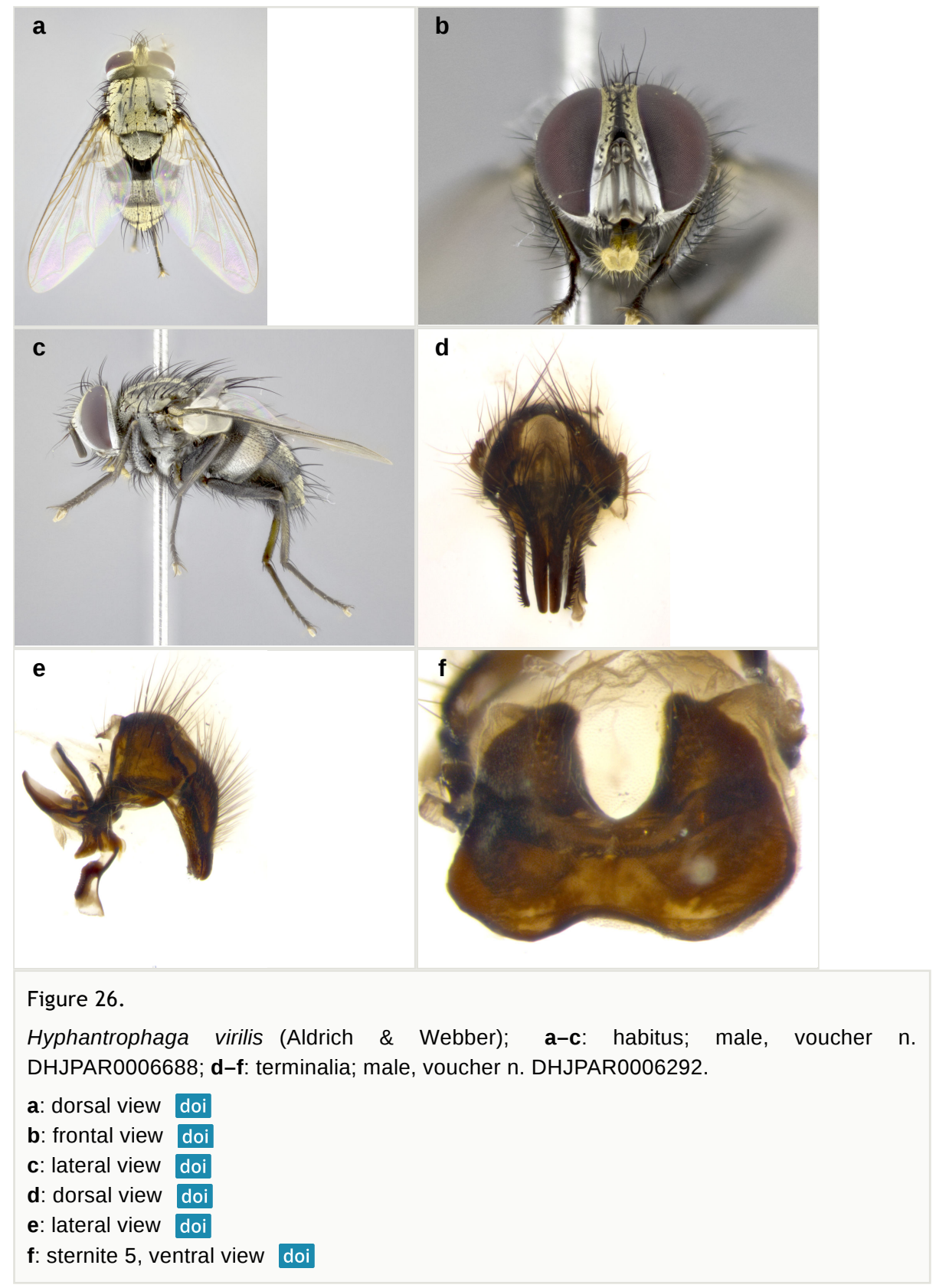

Female. Length: 6-10 $\mathrm{mm}$. As male, differing only by the presence of two pairs of proclinate orbital setae. 


\section{Diagnosis}

Hyphantrophaga virlis can be distinguished from all other Hyphantrophaga species by the following combination of traits: three postsutural dorsocentral setae, hind coxa setose, median marginal setae present on ST1+2 and discal setae present on T3-T5.

\section{Distribution}

From south-eastern Canada and north-eastern USA, west to Kansas and south to Costa Rica; Costa Rica, ACG (Provinces of Alajuela and Guanacaste), 2-1220 m elevation.

\section{Ecology}

Within the ACG inventory, Hyphantrophaga virilis has been reared 669 times from species spanning 30 families of Lepidoptera, as follows: one species in the family Apatelodidae; two species in the family Bombycidae; 18 species in the family Crambidae; four species in the family Depressariidae; 24 species in the family Erebidae; one species in the family Euteliidae; one species in the family Gelechiidae; 13 species in the family Geometridae; one species in the family Hedylidae; 64 species in the family Hesperiidae; one species in the family Immidae; two species in the family Lasiocampidae; one species in the family Limacodidae; two species in the family Megalopygidae; one species in the family Mimallonidae; 12 species in the family Noctuidae; four species in the family Nolidae; 24 species in the family Notodontidae; 18 species in the family Nymphalidae; three species in the family Papilionidae; one species in the family Phiditiidae; eight species in the family Pieridae; one species in the family Pterophoridae; six species in the family Pyralidae; six species in the family Riodinidae; four species in the family Saturniidae; five species in the family Sphingidae; three species in the family Thyrididae; and one species in the family Tortricidae; in rain forest, dry forest and dry-rain lowland intergrade.

\section{Identification keys}

\section{Key to the species of Hyphantrophaga Townsend, of the Mesoamerican region}

This key was written, based on characters present in males; in cases where sexual dimorphism is present, the species keys to males and females separately. This key is confined to the Mesoamerican biogeographical region, defined as ranging from the Isthmus of Tehuantepec in the north to the Colombian border with Panama in the south.

\begin{tabular}{|l|l|l|}
\hline 1 & Hind coxa setose with at least 1 hair (Fig. 1b) & 2 \\
\hline- & Hind coxa bare & 18 \\
\hline
\end{tabular}




\begin{tabular}{|c|c|c|}
\hline 2 & $\begin{array}{l}\text { Tomentum on T5 bright gold, contrasting with tomentum of T1 } \\
+2-\mathrm{T} 4\end{array}$ & $\begin{array}{l}\text { Hyphantrophaga myersi } \\
\text { (Aldrich, 1933) }\end{array}$ \\
\hline- & Tomentum on T5 matching that on rest of abdominal tergites & 3 \\
\hline 3 & Discal setae present on T3-T5 & 4 \\
\hline- & Discal setae present only on T5 & 13 \\
\hline 4 & Thorax with 3 postsutural dorsocentral setae & $\begin{array}{l}\text { Hyphantrophaga virilis } \\
\text { (Aldrich \& Webber, } \\
\text { 1924) }\end{array}$ \\
\hline- & Thorax with 4 postsutural dorsocentral setae & 5 \\
\hline 5 & $\begin{array}{l}\text { Fronto-orbital plate silver tomentose; abdomen and thorax } \\
\text { entirely silver/grey tomentose }\end{array}$ & 6 \\
\hline- & $\begin{array}{l}\text { Fronto-orbital plate brassy/gold tomentose; abdomen and } \\
\text { thorax either with both silver and gold tomentum or brassy-grey } \\
\text { tomentose }\end{array}$ & 8 \\
\hline 6 & Pedicel bright orange; legs bright orange with black tarsi & $\begin{array}{l}\text { Hyphantrophaga } \\
\text { autographae (Sellers, } \\
\text { 1943) }\end{array}$ \\
\hline- & Pedicel black; legs dark brown/black & 7 \\
\hline 7 & $\begin{array}{l}\text { Transverse abdominal brassy tomentose bands along anterior } \\
\text { edges of T3-T5 unbroken medially, covering almost } 70 \% \text { of } \\
\text { tergites; T5 with silver tomentum throughout }\end{array}$ & $\begin{array}{l}\text { Hyphantrophaga } \\
\text { duniagarciae sp. } \mathbf{n} .\end{array}$ \\
\hline- & $\begin{array}{l}\text { Distinct brassy-grey tomentum covering all but posterior margin } \\
\text { of T3 and T4; T3 and T4 with darkened patches dorsolaterally, } \\
\text { patches concolorous with ST1+2; T5 with brassy-grey } \\
\text { tomentum throughout }\end{array}$ & $\begin{array}{l}\text { Hyphantrophaga } \\
\text { osvaldoespinozai sp. } \mathbf{n} \text {. }\end{array}$ \\
\hline 8 & Pedicel and arista orange-brown, lighter than postpedicel & 9 \\
\hline- & Pedicel and arista black, concolorous with postpedicel & 10 \\
\hline 9 & $\begin{array}{l}\text { Fronto-orbital plate and parafacial entirely gold; fronto-orbital } \\
\text { plate sparsely setulose along upper half }\end{array}$ & $\begin{array}{l}\text { Hyphantrophaga } \\
\text { blandoides Thompson, } \\
1963\end{array}$ \\
\hline- & $\begin{array}{l}\text { Fronto-orbital plate gold, parafacial silver; fronto-orbital plate } \\
\text { sparsely setulose, setulae extending to lowest frontal seta }\end{array}$ & $\begin{array}{l}\text { Hyphantrophaga } \\
\text { guillermopereirai sp. } \mathbf{n} \text {. }\end{array}$ \\
\hline
\end{tabular}




\begin{tabular}{|c|c|c|}
\hline 10 & Median pair of thoracic vittae interrupted at suture & $\begin{array}{l}\text { Hyphantrophaga } \\
\text { ciriloumanai sp. } \mathbf{n} .\end{array}$ \\
\hline- & Median pair of thoracic vittae continuous across suture & 11 \\
\hline 11 & $\begin{array}{l}\text { Tomentose bands on T3 and T4 extending only on basal half } \\
\text { (up to } 50 \% \text { ) of tergites; T5 gold tomentose with a black apex }\end{array}$ & $\begin{array}{l}\text { Hyphantrophaga } \\
\text { nigricauda sp. } \mathbf{n} \text {. }\end{array}$ \\
\hline- & $\begin{array}{l}\text { Tomentose bands on T3 and T4 extending over } 70 \% \text { of tergites; } \\
\text { T5 entirely covered in gold tomentum }\end{array}$ & 12 \\
\hline 12 & $\begin{array}{l}\text { Dorsal tomentum of thorax of brilliant yellow-gold colour; } \\
\text { chaetotaxy as follows: acrostichal setae } 4: 3 \text {, intra-alar setae } \\
3: 3 \text {, three katepisternal setae }\end{array}$ & $\begin{array}{l}\text { Hyphantrophaga } \\
\text { eliethcantillanoae sp. } \mathbf{n} \text {. }\end{array}$ \\
\hline- & $\begin{array}{l}\text { Dorsal tomentum of thorax of pale-brassy colour; chaetotaxy as } \\
\text { follows: acrostichal setae } 3: 3 \text {, intra-alar setae } 2: 3 \text {, two } \\
\text { katepisternal setae }\end{array}$ & $\begin{array}{l}\text { Hyphantrophaga } \\
\text { adrianguadamuzi sp. } \mathbf{n} \text {. }\end{array}$ \\
\hline 13 & 2 katepisternal setae & 14 \\
\hline- & 3 katepisternal setae & 15 \\
\hline 14 & 3 postsutural dorsocentral setae & $\begin{array}{l}\text { Hyphantrophaga calva } \\
\text { sp. } \mathbf{n} \text {. in part (females) }\end{array}$ \\
\hline- & 4 postsutural dorsocentral setae & $\begin{array}{l}\text { Hyphantrophaga } \\
\text { luciariosae sp. } \mathbf{n} .\end{array}$ \\
\hline 15 & $\begin{array}{l}\text { Pedicel brownish orange; entire fly grey tomentose; sex patch } \\
\text { of male indistinct/absent (Fig. 1d) }\end{array}$ & 16 \\
\hline- & $\begin{array}{l}\text { Pedicel brown to black; fly not entirely grey tomentose; sex } \\
\text { patch of male present (Fig. 1c) }\end{array}$ & 17 \\
\hline 16 & $\begin{array}{l}\text { Ocellar triangle gold; fronto-orbital plate sparsely setulose } \\
\text { throughout; abdominal tergites with silver tomentum throughout, } \\
\text { brassy toned at tergal edges }\end{array}$ & $\begin{array}{l}\text { Hyphantrophaga } \\
\text { calixtomoragai sp. } \mathbf{n} .\end{array}$ \\
\hline- & $\begin{array}{l}\text { Ocellar triangle silver, concolorous with fronto-orbital plate and } \\
\text { parafacial; fronto-orbital plate sparsely setulose only along } \\
\text { upper half; abdominal tergites with silver tomentum throughout, } \\
\text { lacking brassy tones }\end{array}$ & $\begin{array}{l}\text { Hyphantrophaga } \\
\text { manuelriosi sp. } \mathbf{n} .\end{array}$ \\
\hline 17 & $\begin{array}{l}\text { Found parasitising only medium-sized, naked, ringed nymphalid } \\
\text { larvae from open heavily insolated grassland and pasture } \\
\text { habitats }\end{array}$ & $\begin{array}{l}\text { Hyphantrophaga } \\
\text { danausophaga sp. } \mathbf{n} \text {. in } \\
\text { part }\end{array}$ \\
\hline
\end{tabular}




\begin{tabular}{|c|c|c|}
\hline- & $\begin{array}{l}\text { Found parasitising only large, hairy, non-ringed cryptic } \\
\text { nymphalid larvae in densely shaded habitat within the forest }\end{array}$ & $\begin{array}{l}\text { Hyphantrophaga } \\
\text { morphophaga sp. } \mathbf{n} \text {. in } \\
\text { part }\end{array}$ \\
\hline 18 & Lateral surface of thorax with pale blond setulae & $\begin{array}{l}\text { Hyphantrophaga } \\
\text { albopilosa sp. } \mathbf{n} \text {. }\end{array}$ \\
\hline- & Lateral surfaces of thorax with only black setulae throughout & 19 \\
\hline 19 & Median marginal setae present on ST1+2 & 20 \\
\hline- & Median marginal setae absent on ST1+2 & 27 \\
\hline 20 & $\begin{array}{l}\text { Ocellar triangle brassy-silver, appearing gold under some } \\
\text { angles of light; } 3 \text { postsutural dorsocentral setae; abdomen } \\
\text { ground colour entirely black, with silver or gold tomentum }\end{array}$ & 21 \\
\hline- & $\begin{array}{l}\text { Ocellar triangle brassy-gold tinged; } 3-4 \text { postsutural } \\
\text { dorsocentral setae; abdomen ranging from dark reddish-brown } \\
\text { to entirely reddish }\end{array}$ & 22 \\
\hline 21 & $\begin{array}{l}\text { Pedicel orange; facial ridge bare; fronto-orbital plate silver over } \\
80 \%\end{array}$ & $\begin{array}{l}\text { Hyphantrophaga } \\
\text { fasciata (Curran, 1934) }\end{array}$ \\
\hline- & $\begin{array}{l}\text { Pedicel black; facial ridge haired over } 1 / 2 \text { its length; fronto- } \\
\text { orbital plate gold over } 60 \%\end{array}$ & $\begin{array}{l}\text { Hyphantrophaga } \\
\text { hazelcambroneroae sp. } \\
\text { n. }\end{array}$ \\
\hline 22 & Thorax with three post-sutural dorsocentral setae & 23 \\
\hline- & Thorax with four post-sutural dorsocentral setae & 24 \\
\hline 23 & $\begin{array}{l}\text { Palpus yellow/orange throughout and thorax with } 2 \\
\text { katepisternal setae }\end{array}$ & $\begin{array}{l}\text { Hyphantrophaga calva } \\
\text { sp. } \mathbf{n} \text {. in part (males) }\end{array}$ \\
\hline- & $\begin{array}{l}\text { Palpus dark brown/black basally with orange at tip and thorax } \\
\text { with } 3 \text { katepisternal setae }\end{array}$ & $\begin{array}{l}\text { Hyphantrophaga } \\
\text { angustata (van der } \\
\text { Wulp) }\end{array}$ \\
\hline 24 & $\begin{array}{l}\text { Ground colour of abdomen light orange-brown throughout; } \\
\text { ground colour of thorax light orange-brown; ST1+2-T5 with } \\
\text { silver tomentum ventrally; coxae and femora orangy-brown, } \\
\text { tibiae yellow }\end{array}$ & $\begin{array}{l}\text { Hyphantrophaga } \\
\text { adamsoni Thompson, } \\
1963\end{array}$ \\
\hline- & $\begin{array}{l}\text { Ground colour of abdomen dark, reddish-brown with black } \\
\text { tones; ground colour of thorax black/dark grey; ground colour of } \\
\text { T4 and T5 ventrally dark brown, only T3 with silver tomentum } \\
\text { on ventral surface; legs dark reddish-brown to black }\end{array}$ & 25 \\
\hline
\end{tabular}




\begin{tabular}{|c|c|c|}
\hline 25 & $\begin{array}{l}\text { Pedicel orange, of distinctly lighter colour than postpedicel; } \\
\text { thorax with } 2 \text { katepisternal setae }\end{array}$ & $\begin{array}{l}\text { Hyphantrophaga } \\
\text { pabloumanai sp. } \mathbf{n} .\end{array}$ \\
\hline- & Pedicel black to dark brown; thorax with 3 katepisternal setae & 26 \\
\hline 26 & $\begin{array}{l}\text { Legs dark reddish-brown; tomentum covering more than } 50 \% \text { of } \\
\text { abdominal } \mathrm{T} 3 \text {, ventral surface of tergite with silver tomentum } \\
\text { reaching edge of tergite }\end{array}$ & $\begin{array}{l}\text { Hyphantrophaga } \\
\text { eldaarayae sp. } \mathbf{n} .\end{array}$ \\
\hline- & $\begin{array}{l}\text { Legs black; tomentum covering less than } 50 \% \text { of abdominal T3, } \\
\text { tomentum on ventral surface of tergite covering up to } 90 \% \text { of } \\
\text { tergite, but not reaching ventral edge }\end{array}$ & $\begin{array}{l}\text { Hyphantrophaga } \\
\text { diniamartinezae sp. } \mathbf{n} .\end{array}$ \\
\hline 27 & Thorax with 2 katepisternal setae & 28 \\
\hline- & Thorax with 3 katepisternal setae & 29 \\
\hline 28 & $\begin{array}{l}\text { Dorsum of thorax brassy tomentose with four distinct unfused } \\
\text { dorsal vittae, inner pair extending up to third postsutural } \\
\text { dorsocentral seta }\end{array}$ & $\begin{array}{l}\text { Hyphantrophaga similis } \\
\text { sp. } \mathbf{n} .\end{array}$ \\
\hline- & $\begin{array}{l}\text { Dorsum of thorax gold tomentose with four dorsal vittae fused } \\
\text { into two prominent dark lines extending across thorax }\end{array}$ & $\begin{array}{l}\text { Hyphantrophaga } \\
\text { gilberthampiei sp. } \mathbf{n} .\end{array}$ \\
\hline 29 & $\begin{array}{l}\text { Legs light coloured, yellow; abdominal tergites entirely brassy- } \\
\text { silver tomentose in the form of bands spanning the tergite; in } \\
\text { males, sex patch not extending dorsally on } \mathrm{T} 4\end{array}$ & $\begin{array}{l}\text { Hyphantrophaga } \\
\text { edwinapui sp. } \mathbf{n} .\end{array}$ \\
\hline- & $\begin{array}{l}\text { Legs dark coloured, if yellow in ground colour then so densely } \\
\text { covered in black setulae as to appear dark; abdominal tergites } \\
\text { not entirely brassy-silver tomentose, but tomentum broken into } \\
\text { bilateral silver patches; in males, sex patch extending dorsally } \\
\text { on T4 }\end{array}$ & 30 \\
\hline 30 & $\begin{array}{l}\text { Legs entirely black; T4 with silver tomentum covering } \\
\text { approximately } 20 \% \text { of dorsal surface; ground colour of } \\
\text { abdomen dark, reddish-brown to black; scutellum black over } \\
20 \% \text { of its surface, with gold tomentum over remainder }\end{array}$ & $\begin{array}{l}\text { Hyphantrophaga } \\
\text { anacordobae sp. } \mathbf{n} .\end{array}$ \\
\hline- & $\begin{array}{l}\text { Legs reddish-brown, covered with dense dark hairs and with } \\
\text { some light coloured spots at base of femora, adjacent to coxae; } \\
\text { T4 with silver tomentum covering more than 50\% of dorsal } \\
\text { surface; ground colour of abdomen light orange-brown; } \\
\text { scutellum gold tomentum covering entire surface margin }\end{array}$ & 31 \\
\hline 31 & $\begin{array}{l}\text { Found parasitising only medium-sized, naked, ringed nymphalid } \\
\text { larvae from open, heavily insolated grassland and pasture } \\
\text { habitats }\end{array}$ & $\begin{array}{l}\text { Hyphantrophaga } \\
\text { danausophaga sp. } \mathbf{n} . \text { in } \\
\text { part }\end{array}$ \\
\hline
\end{tabular}


Found parasitising only large, hairy, non-ringed, cryptic

- $\quad$ nymphalid larvae from densely shaded covered habitats within the forest

Hyphantrophaga

morphophaga sp. $\mathbf{n}$. in

part

\section{Analysis}

\section{Hyphantrophaga morphophaga species-group}

Two of our new species, Hyphantrophaga morphophaga and Hyphantrophaga danausophaga, require a complex explanation, but one that the results of the overall ACG inventory are suggesting will become commonplace as more in-situ exploration of tropical species takes place through the interfacing of genetics with natural history traits. Only two traits are currently known to separate them: their habitat and their sympatric host caterpillars. These traits are not available for typical museum specimens. While the ACG caterpillar and parasitoid inventory began in 1978, the first Morpho Fabricius, 1807 caterpillar (Nymphalidae) was not found until 1981 and the first $H$. morphophaga was reared in 1984. Since then, it has been reared only from $11 \%$ of 1135 wild-caught Morpho helenor (Cramer, 1776) caterpillars feeding on 33 species of woody Fabaceae in dry forest and rain forest interior and from $21 \%$ of 62 caterpillars feeding on two species of Dichapetalaceae in rain forest interior and from $7 \%$ of 123 Morpho polyphemus catalina Correa \& Chacon, 1984 wild-caught caterpillars feeding on five species of Fabaceae and one species of Capparaceae, all in dense shade of interior ACG cloud forest (Janzen and Hallwachs 2018). It has never been reared from the other two common rain forest species of Morpho found by the ACG inventory (408 Morpho amathonte (Deyrolle, 1860), feeding mostly on the same species of Fabaceae and 222 Morpho theseus justitiae (Deyrolle, 1860), feeding primarily on Menispermaceae in the shade of the same forests), though both are parasitised by two other species of Hyphantrophaga that are easily distinguished by morphology and DNA barcodes. The first Danaus plexippus (Linnaeus, 1758) caterpillar was found in 1998 and the first $H$. danausophaga was reared the same year. Since then, it has been reared only from $15 \%$ of 603 wild-caught $D$. plexippus caterpillars, $7 \%$ of 117 wild-caught Danaus gilippus (Cramer, 1775) caterpillars and 1\% of 302 wild-caught Lycorea halia atergatis (Doubleday, 1847) caterpillars. Lycorea $h$. atergatis is not only a Nymphalidae, but it is also similar to Danaus Kluk, 1802 in caterpillar morphology (ringed, naked, ostentatious), pupal morphology and food plants (those with milky latex growing on insolated forest edges) and is phylogenetically closely related (Brower et al. 2010).

These two species of Hyphantrophaga cannot be distinguished by external morphology, terminalia, colour pattern or DNA barcodes. In any NJ tree, they are highly intermingled within a single BIN, with no apparent base pair difference. However, in 1995, the first specimens of $H$. morphophaga were presented to one of us (DMW) with no background information, as just another tachinid fly. They were given the interim name Hyphantrophaga Wood05. When, in 1999, the first specimens of $H$. danausophaga were likewise presented to DMW, again with no background information, they were compared with $\mathrm{H}$. Wood05 and deemed to be different enough to be baptised, at the time, $H$. Wood06; however, today, we 
cannot find the morphological traits that led to this initial discrimination. As the years passed and the number of records accumulated, two hypotheses emerged:

A. The two belong to a single species that parasitises only medium-sized, naked, ringed and ostentatious Danaus and Lycorea Doubleday, 1847 caterpillars feeding on Asclepiadaceae and other latex-generating plants in open, intensely insolated, pastures and their edges in rain forest and dry forest and simultaneously parasitises only large, hairy, non-ringed cryptic caterpillars of Morpho helenor and M. p. catalina (never parasitising the co-occurring $M$. amathonte and $M$. t. justitiae) feeding on Fabaceae and Menispermaceae in the dense forest shade rather than in the open pastures, strongly populated with Asclepias curassavica (Asclepiadaceae), the primary ACG food plant of $D$. plexippus and D. gilippus.

B. These are two extremely similar species, one living in fully insolated habitats and parasitising just a few species of ostentatious, naked Danaus and Lycorea caterpillars (Fig. 27) that feed on Asclepiadaceae and other latex-baring plants and the other living in dense shade and parasitising just two species of large hairy cryptic Morpho caterpillars (Fig. 28) that feed almost entirely on Fabaceae in dense shade.
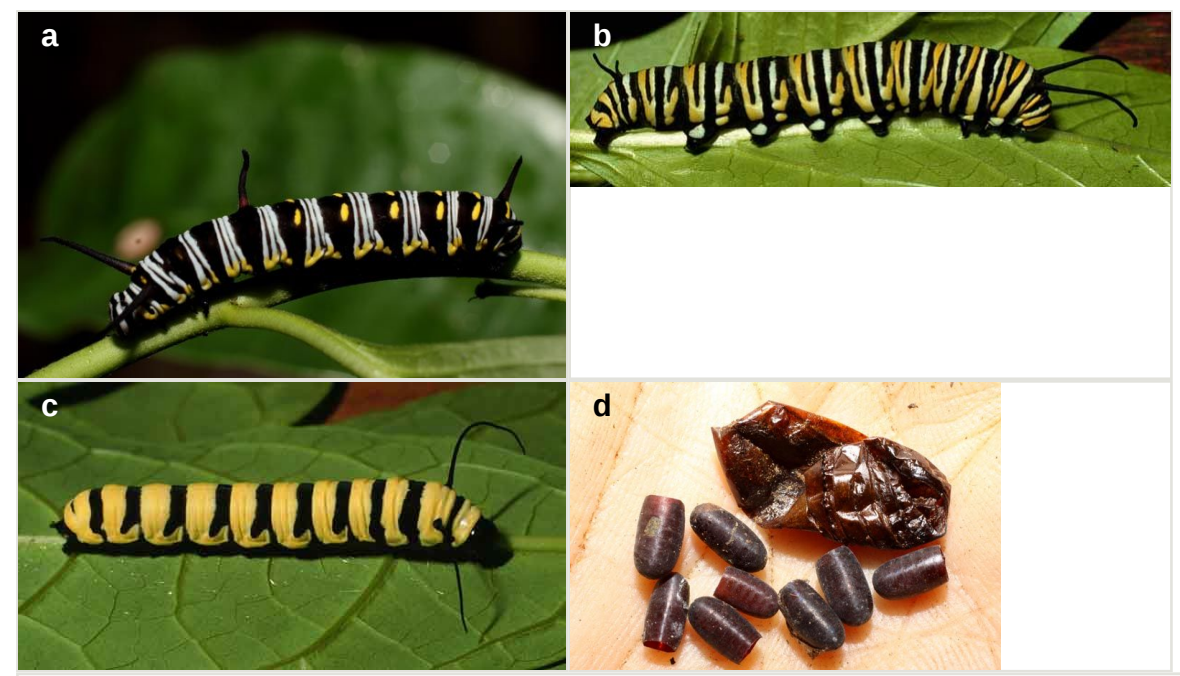

d

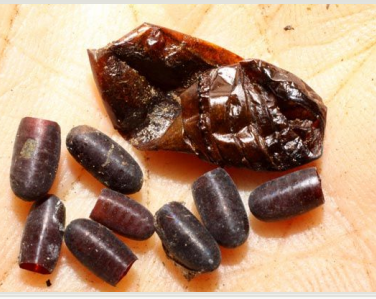

Figure 27.

The ostentatious, naked caterpillar hosts of Hyphantrophaga danausophaga sp. $\mathbf{n}$.

a: Danaus gilippusDHJ02 voucher n. 13-SRNP-10060, feeding on Asclepiadaceae. Image voucher n. DHJ498910.jpg doi

b: Danaus plexippus voucher n. 98-SRNP-7073, feeding on Asclepiadaceae. Image voucher n. DHJ46548.jpg doi

c: Lycorea $h$. atergatis voucher n. 99-SRNP-5616, feeding on Caricaceae. Image voucher n. DHJ51243.jpg doi

d: Puparia of Hyphantrophaga danausophaga voucher n. DHJPAR0034375, reared from Danaus plexippus voucher n. 09-SRNP-35341. Image voucher n. DHJ470083.jpg doi 

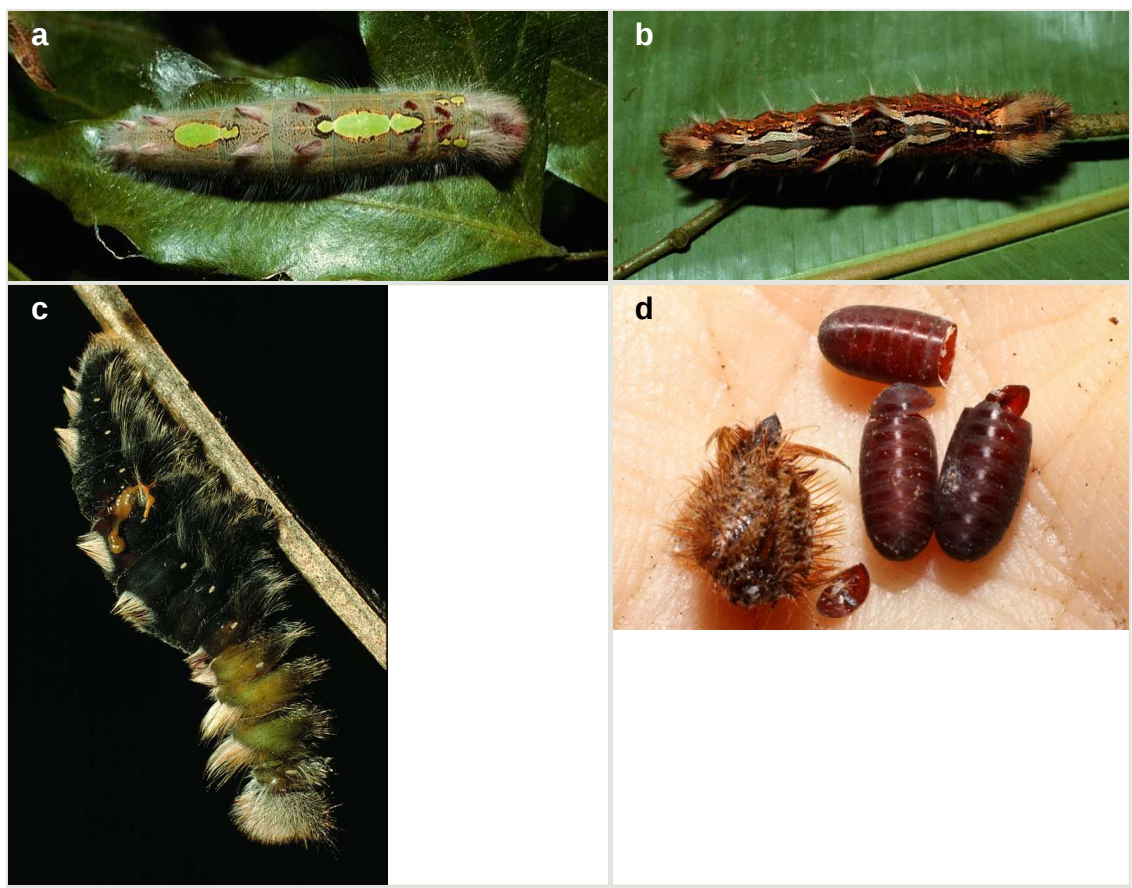

d

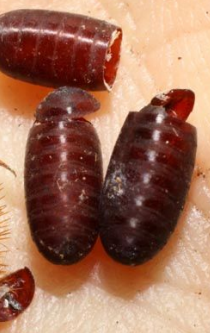

Figure 28.

The large, hairy cryptic Morpho caterpillar hosts of Hyphantrophaga morphophaga sp. $\mathbf{n}$.

a: Morpho p. catalina voucher n. 02-SRNP-8076, feeding on Fabaceae. Image voucher n. DHJ66311.jpg doi

b: Morpho helenor voucher n. 89-SRNP-735a, feeding on Fabaceae. Image voucher n. DHJ11620.jpg doi

c: Morpho p. catalina voucher n. 95-SRNP-4612, feeding on Fabaceae, parasitized by Hyphantrophaga morphophaga voucher n. DHJPAR0007354. Image voucher n. DHJ23257.jpg doi

d: Puparia of Hyphantrophaga morphophaga voucher n. DHJPAR0016681, reared from Morpho helenor voucher n. 06-SRNP-36610. Image voucher n. DHJ469258.jpg doi

We take hypothesis B to represent the more likely natural history and therefore describe two new species, inconvenient as that is for curators and other users of specimens of these flies, who do not have rearing records for them. When funds are available for deep exploration of their nuclear genomes, they will be amongst the first species to be explored. If they are found to be different at the nuclear genome level, that will not change how inconvenient cases like these are for identification systems that are based solely on morphology or DNA barcodes.

\section{DNA Barcoding}

A phylogenetic tree based on $\mathrm{CO} 1$ barcodes was used to visually confirm the variation present within and between each species and is presented in Fig. 29. For the Hyphantrophaga CO1 barcodes, we calculated the best DNA substitution model using the 
model test module in MEGA6 (Tamura et al. 2013) to find the model with the lowest BIC scores (Bayesian Information Criterion). We found that in this case, the TN92-G model (Tamura 1992) best described the data. We then built a Neighbour-Joining tree (Saitou and Nei 1987) based on TN92-G distances, using 25 nucleotide sequences from each species. All ambiguous positions were removed for each sequence pair. There were a total of 693 positions in the final dataset. For each species of Hyphantrophaga, we used a single CO1 DNA sequence for this tree. Twenty-two of the 25 sequences selected belonged to the holotypes designated herein, the remaining three being from ACG vouchers of previously known species.

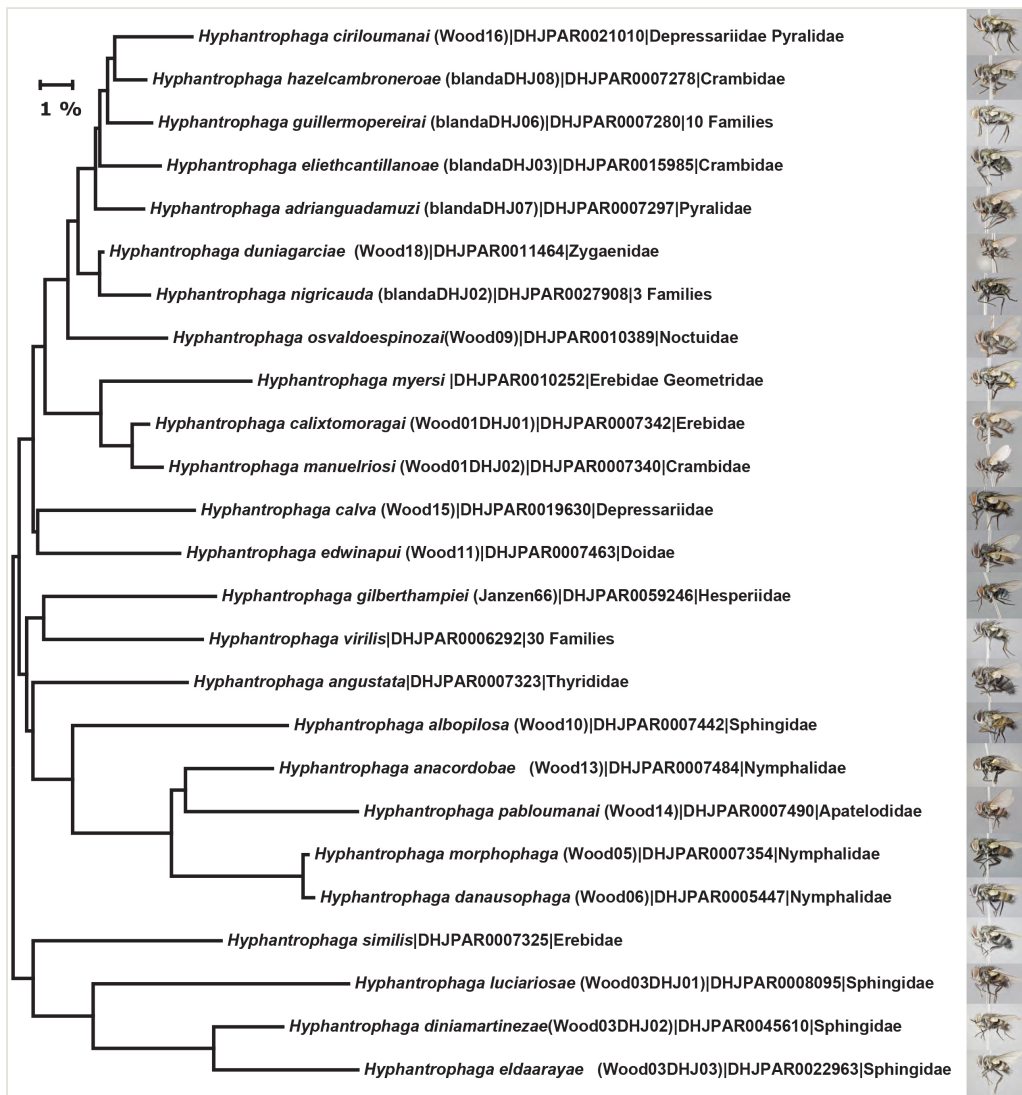

Figure 29. doi

CO1 NJ tree for the Hyphantrophaga spp. of ACG (Costa Rica). Tip labels are the species names [(older interim name)|sample accession|host family(ies)].

For a sub-set of the ACG Hyphantrophaga specimens, we also sequenced the ribosomal spacer region ITS2 (Fig. 30). The NJ tree was constructed based on p-dist (Nei and Kumar 2000). The average p-dist between species was 0.25 (all species pairs included). For one species pair $(H$. morphophaga and $H$. danausophaga), there were no observed differences between species. 


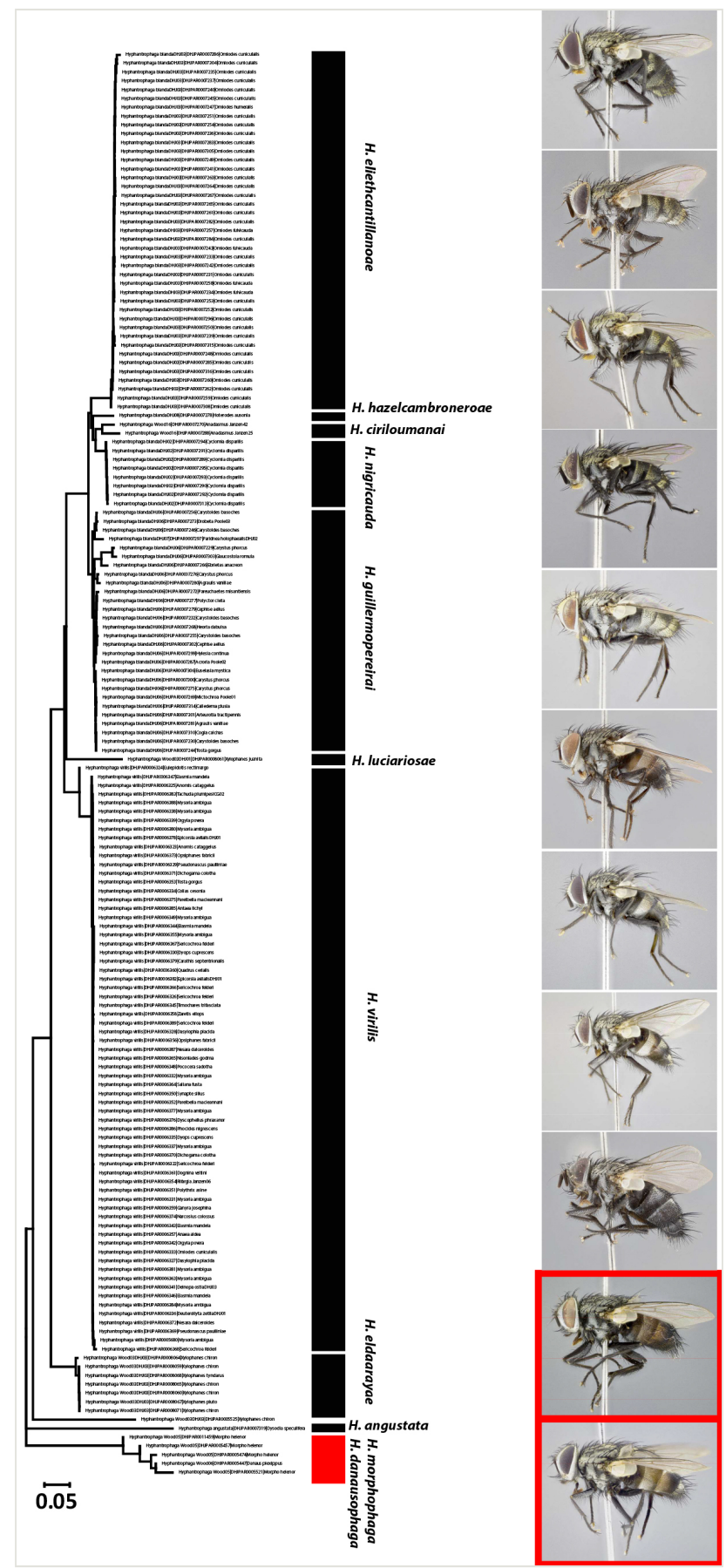

Figure 30 . doi

ITS2 NJ tree for Hyphantrophaga of ACG. Tip labels are interim species name|sample accession|host family. 


\section{Acknowledgements}

We gratefully acknowledge the unflagging support of the team of ACG parataxonomists (Janzen et al. 2009, Janzen and Hallwachs 2011) who found and reared the specimens used in this study and the team of biodiversity managers who protect and manage the ACG forests that are home to these tachinids and their caterpillar hosts. The study has been supported by U.S. National Science Foundation grants BSR 9024770 and DEB 9306296 , 9400829, 9705072, 0072730, 0515699 and grants from the Wege Foundation, International Conservation Fund of Canada, Jessie B. Cox Charitable Trust, Blue Moon Fund, Guanacaste Dry Forest Conservation Fund, Area de Conservación Guanacaste, Permian Global and University of Pennsylvania ( $\mathrm{DHJ} \& \mathrm{WH})$. This study has been supported by the Government of Canada through its ongoing support of the Canadian National Collection, Genome Canada, the Biodiversity Institute of Ontario and the Ontario Genomics Institute (2008-0GI-ICl-03) (MAS) and by a Discovery Grant from Natural Sciences and Engineering Research Council of Canada (MAS). Acknowledgements to Dr. James E. O'Hara for his support and many helpful discussions on nomenclature and history (MW \& AJF).

\section{References}

- $\quad$ Aldrich JM, Webber R (1924) The North American species of parasitic two-winged flies belonging to the genus Phorocera and allied genera. Proceedings of the United States National Museum 63 (2486): 1-90. https://doi.org/10.5479/si.00963801.63-2486.1

- $\quad$ Arnaud P (1963) The type locality of Eusisyropa virilis (Aldrich and Webber) (Diptera: Tachinidae). Proceedings of the Entomological Society of Washington 65 (2): 116-116.

- $\quad$ Brower AZ, Wahlberg N, Ogawa J, Boppré M, Vane-Wright RI (2010) Phylogenetic relationships among genera of danaine butterflies (Lepidoptera: Nymphalidae) as implied by morphology and DNA sequences. Systematics and Biodiversity 8 (1): 75-89. https:// doi.org/10.1080/14772001003626814

- $\quad$ Cumming JM, Wood DM (2009) Adult morphology and terminology. 9-50 pp. In: Brown B, Borkent A, Cumming J, Wood D, Woodley N, Zumbado M (Eds) Manual of Central American Diptera. 1. NRC Research Press, Ottawa, Canada, 714 pp.

- DeVries PJ (1984) Butterflies and Tachinidae: does the parasite always kill its host? Journal of Natural History 18 (2): 323-326. https://doi.org/10.1080/00222938400770251

- $\quad$ Edgar RC (2004) MUSCLE: multiple sequence alignment with high accuracy and high throughput. Nucleic Acids Research 32 (5): 1792-1797. https://doi.org/10.1093/nar/gkh340

- Fernandez-Triana J, Whitfield J, Rodriguez J, Smith MA, Janzen D, Hallwachs W, Hajibabaei M, Burns J, Solis A, Brown J, Cardinal S, Goulet H, Hebert P (2014) Review of Apanteles sensu stricto (Hymenoptera, Braconidae, Microgastrinae) from Area de Conservación Guanacaste, northwestern Costa Rica, with keys to all described species from Mesoamerica. ZooKeys 383: 1-565. https://doi.org/10.3897/zookeys.383.6418

- $\quad$ Fleming AJ, Wood DM, Smith MA, Hallwachs W, Janzen DH (2014a) Revision of the New World species of Houghia Coquillett (Diptera, Tachinidae) reared from caterpillars in Area 
de Conservación Guanacaste, Costa Rica. Zootaxa 3858 (1): 1-90. https:// doi.org/10.11646/zootaxa.3858.1.1

- $\quad$ Fleming AJ, Wood D, Smith M, Janzen D, Hallwachs W (2014b) A new species of Cordyligaster Macquart, reared from caterpillars in Area de Conservacion Guanacaste, northwestern Costa Rica. Biodiversity Data Journal 2: e4174. https://doi.org/10.3897/ bdj.2.e4174

- $\quad$ Fleming AJ, Wood DM, Janzen D, Hallwachs W, Smith MA (2015a) Seven new species of Spathidexia Townsend (Diptera: Tachinidae) reared from caterpillars in Area de Conservación Guanacaste, Costa Rica. Biodiversity Data Journal 3: e4597. https:// doi.org/10.3897/bdj.3.e4597

- $\quad$ Fleming AJ, Wood DM, Janzen D, Hallwachs W, Smith MA (2015b) Three new species of Trigonospila Pokorny (Diptera: Tachinidae), from Area de Conservación Guanacaste, northwestern Costa Rica, with a key for their identification. Biodiversity Data Journal 3: e4595. https://doi.org/10.3897/bdj.3.e4595

- $\quad$ Fleming AJ, Wood DM, Smith MA, Janzen D, Hallwachs W (2015c) Nine new species of Itaplectops (Diptera: Tachinidae) reared from caterpillars in Area de Conservación Guanacaste, northwestern Costa Rica, with a key to Itaplectops species. Biodiversity Data Journal 3: e4596. https://doi.org/10.3897/bdj.3.e4596

- $\quad$ Fleming AJ, Wood D, Smith MA, Hallwachs W, Janzen D (2015d) Three new species of Ametadoria Townsend (Diptera: Tachinidae) from Area de Conservación Guanacaste, Costa Rica. Biodiversity Data Journal 3: e5039. https://doi.org/10.3897/bdj.3.e5039

- $\quad$ Fleming AJ, Wood DM, Smith MA, Hallwachs W, Janzen D, Dapkey T (2016a) Two new species of Erythromelana Townsend, 1919 (Diptera: Tachinidae) from Area de Conservación Guanacaste in northwestern Costa Rica. Biodiversity Data Journal 4: e7386. https://doi.org/10.3897/bdj.4.e7386

- $\quad$ Fleming AJ, Wood DM, Smith MA, Janzen D, Hallwachs W, Dapkey T (2016b) A new species of Phosocephala Townsend, 1908 (Diptera: Tachinidae) from Area de Conservación Guanacaste in northwestern Costa Rica. Biodiversity Data Journal 4: e7863. https://doi.org/10.3897/bdj.4.e7863

- $\quad$ Fleming AJ, Wood DM, Smith MA, Hallwachs W, Janzen D, Dapkey T (2017) Nine new species of Uramya Robineau-Desvoidy (Diptera: Tachinidae) from Area de Conservación Guanacaste in northwestern Costa Rica, with a key to their identification. Biodiversity Data Journal 5: e9649. https://doi.org/10.3897/bdj.5.e9649

- Guimaraes J (1971) Family Tachinidae (Larvaevoridae). A catalogue of the Diptera of the Americas south of the United States 104: 1-333.

- Hall TA (1999) BioEdit: a user-friendly biological sequence alignment editor and analysis program for Windows 95/98/NT. Nucleic Acids Symposium Series 41: 95-98.

- Ivanova NV, Dewaard JR, Hebert PDN (2006) An inexpensive, automation-friendly protocol for recovering high-quality DNA. Molecular Ecology Notes 6 (4): 998-1002. https:// doi.org/10.1111/j.1471-8286.2006.01428.x

- Janzen DH, Hallwachs W, Blandin P, Burns JM, Cadiou J-, Chacon I, Dapkey T, Deans AR, Epstein ME, Espinoza B, Franclemont JG, Haber WA, Hajibabaei M, Hall JP, Hebert PD, Gauld ID, Harvey DJ, Hausmann A, Kitching IJ, LaFontaine D, Landry J-, Lemaire C, Miller JY, Miller JS, Miller L, Miller SE, Montero J, Munroe E, Green SR, Ratnasingham S, Rawlins JE, Robbins RK, Rodriguez JJ, Rougerie R, Sharkey MJ, Smith MA, Solis MA, Sullivan JB, Thiacourt P, Wahl DB, W SJ, Whitfield JB, Willmott KR, Wood DM, Woodley $\mathrm{NE}$, Wilson JJ (2009) Integration of DNA barcoding into an ongoing inventory of complex 
tropical biodiversity. Molecular Ecology Resources 9: 1-26. https://doi.org/10.1111/ j.1755-0998.2009.02628.x

- Janzen DH, Hallwachs W (2011) Joining inventory by parataxonomists with DNA barcoding of a large complex tropical conserved wildland in northwestern Costa Rica. PLoS ONE 6 (8): e18123. https://doi.org/10.1371/journal.pone.0018123

- J Janzen DH, Hallwachs W, Dincă V (2016) DNA barcoding the Lepidoptera inventory of a large complex tropical conserved wildland, Area de Conservacion Guanacaste, northwestern Costa Rica 1. Genome 59 (9): 641-660. https://doi.org/10.1139/ gen-2016-0005

- Janzen DH, Hallwachs W (2018) Dynamic database for an inventory of the macrocaterpillar fauna, and its food plants and parasitoids, of Area de Conservacion Guanacaste (ACG), northwestern Costa Rica (nn-SRNP-nnnnn voucher codes). http:// janzen.sas.upenn.edu. Accessed on: 2018-3-21.

- $\quad$ Ji Y, Zhang D, He L (2003) Evolutionary conservation and versatility of a new set of primers for amplifying the ribosomal internal transcribed spacer regions in insects and other invertebrates. Molecular Ecology Notes 3 (4): 581-585. https://doi.org/10.1046/ j.1471-8286.2003.00519.x

- $\quad$ Nei M, Kumar S (2000) Molecular Evolution and Phylogenetics. Oxford University Press, New York, NY, 333 pp. [ISBN 0-19-513584-9]

- O'Hara JE, Wood DM (1998) Tachinidae (Diptera): Nomenclatural review and changes, primarily for America north of Mexico. The Canadian Entomologist 130: 751-774. https:// doi.org/10.4039/Ent130751-6

- $\quad$ Ratnasingham S, Hebert PDN (2007) BARCODING: bold: The Barcode of Life Data System (http://www.barcodinglife.org). Molecular Ecology Notes 7 (3): 355-364. https:// doi.org/10.1111/j.1471-8286.2007.01678.x

- $\quad$ Rodriguez J, Fernández-Triana J, Smith MA, Janzen D, Hallwachs W, Erwin T, Whitfield J (2012) Extrapolations from field studies and known faunas converge on dramatically increased estimates of global microgastrine parasitoid wasp species richness (Hymenoptera: Braconidae). Insect Conservation and Diversity 6 (4): 530-536. https:// doi.org/10.1111/icad.12003

- $\quad$ Sabrosky CW, Arnaud PH (1965) Family Tachinidae (Larvaevoridae). 961-1108 pp. In: Stone A, Sabrosky CW, Wirth WW, Foote RH, Coulson JR (Eds) A catalog of the Diptera of America north of Mexico Agriculture Handbook. United States Department of Agriculture, $1696 \mathrm{pp}$.

- $\quad$ Sabrosky CW (1983) The Type Specimen Of Eusisyropa boarmiae (Coquillett) And A New Specific Name For The Species (Diptera, Tachinidae). Proceedings of The Entomological Society of Washington 85: 251-255.

- $\quad$ Saitou N, Nei M (1987) The neighbor-joining method: a new method for reconstructing phylogenetic trees. Molecular Biology and Evolution 4 (4): 406-425. https://doi.org/10.1093/ oxfordjournals.molbev.a040454

- $\quad$ Sellers W (1943) The Nearctic species of parasitic flies belonging to Zenillia and allied genera. Proceedings of the United States National Museum 93 (3157): 1-108. https:// doi.org/10.5479/si.00963801.3157

- $\quad$ Smith D, Janzen D, Hallwachs W, Smith MA (2012) Hyperparasitoid wasps (Hymenoptera, Trigonalidae) reared from dry forest and rain forest caterpillars of Area de Conservación Guanacaste, Costa Rica. Journal of Hymenoptera Research 29: 119-144. https:// doi.org/10.3897/jhr.29.3233 
- $\quad$ Smith MA, Woodley NE, Janzen DH, Hallwachs W, Hebert PDN (2006) DNA barcodes reveal cryptic host-specificity within the presumed polyphagous members of a genus of parasitoid flies (Diptera: Tachinidae). Proceedings of the National Academy of Sciences 103 (10): 3657-3662. https://doi.org/10.1073/pnas.0511318103

- $\quad$ Smith MA, Wood DM, Janzen DH, Hallwachs W, Hebert PDN (2007) DNA barcodes affirm that 16 species of apparently generalist tropical parasitoid flies (Diptera, Tachinidae) are not all generalists. Proceedings of the National Academy of Sciences 104 (12): 4967-4972. https://doi.org/10.1073/pnas.0700050104

- $\quad$ Smith MA, Rodriguez JJ, Whitfield JB, Deans AR, Janzen DH, Hallwachs W, Hebert PDN (2008) Extreme diversity of tropical parasitoid wasps exposed by iterative integration of natural history, DNA barcoding, morphology, and collections. Proceedings of the National Academy of Sciences 105 (34): 12359-12364. https://doi.org/10.1073/pnas.0805319105

- Smith MA, Fernandez-Triana J, Roughley R, Hebert PN (2009) DNA barcode accumulation curves for understudied taxa and areas. Molecular Ecology Resources 9: 208-216. https:// doi.org/10.1111/j.1755-0998.2009.02646.x

- Tamura K (1992) Estimation of the number of nucleotide substitutions when there are strong transition-transversion and $\mathrm{G}+\mathrm{C}$-content biases. Molecular Biology and Evolution 9: 678-687. https://doi.org/10.1093/oxfordjournals.molbev.a040752

- $\quad$ Tamura K, Stecher G, Peterson D, Filipski A, Kumar S (2013) MEGA6: Molecular Evolutionary Genetics Analysis Version 6.0. Molecular Biology and Evolution 30 (12): 2725-2729. https://doi.org/10.1093/molbev/mst197

- Townsend CHT (1891) A parasite of the fall web-worm. Psyche: A Journal of Entomology 6 (187): 176-177. https://doi.org/10.1155/1891/49230

- $\quad$ Townsend CHT (1892) A new genus of Tachinidae. Psyche: A Journal of Entomology 6 (192): 247-247. https://doi.org/10.1155/1892/78953

- Townsend CHT (1912) Descriptions of new genera and species of muscoid flies from the Andean and Pacific coast regions of South America. Proceedings of the United States National Museum 43: 301-367.

- $\quad$ Townsend CHT (1941) Manual of Myiology, in twelve parts. Part XI. Charles Townsend \& Filhos, Itaquaquecetuba, São Paolo, Brasil, 342 pp.

- Wood DM (1987) Chapter 110. Tachinidae pp. 1193-1269. In: McAlpine JF, Peterson BV, Shewell GE, Teskey HJ, Vockeroth JR, Wood DM (Eds) Manual of Nearctic Diptera. 2. Agriculture Canada, Ottawa, Canada.

- Wood DM, Zumbado M (2010) Tachnidae (tachinid flies, parasitic flies) pp. 1343-1419. In: Brown B, Borkent A, Cumming J, Wood D, Woodley N, Zumbado M (Eds) Manual of Central American Diptera. 2. NRC Research Press, Ottawa, Canada, 715-1442 pp. 